\title{
Selektive Halogenierungen unter Phasentransferbedingungen: Mechanistische Untersuchungen und Synthetische Anwendungen
}

\author{
Dissertation \\ Zur Erlangung des Doktorgrades \\ der Mathematisch-Naturwissenschaftlichen Fakultäten \\ der Georg-August-Universität zu Göttingen
}

vorgelegt von

Oliver Lauenstein

aus Kassel

Göttingen 2001 
D 7

Referent: Prof. Dr. P. R. Schreiner

Korreferent: Prof. Dr. H. Lackner

Tag der mündlichen Prüfung: 
Die vorliegende Arbeit wurde im Institut für Organische Chemie der Universität Göttingen von November 1998 bis Juni 2001 unter der Leitung von Herrn Prof. Dr. P. R. Schreiner Ph.D. durchgeführt.

Meinem Lehrer Herrn Prof. Dr. P. R. Schreiner möchte ich herzlich für die interessante Themenstellung, die vielen anregenden Diskussionen und die erwiesene Unterstützung sowie die gewährte forscherische Freiheit im Verlauf der Arbeit danken. 
Meiner Familie 
1. EINLEITUNG UND AUFGABENSTELLUNG

1.1. ZUSAMMENFASSUNG BISHERIGER ERGEBNISSE DER PT-HALOGENIERUNGEN

1.2. REAKTIONEN UNTER PHASENTRANSFERBEDINGUNGEN

1.3. Die Regioselektivität von Radikalischen Substitutionen in AlKanen 14

1.4. COMPUTERCHEMIE: GRUNDLAGEN DER VERWENDETEN METHODEN UND BASISSÄTZE 18

\section{MECHANISTISCHE UNTERSUCHUNGEN}

2.1. SYSTEMATISCHE UNTERSUCHUNG DER ALLGEMEINEN REAKTIONSBEDINGUNGEN 22

2.1.1. Einleitung 22

2.1.2. Die Bromierung 23

2.1.2.1. Variation des Lösungsmittels 23

2.1.2.2. Variation des Katalysators 25

2.1.2.3. Variation des Basenkonzentration 26

2.1.2.4. Variation der Katalysatorkonzentration 27

2.1.2.5. Weitere allgemeine Beobachtungen bei unsystematischer Variation diverser Reaktionsbedingungen 28

2.1.3. Die Iodierung 31

2.1.3.1. Variation des Lösungsmittels 31

2.1.3.2. Variation des Katalysators 32

2.1.3.3. Variation der Basenkonzentration 33

2.1.3.4. Variation der Katalysatorkonzentration 34

2.1.3.5. Variation der Base 35

2.1.3.6. Variation des Volumens der wäßrigen Phase bei konstanter absoluter Basenmenge 36

2.1.4. Die Chlorierung 37

2.2. SPEZIELLE MECHANISTISCHE UNTERSUCHUNGEN 38

\subsubsection{Einleitung} 38

2.2.2. Vergleich der Regioselektivität der Halogenierung von Adamantan unter PT-Bedingungen und klassisch thermisch initiiert durch AIBN 38

2.2.3. Nachweis von molekularem Brom im Rahmen der PT-Bromierung 40

2.2.4. Abfangen des Trihalomethylradikals im Rahmen der PT-Bromierung 41

2.2.5. Die kinetischen H/D-Isotopeneffekte im Rahmen der PT-Halogenierungen 42

2.2.5.1. Einleitung 42

2.2.5.2. Experimentelle Voraussetzungen zur Bestimmung des KIE 46

2.2.5.3. Experimentelle Bestimmung des KIE 49

2.2.5.4. Die Berechnung des kinetischen Isotopeneffektes 52

2.3. SCHLUßFOLGERUNGEN AUS DEN MECHANISTISCHEN UNTERSUCHUNGEN ___ 38

\section{VERSCHIEDENE ANWENDUNGEN DER PT-HALOGENIERUNGEN__ 59}

3.1. Die PT-Halogenierung VON CUBAN UNTER ERHALtung DER KÄFIGSTRUKTUR ___ 59

3.1.1. Einleitung _ 59

3.1.2. Die PT-Halogenierung $(\mathrm{Cl}, \mathrm{Br}, \mathrm{I})$ von Cuban unter PT-Bedingungen ___ 61

3.1.3. Computerberechnungen zur Erklärung der Produktverteilung bei der Halogenierung von Cuban $(\mathrm{Cl}, \mathrm{Br})$ 
3.1.4. Die PT-Halogenierung von Monohalocubanen

3.1.5. Computerberechnungen zur Erklärung der Produktverteilung bei den PT-Halogenierungen $(\mathrm{Cl}, \mathrm{Br}, \mathrm{I})$ von Monohalocubanen

3.1.6. Ergebnis 71

3.2. Die Synthese von Polyhaloadamantanen 72

3.2.1. Einleitung 72

3.2.2. Die PT-Halogenierung von Adamantan 73

3.2.3. Die PT-Halogenierung von 1-Fluoradamantanen 76

3.2.4. Die PT-Halogenierung von 1-Phenyladamantanen 78

3.2.5. Die PT-Halogenierungen von 1-Methoxyadamantanen 81

3.2.6. Weitere Versuche der Synthese von 1-Iod-3-brom-5-chlor-7-fluoradamantan 83

3.2.7. Racemattrennungen von chiralen Polyhaloadamantanverbindungen 84

3.2.7.1. Versuche mit Lanthanide-Shift Reagenzien (LSR) zur Analyse der racemischen Gemische 84

3.2.7.1.1. Einleitung 84

3.2.7.1.2. Durchführung der Shiftexperimente 86

3.2.7.1.3. Ergebnis der Analysen mit LSR 89

3.2.7.2. Analytische Enantiomerentrennung durch chirale Gaschromatographie 90

3.2.7.3. Analytische und präparative Enantiomerentrennung durch chirale HPLC 92

3.3. Die PT-Halogenierung VON ACETALEN___ 94

3.4. DIE PT-IODIERUNG VON 1-ISOPROPYLADAMANTAN___

4. ERGEBNISSE UND AUSBLICK _ 98

5. EXPERIMENTELLER TEIL 103

ANHANG A. DIE ENTHALPIEN UND ENERGIEN ALLER COMPUTEROPTIMIERTEN STRUKTUREN 145

ANHANG B. MOLEKÜLVERZEICHNIS 149

ANHANG C. ABBILDUNGEN DER ${ }^{1}$ H-NMR-SPEKTREN 151

ANHANG D. ABBILDUNGEN DER ${ }^{13}$ C-NMR-SPEKTREN 167

LITERATURVERZEICHNIS 183

ABKÜRZUNGEN UND AKRONYME 192

PUBLIKATIONSLISTE 194

DANKSAGUNGEN 195

LEBENSLAUF 196 


\section{Einleitung und Aufgabenstellung}

Eine der herausragenden Aufgabenstellungen an die Chemie ist die selektive Aktivierung von C-H-Bindungen[1-5] in gesättigten Kohlenwasserstoffen und deren damit einhergehende Funktionalisierung, um sie für die organische Synthese nutzbar zu machen. Halogenierungen mit freien Radikalen sind lange bekannt, ${ }^{[6,7]}$ allerdings werden hier nur geringe Selektivitäten erzielt und Fragmentierungen sowie Überfunktionalisierungen treten auf. Aufgrund des Fehlens von Mehrfachbindungen und der Stärke der kovalenten C-H- $\sigma-$ Bindung galten die "Parafine" zunächst bezüglich alternativer Reaktionsbedingungen als unreaktiv, $[1,4,5]$ bis durch erste Arbeiten von Shilov durch die stöchiometrische und auch katalytische Verwendung von Platinsalzen erste Erfolge in dieser Hinsicht zu verzeichnen waren und H/D-Austausch sowie Chlorierungen durchgeführt werden konnten.[8] Dies war der Initiator für die Entwicklung diverser Strategien der C-H-Aktivierung und es haben sich verschiedene erfolgreiche Ansätze auf diesem Gebiet etabliert. So sind verschiedene homogene Katalysatoren, die auf Übergangsmetallkomplexen basieren, besonders effizient, ${ }^{[9-}$ 12] wobei die von Bergman entwickelten Metallhydridkomplexe[13, 14] besonders hervorgehoben werden müssen. Andere Methoden beruhen auf der Verwendung von Enzymen[12, 15] oder den von ihnen abgeleiteteten Metallporphyrinen, $[1,4,16]$ die sich teilweise durch eine sehr hohe Selektivität auszeichnen. Eine metallfreie Alternative der C-HAktivierung ist die von Olah entwickelte und intensivst untersuchte elektrophile Aktivierung in superaciden Media.[1-3, 17] Nachteilig an allen genannten Methoden ist, daß sie entweder nur mit den heutzutage im großtechnischen Maßstab ökologisch nicht vertretbaren Schwermetallen arbeiten oder mit aufwendigen Reaktionsdurchführungen verbunden sind.

Das Ziel der vorliegenden Arbeit war, verschiedene Aspekte und Anwendungen eines neuen Konzeptes ${ }^{[18]}$ genauer zu untersuchen, das die eben genannten Nachteile bisheriger $\mathrm{C}-\mathrm{H}$-Aktivierungen vermeiden soll. Es beinhaltet die Kombination von zwei lange bekannten Methoden der organischen Chemie, nämlich die der Radikalreaktionen[6, 7, 19, 20] und die der unter Phasentransfer (PT) ausgeführten Reaktionen. [21-27] Dabei wird durch einen PT ein SET-Reduktionsmittel (SET $=$ single-electron transfer $=$ Einelektronentransfer $)$ in die organische Phase überführt, wo dieses durch einen Redoxprozeß ein Radikal erzeugt, welches seinerseits einen radikalischen Propagationszyklus initiiert. Bei der ersten Applikation dieses Konzeptes fungiert das Hydroxidion als Reduktionsmittel, das transferiert in die organische Phase, in fast unsolvatisierter Form vorliegt und dort eine solche reduktive Eigenschaft 
entfaltet, ${ }^{[28]}$ während ein Tetrahalomethan $(\mathrm{Cl}, \mathrm{Br}, \mathrm{I})$ als Radikalvorstufe verwendet wird und auf diese Weise Halogenierungen durchgeführt werden können (Abb. 1).[29, 30]

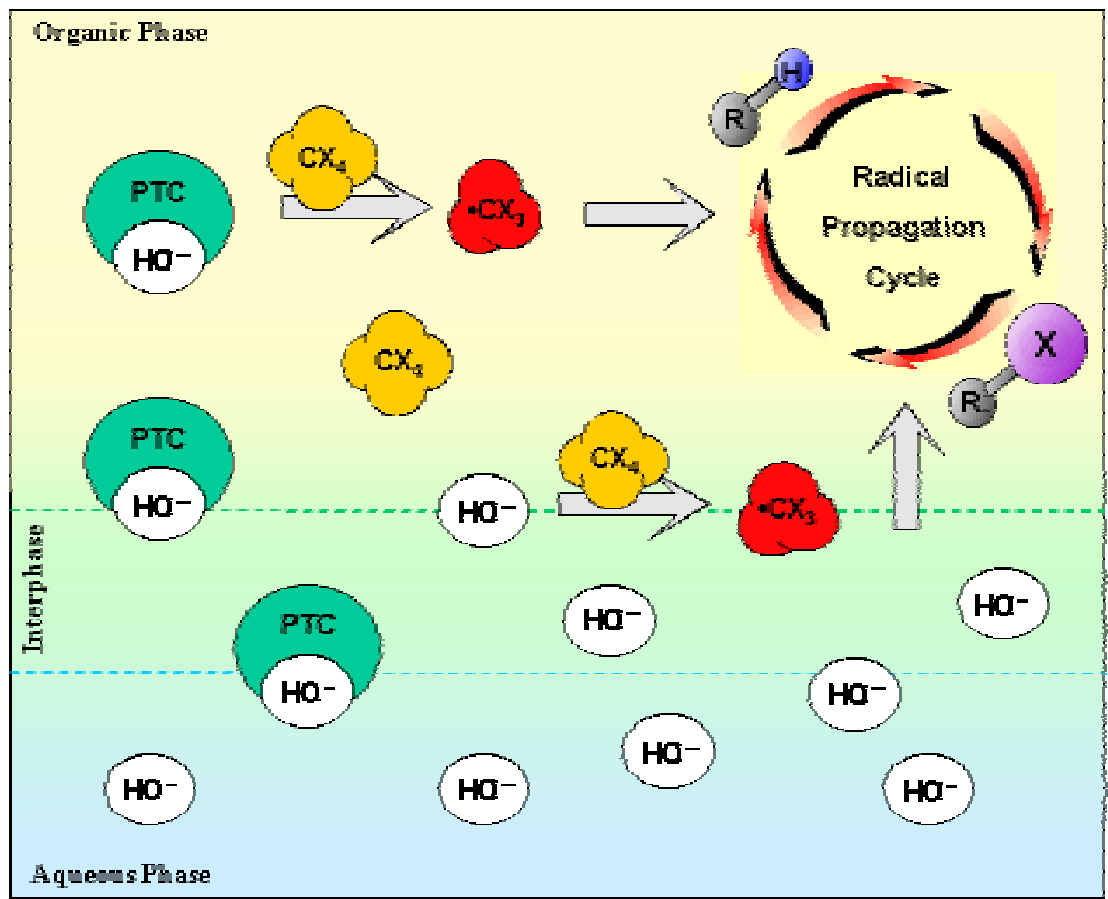

Abbildung 1. Allgemeines Schema des neuen Konzeptes zur Halogenierungen von nicht aktivierten Kohlenwasserstoffen $(\mathrm{X}=\mathrm{Cl}, \mathrm{Br}$; I).

In der folgenden Einleitung sollen die im Rahmen meiner Diplomarbeit ${ }^{[31]}$ erhaltenen bisherigen Resultate und eine Arbeitshypothese kurz dargestellt und auf die für die neuen Reaktionen grundlegenden Teilaspekte, wie die wichtigsten Mechanismen der PTK und die Selektivität verschiedener Radikale eingegangen, sowie eine kurze Einführung in die bei den Computerberechnungen verwendeten Methoden gegeben werden. Die Schwerpunkte in dieser Arbeit sollten bezüglich mechanistischer Untersuchungen und verschiedener Anwendungen zur Verdeutlichung der Vorteile der neuen Methodik gesetzt werden.

Dafür sollten zunächst die verschiedenen Reaktionsparameter im großen Ausmaß für die Bromierungen und Iodierungen variiert werden, um so einen besseren mechanistischen Einblick in das Reaktionsgeschehen zu gewinnen und um die neuen Reaktionen möglichst optimal nutzen zu können. Diese mechanistischen Untersuchungen sollten noch durch einige spezielle Experimente wie u.a. die Messung des KIE (Kinetischer Isotopeneffekt) und Radikalabfangreaktionen unterstützt werden, um weitere Bestätigungen für eine allgemeine Arbeitshypothese (Kap 1.1) zu erhalten. Da durch Vorabexperimente festgestellt worden ist, 
daß auch hochgespannte Kohlenwasserstoffe ohne Verlust der Grundstruktur halogeniert werden können, sollte dies systematisch im Rahmen der Mono- und Polyhalogenierung von Cuban, einem der gespanntesten Kohlenwasserstoffe (Ringspannung $=166 \mathrm{kcal} \mathrm{mol}^{-1}$ ), $\left.{ }^{[32}\right]$ untersucht werden. Die eben angesprochene Polyhalogenierung wird durch den Vorteil der PT-Halogenierungen ermöglicht, daß bei einer Zweithalogenierung ein bereits im Substratmolekül vorhandener Halogensubstituent, der von dem neu eingeführten verschieden ist, nicht ausgetauscht wird. Dieser Vorteil sollte anhand der Polyhalogenierung von verschiedenen in der $3^{\circ}$-Position monosubstituierten Adamantanderivaten $(\mathrm{R}=-\mathrm{F},-\mathrm{Cl}$, $\mathrm{OMe},-\mathrm{Ph})$ systematisch ausgenutzt werden, um einerseits die Kompatibilität zu weiteren funktionellen Gruppen zu dokumentieren und andererseits chirale Adamantanverbindungen mit einem stereogenen Zentrum zu synthetisieren, das nicht in einem Atom lokalisiert ist. Letztere Verbindungen gehören zu einer Verbindungsklasse die bislang nur wenig untersucht wurde und zu der durch die neue Halogenierungsmethode ein besonders einfacher Zugang geschaffen wurde. Um deren chiroptischen Eigenschaften erforschen und etwaige Enantiomerenüberschüsse im Rahmen späterer enantio- oder diastereoselektiver PTHalogenierungen (in weiterführenden Arbeiten) bestimmen zu können, sollte nach Möglichkeiten zur analytischen und präparativen Enantiomerentrennung gesucht werden. Schließlich sollten die experimentellen Ergebnisse teilweise durch Computerberechnungen untermauert werden. Dies erschien gerade bei der Halogenierung von Cuban und der Messung des KIE informativ und aufschlußreich.

\subsection{Zusammenfassung bisheriger Ergebnisse der PT-Halogenierungen}

Bei dem Versuch der Funktionalisierung von Adamantan 1 über Dibromcarbeninsertion unter phasentransferkatalytischen Bedingungen $\left[\mathrm{HCBr}_{3} / \mathrm{NaOH}\right.$ (aq. $50 \%$ ) / $\mathrm{CH}_{2} \mathrm{Cl}_{2} /$ Triethylbenzylammoniumchlorid, 16h, Rückfluß] in die Brückenkopf C-HBindung konnten neben dem gewünschten 1-Dibrommethyladamantan 5 (33\%) auch 1-Bromadamantan 2 (41\%), 1,3-Dibromadamantan 3 (16\%) und Spuren von 2-Bromadamantan 4 isoliert werden (Schema 1). 


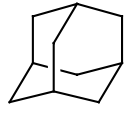

1
Rfx. / 16 h

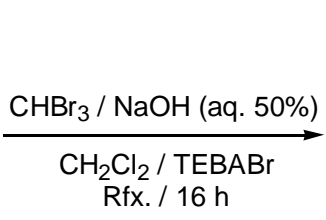

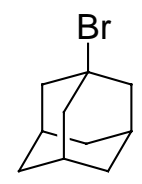

2

$41 \%$

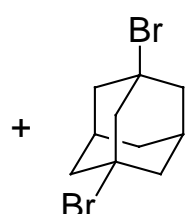

3

$16 \%$

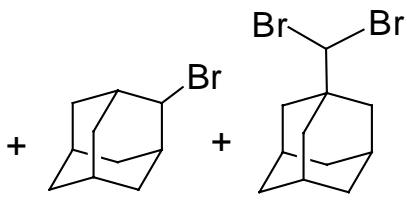

5

$33 \%$

Schema 1. Bromierung von Adamantan mit Bromoform unter PTK-Bedingungen

Zum einen war dabei erstaunlich, daß die Bildung der Substitutionsprodukte nicht aufgefallen ist, obwohl Dibromcarbeninsertionen schon seit langer Zeit bekannt und Gegenstand zahlreicher Untersuchungen waren, ${ }^{[33-36]}$ zum anderen stellte sich die Frage über den Bildungsmechanismus dieser Produkte. Wichtig war zunächst, ob die Reaktionsbedingungen so geändert werden könnten, daß eine Umverteilung der Produktbildung zugunsten der Substitutionsprodukte erfolgt. Durch Verwendung von Tetrabrommethan anstelle von Bromoform unter ansonsten gleichen Reaktionsbedingungen konnte die Bildung der Insertionsprodukte komplett unterdrückt werden. Damit war ein neue Methode zur Bromierung von nichtaktivierten Kohlenwasserstoffen entdeckt.[29] Im nächsten Schritt wurde überprüft, wie allgemein diese neue Reaktion anwendbar ist, d.h. es wurden neben dem tricyclischen Adamantan auch bicyclische, cyclische und aliphatische Kohlenwasserstoffe der neuen Bromierungsmethode unterworfen (Tab. 1). Dabei wurde festgestellt, daß Methylgruppen grundsätzlich nicht angegriffen werden und sekundäre Wasserstoffatome weniger reaktiv als tertiäre sind. Im Fall von mehrfach methylsubstituierten Kohlenwasserstoffen kann eine Reaktion ganz ausbleiben (Isooctan, Camphan). Ein Grund dafür könnte in der durch die Methylgruppen bedingte verstärkte sterische Hinderung zu suchen sein. Mehrfach methylsubstituierte Substrate neigen auch stärker zu Eliminierungen, was ebenfalls für das Fehlen von bromierten Produkten verantwortlich sein könnte. Der Nachweis von Alkenen unter den Reaktionsbedingungen gelang allerdings nicht, eventuell aufgrund nachfolgender radikalisch initiierter Polymerisierungen. 
Tabelle 1. Bromierung von ausgewählten Kohlenwasserstoffen unter PTK Bedingungen $\left[\mathrm{CBr}_{4} /\right.$ TEBABr (10 mol\%) / NaOH (aq. 50\%)]

\begin{tabular}{|c|c|c|c|c|}
\hline \# & Edukt & $\mathbf{T}\left[{ }^{\circ} \mathbf{C}\right] / \mathrm{t}[\mathrm{h}] /$ Solvens & Produkt 1 [\%] & Produkt $2[\%]$ \\
\hline 1 & 1 & $40 / 87 / \mathrm{CH}_{2} \mathrm{Cl}_{2}$ & $2 \quad[68]$ & 3 [14] \\
\hline 2 & & $40 / 64 / \mathrm{CH}_{2} \mathrm{Cl}_{2}$ & {$[38]$} & {[} \\
\hline 3 & & $40 / 48 / \mathrm{CH}_{2} \mathrm{Cl}_{2}$ & {$[50]$} & - \\
\hline 4 & & $40 / 80 /[a]$ & & - \\
\hline 5 & & 23 / 42 / [a] & & - \\
\hline 6 & & $23 / 16 /[a]$ & [38 & - \\
\hline & 14 & & 15 & \\
\hline
\end{tabular}

[a] Das Edukt selbst wurde als Lösungsmittel verwendet. Die Ausbeuten beziehen sich auf eingesetztes Tetrabrommethan und sind präparativ (Die Produktverhältnisse wurden durch Integration separater Signale in ${ }^{1} \mathrm{H}-\mathrm{NMR}$ Spektrum bestimmt).

Nachdem die Experimente der Bromierung so erfolgreich verliefen, lag es nahe zu überprüfen, ob auch andere Polyhalomethanverbindungen als Halogenquelle für Halogenierungen geeignet wären. Erstaunlicherweise ließen sich auch Iodierungen mit Tetraiodmethan auf diese Weise durchführen.[30] Allerdings ist diese Iodverbindung sehr teuer und instabil. Aus diesem Grund stellte sich Iodoform als Iodquelle der Wahl für die Iodierungen unter PT-Bedingungen heraus (Tab. 2.), ${ }^{[30]}$ denn im Gegensatz zu den Reaktionen mit Bromoform findet mit Iodoform fast keine Carbeninsertion statt (es konnten nur Spuren von Insertionsprodukt massenspektrometrisch in frühen Fraktionen der Säulenchromatographie im Rahmen einer Iodierung mit Iodoform von 1 nachgewiesen werden.). Des weiteren wurde festgestellt, daß nicht eine wäßrige Lösung, sondern festes 
Natriumhydroxid für Iodierungen besser geeignet ist. Wahrscheinlich ist auch hier der Grund dafür in Nebenreaktionen in Form von Eliminierungen zu suchen, die bei Verwendung von festem $\mathrm{NaOH}$ nicht in dem Maß auftritt.

Tabelle 2. Iodierung von ausgewählten Kohlenwasserstoffen unter PT-Bedingungen $\left[\mathrm{CHI}_{3} /\right.$ $\mathrm{NaOH}(\mathrm{s})]$

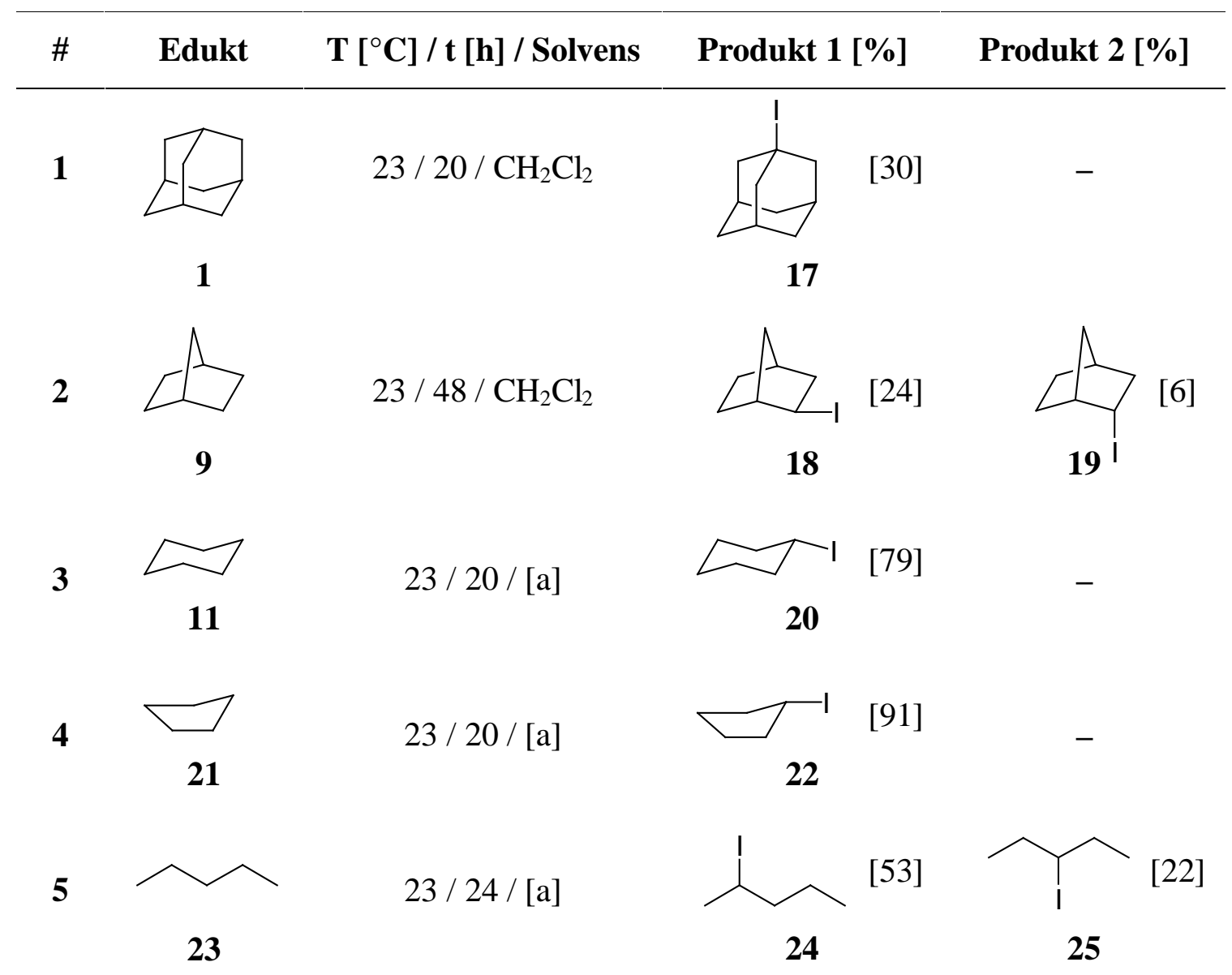

[a] Das Edukt selbst wurde als Lösungsmittel verwendet. Die Ausbeuten beziehen sich auf eingesetztes Iodoform und sind präparativ (Die Produktverhältnisse wurden durch Integration separater Signale in ${ }^{1} \mathrm{H}-$ NMR Spektrum bestimmt).

Nachdem durch die eben erwähnten Experimente die allgemeine Relevanz der neuen Methode zur Bromierung und Iodierung von Kohlenwasserstoffen aufgezeigt wurde, mußte die Frage nach dem Reaktionsmechanismus eingehender überdacht und untersucht werden. Einen dahingehenden Hinweis liefert die beobachtete Regioselektivität z.B. bei der Halogenierung von Adamantan. So wird bei der radikalischen Bromierung mit elementarem Brom eine $3^{\circ} / 2^{\circ}$ Selektivität von $52: 48$ beobachtet.[37, 38] Somit ist wahrscheinlich, daß 
Halogenradikale nicht für die Substitution verantwortlich sein können, denn im Rahmen der PT-Halogenierungen werden tertiäre Produkte mit weitaus höherer Präferenz (> 90\%) gebildet. Diese Annahme wird zusätzlich durch die Durchführbarkeit von Iodierungen bestätigt, denn ein Propagationszyklus getragen von Iodradikalen ist endotherm und eine Kettenpropagation über diese Radikale deswegen thermodynamisch nicht möglich. Eine Arbeitshypothese (Schema. 3), die durch die bisherigen Beobachtungen gestützt wird, beruht auf einem radikalischen Mechanismus, bei dem das Trihalomethylradikal die Propagation der Radikalreaktion trägt.

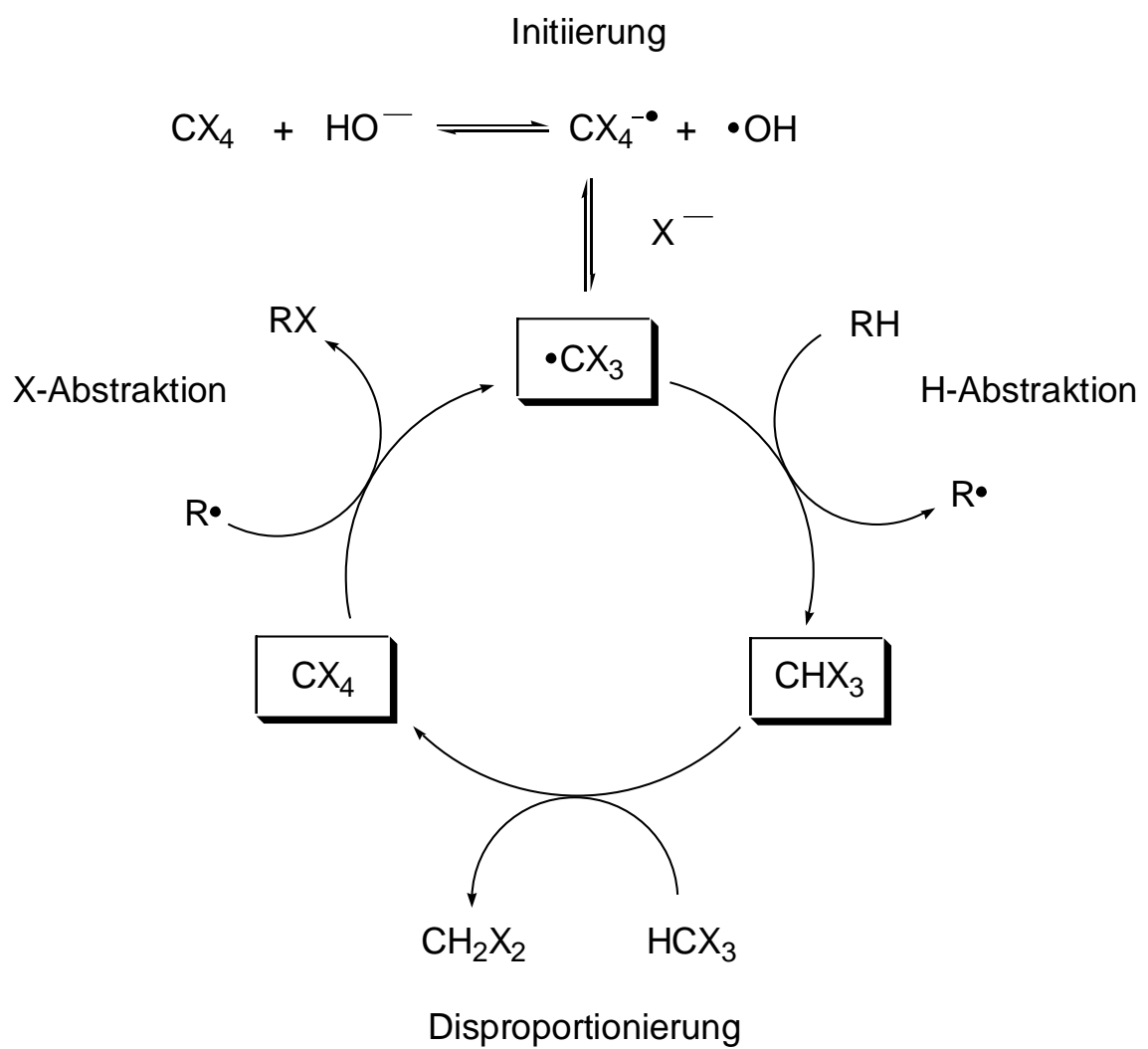

Schema 2. Allgemeine Arbeitshypothese für den Mechanismus der Halogenierung unter PTK Bedingungen

Dabei erfolgt die Initiierung der Reaktion über einen SET vom Hydroxidanion auf das Tetrahalomethan unter Bildung eines Radikalanions und eines Hydroxylradikals, das zum Halogenanion und dem Trihalomethylradikal fragmentiert (dissoziativer Elektronentransfer). Denkbar wäre auch, daß keine Fragmentierung auftritt, sondern das Hydroxylradikal vom zu halogenierenden Substratmolekül ein Wasserstoffatom abstrahiert und so der Radikalcyclus gestartet wird. Dies näher zu untersuchen wird Gegenstand weiterer Untersuchungen sein. 
Da auch die Haloforme als Halogenquellen eingesetzt werden konnten, wird hierbei eine basisch initiierte vorgelagerte Disproportionierung zum Dihalo- und Tetrahalomethan angenommen. Solche Disproportionierungen sind für Chloroform und Bromoform bereits eingehend untersucht worden und literaturbekannt. [39-42] Im Fall der Disproportionierung von Iodoform $\left[\mathrm{CHI}_{3} / \mathrm{CH}_{2} \mathrm{Cl}_{2} / \mathrm{NaOH}(\mathrm{s}) / 22 \mathrm{~h} / \mathrm{RT}\right]$ konnten Diiodmethan ${ }^{1} \mathrm{H}-\mathrm{NMR}$ - und Tetraiodmethan massenspektroskopisch in der Reaktionsmischung nachgewiesen werden. [30]

Als weiteren Hinweis, daß es sich insgesamt um eine Radikalreaktion handelt, kann aufgefaßt werden, daß durch Zusatz von 2,2,6,6-Tetramethylpiperidyl- $N$-oxid (TEMPO), einem stabilen Radikal, eine Halogenierung vollständig unterdrückt wird.[43] Werden die bislang erhaltenen Ergebnisse zusammenfassend betrachtet, so wurde hier eine neue Methode zur selektiven Halogenierung von Kohlenwasserstoffen entwickelt, die, wie eingangs erwähnt, auch als erste Applikation eines neuen allgemeineren Konzeptes aufgefaßt werden kann.[18] Die Vorteile der neuen Methodik werden aber schon bei Betrachtung der Halogenierungen deutlich.

- Billige übergangsmetallfreie Reagenzien

- Hohe Selektivitäten

- Niedrige Temperaturen.

- Kein Halogenaustausch

- Neue Reaktionen

- Einfache Reaktionsdurchführung

\subsection{Reaktionen unter Phasentransferbedingungen}

Unter PT-Bedingungen durchgeführte Reaktionen sind solche, bei denen die an dieser Reaktion partizipierenden Teilchen (Moleküle, Ionen, etc.) nicht aus der selben Phase entstammen, was ein mehrphasiges Reaktionssystem bedingt. Diese Methodik hat sich zu einem sehr großen Gebiet in der organischen Chemie entwickelt und mittlerweile sind zahllose Bücher und Übersichtsartikel zu diesem Thema erschienen.[21-27] In der folgenden kurzen Übersicht können deswegen unmöglich alle Methoden und Anwendungen behandelt werden, und es wird hier nur auf die für diese Arbeit relevanten bzw. die wichtigsten in der Literatur bekannten Aspekte der Reaktionen eingegangen. Die PT-Reaktionssysteme bestehen 
fast immer aus einer festen oder flüssigen (meist wäßrigen) anorganischen und einer flüssigen organischen Phase und können, müssen aber nicht notwendigerweise die folgenden Vorteile bieten. $[21-25,27]$

- Manche Reaktionen können nur unter PT-Bedingungen durchgeführt werden

- Verkürzte Reaktionszeiten

- Erhöhte Ausbeuten durch Unterdrückung von Nebenreaktionen

- Verschiebung von Selektivitäten

- Änderung von Produktverhältnissen

- Mildere Reaktionsbedingungen

- Vereinfachte Aufarbeitung

- Vereinfachte Durchführung, da nicht wasserfrei gearbeitet werden muß

Für eine bestimmte Reaktion muß immer von Fall zu Fall entschieden werden, ob sich eine Durchführung unter PT-Bedingungen lohnt. Wie schon erwähnt, sind diese auf viele "klassische" Reaktionen der organischen Chemie angewendet worden und es soll hier nur eine kleine Auswahl der wichtigsten angegeben werden. [21-25, 27]

- Halogenaustausch

- $\mathrm{S}_{\mathrm{N}}$-Reaktionen

- C-Alkylierungen von $\mathrm{C}-\mathrm{H}$-aziden Verbindungen

- Verester- und Veretherungen

- $\alpha$-, $\beta$ - und $\gamma$-Eliminierungen

- Addition an C-C-Mehrfachbindungen

- Wittig und Wittig-Horner Reaktionen

- Umlagerungen

- Übergangsmetallvermittelte Kupplungsreaktionen

- Reduktionen

- Oxidationen

Viele dieser Reaktionen zeichnen sich durch unterschiedliche Abhängigkeiten von der Art des Phasentransferkatalysators (PTK), des Lösungsmittels, des Gegenions des Katalysators (falls dieser über eine Ladung verfügt) und der Rührgeschwindigkeit u.a. aus.[21-25, 27] 
Zur Erklärung dieser verschiedenen Abhängigkeiten werden grundsätzlich drei verschiedene Reaktionsmechanismen postuliert: Der Extraktions-, ${ }^{[44]}$ der Grenzflächen-[45, 46] und der Interphasenmechanismus (Abb. 2).[22] Beim Extraktionsmechanismus wird das reaktive Agens vom Phasentransferkatalysator an der Phasengrenze aufgenommen und in die organische Phase überführt, wo dann die eigentliche Reaktion stattfindet. Im zweiten Fall, dem Grenzflächenmechanismus, ${ }^{4}$, 46] findet in einem vorgelagerten Gleichgewicht eine Reaktion zwischen einem Substratmolekül und dem aus der anorganischen Phase stammenden reaktiven Agens an der Phasengrenze statt. Aufgrund des ambivalenten Löslichkeitsverhalten des entstehenden Komplexes bleibt dieser an der Phasengrenze so lange verankert bis der PTK das aktivierte Substratmolekül in die organische Phase überführt, wo es dann weiterreagieren kann. Im Gegensatz zum Extraktionsmechanismus findet demzufolge ein Teil der Reaktion im Phasengrenzbereich statt. Der dritte Mechanismus, der als Interphasenmechanismus bezeichnet wird, wurde postuliert, weil bestimmte Reaktionen auch ohne Zusatz eines Katalysators genauso schnell ablaufen wie mit einem PTK. Dabei wird davon ausgegangen, daß der gesamte Ablauf der Reaktion im Phasengrenzbereich vonstatten geht. ${ }^{[22]}$ Es wird in der Literatur auch noch ein weiterer Mechanismus, bei dem der Transfer eines Teilchens von der organischen in die anorganische Phase stattfindet, diskutiert, nämlich der inverse Extraktionsmechanismus. ${ }^{[21]}$ Er soll hier aber nicht näher erläutert werden, weil dieser Mechanismus für die in dieser Arbeit betrachteten Systeme nicht von Bedeutung ist.

Mechanismus

Extraktion

Grenzfläche

Interphase

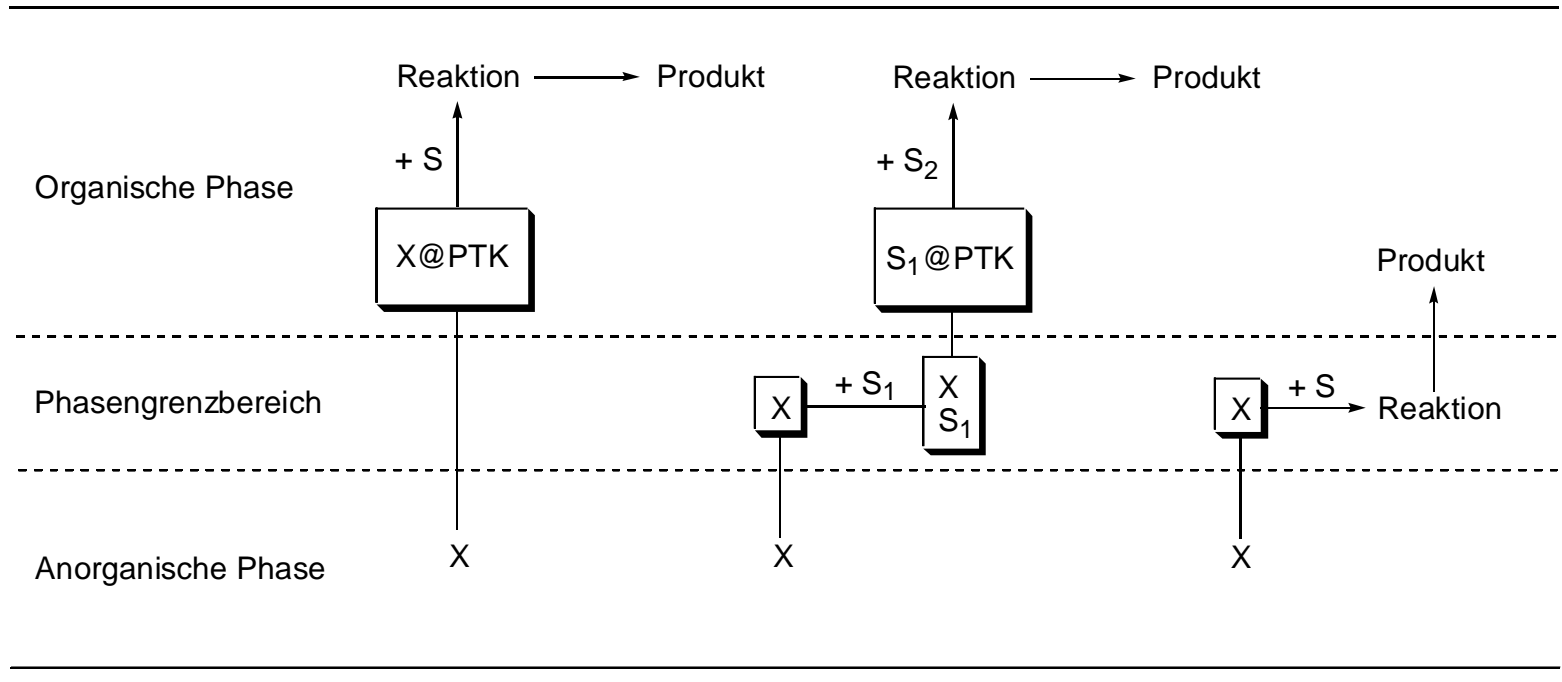

Abbildung 2. Allgemeine Mechanismen von PT-Reaktionen: $S=$ Substrat, $X=$ initiierendes Agens 
Da beim Extraktionsmechanismus das reaktive Teilchen in die organische Phase überführt wird, werden Reaktionen, die diesem Mechanismus gehorchen, besonders effektiv durch möglichst lipophile PTK gefördert. Dies äußert sich z.B. bei Verwendung von quartären Ammoniumsalzen als PTK in einer signifikanten Steigerung der Reaktionsgeschwindigkeit mit steigender Kettenlänge bei ansonsten gleichen Reaktionsbedingungen. Im Gegensatz dazu wird ein Grenzflächenmechanismus durch Katalysatoren maximal beschleunigt, die über eine gut zugängliche Ladung verfügen. Der Grund dafür ist darin zu suchen, daß meistens ein raumbeanspruchendes organisches Anion in die organische Phase überführt werden muß. Das bedeutet, daß für den Fall von quartären Ammoniumsalzen als Katalysatoren, die kurzkettigen sich am effektivsten erweisen. Wie schon erwähnt ist ein Zusatz von Katalysatoren im Fall eines Interphasenmechanismus unnötig, weil hier beide Komponenten einer Reaktion einigermaßen löslich in einem Phasengrenzbereich (Interphase) sind und dort auch ohne Hilfe eines Katalysators miteinander reagieren können. Die Feststellung der Effektivität einer Auswahl verschiedener Katalysatoren ist eines der wichtigsten Indizien für einen bestimmten Mechanismus.

Im Fall von geladenen PTK wie z.B. quartären Ammoniumsalzen kann sich auch das Gegenion stark auf die Reaktionsgeschwindigkeit auswirken. Wenn gemäß dem PearsonKonzept ${ }^{[47]}$ harte Basen (z.B. $\mathrm{OH}^{-}$) durch den PTK transferiert werden müssen, wird eine Reaktion verlangsamt, wenn diese mit einem weichen Anion (z.B. $\mathrm{I}^{-}$) konkurrieren müssen, weil letzteres gemäß dieses Konzeptes besser mit dem weicheren Katalysatorkation koordinieren. Hauptsächlich dem Extraktionsmechanismus gehorchende Reaktionen werden durch diesen Effekt beeinflußt. [44]

Neben den eben beschriebenen gibt es noch weitere spezielle Reaktionsbedingungen die auf einen bestimmten Reaktionsmechanismus hinweisen. Sie dürfen allerdings nicht zu stark bewertet werden, weil sie alle PTK-Reaktionen in gleicher Weise, nur in abgestufter Intensität beeinflussen. Aufgrund dieser Hinweise ist es dann sehr schwierig auf einen bestimmten Mechanismus zu schließen.[21-25, 27] Ein Beispiel dafür ist die Rührgeschwindigkeit. Während dem Extraktionsmechanismus gehorchende Reaktionen nur bis zu einem gewissen Schwellenwert der Rührgeschwindigkeit beschleunigt werden, können durch eine weitere Erhöhung die Umsätze bei den anderen PT-Reaktionen weiter gesteigert werden, weil hier eine direkte Abhängigkeit von der Größe der Phasengrenzfläche gegeben ist. Weitere Abhängigkeiten, wie z.B. die Polarität des Lösungsmittels sind noch unspezifischer und können kaum mehr als Befund für einen bestimmten PT-Mechanismus angesehen werden. 
Schließlich soll noch ein Faktor angesprochen werden, der gerade bei hydroxidvermittelten PT-Reaktionen verstärkt auftritt.[48] Das Hydroxidion ist ein sehr kleines Anion, fast ohne jegliche Ladungsdispersion und liegt deswegen in stark polaren protischen Lösungsmitteln hoch solvatisiert vor. Beim Übergang in die organische Phase wird dann ein gewisses Kontingent an Lösungsmittelmolekülen mitgeführt. Bei Steigerung der Konzentration der Base in der anorganischen Phase verkleinert sich dieses Kontingent zunehmend,[49] wodurch eine enorme Steigerung der Reaktivität (Basizität, Nukleophilie, Reduktionspotential)[28] erreicht wird. Deswegen werden viele durch Hydroxidanionen initiierte Reaktionen unter PT-Bedingungen durch steigende Basenkonzentration stark beschleunigt.[50]

Werden Reaktionen katalysiert durchgeführt, so ist es immer naheliegend, mit Hilfe des Katalysators gleichzeitig eine chirale Induktion zu bewirken.[22, 26, 27] Eine Möglichkeit chirale PTK herzustellen beruht auf der Derivatisierung und Quarternisierung von Alkaloiden aus dem chiral pool, der solche Ausgangsverbindungen billig und in enantiomerenreiner Form anbietet. Erste Versuche in Form von $C$-Alkylierungen von $\beta$-Ketoestern oder $\beta$-Diketonen mit von Ephidrinen abgeleiteten Katalysatoren verliefen mit enttäuschenden Ergebnissen.[51] Es wurden nur geringe Enantiomerenüberschüsse beobachtet, und es ist sogar zu vermuten, daß die geringen Drehwerte der Produktgemische auf Verunreinigungen, die durch den Zerfall des Katalysators entstanden sein könnten, zurückzuführen sind. ${ }^{[52]}$ Als sehr viel erfolgreicher stellte sich die Verwendung von chiralen Katalysatoren basierend auf den Cinchona-Alkaloiden heraus (Schema 3).[53-60] So konnten Alkylierungen von C-Haziden Verbindungen (Nr. 2, 3; Schema 3), [55, 56] Epoxidierungen von Alkenen (Nr. 4; Schema 3) ${ }^{[58]}$ und Darzens Kondensationen (Nr. 5; Schema 3)[59] mit guten Ausbeuten bei gleichzeitig hohen Enantiomerenüberschüssen (bis zu 92\% ee) durchgeführt werden. 
<smiles>COc1cc2c(c(Cl)c1Cl)C(=O)C(c1ccccc1)C2</smiles><smiles>CC(C)(C)OC(=O)CN=C(c1ccccc1)c1ccccc1</smiles>

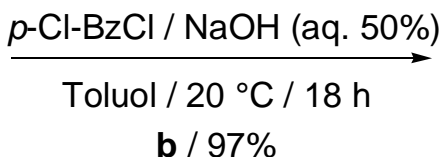<smiles>CC(C)(C)OC(=O)[C@H](Cc1ccc(Cl)cc1)N=C(c1ccccc1)c1ccccc1</smiles><smiles>CC(C)(C)C(=O)/C=C/c1ccccc1</smiles>

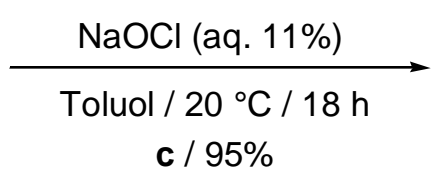<smiles>CC(C)(C)C(=O)C1OC1c1ccccc1</smiles>
c / $95 \%$<smiles>CC(C)C1O[C@H]1C(=O)c1ccccc1</smiles><smiles>CC(C)C=[O+]</smiles><smiles>CCOCCOCCO</smiles>

a / $73 \%$

$69 \%$ ee<smiles>[R8]C[N+]12CCC(C(C=C)C1)C2[C@H](Cl)c1ccnc2ccc([R3])cc12</smiles>

a: $R_{1}=$<smiles>Cc1ccc(C(F)(F)F)cc1</smiles>
$\mathrm{R}_{2}=\mathrm{R}_{3}=\mathrm{H}$

b: $\mathrm{R}_{1}$<smiles>Cc1ccccc1</smiles>
$\mathrm{R}_{2}=\mathrm{R}_{3}=\mathrm{H}$

c: $\mathrm{R}_{1}=$<smiles>Cc1c2ccccc2cc2ccccc12</smiles>
$\mathrm{R}_{2}=$ $\mathrm{R}_{3}=\mathrm{H}$

Schema 3. Beispiele von asymmetrischen Reaktionen unter Verwendung chiraler CinchonaAlkaloidkatalysatoren

Dabei wurde festgestellt, daß eine gewisse Einschränkung bezüglich der Substratauswahl besteht. Sie müssen über mindestens einen aromatischen Substituenten verfügen, weil es ansonsten nicht zu einer koordinierenden Wechselwirkung mit dem Katalysator kommen kann, und dann keine nennenswerte asymmetrische Induktion erfolgt. Ein weiterer Nachteil der Cinchona-Alkaloidkatalysatoren ist, daß sie wie auch die 
Ephidrinkatalysatoren unter den stark basischen Reaktionsbedingungen nicht stabil zu sein scheinen. [52] Diese Nachteile führten zu der Entwicklung von chiralen Hilfsreagenzien[61] und u.a. ${ }^{[62]}$ von neuen $C_{2}$-symmetrischen Katalysatoren die chemisch von den enantiomerenreinen $R$ - oder $S$-Binaphtholen abgeleitet wurden (Abb. 3). [63]

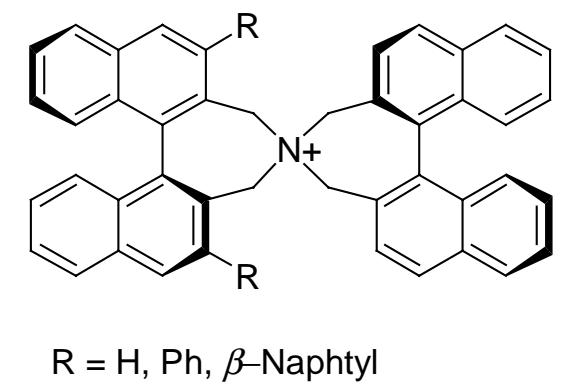

Abbildung 3. Beispiel für eine Klasse enantiomerenreinr $C_{2}$-symmetrischen Katalysatoren $(S)$

Durch Anwendung solcher Katalysatoren im Rahmen der Alkylierung von Schiffschen Basen (G1. 2, Schema 3) werden durchweg Enantioselektivitäten um 95\% ee erzielt und diese Katalysatoren sind auch unter den stark basischen Reaktionsbedingungen stabil. Allerdings wurden sie bislang nur an aromatischen Substraten getestet. Neben den erwähnten chiralen quartären Ammoniumsalzen fanden auch asymmetrische Kronenether und Kryptanden als ungeladene PTK Anwendung in der chiralen Synthese.[21] Sie sollen hier aber nicht weiter erläutert werden, weil solche PTK für die vorliegenden Arbeit keine Relevanz haben. Es wurde auch überprüft, wie sich chirale Lösungsmittel im Rahmen der Phasentransferkatalyse auswirken. Dabei wurden aber nur geringste Enantiomerenüberschüsse erzielt.[22] Abschließend muß festgestellt werden, daß das Gebiet der chiralen PT-Katalyse bislang noch nicht sehr ausführlich untersucht worden ist und ein großes Feld für weitere Forschungen auch im Rahmen der PT-Halogenierungen darstellt (s. Ausblick).

\subsection{Die Regioselektivität von radikalischen Substitutionen in Alkanen}

Wie bereits erwähnt wurde, handelt es sich bei der neuen Methodik um die Möglichkeit zur Generierung von Radikalen unter PT-Bedingungen. Radikalreaktionen sind ein umfangreiches Thema der organischen Chemie, $[6,7,19,20,64]$ so daß auch in der 
folgenden Einleitung Einschränkungen bezüglich der Erklärung der verschiedenen Aspekte dieser Reaktionen gemacht werden mußten und nur auf die für die Arbeit relevanten, speziell auf die Regioselektivitäten von Radikalreaktionen, eingegangen werden konnte.[65] Ein wichtiger Grund für die Entscheidung für eine bestimmte Reaktion ist zunächst deren Einfachheit bei gleichzeitig möglichst hoher Ausbeute, wobei Radikalreaktionen genau diese Vorteile bieten. Der entscheidende Schritt ein jeder Radikalreaktion ist die Freisetzung von Radikalen aus geeigneten Vorläufern. Dies beruht auf zwei verschiedenen allgemeinen Methoden, den homolytischen Bindungsspaltungen und den Redoxprozessen.[20] Homolytische Bindungsspaltungen können durch Thermolyse oder Photolyse erreicht werden. Dabei wird auf die eine oder andere Weise einer Bindung soviel Energie zugeführt, daß sie schließlich bricht, wodurch zwei Radikale entstehen. Im anderen Fall, der Generierung von Radikalen durch Redoxprozesse, sind sowohl Reduktionen als auch Oxidationen, meist durch Übertragung nur eines Elektrons, für die Generierung von radikalanionischen oder radikalkationischen Intermediaten verantwortlich, aus denen dann die Radikale hervorgehen, wobei der SET über einen Inner-sphere- oder Outer-sphere-Mechanismus verlaufen kann.[66]

Ein entscheidender Nachteil von Radikalreaktionen ist jedoch, daß sie oftmals mit mangelhafter Regioselektivität verlaufen. Dies wird gerade bei den radikalischen Aktivierungen von Kohlenwasserstoffen deutlich.[6, 7, 19, 20, 64] Anhand dieser Reaktionen soll verdeutlicht werden, welche Faktoren für den für jeweiligen Grad der Selektivitäten verantwortlich sind. Das ist im untergeordneten Maß die Reaktionstemperatur und die Art des Lösungsmittels aber hauptsächlich die Wahl der radikalischen Spezies, worauf im folgenden eingegangen werden soll.

$\mathrm{Zu}$ den am längsten bekannten und auch am besten untersuchten Reaktionen gehören die Substitutionen von $\mathrm{H}-$ Atomen in Kohlenwasserstoffen durch Halogene (F, Cl, Br), die als Kettenreaktionen ablaufen und bei denen Halogenradikale die Propagationen tragen. (Schema 4).

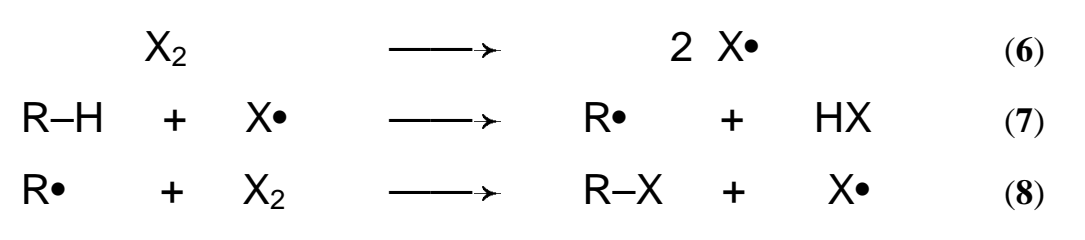

Schema 4. Allgemeines Reaktionsschema der radikalischen Substitution von Wasserstoff- durch Halogenatome (X = F, Cl, Br) 
Bei Fluorierungen wird keine Selektivität bezüglich $3^{\circ}-, 2^{\circ}-$, und $1^{\circ}$-Positionen in entsprechenden Substraten festgestellt.[67] Weiterhin sind diese Reaktionen stark exotherm, was zu Nebenreaktionen wie Fragmentierungen des Kohlenstoffgerüstes führt. Schon etwas anders gelagert ist der Fall der Chlorierungen.[68] Hier können merkliche Selektivitäten erreicht werden. So wird bei der Chlorierung von 2,3-Dimethylbutan eine $3^{\circ}: 1^{\circ}$ Selektivität von 38.0 und $2^{\circ}: 1^{\circ}=8.8$ an relativer Selektivität für $n$-Alkane beobachtet.[69] Noch höhere Selektivitäten werden bei radikalischen Bromierungen erreicht. Dies wird bei der Reaktion von 2-Methylbutan deutlich. Die Methylgruppen werden so gut wie nicht angegriffen und ein großer Überschuß an tertiären Produkten erzielt $\left(3^{\circ}: 2^{\circ}=12.5\right) .{ }^{[70,71]}$ Dieser Trend setzt sich auch bei der Bromierung von anderen Alkanen wie Isobutan und Propan fort.[72] Für den Grad der jeweiligen Selektivität sind verschiedene Faktoren ausschlaggebend. Bei der Bromierung ist die Wasserstoffabstraktion der geschwindigkeitsbestimmende und zudem immer endotherme Schritt, der in der Reihenfolge der Positionen $3^{\circ}, 2^{\circ}, 1^{\circ}$ kinetisch zunehmend ungünstiger wird, d.h. die Aktivierungsenergie nimmt zu.[70, 71] Gemäß dem Hammond-Postulat[73] wird hier ein später, also produktähnlicher ÜZ durchlaufen, so daß auch die Stabilität der entstehenden Radikale, die in der vorher genannten Reihenfolge abnimmt und so die beobachtete Selektivität mitbestimmt, eine Rolle spielt. Im Kontrast dazu ist die Wasserstoffabspaltung durch ein Chlorradikal aufgrund dessen höherer Reaktivität in allen möglichen Positionen exotherm. Dies bedingt gemäß dem Hammond-Postulat einen frühen ÜZ, wodurch die Radikalstabilitäten einen nur geringen Einfluß auf die Aktivierungsenergien haben und dadurch eine geringere Selektivität im Vergleich zu den Bromierungen resultiert. Im Fall einer Fluorierung sind beide Teilschritte der Propagation so stark exotherm, daß wie eingangs gesagt keinerlei Selektivität mehr resultiert.

Neben den Halogenatomen gib es noch eine ganze Reihe anderer Radikale, die zur Aktivierung von einfachen Kohlenwasserstoffen herangezogen werden können, und anhand der Lokalisierung des radikalischen Zentrums charakterisiert werden. ${ }^{[19]}$ Dazu gehören z.B. die sauerstoffzentrierten Radikale. Neben Sauerstoffatomen, [74] die hier nicht weiter besprochen werden sollen, ist das Hydroxylradikal der einfachste und zugleich ein sehr wichtiger Vertreter dieser Spezies.[20] Es kann auf verschiedene Weisen, z.B. durch $\gamma$-Strahlung aus Wasser,[20] chemisch durch Zerfall von Peroxosalpeteräure ${ }^{[75]}$ oder durch Zerfall von Wasserstoffperoxid unter reduktiven Fenton-[76] oder Gif-[4] Reaktionsbedingungen generiert werden. Dabei ist aufgrund der hohen Reaktivität des Hydroxylradikals nur eine geringe Selektivität bei der $\mathrm{H}-$ Abstraktion in der $3^{\circ}-2^{\circ}$ - und $1^{\circ}$-Position von Alkanen zu verzeichnen. ${ }^{[75] ~ W i r d ~ d a s ~ W a s s e r s t o f f a t o m ~ i m ~ H y d r o x y l r a d i k a l ~}$ 
durch einen Alkylrest substituiert ergeben sich Alkoxyradikale, wobei das $t$-Butoxyradikal am besten untersucht wurde (Schema 5). Es kann sehr leicht durch Photolyse oder Thermolyse von Di-t-butylperoxid oder $t$-Butylhydroperoxid generiert werden.[77] Die beobachtete Selektivität der H-Abstraktionen an Alkanen bewegt sich im Bereich der Bromierungen $\left(3^{\circ}\right.$ : $2^{\circ}=12$ und $\left.3^{\circ}: 1^{\circ}=50\right) .[78,79]$ Entsteht $t-\mathrm{BuO} \bullet$ nicht aus den entsprechenden Peroxoverbindungen sondern aus dem $t$-Butoxyhalogenid $(t-\mathrm{BuOX}, \mathrm{X}=\mathrm{Cl}, \mathrm{Br}$, I), so können die entsprechenden Halogenierungen (auch Iodierungen) mit relativ hohen Regioselektivitäten durchgeführt werden. $[80,81]$ Dabei erfolgt die Initiierung durch Zusatz geringer Mengen eines Radikalstarters (Azobisisobutyronitril = AIBN ) oder photolytisch. $[80,81]$

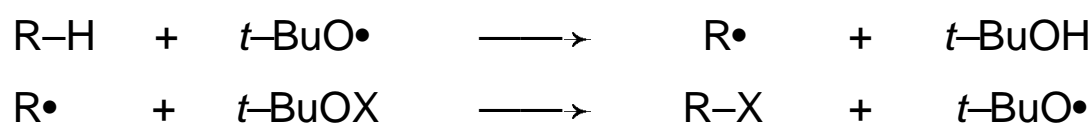

Schema 5. Allgemeines Reaktionsschema der radikalischen Substitution von Wasserstoff- durch Halogenatome über t-Butoxyradikale $(\mathrm{X}=\mathrm{Cl}, \mathrm{Br}, \mathrm{I})$

Eine weitere Gruppe von Radikalen sind die stickstoffzentrierten Radikale. Hier gehören die $\mathrm{N}$-Halogensuccinimide zu den wichtigsten Ausgangssubstanzen.[64] Das korrespondierende Succinimidylradikal wird durch kleine Mengen an Radikalstartern erzeugt und so die Kettenreaktion eingeleitet. Die auf diese Weise erzielten Regioselektivitäten bei der Bromierung von Alkanen sind allerdings erheblich geringer als mit Bromradikalen, [82] während im Vergleich zu den Chlorierungen über Chlorradikale auf diese Weise höhere Selektivitäten beobachtet werden. ${ }^{[83]}$

Für die vorliegende Arbeit sind aber die Eigenschaften von kohlenstoffzentrierten Radikalen am bedeutendsten (Schema 6), weil hierbei die höchsten Selektivitäten erzielt werden können. Bislang haben hauptsächlich die halogensubstituierten Alkylradikale $\left(\bullet \mathrm{CCl}_{3}\right.$, $\left.\cdot \mathrm{CBr}_{3}, \cdot \mathrm{C}_{\mathrm{n}} \mathrm{F}_{2 \mathrm{n}+1}\right)$ aufgrund der, im Vergleich zu reinen Alkylradikalen, erhöhten Stabilität und Regioselektivität Anwendung in der Aktivierung von Kohlenwasserstoffen gefunden. [84-87] 


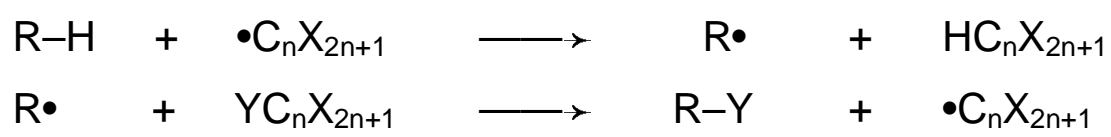

Schema 6. Allgemeines Reaktionsschema der radikalischen Substitution von Wasserstoff- durch Halogenatome über halogensubstituierte Alkylradikale (X = $\mathrm{F}, \mathrm{Cl}, \mathrm{Br} ; \mathrm{Y}=\mathrm{Cl}, \mathrm{Br}, \mathrm{I})$

Die Chlorierungen mit $\cdot \mathrm{CCl}_{3}$ sind dabei am intensivsten untersucht worden. ${ }^{[85]}$ Die bereits erwähnte Selektivität von Haloalkylradikalen manifestiert sich hier durch eine $3^{\circ}: 2^{\circ}$ Selektivität von $24.3: 1.0$ bei der Chlorierung von Adamantan ${ }^{[37]}$ und ist damit im Vergleich zu Chlorierungen über andere Radikale (z.B. $\cdot C l, N$-Succinimidyl) unerreicht. Erstaunlicherweise wurde $\cdot \mathrm{CBr}_{3}$ nicht so gut untersucht, obwohl es sehr leicht aus Tetrabrommethan photolytisch, durch übergangsmetallkatalysierten homolytischen Zerfall oder durch thermischen Zerfall von Radikalstartern generiert und eine noch höhere Regioselektivität als im Fall von $\cdot \mathrm{CCl}_{3}$ erwartet werden kann. [86] Zahlreich variiert und untersucht wurden die fluorierten Alkylradikale. ${ }^{[88]}$ Der einfachste Vertreter seiner Art ist $\cdot \mathrm{CF}_{3}$, das leicht durch den thermischen Zerfall von $\mathrm{CF}_{3} \mathrm{COOOH}$ generiert werden kann. [89] Die Selektivität der H-Abstraktion von $n$-Heptan durch $i-\mathrm{C}_{3} \mathrm{~F}_{7}$ ist $2^{\circ}: 1^{\circ}=12$ und liegt damit im Bereich der Regioselektivität des $t$-BuO-Radikals. [87, 88] Die photolytisch initiierte Iodierung von Adamantan mit $n-\mathrm{C}_{4} \mathrm{~F}_{9} \mathrm{I}$, wobei das primäre $n-\mathrm{C}_{4} \mathrm{~F}_{9}$-Radikal die Propagation der Radikalreaktion trägt, zeigt eine Selektivität von $3^{\circ}: 2^{\circ}=17 .{ }^{[90]}$

Abschließend sei nochmals darauf hingewiesen, daß in diesem Kapitel nur auf Teilaspekte, insbesondere die Regioselektivitäten von Radikalreaktionen bei der AlkanAktivierung, eingegangen werden sollte. Dadurch soll ein Vergleich mit den unter PTHalogenierungen erzielten Selektivitäten ermöglicht werden und gleichzeitig zum Nachdenken über die Applikation anderer Radikale für das PT-Konzept angeregt werden.

\subsection{Computerchemie: Grundlagen der verwendeten Methoden und Basissätze}

In der vorliegenden Arbeit wurden sämtliche Berechnungen unter Verwendung von Methoden durchgeführt, welche die DFT (Density Functional Theory = Dichtefunktionaltheorie) zur Grundlage haben.[91-93] Sie beruht auf der Substitution der klassischen Wellenfunktionen durch die Elektronendichte, um ein elektronisches System 
mathematisch $\mathrm{zu}$ beschreiben bzw. $\mathrm{zu}$ approximieren und ist somit ein rein quantenmechanisches Modell. Die absolute Energie eines chemischen Systems kann durch die folgende Gleichung dargestellt werden.

$$
E=E^{T}+E^{V}+E^{J}+E^{X}+E^{C}
$$

Hierbei wird die Gesamtenergie in verschiedene Terme aufgeteilt. $\mathrm{E}^{\mathrm{T}}$ ist die kinetische Energie der Elektronen und der Kerne; $\mathrm{E}^{\mathrm{V}}$ ist die Energie, die aus der Anziehung der Elektronen durch die Atomkerne resultiert; $\mathrm{E}^{\mathrm{J}}$ ist die Energie, die die Wechselwirkung der Elektronen mit ihrem, durch ihre Eigenbewegung induzierten, magnetischen Feld hervorruft. $\mathrm{E}^{\mathrm{X}}$ und $\mathrm{E}^{\mathrm{Z}}$ sind Energieterme, die aus der Störung der "Flugbahnen" der Elektronen bei Annäherung an andere Elektronen resultieren. Dabei ist $\mathrm{E}^{\mathrm{X}}$ die sogenannte Austauschenergie, die aus der Abstoßung von Elektronen mit gleichem Spin entsteht, denn gemäß dem Pauli Prinzip können sich Elektronen mit gleichem Spin nicht an der gleichen Stelle im Raum aufhalten. Dieses Phänomen wird als Fermi-Korrelation bezeichnet. ${ }^{[91]} \mathrm{E}^{\mathrm{C}}$ ist die Korrelationsenergie der Elektronen mit gegensätzlichem Spin und ist attraktiv. Die verschiedenen quantenmechanischen Methoden unterscheiden sich nur in der Evaluierung der letzten drei Energieterme $\mathrm{E}^{\mathrm{J}}, \mathrm{E}^{\mathrm{X}}$ und $\mathrm{E}^{\mathrm{C}}$. Sie sind das eigentliche Problem aller $a b$ initio Methoden, da sie jeweils ein Vielkörperproblem darstellen, das analytisch nicht gelöst werden kann. Bei reinen DFT-Methoden[91] werden alle drei Terme durch ein sogenanntes Funktional der Elektronendichte ausgedrückt, das leichter als die Vielelektronen-Wellenfunktionen der Hartree-Fock-Theorie ${ }^{[94]}$ zu berechnen ist. Die Entwicklung von verbesserten Funktionalen ist die Herausforderung an alle Neuentwicklungen in der DFT-Methodik. Neben verschiedenen anderen Theorien haben sich Methoden basierend auf der Kohn-ShamTheorie[95] durchgesetzt. Hierbei werden Hartree-Fock-Theorie und DFT miteinander verbunden. Dabei hat sich die BLYP-Methode (von Becke, Lee, Yang und Parr) ${ }^{[96,97]}$ als für viele Probleme allgemein anwendbar herausgestellt. Bei dieser Methode wird der Energieterm der kinetischen Energie $E^{\mathrm{T}}$ durch das klassische Hartree-Kinetik-Funktional[98] beschrieben, die $\mathrm{E}^{\mathrm{T}}$ als die Summe der kinetischen Einzelenergien der Elektronen unbeeinflußt von anderen ansieht. Die Austauschenergie $\mathrm{E}^{\mathrm{X}}$ wird über das Becke DFT-[97] und die Korrelationsenergie $\mathrm{E}^{\mathrm{C}}$ mit dem Lee-Yang-Parr DFT-Funktional [99] definiert, die beide die Gradienten der Elektronendichte mitberïcksichtigen. Eine Weiterentwicklung dieses Ansatzes sind die Hybrid-DFT Methoden, wo mehrere DFT-Funktionale entsprechend parametrisiert miteinander kombiniert werden. Die Koeffizienten dieser Parametrisierung wurden durch 
Angleichung von Berechnungen an experimentelle Daten erhalten. Am populärsten ist dabei die B3LYP-Methode,[97] bei der die Parametrisierung durch drei empirisch ermittelte Konstanten erreicht wird. Die DFT-Methoden, insbesondere die letztgenannte, bieten große Vorteile gegenüber den hochkorrelierten Post-SCF-Methoden. Sie benötigen gerade bei großen chemischen Systemen sehr viel weniger Rechenzeit und Festplattenkapazität, wobei trotzdem ähnlich gute Ergebnisse erzielt werden. Ein weiterer Vorteil ist der sehr viel geringere BSSE (Basis Set Superposition Error),[100] der mit Hilfe der "Counterpoise Correction“ Methode abgeschätzt werden kann.[101] Außerdem ist das Ausmaß der Spinvergiftung, die durch den Erwartungswert für den Spinoperator $\left\langle\mathrm{S}^{2}\right\rangle$ ausgedrückt wird, für offenschalige Systeme im Vergleich zu anderen Methoden sehr niedrig, was die DFT auch für die Beschreibung von einfachen Radikalen, Biradikalen und carbenoiden Strukturen eignet. [93]

Auch wenn die Computermethoden, die die DFT zur Grundlage haben, nicht so stark basissatzabhängig sind wie andere, muß hier eine Diskussion der verwendeten Basissätze erfolgen, denn die Qualität der Berechnungen hängt immer stark von deren Wahl ab. Bei der Wahl eines Basissatzes ist immer zwischen der Eignung für das entsprechende chemische System und dem bei größeren Basissätzen erhöhten Rechenaufwand abzuwägen. So haben sich für bestimmte Problematiken entsprechende Basissätze bewährt oder wurden sogar extra dafür entwickelt. Die kleinsten Basissätze für ein bestimmtes Atom, wie z.B. der STO-3G Basissatz, ${ }^{[102]}$ bei dem jedes Slater-Orbital durch drei einfache Gauss-Funktionen angenähert wird, beinhalten nur eine minimale Anzahl von Basisfunktionen. Mit Basissätzen dieser Art werden i.a. schlechte Ergebnisse erzielt und sollten nur verwendet werden, wenn größere Basissätze aufgrund des Umfangs des zu berechnenden Systems nicht eingesetzt werden können. Bessere Resultate werden mit Basissätzen erreicht, bei denen die Anzahl der Basisfunktionen pro Atom erhöht wurde. 3-21G und 6-31G sind Vertreter dieser Art.[103] Sie werden als Split-Valence Basissätze (Double-Zeta-Basissätze) bezeichnet, da zu jedem äußeren Orbital zwei Basisfunktionen (zwei Zeta-Funktionen) hinzugefügt wurden, wobei die besetzten Orbitale durch "voluminösere" Funktionen angenähert werden als die unbesetzten. Insgesamt resultiert hieraus eine Flexibilität in der Größe der Orbitale. Eine Flexibilität in Bezug auf die Form erlauben Basissätze mit Polarisationsfunktionen wie z.B. 6-31G(d), bei denen zusätzlich d-Funktionen zu den Basisfunktionen der Schweratome hinzugefügt werden oder 6-31G(d,p) mit zusätzlichen p-Funktionen auf den Wasserstoffatomen und d-Funktionen auf den Schweratomen.[104, 105] Diffuse Funktionen erlauben die Beschreibung von weitreichenden Wechselwirkungen wie sie beispielsweise bei Wasserstoffbrückenbindungen 
auftreten. Hierbei werden z.B. im Fall vom 6-31++G(d) Basissatz weitere Orbitale (mit kleineren Koeffizienten) zu Schwer- und Wasserstoffatomen addiert.[106] Eine weitere Möglichkeit der genaueren Beschreibung des chemischen Systems ist die Verwendung von Triple-Zeta-Basissätzen wie 6-311G.[107] Dabei werden drei Funktionen unterschiedlicher Größe für jeden Orbitaltyp benutzt.

Alle Strukturoptimierungen wurden mit dem GAUSSIAN98 Programmpaket[108] auf DFT-Niveau mit dem drei Parameter Hybridfunktional B3LYP (Becke-3-Lee-Yang-Parr) durchgeführt. Zur Überprüfung der Natur aller stationären Punkte (NIMAG $=0$ für Minima und = 1 für Übergangszustände) wurden die harmonischen Vibrationsfrequenzen (298 K, nicht skaliert) berechnet. Daraus gehen auch die Nullpunktsenergien (ZPVE, unkorrigiert) und die thermischen Korrekturen der Enthalpien sowie Gibbs'sche Energien hervor, die als Grundlage für die Berechnungen dienen. Exaktere absolute Energien wurden gegebenenfalls durch Berechnungen mit größeren Basissätzen für alle stationären Punkte erhalten. Da DFTMethoden auf B3LYP-Niveau schon für die Berechnung vieler Radikalreaktionen herangezogen wurden und gute Ergebnisse erbrachten,[109-111] aber mit dieser Methode eine Berechnung von kohlenstoffzentrierten halogensubstituierten Radikalen bislang nicht auf diesem Niveau durchgeführt wurde, sollten experimentelle Daten durch Computerberechnungen nachvollzogen werden. Die berechneten $\Delta H_{298}^{*}$ Werte für die H-Abstraktion von Cyclohexan und Isobutan durch das Trichlormethylradikal sind in guter Übereinstimmung mit den entsprechenden experimentellen Werten (Schema 7).[112, 113] Das genaue Niveau für eine Rechnung wird immer an entsprechender Stelle angegeben.

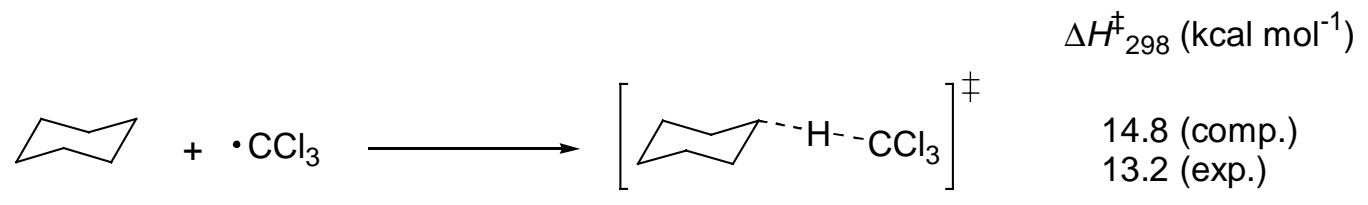

$$
\begin{aligned}
& \lambda+\cdot \mathrm{CCl}_{3} \longrightarrow\left[\begin{array}{ll}
\left.--\mathrm{H}^{-}-\mathrm{CCl}_{3}\right]^{\ddagger} & 9.9 \text { (comp.) } \\
10.2 \text { (exp.) }
\end{array}\right.
\end{aligned}
$$

Schema 7. Vergleich der computerberechneten [B3LYP/6-311+G** $(\mathrm{C}, \mathrm{H}, \mathrm{Cl}, \mathrm{Br}) / /$ B3LYP/6-31G** $(\mathrm{C}, \mathrm{H}, \mathrm{Cl}, \mathrm{Br})]$ und experimentellen $\Delta \mathrm{H}_{298}^{\ddagger}$ Werte für die $\mathrm{H}-$ Abstraktion von Cyclohexan ${ }^{[112]}$ und Isobutan ${ }^{[113]}$ durch ein $\mathrm{CCl}_{3}$ Radikal. 


\section{Mechanistische Untersuchungen}

\subsection{Systematische Untersuchung der allgemeinen Reaktionsbedingungen}

\subsubsection{Einleitung}

Die Halogenierung unter PT-Bedingungen ist konzeptionell eine neue Reaktion. Deswegen ist es notwendig, zunächst die optimalen Reaktionsbedingungen zu ermitteln. Zum einen ermöglicht eine genaue Kenntnis über die Abhängigkeiten der Reaktionen die effiziente Anwendung und zum anderen lassen sich daraus auch Schlüsse auf den Reaktionsmechsnismus ziehen. Die wichtigste und auffälligste Eigenschaft der neuen Halogenierungsmethode ist, daß sie in einem Mehrphasensystem abläuft. Dieses System kann aus zwei nicht miteinander mischbaren flüssigen oder aus einer flüssigen und einer festen Phase bestehen. Eine dieser Phasen ist immer eine organische Phase, in der das zu halogenierende Edukt gelöst ist oder besteht aus dem zu halogenierenden Substrat (z.B. ein KW) selbst. Die andere Phase ist eine wäßrige Hydroxidlösung kann aber auch nur aus dem festen Metallhydroxid ( $\mathrm{M}=$ Alkali oder Erdalkali) bestehen. Die Reaktionen müssen teilweise katalysiert durch PTK durchgeführt werden; in manchen Fällen, läuft die Reaktion aber auch sehr effektiv unkatalysiert ab. Zusätzlich $\mathrm{zu}$ den bislang beobachteten möglichen Reaktionsbedingungen führt der Umstand, daß Bromierungen, Iodierungen und sogar Chlorierungen mit der neuen Methodik durchgeführt werden können, zu einer sehr großen Anzahl von Kombinationsmöglichkeiten, die es zu untersuchen galt. Eine systematische Analyse darüber, welche Bedingungen für die Halogenierung eines bestimmten Substrates ideal sind, war deshalb unvermeidlich. Allerdings mußten einige der eben angesprochenen Variablen festgelegt werden, weil ansonsten die Untersuchungen zu umfangreich gewesen wären.

Die Möglichkeit dazu war durch die Selektion nur eines bestimmten Substrates gegeben, wobei die Wahl auf Tricyclo[3.3.1.1 $\left.1^{3,7}\right]$ decan (Adamantan 1) als Modellverbindung fiel, da dieser Käfigkohlenwasserstoff eine Reihe von Vorteilen bietet. In diesem Molekül gibt es nur zwei verschiedene Positionen, wo eine Halogenierung stattfinden kann. Dies ermöglichte eine schnelle Analyse der Produktgemische. Des weiteren können aufgrund der Regioselektivität der Substitution, auch durch Vergleich mit anderen gut untersuchten Reaktionen an diesem Molekül, Rückschlüsse auf den Reaktionsmechanismus gezogen werden. Ein weiterer Vorteil ist, daß die gebildeten Haloadamantanverbindungen sehr stabil 
gegenüber den basischen Reaktionsbedingungen sind, da keine Eliminierung möglich ist (Bredt'sche Regel) ${ }^{[114]}$ und auch die Hydrolyse im Vergleich zu anderen Halogenalkanen relativ langsam vonstatten geht.[115] Im folgenden wurden die verschiedenen Halogenierungen getrennt voneinander untersucht.

\subsubsection{Die Bromierung}

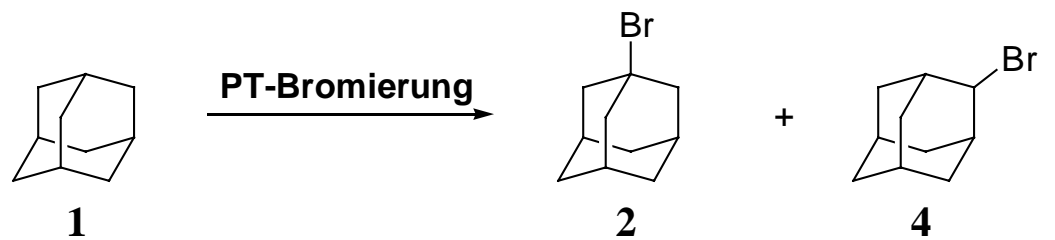

Schema 8. Allgemeines Reaktionssystem zur Untersuchung der optimalen Reaktionsbedingungen für die PT-Bromierung (AV1)

Wie erste Untersuchungen gezeigt haben, verlaufen die Bromierungen von Adamantan mit festen Hydroxiden ausgesprochen langsam. Deswegen wurden die folgenden Abhängigkeiten nur für rein "flüssige" PT-Systeme ermittelt. Alle Bromierungen wurden bei $40^{\circ} \mathrm{C}$ Ölbadtemperatur durchgeführt, damit auch Reaktionssysteme, in denen die Halogenierung nur langsam abläuft, in einem angemessenen Zeitrahmen untersucht werden konnten. Die ermittelten Umsätze beziehen sich auf die Bildung von 2 und $\mathbf{4}$ und wurden durch GC/MS-Analysen der entsprechenden Reaktionsmischungen bestimmt (AV1).

\subsubsection{Variation des Lösungsmittels}

Zunächst erschien es sinnvoll, einen Augenmerk auf die Abhängigkeit vom Lösungsmittel mit und ohne den Einfluß eines Katalysators zu legen (Abb. 4). Für die katalysierten Reaktionen wurde ein PTK gewählt, der sich bereits bei vielen verschiedenen PTK-Reaktionen als effektiv erwiesen hat,[21] nämlich Tetra- $n$-butylammoniumbromid (TBABr). Die Lösungsmittel mußten die Bedingung erfüllen, gegenüber radikalischen Reaktionsbedingungen stabil zu sein. Neben Dichlormethan $(\varepsilon=8.9)^{[116]}$ und Benzol $(\varepsilon=$ 2.3),[116] die klassischerweise bei Radikalreaktionen Anwendung finden, $[6,7,64]$ erschienen fluorierte Lösungsmittel wie Fluorbenzol $(\mathrm{PhF}, \varepsilon=5.3)^{[116]}$ und 1,1,1-Trifluortoluol (TFT, $\varepsilon$ 
$=9.2)^{[116]}$ aufgrund der Tatsache ideal, daß sie normalerweise sehr inert sind. Dabei zeigte sich (Abb. 4), daß die Produktbildungsgeschwindigkeit der Reaktionen zumindest qualitativ aber nicht linear von der Polarität des Solvens abhängt. Je höher die Polarität desto höher der Umsatz. Dies könnte einerseits seine Ursache in der besseren Löslichkeit des PTKHydroxidionenpaares in der organischen Phase haben, was einen Extraktionsmechanismus ${ }^{[44]}$ wahrscheinlich machen würde. Allerdings könnte eine höhere Polarität des Solvens auch eine Vergrößerung der Phasengrenze zur Folge haben, was auf einen Grenzflächenmechanismus ${ }^{[45]}$ oder Interphasenmechanismus ${ }^{[46]}$ hindeuten könnte. Letzterer Mechanismus kann zumindest für alle Lösungsmittel bis auf Dichlormethan ausgeschlossen werden, da dieser sich durch Unabhängigkeit von Katalysatoreinflüssen auszeichnet.[46] Die Rolle von Dichlormethan bedurfte gesonderter Untersuchungen (s.u.).

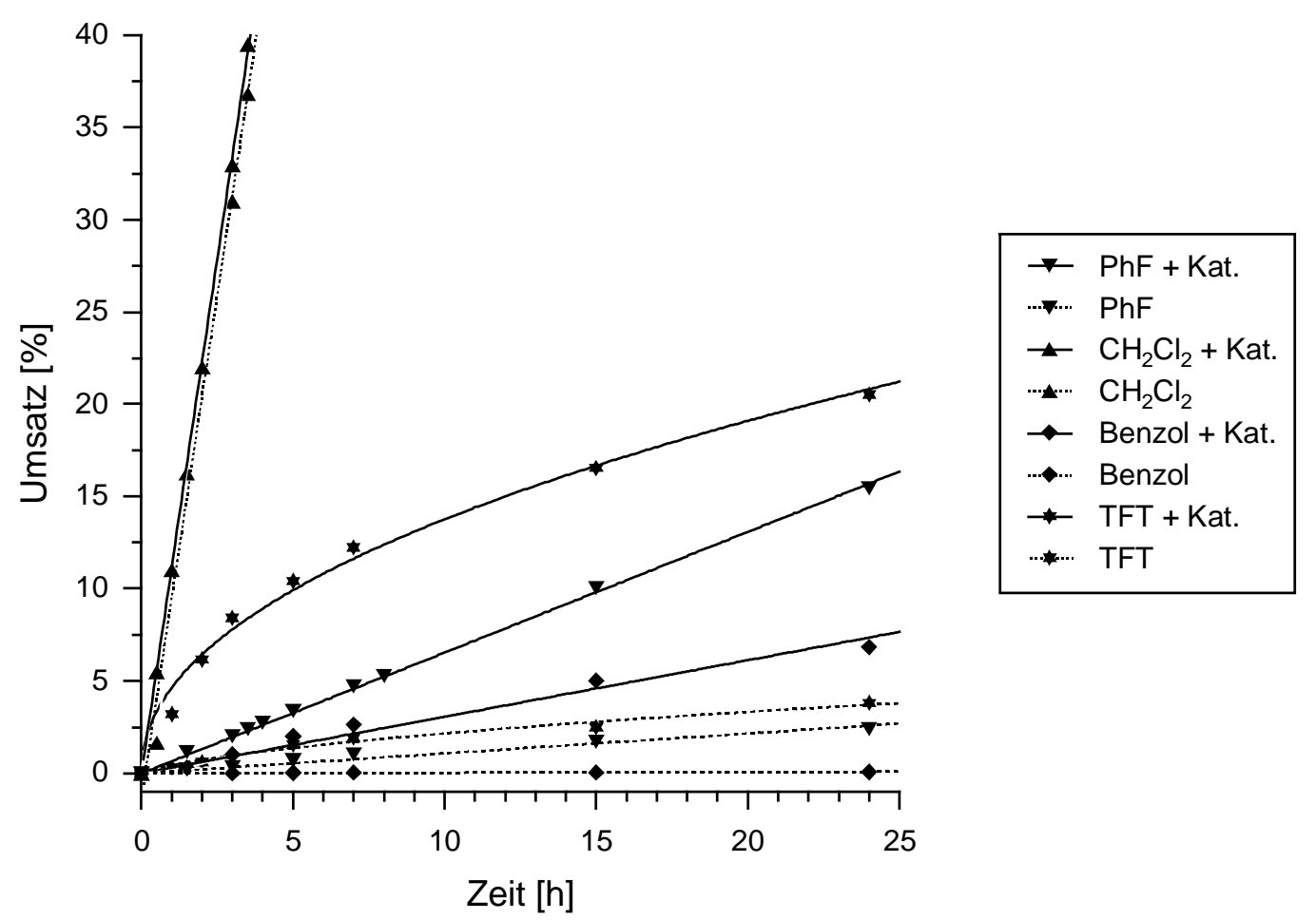

Abbildung 4. Bestimmung der Lösungsmittelabhängigkeit [AV1a, $40^{\circ} \mathrm{C} /$ Solvens \pm TBABr (10 mol\%) / $\mathrm{CBr}_{4} /$ aq. $\left.\mathrm{NaOH}(50 \%)\right]$ bei der Bromierung von 1 mit und ohne Einfluß eines PTK.

Da Dichlormethan eine Sonderrolle zu spielen scheint, Benzol ineffektiv ist und Trifluortoluol in einer nicht weiter untersuchten Weise in das Reaktionsgeschehen eingreift (erkennbar an der gekrümmten Form der Kurve, TFT + Kat., Abb. 4), war Fluorbenzol das geeignetste 
Lösungsmittel für die folgenden Untersuchungen, da die PT-Bromierung in diesem Lösungsmittel auch die größte Abhängigkeit vom Katalysator zeigt.

\subsubsection{Variation des Katalysators}

Im Hinblick auf die Verwendung chiraler PTK ist ein wichtiger Faktor zwischen einem Extraktionsmechanismus[44] oder Grenzflächenmechanismus[45] unterscheiden $\mathrm{zu}$ können. Hierfür ist die Auswirkung einer Variation des PTK aufschlußreich. Eine Reaktion, die dem Extraktionsmechanismus gehorcht, wird am stärksten durch PTK katalysiert, deren Kationen einen möglichst lipophilen Charakter aufweisen, da das reaktive Agens in die organische Phase transportiert werden muß. Im Gegensatz dazu zeichnen sich Grenzflächenmechanismen durch eine Katalyse mit PTK mit gut zugänglicher Ladung aus, weil hier eine Fixierung eines PTK-Substratkomplexes an der Phasengrenze notwendig ist.

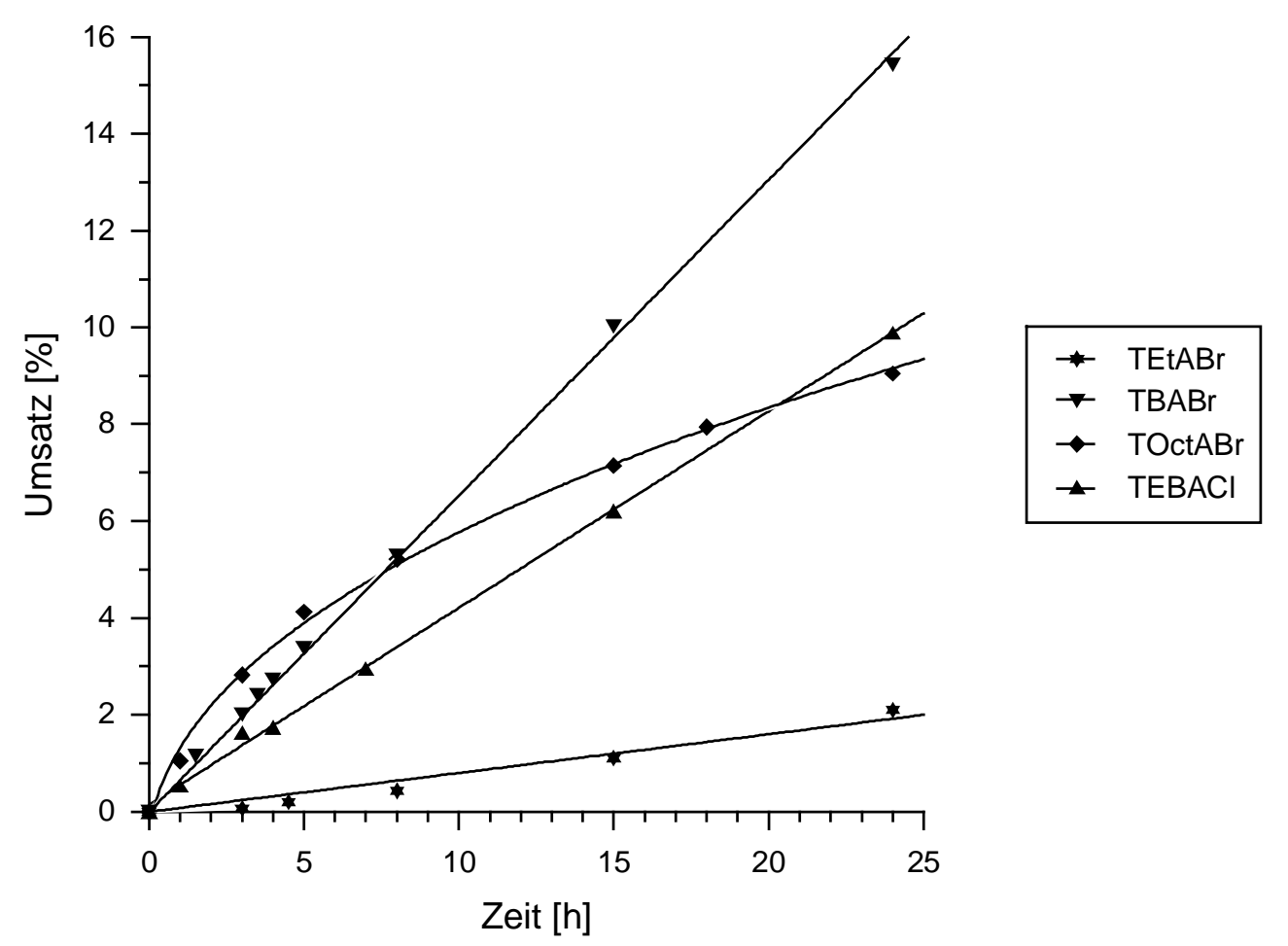

Abbildung 5. Bestimmung der PTK Abhängigkeit [AV1b, 40 ${ }^{\circ} \mathrm{C} / \mathrm{PhF} / \mathrm{PTK}(10 \mathrm{~mol} \%)$ / $\mathrm{CBr}_{4} /$ aq. $\left.\mathrm{NaOH}(50 \%)\right]$ bei der Bromierung von 1

Da die Reaktionsgeschwindigkeit mit steigender Kettenlänge also steigender Lipophilie des PTK-Kations zunahm (Abb. 5), ist ein Extraktionsmechanismus wahrscheinlich. Bei dieser 
Untersuchungsreihe zeigte sich auch, daß $\mathrm{TBABr}$ der geeignetste Katalysator für die PTK Bromierungen ist. Bemerkenswert ist auch, daß TOctABr wahrscheinlich an der Reaktion partizipiert. Eine Nebenreaktion in Form der Bromierung der Seitenketten dieses PTK und/ oder der Zerfall des Katalysators unter den stark basischen Bedingungen durch eine Art Hoffmannabbau ${ }^{[50]}$ ist vermutlich der Grund für das Abfallen der Reaktionsgeschwindigkeit (TOctABr, Abb. 5). TEBACl wurde auch überprüft, weil viele durch Hydroxidionen initiierte PTK-Reaktionen durch diesen PTK effektiv beschleunigt werden.[48] In dem Fall der PTKBromierungen ist er aber nicht besonders effizient.

\subsubsection{Variation der Basenkonzentration}

Die Untersuchung der Reaktion in Fluorbenzol mit TBABr als PTK bezüglich der Abhängigkeit von der Basenkonzentration (Abb. 6) zeigte, daß die Produktbildungsgeschwindigkeit mit steigender Basenkonzentration zunimmt und ihr Maximum mit der maximalen Konzentration der Base erreicht. Dies deutet darauf hin, daß das Hydroxidion tatsächlich eine entscheidende Rolle bei den PTK-Bromierungen spielt.

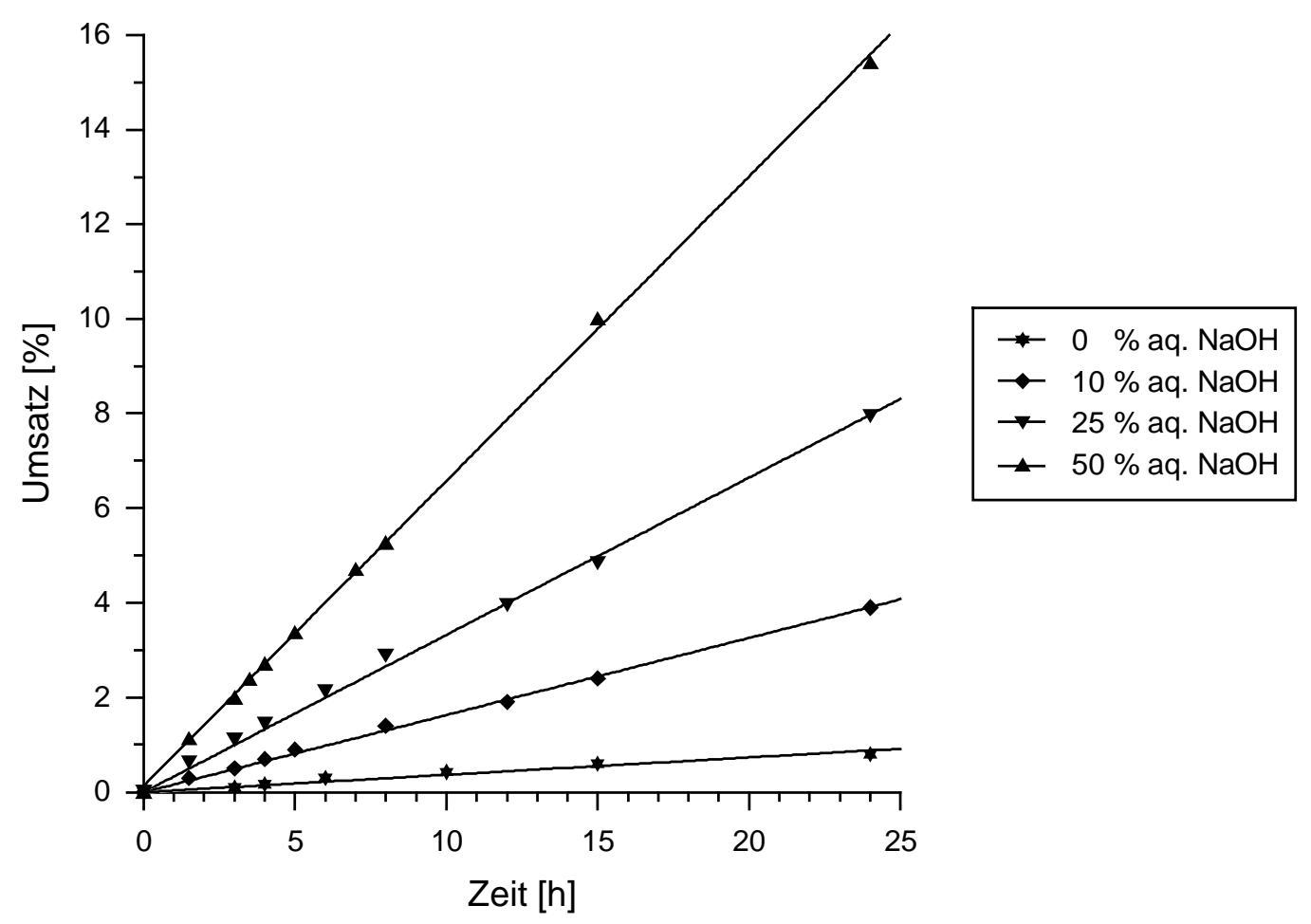

Abbildung 6. Bestimmung der Abhängigkeit von der Basenkonzentration [AV1c, $40^{\circ} \mathrm{C} /$ $\mathrm{PhF} / \mathrm{TBABr}(10 \mathrm{~mol} \%) / \mathrm{CBr}_{4} /$ aq. $\left.\mathrm{NaOH}(\mathrm{X} \%)\right]$ bei der Bromierung von 1 
Zwei Faktoren können für die gemachten Beobachtungen verantwortlich sein. Zum einen könnte die steigende Basenkonzentration in der wäßrigen Phase eine Erhöhung der Hydroxidionenkonzentration in der organischen Phase zur Folge haben und deswegen die Reaktionsgeschwindigkeit erhöhen. Zum anderen würde eine gesteigerte Basenkonzentration dazu führen, daß das PTK-Hydroxidionenpaar weniger stark solvatisiert in die organische Phase übergeht $[48,49]$ und damit die Reaktivität des Hydroxidions entscheidend gesteigert wird, was ebenfalls die Reaktionsrate erhöhen würde. Welche dieser Faktoren ausschlaggebend ist, oder ob eventuell beide Faktoren für die Beschleunigung der Reaktion verantwortlich sind, konnte anhand der bisherigen Experimente nicht ermittelt werden. Diese Entscheidung zu treffen wäre insofern wichtig, als daß diese über das genaue Reaktionsverhalten des Hydroxidions Aufschluß geben könnte, also ob wenig solvatisierte Hydroxidionen unter PT-Halogenierungsbedingungen eher als SET-Reduktionsmittel reagieren oder die solvolytischen und basischen Eigenschaften für die Initiierung der Reaktion verantwortlich sind.

\subsubsection{Variation der Katalysatorkonzentration}

Schließlich wurde noch der Einfluß der Katalysatorkonzentration ebenfalls in Fluorbenzol als Lösungsmittel, $50 \%$ aq. $\mathrm{NaOH}$ und variabler Konzentration an $\mathrm{TBABr}$ näher untersucht (Abb. 7). Dabei wurde festgestellt, daß eine Erhöhung der Konzentration an $\mathrm{TBABr}$ auch eine Erhöhung der Reaktionsgeschwindigkeit zur Folge hat. Es zeigte sich, daß die Zugabe von 10 mol\% TBABr den größten relativen Anstieg der Reaktionsrate nach sich zieht. Eine höhere Konzentration an $\mathrm{TBABr}$ erhöht zwar immer noch die Reaktionsgeschwindigkeit, allerdings nicht mehr so effektiv. Aus diesem Grund sollte für PTBromierungen im allgemeinen eine Konzentration von 10 mol\% des entsprechenden PTK (i.A. TBABr) verwendet werden. 


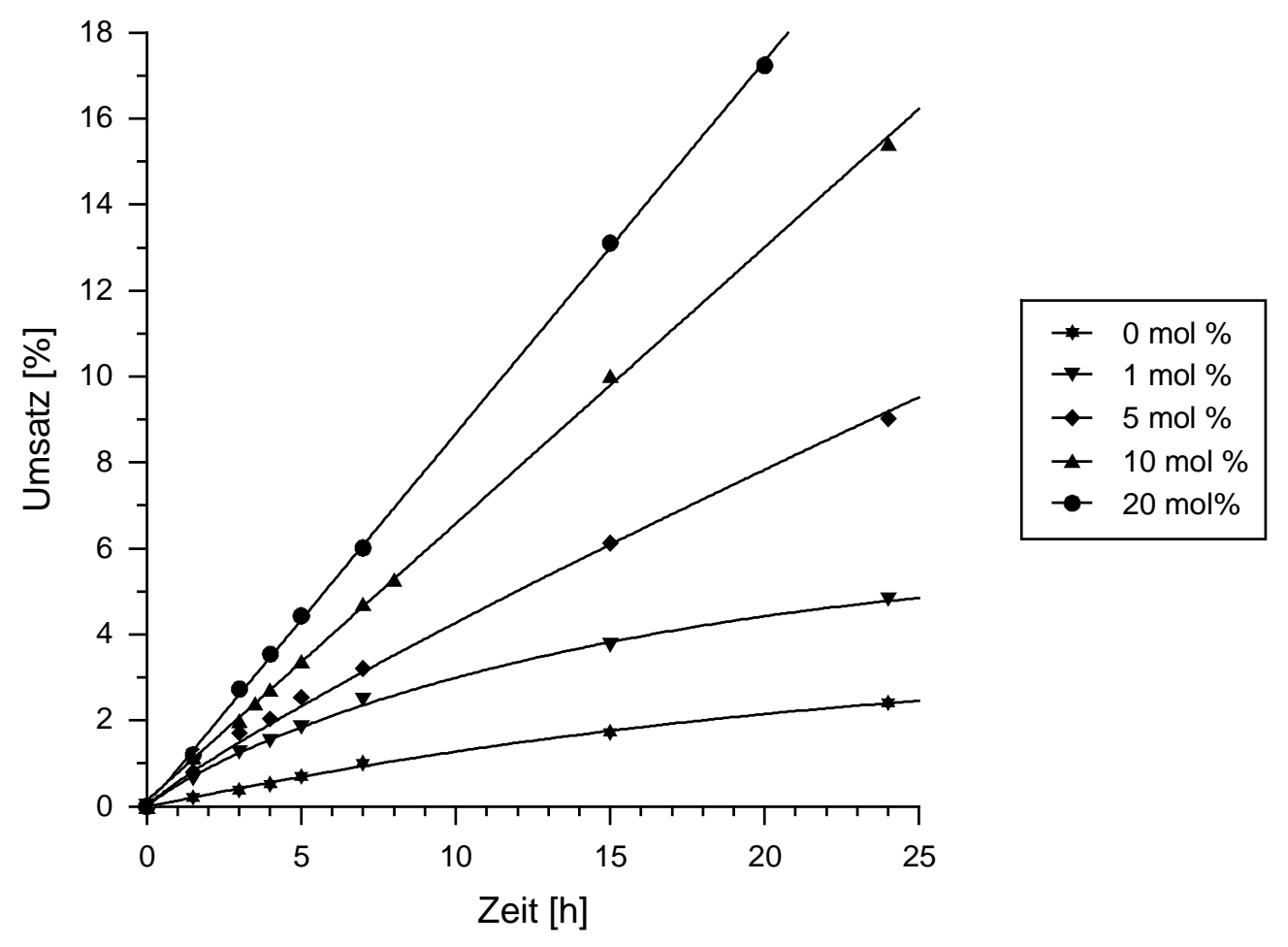

Abbildung 7. Bestimmung der Abhängigkeit von der Katalysatorkonzentration [AV1d, $40^{\circ} \mathrm{C} / \mathrm{PhF} / \mathrm{TBABr}\left(\mathrm{X}\right.$ mol\%) $/ \mathrm{CBr}_{4} /$ aq. $\left.\mathrm{NaOH}(50 \%)\right]$ bei Bromierung von 1

\subsubsection{Weitere allgemeine Beobachtungen bei unsystematischer Variation diverser Reaktionsbedingungen}

Weitere Faktoren, die die Bromierung unter PTK-Bedingungen beeinflussen können, wurden nicht systematisch untersucht. Allerdings konnten u.a. bei der Anwendung der Bromierung weitere Beobachtungen gemacht werden, die schon an dieser Stelle Erwähnung finden sollten. So kann durch Erhöhung der Reaktionstemperatur die Reaktionsgeschwindigkeit signifikant gesteigert werden und auch im Vergleich zu 1 weniger reaktive Edukte (wie z.B. halogenierte Adamantane, Kap. 3.2) zur Reaktion gebracht werden. Auch durch die Erhöhung der Konzentration von Tetrabrommethan kann die Reaktionszeit stark verkürzt werden.

Die im Rahmen der vorangegangenen Untersuchungen gemachte Beobachtung, daß die Bromierung von 1 in Dichlormethan sehr viel schneller abläuft als in allen anderen überprüften Lösungsmitteln, und dies auch ohne den Einfluß eines Katalysators, sollte jetzt genauer untersucht werden. Prinzipiell könnten zwei Gründe für diese Beobachtungen verantwortlich gemacht werden. Entweder verläuft die Reaktion in $\mathrm{CH}_{2} \mathrm{Cl}_{2}$ nicht mehr gemäß eines Extraktionsmechanismus oder das Hydroxidanion ist nicht mehr die reaktive Spezies 
und es tritt in Dichlormethan ein anderer Reaktionsmechanismus auf. Denkbar wäre auch, daß hier zwei verschiedenen Mechanismen nebeneinander ablaufen (s.u.).

Um dies zu überprüfen wurde die Reaktion in demineralisiertem Wasser mit dem Ergebnis durchgeführt, daß auch ohne jeglichen Zusatz von Base die Reaktion ohne wesentliche Änderung der Produktbildungsgeschwindigkeit im Vergleich zu den basischen Reaktionsbedingungen abläuft (Nr. 4, 5; Tab. 3). Allerdings wurde auch die Bildung einer großen Menge an Hydrolyseprodukt, nämlich 1-Adamantanol (AdOH, Tab. 3), beobachtet. Als Kontrollversuch wurde 1 ohne jeglichen Zusatz einer wäßrigen Phase, den gleichen Reaktionsbedingungen ausgesetzt, wobei keinerlei Reaktion auftrat (Nr. 6; Tab. 3). Die Hydrolyse der Bromadamantane kann durch Zusatz geringer Mengen an Base weitestgehend zurückgedrängt werden (Nr. 9, 10; Tab. 3), gleichzeitig wird die Produktbildungsgeschwindigkeit wiederum stark erhöht.

Tabelle 3. Bromierung von 1 bei Variation der Reaktionsbedingungen $\left(40^{\circ} \mathrm{C} / \mathrm{CBr}_{4}\right)$; die Prozentangaben entsprechen der Summe der $3^{\circ}$ u. $2^{\circ}$ substituierten Produkte (AV1e).

\begin{tabular}{ccccccc}
\hline \# & Solvens & Wäßrige Phase & Zeit [h] & AdBr [\%] & AdCl [\%] & AdOH [\%] \\
\hline $\mathbf{1}$ & $\mathrm{CH}_{2} \mathrm{Cl}_{2}$ & $\mathrm{NaOH}($ aq. 50\%) & 5 & 39 & 2 & $<1$ \\
$\mathbf{2}$ & $\mathrm{CH}_{2} \mathrm{Cl}_{2}$ & $\mathrm{H}_{2} \mathrm{O}($ kat. NaOH) & 5 & 43 & 1 & 4 \\
$\mathbf{3}$ & $\mathrm{CH}_{2} \mathrm{Cl}_{2}$ & $\mathrm{H}_{2} \mathrm{O}($ kat. $\mathrm{NaOH})$ & 24 & 75 & 2 & 5 \\
$\mathbf{4}$ & $\mathrm{CH}_{2} \mathrm{Cl}_{2}$ & $\mathrm{H}_{2} \mathrm{O}$ & 5 & 17 & $<1$ & 1 \\
$\mathbf{5}$ & $\mathrm{CH}_{2} \mathrm{Cl}_{2}$ & $\mathrm{H}_{2} \mathrm{O}$ & 20 & 23 & 1 & 18 \\
$\mathbf{6}$ & $\mathrm{CH}_{2} \mathrm{Cl}_{2}$ & ohne & 20 & - & - & - \\
$\mathbf{7}$ & $\mathrm{CH}_{2} \mathrm{Cl}_{2}$ & $\mathrm{HBr}(\mathrm{aq} .10 \%)$ & 5 & 10 & - & 1 \\
$\mathbf{8}$ & $\mathrm{CH}_{2} \mathrm{Cl}_{2}$ & $\mathrm{HBr}(\mathrm{aq} .10 \%)$ & 20 & 40 & 2 & 5 \\
$\mathbf{9}$ & $\mathrm{CH}_{2} \mathrm{Br}_{2}$ & $\mathrm{H} 2 \mathrm{O}(\mathrm{kat} . \mathrm{NaOH})$ & 20 & 28 & - & 2 \\
$\mathbf{1 0}$ & $\mathrm{CHCl}_{3}$ & $\mathrm{H} 2 \mathrm{O}(\mathrm{kat} . \mathrm{NaOH})$ & 20 & 29 & 1 & 2 \\
$\mathbf{1 1}$ & $\mathrm{CF}_{2} \mathrm{Br}_{2}$ & $\mathrm{H} \mathrm{H}_{2} \mathrm{O}$ & 24 & $<1$ & - & - \\
$\mathbf{1 2}$ & $\mathrm{C}_{6} \mathrm{H}_{5} \mathrm{~F}$ & $\mathrm{H}$ & 20 & $<1$ & - & - \\
\hline
\end{tabular}


Wird die Hydroxidionenkonzentration in der wäßrigen Phase durch Verwendung von $\mathrm{HBr}$ (aq. 10\%) noch weiter erniedrigt, findet trotzdem eine Reaktion ebenfalls mit annähernd der gleichen Produktbildungsgeschwindigkeit wie mit der rein wäßrigen Phase statt; erstaunlicherweise unter relativ geringer Bildung von $\mathrm{AdOH}$ (Nr. 7, 8; Tab. 3). Erstaunlich ist das deshalb, weil normalerweise eine solche Hydrolyse schon durch geringe Mengen an Säure katalysiert wird.[115] Die Bildung der Bromide unter den sauren Bedingungen könnte auch auf eine Art elektrophiler Aktivierung zurückzuführen sein, wie sie von Olah et al. bereits bei Alkanaktivierungen mit Supersäuren beobachtet wurde. ${ }^{[117]}$ Da sich Adamantan durch eine besondere Topologie und damit einhergehender Stabilisierung des 1-Adamantylkations auszeichnet, könnte hier vielleicht schon $\mathrm{HBr}$ (aq. 10\%) für eine solche Aktivierung ausreichen (Schema 9). Auch diese Beobachtung bedarf weiterer Untersuchungen (s. Ausblick) in weiterführenden Arbeiten.

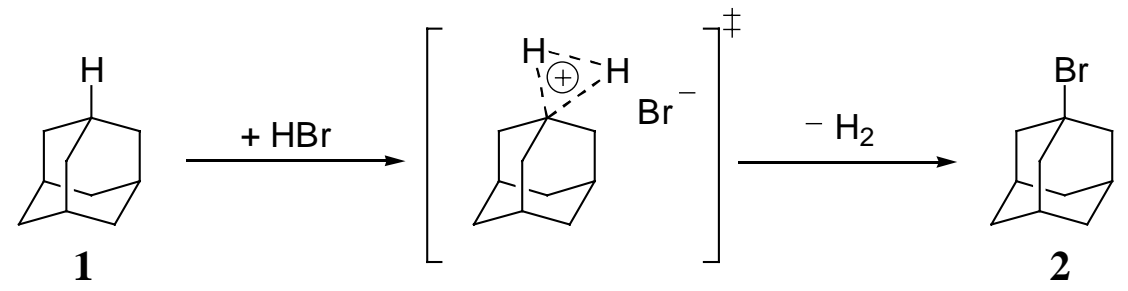

Schema 9. Möglicher elektrophiler Bildungsmechanismus bei der PTBromierung von 1 unter sauren Bedingungen ( $\mathrm{HBr}$, aq. $10 \%$ ) in $\mathrm{CH}_{2} \mathrm{Cl}_{2}$

Um zu überprüfen, ob das unterschiedliche Reaktionsverhalten in Dichlormethan in dessen Struktur begründet liegt, wurde das Lösungsmittel weiter variiert. Dabei wurde zunächst Dibrommethan als Solvens eingesetzt, mit dem Ergebnis, daß eine vergleichbar schnelle Reaktion wie in Dichlormethan auftrat (Nr. 9; Tab. 3). Im Gegensatz dazu konnten in Difluordibrommethan nach 24 h nur Spuren von Produkten detektiert werden (Nr. 11; Tab. 3). Auch in Chloroform ist die Bromierung durchführbar (Nr. 10; Tab. 3). Allerdings stagnierte die Reaktion bei ca. 30\% Umsatz durch eine basisch initiierte Disproportionierung von Halomethanen (z.B. $\mathrm{CBr}_{4}+\mathrm{CHCl}_{3} \rightarrow \mathrm{CHBr}_{3}+\mathrm{CBrCl}_{3}$ ). ${ }^{[39,42]}$ Erkennbar wird das dadurch, daß nach $20 \mathrm{~h}$ in der Reaktionsmischung im Gegensatz zu den anderen Bromierungen nicht einmal mehr Spuren von Tetrabrommethan nachweisbar (GC/MS-Analyse) waren. Fazit dieser Experimente ist, daß auch durch die speziellen Experimente, die besondere Rolle von $\mathrm{CH}_{2} \mathrm{Cl}_{2}$ bei den PT-Halogenierungen nicht geklärt werden konnte. 


\subsubsection{Die Iodierung}

Auch die Iodierungen wurden hinsichtlich der optimalen Reaktionsbedingungen untersucht. Wiederum war das Ziel dieser Untersuchungen zum einen die Reaktion möglichst effektiv nutzen zu können und zum anderen mehr über den Mechanismus der Iodierung herauszufinden. Im Gegensatz zu den Bromierungen wurden dabei aber nicht der Reaktionsverlauf über einen gewissen Zeitraum beobachtet, sondern stichprobenartig jeweils $\mathrm{zu}$ einem bestimmten Zeitpunkt. Als Reaktionssystem wurde aus den schon erwähnten Gründen (Kap 2.1.1) wieder die Halogenierung von Adamantan gewählt. Die Bestimmung des Umsatzes erfolgte durch die Integration separater Signale im ${ }^{1} \mathrm{H}-\mathrm{NMR}$ Spektrum der Reaktionsmischung (s. AV2).

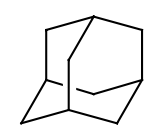

1
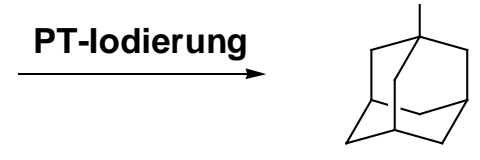

17

Schema 10. Allgemeines Reaktionssystem zur Untersuchung der optimalen Reaktionsbedingungen für die PT-Iodierung (AV2)

\subsubsection{Variation des Lösungsmittels}

Zunächst wurde die Lösungsmittelabhängigkeit bei einer konstanten Konzentration an $\operatorname{TBABr}(10 \mathrm{~mol} \%)$ durchgeführt, einem Katalysator, der sich bereits bei den PTBromierungen als am effektivsten erwiesen hat (Abb. 5). Dabei zeigte sich, daß die Iodierung in $\mathrm{PhF}$ am schnellsten abläuft (Abb. 8). Insgesamt konnte hier wie auch bei den Bromierungen eine "grobe" Korrelation mit der Polarität des Lösungsmittels festgestellt werden. Zwei der Lösungsmittel, nämlich Dichlormethan und Fluorbenzol wurden auch dahingehend überprüft, ob überhaupt eine Katalysatorabhängigkeit vorhanden ist. Während ohne Zusatz eines Katalysators der Umsatz in Dichlormethan um ca. die Hälfte reduziert wurde, fand in Fluorbenzol so gut wie keine Reaktion statt. Diese Beobachtung machte letzteres für alle folgenden Untersuchungen wieder zum Lösungsmittel der Wahl. 


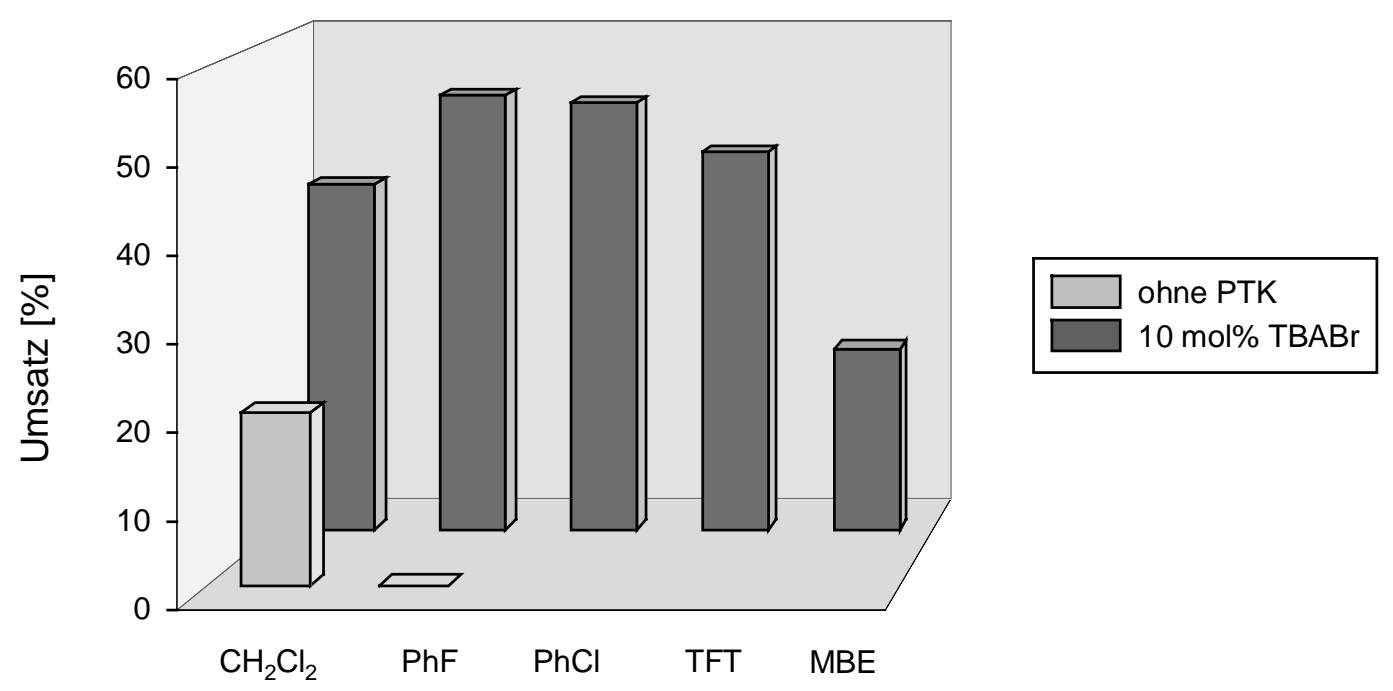

Abbildung 8. Bestimmung der Lösungsmittelabhängigkeit $\left[\mathrm{AV} 2 \mathrm{a}, 40^{\circ} \mathrm{C} /\right.$ Solvens \pm $\operatorname{TBABr}(3 \mathrm{~mol} \%) / \mathrm{CHI}_{3} /$ aq. $\mathrm{NaOH}(50 \%) / 48 \mathrm{~h}$ ] bei der Iodierung von 1 mit und ohne Einfluß eines Phasentransferkatalysators $(\mathrm{MBE}=$ Methyl-t-Butylether $)$

Da die Bromierung in Dichlormethan auch ohne jeglichen Zusatz von Base und sogar im sauren Milieu abläuft, mußte dies auch bei den Iodierungen überprüft werden. Während in Dichlormethan ohne Zusatz einer wäßrigen Phase überhaupt keine Reaktion stattfindet, konnten bei Zusatz von demineralisiertem Wasser nach 24 h nur Spuren von 17 (GC/MSAnalyse) nachgewiesen werden.

\subsubsection{Variation des Katalysators}

Als nächster Faktor wurde die Katalysatorabhängigkeit der Iodierung näher untersucht (Abb. 9) Dabei war wiederum von Interesse, ob es sich mechanistisch wie bei den Bromierungen um eine Extraktion der reaktiven Spezies in die organische Phase handelt oder ob eher ein Grenzflächenmechanismus vorliegt. Die größere Effizienz der Katalysatoren mit steigender Kettenlänge deutet auf einen Extraktionsmechanismus hin. Der leicht reduzierte Umsatz mit TOctABr liegt wahrscheinlich wie bei den PT-Bromierungen in einer Teilnahme dieses Katalysators an der Reaktion begründet, eventuell in Form einer Seitenketteniodierung, was mit steigender Kettenlänge stärker zum Tragen kommt.

Wie bei den Bromierungen ist auch $\mathrm{TBABr}$ der geeignetste Katalysator für die Iodierungen. TEBACl wurde ebenfalls überprüft, da wie bereits angemerkt, viele durch 
Hydroxidionen initiierte PTK-Reaktionen, durch diesen Katalysator effektiv beschleunigt werden, ${ }^{[48]}$ zeigt aber im Rahmen der Iodierungen nur mittelmäßige Aktivität.

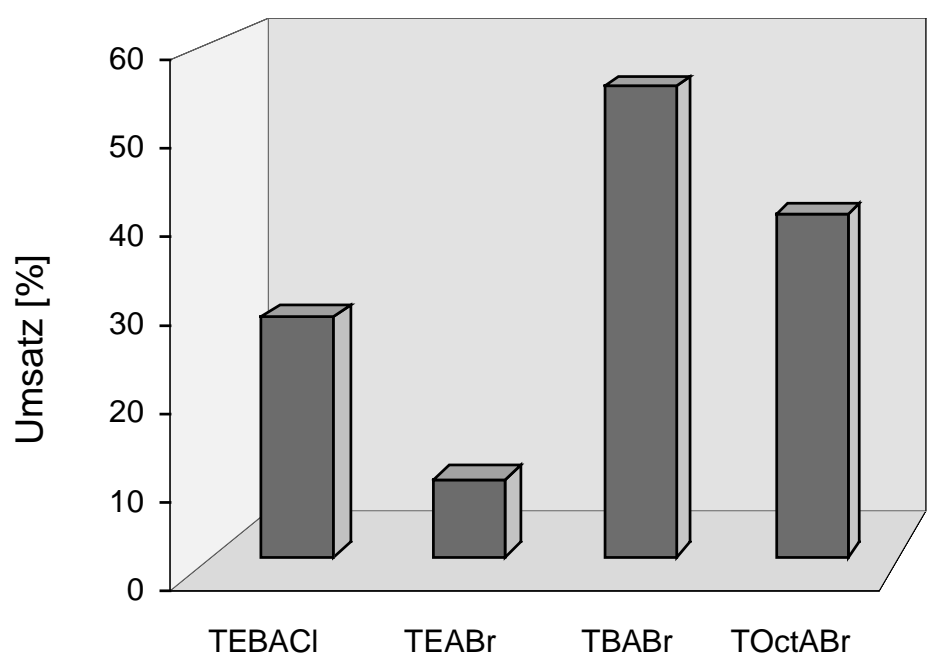

Abbildung 9. Bestimmung der Katalysatorabhängigkeit [AV2b, $40^{\circ} \mathrm{C} / \mathrm{PhF} / \mathrm{Kat}$. (3 $\mathrm{mol} \%) / \mathrm{CHI}_{3} /$ aq. $\mathrm{NaOH}(50 \%) / 48 \mathrm{~h}$ ] bei der Iodierung von 1

\subsubsection{Variation der Basenkonzentration}

Nachdem PhF als geeignetstes Lösungsmittel und TBABr als effektivster Katalysator ermittelt wurden, sollte jetzt der Einfluß der Basenkonzentration untersucht werden. Dabei stellte sich heraus, daß mit steigender Basenkonzentration auch der Umsatz der Reaktion anstieg (Abb. 10). Dieser sprunghafte Anstieg des Umsatzes zwischen 14\% und 20\% Basenkonzentration ist schwer nachvollziehen. Eventuell hängt das mit einer einhergehenden stufenweisen Desolvatisierung des PTK-Hydroxidionenpaares zusammen, was zu einer ebenfalls stufenweisen Steigerung der Reaktivität des Hydroxidions führen könnte, verursacht durch den Abbau einer Solvathülle in diesem Konzentrationsbereich. Diese Beobachtung könnte als Hinweis für einen SET-Mechanismus angesehen werden, bedarf aber noch weiterer Untersuchungen. 


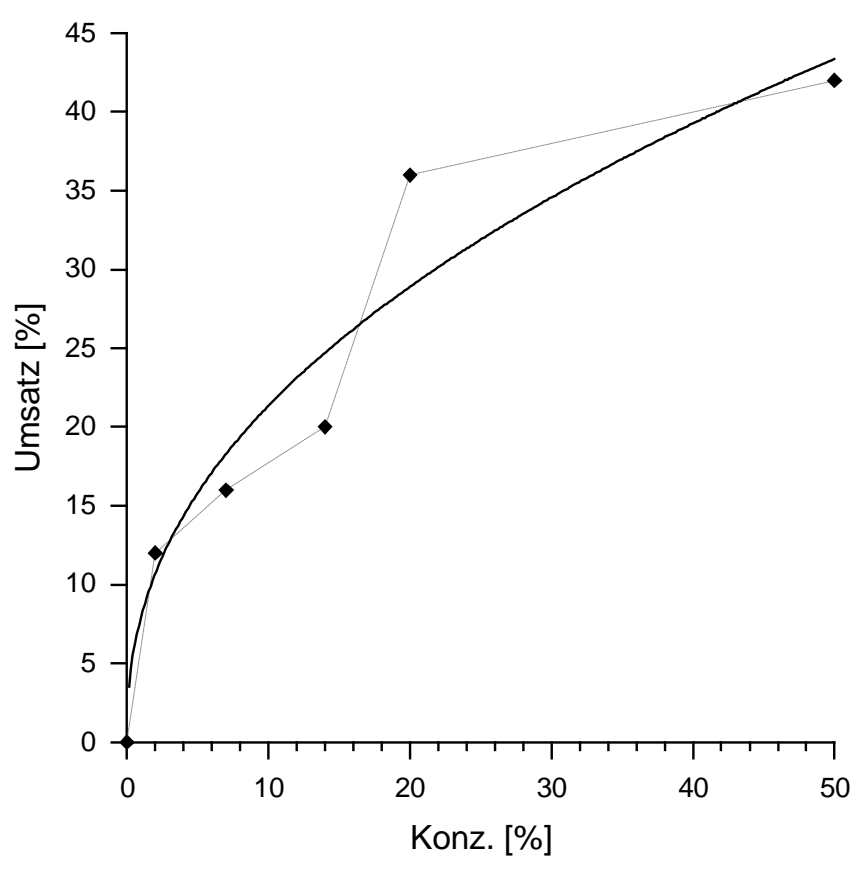

Abbildung 10. Umsatz bei Variation der Basenkonzentration [AV2c, $40^{\circ} \mathrm{C} / \mathrm{PhF} /$ $\operatorname{TBABr}(3 \mathrm{~mol} \%) / \mathrm{CHI}_{3} /$ aq. $\mathrm{NaOH}(\mathrm{X} \%) / 48 \mathrm{~h}$ ] bei der Iodierung von 1

\subsubsection{Variation der Katalysatorkonzentration}

Bei der Ermittlung der effektivsten Katalysatorkonzentration wurde festgestellt, daß mit einer Konzentration von 3 bis 10 mol\% die besten Ergebnisse erzielt werden (Abb. 11). Dies ist nicht ganz im Einklang mit den Bromierungen, wo auch noch mit 20 mol\% Katalysatorkonzentration eine signifikante Steigerung des Umsatzes erzielt werden konnte. Eventuell liegt dies an einer höheren Instabilität des Katalysators bezüglich der Reaktionsbedingungen der Iodierungen und den damit verbundenen Nebenreaktionen, die in einem Zerfall des Katalysators enden. Eine andere Möglichkeit diesen Sachverhalt zu erklären, wäre, daß wie bei der Bromierung bereits vermutet, eine Seitenkettenhalogenierung des Katalysators erfolgt, der bromierte Katalysator aber nach wie vor der Reaktion zur Verfügung steht, während der iodierte Katalysator leichter durch Eliminierung oder andere Nebenreaktionen zerfällt. Insgesamt können aber nur Vermutungen angestellt werden, wodurch der unerwartete Kurvenverlauf verursacht wird. 


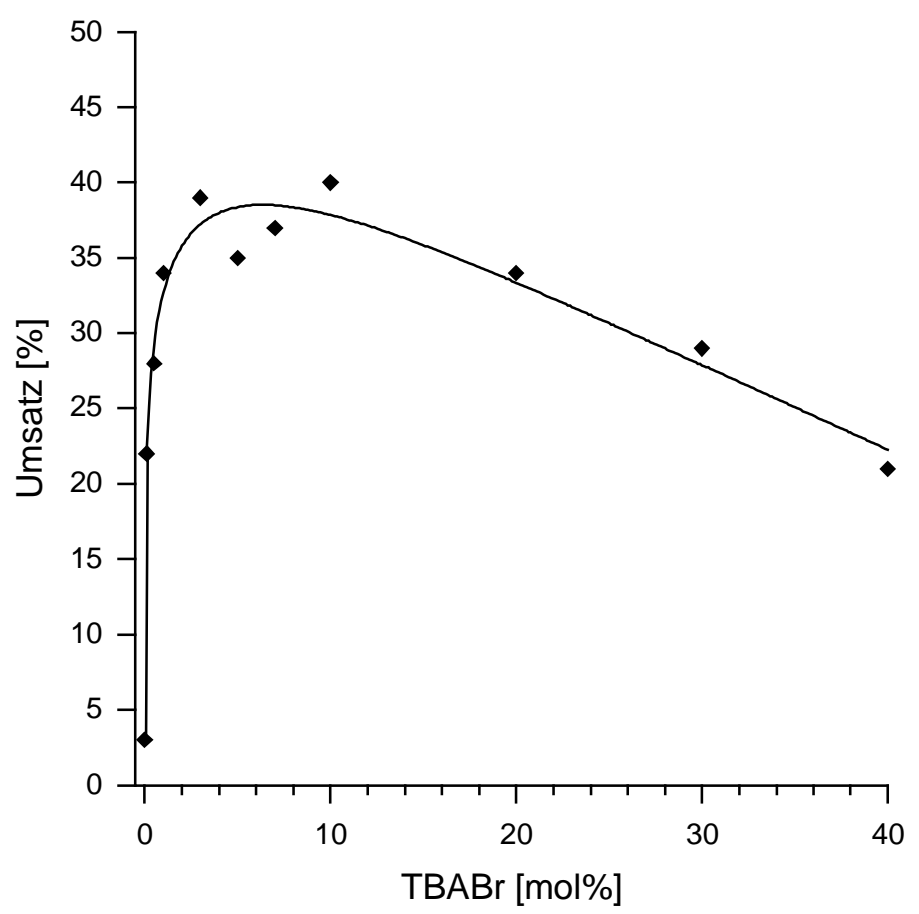

Abbildung 11. Umsatz bei Variation der Katalysatorkonzentration [AV2d, $40^{\circ} \mathrm{C} / \mathrm{PhF}$ / $\mathrm{TBABr}(\mathrm{X} \mathrm{mol} \%) / \mathrm{CHI}_{3} /$ aq. $\mathrm{NaOH}(50 \%) / 20 \mathrm{~h}$ ] bei der Iodierung von 1

\subsubsection{Variation der Base}

Im Rahmen der Untersuchung der optimalen Reaktionsbedingungen der Iodierung wurden im Gegensatz zu den PTK-Bromierungen noch weitere Faktoren, die die Reaktion beeinflussen können, überprüft. Hierzu gehört z.B. die Variation der Base (Abb. 12). Dabei wurde das Kation der Base in der Folge Li, Na, K und Cs variiert und eine Steigerung des Umsatzes in der eben genannten Reihenfolge festgestellt. Ähnliche Steigerungen der Ausbeute und der Enantioselektivitäten konnten von Corey bei Alkylierungen mit chiralen PTK unter Verwendung von $\mathrm{CsOH}$ beobachtet werden.[53, 60] Allerdings lassen sich diese Reaktionen nicht direkt mit den PT-Halogenierungen vergleichen.

Dieser Effekt könnte darin begründet liegen, daß mit steigendem Kationenradius die Ladungsverteilung diffuser ist und damit eine höhere Lipophilie durch geringere Solvatisierung einhergeht. Das würde eine bessere Löslichkeit der Base in der organischen Phase bedingen. Allerdings wäre dies nur dann eine Begründung für die gesteigerte Reaktivität, wenn der PTK keine starke Wirkung auf die Reaktion haben würde, denn bei dem Transport des Hydroxidions in die organische Phase hat das Gegenion nur geringen Einfluß. Wahrscheinlicher ist deswegen, daß die Steigerung der Basizität der Base in der o.a. 
Reihenfolge von Bedeutung ist. Dies wäre wiederum ein Indiz, daß das Hydroxidion die eigentliche reaktive Spezies für die Halogenierung ist. Aufgrund der hohen Molmasse war es nicht möglich mit $\mathrm{CsOH}$ eine $25 \mathrm{M}$ (entspricht der Konzentration einer 50 proz. wäßrigen $\mathrm{NaOH}-L s g$. .) wäßrige Lösung herzustellen. Deshalb war es notwendig die Konzentration der Basen auf $3.5 \mathrm{~mol} / \mathrm{L}$ zu erniedrigen, um sie miteinander vergleichen zu können. Dadurch ist aber keine direkte Vergleichsmöglichkeit mit den anderen Experimenten gegeben, die mit 25 molarer $\mathrm{NaOH}$ Lösung (aq. 50\%) durchgeführt wurden.

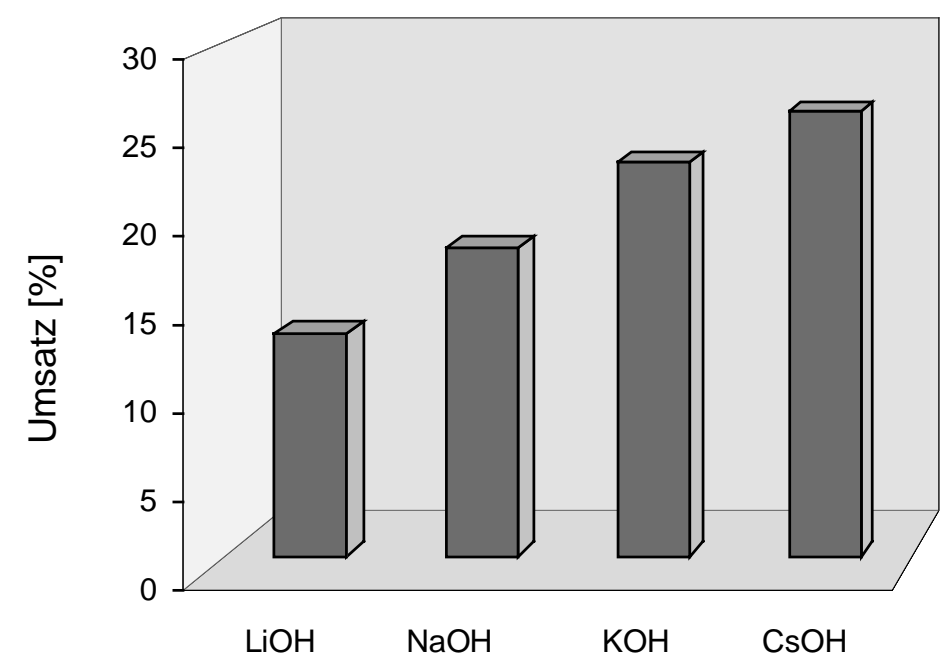

\begin{abstract}
Abbildung 12. Umsatz bei Variation der Base $\left[\mathrm{AV} 2 \mathrm{e}, 40^{\circ} \mathrm{C} / \mathrm{PhF} / \mathrm{TBABr}\right.$ $(3 \mathrm{~mol} \%) / \mathrm{CHI}_{3} /$ aq. $\left.\mathrm{MOH}(3.5 \mathrm{M}) \mathrm{M}=\mathrm{Li}, \mathrm{Na}, \mathrm{K}, \mathrm{Cs} / 48 \mathrm{~h}\right]$ bei der Iodierung von 1
\end{abstract}

\title{
2.1.3.6. Variation des Volumens der wäßrigen Phase bei konstanter absoluter Basenmenge
}

Um Grenzflächeneffekte genauer untersuchen zu können, wurden Experimente durchgeführt, wo die absolute Menge an Hydroxidionen in der wäßrigen Phase konstant gehalten, dessen Volumen aber erhöht wurde (Abb. 13). Dies steht im Gegensatz zu den anderen konzentrationsabhängigen Experimenten, bei denen das Volumen der wäßrigen Phase konstant gehalten wurde, die Konzentration an Hydroxidionen jedoch erhöht wurde. Dabei mußten jedoch aufgrund der steigenden Verdünnung lange Reaktionszeiten in Kauf genommen werden. Durch das gesteigerte Volumen der wäßrigen Phase, sollte nun überprüft werden, ob die damit einhergehende vergrößerte Phasengrenzfläche eine Steigerung des Umsatzes bedingen würde. Dies konnte nicht beobachtet werden, was, wie schon bei anderen 
Experimenten, gegen einen Grenzflächenmechanismus spricht. Die höchste Reaktionsrate konnte mit der höchsten Konzentration an Hydroxidionen, d.h. mit dem geringsten Volumen der wäßrigen Phase, erreicht werden. Hier liegt wahrscheinlich wieder eine geringere Solvatisierung des Hydroxidions bzw. des PTK-Hydroxidionenpaares vor und dadurch wird eine höhere Reaktivität bewirkt. Bei der Erhöhung der absoluten Basenmenge gleicher Konzentration (hier nicht dargestellt, AV2g) wurde wie erwartet auch eine Erhöhung des Umsatzes beobachtet

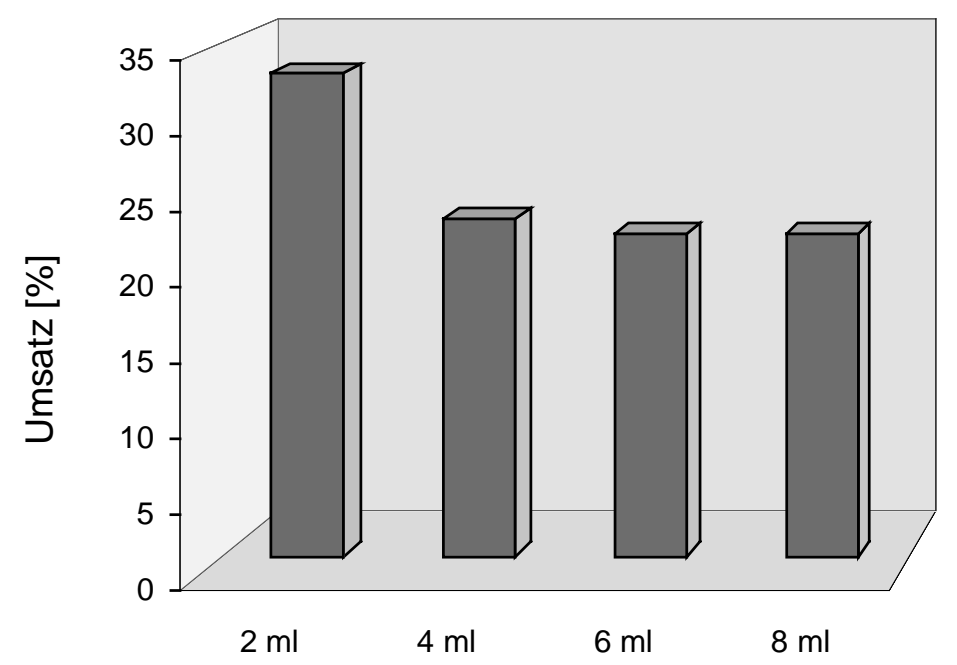

\begin{abstract}
Abbildung 13. Umsatz bei Variation des Volumens der wäßrigen Phase [AV2f, $40^{\circ} \mathrm{C} / \mathrm{PhF} / \mathrm{TBABr}(3 \mathrm{~mol} \%) / \mathrm{CHI}_{3} / \mathrm{aq} . \mathrm{NaOH}(\mathrm{X} \mathrm{mol} / \mathrm{Y} \mathrm{mL}) /$ $48 \mathrm{~h}$ ] bei der Iodierung von 1 und konstanter Menge an Hydroxidionen
\end{abstract}

\title{
2.1.4. Die Chlorierung
}

An dieser Stelle soll nun noch die Chlorierung Erwähnung finden. Auch sie ist durchführbar, und zwar mit ähnlich hohen Selektivitäten wie die Bromierungen und die Iodierungen. Dabei wird beispielsweise 1 und ca. 5 mol\% TBABr in Tetrachlormethan gelöst und $\mathrm{NaOH}$ (aq. 50\%) zugesetzt. Die Mischung mußte über einen langen Zeitraum (10 Tage) refluxiert werden (AV3, Schema 11). Die Chlorierung wurde nicht so systematisch untersucht wie die anderen Halogenierungen. Dies liegt hauptsächlich in den langen Reaktionszeiten begründet. Ein weiterer Faktor ist, daß die Chlorierungen immer bei höheren Temperaturen (am besten unter Rückfluß) durchgeführt werden müssen, damit überhaupt eine merkliche Reaktion stattfindet. Deswegen ist es vorteilhafter, die Chlorierungen thermisch durch Radikalkettenstarter mit Tetrachlormethan als Halogenquelle zu initiieren, da sowieso auf den 
Vorteil der niedrigen Reaktionstemperaturen verzichtet werden muß. Die Reaktionen sind in diesem Fall dann schon nach wenigen Tagen abgeschlossen (s. Kap. 3) und es werden die gleichen Selektivitäten erzielt wie mit den PT-Chlorierungen (s.u.). Nichtsdestotrotz sind letztere im Hinblick auf das Gesamtkonzept der PT-Halogenierungen von großem mechanistischen Interesse.

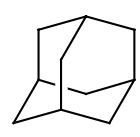

1 $10 \mathrm{~d}$

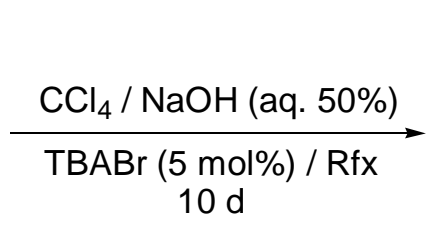

$$
43 \%
$$

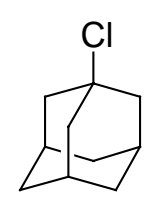

26

$43 \% / 60 \%$

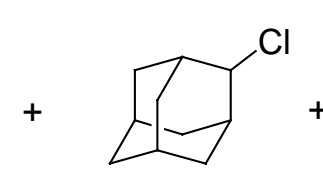

27

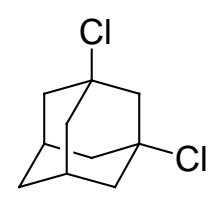

28

Schema 11. Die PT-Chlorierung von 1 (isolierte Ausbeute / Ausbeute ermittelt durch GC/MS-Analyse der Reaktionsmischung)

\subsection{Spezielle mechanistische Untersuchungen}

\subsubsection{Einleitung}

Nachdem die PT-Bedingungen der neuen Halogenierungsmethode eingehend untersucht und optimiert wurden, sollten speziellere Experimente weiteren Aufschluß über den Reaktionsmechanismus geben. Dabei wurde einerseits ein Augenmerk auf die Vorgänge in der organischen Phase gelegt, weil dort vermutlich die Propagation der Reaktion abläuft. $\mathrm{Ob}$ es sich überhaupt um eine Radikalreaktion handelt und wenn, welche Radikale für die Propagation der Reaktion verantwortlich sind, sollte dabei genauer untersucht werden. Zum anderen sollten weitere Hinweise für den Mechanismus der Initiierung gefunden werden.

\subsubsection{Vergleich der Regioselektivität der Halogenierung von Adamantan unter PT- Bedingungen und klassisch thermisch initiiert durch AIBN}

Beide Positionen in Adamantan sind bei radikalischen Substitutionen sehr empfindlich bezüglich elektronischer aber hauptsächlich bezüglich sterischer Effekte. Obwohl das 2-Adamantylradikal um ca. $2.5 \mathrm{kcal}^{\mathrm{mol}^{-1}}$ thermodynamisch stabiler als das 1-Adamantylradikal ist, $[118,119]$ ist die sekundäre C-H-Bindung aufgrund der Orientierung 
des einfach besetzten $p$-Orbitals sterisch deutlich stärker gehindert und reagiert deswegen sensitiv auf die Größe der Radikalvorstufe (z.B. ein Halomethan, Abb. 14). Gleiches gilt auch für Adamantan bezüglich des Angriffs eines sterisch anspruchsvollen Radikals. Darum kann die Selektivität einer Substitution zwischen den besagten Positionen als ein Indikator für die Natur der verantwortlichen radikalischen Spezies angesehen werden (s. Kap. 1.3).

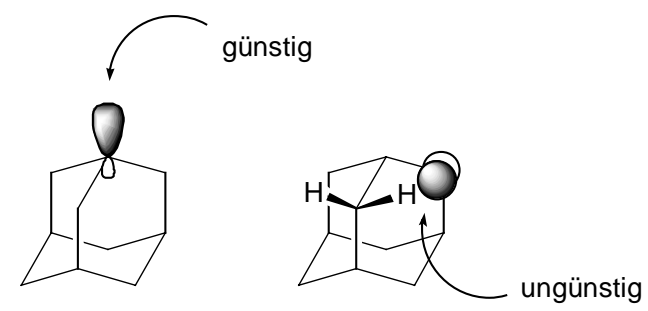

Abbildung 14. Das 1- und 2-Adamantylradikal

Dies sollte bei den folgenden Experimenten ausgenutzt werden, indem unabhängig voneinander 1 unter PT-Bedingungen und klassisch thermisch initiiert durch einen Radikalkettenstarter (AIBN) halogeniert wurde. Sind die Regioselektivitäten gleich, so kann dies als ein starker Hinweis auf die gleiche radikalische Spezies aufgefaßt werden. Zunächst wurden die Bromierungen untersucht. Dabei konnte festgestellt werden, daß das Produktverhältnis zwischen der 1- und 2- Positionen sowohl bei der radikalisch initiierten als auch bei der PT-Reaktion fast gleich ist (Nr. 2, 3; Tab. 4.). Da als gesichert anzunehmen ist, daß bei der durch Kettenstarter initiierten Reaktion das Tribrommethylradikal die Propagation der Radikalreaktion trägt ist, kann gefolgert werden, daß dies auch für PTK-Bromierungen gilt. Lediglich bei geringeren Reaktionstemperaturen wurde eine etwas höhere Regioselektivität bezüglich der 1-Position festgestellt, was aber auf energetische Effekte zurückzuführen sein dürfte (Nr. 1; Tab. 4.). Anders verhält sich die Situation bei der Iodierung mit Iodoform als Halogenquelle. Während bei der radikalisch, thermisch initiierten Reaktion eine Regioselektivität beobachtet wurde, die im Bereich der Bromierung lag, war die unter PTK-Bedingungen erreichte erheblich größer (Nr. 4, 5 ; Tab. 4.). Da unter PTKBedingungen eine vorgelagerte Disproportionierung von Iodoform $\mathrm{zu}$ Diiod- und Tetraiodmethan postuliert wird, wobei letzteres als das eigentliche iodierende Reagenz angesehen wird, ist hier ein weiterer Hinweis auf die Richtigkeit dieser Hypothese gefunden worden.

Für die Chlorierungen gelten die gleichen Schlußfolgerungen wie für die Bromierungen. Es kann allerdings eine etwas höhere Regioselektivität bei der PTKChlorierung festgestellt werden (Nr. 6, 7; Tab. 4.), wobei der Unterschied aber längst nicht so 
ausgeprägt ist wie bei den Iodierungen und könnte auf eine Teilnahme des Isobutyronitrilradikals (aus AIBN) an der $\mathrm{H}$-Abstraktion mit geringer Selektivität zurückzuführen sein.

Tabelle 4. Statistisches Produktverhältnis (stat. $3^{\circ}: 2^{\circ}$ ) bei der Halogenierung von 1 initiiert durch PTK Bedingungen, AIBN und Wasser (AV4)

\begin{tabular}{|c|c|c|c|c|c|c|c|}
\hline \# & Halogenquelle & Rkt.Bed. & Zeit [h] & Solvens & $\mathbf{T}\left[{ }^{\circ} \mathbf{C}\right]$ & $3^{\circ}: 2^{\circ}$ & stat. $3^{\circ}: 2^{\circ}$ \\
\hline 1 & $\mathrm{CBr}_{4}$ & PTK & 60 & $\mathrm{CH}_{2} \mathrm{Cl}_{2}$ & 40 & 8.4 & 25.2 \\
\hline 2 & $\mathrm{CBr}_{4}$ & PTK & 20 & $\mathrm{C}_{6} \mathrm{H}_{5} \mathrm{~F}$ & 75 & 10.0 & 30.1 \\
\hline 3 & $\mathrm{CBr}_{4}$ & AIBN & 20 & $\mathrm{C}_{6} \mathrm{H}_{5} \mathrm{~F}$ & 75 & 10.1 & 30.2 \\
\hline 4 & $\mathrm{CHI}_{3}$ & PTK & 72 & $\mathrm{C}_{6} \mathrm{H}_{5} \mathrm{~F}$ & 75 & 44.0 & 132.0 \\
\hline 5 & $\mathrm{CHI}_{3}$ & AIBN & 72 & $\mathrm{C}_{6} \mathrm{H}_{5} \mathrm{~F}$ & 75 & 22.3 & 66.9 \\
\hline 6 & $\mathrm{CCl}_{4}$ & PTK & 72 & $\mathrm{CCl}_{4}$ & 70 & 9.5 & 28.5 \\
\hline 7 & $\mathrm{CCl}_{4}$ & AIBN & 72 & $\mathrm{CCl}_{4}$ & 50 & 5.7 & 17.2 \\
\hline 8 & $\mathrm{CBr}_{4}$ & $\mathrm{H}_{2} \mathrm{O}$ & 20 & $\mathrm{CH}_{2} \mathrm{Cl}_{2}$ & 40 & 3.7 & 11.1 \\
\hline
\end{tabular}

Insgesamt bestätigen die Versuche die Arbeitshypothese hinsichtlich der Vorgänge in der organischen Phase im Rahmen der PTK-Halogenierungen mit $\mathrm{NaOH}$ (aq. 50\%). Auffallend ist jedoch die stark erniedrigte Regioselektivität der Bromierung in Dichlormethan nur initiiert durch Wasser unter Zusatz geringer Mengen an Base zur Prävention der Hydrolyse. Hier wird eine Selektivität von $3^{\circ} / 2^{\circ}=11.1$ (statistisch korrigiert) festgestellt (Nr. 8; Tab. 4.), d.h. die Selektivität nimmt erheblich ab. Das ist ein weiterer Hinweis für einen Wechsel des Reaktionsmechanismus oder für das Auftreten verschiedener Mechanismen nebeneinander.

\subsubsection{Nachweis von molekularem Brom im Rahmen der PT-Bromierung}

Erste Anhaltspunkte für eine Erklärung des eben angesprochenen Phänomens (Nr. 8; Tab. 4.) lieferte die Beobachtung, daß sich die Reaktionsmischung einer PT-Bromierung von 1, initiiert durch demineralisiertes Wasser, bräunlich verfärbte und den typischen Geruch 
von Brom abgab (AV5). Es könnte somit sein, daß bei der durch Wasser initiierten Reaktion in Dichlormethan, vornehmlich eine Hydrolyse von Tetrabrommethan auftritt, die schließlich die Bildung von molekularen Brom nach sich zieht. $\mathrm{Br}_{2}$ bzw. dessen Homolyseprodukt, das Bromradikal, konnte experimentell durch eine Abfangreaktion (Schema 12) mit 3,7Bismethylenbicyclo[3.3.1]nonan 29 nachgewiesen werden.[120, 121] Dabei wurde zu einer Probe der Reaktionsmischung der Bromierung von Adamantan in Dichlormethan, die durch reines Wasser initiiert wurde, nach $24 \mathrm{~h}$ soviel der Bismethylenverbindung 29 hinzugefügt (ca. $70 \mathrm{mg}$ ), bis eine komplette Entfärbung eintrat. Durch den Vergleich der GC/MS-Analyse der Reaktionsmischung mit einer, die vor dem Experiment durchgeführt wurde, konnte ein "neuer" Peak mit dem für die Abfangverbindung erwarteten Massenspektrum detektiert werden.

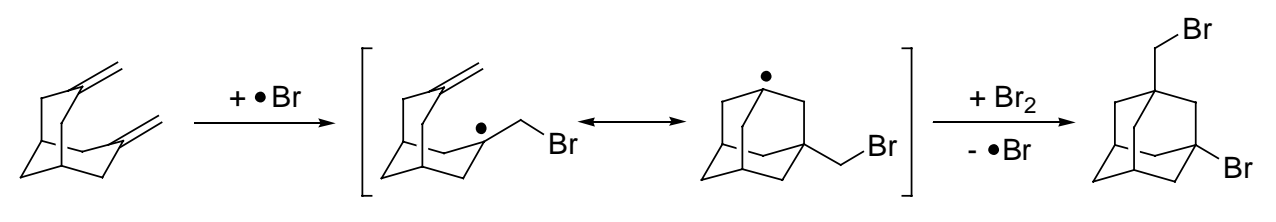

29

30

Schema 12. Mechanismus zur Addition von Brom an 3,7-Dimethylenbicyclo[3.3.1]nonan 29 $\left(\mathrm{CH}_{2} \mathrm{Cl}_{2} / \mathrm{H}_{2} \mathrm{O} / \mathrm{CBr}_{4} / \mathrm{AV} 5\right)$

Zur Verifizierung dieses Ergebnisses wurde ein Vergleichsexperiment durchgeführt, indem zu einer Lösung von Brom in Dichlormethan ebenfalls 29 bis zur völligen Entfärbung hinzugefügt wurde. Die nachfolgende GC/MS-Analyse der Reaktionsmischung ergab ein Hauptprodukt (95\%) mit der gleichen Retentionszeit und dem identischen Massenspektrum. Der Nachweis von Brom in der Reaktionsmischung bedeutet, daß der Mechanismus der Initiierung, für die durch Wasser initiierte PT-Halogenierung, neu überdacht werden muß (s. Kap. 2.2.6).

\subsubsection{Abfangen des Trihalomethylradikals im Rahmen der PT-Bromierung}

Die allgemeine Arbeitshypothese für den Mechanismus der PTK-Halogenierung steht und fällt mit Frage, ob wirklich das Trihalomethylradikal den Propagationszyklus der Radikalreaktion trägt. Eine oftmals angewandte Möglichkeit des Nachweises ist, daß entsprechende Radikal abzufangen. Wie schon bei der Nachweisreaktion des Broms, wurde auch in diesem Fall die Bismethylenverbindung 29 eingesetzt. ${ }^{[120-122]}$ Dazu wurde selbige 
und $\mathrm{CBr}_{4}$ in Dichlormethan gelöst und in Anwesenheit von festem Natriumhydroxid bei RT gerührt. In diesem Fall mußte festes $\mathrm{NaOH}$ verwendet werden, weil zu erwarten war, daß das polyhalogenierte Abfangprodukt im rein flüssigen Zweiphasensystem instabil sein würde (AV6).[123]

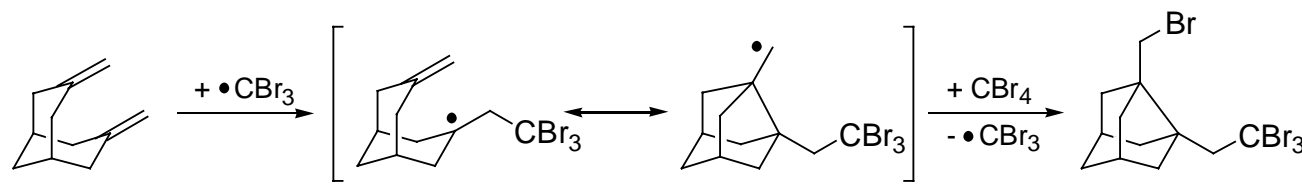

29

$31[60 \%]$

Schema 13. Abfangreaktion von $\cdot \mathrm{CBr}_{3}$ mit nachfolgender Cyclisierung $\left(\mathrm{CH}_{2} \mathrm{Cl}_{2} / \mathrm{NaOH}(\mathrm{s}) /\right.$ $\left.\mathrm{CBr}_{4} / \mathrm{RT} / \mathrm{AV} 6\right)$

Diese Art der Abfangreaktion mit nachfolgender Cyclisierung wurde schon zuvor mit $\mathrm{CBr}_{4}$, allerdings initiiert durch AIBN, durchgeführt.[120, 122] Auch in diesem Fall, konnte das gleiche Produkt isoliert werden. Der Grund für die Bildung des Noradamantangerüstes, im Gegensatz zur Bildung des Adamantangerüstes im Fall der Reaktion mit Brom (Schema 13), liegt darin begründet, daß die Produktbildung bei beiden Reaktionen unter thermodynamischer Kontrolle erfolgt. Durch die Sperrigkeit der Tribrommethylgruppe wird eine Bildung des normalerweise favorisierten Adamantangerüstes thermodynamisch unvorteilhaft.[121, 122] Dieser Trend wird auch dadurch deutlich, daß durch den geringeren räumlichen Anspruch der $\mathrm{CCl}_{3}$-Gruppe bei der radikalisch initiierten Addition von $\mathrm{CCl}_{4}$ an 29 bereits ein Produktverhältnis von 3:1 von Noradamantan- zu Adamantanprodukt erreicht wird. ${ }^{[120]}$ Dieses Experiment ist ein wichtiger Hinweis auf das Tribrommethylradikal als das die Propagation tragende radikalische Spezies. Auch im Rahmen der Iodierung wurde versucht, das Triiodmethylradikal abzufangen. Dies mißlang allerdings, evt. aufgrund der Instabilität der gebildeten Polyiodverbindung.

\subsubsection{Die kinetischen H/D-Isotopeneffekte im Rahmen der PT-Halogenierungen[123]}

\subsubsection{Einleitung}

Ein wichtiges Werkzeug für die mechanistischen Untersuchungen von chemischen Reaktionen ist der kinetische Isotopeneffekt (KIE). ${ }^{[124-128]}$ Dieser Effekt entsteht durch den 
Austausch eines Atoms durch eines seiner Isotope und äußert sich durch eine Änderung der Reaktionsgeschwindigkeit. Der weitaus am häufigsten untersuchte Fall ist der KIE bei Austausch von Wasserstoff durch Deuterium. Dies ist auf gute Verfügbarkeit von angereicherten Deuteriumderivaten zurückzuführen. Seltener findet Tritium Anwendung, weil dieses Element den Nachteil hat radioaktiv zu sein, und dadurch der experimentellen Umgang mit tritiumhaltigen Substanzen stark erschwert wird. Nicht nur Wasserstoffisotope wurden zur Untersuchung von Reaktionsmechanismen herangezogen, sondern auch Isotope von Kohlenstoff, Schwefel, Stickstoff, Chlor, Silizium und sogar Brom. ${ }^{[126-128]}$ In diesen Fällen wurde meist auf eine Anreicherung bestimmter Isotopen verzichtet und eine zu untersuchende Verbindungen mit deren natürlichen Isotopenverhältnissen eingesetzt.[127-130] Dies ist notwendig, weil gerade bei schwereren Elementen der relative Massenunterschied der Isotope verhältnismäßig gering ist und damit deren Anreicherung sehr aufwendig wäre oder gar nicht erst möglich ist. Dabei ist problematisch, daß Isotope nie in den gleichen Konzentrationen in der Natur vorkommen, und das zieht bei der Auswertung der kinetischen Daten aufgrund der unterschiedlichen Startkonzentrationen komplizierte mathematische Näherungen und damit auch einen größeren Fehler im Endergebnis nach sich.[127, 128] Wenn nicht anders vermerkt, beziehen sich alle folgenden Beschreibungen auf den H/D-KIE, da dieser Gegenstand der nachfolgenden Untersuchungen sein wird. Ein weiterer Grund, warum Deuterium am häufigsten für die Bestimmung des KIE eingesetzt wird, ist, daß dabei der maximalste Wert erzielt werden kann (Nur bei ${ }^{1} \mathrm{H} /{ }^{3} \mathrm{H}-\mathrm{KIE}$ können noch stärkere Effekte erreicht werden, allerdings mit den bereits erwähnten Nachteilen). Die Ursache dafür ist in den physikalischen Grundlagen des KIE zu suchen. Jede Bindung in einem Molekül besitzt eine für sie typische Schwingung, die zur Gesamtenergie des Moleküls im Grundzustand beiträgt, d.h. zur Nullpunktsenergie. Dabei ist der Beitrag zu dieser Energie abhängig von der Masse der beteiligten Atome, denn je höher die Masse ist, desto geringer ist der energetiche Beitrag aufgrund der kleineren Frequenz der Schwingung, und die Nullpunktsenergie sinkt. Wird im Rahmen einer Reaktion diese Bindung gebrochen, dann wandelt sich der Schwingungsfreiheitsgrad dieser Bindung in einen Translationsfreiheitsgrad um, wobei die Übergangszustände für beide isotopomeren Moleküle in erster Näherung als gleich angenommen werden. Durch die niedrigere Nullpunktsenergie des schwereren Isotopomers wird die Aktivierungsenergie größer und die Reaktion verlangsamt sich in diesem Fall (Abb. 15). Dies ist nur die einfachste theoretische Betrachtungsweise des KIE, die aber an entsprechender Stelle (s.u.) noch erweitert wird, da meistens auch die Energetik der ÜZ mitberücksichtigt werden muß (Abb. 15, ÜZ). 


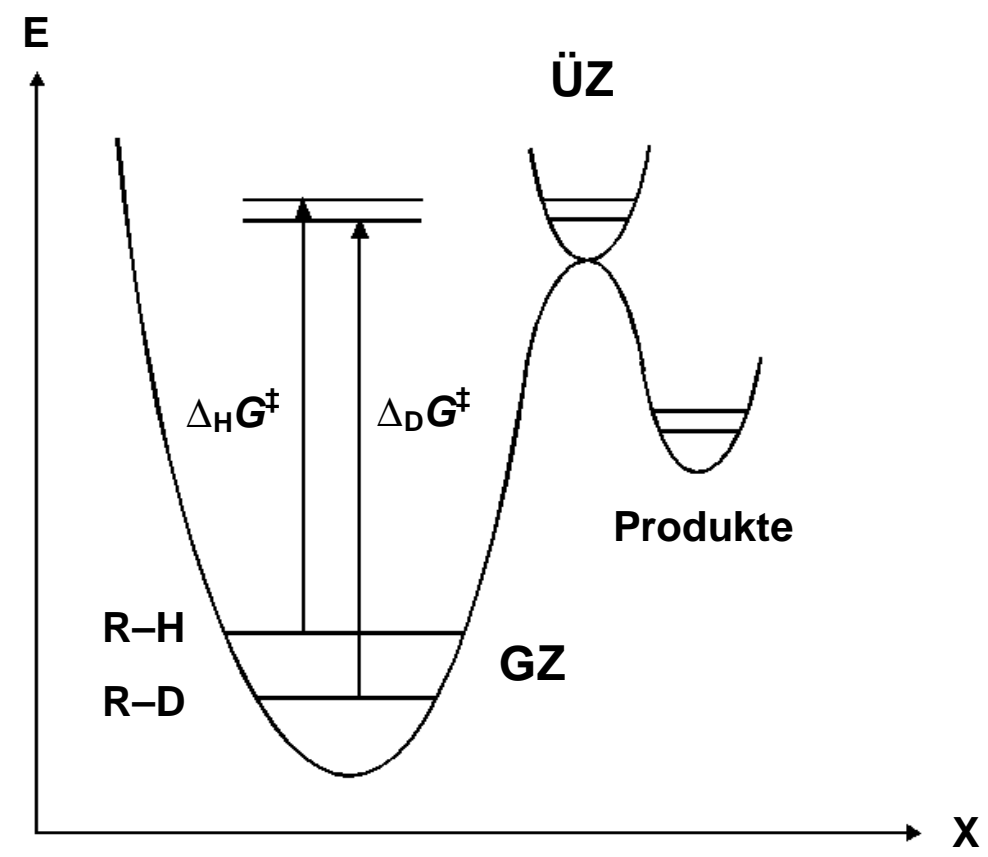

Abbildung 15. Schematischer Schnitt durch die Energiehyperfläche des Bindungsbruchs einer H- und D-Bindung zur Veranschaulichung des KIE

Da für die Wasserstoffisotope der relative Massenunterschied maximal ist, werden hier auch die höchsten KIEs beobachtet, wodurch im Fall eines H/D-KIE gemäß obiger Betrachtungsweise ein theoretischer Wert von ca. 7 resultiert.[124-128] Die Grundvoraussetzung für das Auftreten eines KIE ist jedoch, daß der Bindungsbruch im geschwindigkeitsbestimmenden Schritt der Reaktion erfolgt und die Reaktion unter kinetischer Produktkontrolle erfolgt. Ist die Reaktion reversibel, d.h. die Produktbildung erfolgt unter thermodynamischer Kontrolle, so kann der Gleichgewichtsisotopeneffekt (GIE) beobachtet werden. Dieser ist naturgemäß sehr gering, denn die Unterschiede der thermodynamischen Stabilitäten isotopomerer Produkte ist i.A. nicht sehr groß.[128]

Die meisten Fälle sind allerdings nicht so einfach gelagert, denn es gibt noch weitere Faktoren die das Ausmaß eines KIE beeinflussen können. Dazu gehören die Geometrie des Übergangszustands, Lösungsmittel- und nicht zuletzt Tunneleffekte.[127, 128] Diese Faktoren sollen an dieser Stelle etwas genauer betrachtet werden, um die experimentell erhaltenen Werte besser einschätzen zu können. Der theoretisch maximale Wert von 7 für den H/D-KIE kann dann beobachtet werden, wenn der Übergangszustand (ÜZ) genau symmetrisch zwischen den Bindungspartnern liegt. Ist der ÜZ früher oder später, so verkleinert sich der 
KIE dementsprechend. Wodurch dieser Effekt hervorgerufen wird, kann mit dem theoretischen Ansatz des Dreizentren-Übergangszustandsmodell von Melander und Westheimer[124, 128, 131] erklärt werden, das das abstrahierende Teilchen mitberücksichtigt. Die resultierenden mathematischen Ableitungen sind ziemlich umfangreich und sollen hier nicht wiedergegeben werden. Aus dem eben angesprochenen Modell kann auch abgeleitet werden, warum im Fall eines nicht linearen ÜZ eine Verkleinerung des KIE resultiert, der um so geringer wird, je größer die Abweichung vom linearen ÜZ ist.[127, 128] Lösungsmitteleffekte können hauptsächlich dann erwartet werden, wenn eine relativ starke Wechselwirkung zwischen Reaktanden und Solvens stattfindet. Eine Voraussage über den Trend der Beeinflussung des KIE ist hierbei schwierig zu treffen. Als wichtigster Faktor für einen KIE, größer als erwartet, müssen Tunneleffekte angesehen werden.[127, 128] Diese kommen dadurch zustande, daß ein reagierendes Teilchen aufgrund der Unschärfe seines Ortes mit einer gewissen Wahrscheinlichkeit eine Energiebarriere durchdringen kann, ohne die für das Überschreiten der Barriere notwendige Energie zu besitzen. Der Tunneleffekt ist im starken $\mathrm{Maß}$ abhängig von der Höhe und Breite der zu durchschreitenden Energiebarriere, von der Geometrie des ÜZ, aber hauptsächlich von der Masse des im ÜZ transferierten Teilchens. Dabei gilt, daß der Tunneleffekt um so größer sein kann, je geringer die Masse dieses Teilchens ist. Das hat zur Folge, daß für schwerere Atome als die verschiedenen Wasserstoffisotope, die Beteiligung eines Tunneleffektes im allgemeinen nicht diskutiert werden muß. Ferner ist der Tunneleffekt temperaturabhängig, was als Überprüfung für dessen Auftreten ausgenutzt werden kann. Wird in einem bestimmten Temperaturbereich eine starke Abweichung von der Arrheniusgleichung festgestellt, so kann vermutet werden, daß bei der entsprechenden Reaktion ein Tunneleffekt beteiligt ist.

Neben dem eben beschriebenen primären KIE kann auch ein sekundärer beobachtet werden. Es wird dann von einem sek. KIE gesprochen, wenn das durch ein Isotop substituierte Atom nicht direkt an der Reaktion beteiligt ist $[127,128]$ und wurde bislang nur genauer im Rahmen von H/D-Isotopeneffekten untersucht. Die Werte für den sekundären $\mathrm{H} / \mathrm{D}-\mathrm{KIE}$ sind kleiner als für den primären und bewegen sich im Bereich von $\mathrm{k}_{\mathrm{H}} / \mathrm{k}_{\mathrm{D}}=0.7-$ 1.5. Ist der sek. H/D-KIE größer eins, so wird von einem normalen und im anderen Fall von einem inversem Effekt gesprochen. Ein sekundärer Effekt ist darin begründet, daß sich im ÜZ die Bindungsverhältnisse ändern und benachbarte H- bzw. D-Atome, abhängig von der Art und Weise wie sich die Bindungsverhältnisse ändern, hyperkonjugativ mehr oder weniger stabilisierend wirken können. Wird z.B. ein ÜZ durch eine im Vergleich zur C-H-Bindung verkürzte C-D-Bindung hyperkonjugativ stärker stabilisiert, dann wird ein inverser sek. H/D- 
KIE beobachtet. Im umgekehrten Fall wird der Effekt normal. Dieser sekundäre Isotopeneffekt ist somit nicht-klassisch und kann nur durch die energetische Berücksichtigung der ÜZ erfaßt werden (Abb. 15, ÜZ).

\subsubsection{Experimentelle Voraussetzungen zur Bestimmung des KIE}

Grundsätzlich ist wichtig, einen allgemeinen Ansatz für die Ermittlung des KIE aus den experimentellen Rohdaten zu finden. Dabei wurden die folgenden Voraussetzungen zugrunde gelegt. Die Startkonzentrationen der deuterierten sowie nichtdeuterierten Verbindungen sind gleich und das jeweilige Halomethan $\left(\mathrm{CHI}_{3}, \mathrm{CBr}_{4}\right)$, das als Halogenquelle dient, wird im großen Überschuß eingesetzt. In einem frühen Stadium einer bestimmten Reaktion (Umsatz < 7 \%) können dann näherungsweise die Konzentrationen der Substrate als konstant angesehen werden. Das bedeutet, daß dadurch eine Extrapolation für $\mathrm{t} \rightarrow 0$ aus einer Auftragung des Verhältnisses deuterierter zu nichtdeuterierter Produkte direkt der KIE ermittelt werden könnte. Dieser Ansatz kann gemäß der Regel von l'Hospital[132] begründet werden, die besagt, daß für den Grenzwert eines Quotienten, in Abhängig von einer Variablen, für diese Variable gegen Null, gilt, daß dieser gleich dem Quotienten ist (Gl. I), was in dem zu untersuchenden speziellen Fall dem KIE entspricht. Allerdings ist dies ein rein mathematischer Ansatz, ohne genauen physikalisch chemischen Bezug zum Experiment.

$$
\lim _{t \rightarrow 0} \frac{[P]_{t}^{H}}{[P]_{t}^{D}}=\lim _{t \rightarrow 0} \frac{\frac{d[P]^{H}}{d t}}{\frac{d[P]^{D}}{d t}}=\frac{[P]_{t}^{H}}{[P]_{t}^{D}}=K I E
$$

$[\mathrm{P}]^{\mathrm{H} / \mathrm{D}}=$ Konzentration der nicht deuterierten / deuterierten Produkte

Deswegen mußte ein mathematischer Beweis basierend auf einer kinetischen Ableitung für das geplante Experiment gefunden werden. Für die beiden isotopomeren Reaktanden $\mathrm{R}_{\mathrm{H}}$ und $\mathrm{R}_{\mathrm{D}}$ gelten die folgenden Geschwindigkeitsgesetze:

$$
-\frac{\left[R_{H}\right]}{d t}=k_{H}\left[R_{H}\right] \cdot f([B],[C], \ldots . .) \text { und }-\frac{\left[R_{D}\right]}{d t}=k_{D}\left[R_{D}\right] \cdot f([B],[C], \ldots \ldots)
$$

Dabei bedeuten $f([B],[C], \ldots .$.$) die für die betreffende Reaktion geltende Funktion der$ Konzentrationen der Reaktanden bzw. Katalysatoren in denen keine Isotopensubstitution 
vorgenommen worden ist. Durch Division der beiden Gleichungen, anschließende Umformung und bestimmte Integration $[128]$ in den Grenzen der Startkonzentrationen $\left[R_{H}\right]_{0}$, $\left[R_{D}\right]_{0}$ bis zu den Konzentrationen $\left[R_{H}\right],\left[R_{D}\right]$ ergibt sich:

$$
\frac{k_{H}}{k_{D}}=\frac{\ln \frac{\left[R_{H}\right]}{\left[R_{H}\right]_{0}}}{\ln \frac{\left[R_{D}\right]}{\left[R_{D}\right]_{0}}}=\frac{\ln \left(1-x_{H}\right)}{\ln \left(1-x_{D}\right)} ; \text { mit } x=1-\frac{[R]}{[R]_{0}}
$$

Da die Experimente unter Verfolgung der Produktverhältnisse (H/D) erfolgten, war es sinnvoll den Quotienten der Molenbrüche $Q=x_{H} / x_{D}$ zu betrachten und dann $x_{H} \mathrm{zu}$ substituieren.[127]

$$
\frac{k_{H}}{k_{D}}=\frac{\ln \left(1-Q \cdot x_{D}\right)}{\ln \left(1-x_{D}\right)}
$$

Für kleine Werte von $x_{D}$, was in einem frühen Stadium der jeweils betrachteten Reaktion zutrifft, läßt sich für $\ln (1-x)$ eine Reihenentwicklung durchführen, die nach dem ersten Glied abgebrochen werden kann. Dies vereinfacht die vorherige Gleichung:

$$
\frac{k_{H}}{k_{D}}=Q=\frac{x_{H}}{x_{D}}
$$

Da für den Molenbruch definitionsgemäß weiterhin gilt: $x=[P] /[R]_{0}$ wobei $[P]$ der jeweiligen Produktkonzentration entspricht und weiterhin gilt, daß $\left[R_{H}\right]_{0}=\left[R_{D}\right]_{0}$ ist, kann schließlich folgende einfache Gleichung (Gl. VII) abgeleitet werden:

$$
\frac{k_{H}}{k_{D}}=K I E=\frac{\frac{\left[P_{H}\right]}{\left[R_{H}\right]_{0}}}{\frac{\left[P_{D}\right]}{\left[R_{D}\right]_{0}}}=\frac{\left[P_{H}\right]}{\left[P_{D}\right]}
$$

Damit wäre belegt, daß in einem frühen Stadium der Reaktion der KIE direkt dem Verhältnis der isotopomeren Produktkonzentrationen entspricht. Der ermittelte Wert wird um so ungenauer, je weiter die Reaktion fortschreitet, weswegen die Bestimmung des KIE aus einer 
Extrapolation des y-Achsenabschnittes aus einer Auftragung des Produktverhältnisses gegen die Zeit erfolgen sollte.

Des weiteren mußte ein adäquater Weg zur Ermittlung der Produktkonzentrationen gefunden werden. Dabei bot sich die GC/MS-Analyse als einzige probate Methode an, denn es bestand die Schwierigkeit, daß die deuterierten und nichtdeuterierten Produkte fast die gleichen Retentionszeiten in einfachen GC-Analysen aufweisen. Die GC/MS-Analyse bietet aber eine massenselektive Integration spezifischer Ionenpeaks der Produkte an, wodurch das Problem beseitigt werden konnte. Eine so durchgeführte Analyse bedingt allerdings auch eine Kalibrierung der verschiedenen Produkte. Als wichtigstes Vorabexperiment wurde 1,3,5,7Tetradeuteroadamantan konkurrierend mit undeuterierten Adamantan einer Bromierung unterworfen. Die GC/MS-Analyse nach einer bestimmten Reaktionszeit (8 h) ergab einen signifikanten Isotopeneffekt, wodurch primär gezeigt wurde, daß die H-Abstraktion den geschwindigkeitsbestimmenden Schritt der Reaktion darstellt. Wäre das nicht der Fall gewesen, hätten Isotopeneffekte nicht zur weiteren Untersuchung des Reaktionsmechanismus herangezogen werden können.

Weiterhin mußte eine Auswahl der zu untersuchenden Ausgangsverbindungen getroffen werden. Die Wahl viel auf Cyclohexan 10 und wiederum auf Adamantan 1 als repräsentative Kohlenwasserstoffe. Die korrespondierenden deuterierten Verbindungen waren $D_{12}$-Cyclohexan und 1,3,5,7-Tetradeuteroadamantan. Die Produkte, die aus der Halogenierung der eben genannten Kohlenwasserstoffe hervorgehen, mußten separat hergestellt und eine Kalibrierung dieser Substanzen durchgeführt werden. Durch direkte Bromierung von $D_{12}-$ Cyclohexan mit NBS und AIBN als Radikalkettenstarter wurde $D_{11}-$ Bromcyclohexan dargestellt (AV7). Neben der Kalibrierung sollte diese Verbindung auch als Ausgangsubstanz für die Synthese $D_{11}$-Iodcyclohexan dienen. Dazu wurde $\mathrm{C}_{6} \mathrm{D}_{11} \mathrm{Br}$ mit konzentrierter HI-Lösung (aq. ca. $50 \%$ ) bei $105{ }^{\circ} \mathrm{C}$ umgesetzt.[133, 134] Eine GC/MSAnalyse des erhaltenen Produktes ergab, daß ein umfangreicher H/D-Austausch im Cyclohexanring stattgefunden hat und das Gemisch von Iodcyclohexanen mit unterschiedlichem Deuterierungsgrad für eine Kalibrierung nicht zu gebrauchen war. Es mußte deswegen ein anderer Weg zur Synthese von $D_{11}$-Iodcyclohexan gefunden werden. Vielversprechend erschien die Umsetzung von $D_{12}-$ Cyclohexanol mit PSSE (PolyPosphatetrimethylSilylEther $=$ Trimethylsilylpolyphosphat $)$ und NaI (s). ${ }^{[135]}$ Durch das PPSE wird eine Hydroxygruppe wahrscheinlich zu einen Siloxyether polymerer Struktur umgewandelt und dann kann unter sehr milden Bedingungen eine einfache $\mathrm{S}_{\mathrm{N}}$-Reaktion mit dem Iodid stattfinden, wodurch hier das $D_{11}$-Iodcyclohexan generiert wurde (AV8). Eine 
nachfolgende GC/MS-Analyse des Produktes zeigte, daß in diesem Fall keinerlei Deuteriumaustausch stattgefunden hat. 1-Brom-3,5,7-trideutero- und 1-Iod-3,5,7trideuteroadamantan wurden durch die entsprechende PTK-Halogenierung dargestellt (AV9, 10). Die sekundären Halogenadamantanverbindungen wurden nicht in reiner Form hergestellt und auch keine Kalibrierung durchgeführt, da diese Produkte für die folgenden Untersuchungen nur eine untergeordnete Rolle spielten.

Tabelle 5. Kalibrierungsfaktoren (KF) für die GC/MS-Analyse zwischen den deuterierten und nichtdeuterierten Verbindungen zur Ermittlung des KIE

\begin{tabular}{ccc}
\hline$\#$ & Produkt & KF \\
\hline $\mathbf{1}$ & $\mathrm{C}_{6} \mathrm{H}_{11} \mathrm{Br} / \mathrm{C}_{6} \mathrm{D}_{11} \mathrm{Br}$ & 1.15 \\
$\mathbf{2}$ & $\mathrm{C}_{6} \mathrm{H}_{11} \mathrm{I} / \mathrm{C}_{6} \mathrm{D}_{11} \mathrm{I}$ & 1.18 \\
$\mathbf{3}$ & 1-Br- / 1-Br-3,5,7-D-Adamantan & 1.01 \\
$\mathbf{4}$ & 1-I- / 1-I-3,5,7-D-Adamantan & 1.00 \\
\hline
\end{tabular}

Für die Kalibrierungen wurde ein Konzentrationsverhältnis von der jeweiligen deuterierten zur nichtdeuterierten Substanz von ca. 1 : 3 eingesetzt, um die Konzentrationsverhältnisse während einer Reaktion annähernd zu simulieren. Anschließend wurde die Lösung Schritt für Schritt bis zur Nachweisgrenze verdünnt und jeweils eine GC/MS-Messung durchgeführt. Dabei wurde eine massenselektive Integration der produktspezifischen Ionenpeaks durchgeführt. Der Mittelwert aller gemessenen Produktverhältnisse wurde mit dem der Ausgangskonzentration verglichen und aus diesem Verhältnis der jeweilige Kalibrierungsfaktor (KF, AV11) ermittelt.

\subsubsection{Experimentelle Bestimmung des KIE}

Nachdem nun die theoretischen sowie experimentellen Voraussetzungen für die eigentliche Bestimmung der kinetischen Isotopeneffekte geschaffen waren, wurden zunächst einige Vorabexperimente für die Bromierungen und Iodierungen der verschiedenen undeuterierten Kohlenwasserstoffe durchgeführt, um zu ermitteln, welche Reaktionsbe- 
dingungen nach einer bestimmten Zeit zu einem Umsatz von ca. $7 \%$ führen. Diese Bedingungen wurden anschließend auf die eigentlichen Experimente angewendet. Grundsätzlich wurden alle Reaktionen als Konkurrenzreaktionen in einem Reaktionsgefäß durchgeführt. Nur in einem Fall (Bromierung von Cyclohexan) wurde ein Experiment, d.h. die Halogenierung von deuterierten und nichtdeuterierten Kohlenwasserstoffen unabhängig voneinander durchgeführt, was aber $\mathrm{zu}$ einem ähnlichen Ergebnis führte (ca. 15\% Abweichung; der Fehler eines nicht direkt kompetativen Experimentes ist sehr viel größer). Allgemein wurde festgestellt, daß sich das Produktverhältnis von deuteriertem zu nichtdeuteriertem Produkt, also dem KIE, während dem Fortgang der Reaktion änderte. $\mathrm{Zu}$ Beginn war der KIE sehr gering (ca. 2) und stieg kontinuierlich innerhalb einer halben Stunde, um dann für einige Stunden konstant zu bleiben. Anschließend stieg er erneut, in Abhängigkeit vom betrachteten Reaktionssystem, bis auf einen Wert um 15. Der geringe KIE zu Anfang der Reaktion könnte auf das Auftreten eines während der Induktionsphase vom $\cdot \mathrm{CX}_{3}$ verschiedenen Radikals zurückzuführen sein $(\cdot \mathrm{OH}, \cdot \mathrm{Br}$, o.a.). Da über die Initiierung der Reaktion bislang nur Vermutungen angestellt werden können, konnte auch diese Beobachtung nicht eindeutig geklärt werden.
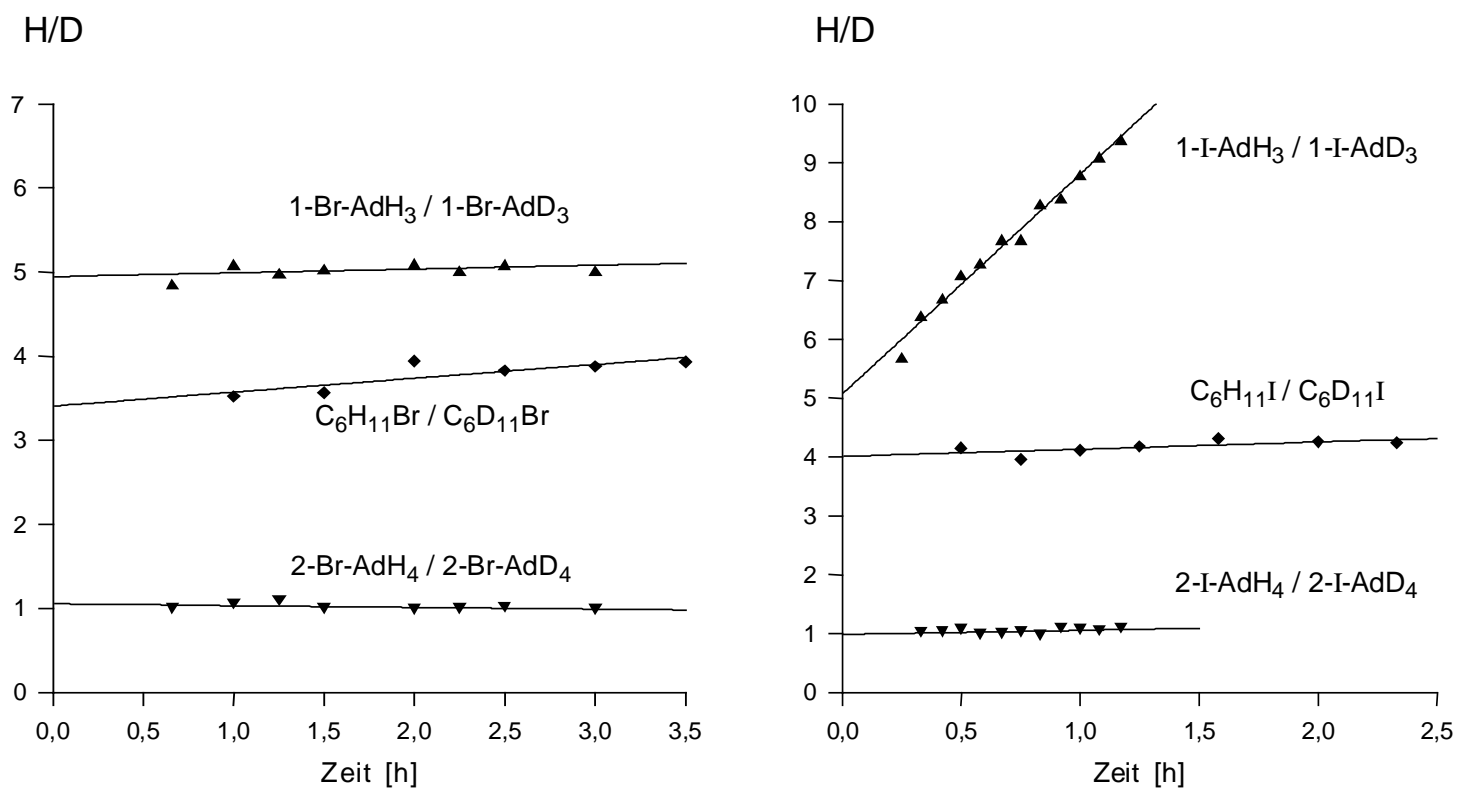

Abbildung 16. Das Verhältnis von deuteriertem zu nicht-deuteriertem Produkt für die Bromierung (links) und Iodierung (rechts) von Cyclohexan sowie der tertiären und sekundären Position von Adamantan unter PTK Bedingungen. Jeder Meßpunkt ist ein Mittelwert aus fünf Experimenten (AV12).

Der extreme Anstieg des KIE nach dem Erreichen von ca. 10\% Umsatz könnte auch auf das Auftreten von Nebenreaktionen, wie z.B. Eliminierungen zurückgeführt werden. 
Allerdings kann auch nicht erklärt werden wie diese Nebenreaktionen einen Erhöhung des KIE bewirken können. So wurde als entscheidende Zeitphase der Reaktion für die Bestimmung des KIE diejenige angesehen, in der dieser über mehrere Stunden konstant blieb (AV11, Abb. 16).

Die Fehlergrenzen wurden durch eine einfache Standartabweichung mit einem 95\% Konfidenzintervall für die jeweilige Meßreihe bestimmt. Eine genaue Berechnung des Folgefehlers verursacht durch Meßungenauigkeiten wie z.B. Einwaagefehler oder Fehler bei der Volumenbestimmung, konnte nicht durchgeführt werden, da naturgemäß keine mathematische Abhängigkeit von den Reaktionsvariablen gegeben ist, weil es sich bei den Experimenten um direkte Konkurrenzreaktionen handelt.

Tabelle 6. Experimentelle KIE bei der PTK Halogenierung (Br, I) von Cyclohexan sowie der tert. und sek. Position von Adamantan (AV12)

\begin{tabular}{cll}
\hline$\#$ & \multicolumn{1}{c}{ Reaktionssystem } & KIE \\
\hline $\mathbf{1}$ & Bromierung von Adamantan $\left(3^{\circ}\right)$ & $4.9 \pm 0.3$ \\
$\mathbf{2}$ & Bromierung von Adamantan $\left(2^{\circ}\right)^{[\mathrm{b}]}$ & $1.1 \pm 0.1$ \\
$\mathbf{3}$ & Iodierung von Adamantan $\left(3^{\circ}\right)$ & $5.1 \pm 0.7$ \\
$\mathbf{4}$ & Iodierung von Adamantan $\left(2^{\circ}\right)^{[\mathrm{b}]}$ & $1.0 \pm 0.1$ \\
$\mathbf{5}$ & Bromierung von Cyclohexan & $4.0 \pm 0.5^{[\mathrm{a}]}$ \\
$\mathbf{6}$ & Iodierung von Cyclohexan & $4.7 \pm 0.4^{[\mathrm{a}]}$
\end{tabular}

[a] Die Werte wurden kalibriert (KF (Nr. 5) $=1.15$ und KF (Nr. 6) $=1.18) \quad$ [b] Der ermittelte KIE ist sekundär, da 1,3,5,7-Tetradeuteroadamantan als Edukt verwendet wurde.

Die Reaktionsbedingungen wurden in weitem Umfang variiert, um zu belegen, daß der Isotopeneffekt in keiner Weise von anderen Faktoren als von dem die Geschwindigkeit bestimmenden, der H-Abstraktion, abhängt. Dazu wurde sowohl die Menge an Base als auch die Eduktkonzentration erhöht und erniedrigt, mit dem Ergebnis, daß nur marginale, im Bereich der Fehlergrenzen befindliche Differenzen zu den unter den Standartbedingungen gemessenen KIEs auftraten. Bei Reaktionen, deren Geschwindigkeit durch Zusatz größerer Menge an Base beschleunigt abliefen, wurde festgestellt, daß sich die Induktionszeit, d.h. der Zeitraum, in dem eine geringerer KIE festgestellt wurde, verkürzte. Allerdings wurde auch die 
Phase der Reaktionen mit konstantem KIE verkürzt. Bei Verlangsamung der Reaktionen wurde der umgekehrte Trend festgestellt.

\subsubsection{Die Berechnung des kinetischen Isotopeneffektes}

Um einen Vergleich zu den experimentell erhaltenen Daten ziehen zu können, sollten die KIEs computerberechnet werden. Wie einleitend gezeigt wurde, eignen sich auf der Dichtefunktionaltheorie basierenden Computerberechnungen gut zur Simulation von Radikalreaktionen. Abgesehen davon ist aufgrund der Größe der betrachteten Reaktionssyteme mit bis zu 14 Schweratomen, die Verwendung von ab initio Methoden die z.B. auf dem störungstheoretischen Ansatz beruhen (Møller-Plesset) oder noch aufwendigeren Methoden nicht möglich. So wurden sämtliche Computerberechnungen in diesem Kapitel auf dem B3L YP/6-31G** (C, H, Br), 3-21G* (I) durchgeführt.

Neben der Wahl einer bestimmten Methode mußte auch eine Entscheidung über den theoretischen Ansatz getroffen werden, mit dem, aus den durch die Computerberechnungen erhaltenen Daten, der KIE berechnet werden sollte. Der einfachste Ansatz ist derjenige, in dem nur der energetische Unterschied zwischen einer einzigen $\mathrm{C}-\mathrm{H}-$ und $\mathrm{C}-\mathrm{D}-$ Streckschwingung $\left(\nu_{H / D}\right)$ des Reaktanden berücksichtigt wird. Im ÜZ werden diese imaginär, da aus ihnen diejenigen Reaktionsmodi geworden sind, die den Geschwindigkeiten entsprechen, mit der die Energiebarrieren des ÜZ überschritten werden.[136] So kann ohne eine Berücksichtigung von Symmetriezahlen eine sehr einfache Gleichung abgeleitet werden, die allerdings nur zur Abschätzung eines KIEs geeignet ist und als die Streckfrequenznäherung bezeichnet wird (Gl. IIX).[127, 128]

$$
\frac{k_{H}}{k_{D}}=\exp \left[\frac{h c}{2 k T}\left(v_{H}-v_{D}\right)\right]
$$

$$
\begin{aligned}
& h=\text { Planksche Konstante } \\
& c=\text { Lichtgeschwindigkeit } \\
& k=\text { Boltzmann-Konstante } \\
& \mathrm{T}=\text { Temperatur }
\end{aligned}
$$

In vielen Fällen muß allerdings auch berücksichtigt werden, daß sich die Beiträge der Deformationsschwingungen im ÜZ und im Reaktanden sich nicht gegenseitig ausgleichen, weil sie zu stark unterschiedlich sind. Näherungsweise werden dann im Reaktanden zwei Deformationsschwingungen und eine Streckschwingung und im ÜZ nur diese zwei Deformationschwingungen berücksichtigt.[127, 128] Wird als Reaktionsbedingung RT 
angenommen, so sind die Deformationsschwingungen bereits stark angeregt und verhalten sich wie klassische Oszillatoren und es kann die folgenden Gleichung (IX) abgeleitet werden.

$$
\frac{k_{H}}{k_{D}}=\frac{m_{H}}{m_{D}} \exp \left[\frac{h c}{2 k T} \sum_{i=1}^{3}\left(v_{i H}-v_{i D}\right)\right]
$$

$$
\begin{aligned}
& h=\text { Planksche Konstante } \\
& c=\text { Lichtgeschwindigkeit } \\
& k=\text { Boltzmann-Konstante } \\
& \mathrm{T}=\text { Temperatur } \\
& \mathrm{m}=\text { Masse }
\end{aligned}
$$

Beide Ansätze sind allerdings grobe Näherungen und nicht sehr exakt. Sie wurden deshalb entwickelt, um aus spektroskopischen ermittelbaren Größen schnell einen KIE abschätzen zu können. Heutzutage können die entsprechenden Frequenzen auch durch Computerberechnungen mit hoher Genauigkeit ermittelt werden. Diese führen aber aufgrund der Näherungen des mathematischen Ansatzes (IIX, IX) ebenfalls nur zu relativ schlechten Ergebnissen.

Eine Berechnung des KIE kann auch auf der Basis von Geschwindigkeitskonstanten erfolgen, wobei auch die Energetik der ÜZ miteinbezogen wird und am einfachsten durch den Ansatz von Arrhenius erfolgen kann (X). In diesem Fall basiert die Berechnung der Geschwindigkeitskonstanten einzig auf den Aktivierungsenergien der betrachteten Reaktionen, die für die isotopomeren Edukte, wie einleitend erwähnt, unterschiedlich sind.

$$
k_{o b s}=A \exp \left[-\frac{E_{a}}{R T}\right]
$$

$$
\begin{aligned}
& A=\text { Präexponentieller Faktor } \\
& R=\text { Allgemeine Gaskonstante } \\
& \mathrm{T}=\text { Temperatur } \\
& E_{a}=\text { Aktivierungsenergie }
\end{aligned}
$$

Der Quotient der Geschwindigkeitskonstanten für das deuterierte und nichtdeuterierte Produkt entspricht dem KIE, wobei die präexponentiellen Faktoren $A_{H}$ und $A_{D}$ als gleich groß angenommen werden. Diese Näherung kann aufgrund zweier Umstände gemacht werden; T ist Relativ niedrig (RT) und die optimierten ÜZ Strukturen sind annähernd linear (d.h. der Winkel zwischen brechender und sich bildender Bindung ist ca. $180^{\circ}$, Abb. 17).[127, 128] Dadurch resultiert die folgende Gleichung (XI). 


$$
\begin{aligned}
\frac{k_{H}}{k_{D}} & =K I E=\exp \left[-\frac{\left(E_{a H}-E_{a D}\right)}{R T}\right] & & ; \text { mit } E_{a}=\Delta H_{298}^{\ddagger}-R T \\
& =\exp \left[-\frac{\left(\Delta H_{298(H)}^{\ddagger}-\Delta H_{298(D)}^{\ddagger}\right)}{R T}\right] & & \text { (XI) }
\end{aligned}
$$

Diese Gleichung wurde für alle folgenden Berechnungen zugrunde gelegt. Dabei wurde $E_{a}$ als die Differenz $\Delta H^{\ddagger}{ }_{298}$ der thermisch korrigierten Aktivierungsenthalpien $\left(\Delta H_{0}-\right.$ $\left.\Delta H_{298}\right)$ der deuterierten und nichtdeuterierten Spezies in Relation zu deren Grundzuständen angesehen. Für die Berechnungen der H-Abstraktion von Cyclohexan sind zwei verschiedene ÜZ denkbar. Dabei ist der axiale ÜZ energetisch unvorteilhafter (Vorabberechnungen auf HFNiveau) und deshalb wurde der äquatoriale ÜZ für die Computerberechnungen optimiert.

Tabelle 7. Computerberechnete KIEs bei der PT-Halogenierung (Br, I) von Cyclohexan sowie der tert. und sek. Position von Adamantan [B3LYP/6-31G** (C,H,Br); 3-21G* (I)], s. Abb. 17.

\begin{tabular}{cccc}
\hline$\#$ & \multicolumn{1}{c}{ TS } & KIE \\
\hline $\mathbf{1}$ & $\mathrm{Br}_{3} \mathrm{C} \cdots \mathrm{H} \cdots$ Adamant-1-yl & $(\ddot{\mathbf{U Z Z 1}})$ & 4.9 \\
$\mathbf{2}$ & $\mathrm{Br}_{3} \mathrm{C} \cdots \mathrm{H} \cdots$ Adamant-2-yl & $(\ddot{\mathbf{U Z Z 2}})$ & 1.0 \\
$\mathbf{3}$ & $\mathrm{I}_{3} \mathrm{C} \cdots \mathrm{H} \cdots$ Adamant$-1-\mathrm{yl}$ & $(\ddot{\mathbf{U Z Z 3}})$ & 5.3 \\
$\mathbf{4}$ & $\mathrm{I}_{3} \mathrm{C} \cdots \mathrm{H} \cdots$ Adamant$-2-\mathrm{yl}^{[\mathrm{a}]}$ & $(\ddot{\mathbf{U Z Z}})$ & 1.0 \\
$\mathbf{5}$ & $\mathrm{Br}_{3} \mathrm{C} \cdots \mathrm{H} \cdots \ddot{a} q \cdot-c-\mathrm{C}_{6} \mathrm{H}_{11}$ & $(\ddot{\mathbf{U Z Z 5}})$ & 4.7 \\
$\mathbf{6}$ & $\mathrm{I}_{3} \mathrm{C} \cdots \mathrm{H} \cdots \ddot{a} q \cdot-c-\mathrm{C}_{6} \mathrm{H}_{11}$ & $(\ddot{\mathbf{U Z Z 6}})$ & 5.0
\end{tabular}

[a] Der berechnete KIE ist sekundär, da 1,3,5,7-Tetradeuteroadamantan verwendet wurde

Die berechneten relativ hohen primären KIEs im Bereich von 4.7 - 5.3 sind plausibel, da die Länge der brechenden und sich bildenden Bindung in den optimierten ÜZ-Strukturen (ت̈ZZ1 ÜZ6, Abb. 17) sehr symmetrisch sind sowie annähernd einen Winkel von $180^{\circ}$ einnehmen. Frühere, spätere oder gewinkelte ÜZ würden niedrigere KIEs verursachen.[127, 128] Auch wenn die Computerberechnungen aufgrund der Größe der betrachteten Systeme nicht auf höchstem Niveau durchgeführt wurden [B3LYP/6-31G** (C,H,Br); 3-21G* (I)], und die absoluten Aktivierungsenergien (17 - $\left.19 \mathrm{kcal} \mathrm{mol}^{-1}\right)$ vielleicht etwas zu hoch ausfallen,[112, 113] kann doch der Schluß gezogen werden, daß aufgrund deren Höhe Tunneleffekte nur im geringen Maß eine Rolle spielen. 


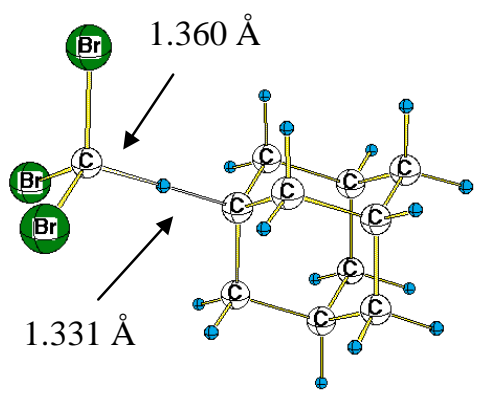

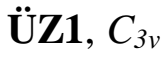

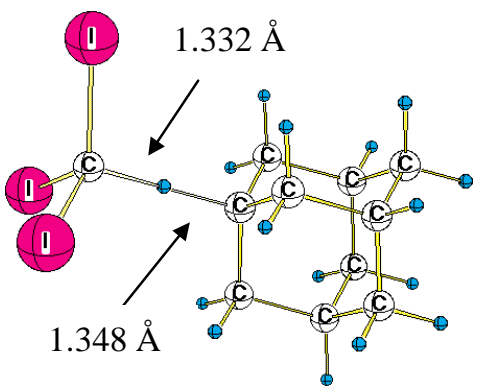

$\ddot{\mathbf{U Z Z}}, C_{3 v}$

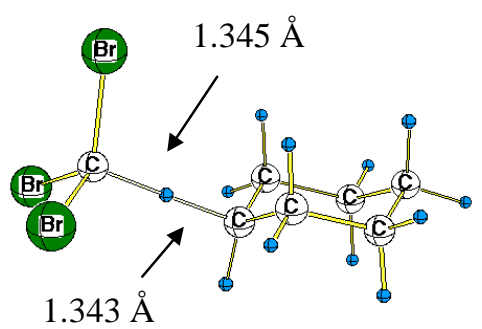

ÜZ5, $C_{s}$

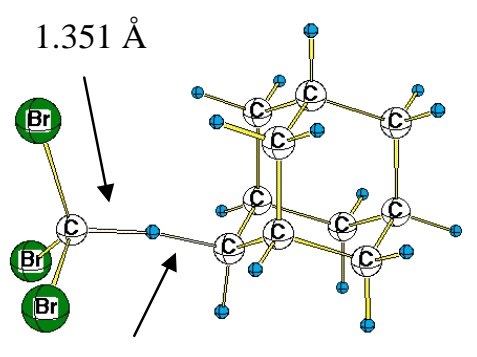

$1.353 \AA$

$\ddot{\mathbf{U Z 2}}, C_{s}$

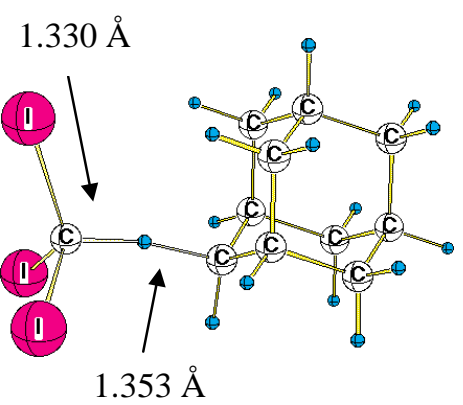

$\ddot{\mathbf{U Z Z}}, C_{s}$

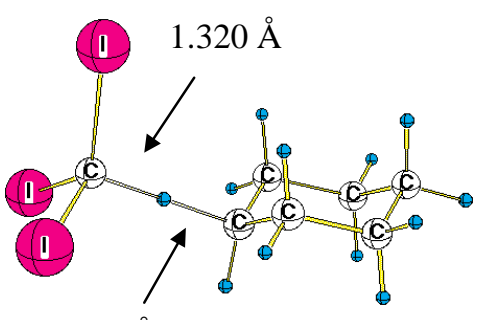

$1.361 \AA$

ÜZ6, $C_{s}$

\begin{abstract}
Abbildung 17. Computerberechnete ÜZ für die H-Abstraktion in der tertiären (ÜZ1,

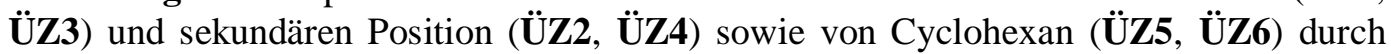
$\cdot \mathrm{CBr}_{3}$ und $\cdot \mathrm{CI}_{3}\left[\mathrm{~B} 3 \mathrm{LYP} / 6-31 \mathrm{G}^{* *}(\mathrm{C}, \mathrm{H}, \mathrm{Br}) ; 3-21 \mathrm{G}^{*}(\mathrm{I})\right]$
\end{abstract}

Weiterhin kann angenommen werden, daß sich der Fehler der überhöhten absoluten Energiebarrieren bei Vergleich zwischen der jeweiligen deuterierten und nichtdeuterierten Spezies herausmittelt, und die relativen Energiedifferenzen für die Berechnung der KIEs exakt genug sind, was durch die gute Übereinstimmung mit den experimentellen KIEs bestätigt wird (Kap. 2.3.). Dies erlaubt auch eine Diskussion der relativen Aktivierungsenthalpien bei der Substitution in der $3^{\circ}$ - und $2^{\circ}$-Position. So wurden relative Unterschiede bei der H-Abstraktion von $\mathbf{1}$ durch ein Tribrommethylradikal von $\Delta \Delta E_{\mathrm{a}}(\ddot{\mathbf{U} Z \mathbf{Z}}$ - 
$\ddot{\mathbf{U Z 2}})=2.6 \mathrm{kcal} \mathrm{mol}^{-1}$ und durch ein Triiodmethylradikal von $\Delta \Delta E_{\mathrm{a}}(\ddot{\mathbf{U Z Z 3}}-\ddot{\mathbf{U} Z \mathbf{Z 4}})=2.9 \mathrm{kcal}$ $\mathrm{mol}^{-1}$ berechnet, was einerseits die hohe Regioselektivität der Halogenierungen im allgemeinen und die dabei noch höhere Regioselektivität der Iodierung verständlich macht.

\subsection{Schlußfolgerungen aus den mechanistischen Untersuchungen}

Die Ergebnisse der mechanistischen Untersuchungen bestätigen im großen und ganzen die in der Einleitung vorgestellte Arbeitshypothese (Schema 2). Die Variation der PTBedingungen hat ergeben, daß sowohl bei der Bromierung als auch der Iodierung in den untersuchten Lösungsmitteln bis auf einen Fall $\left(\mathrm{CH}_{2} \mathrm{Cl}_{2}\right.$ s.u. $)$ eine starke Katalysatorabhängigkeit zu verzeichnen ist. Dabei gilt, je lipophiler der PTK, desto größer seine Effektivität (d.h. steigt mit steigender Kettenlänge der Alkylreste des Tetraalkylammoniumsalzes). Das macht einen Extraktionsmechanismus wahrscheinlich, denn eine Reaktion, die einem Interphasenmechanismus gehorcht, würde ohne einen PTK auskommen und bei einem Grenzflächenmechanismus ist die Zugänglichkeit der positiven Ladung am PTK entscheidend. So wird gemäß eines Extraktionsmechanismus ein Hydroxidion durch den PTK in die organische Phase transferiert, wo dieses dann die eigentliche Reaktion auslösen kann.

Über die Art und Weise wie die Initiierung stattfindet, können momentan nur Vermutungen angestellt werden. Ein Hinweis darauf wird durch die Tatsache erbracht, daß durch eine Erhöhung der Basenkonzentration in der wäßrigen Phase, das Hydroxidion verstärkt desolvatisiert in die organische Phase übergeht, wodurch dessen Basizität, Nukleophilie und Oxidationsspotential gesteigert wird. Ein Reaktionsweg der Initiierung, die auf die basischen Eigenschaften von ${ }^{-} \mathrm{OH}$ zurückzuführen ist kann ausgeschlossen werden, denn die Deprotonierung von Adamantan oder gar Cyclohexan erscheint äußerst unwahrscheinlich. Auch eine $\mathrm{S}_{\mathrm{N}}$-Reaktion von ${ }^{-} \mathrm{OH}$ mit dem Halomethan ist aufgrund der höheren Nukleophilie der Halogenatome eigentlich nicht denkbar. So ist wahrscheinlich die Erhöhung des Reduktionsspotentials, verursacht durch die Desolvatisierung, für den gesteigerten Umsatz verantwortlich, was für die postulierte Initiierung über einen SET vom Hydroxidion zum Tetrahalomethan sprechen würde. Ein weiterer Hinweis auf die Richtigkeit dieser Hypothese liefert die Tatsache, daß auch Kalium- $t$-butylat, daß für Nebenreaktionen, die auf SET-Prozessen beruhen, im Rahmen von $S_{N^{-}}$Reaktionen bekannt ist, die Halogenierung initiieren kann. 
Das die Reaktion eine Radikalreaktion ist, bei der das Trihalomethylradikal als Protagonist der Reaktion den Propagationszyklus trägt, kann als gesichert angenommen werden, da dafür zahlreiche experimentelle Hinweise gefunden wurden. So wird beim Vergleich der erzielten Regioselektivitäten bei der PT-Chlorierung und PT-Bromierung von Adamantan unter PT-Bedingungen mit der durch Kettenstarter (AIBN / $\mathrm{CCl}_{4}$ bzw. $\mathrm{CBr}_{4}$ ) initiierten fast gleiche Produktverhältnise $\left(3^{\circ}: 2^{\circ}\right)$ festgestellt (Tab. 4). Nur bei der Iodierung ist die Selektivität der PT-Halogenierung höher, was neben den in vorangegangenen Arbeiten durchgeführten Nachweis von $\mathrm{CI}_{4}$ durch EI/MS-Spektroskopie, [30, 31] einen Hinweis auf die Richtigkeit der Hypothese der Äquilibrierung von Iodoform zu Diiodmethan und Tetraiodmethan darstellt, wobei letzteres als die eigentliche Halogenquelle agiert. Ferner konnte das Tribrommethylradikal durch ein Abfangexperiment (Kap. 2.4) nachgewiesen werden

Weitere Bestätigung für die Existenz des Trihalomethylradikals lieferte eine systematische Untersuchung der KIEs bei der Halogenierung (Br, I) unter PT-Bedingungen. Sowohl die Bromierung als auch die Iodierung von Cyclohexan vs. $D_{12}-$ Cyclohexan und Adamantan vs. $D_{4}$-Adamantan (Kap. 2.5) zeigen einen merklichen primären KIE, woraus gefolgert werden kann, daß der Schritt der H-Abstraktion geschwindigkeitsbestimmend sein muß. Die mit der B3LYP Methode computerberechneten KIEs sind in hervorragender Übereinstimmung mit den experimentellen Werten. Daraus läßt sich folgern, daß die computeroptimierten Strukturen der ÜZ wahrscheinlich den entscheidenden Schlüsselschritt des Bindungsbruches und gleichzeitiger Bindungsbildung der Reaktionen gut repräsentieren. So bestätigen die Geometrien der ÜZ, deren Längen der brechenden und sich bildenden Bindung in den optimierten ÜZ-Strukturen sehr symmetrisch sind, d.h. annähernd zwischen entsprechenden Kohlenwasserstoff und dem Trihalomethylradikal lokalisiert sind, die experimentellen KIEs mit Werten um 5. Ein sekundärer KIE konnte nicht beobachtet werden. Die experimentellen KIEs unterscheiden sich auch deutlich von denen anderer Halogenierungsmethoden. Im Rahmen der Bromierung von Cyclohexan unter GifBedingungen werden KIEs zwischen 2.1 und 2.4 und unter Fenton Bedingungen ein KIE von 1.5 gemessen.[137] Allerdings resultiert bei der radikalisch initiierten Bromierung von Cyclohexan vs. $D_{12}-$ Cyclohexan mit elementarem Brom ein KIE von 3 - 4 (abhängig von den Reaktionsbedingungen) ${ }^{[138]}$ und unterscheidet sich somit nur geringfügig von dem der PTBromierung (4.0). Durch die hohe Regioselektivität bei der PT-Bromierung von 1 kann aber eine Verantwortlichkeit von Bromradikalen für den Fortgang der Propagation unter basischen Bedingungen ausgeschlossen werden. 
Abschließend soll hier noch auf die besondere Rolle von Dichlormethan bei den PTHalogenierungen eingegangen werden. Eine Bromierung von 1 in $\mathrm{CH}_{2} \mathrm{Cl}_{2}$ als Lösungsmittel kann im "klassisch" basischen Milieu [aq. $\mathrm{NaOH}$ (50\%)], mit katalytischen Mengen an $\mathrm{NaOH}$, im sauren Milieu [aq. $\mathrm{HBr}(10 \%)]$ und sogar mit reinem Wasser durchgeführt werden. Ohne den Zusatz einer wäßrigen Phase findet keine Reaktion statt und der Zusatz eines PTK ist nicht notwendig. Um die allgemeine Gültigkeit dieser Beobachtung zu verifizieren, wurde auch versucht die Iodierung von 1 in $\mathrm{CH}_{2} \mathrm{Cl}_{2}$ nur initiiert durch Wasser durchzuführen. Allerdings konnten hier nur Spuren von 17 durch eine GC/MS-Analyse nachgewiesen werden. Auffällig ist, daß bei Bromierungen von 1 unter nicht basischen Reaktionsbedingungen eine geringere $3^{\circ}: 2^{\circ}$ Selektivität resultiert, was auf einen Wechsel des Reaktionsmechanismus hindeuten könnte. So würde eine einfache Hydrolyse von Tetrabrommethan zu molekularem Brom als Endprodukt führen (Schema 14), das auch durch eine Abfangreaktion in der Reaktionsmischung nachgewiesen wurde. Die Homolyse von Brom, die auch schon bei RT im geringen Maß stattfinden kann, oder die Homolyse eines der primären Hydrolyseprodukte von $\mathrm{CBr}_{4}$ (Schema 14) könnte dann die Reaktion initiieren, womit auch die geringere Regioselektivität der Substitution unter diesen Reaktionsbedingungen erklärt werden könnte. Es muß an dieser Stelle vermerkt werden, daß diese Annahmen ziemlich spekulativ sind und es weiterer systematischer Untersuchungen bezüglich der Lösungsmittelabhängigkeit der Initiierung bedarf, sie aber die gemachten experimentellen Beobachtungen gut erklären können.

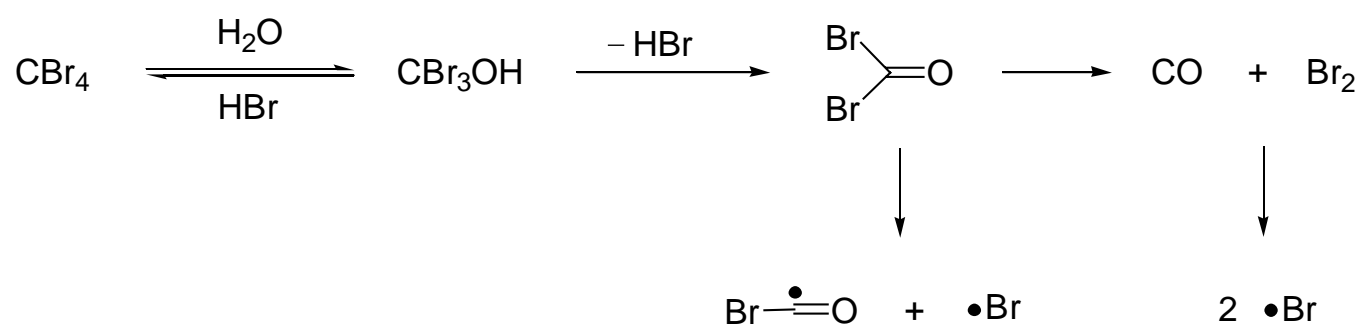

Schema 14. Mögliche Mechanismen der Hydrolyse von Tetrabrommethan und Homolyse der Folgeprodukte

Es stellt sich allerdings auch die Frage, warum keine Iodierung mit Iodoform, nur initiiert durch Wasser, durchgeführt werden kann. Ein Grund dafür könnte sein, daß für die Äquilibrierung von $\mathrm{CHI}_{3}$ zu $\mathrm{CH}_{2} \mathrm{I}_{2}$ und $\mathrm{CI}_{4}$ basische Reaktionsbedingungen notwendig sind, 
so daß kein Tetrahalomethan für eine Hydrolyse zur Verfügung steht. Möglich wäre auch, daß Iodoform zu schnell hydrolysiert wird, als das eine signifikante Bildung von Tetraiodmethan stattfinden könnte.

\section{Verschiedene Anwendungen der PT-Halogenierungen}

3.1. Die PT-Halogenierung (Cl, Br, I) von Cuban unter Erhaltung der Käfigstruktur[139]

\subsubsection{Einleitung}

Als erster Vertreter der platonischen Kohlenwasserstoffe wurde Cuban (Pentacyclo[4.2.0.0. $\left.{ }^{2,5} 0 .^{3,8} \cdot 0^{4,7}\right]$ octan) 1964 von Eaton et al. zum ersten Mal synthetisiert[140] und stellt aufgrund seiner außergewöhnlichen Struktur und der damit einhergehenden extremen Ringspannung (166 kcal mol$\left.{ }^{-1}\right)^{[141]}$ die ultimative Modellverbindung für gespannte Systeme dar. Trotzdem ist Cuban kinetisch stabil, da alle konzertierten Reaktionswege, die zu einer Fragmentierung der Gerüststruktur führen würden, symmetrieverboten und deswegen mit einer hohen Aktivierungsbarriere verbunden sind. ${ }^{[142]}$ Die meisten Synthesestrategien der Cubanderivate leiten sich von einer Zwischenstufe der Cubansynthese, der 1,4Cubandicarbonsäure $a b,[143,144]$ so daß die direkte Derivatisierung von Cuban einen Umweg darstellt, der bislang nur in bestimmten Fällen eingeschlagen wurde (s.u.). Durch Umwandlung dieser funktionellen Gruppen in Amide[145] und nachfolgende orthoMetallierungen[146-148] konnten die verbleibenden Positionen im Cubangerüst sehr selektiv aktiviert und ein Zugang zu zahlreichen Cubanverbindungen geschaffen werden.[143, 144] Aus der 1,4-Cubandicarbonsäure kann auch direkt das Diiodid gewonnen werden, ${ }^{[149,150]}$ das als Ausgangsverbindung für das 1,4-Dedehydrocuban[151] dient und u.a. zur Synthese von arylsubstituierten und verschiedenen $p$-[n]polymeren $(\mathrm{n}=2-4)$ Cubanen verwendet wurde. ${ }^{[151]}$ Eine der Intentionen für die Anstrengungen zur Polyfunktionalisierung von Cuban war die Synthese von Octanitrocuban, einem der potentiell vielversprechendsten Sprengstoffe, ${ }^{[152]}$ die schließlich nach 20 Jahren Forschungsarbeit durch Eaton et al. jüngst realisiert werden konnte.[153]

Die direkte Funktionalisierung von Cuban war bislang aus den eben genannten Gründen bislang nur von mechanistischem Interesse. Obwohl die Acididät von Cuban im Vergleich von z.B. Cyclohexan (63000 fach) durch den hohen $s$-Charakter (ca. 31\%) der C- 
H-Bindung erheblich verstärkt ist, ${ }^{[154]}$ reicht dies nicht zur präparativen Aktivierung von Cuban aus. Übergangsmetallkomlexe (Rh, Pd) ${ }^{[155-157]}$ und das durch Photooxidation z.B. mit TCB (1,2,4,5-Tetracyanobenzol) generierte Radikalkation[158] führen $\mathrm{zu}$ einer Fragmentierung der Cubanstruktur.

Auch die radikalische Halogenierung $(\mathrm{Cl}, \mathrm{Br})$ von Cuban (32) führt zu Umlagerungen des Cubangerüstes und Mehrfachhalogenierungen. So entsteht bei der Bromierung mit elementarem Brom unter photolytischen Reaktionsbedingungen als Hauptprodukt das Tetrabromid 33 (Schema 15)[159] und unter den gleichen Reaktionsbedingungen mit elementarem Chlor eine komplexe Mischung von Polychlorcubanen und einer geringen Menge des Tetrachlorids 34 (Schema 15).[159] Dies ist darauf zurückzuführen, daß die Halogenradikale gemäß einem $S_{\mathrm{H}}$ 2-Mechanismus reagieren können, wie es auch schon bei der radikalischen Bromierung von Bicyclo[2.2.0]hexan ${ }^{[160]}$ und verschiedenen [n.2.2.]Propellanen[161] beobachtet wurde. Während dies bei der Bromierung die Hauptreaktion ist, konkurriert bei der Chlorierung die $\mathrm{S}_{\mathrm{H}}$ 2-Reaktion mit der C-H-Abstraktion, wobei die Produktverteilung eine kinetische Reaktionskontrolle wahrscheinlich macht.

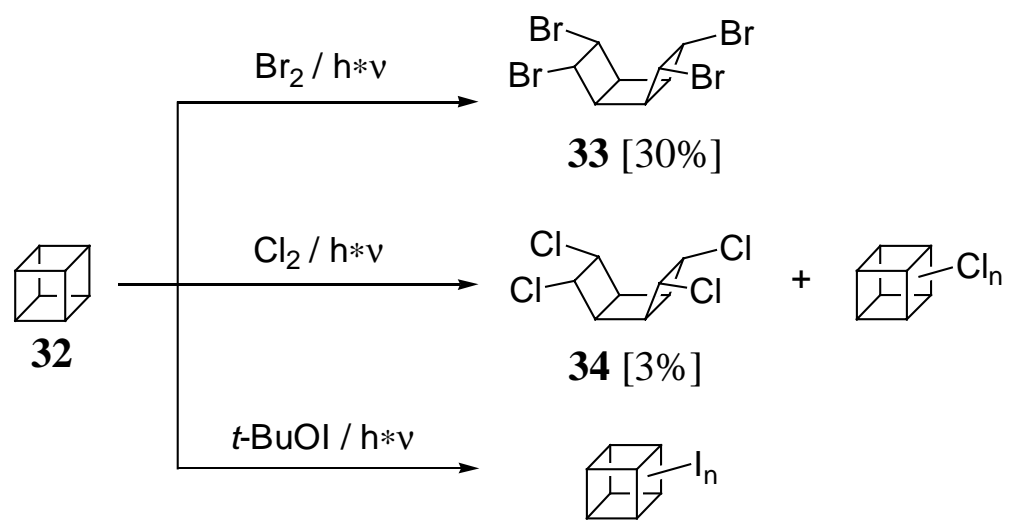

Schema 15. Die Produktbildung bei der photoinitiierten radikalischen Halogenierung von Cuban

Der einzige bislang beschriebene Fall der direkten Halogenierung von 32 ohne jeglichen Zerfall des Cubangerüstes ist die photoinitiierte Iodierung mit $t$-Butylhypoiodid, wobei das $t$-Butoxyradikal für die H-Abstraktion verantwortlich ist (Schema 15). Allerdings kann auch hier die Reaktion nicht auf der Stufe der Monohalogenierung angehalten werden, was $\mathrm{zu}$ einer komplexen Mischung von Polyiodcubanen führt.[162] Aufgrund der mangelhaften Chemoselektivität der Produktbildung, ist die photoinitiierte Halogenierung von 
Cuban für Synthesen im präparativen Maßstab uninteressant. Deswegen sollte überprüft werden, ob die Halogenierung unter PT-Bedingungen bessere Ergebnisse erbringen würde. Sollte dies der Fall sein, wäre hier eine neue allgemeine Möglichkeit zur Funktionalisierung von hochgespannten Kohlenwasserstoffen gefunden worden, denn was bei Cuban als einem der gespanntesten Kohlenwasserstoffe durchführbar ist, sollte auch bei anderen gespannten Systemen Anwendung finden können.

\subsubsection{Die PT-Halogenierung (Cl, Br, I) von Cuban unter PT-Bedingungen}

Es wurde festgestellt, daß die Monohalogenierungen (Cl, Br, I, Schema 16, AV13a-c) von Cuban unter PT-Bedingungen mit guten bis sehr guten Ausbeuten unter Erhaltung des Cubangerüstes durchführbar sind. Im Rahmen von GC/MS-Untersuchungen der Reaktionsmischungen, zur Verfolgung des Fortschritts der jeweiligen Reaktion, konnten als einzige Nebenprodukte geringe Mengen $(<5 \%)$ an dihalogenierten Cubanen nachgewiesen werden.

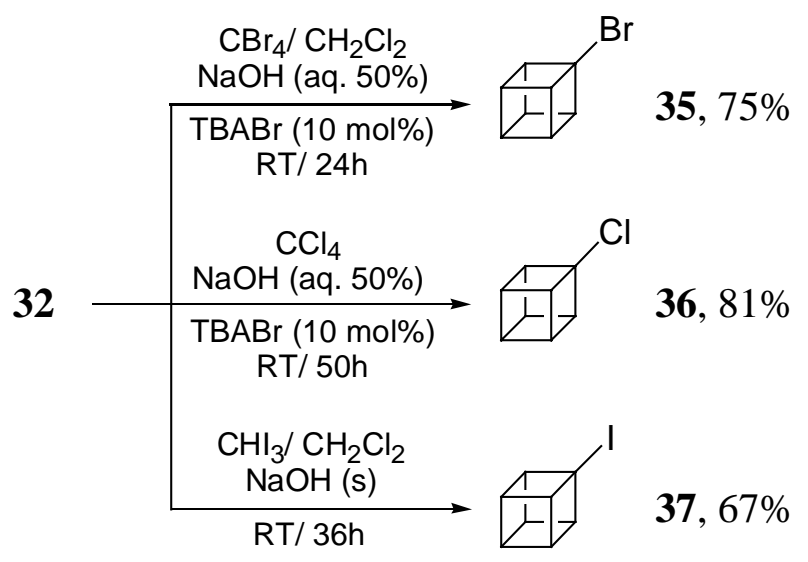

Schema 16. Die präparativen Ausbeuten bei Halogenierung von 32 unter PTBedingungen (Cl, Br, I, AV13a-c)

Die Bromierung unter PTK Bedingungen [AV13a, $\mathrm{CBr}_{4} / \mathrm{NaOH}$ (aq. 50\%)/ $\mathrm{TBABr}$ (10 mol\%), $\mathrm{CH}_{2} \mathrm{Cl}_{2} / \mathrm{RT} / 36 \mathrm{~h}$ ] führte zu einer präparativen Ausbeute von $75 \%$ an reinem Monobromid 35. Anstelle von Dichlormethan wurde bei der Chlorierung $\mathrm{CCl}_{4}$ als Lösungsmittel eingesetzt, das gleichzeitig auch als Halogenquelle dient, wobei $81 \%$ an 
Monochlorcuban (36) isoliert werden konnten [AV13b, $\mathrm{CCl}_{4} / \mathrm{NaOH}$ (aq. 50\%)/ $\mathrm{TBABr}(10$ mol\%), RT/ 50h].

Um die Iodierung von Cuban mit guten Ausbeuten durchführen zu können, mußten die Reaktionsbedingungen leicht modifiziert werden. So kann auf die Verwendung eines PTK ganz verzichtet und anstelle von wäßriger Natriumhydroxidlösung sollte festes $\mathrm{NaOH}$ verwendet werden. Anderenfalls konnten nur Spuren von Produkt isoliert werden und die Reaktion wies eine negative Massenbilanz auf. Dies ist wahrscheinlich auf eine geringere Stabilität der Iodide im Gegensatz zu den Bromiden und Chloriden unter den stark basischen, wäßrigen Bedingungen zurückzuführen. Mit diesen Modifikationen der Reaktionsbedingungen $\left[\mathrm{AV} 13 \mathrm{c}, \mathrm{CHI}_{3} / \mathrm{NaOH}(\mathrm{s}) / \mathrm{CH}_{2} \mathrm{Cl}_{2}\right.$ ] konnten $67 \%$ an Monoiodid 37 erhalten werden.

Aufgrund einer starken Deaktivierung des Cubangerüstes durch den Erstsubstituenten, tritt eine Mehrfachhalogenierung in den jeweils angegebenen Reaktionszeiten nur im untergeordneten Maß auf. Das Ausmaß und die genauen Gründe hierfür werden im Rahmen der Mehrfachhalogenierungen noch genauer untersucht und beschrieben (s. Kap. 3.1.5.).

\subsubsection{Computerberechnungen zur Erklärung der Produktverteilung bei der Halogenierung von Cuban (Cl, Br)}

Wie bereits einleitend erwähnt wurde, verlaufen die PT-Halogenierungen im Gegensatz zu den photolytisch initiierten Reaktionen unter Erhaltung des Cubangerüstes. Dies muß auf die Natur des abstrahierenden Radikals zurückzuführen sein, also auf ein unterschiedliches Reaktionsverhalten des $\mathrm{CX}_{3}-$ und $\mathrm{X}$-Radikals $(\mathrm{X}=\mathrm{Cl}, \mathrm{Br})$. Um diesen Sachverhalt genauer $\mathrm{zu}$ untersuchen, wurden Computerberechnungen auf DFT-Niveau (B3LYP/6-311+G**//B3LYP/6-31G**) durchgeführt. Mit Hilfe der Computerberechnungen sollten die verschiedenen Reaktionswege eines Halogenradikals $(\bullet X)$ und des Trihalomethylradikals $\left(\bullet \mathrm{CX}_{3}\right)$ mit Cuban verglichen werden $(\mathrm{X}=\mathrm{Cl}, \mathrm{Br}$, Schema 17$)$, um das unterschiedliche Reaktionsverhalten der Radikale erklären zu können. Es sind hierbei zwei verschiedenen Reaktionswege denkbar. 

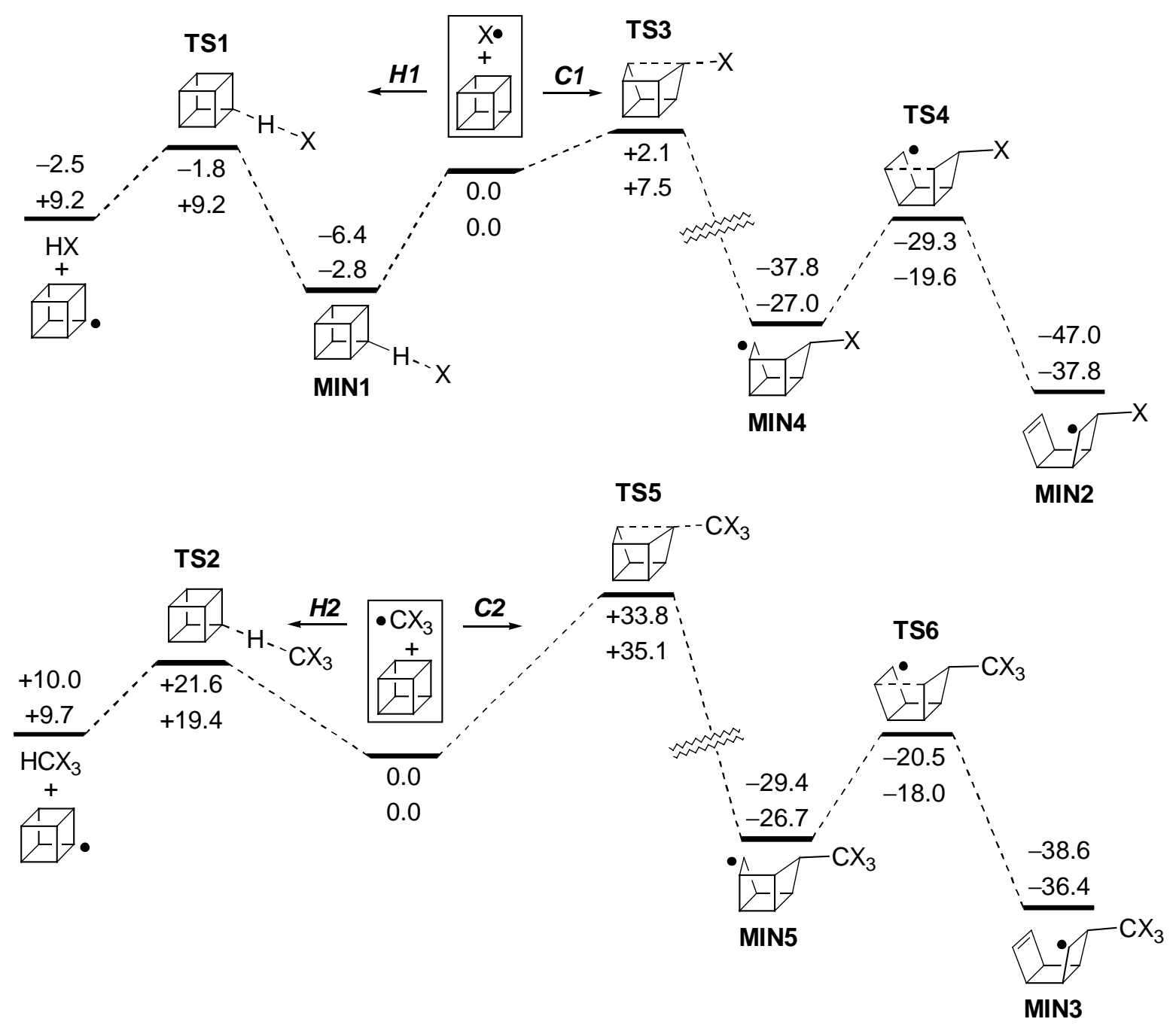

Schema 17. Computerberechnete Reaktionswege der H-Abstraktion $(\boldsymbol{H 1}, \boldsymbol{H 2})$ sowie C-Angriff (Fragmentierung: $\boldsymbol{C 1}, \boldsymbol{C 2})$ von Cuban durch ein Halogen bzw. Trihalomethylradikal $(\mathrm{X}=\mathrm{Cl}$ : erste Reihe, $\mathrm{X}=\mathrm{Br}$ : zweite Reihe; $\Delta G_{298}\left(\mathrm{kcal} \mathrm{mol}^{-1}\right)$, B3LYP/6-311+G** // B3LYP/6-31G**)

Zum einen kann der Angriff eines dieser Radikale unter Abstraktion eines H-Atoms erfolgen (Reaktionswege: $\boldsymbol{H} \boldsymbol{1}=\mathrm{H}-$ Abstraktion durch $\bullet \mathrm{X}$ und $\boldsymbol{H} \mathbf{2}=\mathrm{H}-$ Abstraktion durch $\left.\cdot \mathrm{CX}_{3} ; \mathrm{X}=\mathrm{Cl}, \mathrm{Br}\right)$. Dabei bildet Cuban exergonisch einen $\eta^{1}$-Komplex mit dem Halogenradikal (MIN1, Schema 17), der bei der H-Abstraktion als Grundzustand angesehen wird. Im Gegensatz dazu ist die Bildung eines solchen Komplexes mit $\bullet \mathrm{CX}_{3}$ thermoneutral und muß deswegen nicht berücksichtigt werden. Der andere mögliche Reaktionsweg ist der direkte Angriff auf ein $\mathrm{C}$-Atom gemäß eines $\mathrm{S}_{\mathrm{H}} 2$-Mechanismus (Reaktionswege: $\boldsymbol{C l}=\mathrm{C}$ Angriff durch $\bullet \mathrm{X}$ und $\boldsymbol{C 2}=\mathrm{C}-$ Angriff durch $\cdot \mathrm{CX}_{3} ; \mathrm{X}=\mathrm{Cl}$, Br, Schema 17), der zu einer Fragmentierung des Cubangerüstes führt. Die C-H-Bindungsaktivierung vollzieht sich über die Übergangszustände TS1 und TS2 (Reaktionswege $\boldsymbol{H 1}$ und $\boldsymbol{H 2}$, Schema 17) und sind 
aufgrund der geringen Stabilität des Cubanradikals leicht exergonisch. Dagegen sind die Reaktionswege $\boldsymbol{C 1}$ und $\boldsymbol{C 2}$, die zur Ringöffnung führen, aufgrund des Nachlassens der Ringspannung stark exergonisch und verlaufen über die Übergangszustände TS3-TS6 und die Zwischenprodukte MIN4 und MIN5 (Schema 17). Im Fall der Halogenradikale haben die Reaktionswege der H-Abstraktion (H1) und des C-Angriffs (C1) allgemein relativ geringe Aktivierungsbarrieren (entsprechend für $\mathrm{Cl}, \mathrm{Br}: \boldsymbol{C l}=2.1,7.5$ u. $\boldsymbol{H 1}=4.6,12.0 \mathrm{kcal} \mathrm{mol}^{-1}$, Schema 17). Der Reaktionsweg der Fragmentierung (C1) für das Bromradikal über MIN4 ist somit sowohl kinetisch als auch thermodynamisch favorisiert.

Wird der Reaktionsweg der H-Abstraktion durch ein Chlor- mit dem des Bromradikals verglichen, so kann festgestellt werden, daß im ersten Fall die Aktivierungsbarriere erheblich geringer ist (entsprechend 4.6 gegenüber $12.0 \mathrm{kcal} \mathrm{mol}^{-1}$ ). Das entspricht auch den experimentellen Ergebnissen (s.o.), wo bei der photolytisch initiierten Chlorierung sowohl Chlorcubane als auch das Fragmentierungsprodukt erhalten wurden, während die Bromierung unter den gleichen Bedingungen ausschließlich zu letzterem führt.

Anders als im Fall der Halogenierung von Cuban mit Halogenradikalen sind die Barrieren der H-Abstraktionen im Fall der Trihalomethylradikale $\left[\boldsymbol{H} 2,21.6\left(\cdot \mathrm{CCl}_{3}\right)\right.$ und 19.4 $\left(\bullet \mathrm{CBr}_{3}\right) \mathrm{kcal} \mathrm{mol}^{-1}$ ] über den ÜZ TS2 erheblich geringer als der direkte C-Angriff $(\boldsymbol{C} 2,33.8$ $\left(\bullet \mathrm{CCl}_{3}\right)$ und $\left.35.1\left(\cdot \mathrm{CBr}_{3}\right) \mathrm{kcal} \mathrm{mol}^{-1}\right)$ über den ÜZ TS5 (Schema 17). So können durch die Computerberechnungen alle experimentellen Ergebnisse der PT-Halogenierungen auch im Vergleich mit den photolytisch initiierten Halogenierungen mit elementarem Halogen gut nachvollzogen werden. Der C-Angriff von $\bullet \mathrm{CX}_{3}$ ist zwar thermodynamisch begünstigt, die beobachtete hohe Selektivität der H-Abstraktion, die zu den Substitutionsprodukten führt, dürfte jedoch auf eine kinetische Produktkontrolle der Reaktion zurückzuführen sein.

\subsubsection{Die PT-Halogenierung von Monohalocubanen}

Wie bereits erwähnt, hat die Erstsubstitution eines Wasserstoffatoms durch ein Halogenatom am Cubangerüst starke Auswirkungen auf die Zweithalogenierung. Dies wird dadurch deutlich, daß im Rahmen der Ersthalogenierung auch nach längeren Reaktionszeiten die Bildung nur geringer Mengen an Dihalogeniden beobachtet wurde. Dies hat einerseits den Vorteil, daß die Monohalogenierung sehr selektiv erfolgt, andererseits ist eine unter Umständen erwünschte Zweithalogenierung mit sehr viel längeren Reaktionszeiten und drastischeren Reaktionsbedingungen (höhere Temperaturen) verbunden. 

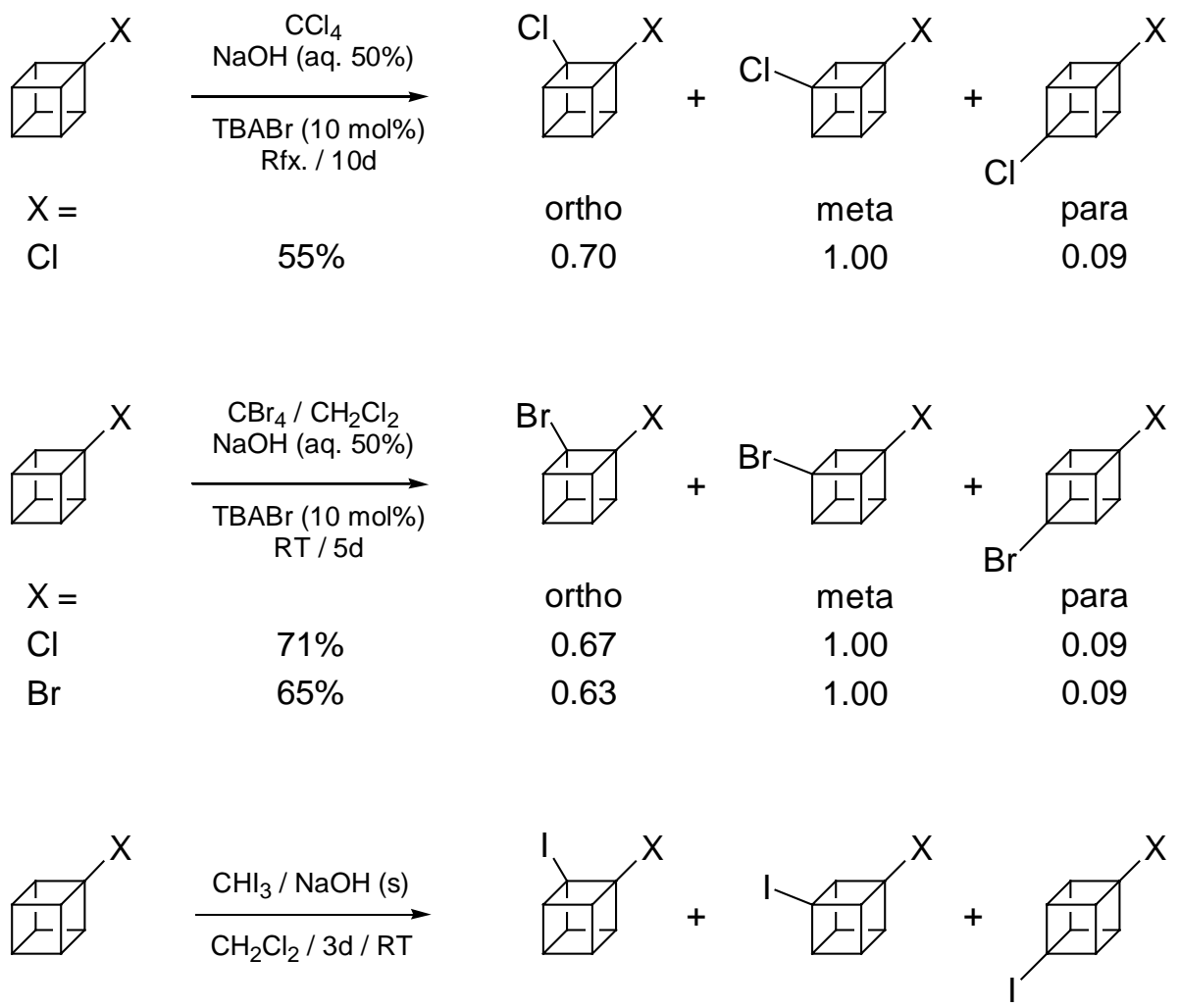

$X=$

ortho

meta

para

Cl

$54 \%$

$\mathrm{Br}$

$49 \%$

0.65

1.00

0.09

I

$69 \%$

0.68

1.00

0.09

0.70

1.00

0.08

Schema 18. Die isolierten Gesamtausbeuten sowie Produktverteilung und Reaktionsbedingungen bei den PT-Halogenierungen $(\mathrm{Cl}, \mathrm{Br}, \mathrm{I})$ von Monohalocubanen (AV13d-13i)

Um die Zweithalogenierung von Cuban genauer $\mathrm{zu}$ untersuchen, wurden Homohalogenierungen sowie Heterohalogenierungen von 35, 36 und 37 (AV13d-13i) durchgeführt, wodurch zum ersten Mal alle möglichen Produktpermutationen der Dihalocubane synthetisiert werden konnten (Schema 18).

Zur Analyse der jeweiligen Produktmischung wurde zunächst eine GC/MS-Analyse durchgeführt, wodurch das Verhältnis vom ortho- zum meta- und para-Produkt bestimmt werden konnte. Letzteres Verhältnis ( $\left.m^{-}: o^{-}\right)$wurde nicht durch eine solche Analyse bestimmt, da keine Separation der Signale erreicht werden konnte. Deswegen wurde nachfolgend eine Säulenchromatographie durchgeführt, bei der neben der reinen $\mathrm{m}$ Verbindung auch das Gemisch von $o$ - und $p$-Produkt isoliert wurde. Anschließend konnte die Produktanalyse durch Integration separater Signale im ${ }^{1} \mathrm{H}-\mathrm{NMR}$ Spektrum erfolgen. Die 
ermittelten ortho- : metha- : para-Verhältnisse liegen in den Bereichen $0.63-0.70: 1.00$ : 0.08 - 0.09 mit einer starken Präferenz für das meta-Produkt (Schema 18).

\subsubsection{Computerberechnungen zur Erklärung der Produktverteilung bei der PT- Halogenierungen (Cl, Br, I) von Monohalocubanen (Cl, Br, I)}

Die Auswirkungen eines Halogensubstituenten am Cubangerüst sind sehr weitreichend, wie schon die Deaktivierung durch den ersten Halogensubstituenten bei einer Zweithalogenierung experimentell gezeigt hat. Daneben hat ein solcher auch einen starken Einfluß auf die Regioselektivität der Zweitsubstitution. Es können verschiedene Faktoren für diese Selektivität verantwortlich sein. Dazu wurden zunächst die Radikalstabilitäten in der ortho-, meta- und para-Position des jeweiligen Halocubans berechnet [B3LYP/6-311+G** $(\mathrm{C}, \mathrm{H}, \mathrm{Cl}, \mathrm{Br}), 3-21 \mathrm{G}^{*}(\mathrm{I}) / / \mathrm{B} 3 \mathrm{LYP} / 6-31+\mathrm{G}^{*}(\mathrm{C}, \mathrm{H}, \mathrm{Cl}, \mathrm{Br}), 3-21 \mathrm{G}^{*}$ (I)]. Die durchschnittlichen, entsprechend statistisch korrigierten Mittelwerte für die Produktverhältnisse im Rahmen einer Zweithalogenierung sind ortho : meta $:$ para $=0.68$ : 1.00 : 0.36. Somit korrelieren die experimentell ermittelten Selektivitäten gut mit den computerberechneten Radikalstabilitäten (Schema 19), wo durchschnittlich das Radikal lokalisiert in der ortho-Position um 0.8 und in der para-Position um $1.1 \mathrm{kcal} \mathrm{mol}^{-1}$ instabiler ist als in der meta-Position. Neben der Stabilität der radikalischen Intermediate müssen aber auch noch andere Faktoren für die Produktverteilung verantwortlich sein.

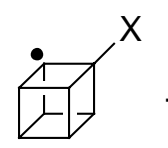

$\mathrm{X}=$

$\mathrm{Cl}$

$\mathrm{Br}$

I ortho

0.8

0.9

0.6

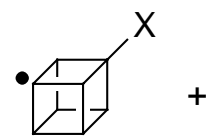

meta

0.0

0.0

0.0

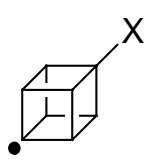

para

1.2

1.1

1.0

Schema 19. Relative Radikalstabilitäten der Halocubanradikale $\left[\Delta H_{298}\left(\mathrm{kcal} \mathrm{mol}^{-1}\right)\right.$, B3LYP/6-311+G**(C, H, Cl, Br), 3-21G*(I)//B3LYP/6-31+G*(C, H, Cl, Br),3-21G*(I)]

In den Monohalocubanen (auch in Cuban) tragen die Wasserstoffatome eine positive Partialladung [Abb. 18, NBO-Ladungen $(32,36)$ ], was durch das Reaktionsverhalten der Trihalomethylradikale, nämlich einer Präferenz der Wasserstoffabstraktion, eine gewisse Nukleophilie wahrscheinlich macht. Allerdings haben nur die Wasserstoffatome in ortho- und 
meta-Position aufgrund der $C_{3 \mathrm{v}}$-Symmetrie der Monohalocubane einen signifikanten Orbitalkoeffizienten im LUMO-Molekülorbital [Abb. 18, LUMO (36)], wodurch das geringe Ausmaß der Bildung von para-Produkten erklärt werden kann; aber nur wenn die $\bullet \mathrm{CX}_{3}-$ LUMO-Wechselwirkung entscheidend ist. Das ist mit hoher Wahrscheinlichkeit der Fall, da im HOMO [Abb. 18, HOMO (36)] die größten Orbitalkoeffizienten auf der C-C-Bindung lokalisiert ist, mit den Trihalomethylradikalen jedoch kein C-Angriff stattfindet.

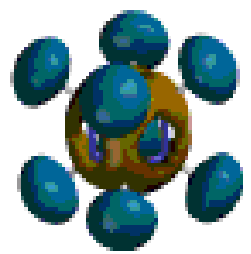

LUMO (32), $O_{\mathrm{h}}$

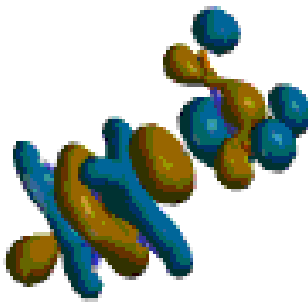

LUMO (ய̈Z1), $C_{3 \mathrm{v}}$

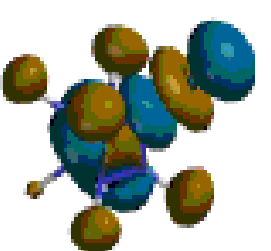

LUMO (36), $C_{3 \mathrm{v}}$

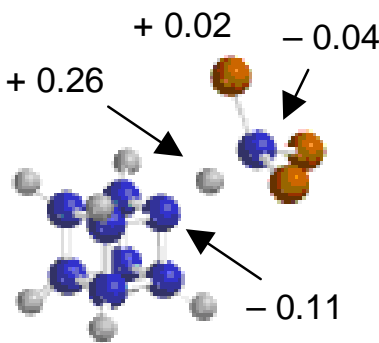

NBO Ldg ( ̈̈Z1)

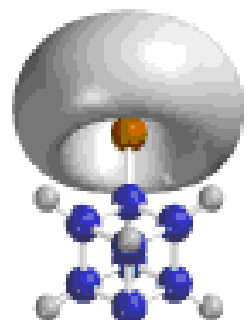

$\operatorname{ESP}(36)$

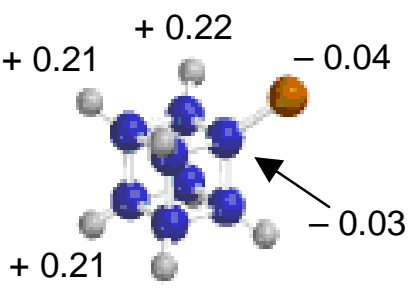

$-0.20$

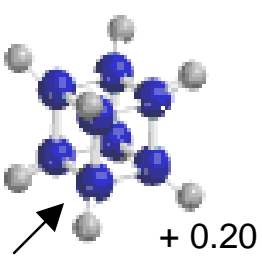

NBO Ldg. (32)

Abbildung 18. Ausgewählte MOs von 32, 36 und ÜZ1 (links); NBO-Ladungen von 32, 36 und ÜZ1 sowie elektrostatisches Potential (ESP) um das Chloratom in $\mathbf{3 6}$ (rechts)

Weiterhin können sterische Aspekte zur Erklärung der Bevorzugung der meta- vor der ortho-Produktbildung herangezogen werden. So wird die H-Abstraktion in der meta- im Vergleich zur ortho-Position aufgrund einer repulsiven Wechselwirkung zwischen dem Trihalomethylradikal und dem Halogensubstituenten (Abb. 18, elektrostatisches Potential von 36) begünstigt. Dieser sterische Anspruch macht sich auch in einer Abweichung des $\mathrm{C}-\mathrm{H}-\mathrm{C}$ Bindungswinkels der gebrochenen und sich bildenden Bindung (bis $5^{\circ}$ ) in den computerberechneten ÜZ [B3LYP/6-311+G** (C, H, Cl, Br), 3-21G* (I)// B3LYP/6-31+G* (C, H, $\mathrm{Cl}, \mathrm{Br}$ ), 3-21G* (I)] der H-Abstraktionen in der ortho-Position zu einem Halogensubstituenten bemerkbar (ÜZ4-12, Abb. 19), während in den meta-ÜZ nur eine geringe Abweichungen von max. $1^{\circ}$ resultiert (ÜZ13-21, Abb. 19). 
Die auf dem gleichen Niveau wie die Radikalstabilitäten computerberechneten Aktivierungsenthalpien der H-Abstraktionen von Cuban und in allen isomeren Positionen der Halocubane durch ${ }^{-} \mathrm{CX}_{3}(\mathrm{X}=\mathrm{Cl}, \mathrm{Br}, \mathrm{I} ; \mathrm{Abb}$. 20) sind für das Trichlor- und Tribrommethylradikal sehr ähnlich und liegen im Bereich von $\Delta H_{298 \mathrm{~K}}^{\ddagger}=11-15 \mathrm{kcal} \mathrm{mol}^{-1}$, während sie für das Triiodmethylradikal substantiell geringer ausfallen $\left(\Delta H_{298 \mathrm{~K}}^{\ddagger}=6-8 \mathrm{kcal}\right.$ $\mathrm{mol}^{-1}$ ). Dieser Unterschied könnte auf die bereits erwähnte Polarisierung der ÜZ der HAbstraktion (Abb. 18, NBO-Ladungen, ÜZ1) zurückzuführen sein, wo eine negative Partialladung am $\mathrm{C}$-Atom von $\bullet \mathrm{CX}_{3}$ für das Triiodmethylradikal $(\mathrm{NBO}(\mathrm{I})=-0.37, \mathrm{~B} 3 \mathrm{LYP})$ erheblich größer ausfällt als für die beiden anderen Halomethylradikale $(\mathrm{NBO}(\mathrm{Cl}, \mathrm{Br})=$ $0.19,+0.07$, B3LYP), wodurch eine Bindungsbildung zu dem zu abstrahierenden und positiv polarisierten $\mathrm{H}$-Atom durch $\pi$-Backbonding mit den Iodatomen besser unterstützt werden kann.

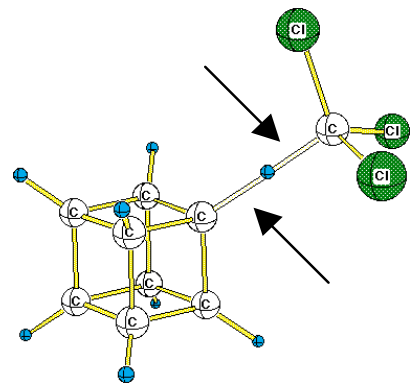

ÜZ1, $C_{3 v}\left(180^{\circ}\right)$

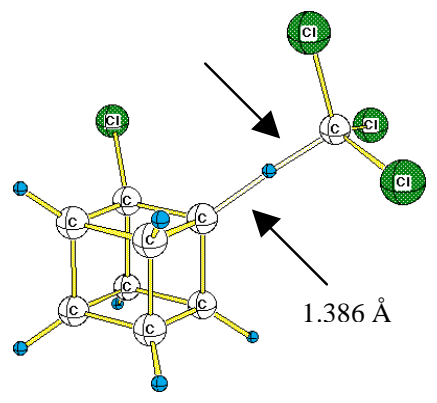

ÜZ4, $C_{s}\left(176^{\circ}\right)$

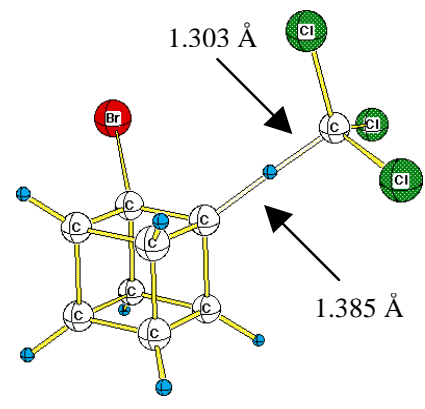

ÜZ7, $C_{s}\left(176^{\circ}\right)$

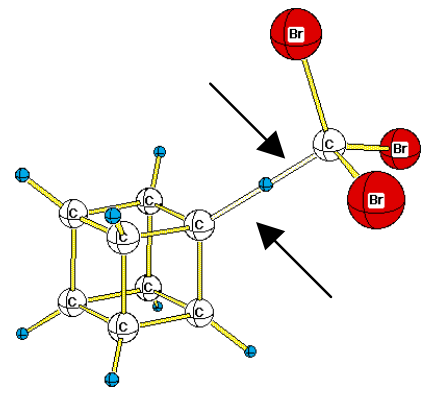

ÜZ2, $C_{3 v}\left(180^{\circ}\right)$

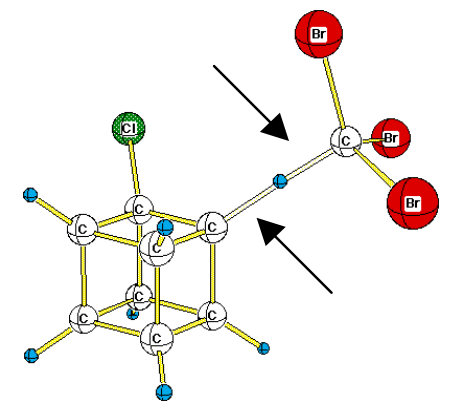

ÜZ5, $C_{s}\left(176^{\circ}\right)$

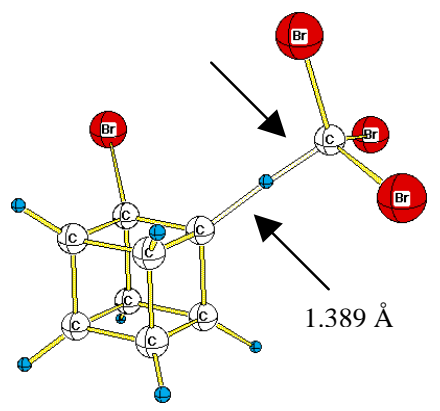

ÜZ8, $C_{s}\left(176^{\circ}\right)$

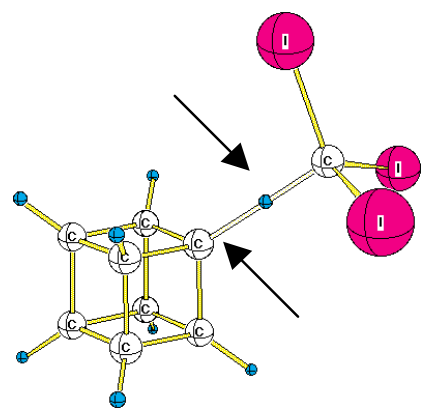

$\ddot{\mathbf{U Z Z 3}} C_{3 v}\left(180^{\circ}\right)$

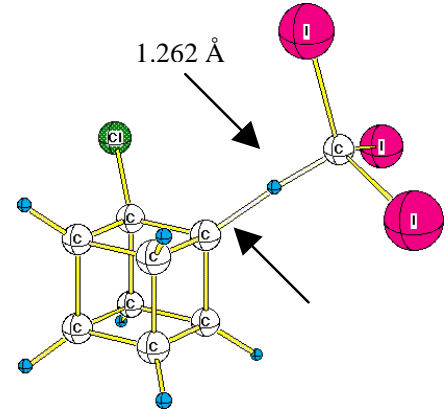

ÜZ6, $C_{s}\left(175^{\circ}\right)$

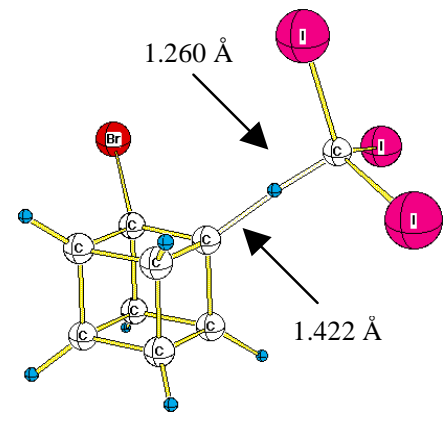

ÜZ9, $C_{s}\left(175^{\circ}\right)$

Abbildung 19. s.u. 


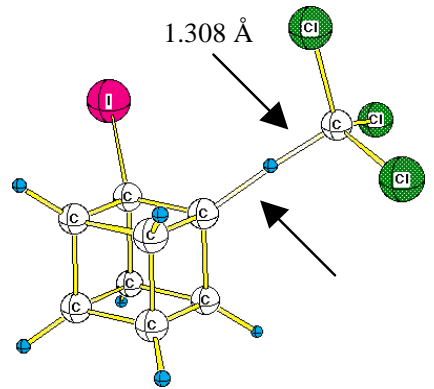

ÜZ10, $C_{s}\left(176^{\circ}\right)$

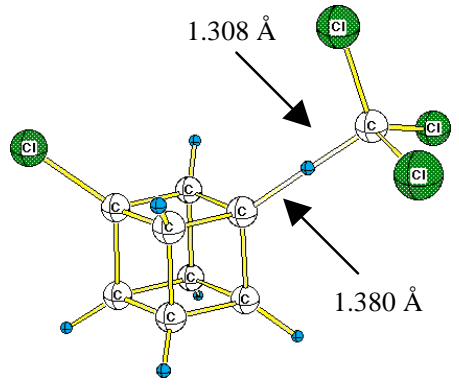

ÜZ13, $C_{s}\left(180^{\circ}\right)$

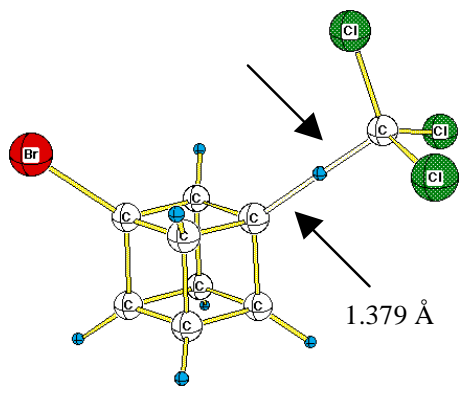

ÜZ16, $C_{s}\left(179^{\circ}\right)$

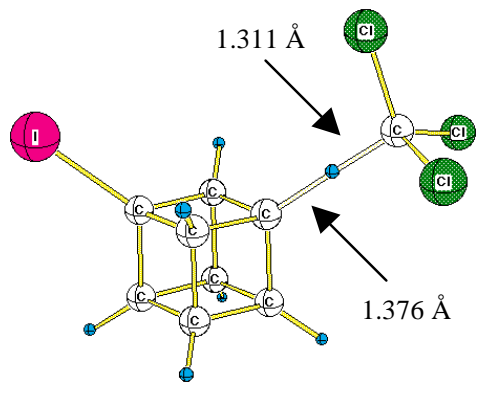

ÜZ19, $C_{s}\left(179^{\circ}\right)$

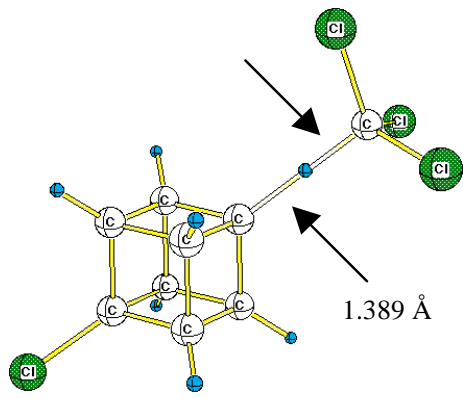

ÜZ22, $C_{3 v}\left(180^{\circ}\right)$

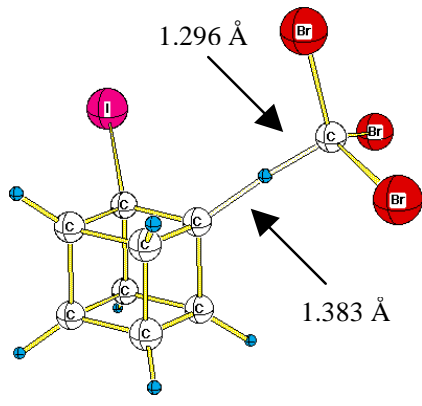

ÜZ11, $C_{s}\left(175^{\circ}\right)$
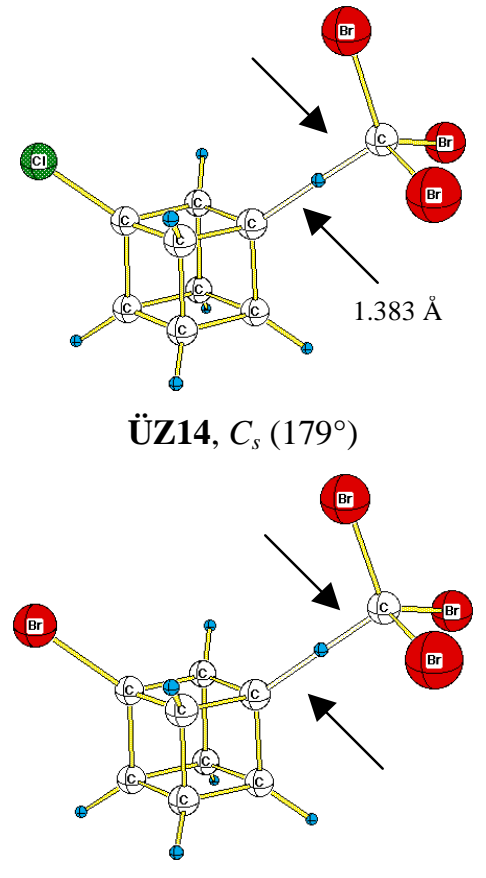

ÜZ17, $C_{s}\left(179^{\circ}\right)$

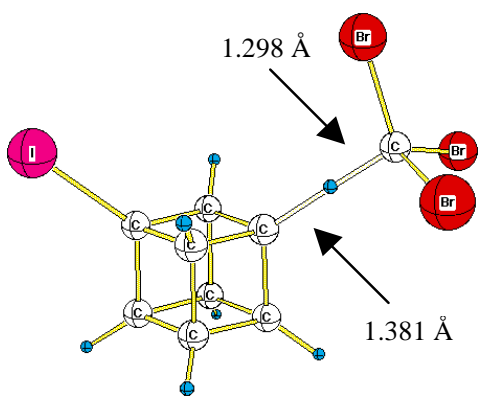

ÜZ20, $C_{s}\left(179^{\circ}\right)$

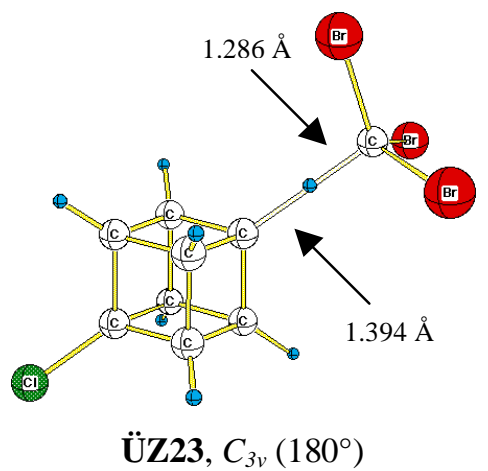

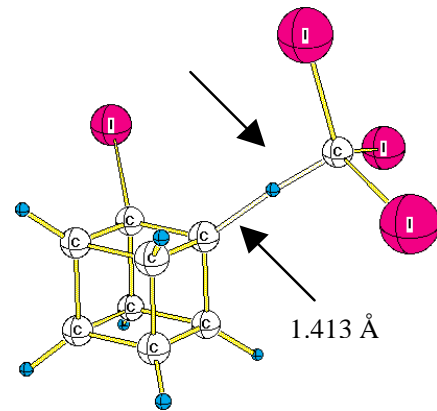

ÜZ12, $C_{s}\left(175^{\circ}\right)$

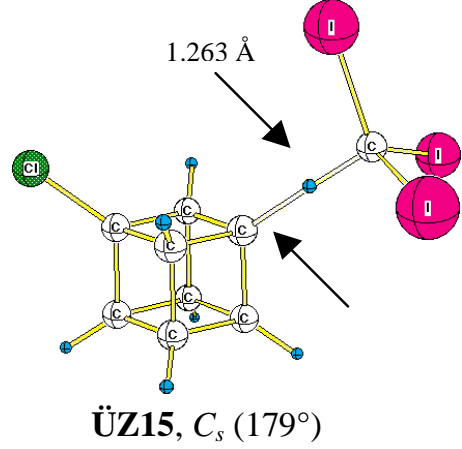

(Br)

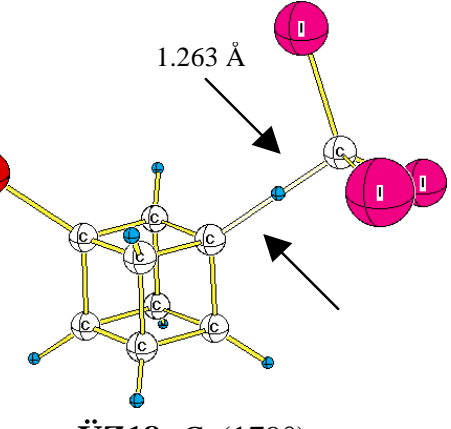

ÜZ18, $C_{s}\left(179^{\circ}\right)$
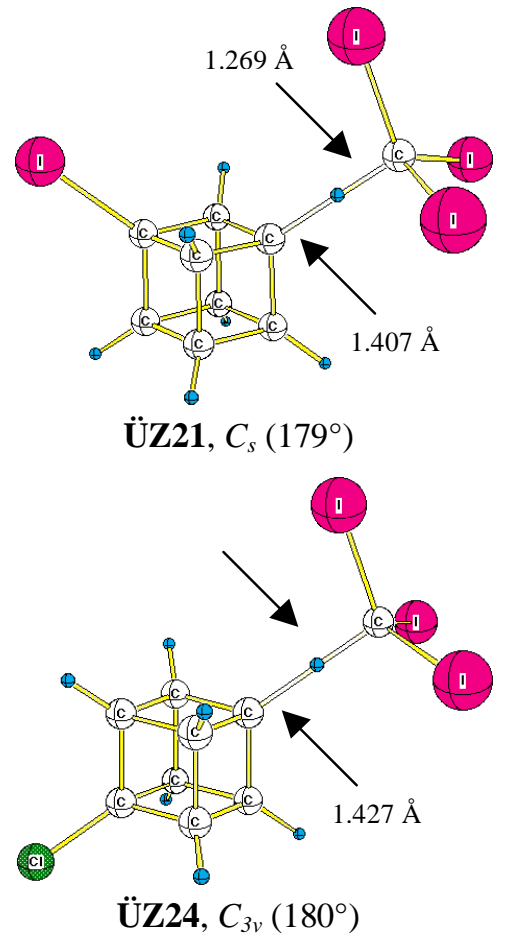

Abbildung 19. s.u. 

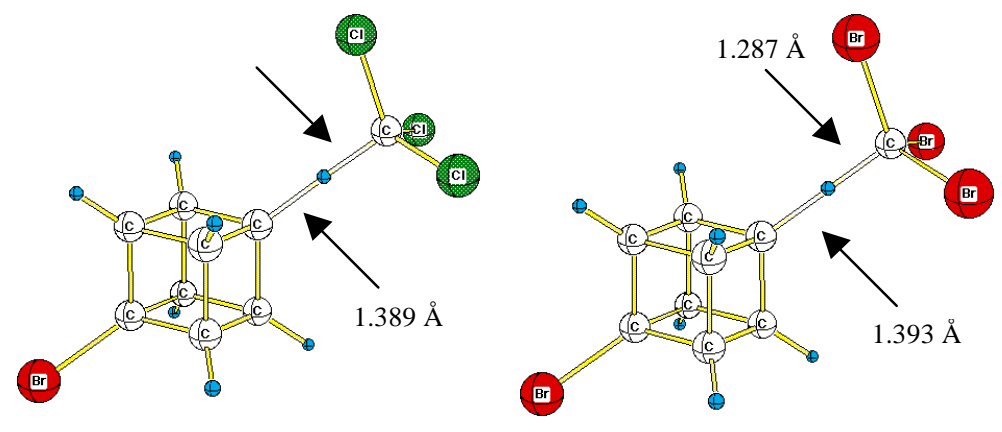

ÜZ26, $C_{3 v}\left(180^{\circ}\right)$

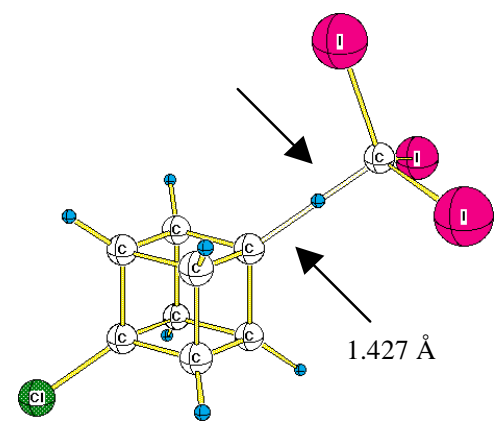

ÜZ25, $C_{3 v}\left(180^{\circ}\right)$
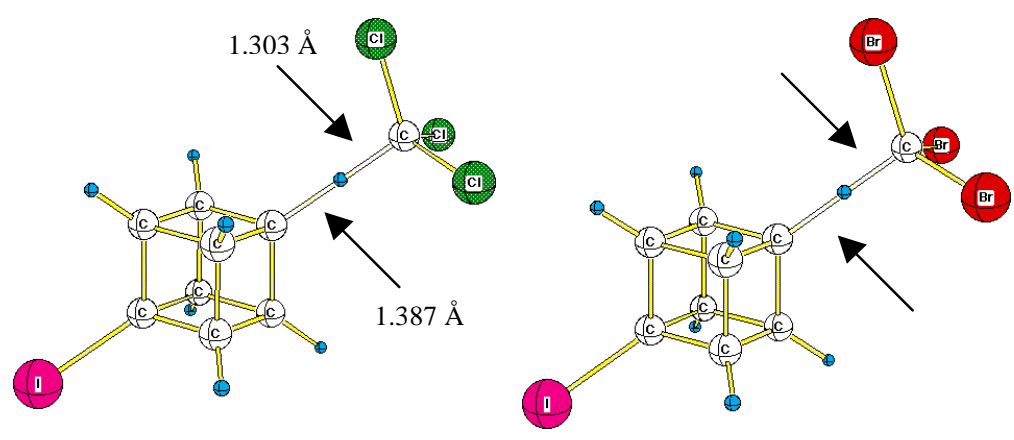

ÜZ28, $C_{3 v}\left(180^{\circ}\right)$

ÜZ29, $C_{3 v}\left(180^{\circ}\right)$

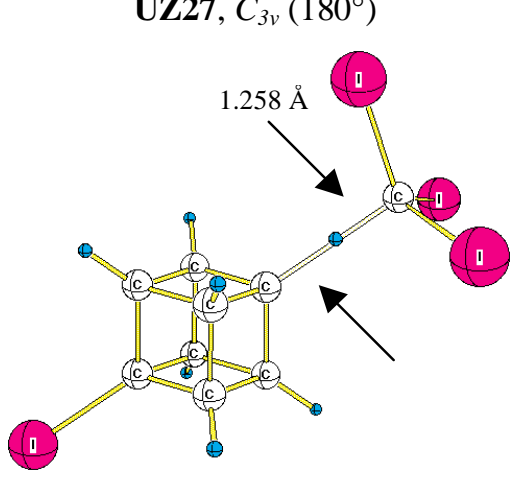

$\ddot{\mathbf{U Z 3 0}}, C_{3 v}\left(180^{\circ}\right)$

Abbildung 19. Die computerberechneten ÜZ der H-Abstraktionen von Cuban und den Halocubanen durch $\cdot \mathrm{CX}_{3}$ [Winkel $(\mathrm{C}-\mathrm{H}-\mathrm{C})$; B3LYP/6-311+G** $(\mathrm{C}, \mathrm{H}, \mathrm{Cl}, \mathrm{Br}), 3-21 \mathrm{G}^{*}$ (I)//B3LYP/6-31+G* (C, H, Cl, Br), 3-21G* (I)]

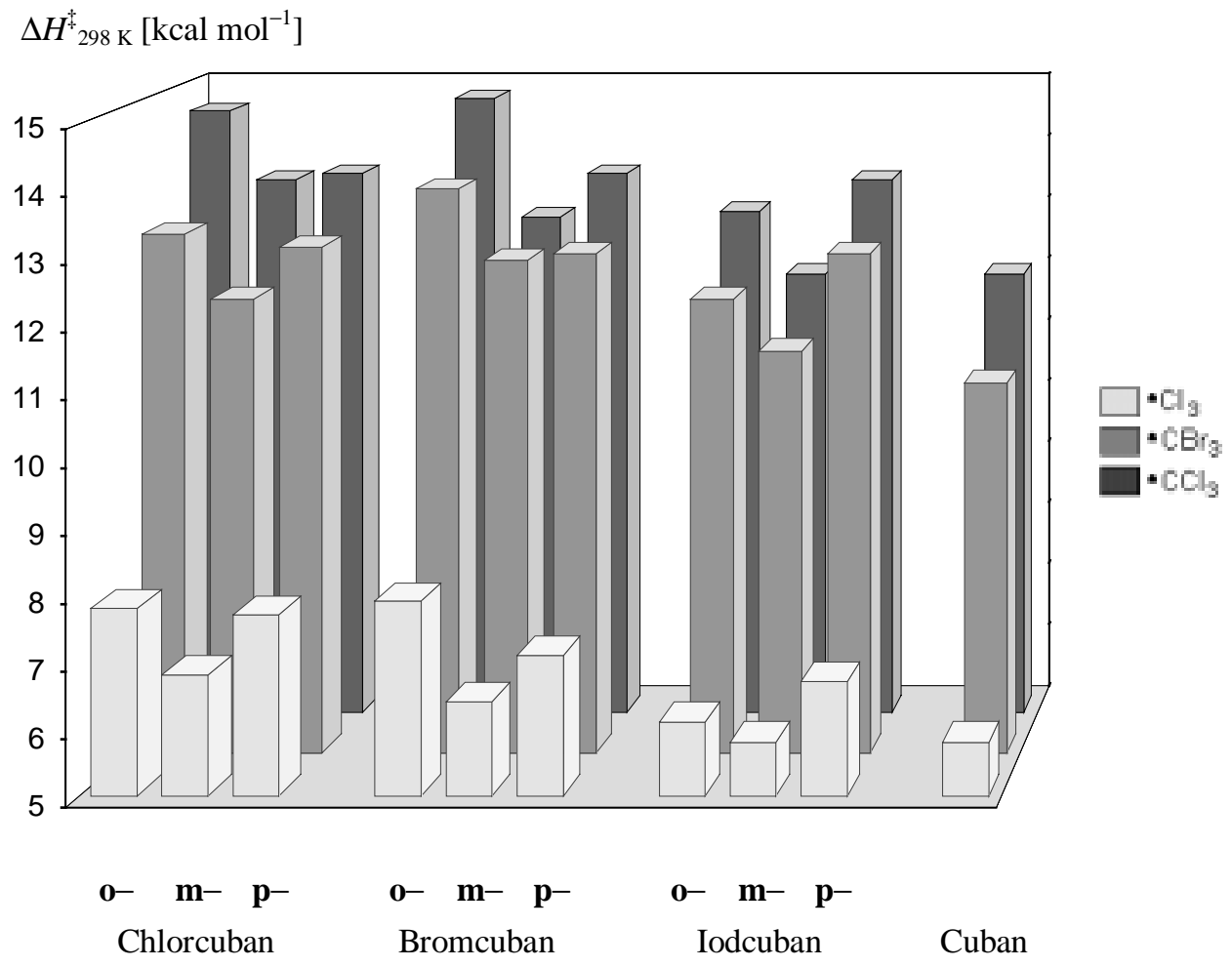

Abbildung 20. Die computerberechneten Aktivierungsenthalpien $\left(\Delta H_{298 \mathrm{~K}}^{\ddagger}, \mathrm{kcal} \mathrm{mol}^{-1}\right)$ für die H-Abstraktion von Cuban und von den Halocubanen durch Trihalomethylradikale [B3LYP/6$\left.311+\mathrm{G}^{* *}(\mathrm{C}, \mathrm{H}, \mathrm{Cl}, \mathrm{Br}), 3-21 \mathrm{G}^{*}(\mathrm{I}) / / \mathrm{B} 3 \mathrm{LYP} / 6-31+\mathrm{G}^{*}(\mathrm{C}, \mathrm{H}, \mathrm{Cl}, \mathrm{Br}), 3-21 \mathrm{G}^{*}(\mathrm{I})\right]$ 
Dies geht einher mit den Stabilitäten der entsprechenden Halomethylanionen, bei denen das Triiodmethylanion den stabilsten Vertreter repräsentiert $\left(\mathrm{I}_{3} \mathrm{C}^{-}>\mathrm{Br}_{3} \mathrm{C}^{-}>\mathrm{Cl}_{3} \mathrm{C}^{-}\right)$: Die isodesmische Gleichung $\mathrm{Br}_{3} \mathrm{CH}+\mathrm{Cl}_{3} \mathrm{C}^{-} \rightarrow \mathrm{Br}_{3} \mathrm{C}^{-}+\mathrm{Cl}_{3} \mathrm{CH}$ ist zwar leicht exotherm $(\Delta E=-$ $\left.6.3 \mathrm{kcal} \mathrm{mol}^{-1}, \mathrm{~B} 3 \mathrm{LYP}\right)$, fällt jedoch für $\mathrm{I}_{3} \mathrm{CH}+\mathrm{Cl}_{3} \mathrm{C}^{-} \rightarrow \mathrm{I}_{3} \mathrm{C}^{-}+\mathrm{Cl}_{3} \mathrm{CH}\left(\Delta \mathrm{E}=-8.3 \mathrm{kcal} \mathrm{mol}^{-1}\right.$, B3LYP) noch um $2 \mathrm{kcal} \mathrm{mol}^{-1}$ größer aus. Aus diesen Gründen sind allgemein die Barrieren der $\mathrm{H}-\mathrm{Abstraktion}$ für $\bullet \mathrm{CI}_{3}$ am niedrigsten und für $\bullet \mathrm{CCl}_{3}$ am höchsten.

\subsubsection{Ergebnis}

Die Methode zur Halogenierung unter PT-Bedingungen eignet sich hervorragend zur selektiven Funktionalisierung von Cuban, wobei gute bis sehr gute Ausbeuten an monohalogenierten Cubanen $(\mathrm{Cl}, \mathrm{Br}, \mathrm{I})$ erhalten werden. Eine Polyhalogenierung wird dadurch vermieden, daß das zuerst gebildete Monohalogencuban gegenüber dem Trihalomethylradikal stark deaktiviert ist. Eine Zweithalogenierung, die aus den eben genannten Gründen mit längeren Reaktionszeiten einhergeht, ließen sich zum ersten Mal alle isomeren hetero- und homosubstituierten Dihalocubane synthetisieren. Dabei wird eine hohe meta-Selektivität beobachtet, die auf sterische Effekte, Polarisationseffekte sowie MO Kontrolle der Reaktion zurückzuführen sein dürfte. Wahrscheinlich sind die Polarisationseffekte auch für das unterschiedliche Reaktionsverhalten bei der Halogenierung von Cuban über Trihalomethylradikale bzw. Halogenradikale verantwortlich. Während ein Trihalomethylradikal eher ein nukleophiles Reaktionsverhalten aufweist und die im Cubanmolekül positivierten Wasserstoffatome angreift, sind Halogenradikale elektrophiler und über einen C-Angriff bilden sich hauptsächlich Ringöffnungsprodukte. Der andere Grund für das unterschiedliche Reaktionsverhalten ist, daß im ÜZ die Halogenradikale mit den HOMO-MOs wechselwirken, beoi denen große Orbitalkoeffizienten auf den $\mathrm{C}-\mathrm{C}$-Bindungen lokalisiert sind, während für die Trihalomethylradikale die Wechselwirkung mit den LUMOMOs entscheidend ist. Auch die für das Trihalomethylradikal leichter zugänglichen H-Atome bewirken eine Präferenz der H-Abstraktion. Insgesamt ist davon auszugehen, daß die Methode der PT-Halogenierung auch für die Aktivierung anderer hochgespannter Systeme Anwendung finden kann. 


\subsection{Die Synthese von chiralen Polyhaloadamantanen}

\subsubsection{Einleitung}

Adamantan wurde vor ca. 70 Jahren von Landa in Fraktionen der Erdöldestillation entdeckt. ${ }^{[163]}$ Nachdem durch Schleyer ein äußerst effizienter Zugang über eine kationische Umlagerungskaskade von Tetrahydrodicyclopentadien zur Synthese von Adamantan gefunden wurde, ${ }^{[164]}$ ist diese Verbindung (und seine Derivate) aufgrund deren besonderer Eigenschaften, immer wieder in den Mittelpunkt des wissenschaftlichen Interesses gerückt. So diente Adamantan und Verbindungen, die auf dem Adamantangerüst basieren, zur Überprüfungen zahlreicher theoretischer Konzepte in der organischen Chemie (StrukturReaktivitätsbeziehungen, Hyperkonjugationseffekte, Umlagerungen, etc.).[32, 120, 121] Weiterhin konnte durch Inkorporation des Adamantangerüstes in Polymere deren Eigenschaften (Härte, thermischer Ausdehnugskoeffizient, etc.) positiv beeinflußt werden.[165-168] Auch haben sich viele Adamantanverbindungen als pharmakologisch wirksam erwiesen und wurden als Antidepressiva, Antitumormedikamente, Antibiotika[121, 169] und zur Behandlung von Virusinfektionen[170] und der Parkinsonkrankheit [171] eingesetzt. Dabei hängt die Wirksamkeit solcher Medikamente stark vom Substitutionsmuster am Adamantangerüst ab, weil dessen physikalischen Eigenschaften wie Lipophilie, Acidität etc. und somit deren metabolische Stabilität dadurch beeinflußt werden.[172] Die PTHalogenierungen bieten nun einen einfachen Zugang zu einer Substanzklasse im Bereich der Adamantanverbindungen, nämlich den chiralen Polyhaloadamantanen, die sich durch ein Stereozentrum auszeichnen, das nicht in einem Atom lokalisiert ist. Solche $C_{1}$-symmetrischen Verbindungen sind bislang nur oberflächlich untersucht worden.[173-175] Neben ihren unbekannten chiroptischen Eigenschaften erscheinen sie bezüglich einer pharmakologischen Wirksamkeit äußerst vielversprechend, weil das Stereozentrum isomerisierungsstabil ist. Außerdem können sie als Vorstufe für die eben erwähnten chiralen pharmakologischen Wirkstoffe dienen, da zahlreiche chemische Modifikationen wie z.B. Kreuzkupplungen aufgrund der abgestuften Reaktivität der verschiedenen Halogenatome chemoselektiv erfolgen können, und so die chiralen Haloadamantane schrittweise auf verschiedenste Weisen modifiziert werden könnten.[176-178] Das wäre auch eine Möglichkeit zum Aufbau ganzer Substanzbibliotheken für diverse kombinatorische Ansätze (siehe Ausblick).

Der einfache Zugang zu den Polyhaloadamantanen beruht auf der Tatsache, daß die Halogenierungen unter PT-Bedingungen ohne Austausch eines bereits vorhandenen 
Halogenatoms im Eduktmolekül verlaufen, und es so ermöglicht wird sukzessive mehrere verschiedene Halogenatome in ein Adamantanmolekül einzuführen. Nicht nur, daß kein Halogenaustausch stattfindet, auch wird eine extrem hohe Regioselektivität bei der Zweitund Drittsubstitution bezüglich der $3^{\circ}$-Position beobachtet (Sekundäre Produkte $=0.5-3 \%$, nur durch GC/MS-Analyse detektierbar). Außerdem toleriert die PT-Halogenierung neben Halogensubstituenten noch weitere funktionelle Gruppen. Diese Faktoren sollen für die folgenden Darstellung verschiedener Polyhaloadamantanverbindungen systematisch ausgenutzt werden. An dieser Stelle muß noch vermerkt werden, daß alle möglichen Dihaloadamantane $($ Halogen $=\mathrm{F}, \mathrm{Cl}, \mathrm{Br}, \mathrm{I})$ bereits dargestellt wurden, [179] wobei allerdings z.T. mehrstufige Synthesen in Kauf genommen werden mußten, für die die PT-Halogenierung eine gute Alternative darstellen würde.

\subsubsection{Die PT-Halogenierung von Adamantan}

Zunächst wurden verschiedene monosubstituierte Haloadamantane den PTHalogenierungen unterworfen (Tab. 8.) um zu überprüfen, wie groß das Ausmaß der Kompatibilität bezüglich verschiedener Halogensubstituenten ist. Dabei wurde festgestellt, daß auch hierbei keinerlei Halogenaustausch stattfand. Allerdings wurden aufgrund der hohen Anzahl an verschieden Möglichkeiten der Zweithalogenierung nicht alle Permutationen getestet. Es kann aber davon ausgegangen werden, daß auch die nicht überprüften Reaktionsmöglichkeiten nicht mit einem Halogenaustausch verbunden sein würden.

Drei Experimente (Nr. 1-3; Tab. 8.) wurden solange fortgeführt bis die GC/MSAnalysen dieser Reaktionen ca. 80\% Umsatz anzeigten. Dabei erwies sich, daß sich die als Nebenprodukte gebildeten Trihalogenadamantane säulenchromatografisch nur sehr schlecht von den Dihalogenadamantanen trennen lassen. Selbst bei sehr sorgfältiger Durchführung der Trennung wurde ein Großteil der Produkte als Mischfraktionen erhalten und dadurch die präparative Ausbeute stark reduziert. Zudem sind die Reaktionsbedingungen sehr aggressiv ( $\mathrm{NaOH}$ aq. 50\%), was bei langen Reaktionszeiten zu einer negativen Massenbilanz führt; d.h. die Edukte oder Produkte zersetzen sich bei längerer Reaktionsführung. Deshalb erschien es bei weiteren Synthesen vorteilhafter, die Zweithalogenierungen von Haloadamantanen bei 30 - $40 \%$ Umsatz zu beenden. Die als einzige Produkte (Nr. 4; Tab. 8) gebildeten Dihaloadamantane lassen sich dann sehr gut säulenchromatographisch von den 
Monohaloadamantanen trennen und letztere können dabei zum größten Teil zurückgewonnen werden.

Tabelle 8. Exemplarische Zweithalogenierung von Haloadamantanen unter PT-Bedingungen

\# Edukt AV / Halogenierung Produkt 1 [\%] Produkt 2 [\%]

[a] nicht isoliert, nur durch GC/MS-Analyse der Reaktionsmischung detektiert (ca.12\%)

Aufgrund der Ergebnisse, daß bei Halogenierungen unter PT-Bedingungen erstens kein Halogenaustausch stattfindet und zweitens auch dreifach halogenierte Adamantane isoliert werden konnten, war es naheliegend auch die Dreifachhalogenierung systematischer $\mathrm{zu}$ untersuchen. Hierfür wurde zunächst versucht, ausgehend von den bisher dargestellten Dihaloadamantanen, durch direkte Halogenierung ( \pm )-1-Chlor-3-Brom-5-Iodadamantan (44) als den am einfachsten zugänglichen Vertreter der chiralen Polyhaloadamantane zu synthetisieren (Tab. 9.). Die Chlorierung von 42 (Nr.1; Tab. 9.) war aufgrund der langen Reaktionszeit (8 Wochen) mit einer schlechten Ausbeute verbunden. Deswegen wurde nach einer alternativen Möglichkeit zu der PT-Initiierung gesucht. Dabei erschien eine klassische 
thermisch initiierte radikalische Chlorierung mit Tetrachlormethan als Halogenquelle am erfolgversprechendsten. Es wurden drei verschiedene Kettenstarter getestet, nämlich AIBN (Azobisisobutyronitril), DBO (Di-t-butylperoxid) und DBPO (Dibenzoylperoxid). Sowohl mit AIBN als auch mit DBO fand überhaupt keine Reaktion statt. Die Reaktion mit DBPO führte ausschließlich zum Austausch von Iod durch Chlor. Somit war ein effektiver Syntheseweg ausgehend von 42 versperrt. Die Iodierung von 38 (Nr. 2; Tab. 9) verlief sehr langsam, was auf die Deaktivierung des Adamantangerüstes durch die Erstsubstituenten und zugleich hohe Selektivität der H-Abstraktion des Triiodmethylradikals zurückzuführen war. Wahrscheinlich aufgrund sich annähender Reaktionsgeschwindigkeiten des Zerfalls und der Bildung des gewünschten Produktes stagnierte die Reaktion bei ca. 3\% Umsatz (GC/MS-Analyse). Auf eine Isolierung der Produkte wurde daher verzichtet.

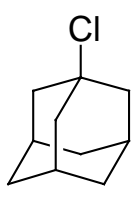

26

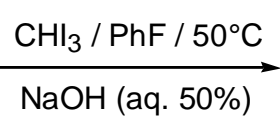

$5 \mathrm{~d} / 30 \%$

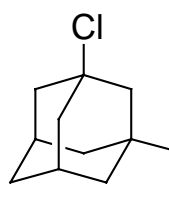

40

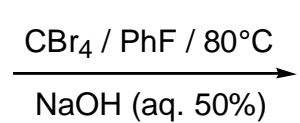

$7 \mathrm{~d} / 35 \%$

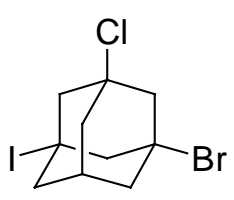

$( \pm)-44$

Schema 20. Effektivste Synthesesequenz zur Darstellung von ( \pm )-1-Chlor-3brom-5-iodadamantan (44) ausgehend von 26

Tabelle 9. Halogenierung von Dihaloadamantanen zur Synthese von $( \pm)-44$

\begin{tabular}{|c|c|c|c|c|}
\hline \# & Edukt & AV / Halogenierung & Produkt 1 [\%] & Produkt $2[\%]$ \\
\hline 1 & 42 & AV14e / Cl & $( \pm)-44 \quad[22]$ & - \\
\hline 2 & 38 & $\mathrm{I}$ & $( \pm)-44 \quad[3]^{[\mathrm{a}]}$ & - \\
\hline 3 & 40 & $\mathrm{AV} 14 \mathrm{f} / \mathrm{Br}$ & $( \pm)-44 \quad[35]$ & \\
\hline
\end{tabular}

[a] nicht isoliert, nur durch GC/MS-Analyse der Reaktionsmischung detektiert. 
Die Bromierung von 40 (Schema 20/ Nr. 3; Tab. 9.) erwies sich als effektivster Syntheseweg, um zum gewünschten Zielmolekül zu gelangen. Auch in diesem Fall wurde die Reaktion bis zu einem Umsatz von ca. 90\% (GC/MS-Analyse) fortgesetzt, um zu vermeiden, daß sich noch größere Mengen an Edukt in der Reaktionsmischung befinden. Dabei wurde, in der Annahme einer leichteren Separierbarkeit vom Hauptprodukt, die Bildung von tetrahalogeniertem Adamantan und eine durch die lange Reaktionszeit reduzierte Ausbeute in Kauf genommen. ( \pm )-1-Chlor-3-brom-5-iodadamantan (44) konnte dann in mäßiger Ausbeute isoliert werden.

In der Tat stellte sich auch bei noch folgenden Synthesen dieser Art heraus, daß tetrahalogenierte Adamantane eine Retentionszeit aufweisen, die zwischen den monohalogenierten und dihalogenierten Adamantanen liegt. Entscheidend für die Retentionszeiten sind hauptsächlich die Anzahl der Halogensubstituenten, wobei sich die Art der Halogenatome nur geringfügig auswirkt (Schema 21).

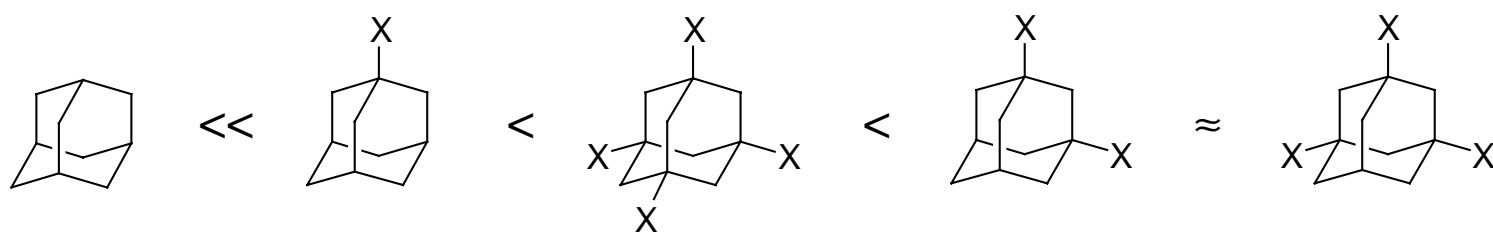

Schema 21. Allgemeine Darstellung der Retentionszeiten von verschiedenzahlig substituierten Haloadamantanen.

\subsubsection{Die PT-Halogenierung von 1-Fluoradamantanen}

Nachdem ( \pm )-44 als erster Vertreter der chiralen Polyhaloadamantane erfolgreich dargestellt wurde, sollte das verbleibende Brückenkopfwasserstoffatom durch andere Substituenten ersetzt werden, um so eine systematische Untersuchung der Substanzgruppe der chiralen Polyhaloadamantane zu ermöglichen. Zunächst wurden deshalb, ausgehend von 1Fluoradamantan (47, Schema 22), das sehr bequem aus 1-Adamantanol (46) gewonnen werden kann (AV14g), ${ }^{[180]}$ die bereits bekannten Verbindungen 1-Fluor-3-chlor- (48) und 1Fluor-3-iodadamanatan (49) ${ }^{[179]}$ durch PT-Halogenierung synthetisiert werden. Auch wenn hierbei die Ausbeuten aus den schon genannten Gründen nur mäßig sind, sind die Synthesewege im Vergleich zu den literaturbekannten verkürzt.[179] Die nachfolgende Bromierung von 48 und 49 führte zu den entsprechenden chiralen Bromverbindungen $( \pm)-1-$ Fluor-3-chlor-5-brom- (50) und ( \pm )-1-Fluor-3-brom-5-iodadamantan (51). In beiden Fällen 
konnten auch die entsprechenden tetrahalogenierten Verbindungen 52 und 53 (Tab. 10) isoliert werden. Die Iodierung von $\mathbf{4 8}$ verlief allerdings mit negativem Ergebnis.<smiles>OC12CC3CC(CC(C3)C1)C2</smiles>

46

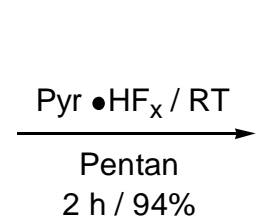

$2 \mathrm{~h} / 94 \%$<smiles>FC12CC3CC(CC(C3)C1)C2</smiles>

47

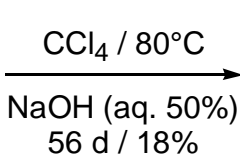

$56 \mathrm{~d} / 18 \%$<smiles>CC12CC3CC(C1)CC(F)(C3)C2</smiles>

48

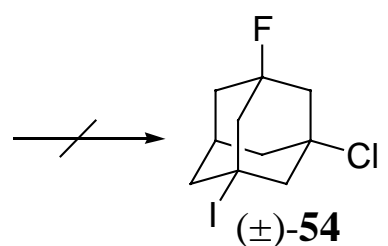

$( \pm)-54$

$$
\begin{gathered}
\mathrm{CHI}_{3} / \mathrm{CH}_{2} \mathrm{Cl}_{2} \\
\mathrm{RT} / \mathrm{NaOH}(\mathrm{s}) \\
8 \mathrm{~d} / 24 \%
\end{gathered}
$$<smiles>FC12CC3CC(C1)C(Br)(C3)C(Br)(I)C2</smiles>

$( \pm)-51$

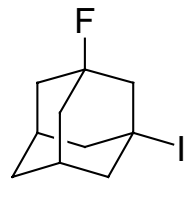

49

$$
\begin{gathered}
\mathrm{CBr}_{4} / \mathrm{PhF} / 80^{\circ} \mathrm{C} \\
\mathrm{NaOH}(\text { aq. } 50 \%) \\
42 \mathrm{~d} / 35 \%
\end{gathered}
$$

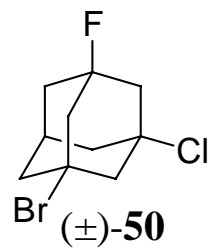

\begin{tabular}{|c|c|c|c|c|}
\hline \# & Edukt & AV / Halogenierung & Produkt 1 [\%] & Produkt 2 [\%] \\
\hline 1 & 47 & $\mathrm{AV} 14 \mathrm{~h} / \mathrm{Cl}$ & 48 [18] & - \\
\hline 2 & 47 & AV14j / I & 49 [24] & - \\
\hline 3 & 48 & AV14k / Br & $( \pm)-\mathbf{5 0} \quad[21]$ & {$[2]$} \\
\hline 4 & 49 & AV14l / Br & $( \pm)-\mathbf{5 1} \quad[35]$ & [7] \\
\hline
\end{tabular}

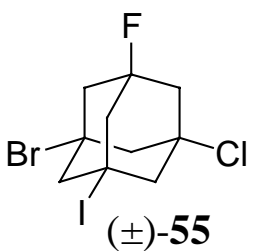

Schema 22. Synthesewege zu fluorsubstituierten chiralen Adamantanen Alle Reaktionen wurden mit 3-10 mol\% TBABr durchgeführt. Manche Reaktionen mußten mehrere Male einem Neustart unterworfen werden (s. exp. Teil).

Tabelle 10. Die PT-Halogenierung von Fluoradamantanen 
Es wurden dafür ohne Erfolg diverse Reaktionsbedingungen getestet [Variation der Eduktkonzentrationen, Temperatur, Lösungsmittel, $\mathrm{NaOH}$ (s) bzw. $\mathrm{NaOH}$ (aq. 50\%)]. Anscheinend ist das Adamantangerüst durch den gleichzeitigen Einfluß der Fluor- und Chlorsubstituenten so stark deaktiviert, daß das sehr selektiv reagierende Triiodmethylradikal nicht mehr in der Lage ist ein Wasserstoffatom zu abstrahieren und so den Propagationszyklus fortzuführen. Somit gab es dann auch keine Möglichkeit das ästhetisch sehr reizvolle ( \pm )-1Fluor-3-chlor-5-brom-7-iodadamanatan (55) auf diese Weise darzustellen. Die direkte Iodierung von $\mathbf{5 0}$ erschien auch nicht erfolgversprechend, da bereits $\mathbf{3 8}$ nur sehr langsam unter diesen Reaktionsbedingungen reagierte und wurde deshalb nicht überprüft.

Auch bei dieser Synthesesequenz wurde versucht, die sehr zeitintensive PT-initiierte Chlorierung von 47 durch eine thermisch durch Kettenstarter initiierte in der Annahme zu ersetzen, daß eine C-F-Bindung stabiler als eine C-I-Bindung (Nr.1, Tab. 9; radikalisch initiiert) sein würde. Wiederum wurden verschiedene Initiatoren getestet, wobei sich DBPO als am effektivsten erwies (AV14i) und auch kein Halogenaustausch festzustellen war. In diesem Fall war es somit möglich durch den Wechsel der Reaktionsbedingungen die Reaktionszeit der Chlorierung auf zwei Tage zu verkürzen.

\subsubsection{Die PT-Halogenierung von 1-Phenyladamantanen}

In einer weiteren Synthesesequenz (Schema 23) wurde die multiple Halogenierung des leicht zugänglichen 1-Phenyladamantans $(\mathbf{5 6})^{[181]}$ näher untersucht. Dabei fiel schon bei den Ersthalogenierungen auf, daß sich die Phenylgruppe im Gegensatz zu Halogenatomen aktivierend auf das Adamantangerüst auswirkt und diese Tendenz wird auch bei den Polyhalogenierungen beobachtet. Die Reaktionszeiten von Phenyladamantan sind im Vergleich zu den entsprechenden halogensubstituierten Analoga um ca. die Hälfte verkürzt. Der Grund und das genaue Ausmaß für diese Aktivierung bedarf allerdings weiterer Untersuchungen (z.B. Konkurrenzexperimente, s. Ausblick). Die phenylsubstituierten Haloadamantane unterscheiden sich von den reinen Haloadamantanen auch durch ihre leichtere Separierbarkeit. So haben die verschiedenzahlig halosubstituierten Phenyladamantane signifikant unterschiedliche Retentionszeiten und können somit einfacher als die reinen Haloadamantane voneinander getrennt werden. So ließ sich im Fall von 56 als Ausgangsmolekül im Gegensatz zu den reinen Polyhaloadamantanen die gesamte geplante Synthesesequenz (Schema 23) mit z.T. guten Ausbeuten realisieren, wobei auch vier 
verschiedene chirale Polyahalodamantane im jeweiligen racemischen Gemisch isoliert werden konnten $(( \pm)-\mathbf{5 8}, \mathbf{6 1}, \mathbf{6 2}, \mathbf{6 3})$. Um sich die kürzeren Reaktionszeiten zu Nutze zu machen, wurde die Chlorierung von Phenyladamantan alternativ auch initiiert durch DBPO durchgeführt (AV14v), wobei ebenfalls gute Ausbeuten erzielt werden konnten.<smiles>CC(C)(C)C12CC3CC(Br)(CC(c4ccccc4)(C3)C1)C2</smiles>

$( \pm)-61$

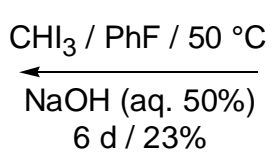

$6 \mathrm{~d} / 23 \%$

$\mathrm{CBr}_{4} / \mathrm{PhF} / 70^{\circ} \mathrm{C}$
$\mathrm{NaOH}(\mathrm{aq} .50 \%)$
$4 \mathrm{~d} / 61 \%$

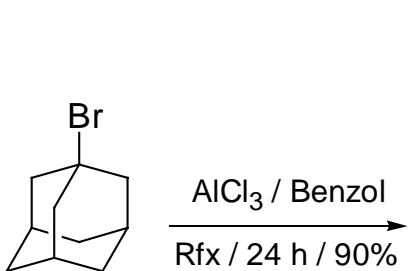

2<smiles>c1ccc(C23CC4CC(CC(C4)C2)C3)cc1</smiles>

56

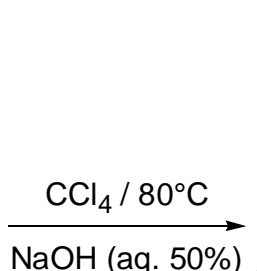

$8 \mathrm{~d} / 56 \%$<smiles>CC12CC3CC(C1)CC(c1ccccc1)(C3)C2</smiles>

57
$\stackrel{\mathrm{NaOH}(\text { aq. } 50 \%)}{\mathrm{CH}}$ $5 \mathrm{~d} / 23 \%$

$$
\begin{gathered}
\mathrm{CBr}_{4} / \mathrm{PhF} / 60^{\circ} \mathrm{C} \\
\mathrm{NaOH}(\mathrm{aq} .50 \%) \\
4 \mathrm{~d} / 7 \%
\end{gathered}
$$<smiles>BrC12CC3CC(C1)CC(c1ccccc1)(C3)C2</smiles>

$( \pm)-61$
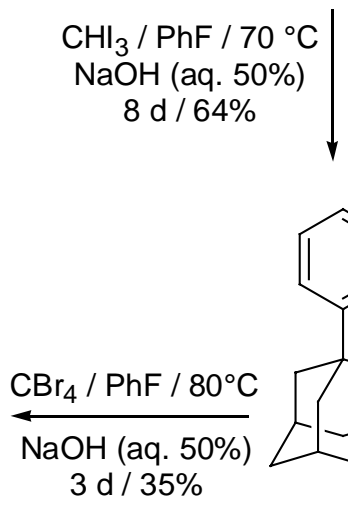

$3 \mathrm{~d} / 35 \%$

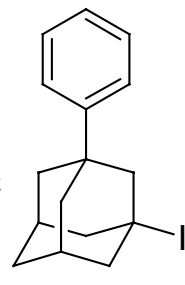

60

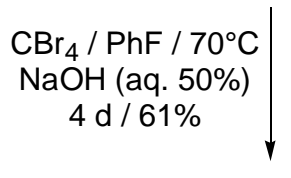<smiles>ClC12CC3CC(Br)(C1)CC(c1ccccc1)(C3)C2</smiles>

$( \pm)-62$

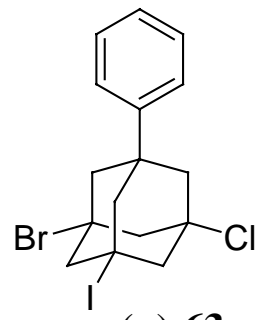

$( \pm)-63$

Schema 23. Synthesewege zu phenylsubstituierten chiralen Adamantanen. Alle Reaktionen wurden mit 3-10 mol\% TBABr durchgeführt. Manche Reaktionen mußten mehrere Male einem Neustart unterworfen werden (s. exp. Teil). 
Tabelle 11. PT-Halogenierungen von Phenyladamantanen

\begin{tabular}{|c|c|c|c|c|}
\hline$\#$ & Edukt & AV / Halogenierung & Produkt 1 [\%] & Produkt 2 [\%] \\
\hline 1 & 56 & $\mathrm{AV} 14 \mathrm{~m} / \mathrm{Cl}$ & $57[56]$ & \\
\hline 2 & 56 & $\mathrm{AV} 14 \mathrm{n} / \mathrm{Br}$ & $59[61]$ & \\
\hline 3 & 56 & $\mathrm{AV} 14 \mathrm{n} / \mathrm{Br}$ & $65[32]$ & {$[24$} \\
\hline 4 & 56 & AV14o / I & 60 & \\
\hline 5 & 57 & AV14p / I & $( \pm)-58 \quad[23]$ & - \\
\hline 6 & 57 & $\mathrm{AV} 14 \mathrm{q} / \mathrm{Br}$ & $( \pm)-62 \quad[61]$ & [17 \\
\hline 7 & 59 & AV14r / I & $( \pm)-61$ & - \\
\hline 8 & 60 & $\mathrm{AV} 14 \mathrm{~s} / \mathrm{Br}$ & $( \pm)-61$ & - \\
\hline 9 & 58 & $\mathrm{AV} 14 \mathrm{t} / \mathrm{Br}$ & $( \pm)-63$ & - \\
\hline 10 & 62 & AV14u / I & $( \pm)-63 \quad[3]$ & - \\
\hline
\end{tabular}




\subsubsection{Die PT-Halogenierungen von 1-Methoxyadamantanen}

Schließlich wurde die Polyhalogenierung auch noch an einem weiteren basenstabilen Adamantanderivat nämlich dem 1-Methoxyadamantan (69) durchgeführt, um zu einer möglichst großen Kollektion an verschieden substituierten Polyhaloadamantanen zu gelangen. Die Methoxygruppe ist zum einen auch deswegen besonders interessant, weil es sich hierbei um eine polarere Gruppe handelt als die Phenyl- oder Halogensubstituenten und so eine Enantiomerentrennung mittels analytischer und präparativer chiraler Chromatographie (GC, oder HPLC) evt. leichter ermöglicht werden könnte. Zum anderen wird durch das Sauerstoffatom der Ethergruppe u.U. eine stärkere Koordination an chirale Shiftreagenzien erlaubt. Dies würde eine schnelle Analyse etwaiger Enantiomerenüberschüsse im Fall enantioselektiv durchgeführter Halogenierungen erlauben (s. Ausblick). Zunächst wurde 1-Methoxyadamantan (69) aus 46 durch Deprotonierung mit $\mathrm{NaH}$ und anschließende Alkylierung mit Methyliodid synthetisiert (Diese Verbindung ist zwar literaturbekannt, die Autoren geben aber keine genaue Synthesevorschrift an. Deswegen wird im experimentellen Teil diese (AV14w) detailliert wiedergegeben.). [182]<smiles>OC12CC3CC(CC(C3)C1)C2</smiles>

46

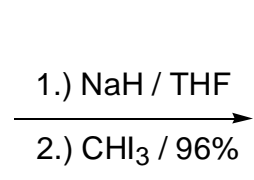

2.) $\mathrm{CH}_{3} / 96 \%$

$$
\begin{gathered}
\mathrm{CHI}_{3} / \mathrm{PhF} / 50^{\circ} \mathrm{C} \\
\mathrm{NaOH}(\text { aq. } 50 \%) \\
3 \mathrm{~d} / 15 \%
\end{gathered}
$$<smiles>COC12CC3CC(C1(Br)Br)C(Br)(C3)C2(Br)Br</smiles>
$( \pm)-74$

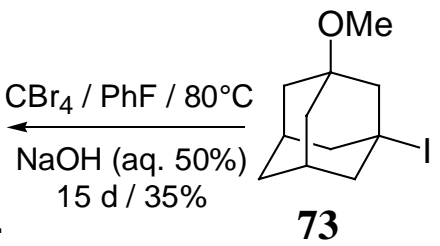

73

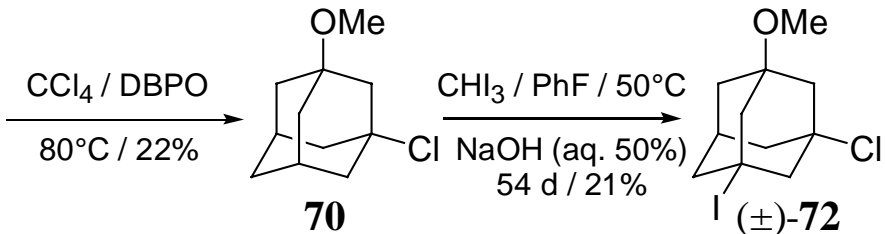

70

$$
\begin{gathered}
\mathrm{CBr}_{4} / \mathrm{PhF} / 75^{\circ} \mathrm{C} \\
\mathrm{NaOH}(\mathrm{aq} .50 \%) \\
12 \mathrm{~d} / 22 \%
\end{gathered}
$$

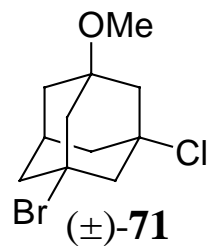

Schema 24. Synthesewege zu methoxysubstituierten chiralen Adamantanen Alle Reaktionen wurden mit 3-10 mol\% TBABr durchgeführt. Manche Reaktionen mußten mehrere Male einem Neustart unterworfen werden (s. exp. Teil). 
Tabelle 12. PT-Halogenierung von Methoxyadamantanen

\begin{tabular}{|c|c|c|c|c|}
\hline \# & Edukt & AV / Halogenierung & Produkt $1[\%]$ & Produkt 2 [\%] \\
\hline 1 & 69 & $\mathrm{AV} 14 \mathrm{x} / \mathrm{Cl}$ & 70 [22] & - \\
\hline 2 & 69 & AV14y/ I & $73[15]$ & - \\
\hline 3 & 70 & $\mathrm{AV} 14 \mathrm{z} / \mathrm{Br}$ & $( \pm)-71 \quad[22]$ & {$[6]$} \\
\hline 4 & 70 & AV14ä / I & $( \pm)-72 \quad[21]$ & [19] \\
\hline 5 & 73 & AV140̈ / Br & $( \pm)-74 \quad[21]$ & [26] \\
\hline
\end{tabular}

Bei der nachfolgenden Ersthalogenierung wurde im Fall der Chlorierung gleich auf die thermisch durch Radikalkettenstarter initiierte Reaktion zurückgegriffen, um die langen Reaktionszeiten $\mathrm{zu}$ vermeiden. Die Iodierung konnte ebenfalls mit guten Ausbeuten durchgeführt werden, wobei aber in beiden Fällen die Reaktion bei ca. 35\% Umsatz (GC/MSAnalyse) abgebrochen wurde. Dies war notwendig, weil sich die Methoxygruppe wie ein Halogensubstituent auswirkt und deswegen die gleichen säulenchromatografischen Trennprobleme wie bei den reinen Polyhaloadamantanen auftraten (Schema 21). Deswegen mußte bei der Zweithalogenierung die Reaktion auch soweit fortgeführt werden, bis so gut wie kein Monohalogenid mehr nachweisbar war (GC/MS-Analyse < 5\%). Die als Nebenprodukte entstandenen trihalogenierten Methoxyadamantane konnten wiederum leicht separiert werden. Die Ausbeuten bei dieser Synthesesequenz sind annehmbar.

Wiederum ist der Grund für eine beobachtete Aktivierung wie im Fall der Phenyladamantane nicht bekannt und bedarf weiterer Untersuchungen (s. Ausblick). Insgesamt konnten so drei verschiedene chirale methoxysubstituierte Polyhaloadamantane als 
racemische Gemische dargestellt werden. Auf eine dreifache Halogenierung wurde hier verzichtet.

\subsubsection{Weitere Versuche zur Synthese von 1-Iod-3-brom-5-chlor-7-fluoradamantan}

Nicht zuletzt wegen dessen Ästhetik aber auch aufgrund der Eigenschaften, die dieses Molekül verspricht, wurden schließlich noch weitere Versuche unternommen, um ( \pm )-55 doch noch zu synthetisieren. Da der direkte Syntheseweg, ausgehend von 48 durch PT-Iodierung und nachfolgende PT-Bromierung, versperrt war, lag es nahe, durch Halogenaustausch bei verschiedenen tetrahalogenierten Adamantanen zum Ziel zu gelangen. Hierfür bot sich der Austausch eines Bromatoms durch Iod in $\mathbf{5 3}$ an, da dieses Molekül bereits bei vorangegangenen Synthesen als Nebenprodukt angefallen war.

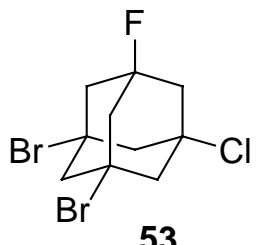

53

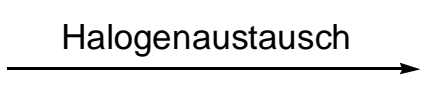

Schema 25. Synthese von ( \pm -55 ausgehend von $\mathbf{5 3}$ durch Halogenaustausch

Es stellte sich heraus, daß mit steigendem Halogengehalt im Adamantanmolekül anscheinend auch eine Steigerung der C-X-Bindungsstärke einhergeht, denn die zahlreich unternommenen Versuche durch klassischen Austauschreaktionen (HI (aq. 50\%), $105^{\circ} \mathrm{C} /[183,184] \mathrm{NaI}$, Aceton ${ }^{[21]} / \mathrm{NaI}$, PTK /[21] 1 . MeLi $\left.\left(-100{ }^{\circ} \mathrm{C}\right) 2 . \mathrm{CH}_{2} \mathrm{I}_{2}{ }^{[185]}\right)$ zum Ziel zu gelangen schlugen fehl. Einzig durch $\mathrm{SmI}_{2}(0.1 \mathrm{M}$ in THF) war es möglich 53 überhaupt zur Reaktion zu bringen.[20, 186, 187] Eine GC/MS-Analyse der Reaktionsmischung ergab allerdings, daß das Hauptprodukt (40\%) wahrscheinlich das Reduktionsprodukt $( \pm)-\mathbf{5 0}$ war und sich $( \pm)-\mathbf{5 5}$ in nur $2 \%$ Ausbeute (GC/MS) neben weiteren Austauschprodukten gebildet hat. Vermutlich wirkt das Lösungsmittel THF als Wasserstoffdonor und fängt so das intermediär durch Zerfall des Radikalanions gebildete Trihaloadamantylradikal ab (Schema 26). Da es nicht möglich ist Iodquellen, wie z.B. Diiodmethan oder gar Iod als Konkurrenz zum THF einzusetzen, da diese auch mit dem $\mathrm{SmI}_{2}$ reagieren würden, bleibt nur die Möglichkeit eines Wechsels des Lösungsmittels. Hier würden sich $t$-Butyl-methylether oder auch Freone wie $\mathrm{CF}_{3} \mathrm{Cl}$ anbieten, 
die sich gegenüber Radikalen relativ inert verhalten. Weitere Versuche zur Synthese dieses eindrucksvollen Moleküls werden Gegenstand weiterführender Arbeiten sein (s. Ausblick).

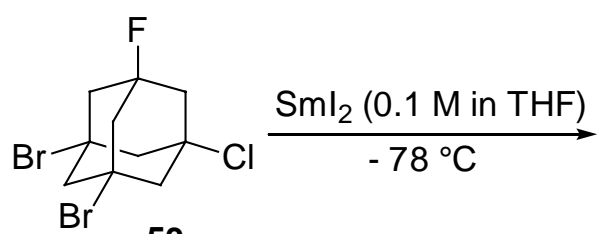

53<smiles>FC12CC3(Cl)CC(Br)(C1)CC(C(F)(F)F)(C2)C3</smiles>
50

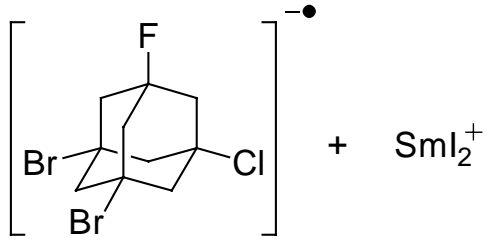

$\downarrow-\mathrm{Br}^{-}$<smiles>FC12CC3(Cl)CC(Cl)(C1)CC(Br)(C2)C3</smiles>

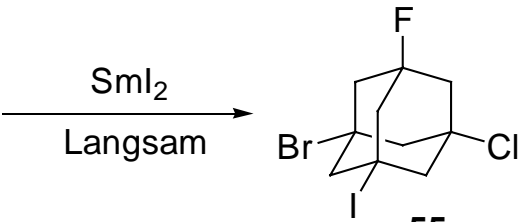
55

Schema 26. Postulierter Bildungsweg von ( \pm -55 in Konkurrenz zur Reduktion infolge des Abfangens des Trihalomethylradikals durch THF

\subsubsection{Racemattrennungen von chiralen Polyhaloadamantanverbindungen}

\subsubsection{Versuche mit Lanthanid-Shift Reagenzien (LSR) zur Analyse der racemischen}

\section{Gemische}

\subsection{Einleitung}

Die Anwendung von LSR in der stereochemischen Analyse von nichtseparierten d.h.

isochronen Signalen in ${ }^{1} \mathrm{H}-\mathrm{NMR}$ Spektren oder zur Analyse von Enantiomerengemischen durch Bildung diastereomerer Komplexe war Gegenstand zahlreicher Untersuchungen und hat sich als außerordentlich nützlich erwiesen. [188-191] Mittlerweile ist diese Methodik aber durch die Entwicklung von NMR-Geräten mit außerordentlich hohen Feldstärken für den ersten Fall und von neuen, sehr leistungsfähigen chiralen Säulenmaterialen für die GC oder HPLC für den zweiten Fall etwas verdrängt worden. In Fällen wo letztere Möglichkeiten nicht zur Verfügung stehen, können Analysen mit LSR aber immer noch sehr nützlich sein. 
Die grundsätzliche Wirkungsweise eines LSR, beruht auf der Koordination seines lewisaciden Metallkations mit einer lewisbasischen funktionellen Gruppe der $\mathrm{zu}$ analysierenden organischen Substanz. Dabei bewirkt die magnetische Anisotropie des Lanthanidmetallions einen Shift im ${ }^{1} \mathrm{H}-\mathrm{NMR}-$ Spektrum für Protonen des organischen Moleküls. Das Ausmaß des Shifts und welches Proton davon betroffen ist beruht auf verschiedenen Faktoren. Die Mehrzahl aller induzierten Verschiebungen $\Delta \delta$ kann durch die Addition zweier Terme, durch den Kontakt- (K) und den Pseudokontakteffekt (PK), beschrieben werden (Gl. XIII). [188]

$$
\Delta \delta=\Delta \delta_{K}+\Delta \delta_{P K}
$$

Der Beitrag durch den Kontakteffekt kann normalerweise für ${ }^{1} \mathrm{H}-\mathrm{NMR}$ Verschiebungen vernachlässigt werden und der Pseudokontakteffekt wird durch die McConnell-Robertson Gleichung (Gl. XIV) angenähert.

$$
\Delta \delta_{P K}=\frac{K\left(3 \cos ^{2} \Theta-1\right)}{r^{3}}
$$

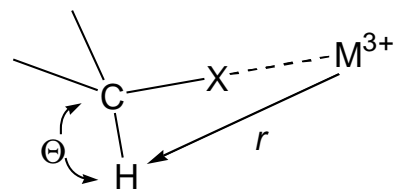

$K=$ empirische Konstante für einen bestimmten Substrat-LSR Komplex

$r=$ Vektor zwischen Metallion und Proton

$\Theta=$ Winkel der Abweichung von $r$ von der Symmetrieachse

Dabei wird deutlich, daß der induzierte Shift für ein bestimmtes Proton hauptsächlich von dessen Entfernung zum LSR Metallion und vom Winkel der Abweichung von $r$ von der Symmetrieachse abhängt. Die Koordination zwischen Substrat und LSR kann als ein sich schnell einstellender reversibler Gleichgewichtsprozeß und der beobachtete Shift als Durchschnitt der Beiträge des Komplexes (LSR•S) und des nicht komplexierten Substrates (S) aufgefaßt werden: $\quad \mathrm{LSR}+\mathrm{S} \rightleftharpoons \mathrm{LSR} \cdot \mathrm{S}$

Verschiedenen Lanthanidmetallionen haben sich je nach Problemstellung zur Verwendung als Shiftreagenzien bewährt, wie z.B. $\mathrm{Eu}^{3+}$, (wird traditionell am häufigsten verwendet, obwohl andere Metallionen u.U. vorteilhafter wären), $\mathrm{Pr}^{3+}, \mathrm{Yb}^{3+}, \mathrm{Dy}^{3+}$ und $\mathrm{Ho}^{3+}$, um nur einige zu nennen. Letztere beiden haben kürzere Elektronenspin-Relaxationszeiten und bewirken deshalb bereits eine merkliche Linienverbreiterung im ${ }^{1} \mathrm{H}-\mathrm{NMR}-$ Spektrum, können aber noch verwendet werden. $[188,190]$ 


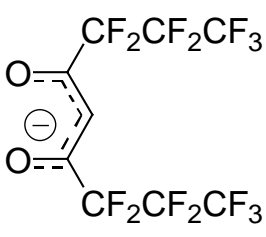

$\mathrm{tfn}^{-}$

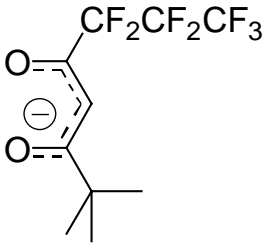

fod $^{-}$

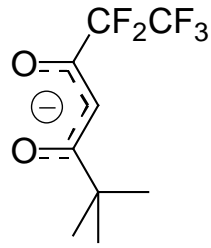

$\operatorname{pfd}^{-}$
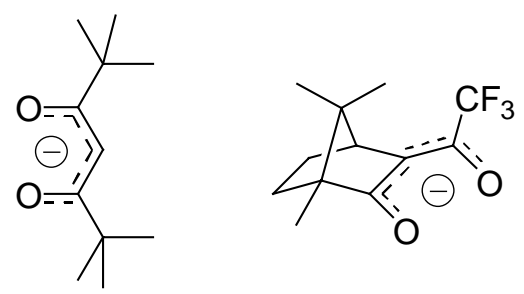

$\mathrm{dpm}^{-}$ $d-\mathrm{tfc}^{-}$

Schema 27. Eine Auswahl von Liganden, die für LSR Verwendung finden, darunter ein chiraler Ligand (rechts).

Ein weiterer wichtiger Faktor eines LSR ist Auswahl eines bestimmten Liganden (Schema 27), der einige Kriterien erfüllen sollte. So muß er eine möglichst hohe Löslichkeit des LSR im organischen Lösungsmittel gewährleisten, den induzierten Shift des Metallions unterstützen, da dieser auch durch den Liganden beeinflußt wird und darf durch seine eigenen Signale im NMR-Spektrum keine des Substrates überlagern. Durch Fluorierung der Liganden kann letzteres Problem weitestgehend umgangen werden. Außerdem gilt, daß je höher fluoriert der Ligand ist, auch die Verschiebung bei gleichem Metallion um so größer wird. [188] Durch Verwendung chiraler Liganden, können auch die Signale von Enantiomeren durch Bildung diastereomerer LSR•S-Komplexe voneinander getrennt werden. Ein solcher Ligand ist $\mathrm{tfc}^{-}$(Schema 27, Trifluorhydroxymethylen- $d$-camphorat) der auch in den folgenden Untersuchungen Verwendung finden soll.[190] Binukleare Komplexe, bestehend aus z.B. Silber(fod) und LSR, finden Anwendung bei der Analyse von aromatischen oder ungesättigten Verbindungen.[192, 193] Auch sekundäre Chloride können auf diese Weise untersucht werden.[194]

\subsection{Durchführung der Shiftexperimente}

Die Shiftexperimente wurden zur Analyse der racemischen Gemische der chiralen Adamantanverbindungen durchgeführt. Es sollte hierbei durch eine Separation der Enantiomeren durch diastereomere Komplexbildung mit einem enantiomerenreinen chiralen LSR die Grundlage geschaffen werden, um in weiterführenden Arbeiten eine apparateunabhängige spezielle Möglichkeit der Bestimmung etwaiger Enantiomerenüberschüsse im Rahmen enantioselektiver PT-Halogenierungen zu schaffen. 
Zunächst sollte durch Vorabexperimente überprüft werden, welche Lanthanidionen sich am besten für die entsprechenden Adamantanverbindungen eignen. Die zu analysierenden Verbindungen enthalten als funktionelle Gruppen $-\mathrm{F},-\mathrm{Cl},-\mathrm{Br},-\mathrm{I},-\mathrm{Ph}-\mathrm{OMe}$. Gut untersucht ist die Anwendung der einleitend erwähnten binuklearen LSR-Komplexe an aromatischen Verbindungen und primären sowie sekundären Halogeniden. So konnten gute Ergebnisse mit $\mathrm{Ag}($ fod $) \bullet \mathrm{Yb}(\text { fod })_{3}$ und $\mathrm{Ag}($ fod $) \bullet \mathrm{Dy}(\text { fod })_{3}$ bei der Untersuchung von primären Chloriden, Bromiden und Iodiden erzielt werden. Dabei stellt die Anwesenheit von Silbersalzen bei gleichzeitigem Vorhandensein von tertiären Halogensubstituenten ein Problem dar, da es zur Ausfällung von Silberhalogenidsalzen kommen kann. Aufgrund der Tatsache das tertiäre Adamantan-Halogenbindungen stabiler sind als tertiäre offenkettige, wurden Shiftexperimente mit $\mathbf{4 8}$ und verschiedenen binuklearen LSR-Komplexen $\left(\mathrm{Ag}(\mathrm{fod}) \bullet \mathrm{Yb}(\mathrm{fod})_{3}\right.$ und $\left.\mathrm{Ag}(\mathrm{fod}) \bullet \mathrm{Dy}(\text { fod })_{3}\right)$ durchgeführt. Allerdings kam es bereits bei der Mischung der verschiedenen Substanzen zu einem Ausfällen, wahrscheinlich von $\mathrm{AgCl}$, was diesen Ansatz für eine Analyse der racemischen Gemische unbrauchbar machte. Weitere Experimente wurde deswegen mit binuklearen LSR-Komplexen nicht durchgeführt. Weiterhin ist literaturbekannt, daß $\mathrm{Yb}(\text { fod })_{3}$, Dy(fod $)_{3}$ und $\mathrm{Eu}(\mathrm{fod})_{3}$ mit Fluorsubstituenten koordinieren können, wobei mit $\mathrm{Yb}$ die größten und mit Eu die geringsten Verschiebungen erzielt werden. ${ }^{[195]}$ Deswegen wurden zunächst Experimente mit 47 als Modellverbindung und $\mathrm{Yb}(\text { fod })_{3}$ sowie $\mathrm{Dy}(\mathrm{fod})_{3}$ als LSR durchgeführt, bei denen auch ein Shift beobachtet werden konnte. Allerdings war diese Verschiebung erstens in beiden Fällen sehr gering und zweitens hauptsächlich beschränkt auf ein Signal (siehe Abb. 21, 22).

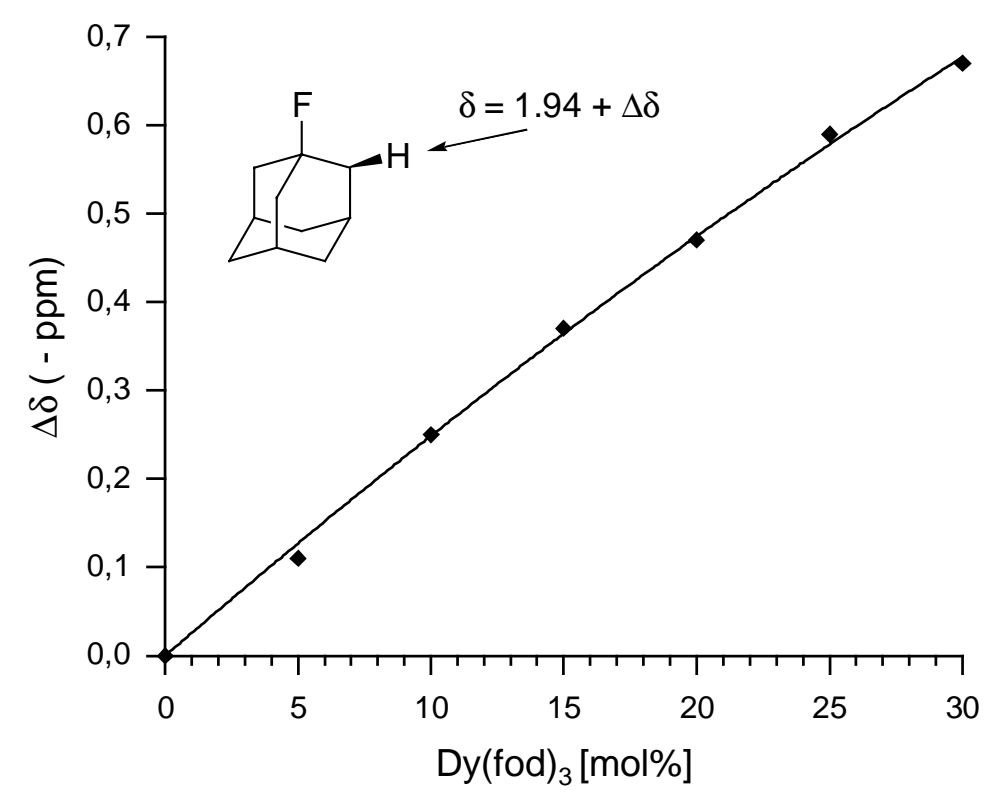

Abbildung 21. Beobachter Shift verursacht durch Dy(fod $)_{3}$ von 2-H in 47 


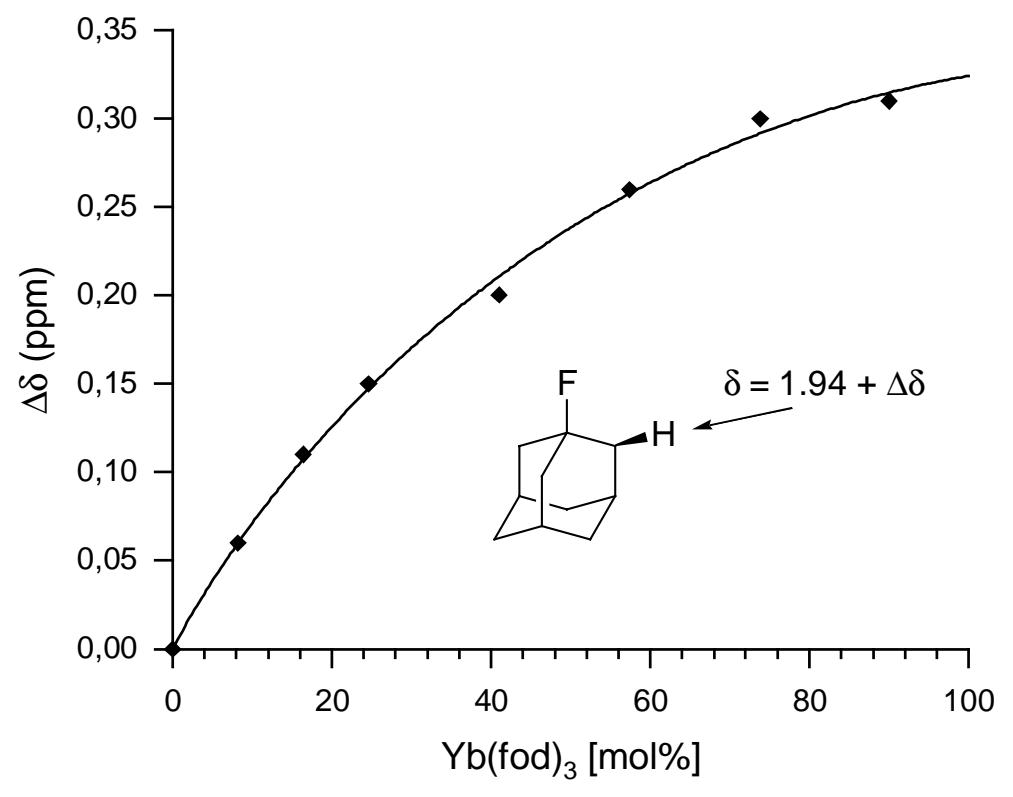

Abbildung 22. Beobachter Shift verursacht durch $\mathrm{Yb}(\text { fod })_{3}$ von $2-\mathrm{H}$ in $\mathbf{4 7}$

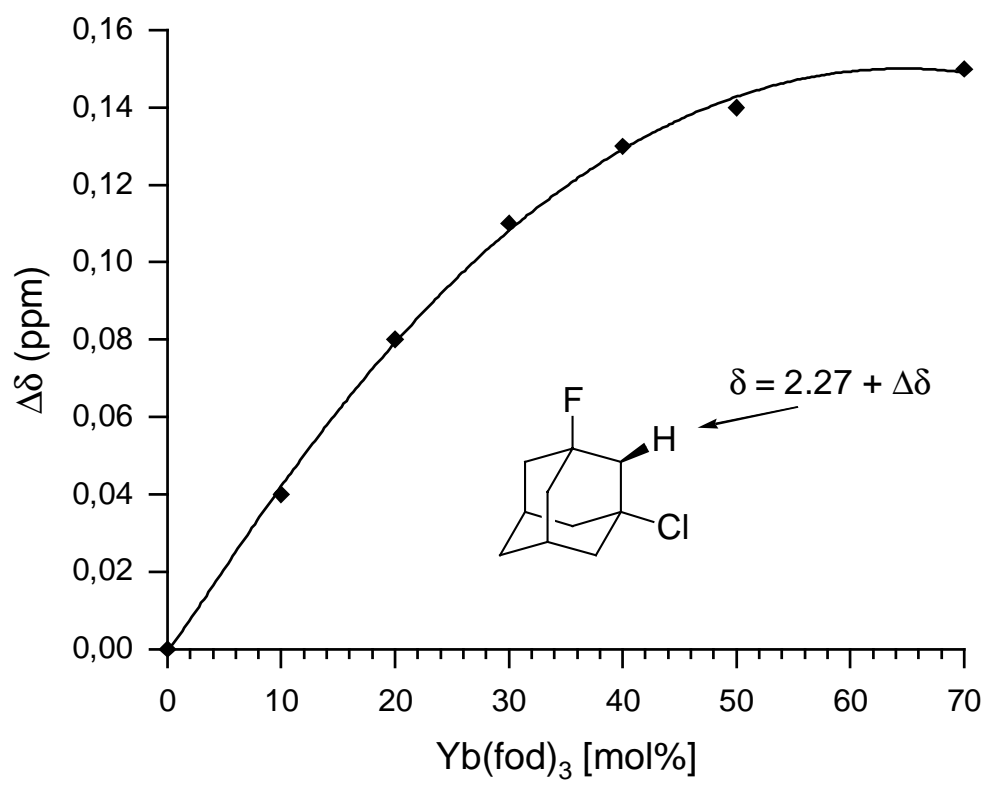

Abbildung 23. Beobachter Shift verursacht durch $\mathrm{Yb}(\text { fod })_{3}$ von $2-\mathrm{H}$ in 48

Der beobachtete Shift war für Dy(fod) $)_{3}$ zwar größer verlief aber ins Tieffeld (Abb. 20), was zur Überlappung mit anderen ${ }^{1} \mathrm{H}-\mathrm{NMR}$-Signalen führte und dieses Lanthanid von vorne herein für weitere Untersuchungen unbrauchbar machte. Um die Auswirkung eines weiteren Halogensubstituenten im Adamantangerüst auf die Verschiebung zu bestimmen wurde 
daraufhin 48 mit $\mathrm{Yb}(\text { fod })_{3}$ untersucht. Auch hier konnte ein Shift beobachtet werden, der aber noch geringer war als im Fall von 47 (Abb. 19). Weiterhin wurde auch noch 2 unter den gleichen Bedingungen untersucht, um festzustellen, wie sich die Änderung des Halogenatoms auf den Shift auswirkt. Es wurde aber keinerlei Shift festgestellt, was wahrscheinlich auf die geringe Koordination des relativ weichen, lewisbasischen Bromsubstituenten mit dem harten lewissauren Lanthanidion zurückzuführen ist. Die bisher erhaltenen Resultate machten weitere Experimente mit chiralen enantiomerenreinen LSR nicht erfolgversprechend. Dennoch wurden mit $\mathrm{Yb}(d \text {-tfc })_{3}$ einige Versuche durchgeführt. Mit 47 als Modellverbindung und ( \pm )-1-Fluor-3-brom-7-iodadamantan (51) wurde keinerlei Verschiebungen festgestellt (die Aktivität des LSR wurde anhand der Koordination mit Adamantancarbonsäuremethylester überprüft).

Am vielversprechendsten erschienen Shiftexperimente mit methoxysubstituierten Adamantanen, da in der Literatur viele Beispiele auch für die Analyse von Enantiomerengemischen solcher Verbindungen beschrieben wurden, $[188,190]$ wobei sich auch wieder Yb-LSR bewährt haben. Hierbei wurde auf Vorabexperimente verzichtet und gleich das chirale $\mathrm{Yb}(d-\mathrm{tfc})_{3}$ eingesetzt. Damit wurde zunächst 1-Chlor-3-methoxyadamantan 70 untersucht, wobei wieder nur geringe Shifts beobachtet wurde $\left[\Delta \delta\left(\mathrm{Yb}(d-\mathrm{tfc})_{3} / 30 \mathrm{~mol} \%\right)=\right.$ $0.14(2-\mathrm{H}) ; 0.15(8-, 10-\mathrm{H}) ; 0.15(-\mathrm{OMe})]$. Schließlich wurde dasselbe LSR noch auf das chirale ( \pm )-1-Brom-3-iod-5-methoxyadamantan 74 angewendet, allerdings auch mit enttäuschendem Ergebnis, denn der gemessene Shift verringerte sich wieder durch die Zunahme an Halogensubstituenten im Adamantangerüst. Der geringe Shift reichte keinesfalls aus, um die Signale der diastereomeren Komplexe aufzulösen $\left[\Delta \delta\left(\mathrm{Yb}(d-\mathrm{tfc})_{3} / 30 \mathrm{~mol} \%\right)=\right.$ $0.08(6-\mathrm{H}) ; 0.08(4-, 10-\mathrm{H}) ; 0.08(-\mathrm{OMe})]$.

\subsection{Ergebnis der Analysen mit LSR}

Es mußte festgestellt werden, daß LSR nicht zur Analyse von enantiomeren Mischungen chiraler Adamantane geeignet sind. Dies ist wahrscheinlich auf die folgenden Gründe zurückzuführen. Die Wechselwirkung zwischen LSR und Substrat erfolgt an einer funktionellen Gruppe, die direkt am Adamantangerüst lokalisiert ist. Somit kommt die Sperrigkeit des Adamantylrestes voll zum Tragen, und die Komplexierung mit dem ebenfalls sehr raumbeanspruchenden LSR erfolgt nur in einem geringen Maß. Ein weiterer Faktor ist, daß die funktionellen Gruppen am Adamantangerüst (mit Ausnahme von -OMe) nicht 
unbedingt $\mathrm{zu}$ den prädestiniertesten für LSR-Analysen gehören und das Komplexbildungsbestreben ohnehin nur schwach ausgeprägt ist. Ein letzter Grund liegt in der Topologie der entsprechenden Adamantanverbindungen begründet. Aus der McConnellRobertson Gleichung geht hervor, daß der induzierte Shift stark vom Abstandsvektor $r$ und vom Winkel dessen Abweichung von der Molekül-LSR Achse abhängt. Durch das starre Adamantangerüst und dadurch, daß es sich um tertiäre Adamantanverbindungen handelt, sind $r$ und $\theta$ relativ groß, so daß der induzierte Shift sehr klein wird (Schema 28).

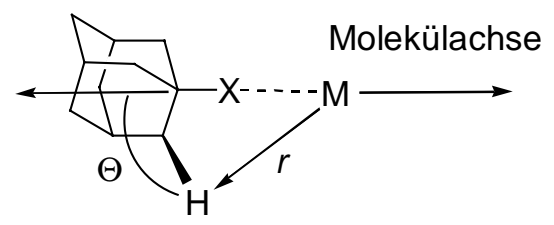

Schema 28. Auswirkung der Adamantangrundstruktur auf die Parameter $r$ und $\theta$ der McConnell-Robertson Gleichung für einen LSR $\bullet$ Substrat Komplex

\subsubsection{Analytische Enantiomerentrennung durch chirale Gaschromatographie}

Eine andere Möglichkeit für die analytische Trennung von Enantiomeren ist die Gaschromatographie unter Verwendung chiraler Säulenmaterialien. Eine solche Trennung sollte auch an den chiralen Polyhaloadamantanen durchgeführt werden. Problematisch ist hierbei, daß eine Trennung am besten bei möglichst niedrigen Säulentemperaturen durchgeführt werden sollte, die aber durch das hohe Molekulargewicht der Adamantanverbindungen nicht zugelassen wird. So ist eine Trennung der Enantiomeren der phenylsubstituierten chiralen Adamantanen von vorne herein aussichtslos.

Als erste Verbindung wurde ( \pm )-1-Fluor-3-brom-5-iodadamantan (51) bei verschieden Temperaturen $\left(90 \rightarrow 110,125,135^{\circ} \mathrm{C}\right.$ Endtemperatur, $1 \% \mathrm{~min}$ : 25m 6-TBDMS2,3,methyl- $\beta$-CD 50\% in OV170) einer Gaschromatographie unterworfen. So konnten eine analytische Trennung der Enantiomeren herbeigeführt werden, wobei aber keine komplette Separation der Signale erreicht wurde (Abb. 24). Der Unterschied in den Retentionszeiten ist somit zu gering, um bei gleichem Säulenmaterial aber präparativer Säule reine Proben der Enantiomeren zu gewinnen, würde aber ausreichen um etwaige Enantiomerenüberschüsse zu extrapolieren. 


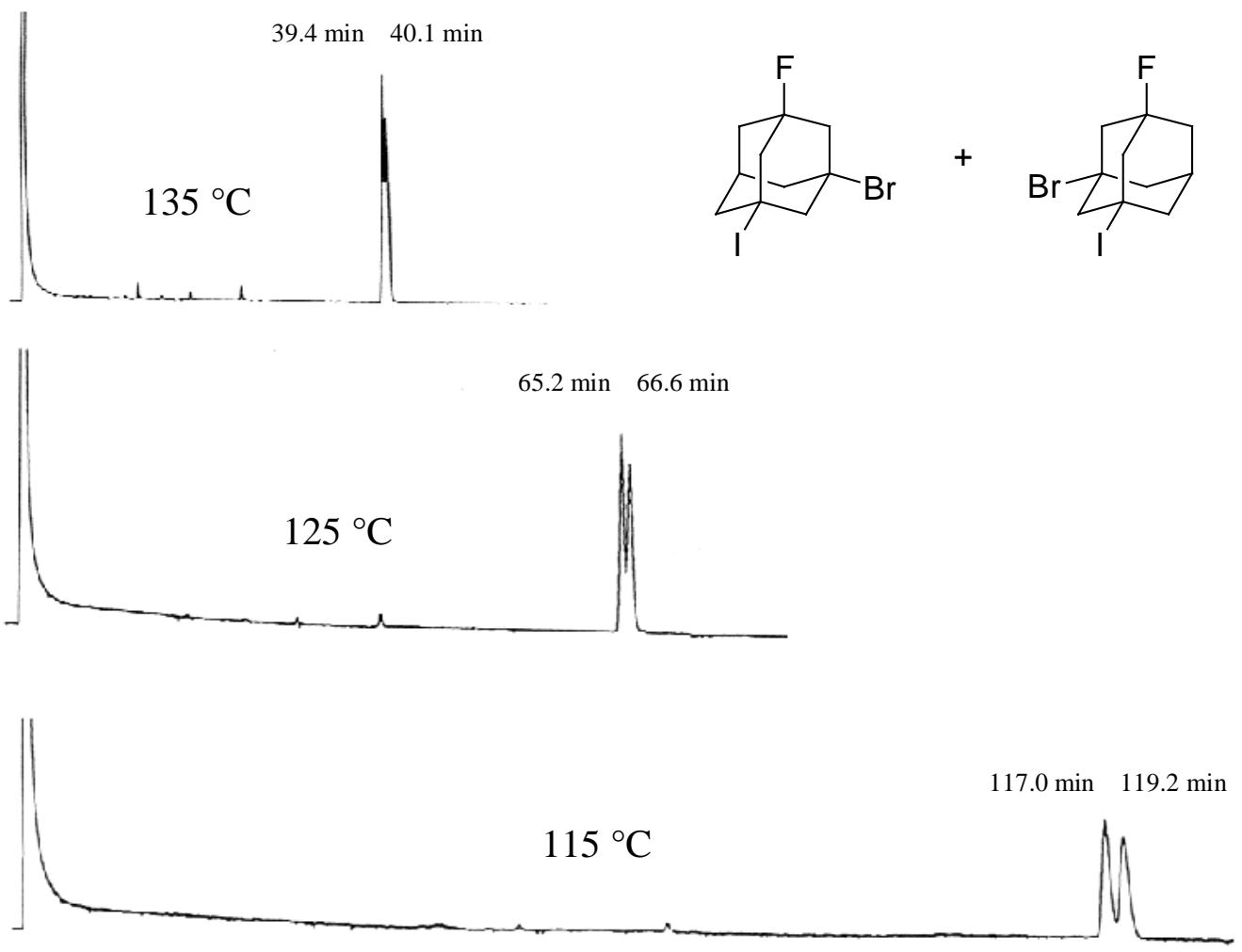

Abbildung 24. GC-Spektren der analytischen Trennung der Enantiomeren von $\mathbf{5 1}$ bei verschiedenen Temperaturen
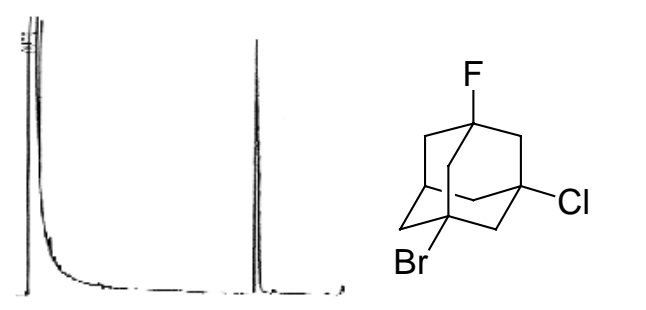<smiles>FC12CC3CC(Cl)(C1)CC(Br)(C3)C2</smiles>
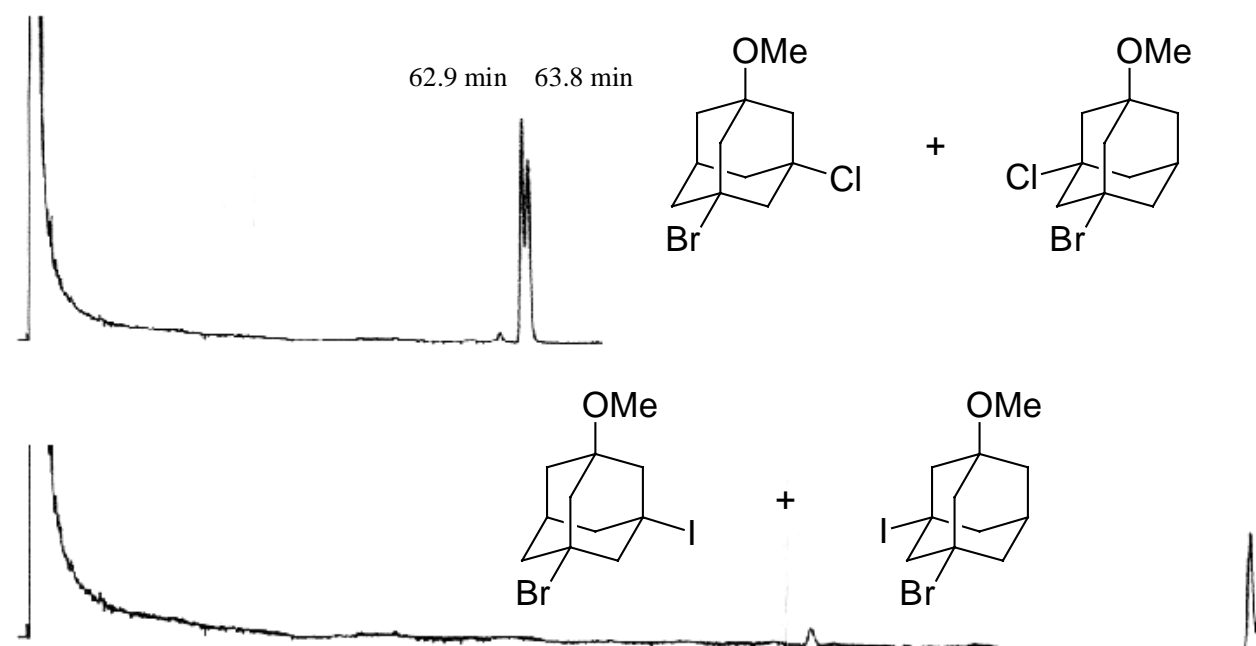

Abbildung 25. GC-Spektren der analytischen Trennung der Enantiomeren von ( \pm )-50, $( \pm)-71$ und $( \pm)-74$ bei $140^{\circ} \mathrm{C}$ 
Um einen weiteren Überblick über das Verhaltenen anderer Polyhaloadamantane zu gewinnen wurden ( \pm )-1-Chlor-3-brom-5-fluor-, ( \pm )-1-Chlor-3-brom-5-methoxy- und ( \pm )1-Brom-3-iod-5-methoxyadamantan $(\mathbf{5 0}, \mathbf{7 1}, \mathbf{7 4})$ analysiert (Abb. 25, $125 \rightarrow 140^{\circ} \mathrm{C}$ Endtemperatur, 1 \% min: 25m 6-TBDMS-2,3, methyl- $\beta$-Cyclodextrin 50\% in OV170). ${ }^{[196]}$ Bei dieser Temperatur konnten die Enantiomeren der ersten Verbindung nicht getrennt werden, während die anderen Verbindungen in etwa gleiches Verhalten aufweisen. Fazit dieser Analysen ist, daß durch chirale GC eine schnelle Analyse von Enantiomerenüberschüssen möglich ist, für präparative Trennung aber andere Wege gefunden werden müssen.

\subsubsection{Analytische und präparative Enantiomerentrennung durch chirale HPLC}

Da eine präparative Enantiomerentrennung der verschiedenen chiralen Adamantanverbindungen durch chirale Gaschromatographie nicht erreicht werden konnte, sollte dies mit chiraler HPLC (High Performance Liquid Chromatography) versucht werden, da diese normalerweise über eine weitaus höhere Trennleistung verfügen. Dabei wurden die von Okamoto et al. entwickelten stationären Phasen CIRALCEL ${ }^{\circledR}$ und CHIRALPAK $^{\circledR}$ eingesetzt,[197] die sich bereits bei vielen Trennproblemen als enorm leistungsfähig herausgestellt haben. ${ }^{[198]}$ Zunächst wurden die chiralen phenylsubstituierten Adamantane einer Trennung unterworfen, wobei UV-Detektoren zum Einsatz kamen (JASCO-UV 1595), da 58, 61 und 62 dafür ausreichend UV-aktiv sind. Es zeigte sich, daß in allen Fällen eine Trennung der Enantiomeren möglich war, diese aber im Fall von 58 und $\mathbf{6 2}$ nur unvollständig erfolgte (Abb. 26). Einzig bei 61 war es möglich eine vollständige Trennung herbeizuführen (Abb. 27) und ein CD-Spektrum aufzunehmen (Abb. 27). Dieses zeigte klar den für die Enantiomere erwarteten spiegelbildlichen Verlauf. Für die Trennungen weiterer racemischen Gemische $(\mathbf{5 0}, \mathbf{5 1}, \mathbf{7 1}, \mathbf{7 4})$ erfolgte die Detektion polarimetrisch (JASCO 830-RI), da keine ausreichende UV-Aktivität zu verzeichnen war. Bei den rein halogensubstituierten chiralen Adamantanen 50 und 51 ließen sich die Enantiomere gut (Abb. 28) und im Fall der methoxysubstituierten Haloadamantane $\mathbf{7 1}$ und $\mathbf{7 4}$ so gut wie gar nicht voneinander trennen (Abb. 29). 

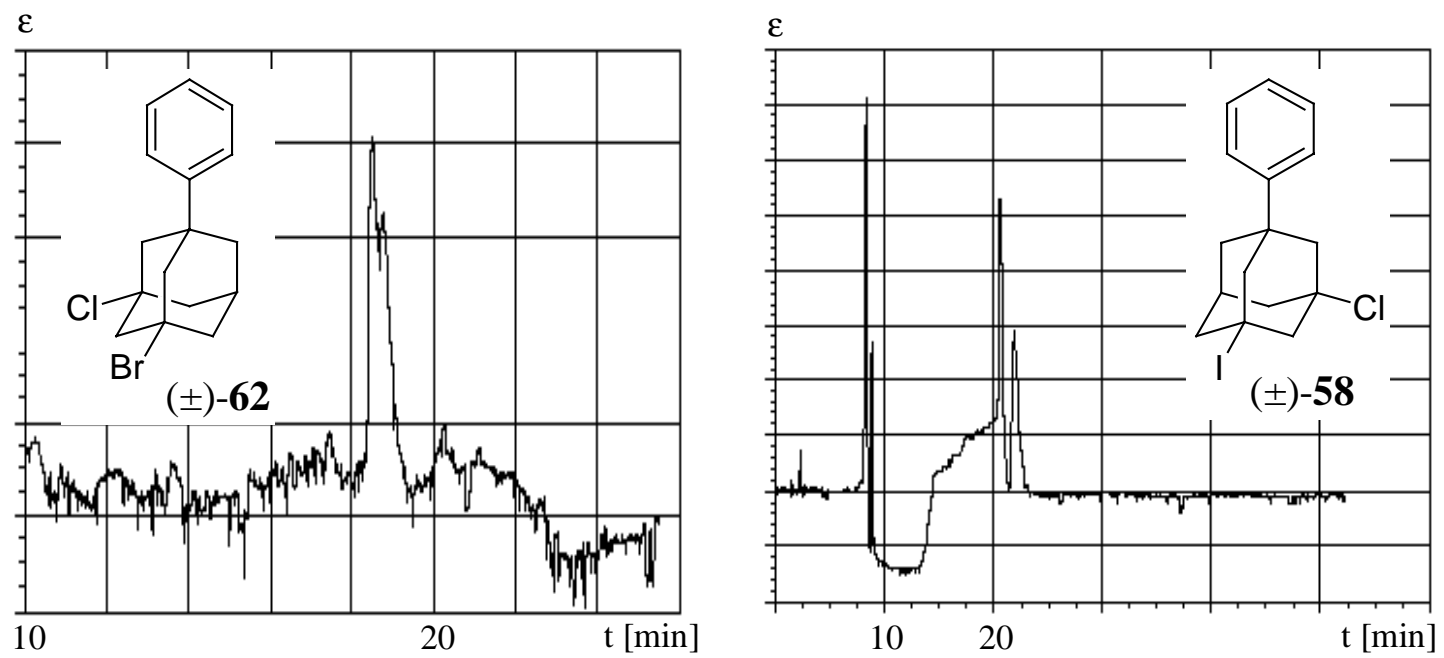

Abbildung 26. HPLC-Trennungen von 62 (links) und 58 (rechts); CHIRALCEL OD (25 cm x $0.46 \mathrm{~cm}, n$-Hexan : Ethanol $=99.9: 0.1,1.0 \mathrm{~mL} / \mathrm{min}$, UV-Detektor JASCO-UV 1595)
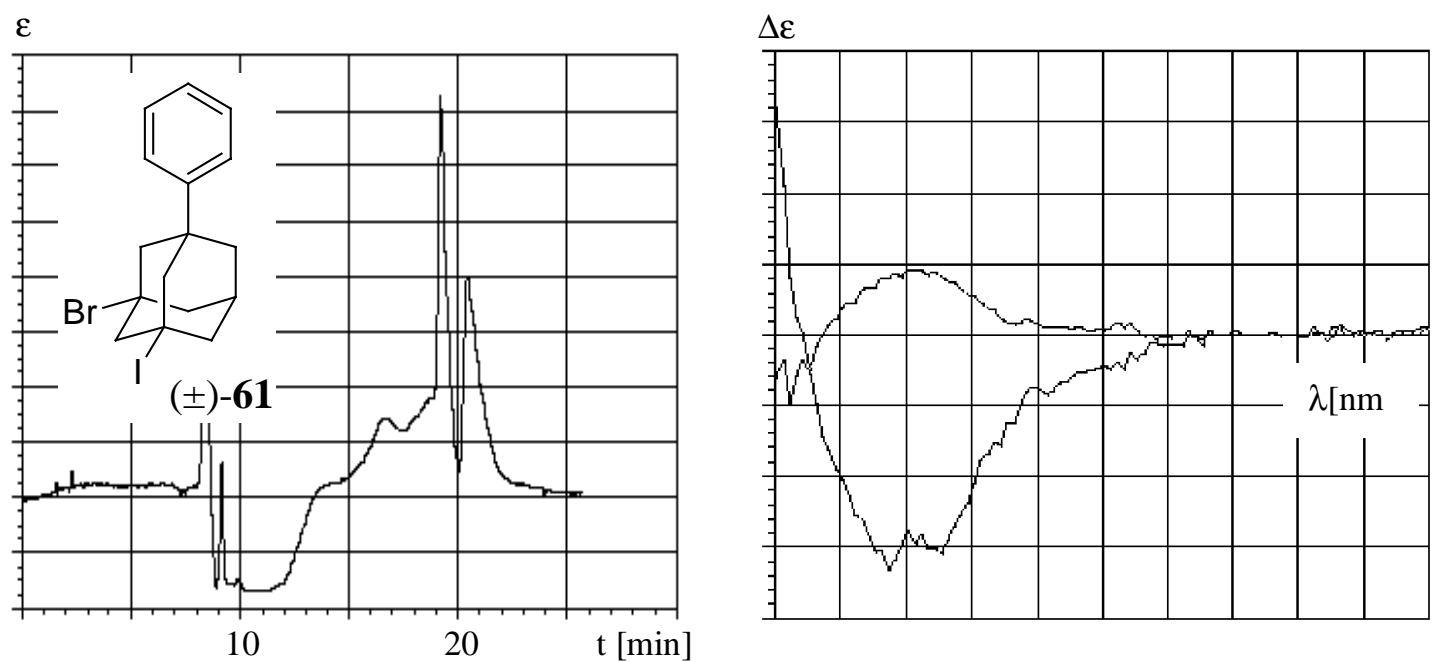

Abbildung 27. HPLC-Trennungen von 61 (links); CHIRALCEL OD (25 cm x $0.46 \mathrm{~cm}, n$ Hexan : Ethanol $=99.9: 0.1,1.0 \mathrm{~mL} / \mathrm{min}$, UV-Detektor JASCO-UV 1595) und die CDSpektren der Enantiomeren (rechts, JASCO-CD 1595, keine Zuordnung möglich)
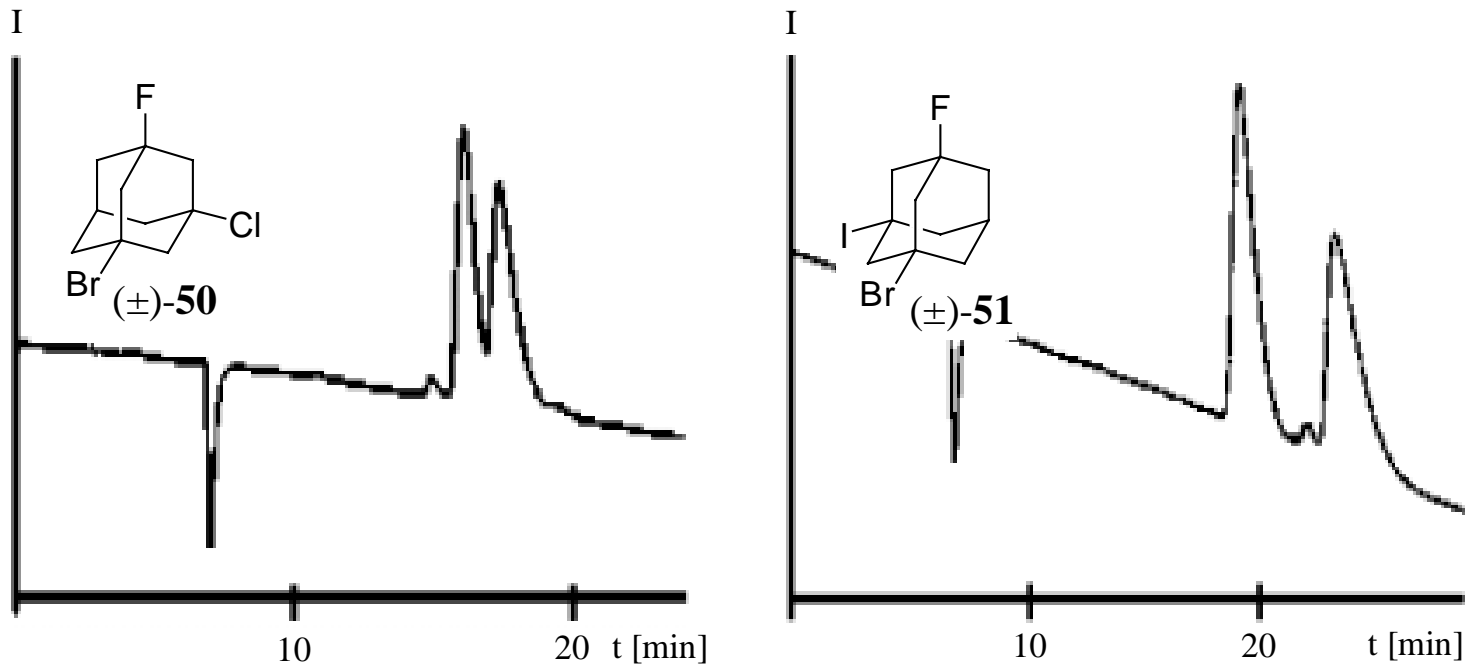

Abbildung 28. HPLC-Trennungen von 50 (links) und $\mathbf{5 1}$ (rechts); CHIRALPAK AD (25 cm x $0.46 \mathrm{~cm}$, Methanol, $0.5 \mathrm{~mL} / \mathrm{min}$, RI-Detektor JASCO-830 RI) 

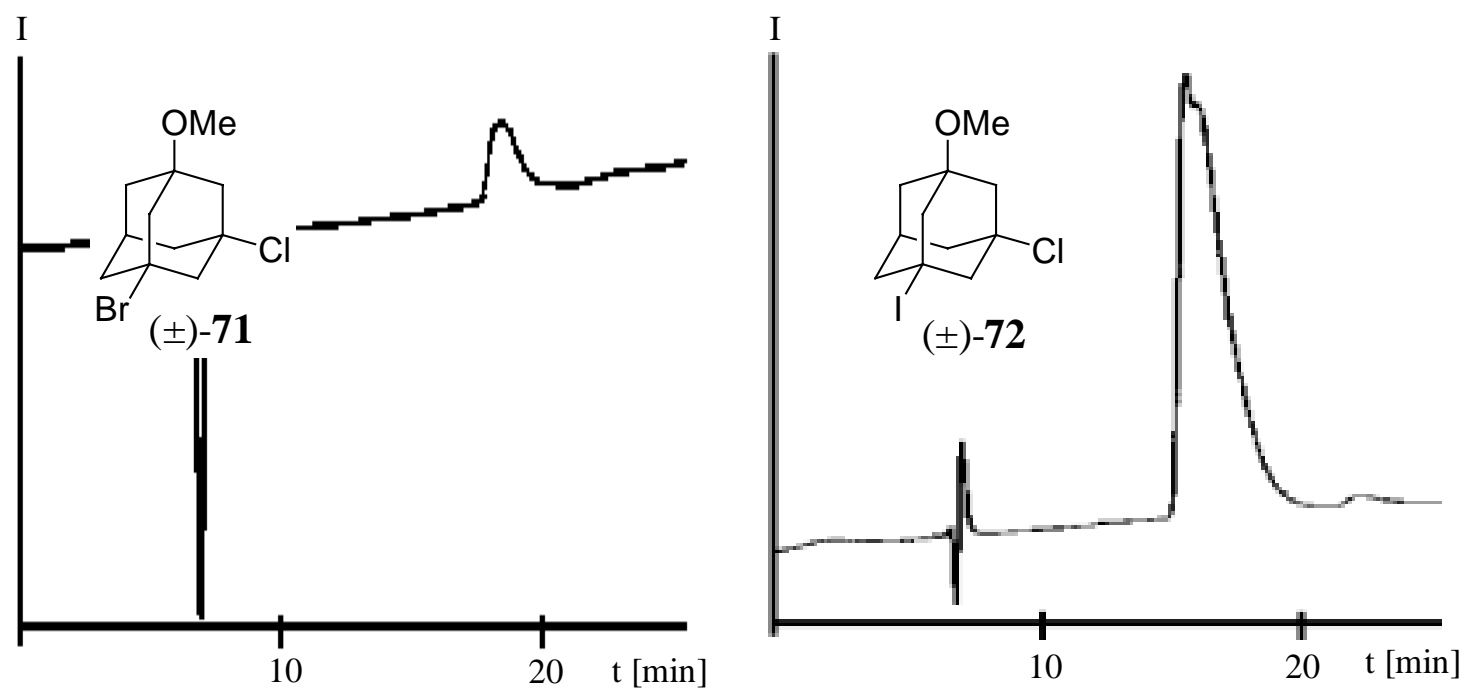

Abbildung 29. HPLC-Trennungen von 71 (links) und 74 (rechts); CHIRALPAK AS (25 cm x $0.46 \mathrm{~cm}, n$-Hexan : Ethanol $=99.9: 0.1,0.5 \mathrm{~mL} / \mathrm{min}, \mathrm{RI}$-Detektor JASCO-830 RI)

Bei den durchgeführten Trennungen konnten die folgenden Beobachtungen gemacht werden. Die Enantiomere chiraler Adamantanderivate, die ausschließlich halogensubstituiert sind oder zusätzlich über einen Phenylsubstituenten verfügen, können durch HPLC aus ihren racemischen Gemischen getrennt werden. Dabei hat sich herausgestellt, daß diejenigen Verbindungen mit Brom- und Iodsubstituenten den höchsten Trennfaktor zeigen, während die Chlor-Bromderivate den niedrigsten aufweisen. Die Racemattrennung der methoxysubstituierten Adamantane hat sich dagegen als schwierig erwiesen. Dies könnte auf einen zu großen Polaritätsunterschied der Methoxygruppe im Vergleich zu den Halogensubstituenten zurückzuführen sein, was dazu führt, daß bei der bei Ethern notwendigen Phase mit höhere Polarität, die chirale Information des untersuchten Moleküls weitestgehend verloren geht. Letztendlich ist die HPLC mit den angegebenen Säulenmaterialien aber eine effektive Möglichkeit sowohl zur analytischen als auch der präparativen Enantiomerentrennung von chiralen Adamantanverbindungen mit pseudotetraedrischer Struktur, deren Substituenten sich bezüglich Polarität nicht zu sehr unterscheiden.

\subsection{Die PT-Halogenierung von Acetalen}

Ketone gehören zu den wichtigsten Ausgangsprodukten für chemische Synthesen und deren Modifikation ist aufgrund der hohen Reaktivität der Carbonylgruppe nur schwierig zu bewerkstelligen. Eine Möglichkeit diese zu schützen besteht in deren Umwandlung in 
basenstabile Acetale, was bedeutet, daß so auch eine Kompatibilität zu den stark basischen Reaktionsbedingungen der PT-Halogenierungen erreicht werden könnte. Dies sollte anhand der Halogenierung von 2-(spiro-1',4'-dioxocyclopentyl)adamantan (79) überprüft werden, das zunächst durch Acetalisierung von Adamantanon (78) als neue Verbindung dargestellt werden konnte (Schema 29, AV16a).

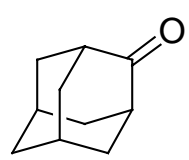

78

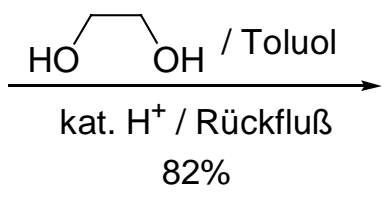

$82 \%$

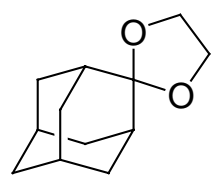

79

Schema 29. Die säurekatalysierte Ketalisierung von Adamantanon

Die PT-Chlorierung verlief unter Bildung eines komplexen Produktgemisches, was wahrscheinlich auf die lange Reaktionszeit von 1.5 Monaten zurückzuführen war. Eine GC/MS-Analyse der Reaktionsmischung hat gezeigt, daß nur noch Spuren des Ausgangsproduktes (79) vorhanden waren und als Hauptprodukte wahrscheinlich die monound dichlorierten Acetale $(\mathbf{8 0}, \mathbf{8 1})$, erkennbar an deren produktspezifischen Massenpeaks, gebildet wurden. Auf eine Separation der Produkte wurde hier verzichtet.
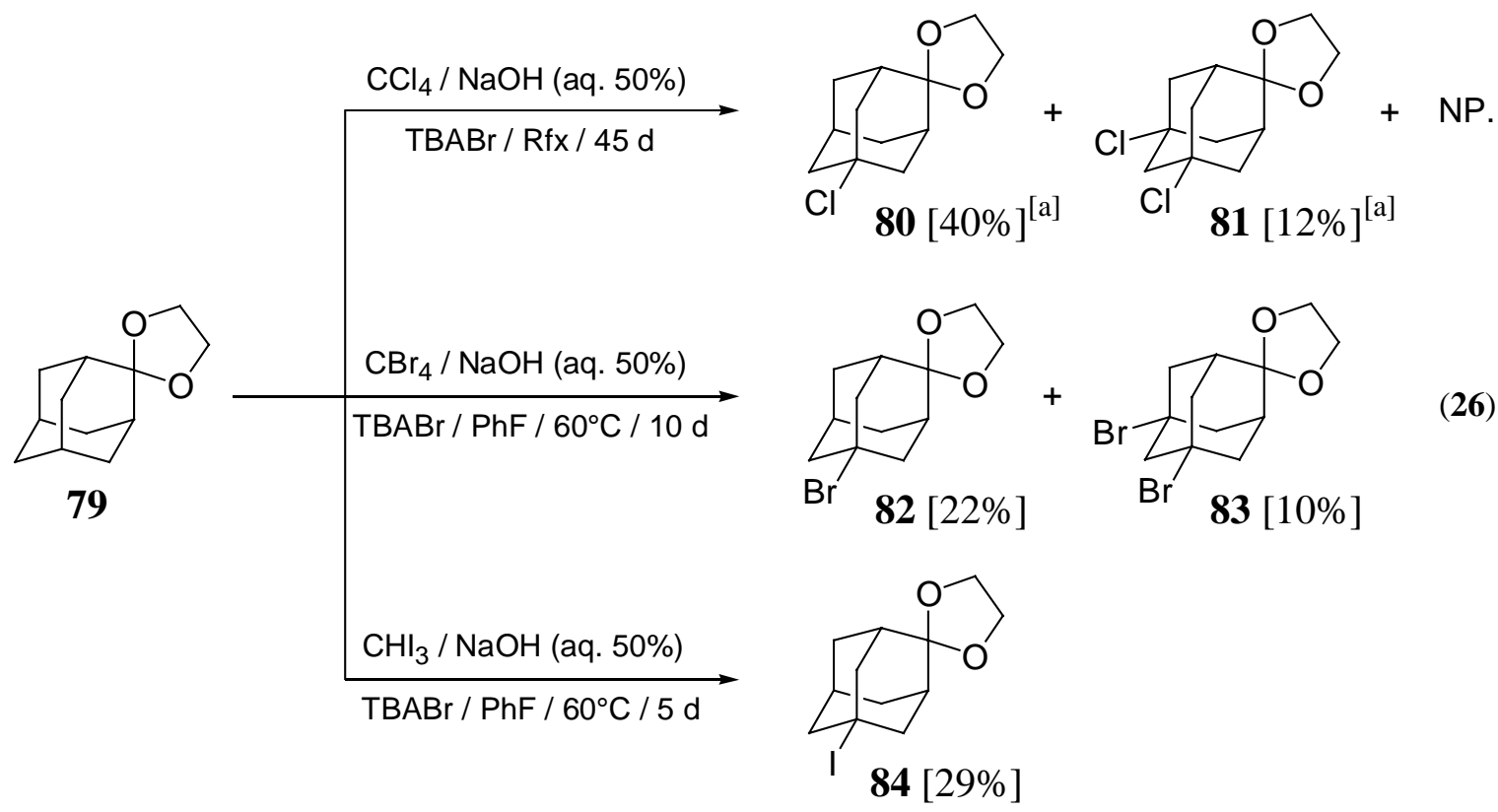

Schema 30. Die PT-Halogenierung von 2-(spiro-1',4'-dioxocyclopentyl)adamantan $(A V 16 b, c)$ [a] Die Ausbeuten wurden durch eine GC/MS-Analyse bestimmt 
Bei der Bromierung waren die Reaktionszeiten sehr viel kürzer und die Halogenierung somit sehr viel chemoselektiver, wie eine auch GC/MS-Analyse der Produktmischung zeigte. Es konnten sowohl das monobromierte 82, als auch das dibromierte Produkt $\mathbf{8 3}$ isoliert werden, wobei auffallend war, daß dabei die Substitution ausschließlich in der 5- und 7-Position stattfand. Dies ist wahrscheinlich auf den sterischen Anspruch des Trihalomethylradikals zurückzuführen, der dazu führt, daß eine Substitution in der Nähe Acetalschutzgruppe in der 1- und 3-Position vermieden wird. Problematisch war die Separation des monobromierten vom dibromierten Produkt, die die gleichen Retentionszeiten bei einer Säulenchromatographie aufwiesen und was dazu führte, daß die präparative Ausbeute sehr reduziert wurde. Deswegen wurde die Iodierung auch nach Bildung von ca. 30\% 84 (GC/MSAnalyse) abgebrochen, da zu diesem Zeitpunkt noch kein diiodiertes Produkt entstanden ist. Ansonsten wurde eine ähnliche Regioselektivität wie bei der Bromierung beobachtet.

\subsection{Die PT-Iodierung von 1-Isopropyladamantan}

Als letztes Beispiel einer PT-Halogenierung wurde noch die Iodierung von Isopropyladamantan (85) durchgeführt (Schema 31, AV17). In diesem Substratmolekül konkurrieren zwei tertiäre Positionen, die aliphatische in der Seitenkette und die am Adamantangerüst, um die Substitution durch Iod. Nach säulenchromatographischer Aufarbeitung konnten sowohl monoiodierte als auch diiodierte Produkte isoliert werden. Die sorgfältige Analyse der NMR-spektroskopischen Daten ergab, daß es sich bei den Monoiodverbindungen um ein nicht trennbares Isomerengemisch, wahrscheinlich bestehend aus 1-Iod-3-(1'-methyl)ethyladamantan 86 und 1-(1'-Iod-1'-methyl)ethyladamantan 87 im Verhältnis $3.4: 1$ (nicht statistisch korrigiert, ermittelt durch Integration separater Integrale der Methylgruppen der Isopropylgruppen der beiden Verbindungen im ${ }^{1} \mathrm{H}-\mathrm{NMR}$ Spektrum) handelt. Die gute Übereinstimmung der experimentellen mit den erwarteten Werten einer Elementaranalyse dieses Gemisches, lieferte einen weiteren Hinweis auf die Richtigkeit dieser Hypothese, während die GC/MS-Analyse auch bei vorsichtiger Durchführung nur einen Produktpeak anzeigte. Die diiodierte Verbindung $\mathbf{8 8}$ konnte rein isoliert werden. Die Charakterisierung von $\mathbf{8 6}$ konnte vollständig durchgeführt werden, während das bei der Minoritätskomponente $\mathbf{8 7}$ durch die teilweise Verdeckung derer Signale im ${ }^{1} \mathrm{H}-\mathrm{NMR}$ Spektrum durch die der Majoritätskomponente nicht möglich war. 


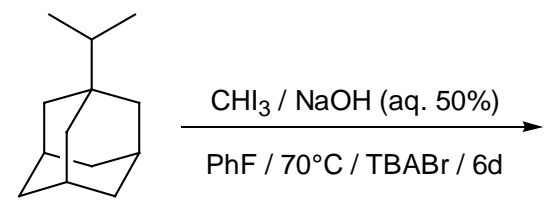

85
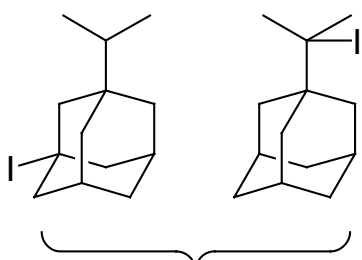

86, $87(3.4: 1)$

$40 \%$

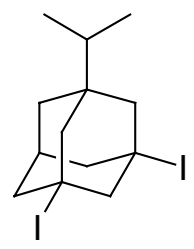

88

Schema 31. Die Produktbildung bei der PT-Iodierung von 1-Isopropyladamantan (AV17) 


\section{Ergebnisse und Ausblick}

Die mechanistischen Untersuchungen der PT-Halogenierungen bestätigen größtenteils die eingangs vorgestellte Arbeitshypothese für den Reaktionsmechanismus. So konnte durch die systematische Variation der Reaktionsbedingungen festgestellt werden, daß es sich bei dem PT-Prozeß wahrscheinlich um einen Extraktionsmechanismus handelt (Kap. 2.1). Ferner konnte durch die Untersuchung der Regioselektivität der Halogenierung (Kap. 2.2.2), durch Abfangreaktionen (Kap. 2.2.4) und durch die gute Übereinstimmung von experimentellen sowie computerberechneten KIEs (Kap. 2.2.5) auf das Trihalomethylradikal als die die Propagation einer Radikalreaktion tragende Spezies geschlossen werden. Auch für die Initiierung der Radikalreaktion durch einen SET vom Hydroxidion auf das Tetrahalomethan und dessen nachfolgende Fragmentierung wurden experimentelle Hinweise gefunden (Kap. 2.1). Allerdings bedarf es hierfür noch weiterer Untersuchungen. Insgesamt sollte auf den so gewonnen Erkenntnissen aufbauend, versucht werden, auch andere SET Reduktionsmittel unter PT-Bedingungen zur Generierung weiterer radikalischer Spezies einzusetzen. Dafür würden sich zum Beispiel andere Basen oder auch $\mathrm{SmI}_{2}$ (fest, im flüssigfest PT-System) eignen, wobei sich als Radikalprecursor z.B. verschiedene perfluorierte Iodalkane eignen würden. Deren Topologie könnte im großen Umfang variiert und so gezielte Selektivitäten bei Radikalreaktionen herbeigeführt werden.

Nachdem festgestellt wurde, daß die Bromierung mit $\mathrm{CBr}_{4}$ durch die Verwendung von Dichlormethan als Lösungsmittel auch in neutralem oder gar saurem Milieu durchführbar ist, eröffnen sich weitere Möglichkeiten der Anwendung der PT-Halogenierungen. Zunächst sollte die Rolle des Lösungsmittels weiter untersucht werden. Bislang konnte die Reaktion auf diese Weise nur in Dichlormethan oder Dibrommethan also halogensubstituierten Methanen durchgeführt werden. Ferner ist bislang nur die Bromierung unter den "neuen" Bedingungen durchführbar. Versucht man die Iodierung mit Iodoform unter neutralen Bedingungen, so findet so gut wie keine Reaktion statt (nur Spuren von Produkt, nachgewiesen durch GC/MSAnalyse), was bedeutet, daß die Äquilibrierung von Iodoform zu Tetraiodmethan wahrscheinlich nur im basischen Millieu stattfindet. Die Äquilibrierung könnte aber auch durch andere Reaktionsbedingungen initiiert werden, wie schon für Bromoform gezeigt wurde.[39, 42] In diesem Fall könnte auch durch Tetrabutylammmoniumfluorid die Äquilibrierung eingeleitet werden. Sollte dies auch im Fall von Iodoform möglich sein, so könnten schon geringe Mengen an Wasser die Reaktion initiieren. In diesem Fall hätte man sehr milde Bedingungen für die direkte Bromierung und sogar Iodierung von sowohl basen- 
als auch säurelabilen Substanzen wie z.B. Ester, bestimmte Ether, Ketone, Aminosäuren, Alkohole etc. gefunden. Eine solche Methode wäre außerordentlich nützlich für die Iodierung mit angereicherten radioaktiven Iodisotopen von Fettsäureestern, die bislang nur sehr schwer zugänglich waren. Ein weiteres einfaches Beispiel für eine Halogenierung, die bislang nicht durchführbar war, wäre die direkte Bromierung von 1-Adamantancarbonsäuremethylester (Schema 32).

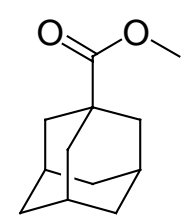

89
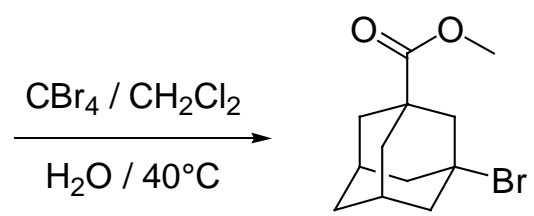

90

Schema 32. Mögliche PT-Halogenierung von 1-Adamantancarbonsäuremethylester

Die Erkenntnis, daß der Phasentransferprozeß in den meisten Lösungsmitteln stark abhängig von dem Zusatz eines PTK ist, rechtfertigt die Annahme, daß unter Umständen durch Verwendung eines enantiomerenreinen chiralen PTK eine chirale Induktion an prochiralen Substraten erreicht werden könnte. Solche Katalysatoren sind bereits bei verschiedenen PT-Reaktionen mit gutem Erfolg zum Einsatz gekommen (Kap. 1.1), wobei hohe Enantiomerenüberschüße erzielt wurden. Es darf aber hierbei nicht verschwiegen werden, daß die Auswahl der Substrate dabei von entscheidender Bedeutung war.

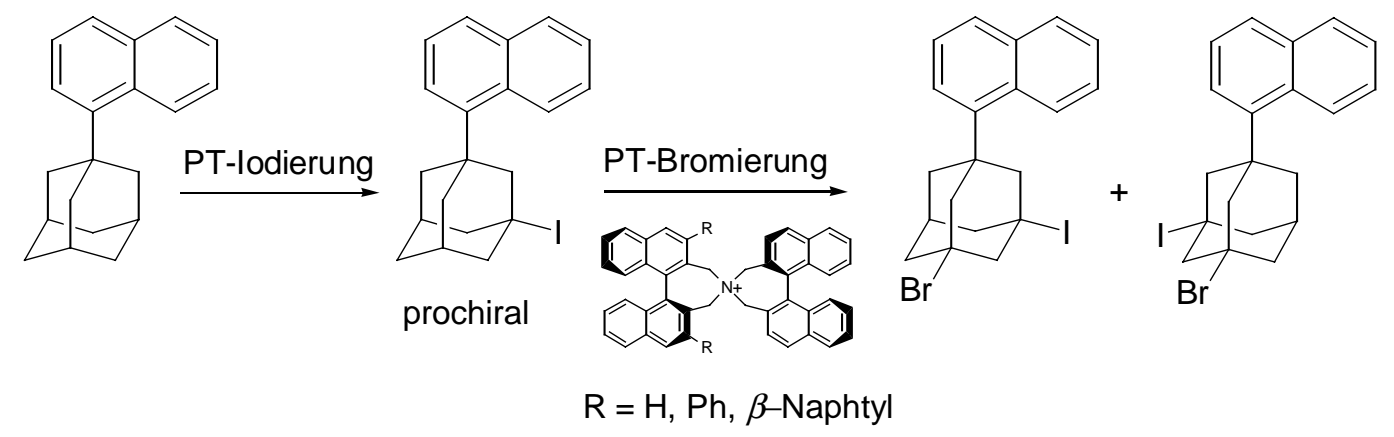

Schema 33. Beispiel einer möglichen chiralen Variante der PT-Bromierung prochiraler Substrate 
So verfügten sowohl die Katalysatoren als auch die Substrate über aromatische Substituenten, so daß es wahrscheinlich zu einer Koordination durch $\pi$-stacking Effekte kam, wodurch eine diastereotope Seite des Substratmoleküls stärker als die andere abgeschirmt wurde und deshalb die hohen ee's resultierten. Dies sollte auch bei zukünftigen PT-Halogenierungen mit chiralen Katalysatoren beachtet werden (Schema 33).

Die allgemeine Relevanz der PT-Halogenierungsmethode konnte vorerst durch zwei systematische Untersuchungen unter Beweis gestellt werden. So konnte Cuban sowohl monoals auch polyhalogeniert werden, wobei durch Ausnutzung des Umstands, daß die PTHalogenierungen ohne Austausch eines bereits im Molekül befindlichen Halogenatoms stattfindet, zum ersten Mal sämtliche Regioisomere aller Kombinationen der Dihalocubane $(\mathrm{Cl}, \mathrm{Br}, \mathrm{I})$ dargestellt wurden (Kap. 3.1). Dabei fanden die Halogenierung unter Erhaltung des Cubangerüstes statt, was im starken Gegensatz zu den Halogenierungen mit molekularem Brom und Chlor steht, wo hauptsächlich Fragmentierungsprodukte erhalten werden. Die PTHalogenierungen sind deswegen auch für die Funktionalisierung anderer hochgespannter Kohlenwasserstoffe vielversprechend und sollten daran getestet werden.

Durch die PT-Halogenierungen wurde auch der erste und zudem ein sehr einfacher Zugang zu verschiedenen chiralen Polyhaloadamantanen durch die PT-Halogenierungen geschaffen, und es konnte eine große Anzahl solcher Verbindungen synthetisiert werden (Kap. 3.2). Dieser Zugang sollte ausgenutzt werden, um durch Variation des Erstsubstituenten $\mathrm{R}$ (Schema 36) noch weitere chirale Adamantanverbindungen darzustellen. Versuche zur Analyse der racemischen Gemische durch chirale Shiftreagenzien scheiterten (Kap.3.2.3). Allerdings wurde festgestellt, daß eine Trennung der Enantiomerenpaare mittels einer chiralen GC-Analyse analytisch durchführbar war (Kap. 3.2.4) und mittels chiraler HPLC (High Pressure Liquid Chromatography) sowohl analytische als auch präparative Racemattrennungen durchgeführt werden konnten (Kap. 3.2.5). .

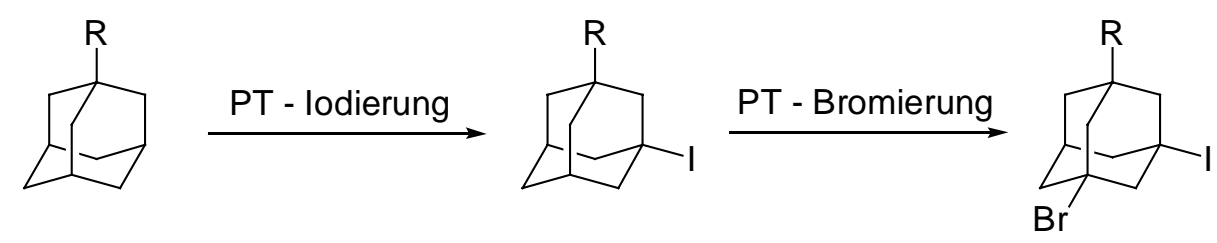

Schema 34. Ein schematischer Syntheseweg zu chiralen Polyhaloadamantane

Somit ist zum einen die Möglichkeit für eine schnelle Analyse etwaiger Enantiomerenüberschüsse im Rahmen von enantioselektiv durchgeführten PTK-Reaktionen 
geschaffen worden und zum anderen ist es nun möglich die chiroptischen Eigenschaften der chiralen Adamantane genauer zu untersuchen. So sollten systematisch deren ORD- und CDSpektren angefertigt werden und wenn möglich nach Kristallisation die absolute Konfiguration durch Röntgenstrukturanalyse bestimmt werden, da bislang über die genauen Eigenschaften dieser pseudotetraedrischen Adamantanverbindungen so gut wie nichts bekannt ist (Substituenteneinflüsse auf die Chiralität, etc.). Ferner würde sich auch ein Vergleich mit der jeweiligen Polyhalomethanverbindung mit gleichem Substitutionsmuster anbieten, um die Auswirkungen eines virtuellen Stereozentrums auf die Chiralität einer Verbindung näher zu untersuchen, die unter Umständen noch enantiomerenrein synthetisiert werden müßten. Eine solche Untersuchung könnte auch durch begleitende Computersimulationen der CD-Spektren unterstützt werden.

Chirale Adamantane können mit Hilfe der PT-Halogenierungen auch durch andere Synthesestrategien erzeugt werden. Auf diese Weise könnten durch eine sequentielle Synthesen z.B. diverse Polyarylverbindungen aus den entsprechenden tricyclischen Käfigkohlenwasserstoffen durch mehrfache Monohalogenierung und jeweils anschließende Kupplung generiert werden kann. Neben Aromaten könnten aber auch andere Kupplungskomponenten eingesetzt werden (Schema 35.).

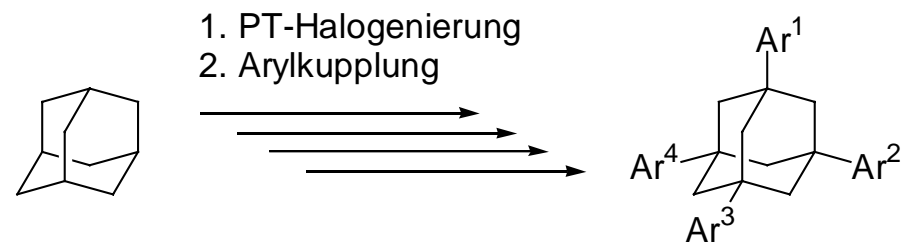

Schema 35. Mögliche sequentielle Synthese chiraler Polyaryladamantane

Alternativ könnten die unterschiedlichen Reaktivitäten der verschiedenen Halogenatome in den chiralen Polyhaloadamantanen für Kreuzkupplungen ausgenutzt werden. Werden verschiedene Kupplungskomponenten gleichzeitig eingesetzt, könnten auf diese Weise ganze Substanzbibliotheken für kombinatorische Untersuchungen z.B. der pharmakologischen Wirksamkeit von Adamantanverbindungen angelegt werden.

Weiterhin bedarf noch eine Beobachtung systematischer Untersuchungen, nämlich die unterschiedliche Reaktivität verschiedener monosubstituierter 1-Adamantylverbindungen bezüglich der PT-Halogenierungen. So benötigen monohalogenierte Adamantane $(\mathrm{F}, \mathrm{Cl}, \mathrm{Br}$, 
I) erheblich längere Reaktionszeiten als die methoxy- oder phenylsubstituierten. Um diesen Sachverhalt genauer zu untersuchen, sollten direkte Konkurrenzexperimente durchgefuihrt werden, um das genaue Ausmaß der Substituenteneffekte zu ermitteln. So können StrukturReaktivitätsbeziehungen aufgestellt werden, die durch begleitende Computerberechnungen erklärt werden könnten.

Schließlich sollte noch ein weiterer Aspekt der PT-Bedingungen im Rahmen der Halogenierungen ausgenutzt werden, nämlich das stark basische Reaktionsmilieu. Durch diese stark basischen Bedingungen und aufgrund der Tatsache das Halogenalkane dabei generiert werden, könnten diese im Rahmen einer Art Tandemreaktion durch Zusatz einer C-H-aziden Verbindung, die in situ deprotoniert wird, C-alkyliert werden (Schema 36), was formal einer direkten Alkylierung nicht aktivierter Kohlenwasserstoffe entsprechen würde, die dann unter Umständen sogar enantioselektiv mit chiralen PTK erfolgen könnte.

$$
\mathrm{R}^{1}-\mathrm{H} \stackrel{\text { PT-Halogenierung }}{\longrightarrow} \mathrm{R}^{1}-\mathrm{X} \stackrel{+\mathrm{R}^{2}-\mathrm{H}_{\text {azid }}}{\longrightarrow} \mathrm{R}^{1}-\mathrm{R}^{2}
$$

Schema 36. Mögliche Tandemreaktion unter Ausnutzung der basischen PT-Reaktionsbedingungen zur direkten Alkylierung nicht aktivierter Kohlenwasserstoffe

Abschließend kann festgehalten werden, daß mit den PT-Halogenierungen nicht nur eine mechanistisch und theoretisch ansprechende sondern auch präparativ äußerst brauchbare neue Synthesemethode entwickelt wurde, die vorhandene Methoden ergänzt aber auch ganz neue Synthesewege eröffnet. Das Konzept zu optimieren und zu erweitern, sowie es auf weitere Problemstellungen der organischen Chemie anzuwenden, wird Gegenstand weiterführender Arbeiten sein. 


\section{Experimenteller Teil}

${ }^{1}$ H-NMR-Spektren wurden auf dem Gerät AM $250(250 \mathrm{MHz})$ der Firma Bruker aufgenommen. Chemische Verschiebungen sind als $\delta$-Werte in ppm angegeben und beziehen sich auf $\delta=7.26$ für Chloroform. Charakterisierung der Signalaufspaltung: $\mathrm{s}=$ Singulett, br. $\mathrm{s}$ $=$ breites Singulett, $\mathrm{d}=$ Dublett, $\mathrm{t}=$ Triplett, $\mathrm{q}=$ Quartett, quint $=$ Quintett, $\mathrm{h}=$ Heptet, $\mathrm{m}=$ Multiplett. Spektren wurden nach erster Ordnung ausgewertet, und die Kopplungskonstanten sind in Hertz angegeben.

${ }^{13}$ C-NMR-Spektren wurden auf dem Gerät AM $250(62.9 \mathrm{MHz})$ der Firma Bruker aufgenommen. Chemische Verschiebungen sind als $\delta$-Werte in ppm angegeben und beziehen sich auf $\delta=77.0$ für Chloroform. Charakterisierung der Signale: $\mathrm{p}=$ primär, $\mathrm{s}=$ sekundär, $\mathrm{t}=$ tertiär, $\mathrm{q}=$ quartär

Massenspektren wurden mit einem Spektrometer Modell MAT 311 der Firma Varian und einem Modell MAT 95 der Firma Finnigan aufgenommen.

Gaschromatographische Arbeiten und Analysen wurden an einem Modell IGC 121 MLR der Firma Delsi Instruments mit Wärmeleitfähigkeitsdetektor durchgeführt. Als Trägergas diente Wasserstoff. Der Wasserstoffstrom betrug jeweils 40 - $80 \mathrm{~mL} / \mathrm{min}$. Angaben über Art und Abmessungen der Säulen, Trägermaterial, Art der stationären Phasen, Belegung in Prozent und Säulentemperatur finden sich in den jeweiligen Versuchsbeschreibungen. Die Integrationen wurden mit einem Gerät D2000 der Firma Merck-Hitachi durchgeführt. Responsefaktoren wurden nicht bestimmt. Weiterhin kam ein analytisches GC der Firma Hewlett Packert HP5890 II ausgestattet mit Massendetektor HP5971A und der Säule HP Ultra 1 Säule (50 m x $0.2 \mathrm{~mm}$ x $0.33 \mathrm{~mm}$ Film) zum Einsatz. Responsefaktoren für bestimmte Substanzen werden an den entsprechenden Stellen angegeben.

Säulenchromatographische Trennungen wurden an Merck Kieselgel 60 (0.0630.200 mm 230400 mesh) durchgeführt. Laufmittel wurden nur destilliert verwendet Angaben über Säulenabmessungen finden sich in den jeweiligen Versuchsbeschreibungen.

Dünschichtchromatographie (DC): Macherey Nagel Fertigfolien: Alugram ${ }^{\odot}$ SIL G/UV ${ }_{254}$; Merck DC Fertigfolien: Kieselgel $60 \mathrm{~F}_{254}$ auf Aluminiumfolie. Detektion unter UV-Licht bei 
254 nm oder durch Entwicklung mit Molybdatophosphorsäure Tauchreagenz (10 proz. Lsg. in Ethanol) und anschließendem Erwärmen.

Schmelzpunkte wurden mit einer Schmelzpunktapparatur nach Dr. Tottoli der Firma Büchi ermittelt; die gemessenen Schmelzpunkte sind unkorrigiert und wurden in verschlossenen Kapillaren bestimmt.

IR-Spektren wurden mit dem Spektrometer IFS 66 (FTIR) der Firma Perkin-Elmer 298 aufgenommen.

\section{AV1. Experimentelle Durchführung der systematischen Untersuchung der allgemeinen Reaktionsbedingungen der PT-Bromierung}

Alle Experimente wurden zur besseren Reproduzierbarkeit und um deren Vergleich untereinander zu ermöglichen, unter möglichst identischen Bedingungen durchgeführt. Das war deshalb notwendig, weil eine PT-Reaktion neben den üblichen Faktoren sehr stark von dem Ausmaß der Durchmischung abhängt, die wiederum durch die Rührgeschwindigkeit (1000 U/min), die Größe des Magnetrührstäbchens (0.5 x $2 \mathrm{~cm})$ und die Kolbengröße $(25 \mathrm{~mL}$ Rundkolben) beeinflußt wird. Die Umgebungstemperatur wurde konstant bei $22^{\circ} \mathrm{C}$ und die Ölbadtemperatur bei $40^{\circ} \mathrm{C}$ gehalten. Obwohl durch vergleichende Versuche festgestellt wurde, daß die Reaktionen dadurch nicht beeinflußt werden, wurden die Reaktionen unter weitestgehender Vermeidung von Lichteinstrahlung durchgeführt.

In einem $25 \mathrm{~mL}$ Rundkolben wurden $0.681 \mathrm{~g} 1$ (0.005 mol), $1.658 \mathrm{~g} \mathrm{CBr}_{4}(0.005 \mathrm{~mol})$ und die entsprechende Menge eines PTK in $15.0 \mathrm{~mL}$ Solvens gelöst und $5 \mathrm{~min}$ auf dem Ölbad unter Rühren auf die Reaktionstemperaratur erwärmt. Anschließend wurde die Reaktion durch Zugabe von $7.0 \mathrm{~mL}$ einer wäßrigen Phase (RT) initiiert. Alle weiteren Angaben finden sich in den folgenden speziellen Vorschriften. Zur Messung des Umsatzes wurden $10 \mu \mathrm{L}$ der Reaktionsmischung entnommen, auf $1 \mathrm{~mL}$ verdünnt und die so erhaltene Lösung einer GC/MS-Analyse unterzogen. Die angegebenen Umsätze beziehen sich auf die Summe der Integrale von $\mathbf{2}$ und $\mathbf{5}$ in Relation zu 1, wobei keine Responsefaktoren bestimmt wurden. 
AV1a. Variation des Lösungsmittels mit und ohne den Einfluß eines PTK: Gemäß AV1 wurden die Reaktionen mit Fluorbenzol, Dichlormethan, Benzol und 1',1',1'-Trifluortoluol mit und ohne Zusatz von $161 \mathrm{mg}$ TBABr (0.0005 mol, $10 \mathrm{~mol} \%)$ durchgeführt.

Tabelle 13. Umsätze bei Bestimmung der Lösungsmittelabhängigkeit mit und ohne PTK

\begin{tabular}{ccccccccc} 
Zeit $[\mathbf{h}]$ & TFT & TFT+PTK & Benzol & Benzol+PTK & $\mathbf{C H}_{\mathbf{2}} \mathbf{C l}_{\mathbf{2}}$ & $\mathbf{C H}_{2} \mathbf{C l}_{\mathbf{2}}+\mathbf{P T K}$ & $\mathbf{P h F}$ & $\mathbf{P h F + P T K}$ \\
\hline $\mathbf{0 . 5}$ & & & & & 1.65 & 5.49 & & \\
$\mathbf{1 . 0}$ & 0.31 & 3.2 & & & & 11.02 & & \\
$\mathbf{1 . 5}$ & & & & 0.31 & 16.25 & & 0.21 & 1.15 \\
$\mathbf{2 . 0}$ & 0.62 & 6.17 & & & & 22 & & \\
$\mathbf{3 . 0}$ & & 8.41 & 0.01 & 1.02 & 31.01 & 32.98 & 0.32 & 2 \\
$\mathbf{3 . 5}$ & & & & & 36.84 & 39.51 & & 2.4 \\
$\mathbf{4 . 0}$ & & & & & & & & 2.72 \\
$\mathbf{5 . 0}$ & 1.54 & 10.41 & 0.02 & 2.01 & & & 0.7 & 3.38 \\
$\mathbf{7 . 0}$ & 1.92 & 12.24 & 0.03 & 2.63 & & & 1.01 & 4.71 \\
$\mathbf{8 . 0}$ & & & & & & & & 5.28 \\
$\mathbf{1 5 . 0}$ & 2.48 & 16.54 & 0.04 & 5.02 & & & 1.72 & 10.02 \\
$\mathbf{2 4 . 0}$ & 3.82 & 20.50 & 0.07 & 6.84 & & & 2.41 & 15.43
\end{tabular}

AV1b. Variation des PTK: Gemäß AV1 wurden die Reaktionen mit Fluorbenzol und unter Zusatz von 10 mol\% (0.0005 mol) verschiedener PTK (105 mg TEBABr, $161 \mathrm{mg}$ TBABr, $273 \mathrm{mg}$ TOctABr, $136 \mathrm{mg}$ TEBACl) durchgeführt.

Tabelle 14. Umsätze bei Variation des PTK

\begin{tabular}{ccccc} 
Zeit $[\mathbf{h}]$ & TEBACl & TOctABr & TBABr & TEtABr \\
\hline $\mathbf{1 . 0}$ & 0.54 & 1.05 & & \\
$\mathbf{1 . 5}$ & & & 1.15 & \\
$\mathbf{3 . 0}$ & 1.63 & 2.82 & 2.00 & 0.07 \\
$\mathbf{3 . 5}$ & & & 2.40 & \\
$\mathbf{4 . 0}$ & 1.74 & & 2.72 & \\
$\mathbf{4 . 5}$ & & & & 0.20 \\
$\mathbf{5 . 0}$ & & 4.12 & 3.38 & \\
$\mathbf{7 . 0}$ & 2.96 & & & \\
$\mathbf{8 . 0}$ & & 5.21 & 5.28 & 0.43 \\
$\mathbf{1 5 . 0}$ & 6.21 & 7.15 & 10.02 & 1.11 \\
$\mathbf{1 8 . 0}$ & & 7.94 & & \\
$\mathbf{2 4 . 0}$ & 9.90 & 9.05 & 15.43 & 2.10
\end{tabular}


AV1c. Variation des Basenkonzentration: Gemäß AV1 wurden die Reaktionen mit Fluorbenzol und unter Zusatz von $161 \mathrm{mg}$ TBABr (0.0005 mol, $10 \mathrm{~mol} \%)$ mit verschiedenen Basenkonzentrationen durchgeführt (aq. NaOH: $0 \%, 10 \%, 25 \%, 50 \%$ ).

Tabelle 15. Umsätze bei Variation der Basenkonzentration

\begin{tabular}{ccccc} 
Zeit $[\mathbf{h}]$ & $\mathbf{5 0} \%$ aq. $\mathbf{~ N a O H}$ & $\mathbf{2 5} \%$ aq. $\mathbf{~ N a O H}$ & $\mathbf{1 0} \%$ aq. $\mathbf{~ N a O H}$ & $\mathbf{0} \%$ aq. $\mathbf{~ N a O H}$ \\
\hline $\mathbf{1 . 5}$ & 1.15 & 0.62 & 0.30 & \\
$\mathbf{3 . 0}$ & 2.00 & 1.10 & 0.50 & 0.10 \\
$\mathbf{3 . 5}$ & 2.40 & & & \\
$\mathbf{4 . 0}$ & 2.72 & 1.44 & 0.70 & 0.17 \\
$\mathbf{5 . 0}$ & 3.38 & & 0.90 & \\
$\mathbf{6 . 0}$ & & 2.12 & & 0.30 \\
$\mathbf{7 . 0}$ & 4.71 & & & \\
$\mathbf{8 . 0}$ & 5.28 & 2.88 & 1.40 & \\
$\mathbf{1 0 . 0}$ & & & & 0.43 \\
$\mathbf{1 2 . 0}$ & & 3.95 & 1.90 & \\
$\mathbf{1 5 . 0}$ & 10.02 & 4.83 & 2.40 & 0.60 \\
$\mathbf{2 4 . 0}$ & 15.43 & 7.94 & 3.90 & 0.80
\end{tabular}

AV1d. Variation des Katalysatorkonzentration: Gemäß AV1 wurden die Reaktionen mit Fluorbenzol und $7 \mathrm{~mL} \mathrm{NaOH}$ (aq. 50\%) unter Zusatz verschiedener Mengen an $\mathrm{TBABr}$ durchgeführt (0 mol\%, $1 \mathrm{~mol} \%$ (16 mg), $5 \mathrm{~mol} \%$ (81 mg), $10 \mathrm{~mol} \%$ (161 mg), $20 \mathrm{~mol} \%$ (322 $\mathrm{mg}$ )

Tabelle 16. Umsätze bei Variation der Katalysatorkonzentration

\begin{tabular}{cccccc} 
Zeit [h] & $\mathbf{2 0} \mathbf{~ m o l} \%$ & $\mathbf{1 0} \mathbf{~ m o l ~ \% ~}$ & $\mathbf{5} \mathbf{~ m o l ~ \%}$ & $\mathbf{1} \mathbf{~ m o l ~ \%}$ & $\mathbf{0} \mathbf{~ m o l ~ \% ~}$ \\
\hline $\mathbf{1 . 5}$ & 1.20 & 1.15 & 0.80 & 0.64 & 0.21 \\
$\mathbf{3 . 0}$ & 2.73 & 2.00 & 1.71 & 1.25 & 0.38 \\
$\mathbf{3 . 5}$ & & 2.40 & & & \\
$\mathbf{4 . 0}$ & 3.54 & 2.72 & 2.04 & 1.52 & 0.52 \\
$\mathbf{5 . 0}$ & 4.43 & 3.38 & 2.54 & 1.84 & 0.70 \\
$\mathbf{7 . 0}$ & 6.01 & 4.71 & 3.21 & 2.47 & 1.01 \\
$\mathbf{8 . 0}$ & & 5.28 & & & \\
$\mathbf{1 5 . 0}$ & 13.11 & 10.02 & 6.12 & 3.74 & 1.72 \\
$\mathbf{2 0 . 0}$ & 17.24 & & & & \\
$\mathbf{2 4 . 0}$ & & 15.43 & 9.02 & 4.80 & 2.41
\end{tabular}


AV1e. Variation verschiedener Reaktionsbedingungen: Gemäß AV1 wurden die Reaktionsverläufe mit den in Tab. 3 angegebenen speziellen Reaktionsbedingungen durchgeführt und analysiert.

\section{AV2. Experimentelle Durchführung der systematischen Untersuchung der allgemeinen Reaktionsbedingungen der PT-Iodierung}

Alle Experimente wurden aus denselben Gründen wie die Bromierungen unter möglichst identischen Bedingungen durchgeführt. Wiederum wurden die Rührgeschwindigkeit (1000 U/min), die Größe des Magnetrührstäbchens $(0.5$ x $2 \mathrm{~cm})$ und die Reaktionsgefäßgröße (25 mL Glasfläschchen (Schott) mit Schraubverschluß) konstant gehalten. Alle Reaktionen wurden bei Raumtemperatur in einem Wasserbad unter weitestgehender Vermeidung von Lichteinstrahlung durchgeführt.

In einem verschließbaren $25 \mathrm{~mL}$ Glasfläschchen wurden $1.362 \mathrm{~g} 1(0.010 \mathrm{~mol}), 3.937$ g CHI 3 (0.010 mol) und die entsprechende Menge eines PTK in $15.0 \mathrm{~mL}$ Solvens gelöst. Anschließend wurde die Reaktion durch Zugabe von $5.0 \mathrm{~mL}$ der wäßrigen Phase initiiert. Alle weiteren Angaben finden sich in den folgenden speziellen Vorschriften. Zur Messung des Umsatzes wurden $0.05 \mathrm{~mL}$ der Reaktionsmischung entnommen, das Lösungsmittel am Rotationsverdampfer unter reduziertem Druck entfernt und von dem Rückstand ein ${ }^{1} \mathrm{H}$ NMR Spektrum angefertigt. Die Analyse erfolgte durch Addition und statistische Korrektur der Integrale von separaten Signalen von $17\left(\delta=2.62\left(\mathrm{~d},{ }^{3} \mathrm{~J}=3.0 \mathrm{~Hz}, 6 \mathrm{H}, \alpha-\mathrm{CH}_{2}\right)\right)$ in Relation zu $\mathbf{1}(\delta=1.90(\mathrm{bs}, 4 \mathrm{H}, 4 \times \mathrm{CH})$.

AV2a. Variation des Lösungsmittels mit und ohne den Einfluß eines PTK: Gemäß AV2 wurde der jeweilige Reaktionsverlauf mit 1',1',1'-Trifluortoluol, Chlorbenzol, Methyl-tButylether (MBE), Dichlormethan und Fluorbenzol unter Zusatz von 322 mg TBABr $(0.0010$ mol, $10 \mathrm{~mol} \%$ ) und $\mathrm{NaOH}$ Lsg. (aq. 50\%) als wäßrige Phase, sowie in den letzten beiden Fällen auch zusätzlich ohne Zusatz eines PTK, bestimmt. 
Tabelle 17. Umsätze bei Bestimmung der Lösungsmittelabhängigkeit mit und ohne PTK

\begin{tabular}{lccc} 
Solvens & $\mathbf{2 4} \mathbf{~ h}$ / Umsatz [\%] & $\mathbf{6 4} \mathbf{~ h}$ / Umsatz [\%] & $\mathbf{6 4} \mathbf{h}$ ohne PTK/ Umsatz [\%] \\
\hline $\mathbf{C H}_{2} \mathbf{C l}_{\mathbf{2}}$ & 26 & 42 & 20 \\
Fluorbenzol & 26 & 53 & 0 \\
Chlorbenzol & 24 & 52 & - \\
Trifluortoluol & 23 & 46 & - \\
MBE & 17 & 22 & -
\end{tabular}

AV2b. Variation des PTK: Gemäß AV2 wurden die Reaktionsverläufe mit Fluorbenzol und unter Zusatz von 5 mol\% (0.0005 mol) verschiedener PTK (105 mg TEtABr, 161 mg TBABr, 273 mg TOctABr, 136 mg TEBACl) und $\mathrm{NaOH} \mathrm{Lsg.} \mathrm{(aq.} \mathrm{50 \% )} \mathrm{als} \mathrm{wäßrige} \mathrm{Phase} \mathrm{bestimmt.}$

Tabelle 18. Umsätze bei Variation des PTK

\begin{tabular}{lccc} 
PTK & $\mathbf{1 6}$ h / Umsatz [\%] & $\mathbf{4 0 ~ h ~ / ~ U m s a t z ~ [ \% ] ~}$ & $\mathbf{6 4}$ h / Umsatz [\%] \\
\hline TEBACl & 10 & 20 & 28 \\
TEtABr & 2 & 6 & 9 \\
TBABr & 25 & 45 & 55 \\
TOctABr & 24 & 32 & 40
\end{tabular}

AV2c. Variation der Basenkonzentration: Gemäß AV2 wurden die Reaktionen mit Fluorbenzol und unter Zusatz von $96.7 \mathrm{mg}$ TBABr $(0.15 \mathrm{mmol}, 3 \mathrm{~mol} \%)$ mit verschiedenen Basenkonzentrationen in der wäßrigen Phase durchgeführt (aq. $\mathrm{NaOH}: 0 \%, 2 \%, 7 \%, 14 \%$, $20 \%, 50 \%)$

Tabelle 19. Umsätze bei Variation der Basenkonzentration

\begin{tabular}{ccccc} 
Konz. [\%] & $\mathbf{2 0}$ h / [\%] & $\mathbf{4 8} \mathbf{h} /$ [\%] & $\mathbf{1 4 4} \mathbf{h} /[\%]$ & $\mathbf{1 9 2 h} /[\%]$ \\
\hline $\mathbf{2 5 . 0}$ & 26 & 42 & 53 & 58 \\
$\mathbf{1 0 . 0}$ & 26 & 36 & 51 & 55 \\
$\mathbf{7 . 0}$ & 16 & 20 & 23 & 25 \\
$\mathbf{3 . 5}$ & 11 & 16 & 19 & 24 \\
$\mathbf{1 . 0}$ & 7 & 12 & 17 & 19 \\
$\mathbf{0 . 0}$ & 0 & 0 & 0 & 0
\end{tabular}

AV2d. Variation der Katalysatorkonzentration: Gemäß AV2 wurden die Reaktionen in Fluorbenzol und unter Zusatz von verschiedenen Konzentrationen von TBABr (3.2 mg (0.1 mol\%), $16.1 \mathrm{mg}(0.5 \mathrm{~mol} \%), 32.2 \mathrm{mg}$ (1.0 mol\%), $96.7 \mathrm{mg}$ (3.0 mol\%), $161.1 \mathrm{mg}$ (5 mol\%), 
$226 \mathrm{mg}$ (7 mol\%), $322 \mathrm{mg}$ (10 mol\%), $644 \mathrm{mg}$ (20 mol\%), $967 \mathrm{mg}$ (30 mol\%), $1289 \mathrm{mg}$ (40 mol\%) und $\mathrm{NaOH}$ Lsg. (aq. 50\%) als wäßriger Phase durchgeführt.

Tabelle 20. Umsätze bei Variation der PTK Konzentration

\begin{tabular}{ccc} 
TBABr [mol\%] & 17h / Umsatz [\%] & 41h/ Umsatz [\%] \\
\hline $\mathbf{0 . 0}$ & 1 & \\
$\mathbf{0 . 1}$ & 22 & 30 \\
$\mathbf{0 . 5}$ & 28 & 36 \\
$\mathbf{1 . 0}$ & 34 & 43 \\
$\mathbf{3 . 0}$ & 39 & 52 \\
$\mathbf{5 . 0}$ & 35 & 46 \\
$\mathbf{7 . 0}$ & 37 & 50 \\
$\mathbf{1 0}$ & 40 & 54 \\
$\mathbf{2 0}$ & 34 & 55 \\
$\mathbf{3 0}$ & 29 & 43 \\
$\mathbf{4 0}$ & 21 & 30
\end{tabular}

AV2e. Variation der Base: Gemäß AV2 wurden die Reaktionen in Fluorbenzol und unter Zusatz von verschiedenen Basen (aq. 3.5 M, LiOH, NaOH, KOH, CsOH) und $96.7 \mathrm{mg}$ TBABr (3.0 mol\%) durchgeführt.

Tabelle 21. Umsätze bei Variation der Base

\begin{tabular}{cccc} 
Base [3.5 M] & $\mathbf{1 8 ~ h}$ / Umsatz [\%] & $\mathbf{9 0}$ h / Umsatz [\%] & $\mathbf{1 6 2}$ h / Ausbeute [\%] \\
\hline LiOH & 8 & 13 & 15 \\
NaOH & 11 & 18 & 22 \\
KOH & 13 & 23 & 26 \\
CsOH & 15 & 26 & 32
\end{tabular}

AV2f. Variation der des Volumens der wäßrigen Phase bei konstanter absoluter Menge an Base: Die Reaktionen wurden gemäß AV2 in Fluorbenzol und unter Zusatz von 96.7 mg $\mathrm{TBABr}(3.0 \mathrm{~mol} \%)$ durchgeführt. Weiterhin wurden die Konzentration der Base und das eingesetzte Volumen wie folgt variiert: $2 \mathrm{~mL}$ einer eingestellten wäßrigen $\mathrm{NaOH}-\mathrm{Lsg}$. (10 M) wurde jeweils unverdünnt (Nr. 1), bzw. verdünnt mit 2 (Nr. 2), 4 (Nr. 3), 6 (Nr. 4) mL Wasser eingesetzt. 
Tabelle 22. Variation von Volumen und Konzentration

\begin{tabular}{cccc}
$\#$ & $\mathbf{1 8 h} /[\%]$ & $\mathbf{9 0 h} /[\%]$ & $\mathbf{1 4 0 h} /[\%]$ \\
\hline $\mathbf{1}$ & 7 & 18 & 33 \\
$\mathbf{2}$ & - & 12 & 23 \\
$\mathbf{3}$ & 8 & 12 & 22 \\
$\mathbf{4}$ & 9 & 12 & 22
\end{tabular}

AV2g. Variation der absoluten Basenmenge: Die Reaktionen wurden gemäß AV2 in Fluorbenzol unter Zusatz von 96.7 mg TBABr (3.0 mol\%) mit $\mathrm{NaOH}$ (aq. 50\%) durchgeführt. Dabei wurden jeweils $1 \mathrm{~mL}, 3 \mathrm{~mL}, 5 \mathrm{~mL}$ und $10 \mathrm{~mL}$ der Base verwendet

Tabelle 23. Umsätze bei Variation der absoluten Basenmenge

\begin{tabular}{ccccc} 
Base $[\mathbf{m l}]$ & $\mathbf{2 0 h} /[\boldsymbol{~ \%}]$ & $\mathbf{4 8 h} /[\boldsymbol{~}]$ & $\mathbf{1 4 4 h} /[\%]$ & $\mathbf{1 9 2 h} /[\%]$ \\
\hline $\mathbf{1 0}$ & 37 & 52 & 58 & 65 \\
$\mathbf{5}$ & 26 & 42 & 53 & 58 \\
$\mathbf{3}$ & 22 & 37 & 48 & 55 \\
$\mathbf{1}$ & 9 & 20 & 27 & 34
\end{tabular}

\section{AV3. Die PT-Chlorierung von Adamantan}

1-Chlortricyclo[3.3.1.1 $\left.1^{3,7}\right]$ decan (26), 1,3-Dichlortricyclo[3.3.1.1 $\left.{ }^{3,7}\right]$ decan (28):

$1.36 \mathrm{~g}$ (0.01 mol) Adamantan 1 und $161 \mathrm{mg}$ (0.005 mmol, 5 mol\%) TBABr wurden in $30 \mathrm{~mL}$ Tetrachlormethan gelöst und $20 \mathrm{~mL}$ Natriumhydroxidlösung (aq. 50\%) zugesetzt. Die Mischung wurde 10 Tage bei intensivem Rühren refluxiert. Nach der Phasentrennung wurde die wäßrige Phase mit $n$-Pentan $(4 \times 30 \mathrm{~mL})$ extrahiert. Die vereinigten organischen Phasen wurden über Natriumsulfat getrocknet und am Rotationsverdampfer unter reduziertem Druck weitestgehend von Lösungsmitteln befreit. Die erhaltenen $2.0 \mathrm{~g}$ Rohprodukt wurden einer säulenchromatographischen Trennung unterzogen ( $n$-Pentan, 1.5 x $40 \mathrm{~cm}, \mathrm{R}_{\mathrm{f}}(\mathbf{2 6})=0.51$, $\left.\mathrm{R}_{\mathrm{f}}(\mathbf{2 8})=0.37\right)$. Es wurden $0.73 \mathrm{~g}(4.3 \mathrm{mmol}, 43 \%)$ von 26 und $0.14 \mathrm{~g}(0.7 \mathrm{mmol}, 7 \%)$ von 28 als weiße Feststoffe erhalten. $0.20 \mathrm{~g}$ von 1 konnten zurückgewonnen werden. Die spektroskopischen Daten sind literaturidentisch. ${ }^{[199]}$ 


\section{AV4. Vorschrift zur Halogenierung von 1 unter PTK Bedingungen oder radikalisch initiiert durch AIBN (Cl, Br, I)}

In einem $10 \mathrm{~mL}$ Rundkolben wurden $0.341 \mathrm{~g} 1$ (2.5 mmol), das entsprechende Halomethan $(2.5 \mathrm{mmol})$ und im Fall der durch Phasentransfer initiierten Reaktion $81 \mathrm{mg}$ TBABr (10 mol\%) oder im Fall der radikalisch initiierten Reaktion 205 mg AIBN (1.25 mmol, 0.5 äq.) in $5 \mathrm{~mL}$ Solvens gelöst. Die durch PT-initiierten Reaktionen werden durch Zugabe von $2 \mathrm{~mL} \mathrm{NaOH} \mathrm{(aq.} \mathrm{50 \% )} \mathrm{initiiert.} \mathrm{Die} \mathrm{genauen} \mathrm{Angaben} \mathrm{bezüglich}$ Reaktionstemperatur, Reaktionszeit und Lösungsmittel können Tab. 4 entnommen werden. Die Bestimmung des Produktverhältnisses erfolgte jeweils durch eine GC/MS-Analyse der Reaktionsmischungen. Dazu wurden $10 \mu \mathrm{L}$ der Reaktionsmischung entnommen, auf $1 \mathrm{~mL}$ verdünnt und die so erhaltenen Lösung der GC/MS-Analyse unterzogen. Responsefaktoren der $2^{\circ}$ - bezüglich der $3^{\circ}$-substituierten Produkte wurde nicht bestimmt.

\section{AV5. Nachweis von elementarem Brom im Rahmen einer nur durch Wasser initiierten Bromierung von 1}

In einem $25 \mathrm{~mL}$ Rundkolben wurden $0.681 \mathrm{~g} 1$ (0.005 mol) und $1.658 \mathrm{~g} \mathrm{CBr}_{4}(0.005 \mathrm{~mol})$ in $10 \mathrm{~mL} \mathrm{CH}_{2} \mathrm{Cl}_{2}$ gelöst und $5 \mathrm{~mL}$ Wasser hinzugegeben. Nach $20 \mathrm{~h}$ wurden zur Analyse der Reaktionsmischung, die mittlerweile eine gelblich braune Färbung angenommen hatte, $10 \mu \mathrm{L}$ der organischen Phase entnommen, auf $1 \mathrm{~mL}$ verdünnt und die so erhaltenen Lösung einer GC/MS-Analyse unterzogen. Anschließend wurde ein weiterer $\mathrm{mL}$ der organischen Phase entnommen, bis zu deren völligen Entfärbung eine Lösung von 29 in $\mathrm{CH}_{2} \mathrm{Cl}_{2}$ (ca. $5 \mathrm{M}$ ) hinzugegeben und eine weitere GC/MS-Analyse durchgeführt. Als Vergleichsexperiment wurden zu $1 \mathrm{~mL}$ der Lösung von 29 in $\mathrm{CH}_{2} \mathrm{Cl}_{2}$ soviel Brom hinzugegeben, bis die Mischung eine leicht gelbliche Färbung annahm. Eine GC/MS-Analyse und Vergleich mit der des Ausgangsproduktes 29 ergab, daß sich zu $97 \%$ ein Hauptprodukt mit exakt dem gleichen Massenspektrum und der gleichen Retentionszeit, wie dem des neuen Peaks in Reaktionsmischung der Bromierung und 30 entspricht gebildet hat. MS (70 eV); m/z (\%): 227 (100) 229 (98) $\left[\mathrm{M}^{+}-\mathrm{Br}\right], 215$ (7) 213 (7) $\left[\mathrm{M}^{+}-\mathrm{CH}_{2} \mathrm{Br}\right], 147$ (32) $\left[\mathrm{M}^{+}-\mathrm{Br}, \mathrm{CH}_{2} \mathrm{Br}\right], 119$ (13), 105 (26), 91 (61), 77 (17), 65 (9), 53 (8) 


\section{AV6. Experiment zum Abfangen des Tribrommethylradikals}

3-(1'-Brommethyl)-7-(2"-,2"-,2"-tribromethyl)tricyclo[3.3.1.0. $\left.{ }^{3,7}\right]$ nonan (31)

Es wurden $200 \mathrm{mg}(1.35 \mathrm{mmol}) 29$ und $1.20 \mathrm{~g} \mathrm{CBr}_{4}(3.6 \mathrm{mmol})$ in $10 \mathrm{~mL} \mathrm{CH}_{2} \mathrm{Cl}_{2}$ gelöst sowie $4 \mathrm{~g} \mathrm{NaOH}$ (s) hinzugefügt und die Mischung $30 \mathrm{~h}$ in einem verschlossenen 25 mL Glasfläschchen bei RT gerührt. Nach Phasentrennung und Extraktion der festen Phase mit Dichlormethan $(3 \times 10 \mathrm{~mL})$ wurden die organischen Phasen vereinigt und die Lösungsmittel unter reduziertem Druck weitestgehend entfernt. Anschließend fand eine säulenchromatographische Trennung ( $n$-Hexan, 1.5 x $\left.50 \mathrm{~cm}, \mathrm{R}_{\mathrm{f}}(\mathbf{3 1})=0.30\right)$ der $0.7 \mathrm{~g}$ an Rohprodukt statt. Es konnten $400 \mathrm{mg}$ (60\%) an $\mathbf{3 1}$ nach Umkristallisation aus $n$-Hexan isoliert werden. ${ }^{\mathbf{1}} \mathbf{H}-\mathbf{N M R}\left(250 \mathrm{MHz}, \mathrm{CDCl}_{3}\right)$ : Ist literaturidentisch;[122] ${ }^{\mathbf{1 3}} \mathbf{C}-\mathbf{N M R}(62.9 \mathrm{MHz}$, $\mathrm{CDCl}_{3}$ ): $\delta=33.46$ (s, C-9); 35.57 (t, C-1, C-5); 36.72 (q, C-2”); 41.58 (s, C-1"); 48.22 (s, C6, C-8); 49.52 (s, C-2, C-4); 53.61 (q, C-7); 54.13 (q, C-3); 64.83 (s, C-1'); EA: $\mathrm{C}_{11} \mathrm{H}_{16} \mathrm{Br}_{4}$ (479.87) Ber.: C 30.03, H 3.36 Gef.: C 30.20, H 3.41; MS (70eV): m/z (\%) = 403 (32) 401 (60) 399 (59) 397 (19) [M+ - Br], 367 (51) 369 (100) 371 (49) $\left[\mathrm{M}^{+}-\mathrm{Br}_{2}\right], 265$ (30) 263 (62) 261 (29), 239 (13) 237 (13) [ $\left.\mathrm{M}^{+}-\mathrm{Br}_{3}\right], 212$ (72), 214 (71) [ $\left.\mathrm{M}^{+}-\mathrm{CH}_{2} \mathrm{CBr}_{3}\right], 184$ (20) 182 (19), 133 (65), 105 (40), 91 (97), 79 (64), 65 (28), 41 (28); IR (KBr): 2910, 2849, 1454, 1244, 1071, 899, 785, 736, 664, 635, $581 \mathrm{~cm}^{-1}$; Schmp.: Ist literaturidentisch[122]

\section{AV7. Die Synthese von $D_{11-1-B r o m c y c l o h e x a n}$}

In $10 \mathrm{~mL} \mathrm{CH} \mathrm{Cl}_{2}$ wurden $2.00 \mathrm{~g} D_{12}$-Cyclohexan $(0.021 \mathrm{~mol}), 2.00 \mathrm{~g}(0.011 \mathrm{~mol}) \mathrm{N}$ Bromsuccinimid und 0.20 g Azobisisobutyronitril (1.2 mmol) gelöst und die Mischung unter Rühren refluxiert. Nach jeweils $24 \mathrm{~h}$ und $48 \mathrm{~h}$ wurden weitere $0.20 \mathrm{~g}$ AIBN zugesetzt und die Reaktion nach 68 h beendet. Die Reaktionsmischung wurde mit $20 \mathrm{~mL}$ wäßriger $\mathrm{NaSO}_{3} \mathrm{Lsg}$. (konz.) gewaschen, die Phasen getrennt und die wäßrige mit $\mathrm{CH}_{2} \mathrm{Cl}_{2}(3 \times 10 \mathrm{~mL})$ extrahiert. Nach dem Trocknen der vereinigten organischen Phasen über $\mathrm{Na}_{2} \mathrm{SO}_{4}$ und destillativer Entfernung des größten Teils der Lösungsmittel unter Normaldruck wurde der Rückstand einer säulenchromatographischen Trennung ( $n$-Pentan, 50 x $\left.2.5 \mathrm{~cm}, \mathrm{R}_{\mathrm{f}}=0.62\right)$ unterzogen. Es konnten $250 \mathrm{mg} D_{11}-1-$ Bromcyclohexan $(7 \%, 1.4 \mathrm{mmol})$ als farblose Flüssigkeit isoliert werden. Eine GC/MS-Analyse ergab einen Deuterierungsgrad von $>99 \%$. 


\section{AV8. Die Synthese von $D_{11}$-1-Iodcyclohexan}

Es wurden $15 \mathrm{~mL}$ Hexamethyldisiloxan und $6.6 \mathrm{~g} \mathrm{P}_{2} \mathrm{O}_{5}$ in $30 \mathrm{~mL}$ Benzol gelöst und 30 min unter Rückfluß gerührt. Anschließend wurden ca. $12 \mathrm{~mL}$ Benzol unter reduziertem Druck (400 Torr) destillativ entfernt, wodurch eine stark viskose farblose Flüssigkeit entstand (PPSE = Polyphosphasiloxyether). Zu Benzol wurden 2 g PPSE, 336 mg $D_{12}$-Cyclohexanol (3.0 mmol), und $540 \mathrm{mg} \mathrm{NaI}$ (3.6 mmol) gegeben und die Mischung bei RT $18 \mathrm{~h}$ gerührt. Nach erneuter Zugabe von $270 \mathrm{mg} \mathrm{NaI}(1.8 \mathrm{mmol})$ und weiterem Rühren der Mischung bei RT (7d) wurde die Reaktionsmischung mit $15 \mathrm{~mL} n$-Pentan versetzt, mit $10 \mathrm{~mL}$ wäßriger $\mathrm{NaHCO}_{3}$ Lsg. (konz, $+0.1 \mathrm{~g} \mathrm{Na}_{2} \mathrm{~S}_{2} \mathrm{O}_{3}$ ) gewaschen, die Phasen getrennt und die wäßrige Phase mit $n$ Pentan extrahiert (3 x $10 \mathrm{~mL})$. Nach weitestgehendem destillativen Abtrennen der Lösungsmittel unter Normaldruck wurde der Rückstand einer Mikrodestillation unterzogen

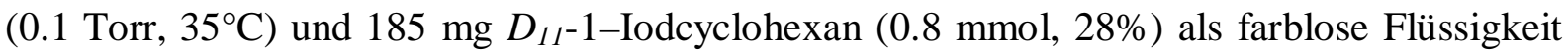
erhalten. Eine GC/MS-Analyse ergab einen Deuterierungsgrad von >99\%.

\section{AV9. Die Synthese von 1-Brom-3,5,7-trideuterotricyclo[3.3.1.1 $\left.{ }^{3,7}\right]$ decan}

Es wurden $100 \mathrm{mg}$ 1,3,5,7-Tetradeuteroadamantan $(0.71 \mathrm{mmol}), 23 \mathrm{mg} \mathrm{TBABr}(0.07$ mmol, $10 \mathrm{~mol} \%)$ sowie $359 \mathrm{mg} \mathrm{CBr} 4(1.1 \mathrm{mmol})$ in $5 \mathrm{~mL}$ Fluorbenzol gelöst und nach Zugabe von $1 \mathrm{~mL} \mathrm{NaOH}$ (aq. 50\%) bei $40^{\circ} \mathrm{C}$ gerührt. Die Reaktion wurde nach $48 \mathrm{~h}$ abgebrochen, als eine GC/MS-Analyse der Reaktionsmischung einen Umsatz von 35\% anzeigte. Nach der Phasentrennung und Extraktion der wäßrigen Phase mit $n$-Pentan, wurden die vereinigten organischen Phasen über $\mathrm{Na}_{2} \mathrm{SO}_{4}$ getrocknet und anschließend der größte Teil der Lösungsmittel am Rotationsverdampfer unter reduziertem Druck entfernt. Der Rückstand wurde einer säulenchromatographischen Trennung unterzogen (n-Pentan, $1.5 \times 45 \mathrm{~cm}, \mathrm{R}_{\mathrm{f}}=$ 0.50). Es wurden $48 \mathrm{mg} 1$-Brom-3,5,7-trideuteroadamantan $(0.22 \mathrm{mmol}, 31 \%)$ als weißer Feststoff erhalten. $50 \mathrm{mg}$ des Ausgangssubstanz konnten zurückgewonnen werden. Eine GC/MS-Analyse ergab einen Deuterierungsgrad von $>99 \%$. 


\section{AV10. Die Synthese von 1-Iod-3,5,7-trideuterotricyclo[3.3.1.1 $\left.{ }^{3,7}\right]$ decan}

Es wurden $100 \mathrm{mg}$ 1,3,5,7-Tetradeuteroadamantan (0.71 mmol), $23 \mathrm{mg}$ TBABr (0.07 mmol, $10 \mathrm{~mol} \%)$ sowie $433 \mathrm{mg} \mathrm{CHI}_{3}(1.1 \mathrm{mmol})$ in $5 \mathrm{~mL}$ Fluorbenzol gelöst und nach Zugabe von 1 $\mathrm{mL} \mathrm{NaOH}$ (aq. $50 \%$ ) bei $40^{\circ} \mathrm{C}$ gerührt. Die Reaktion wurde nach $36 \mathrm{~h}$ abgebrochen, als eine GC/MS-Analyse der Reaktionsmischung einen Umsatz von 32\% anzeigte. Nach der Phasentrennung und Extraktion der wäßrigen Phase mit $n$-Pentan, wurden die vereinigten organischen Phasen über $\mathrm{Na}_{2} \mathrm{SO}_{4}$ getrocknet und anschließend der größte Teil der Lösungsmittel am Rotationsverdampfer unter reduziertem Druck entfernt. Der Rückstand wurde einer säulenchromatographischen Trennung unterzogen ( $n$-Pentan, $1.5 \times 45 \mathrm{~cm}, \mathrm{R}_{\mathrm{f}}=$ 0.45). Es wurden $39 \mathrm{mg}$ 1-Iod-3,5,7-trideuteroadamantan $(0.15 \mathrm{mmol}, 21 \%)$ als weißer Feststoff erhalten. $65 \mathrm{mg}$ der Ausgangssubstanz konnten zurückgewonnen werden. Eine GC/MS-Analyse ergab einen Deuterierungsgrad von $>99 \%$.

\section{AV11. Bestimmung der Responsefaktoren für die halogenierten Produkte im Rahmen der Bestimmung der KIEs bei den PT-Halogenierungen.}

Für die Kalibrierung der halogenierten Produkte wurden jeweils die undeuterierte und deuterierte Verbindung in einem Molverhältnis von ca. $1: 3$ eingewogen (Tab 24), in $1 \mathrm{~mL}$ $\mathrm{CH}_{2} \mathrm{Cl}_{2}$ gelöst und anschließend eine systematische Verdünnung der Mischung vorgenommen. Dazu wurden 10, 5, 1 und $0.1 \mu \mathrm{L}$ der jeweiligen Stammlösung (Einwaage in $1 \mathrm{~mL} \mathrm{CH}_{2} \mathrm{Cl}_{2}$, Tab. 24) auf $1 \mathrm{~mL}$ verdünnt und 7, 3 und $1 \mu \mathrm{L}$ der entstehenden Lösungen durch eine GC/MS Messung durch massenselektive Integration analysiert $\left(\mathrm{C}_{6} \mathrm{H}_{11} \mathrm{Br}: \mathrm{m} / \mathrm{z}=83 ; \mathrm{C}_{6} \mathrm{D}_{11} \mathrm{Br}: \mathrm{m} / \mathrm{z}=\right.$ 94; $\mathrm{C}_{6} \mathrm{H}_{11} \mathrm{I}: \mathrm{m} / \mathrm{z}=83 ; \mathrm{C}_{6} \mathrm{D}_{11} \mathrm{I}: \mathrm{m} / \mathrm{z}=94 ;$ AdI: $\mathrm{m} / \mathrm{z}=135 ; \mathrm{D}_{3}$-AdI: $\mathrm{m} / \mathrm{z}=138$ ). Die Messung der höchstverdünntesten Lösung bewegte sich an der Nachweisgrenze. Es wurden die folgenden Temperaturprogramme verwendet (Analyse der Cyclohexanmischungen: $45 \rightarrow$ $150^{\circ} \mathrm{C}, 5 \% \mathrm{~min}$; Analyse der Adamantanmischungen: $150 \rightarrow 230^{\circ} \mathrm{C}, 5^{\circ} / \mathrm{min}$ ) 
Tabelle 24. Bestimmung der Responsefaktoren für die Produkte der KIE-Untersuchung

\begin{tabular}{ccccc} 
Verd. / Inj. & $\begin{array}{c}\text { Bromcyclohexan } \\
\mathrm{H}: \mathrm{D}\end{array}$ & $\begin{array}{c}\text { Iodcyclohexan } \\
\mathrm{H}: \mathrm{D}\end{array}$ & $\begin{array}{c}\text { 1-Bromadamantan } \\
\mathrm{H}: \mathrm{D}\end{array}$ & $\begin{array}{c}\text { 1-Iodadamantan } \\
\mathrm{H}: \mathrm{D}\end{array}$ \\
{$[\mu \mathrm{L}$ in $\mathrm{mL} / \mu \mathrm{L}]$} & $\begin{array}{c}(56.90: 20.29) \mathrm{mg} \\
\text { theor. Faktor: } 2.995\end{array}$ & $\begin{array}{c}(34.40: 13.70) \mathrm{mg} \\
\text { theor. Faktor: } 2.641\end{array}$ & $\begin{array}{c}(89.44: 30.15) \mathrm{mg} \\
\text { theor. Faktor: 3.008 }\end{array}$ & $\begin{array}{c}(120.69: 35.07) \mathrm{mg} \\
\text { theor. Faktor: } 3.481\end{array}$ \\
\hline 10 in $1 / 7$ & 3.262 & 2.222 & 3.007 & 3.480 \\
10 in $1 / 3$ & 3.239 & 2.230 & 3.004 & 3.478 \\
10 in $1 / 1$ & 3.093 & 2.238 & 3.005 & 3.482 \\
5 in $1 / 7$ & 3.201 & 2.235 & 3.010 & 3.481 \\
5 in $1 / 3$ & 3.163 & 2.241 & 3.011 & 3.485 \\
5 in $1 / 1$ & 3.089 & 2.246 & 3.009 & 3.484 \\
1 in $1 / 7$ & 3.145 & 2.238 & 3.008 & 3.489 \\
1 in $1 / 3$ & 3.104 & 2.246 & 3.001 & 3.490 \\
1 in $1 / 1$ & 3.102 & 2.244 & 3.015 & 3.481 \\
0.1 in $1 / 7$ & 3.151 & 2.245 & 3.009 & 3.487 \\
0.1 in $1 / 3$ & 3.146 & 2.214 & 3.022 & 3.473 \\
0.1 in $1 / 1$ & 3.091 & 2.240 & 3.015 & 3.479 \\
$\varnothing$ & 3.149 & 2.237 & 3.010 & 3.482 \\
\hline Responsefaktor & 1.15 & 1.18 & 1.00 & 1.00
\end{tabular}

\section{AV12. Bestimmung des kinetischen Isotopeneffektes}

Alle Experimente wurden bei gleicher Rührgeschwindigkeit, gleicher Magnetrührstäbchengröße und RT in verschließbaren Glasfläschchen (25 mL, Schott) durchgeführt. Zudem wurden die Experimente als direkte Konkurrenzexperimente durchgeführt. (im gleichen Fläschchen) und wurden fünfmal wiederholt. Alle organischen Komponenten wurden in $\mathrm{CH}_{2} \mathrm{Cl}_{2}$ gelöst und dann die Reaktion durch Zugabe einer entsprechenden Menge $\mathrm{NaOH}$ (aq. 50\%) initiiert. Die Analyse der Reaktionsmischungen erfolgte mit den gleichen Temperaturprogrammen wie die Kalibrierung (AV11), um die Konsistenz der Responsefaktoren zu gewährleisten. Die genauen Ansatzgrößen für die KIE Experimente unter den eben beschriebenen Bedingungen sind die folgenden: Bromierung von 11: $84.16 \mathrm{mg} \mathrm{C}_{6} \mathrm{H}_{12}(0.001 \mathrm{~mol}), 96.27 \mathrm{mg} \mathrm{C}_{6} \mathrm{D}_{12}(0.001 \mathrm{~mol})$, 996.6mg $\mathrm{CBr}_{4}(0.003$ mol), $32.22 \mathrm{mg} \operatorname{TBABr}(0.1 \mathrm{mmol}, 10 \mathrm{~mol} \%), 7 \mathrm{~mL} \mathrm{CH}_{2} \mathrm{Cl}_{2}, 3 \mathrm{~mL} \mathrm{NaOH}$ (aq. 50\%). Bromierung von 1: $68.12 \mathrm{mg}$ Adamantan $(0.5 \mathrm{mmol}),:$ : $70.13 \mathrm{mg} D_{4}$-Adamantan (0.5 mmol), 996.6mg CBr 4 (0.003 mol), $16.11 \mathrm{mg} \mathrm{TBABr} \mathrm{(10} \mathrm{mol \% ),} 7 \mathrm{ml} \mathrm{CH}_{2} \mathrm{Cl}_{2}, 3 \mathrm{ml} \mathrm{NaOH}$ (aq. 50\%); Iodierung von 11: $84.16 \mathrm{mg} \mathrm{C}_{6} \mathrm{H}_{12}(0.001 \mathrm{~mol})$, $96.27 \mathrm{mg} \mathrm{C}_{6} \mathrm{D}_{12}(0.001 \mathrm{~mol}), 1.500 \mathrm{~g} \mathrm{CHI}_{3}$ (0.0038 mol), $32.22 \mathrm{mg} \operatorname{TBABr}(0.1 \mathrm{mmol}, 10 \mathrm{~mol} \%), 20 \mathrm{~mL} \mathrm{CH}_{2} \mathrm{Cl}_{2}, 3 \mathrm{~mL} \mathrm{NaOH}$ (aq. 50\%); Iodierung von 1: $68.12 \mathrm{mg}$ Adamantan (0.5 mmol),: $70.13 \mathrm{mg} D_{4}$-Adamantan (0.5 
mmol), $1.500 \mathrm{~g} \mathrm{CHI}_{3}(0.0038 \mathrm{~mol}), 16.11 \mathrm{mg} \mathrm{TBABr}(10 \mathrm{~mol} \%), 20 \mathrm{~mL} \mathrm{CH} \mathrm{Cl}_{2}, 3 \mathrm{~mL}$ $\mathrm{NaOH}($ aq. $50 \%)$.

Tabelle 25. Die durch GC/MS-Analyse ermittelten Produktverhältnisse bei der PT-Bromierung

\begin{tabular}{|c|c|c|c|}
\hline Zeit $[\mathrm{h}]$ & $\mathrm{C}_{6} \mathrm{H}_{11} \mathrm{Br}: \mathrm{C}_{6} \mathrm{D}_{11} \mathrm{Br}$ & 1-Br-Ad : $1-\mathrm{Br}-3,5,7-\mathrm{D}_{4}-\mathrm{Ad}$ & 2-Br-Ad : 2-Br-1,3,5,7- $\mathrm{D}_{4}-\mathrm{Ad}$ \\
\hline 0.66 & & 4.86 & 1.00 \\
\hline 1.00 & 3.52 & 5.09 & 1.05 \\
\hline 1.25 & & 4.99 & 1.09 \\
\hline 1.50 & 3.56 & 5.04 & 1.00 \\
\hline 2.00 & 3.94 & 5.10 & 0.99 \\
\hline 2.25 & & 5.02 & 1.00 \\
\hline 2.50 & 3.83 & 5.09 & 1.01 \\
\hline 3.00 & 3.88 & 5.02 & 0.99 \\
\hline 3.50 & 3.93 & & \\
\hline KIE & $3.48 \pm 0.5$ & $4.9 \pm 0.3$ & $1.1 \pm 0.1$ \\
\hline
\end{tabular}

Tabelle 26. Die durch GC/MS-Analyse ermittelten Produktverhältnisse bei der PT-Iodierung

\begin{tabular}{|c|c|c|c|}
\hline Zeit [h] & $\mathrm{C}_{6} \mathrm{H}_{11} \mathrm{I}: \mathrm{C}_{6} \mathrm{D}_{11} \mathrm{I}$ & $1-\mathrm{I}-\mathrm{Ad}: 1-\mathrm{I}-3,5,7-\mathrm{D}_{4}-\mathrm{Ad}$ & 2-I-Ad : 2-I-1,3,5,7-D $\mathrm{D}_{4}-\mathrm{Ad}$ \\
\hline 0.25 & & 5.71 & \\
\hline 0.33 & & 6.40 & 1.02 \\
\hline 0.42 & & 6.72 & 1.03 \\
\hline 0.50 & 4.15 & 7.10 & 1.07 \\
\hline 0.58 & & 7.33 & 0.98 \\
\hline 0.67 & & 7.67 & 1.00 \\
\hline 0.75 & 3.96 & 7.71 & 1.03 \\
\hline 0.83 & & 8.30 & 0.97 \\
\hline 0.92 & & 8.40 & 1.09 \\
\hline 1.00 & 4.11 & 8.80 & 1.07 \\
\hline 1.08 & & 9.10 & 1.05 \\
\hline 1.17 & & 9.40 & 1.09 \\
\hline 1.25 & 4.18 & & \\
\hline 1.58 & 4.31 & & \\
\hline 2.00 & 4.26 & & \\
\hline 2.33 & 4.24 & & \\
\hline KIE & $3.98 \pm 0.4$ & $5.1 \pm 0.7$ & $1.0 \pm 0.1$ \\
\hline
\end{tabular}

Die Auswertung der experimentellen Daten erfolgte durch eine einfache lineare Regression, wobei der jeweilige Fehler aus der Standartabweichung und Folgefehler für die Ordinate bestimmt wurde (Tab. 25, 26). Die angegebenen Werte sind die Mittelwerte aus jeweils fünf Experimenten. 


\section{AV13. Die Halogenierung von Cuban}

Im folgenden werden der Vollständigkeit halber nur die Durchführungen für die PTHalogenierungen von Cuban und dessen Monohaloderivate angegeben, da alle spektroskopischen Daten der neuen Verbindungen bereits entsprechend veröffentlicht wurden. ${ }^{[139]}$ Von denen durch die Zweithalogenierung erhaltenen Dihalogencubanen konnte jeweils nur das meta-Isomer säulenchromatographisch isoliert werden während die ortho- und para-Produkte als Gemische erhalten wurden. Hier erfolgte die Analyse durch Integration separater Signale im ${ }^{1}$ H-NMR Spektrum. Ferner wurden für die verschiedenen Produkte keine $\mathrm{R}_{\mathrm{f}}$-Werte bestimmt, da die verschiedenen Halogencubane durch Dünschichtchromatographie nicht separierbar und nur schwer detektierbar waren. Die einzelnen Fraktionen der Säulenchromatographien über Kieselgel wurden durch GC/MS-Analysen identifiziert. Alle Experimente wurden in $25 \mathrm{~mL}$ Glassfläschchen (Schott) mit Schraubverschluß und dem gleichen Magnetrührstäbchen $(2 \times 0.5 \mathrm{~cm})$ durchgeführt.

AV13a. 1-Chlorcuban (36)

In $15 \mathrm{~mL} \mathrm{CCl}{ }_{4}$ wurden $208 \mathrm{mg} 32(2.0 \mathrm{mmol})$ und $25 \mathrm{mg} \operatorname{TBABr}(0.08 \mathrm{mmol}, 4$ mol\%) gelöst und $5 \mathrm{~mL} \mathrm{NaOH}$ (aq. $50 \%$ ) hinzugefügt. Die Reaktionsmischung wurde $5 \mathrm{~d}$ unter Rückfluß gerührt. Anschließend wurde diese mit $10 \mathrm{~mL}$ Wasser verdünnt, die Phasen getrennt und die wäßrige Phase mit $\mathrm{CCl}_{4}(3 \times 10 \mathrm{~mL})$ extrahiert. Die vereinigten organischen Phasen wurden mit Wasser gewaschen und über $\mathrm{Na}_{2} \mathrm{SO}_{4}$ getrocknet. Der größte Teil des Lösungsmittels wurde destillativ (Normaldruck, Vigreuxkolonne $(20 \mathrm{~cm})$ ) entfernt und anschließend eine säulenchromatographische ( $n$-Pentan, 1.5 x $50 \mathrm{~cm}$ ) Trennung der Produkte durchgeführt. Es wurden $227 \mathrm{mg} 36$ (1.64 mmol, $81 \%$ ) als farblose Kristalle erhalten, wobei 10 mg 32 zurückgewonnen werden konnten.

AV13b. 1-Bromcuban (35)

In $5 \mathrm{~mL} \mathrm{CH} \mathrm{Cl}_{2}$ wurden $208 \mathrm{mg} 32$ (2.0 mmol), $25 \mathrm{mg}$ TBABr $(0.08 \mathrm{mmol}, 4 \mathrm{~mol} \%)$ sowie $1.289 \mathrm{~g} \mathrm{CBr}_{4}(4.0 \mathrm{mmol})$ gelöst und $3 \mathrm{~mL} \mathrm{NaOH}$ (aq. $50 \%$ ) hinzugefügt. Die Mischung wurde $24 \mathrm{~h}$ bei RT gerührt. Nach Zugabe von $10 \mathrm{~mL}$ Wasser wurden die Phasen 
getrennt und die wäßrige mit $\mathrm{CH}_{2} \mathrm{Cl}_{2}(3 \times 10 \mathrm{~mL})$ extrahiert. Die vereinigten organischen Phasen wurden mit Wasser gewaschen und über $\mathrm{Na}_{2} \mathrm{SO}_{4}$ getrocknet. Der größte Teil des Lösungsmittels wurde destillativ (Normaldruck) entfernt und anschließend eine säulenchromatographische ( $n$-Pentan, 1.5 x $50 \mathrm{~cm}$ ) Trennung der Produkte durchgeführt. Es wurden $274 \mathrm{mg} 36$ (1.50 mmol, $75 \%$ ) als farblose Kristalle erhalten, wobei $20 \mathrm{mg} 32$ zurückgewonnen werden konnten.

AV13c. 1-Iodcuban (37)

In $5 \mathrm{~mL} \mathrm{CH} \mathrm{Cl}_{2}$ wurden $208 \mathrm{mg} 32$ (2.0 mmol), $25 \mathrm{mg} \mathrm{TBABr}(0.08 \mathrm{mmol}, 4 \mathrm{~mol} \%)$ sowie $1.576 \mathrm{~g} \mathrm{CHI}_{3}(4.0 \mathrm{mmol})$ gelöst und $3 \mathrm{~g} \mathrm{NaOH}$ (s, mit aq. $\mathrm{NaOH}$ findet ein Zerfall der Produkte statt) hinzugefügt. Die Mischung wurde $24 \mathrm{~h}$ bei RT gerührt. Nach Zugabe von weiteren $10 \mathrm{~mL} \mathrm{CH} \mathrm{Cl}_{2}$ wurde das Hydroxid abfiltriert und letzteres mit $\mathrm{CH}_{2} \mathrm{Cl}_{2}$ extrahiert. Die vereinigten organischen Phasen wurden durch Destillation unter Normaldruck vom größten Teil des Lösungsmittels befreit und anschließend eine säulenchromatographische Reinigung der Produkte ( $n$-Pentan, 1.5 x $50 \mathrm{~cm}$ ) durchgeführt. Es wurden $308 \mathrm{mg} 37$ (1.34 mmol, $67 \%$ ) als weißer Feststoff erhalten, wobei 25 mg 32 zurückgewonnen werden konnten.

AV13d. 1,2-, 1,3- und 1,4-Dichlorcuban

Gemäß der Prozedur AV13a wurden 137 mg 36 (1.0 mmol) einer Chlorierung unterworfen, wobei die Reaktion 10 d fortgeführt werden mußte. Nach der entsprechenden Aufarbeitung und der säulenchromatographischen Trennung der Produkte ( $n$-Pentan, $1.5 \mathrm{x}$ $120 \mathrm{~cm})$ wurden $35 \mathrm{mg} 36$ zurückgewonnen und $95 \mathrm{mg}(0.55 \mathrm{mmol}, 55 \%)$ an 1,2-, 1,3- und 1,4-Dichlorcuban im Verhältnis 0.70 : 1.00 : 0.09 als weiße Feststoffe erhalten.

AV13d. 1,2-, 1,3- und 1,4-Dibromcuban

In $3 \mathrm{~mL} \mathrm{CH} \mathrm{Cl}_{2}$ wurden $183 \mathrm{mg} 35$ (1.0 mmol), $10 \mathrm{mg}$ TBABr $(0.03 \mathrm{mmol}, 3 \mathrm{~mol} \%)$ sowie $664 \mathrm{mg} \mathrm{CBr} 4(2.0 \mathrm{mmol})$ gelöst und $1.5 \mathrm{~mL} \mathrm{NaOH}$ (aq. $50 \%$ ) hinzugefügt. Die Mischung wurde $5 \mathrm{~d}$ bei RT gerührt. Nach der Aufarbeitung und Säulenchromatographie 
gemäß Prozedur AV13b wurden $183 \mathrm{mg}(0.69$ mmol, $69 \%)$ an 1,2-, 1,3- und 1,4Dibromcuban im Verhältnis 0.63 : 1.00 : 0.09 als weiße Feststoffe erhalten, wobei 25 mg 35 zurückgewonnen werden konnten.

AV13e. 1,2-, 1,3- und 1,4-Diiodcuban

$115 \mathrm{mg} 37$ (0.5 mmol) und $788 \mathrm{mg} \mathrm{CHI}$ (2.0 mmol) wurden in $5 \mathrm{~mL} \mathrm{CH}_{2} \mathrm{Cl}_{2}$ gelöst und $1.5 \mathrm{~g} \mathrm{NaOH}$ (s) hinzugefügt. Die Mischung wurde $3 \mathrm{~d}$ bei RT gerührt und danach entsprechend Prozedur AV12c aufgearbeitet. Anschließend wurde das erhaltene Rohprodukt erneut den gleichen Reaktionsbedingungen (788 mg CHI 3 (2.0 mmol), $5 \mathrm{~mL} \mathrm{CH}_{2} \mathrm{Cl}_{2}, 1.5 \mathrm{~g}$ $\mathrm{NaOH}$ (s)) ausgesetzt. Nach Fortsetzung der Reaktion für weitere $5 \mathrm{~d}$ wurde die Reaktionsmischung erneut entsprechend der Prozedur AV12c aufgearbeitet und eine Säulenchromatographie (n-Pentan, $1.5 \mathrm{x} \quad 120 \mathrm{~cm}$ ) durchgeführt, wobei $16 \mathrm{mg} 37$ zurückgewonnen und $123 \mathrm{mg}(0.35 \mathrm{mmol}, 70 \%)$ an 1,2-, 1,3- und 1,4-Diiodcuban im Verhältnis $0.70: 1.00: 0.08$ als weiße Feststoffe erhalten wurden.

AV13f. 1-Iod-2-bromcuban, 1-Iod-3-bromcuban, 1-Iod-4-bromcuban

$183 \mathrm{mg}$ Bromcuban (1.0 mmol) und $1.576 \mathrm{~g} \mathrm{CHI}_{3}(4.0 \mathrm{mmol})$ wurden in $7 \mathrm{~mL} \mathrm{CH}_{2} \mathrm{Cl}_{2}$ gelöst und $3 \mathrm{~g} \mathrm{NaOH}$ (s) hinzugefügt. Die Mischung wurde $4 \mathrm{~d}$ bei RT gerührt und danach entsprechend Prozedur AV13c aufgearbeitet. Anschließend wurde das erhaltene Rohprodukt erneut den gleichen Reaktionsbedingungen (1.576 $\mathrm{g} \mathrm{CHI}_{3}$ (4.0 mmol), $7 \mathrm{~mL} \mathrm{CH}_{2} \mathrm{Cl}_{2}, 3 \mathrm{~g}$ $\mathrm{NaOH}$ (s)) ausgesetzt. Nach Fortsetzung der Reaktion für weitere $4 \mathrm{~d}$ wurde die Reaktionsmischung erneut entsprechend der Prozedur AV13c aufgearbeitet und eine Säulenchromatographie (n-Pentan, $1.5 \times 120 \mathrm{~cm}$ ) durchgeführt, wobei $53 \mathrm{mg} 35$ zurückgewonnen und $151 \mathrm{mg}(0.49 \mathrm{mmol}, 49 \%)$ an 1,2-, 1,3- und 1,4-Produkt im Verhältnis $0.68: 1.00: 0.09$ als weiße Feststoffe erhalten wurden. 
AV13g. 1-Brom-2-chlorcuban, 1-Brom-3-chlorcuban, 1-Brom-4-chlorcuban

Entsprechend Prozedur AV13d wurden 137 mg 36 (1.0 mmol) zur Reaktion gebracht, wobei die Reaktion $4 \mathrm{~d}$ fortgeführt werden mußte. Nach der Säulenchromatographie wurden (n-Pentan, 1.5 x $120 \mathrm{~cm}) 17 \mathrm{mg} 36$ zurückgewonnen und $154 \mathrm{mg}$ (0.71 mmol, 71\%) an 1,2-, 1,3- und 1,4-Produkt im Verhältnis $0.67: 1.00: 0.09$ als weiße Feststoffe erhalten.

AV13h. 1-Iod-2-chlorcuban, 1-Iod-3-chlorcuban, 1-Iod-4-chlorcuban

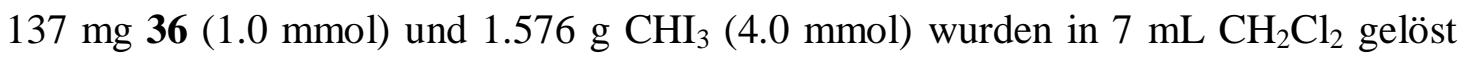
und $3 \mathrm{~g} \mathrm{NaOH}$ (s) hinzugefügt. Die Mischung wurde $4 \mathrm{~d}$ bei RT gerührt und danach entsprechend Prozedur AV13c aufgearbeitet. Anschließend wurde das erhaltene Rohprodukt

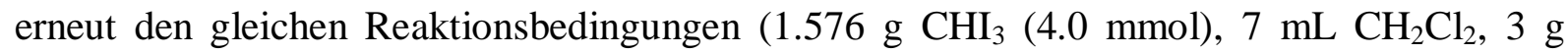
$\mathrm{NaOH}$ (s)) ausgesetzt. Nach Fortsetzung der Reaktion für weitere $4 \mathrm{~d}$ wurde die Reaktionsmischung erneut entsprechend der Prozedur AV13c aufgearbeitet und eine Säulenchromatographie (n-Pentan, $1.5 \mathrm{x} \quad 120 \mathrm{~cm}$ ) durchgeführt, wobei $50 \mathrm{mg} 36$ zurückgewonnen und $143 \mathrm{mg}(0.54 \mathrm{mmol}, 54 \%)$ an 1,2-, 1,3- und 1,4-Produkt im Verhältnis $0.68: 1.00: 0.09$ erhalten wurden.

\title{
AV14. Die Halogenierung verschiedener Adamantanderivate
}

\author{
AV14a. 1-Brom-3-chlortricyclo[3.3.1.1 $\left.{ }^{3,7}\right]$ decan (38), 1,3-Dibrom-5-chlortricyclo- \\ $\left[3.3 .1 .1^{3,7}\right]$ decan $(\mathbf{3 9})$
}

$3.41 \mathrm{~g}(0.02 \mathrm{~mol})$ 26, $13.24 \mathrm{~g}$ (0.04 mol) Tetrabrommethan und $322 \mathrm{mg}$ (0.001 mol, $5 \mathrm{~mol} \%)$ TBABr wurden in $100 \mathrm{~mL}$ Dichlormethan gelöst und $50 \mathrm{~mL}$ Natriumhydroxidlösung (aq. $50 \%$ ) zugesetzt. Die Mischung wurde 8 Tage bei intensivem Rühren refluxiert. Anschließend wurde die schwarze zähflüssige Mischung mit $50 \mathrm{~mL}$ Wasser und $50 \mathrm{~mL} n$-Pentan versetzt, die Phasen getrennt und die wäßrige Phase mit $n$-Pentan (4 x $75 \mathrm{~mL})$ extrahiert. Die vereinigten organischen Phasen wurden über Natriumsulfat getrocknet und am Rotationsverdampfer unter reduziertem Druck weitestgehend vom Lösungsmittel befreit. Die erhaltenen 4.8 g Rohprodukt wurden einer säulenchromatographischen Trennung unterzogen 
$\left(n\right.$-Pentan, $\left.6 \times 40 \mathrm{~cm}, \mathrm{R}_{\mathrm{f}}(\mathbf{2 6})=0.60, \mathrm{R}_{\mathrm{f}}(\mathbf{3 8})=0.35, \mathrm{R}_{\mathrm{f}}(\mathbf{3 9})=0.37\right)$. Es wurden $1.20 \mathrm{~g}(4.8$ mmol, $24 \%$ ) von 38 als weißer Feststoff erhalten. Weitere Fraktionen der Chromatographie waren mit 39 verunreinigt. Die mit 39 angereichertsten (> 80\% ermittelt durch GC/MSAnalyse) wurden vereinigt und siebenmal aus Hexan umkristallisiert. Es wurden $105 \mathrm{mg}$ von 39 als farblose Kristalle erhalten. $0.60 \mathrm{~g}$ von 26 konnten zurückgewonnen werden. 38: Die Spektraldaten sind literaturidentisch. ${ }^{[179]}$ 39: ${ }^{1} \mathbf{H}-\mathbf{N M R}\left(250 \mathrm{MHz}, \mathrm{CDCl}_{3}\right): \delta=2.06\left(\mathrm{~d},{ }^{3} \boldsymbol{J}_{7-\mathrm{H}}\right.$ $=3.1 \mathrm{~Hz}, 2 \mathrm{H}, 6-\mathrm{H}), 2.20\left(\mathrm{~d},{ }^{3} J_{7-\mathrm{H}}=3.1 \mathrm{~Hz}, 4 \mathrm{H}, 8-\mathrm{H}, 9-\mathrm{H}\right), 2.38\left(\mathrm{qq},{ }^{3} J_{6-\mathrm{H}}=3.1 \mathrm{~Hz},{ }^{3} J_{8-\mathrm{H}, 9-}\right.$ $\mathrm{H}=3.1 \mathrm{~Hz}, 1 \mathrm{H}, 7-\mathrm{H}), 2.61(\mathrm{~s}, 4 \mathrm{H}, 4-\mathrm{H}, 10-\mathrm{H}), 2.75(\mathrm{~s}, 2 \mathrm{H}, 2-\mathrm{H}) ;{ }^{13} \mathbf{C}-\mathbf{N M R}(62.9 \mathrm{MHz}$, $\mathrm{CDCl}_{3}$ ): $\delta=35.29$ (t, C-7), 43.68 (s, C-6), 44.95 (s, C-8, C-9), 55.68 (s, C-4, C-10), 58.02 (q, C-1, C-3), 64.59 (q, C-5); IR $\left(\mathrm{CCl}_{4}\right)$ : 959, 1027, 1133, 1315, 1332, 1348, 1456, 2864,

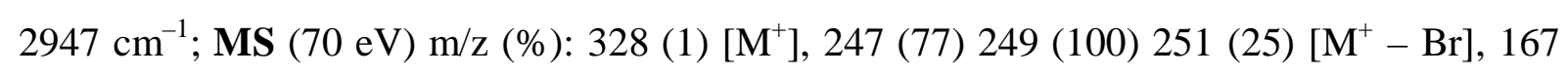
(19) 169 (6) $\left[\mathrm{M}^{+}-\mathrm{Br}_{2}\right], 131$ (22) $\left[\mathrm{M}^{+}-\mathrm{ClBr}_{2}\right], 91$ (27), 77 (12), 65 (9), 51 (5); EA: $\mathrm{C}_{10} \mathrm{H}_{13} \mathrm{Br}_{2} \mathrm{Cl}$ (328.47) Ber.: C 35.57, H 3.99 Gef.: C 34.96, H 3.67; Schmp.: $105-106^{\circ} \mathrm{C}$

AV14b. 1-Iod-3-chlortricyclo[3.3.1.1 $\left.1^{3,7}\right]$ decan (40)

$3.41 \mathrm{~g}$ (0.02 mol) Chloradamantan 26, $11.82 \mathrm{~g}$ (0.03 mol) Iodoform und $320 \mathrm{mg}$ (0.001 mol, 5 mol\%) TBABr wurden in $110 \mathrm{~mL}$ Fluorbenzol gelöst und $40 \mathrm{~mL}$ Natriumhydroxidlösung (aq. $50 \%)$ zugesetzt. Die Mischung wurde 5 Tage bei $50{ }^{\circ} \mathrm{C}$ Ölbadtemperatur intensiv gerührt. Anschließend wurde die schwarze Mischung mit $50 \mathrm{~mL}$ Wasser und $50 \mathrm{~mL} n$-Pentan versetzt, die Phasen getrennt und die wäßrige Phase mit $n$-Pentan $(4 \times 75 \mathrm{~mL})$ extrahiert. Die vereinigten organischen Phasen wurden über Natriumsulfat getrocknet und am Rotationsverdampfer unter reduziertem Druck weitestgehenden vom Lösungsmittel befreit. Die erhaltenen 9 g Rohprodukt wurden unter o.a. Bedingungen erneut zur Reaktion gebracht, nach weiteren 5 Tagen Reaktionszeit die Aufarbeitung in gleicher Weise durchgeführt und das Rohprodukt einer säulenchromatographischen Trennung unterzogen ( $n$-Pentan, 6 x $40 \mathrm{~cm}$, $\left.\mathrm{R}_{\mathrm{f}}(\mathbf{2 6})=0.60, \mathrm{R}_{\mathrm{f}}(\mathbf{4 0})=0.40\right)$. Es wurden $1.75 \mathrm{~g}(5.9 \mathrm{mmol}, 30 \%)$ von 40 als weißer Feststoff erhalten. $1.9 \mathrm{~g}$ von $\mathbf{2 6}$ konnten zurückgewonnen werden. Weitere Fraktionen der Chromatographie enthielten gemäß GC/MS-Analyse in Mischfraktionen auch das diiodierte Produkt 41. Auf eine Isolierung wurde verzichtet. 40: Die Spektraldaten sind literaturidentisch.[179] 
AV14c. 1-Iod-3-bromtricyclo[3.3.1.1 $1^{3,7}$ decan (42), 1,3-Diiod-5-bromtricyclo[3.3.1.1 $\left.{ }^{3,7}\right]$ decan (43)

$4.30 \mathrm{~g}$ (0.02 mol) Bromadamantan 2, $11.82 \mathrm{~g}$ (0.03 mol) Iodoform und $320 \mathrm{mg}$ (0.001 mol, 5 mol\%) TBABr wurden in $110 \mathrm{~mL}$ Fluorbenzol gelöst und $40 \mathrm{~mL}$ Natriumhydroxidlösung (aq. $50 \%$ ) zugesetzt. Die Mischung wurde 4 Tage bei $50{ }^{\circ} \mathrm{C}$ Ölbadtemperatur intensiv gerührt. Anschließend wurde die schwarze Mischung mit $50 \mathrm{~mL}$ Wasser und $50 \mathrm{~mL} n$-Pentan versetzt, die Phasen getrennt und die wäßrige Phase mit $n$-Pentan (4 x $75 \mathrm{~mL})$ extrahiert. Die vereinigten organischen Phasen wurden über Natriumsulfat getrocknet und am Rotationsverdampfer unter reduziertem Druck weitestgehendend von Lösungsmitteln befreit. Die erhaltenen $12 \mathrm{~g}$ Rohprodukt wurden unter o.a. Bedingungen erneut zur Reaktion gebracht, nach weiteren 5 Tagen Reaktionszeit die Aufarbeitung in gleicher Weise durchgeführt und das Rohprodukt einer säulenchromatographischen Trennung unterzogen ( $n$-Pentan, 6 x $40 \mathrm{~cm}$, $\left.\mathrm{R}_{\mathrm{f}}(\mathbf{2})=0.55, \mathrm{R}_{\mathrm{f}}(\mathbf{4 2})=0.34, \mathrm{R}_{\mathrm{f}}(\mathbf{4 3})=0.38\right)$. Es wurden $2.83 \mathrm{~g}(7.0 \mathrm{mmol}, 41 \%)$ von $\mathbf{7}$ als weißer Feststoff erhalten. $1.70 \mathrm{~g}$ von $\mathbf{2}$ konnten zurückgewonnen werden. Weitere Fraktionen der Chromatographie waren mit $\mathbf{4 3}$ verunreinigt. Die mit $\mathbf{4 3}$ angereichertsten (> 70\% ermittelt durch GC/MS-Analyse) wurden vereinigt und fünf Mal aus $n$-Hexan umkristallisiert. Es wurden $85 \mathrm{mg}$ von 43 als weiße Kristalle erhalten. 42: Die Spektraldaten sind literaturidentisch. ${ }^{[179]}$ 43: ${ }^{1} \mathbf{H}-\mathbf{N M R}\left(250 \mathrm{MHz}, \mathrm{CDCl}_{3}\right): \delta=2.07$ (qq, ${ }^{3} J_{6-\mathrm{H}}=3.1 \mathrm{~Hz},{ }^{3} J_{8-\mathrm{H}, 9-}$ $\mathrm{H}=2.2 \mathrm{~Hz}, 1 \mathrm{H}, 7-\mathrm{H}), 2.37\left(\mathrm{~d},{ }^{3} J_{7-\mathrm{H}}=3.1 \mathrm{~Hz}, 2 \mathrm{H}, 6-\mathrm{H}\right), 2.51\left(\mathrm{~d},{ }^{3} J_{7-\mathrm{H}}=2.2 \mathrm{~Hz}, 4 \mathrm{H}, 8-\mathrm{H}\right.$, 9-H), $2.98-3.12$ (m, 4 H, 4-H, 10-H), 3.19 (s, 2 H, 2-H); ${ }^{13}$ C-NMR (62.9 MHz, CDCl 3 ): $\delta$ $=38.41(\mathrm{t}, \mathrm{C}-7), 38.72$ (q, C-1, C-3), 44.98 (s, C-6), 47.61 (s, C-8, C-9), 58.48 (q, C-5), 59.38 (s, C-4, C-10), 61.71 (s, C-2); IR $\left(\mathrm{CCl}_{4}\right)$ : 1132, 1226, 1282, 1312, 1329, 1451, 2862,

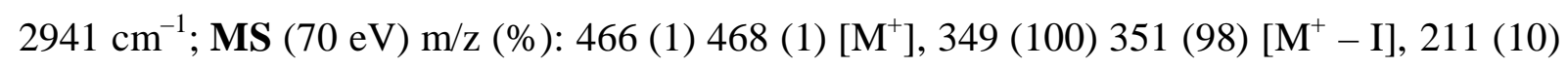
$213(10)\left[\mathrm{M}^{+}-\mathrm{I}_{2}\right], 133$ (70) $\left[\mathrm{M}^{+}-\mathrm{BrI}_{2}\right], 91$ (54), 77 (19), 65 (18), 51 (7); EA: $\mathrm{C}_{10} \mathrm{H}_{13} \mathrm{BrI}_{2}$ (466.92) Ber.: C 25.72, H 2.81 Gef.: C 25.40, H 2.62; Schmp.: $116-117^{\circ} \mathrm{C}$

AV14d. 1-Iod-3-bromtricyclo[3.3.1.1 $\left.1^{3,7}\right]$ decan (42)

$200 \mathrm{mg}$ (0.8 mmol) Iodadamantan 17, $664 \mathrm{mg}$ (2.0 mmol) Tetrabrommethan und $26 \mathrm{mg}(0.08$ mmol, $10 \mathrm{~mol} \%$ ) TBABr wurden in $10 \mathrm{~mL}$ Fluorbenzol gelöst und $7 \mathrm{~mL}$ Natriumhydroxidlösung (aq. 50\%) zugesetzt. Die Mischung wurde 2 Tage bei intensivem Rühren refluxiert, die organische Phase von der wäßrigen getrennt und letztere mit $n$-Pentan 
(4 x $10 \mathrm{~mL}$ ) extrahiert. Die vereinigten organischen Phasen wurden über Natriumsulfat getrocknet und am Rotationsverdampfer unter reduziertem Druck weitestgehendend von Lösungsmitteln befreit. Die erhaltenen $0.7 \mathrm{~g}$ Rohprodukt wurden einer säulenchromatographischen Trennung unterzogen ( $n$-Pentan, 1.5 x $35 \mathrm{~cm}, \mathrm{R}_{\mathrm{f}}(\mathbf{1 7})=0.52$, $\left.\mathrm{R}_{\mathrm{f}}(\mathbf{4 2})=0.34\right)$. Es wurden $90 \mathrm{mg}(0.19 \mathrm{mmol}, 24 \%)$ von 42 als weißer Feststoff erhalten. 100 mg von 17 konnten zurückgewonnen werden. 42: Die Spektraldaten sind literaturidentisch. [179]

AV14e. ( \pm )-1-Iod-3-brom-5-chlortricyclo[3.3.1.1 $\left.{ }^{3,7}\right]$ decan (44):

$341 \mathrm{mg}$ (1.0 mmol) 42, und $32 \mathrm{mg}(0.1 \mathrm{mmol}, 10 \mathrm{~mol} \%) \mathrm{TBABr}$ wurden in $15 \mathrm{~mL}$ Tetrachlormethan gelöst und $5 \mathrm{~mL}$ Natriumhydroxidlösung (aq. 50\%) zugesetzt. Die Mischung wurde sechs Wochen bei intensivem Rühren refluxiert. Anschließend wurde zähflüssige Reaktionsmischung mit $n$-Pentan (5 x $15 \mathrm{~mL})$ extrahiert. Die vereinigten organischen Phasen wurden über Natriumsulfat getrocknet und am Rotationsverdampfer unter reduziertem Druck weitestgehendend von Lösungsmitteln befreit. Die erhaltenen $300 \mathrm{mg}$ Rohprodukt wurden einer säulenchromatographischen Trennung unterzogen ( $n$-Pentan, $1.5 \mathrm{x}$ $\left.45 \mathrm{~cm}, \mathrm{R}_{\mathrm{f}}(\mathbf{4 4})=0.30\right)$. Es wurden $82 \mathrm{mg}(0.22 \mathrm{mmol}, 22 \%)$ von $( \pm)-\mathbf{4 4}$ als weißer Feststoff erhalten. $117 \mathrm{mg}$ von 42 konnten zurückgewonnen werden. ( \pm )-44: ${ }^{1} \mathbf{H}-\mathbf{N M R}(250 \mathrm{MHz}$, $\left.\mathrm{CDCl}_{3}\right): \delta=2.13$ (bs, $\left.2 \mathrm{H}, 6-\mathrm{H}\right), 2.18-2.33(\mathrm{~m}, 1 \mathrm{H}, 7-\mathrm{H}), 2.25\left(\mathrm{~d},{ }^{3} J_{7-\mathrm{H}}=2.1 \mathrm{~Hz}, 2 \mathrm{H}, 10\right.$ H), $2.39\left(\mathrm{~d},{ }^{3} \mathrm{~J}_{7-\mathrm{H}}=2.3 \mathrm{~Hz}, 2 \mathrm{H}, 8-\mathrm{H}\right), 2.59-2.72(\mathrm{~m}, 2 \mathrm{H}, 4-\mathrm{H}), 2.81(\mathrm{~s}, 2 \mathrm{H}, 9-\mathrm{H}), 2.95$ (s, $2 \mathrm{H}, 2-\mathrm{H}) ;{ }^{13} \mathbf{C}-\mathbf{N M R}\left(62.9 \mathrm{MHz}, \mathrm{CDCl}_{3}\right): \delta=36.29$ (t, C-7), 37.64 (q, C-1), 43.72 (s, C-6), 44.99 (s, C-10), 47.62 (s, C-8), 55.73 (s, C-4), 58.13 (q, C-3), 58.31 (s, C-9), 59.37 (s, C-2), 64.44 (q, C-5); IR $\left(\mathrm{CCl}_{4}\right): 1024,1131,1284,1314,1454,2863,2945 \mathrm{~cm}^{-1} ; \mathbf{M S}(70 \mathrm{eV}) \mathrm{m} / \mathrm{z}$

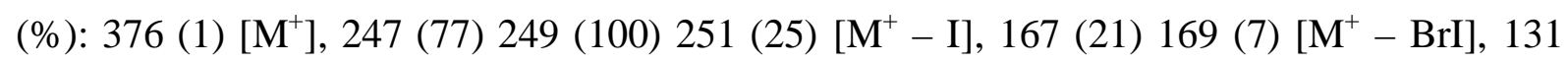
(23) $\left[\mathrm{M}^{+}-\mathrm{ClBrI}\right], 91$ (26), 77 (12), 65 (9), 55 (6); EA: $\mathrm{C}_{10} \mathrm{H}_{13} \mathrm{ClBrI}$ (375.47) Ber.: C 31.99, H 3.49 Gef.: C 32.40, H 3.20; Schmp.: $86-87^{\circ} \mathrm{C}$ 
AV14f. (士)-1-Iod-3-brom-5-chlortricyclo[3.3.1.1 $1^{3,7}$ decan (44), 1-Iod-3,5-dibrom-7-bromtricyclo[3.3.1.1 $\left.1^{3,7}\right]$ decan $(\mathbf{4 5})$ :

$297 \mathrm{mg}$ (1.0 mmol) 1-Iod-3-chloradamantan 40, $1.328 \mathrm{~g}$ (4.0 mol) Tetrabrommethan und 32 $\mathrm{mg}$ (0.1 mmol, $10 \mathrm{~mol} \%)$ TBABr wurden in $15 \mathrm{~mL}$ Fluorbenzol gelöst und $5 \mathrm{~mL}$ Natriumhydroxidlösung (aq. 50\%) zugesetzt. Die Mischung wurde 7 Tage bei $80^{\circ} \mathrm{C}$ intensiv gerührt. Anschließend wurden die Phasen getrennt und die wäßrige Phase mit $n$-Pentan (4 x $10 \mathrm{~mL}$ ) extrahiert. Die vereinigten organischen Phasen wurden über Natriumsulfat getrocknet und am Rotationsverdampfer unter reduziertem Druck weitestgehenden vom Lösungsmittel befreit. Die erhaltenen $600 \mathrm{mg}$ Rohprodukt wurden einer säulenchromatographischen Trennung unterzogen ( $n$-Pentan, 2.5 x $\left.40 \mathrm{~cm}, \mathbf{R}_{\mathbf{f}}(\mathbf{4 4})=0.30, \mathbf{R}_{\mathbf{f}}(\mathbf{4 5})=0.37\right)$. Es wurden 131 mg $(0.35 \mathrm{mmol}, 35 \%)$ von 44 und $35 \mathrm{mg}(0.08 \mathrm{mmol}, 7 \%)$ von $\mathbf{4 5}$ als weiße Feststoffe erhalten. 44: Die Charakterisierung entspricht der unter AV14f wiedergegebenen. 45: ${ }^{1} \mathbf{H}-$ NMR $\left(250 \mathrm{MHz}, \mathrm{CDCl}_{3}\right): \delta=2.59$ (s, 2H, 4-H), 2.60 (s, 2H, 8-H), 2.72 (s, 4H, 6-H, 9-H), 2.84 (s, 4H, 2-H, 10-H); ${ }^{13} \mathbf{C}-\mathbf{N M R}\left(62.9 \mathrm{MHz}, \mathrm{CDCl}_{3}\right): \delta=32.15$ (q, C-1), 53.82 (s, C-6, C-9), 54.55 (q, C-3, C-5), 54.85 (s, C-4), 56.27 (s, C-8), 57.19 (s, C-2, C-10), 62.51 (q, C7); IR $\left(\mathrm{CCl}_{4}\right): 857,1164,1319,1452,1734,2863,2965 \mathrm{~cm}^{-1} ; \mathbf{M S}(70 \mathrm{eV}) \mathrm{m} / \mathrm{z}(\%): 454$ (1) $\left[\mathrm{M}^{+}\right], 325$ (44) 327 (100) 329 (70) 341 (14) [ $\mathrm{M}^{+}$- I], 245 (7) 247 (12) 249 (6) 251 (1) $\left[\mathrm{M}^{+}\right.$$\mathrm{BrI}], 167$ (38) 169 (13) [M+ - $\left.\mathrm{Br}_{2} \mathrm{I}\right], 129$ (25) $\left[\mathrm{M}^{+}-\mathrm{ClBr}_{2} \mathrm{I}\right], 115$ (23), 91 (48), 77 (12), 64 (17), 54 (8); EA: $\mathrm{C}_{10} \mathrm{H}_{12} \mathrm{ClBr}_{2} \mathrm{I}$ (454.37) Ber.: C 26.43, H 2.66 Gef.: C 26.77, H 2.56; Schmp. $121^{\circ} \mathrm{C}$

AV14g. 1-Fluortricyclo[3.3.1.1 $\left.1^{3,7}\right]$ decan (47):

Obwohl literaturbekannt, wird an dieser Stelle die Synthese 47 nochmals wiedergegeben, weil die Durchführung nicht sehr detailliert beschrieben ist und auch kleinere Modifikationen vorgenommen wurden. In einer verschließbaren $200 \mathrm{~mL}$ Polyethylenflasche wurden $10.50 \mathrm{~g}$ $(0.069 \mathrm{~mol}) \quad \mathbf{4 6}$ in $70 \mathrm{~mL} n$-Pentan suspendiert und $60 \mathrm{~mL}$ eines Fluorwasserstoffpyridinkomplexes (ca. $70 \% \mathrm{HF}$ ) zugeben. Nach zweistündigem Rühren wurde die Pyridinphase von der Pentanphase getrennt und erstere mit $n$-Pentan $(3 \times 30 \mathrm{~mL})$ extrahiert. Die vereinigten organischen Phasen wurden mit Salzsäure (aq. $3 \%$ ) gewaschen und über Natriumsulfat getrocknet. Nach der Abtrennung des Lösungsmittels durch Destillation wurde das Rohprodukt aus $n$-Pentan umkristallisiert. Dabei wurden $10.00 \mathrm{~g}$ 
(0.065 mol, $94 \%)$ von 47 als weiße Kristalle erhalten. 47: Die Spektraldaten sind literaturidentisch. [180]

AV14h. 1-Chlor-3-fluortricyclo[3.3.1.1 $\left.1^{3,7}\right] \operatorname{decan}(\mathbf{4 8})$ :

$3.50 \mathrm{~g}(0.023 \mathrm{~mol}) 47$ und $365 \mathrm{mg}(0.012 \mathrm{mmol}, 5 \mathrm{~mol} \%)$ TBABr wurden in $40 \mathrm{~mL}$ Tetrachlormethan gelöst und $10 \mathrm{~mL}$ Natriumhydroxidlösung (aq. 50\%) zugesetzt. Die Mischung wurde sechs Wochen bei intensivem Rühren refluxiert. Nach der Phasentrennung wurde die wäßrige Phase mit $n$-Pentan (4 x $30 \mathrm{~mL}$ ) extrahiert. Die vereinigten organischen Phasen wurden über Natriumsulfat getrocknet und am Rotationsverdampfer unter reduziertem Druck weitestgehend vom Lösungsmittel befreit. Die erhaltenen $3.2 \mathrm{~g}$ Rohprodukt wurden einer säulenchromatographischen Trennung unterzogen ( $n$-Pentan, 6 x $40 \mathrm{~cm}, \mathrm{R}_{\mathrm{f}}(\mathbf{4 7})=0.29$, $\left.\mathrm{R}_{\mathrm{f}}(\mathbf{4 8})=0.19\right)$. Es wurden $0.87 \mathrm{~g}(4.4 \mathrm{mmol}, 19 \%)$ von 48 als weißer Feststoff erhalten. $2.8 \mathrm{~g}$ von 47 konnten zurückgewonnen werden. 48: Die Spektraldaten sind literaturidentisch.[179]

AV14i. 1-Chlor-3-fluortricyclo[3.3.1.1 $\left.1^{3,7}\right]$ decan (48, radikalisch initiiert):

$4.00 \mathrm{~g}(0.026 \mathrm{~mol}) 47$ und $550 \mathrm{mg}(2.3 \mathrm{mmol})$ Dibenzoylperoxid wurden in $60 \mathrm{~mL}$ Tetrachlormethan gelöst. Die Mischung wurde $48 \mathrm{~h}$ refluxiert, wobei nach $16 \mathrm{~h}$ erneut $1 \mathrm{~g}$ (4.1 mmol) Dibenzoylperoxid hinzugefügt wurden. Anschließend wurde am Rotationsverdampfer unter reduziertem Druck der größte Teil des Lösungsmittels entfernt. Die erhaltenen $4.2 \mathrm{~g}$ Rohprodukt wurden einer säulenchromatographischen Trennung unterzogen ( $n$-Pentan, 5 x $\left.60 \mathrm{~cm}, \mathrm{R}_{\mathrm{f}}(\mathbf{4 7})=0.29, \mathrm{R}_{\mathrm{f}}(\mathbf{4 8})=0.19\right)$. Es wurden $1.00 \mathrm{~g}(5.3 \mathrm{mmol}$, $20 \%$ ) von 48 als weißer Feststoff erhalten. 2.4 g von 47 konnten zurückgewonnen werden. 48: Die Spektraldaten sind literaturidentisch.[179]

AV14j. 1-Iod-3-fluortricyclo[3.3.1.1 $\left.1^{3,7}\right]$ decan (49):

In einem verschließbaren $25 \mathrm{~mL}$ Glasfläschchen wurden $1.10 \mathrm{~g}$ (7.1 mmol) 47 und $3.94 \mathrm{~g}$ (10.0 mmol) in $15 \mathrm{~mL}$ Dichlormethan gelöst und $1.5 \mathrm{~g}$ Natriumhydroxid (s, $37.5 \mathrm{mmol}$, Microprills) zugegeben. Das Fläschchen wurde verschlossen und die Mischung 4 Tage bei RT 
intensivst gerührt. Anschließend wurde das $\mathrm{NaOH}$ abfiltriert und dieses $n$-Pentan extrahiert (4 x $10 \mathrm{~mL}$ ). Die vereinigten organischen Phasen wurden über Natriumsulfat getrocknet und am Rotationsverdampfer bei reduziertem Druck von Lösungsmitteln befreit. Das erhaltene Rohprodukt wurde unter den o.a. Bedingungen erneut der Reaktion unterworfen und nach vier weiteren Tagen Reaktionszeit auch wieder entsprechend aufgearbeitet. Das erhaltene Rohprodukt wurde säulenchromatographisch getrennt ( $n$-Pentan, $2.5 \times 50 \mathrm{~cm}, \mathrm{R}_{\mathrm{f}}(\mathbf{4 9})=0.17$ ). Es wurden $1.00 \mathrm{~g}(5.3 \mathrm{mmol}, 20 \%)$ von 49 als weißer Feststoff erhalten. $0.3 \mathrm{~g}$ von 47 konnten zurückgewonnen werden. 49: Die Spektraldaten sind literaturidentisch.[179]

AV14k. ( \pm )-1-Brom-3-chlor-5-fluortricyclo[3.3.1.1 ${ }^{3,7}$ decan (50), 1,3-Dibrom-5-chlor-7fluortricyclo[3.3.1.1 $\left.1^{3,7}\right]$ decan (52):

$580 \mathrm{mg}$ (3.1 mmol) 48, $1.53 \mathrm{~g}$ (4.6 mmol) Tetrabrommethan und $99 \mathrm{mg}$ TBABr (0.3 mmol, $10 \mathrm{~mol} \%$ ) wurden in $10 \mathrm{~mL}$ Fluorbenzol gelöst und $5 \mathrm{~mL}$ Natriumhydroxidlösung (aq. 50\%) zugesetzt. Die Mischung wurde 6 Tage bei $65^{\circ} \mathrm{C}$ intensivst gerührt. Anschließend wurden die Phasen getrennt und die wäßrige Phase mit $n$-Pentan $(4 \times 10 \mathrm{~mL})$ extrahiert. Die vereinigten organischen Phasen wurden über Natriumsulfat getrocknet und am Rotationsverdampfer bei reduziertem Druck vom Lösungsmittel befreit. Das erhaltene Rohprodukt wurde unter o.a. Bedingungen erneut zur Reaktion gebracht. Diese Prozedur wurde noch viermal wiederholt (insgesamt 42 Tage Reaktionszeit) und das letztendlich erhaltene Rohprodukt, daß gemäß einer GC/MS-Analyse kein 48 mehr enthielt, einer säulenchromatographischen Trennung unterzogen ( $n$-Pentan, 2.5 x $\left.60 \mathrm{~cm}, \mathrm{R}_{\mathrm{f}}(\mathbf{5 0})=0.18, \mathrm{R}_{\mathrm{f}}(\mathbf{5 2})=0.27\right)$. Es wurden $170 \mathrm{mg}(0.64$ mmol, 21\%) von ( \pm )-50 und 220 mg (0.64 mmol, 21\%) von 52 als weiße Feststoffe erhalten. (土)-50: ${ }^{1} \mathbf{H}-\mathbf{N M R}\left(250 \mathrm{MHz}, \mathrm{CDCl}_{3}\right): \delta=1.83-1.87(\mathrm{~m}, 2 \mathrm{H}, 6-\mathrm{H}), 1.99$ (bs, $\left.2 \mathrm{H}, 10-\mathrm{H}\right)$, $2.12(\mathrm{bs}, 2 \mathrm{H}, 8-\mathrm{H}), 2.26\left(\mathrm{~d},{ }^{3} J_{\mathrm{F}}=5.0 \mathrm{~Hz}, 2 \mathrm{H}, 4-\mathrm{H}\right), 2.38-2.44(\mathrm{~m}, 3 \mathrm{H}, 7-\mathrm{H}, 9-\mathrm{H}), 2.56$ (s, $2 \mathrm{H}, 2-\mathrm{H}) ;{ }^{13} \mathbf{C}-\mathbf{N M R}\left(62.9 \mathrm{MHz}, \mathrm{CDCl}_{3}\right): \delta=32.83\left(\mathrm{~d},{ }^{3} J_{\mathrm{F}}=10.2 \mathrm{~Hz}, \mathrm{t}, \mathrm{C}-7\right), 39.24\left(\mathrm{~d},{ }^{2} J_{\mathrm{F}}\right.$ $=18.1 \mathrm{~Hz}, \mathrm{~s}, \mathrm{C}-6), 44.07(\mathrm{~s}, \mathrm{C}-10), 45.33(\mathrm{~s}, \mathrm{C}-8), 50.57\left(\mathrm{~d},{ }^{2} J_{\mathrm{F}}=19.9 \mathrm{~Hz}, \mathrm{~s}, \mathrm{C}-4\right), 50.57(\mathrm{~d}$, $\left.{ }^{2} J_{\mathrm{F}}=20.1 \mathrm{~Hz}, \mathrm{~s}, \mathrm{C}-9\right), 56.08(\mathrm{~s}, \mathrm{C}-2), 57.15\left(\mathrm{~d},{ }^{3} J_{\mathrm{F}}=12.2 \mathrm{~Hz}, \mathrm{q}, \mathrm{C}-1\right), 64.21\left(\mathrm{~d},{ }^{3} J_{\mathrm{F}}=12.6\right.$ Hz, q, C-3) 91.64 (d, $\left.{ }^{1} J_{\mathrm{F}}=192.5 \mathrm{~Hz}, \mathrm{q}, \mathrm{C}-1\right)$; IR $\left(\mathrm{CCl}_{4}\right)$ : 927, 1042, 1096, 1137, 1326, 1456, 1734, 2869, $2948 \mathrm{~cm}^{-1}$; MS (70 eV) m/z (\%): 187 (100) 189 (33) $\left[\mathrm{M}^{+}-\mathrm{Br}\right], 151$ (72) $\left[\mathrm{M}^{+}-\right.$ $\mathrm{ClBr}$, 131 (17) [M+ - FClBr], 111 (16), 91 (13), 77 (6), 65 (4), 51 (4); EA: $\mathrm{C}_{10} \mathrm{H}_{13} \mathrm{FClBr}$ (267.57) Ber.: C 44.89, H 4.90 Gef.: C 45.10, H 5.03; Schmp.: $82-85^{\circ} \mathrm{C}$ 52: ${ }^{1}$ H-NMR (250 $\left.\mathrm{MHz}, \mathrm{CDCl}_{3}\right): \delta=2.25\left(\mathrm{~d},{ }^{3} J_{\mathrm{F}}=4.9 \mathrm{~Hz}, 2 \mathrm{H}, 6-\mathrm{H}\right), 2.38\left(\mathrm{~d},{ }^{3} J_{\mathrm{F}}=4.9 \mathrm{~Hz}, 4 \mathrm{H}, 8-\mathrm{H}, 9-\mathrm{H}\right), 2.50$ 
$\left(\mathrm{d},{ }^{5} J_{\mathrm{F}}=0.8 \mathrm{~Hz}, 4 \mathrm{H}, 4-\mathrm{H}, 10-\mathrm{H}\right), 2.63(\mathrm{~s}, 2 \mathrm{H}, 2-\mathrm{H}) ;{ }^{13} \mathbf{C}-\mathbf{N M R}\left(62.9 \mathrm{MHz}, \mathrm{CDCl}_{3}\right): \delta=$ $49.00\left(\mathrm{~d},{ }^{2} J_{\mathrm{F}}=20.1 \mathrm{~Hz}, \mathrm{~s}, \mathrm{C}-6\right), 50.20\left(\mathrm{~d},{ }^{2} J_{\mathrm{F}}=20.3 \mathrm{~Hz}, \mathrm{~s}, \mathrm{C}-8, \mathrm{C}-9\right), 53.00\left(\mathrm{~d},{ }^{3} J_{\mathrm{F}}=12.9\right.$ $\mathrm{Hz}, \mathrm{q}, \mathrm{C}-1, \mathrm{C}-3$ ), 54.23 (s, C-4, C-10), 55.23 (s, C-2), 61.72 (q, C-5), 90.58 (d, ${ }^{1} J_{\mathrm{F}}=196.2$ $\mathrm{Hz}, \mathrm{q}, \mathrm{C}-7)$; IR $\left(\mathrm{CCl}_{4}\right): 547,938,1110,1333,1456,2968,3853 \mathrm{~cm}^{-1} ; \mathbf{M S}(70 \mathrm{eV}) \mathrm{m} / \mathrm{z}$ (\%):165 (77) 267 (100) $269(25)\left[\mathrm{M}^{+}-\mathrm{Br}\right], 229$ (4) 231 (4) $\left[\mathrm{M}^{+}-\mathrm{ClBr}\right], 209$ (8) 211 (8) $\left[\mathrm{M}^{+}\right.$

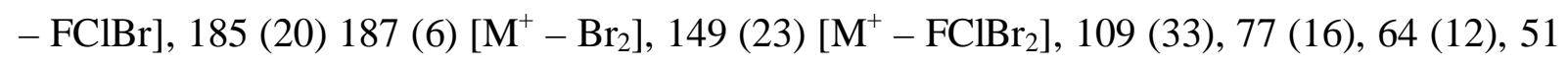
(14); EA: $\mathrm{C}_{10} \mathrm{H}_{12} \mathrm{FClBr}_{2}$ (346.46) Ber.: C 34.67, H 3.49 Gef.: C 34.96, H 3.67; Schmp.: 132 $133^{\circ} \mathrm{C}$

AV14l. ( \pm )-1-Iod-3-brom-5-fluortricyclo[3.3.1.1 $\left.{ }^{3,7}\right]$ decan (51), 1-Iod-3,5-dibrom-7-fluortricyclo[3.3.1.1 $\left.1^{3,7}\right]$ decan $(\mathbf{5 3})$ :

$450 \mathrm{mg}$ (1.3 mmol) 1-Iod-3-fluordamantan 49, $1.25 \mathrm{~g}$ (3.9 mmol) Tetrabrommethan und 42 $\mathrm{mg}(0.13 \mathrm{mmol}, 10 \mathrm{~mol} \%) \mathrm{TBABr}$ wurden in $10 \mathrm{~mL}$ Fluorbenzol gelöst und $3 \mathrm{~mL}$ Natriumhydroxidlösung (aq. 50\%) zugesetzt. Die Mischung wurde 5 Tage bei $70^{\circ} \mathrm{C}$ intensiv gerührt. Anschließend wurden die Phasen getrennt und die wäßrige Phase mit $n$-Pentan $(4 \mathrm{x}$ $10 \mathrm{~mL}$ ) extrahiert. Die vereinigten organischen Phasen wurden über Natriumsulfat getrocknet und am Rotationsverdampfer bei reduziertem Druck vom Lösungsmittel befreit. Die erhaltenen 2 g Rohprodukt wurden unter o.a. Bedingungen erneut zur Reaktion gebracht. Diese Prozedur wurde noch einmal wiederholt (insgesamt 15 Tage Reaktionszeit, bis durch GC/MS-Analyse kein 15 mehr nachweisbar war), die Aufarbeitung in oben beschriebener Weise erneut durchgeführt und das Rohprodukt einer säulenchromatographischen Trennung unterzogen ( $n$-Pentan, 2.5 x $\left.60 \mathrm{~cm}, \mathrm{R}_{\mathrm{f}}(\mathbf{5 1})=0.21, \mathrm{R}_{\mathrm{f}}(\mathbf{5 3})=0.30\right)$. Es wurden $154 \mathrm{mg}(0.43$ mmol, $33 \%)$ von $\mathbf{5 1}$ und $34 \mathrm{mg}(0.08 \mathrm{mmol}, 6 \%)$ von $\mathbf{5 3}$ als weiße Feststoffe erhalten. 51: ${ }^{1} \mathbf{H}-\mathbf{N M R}\left(250 \mathrm{MHz}, \mathrm{CDCl}_{3}\right): \delta=1.91-1.96$ (m, 2 H, 6-H), 2.22 (s, $\left.2 \mathrm{H}, 10-\mathrm{H}\right), 2.21-2.30$ (m, 1 H, 7-H), 2.34 (s, 2 H, 8-H), 2.45-2.49 (m, 2 H, 4-H), 2.61-2.64 (m, 2 H, 9-H), 2.92 (s, $2 \mathrm{H}, 2-\mathrm{H}){ }^{13} \mathbf{C}-\mathbf{N M R}\left(62.9 \mathrm{MHz}, \mathrm{CDCl}_{3}\right): \delta=34.83\left(\mathrm{~d},{ }^{3} J_{\mathrm{F}}=9.6 \mathrm{~Hz}, \mathrm{t}, \mathrm{C}-7\right), 36.23\left(\mathrm{~d},{ }^{3} J_{\mathrm{F}}=\right.$ $11.1 \mathrm{~Hz}, \mathrm{q}, \mathrm{C}-1), 45.31$ (s, C-10), 47.94 (s, C-8), 51.92 (d, $\left.{ }^{2} J_{\mathrm{F}}=20.1 \mathrm{~Hz}, \mathrm{~s}, \mathrm{C}-4\right), 54.57$ (d, $\left.{ }^{2} J_{\mathrm{F}}=19.9 \mathrm{~Hz}, \mathrm{~s}, \mathrm{C}-9\right), 57.45\left(\mathrm{~d},{ }^{3} J_{\mathrm{F}}=11.3 \mathrm{~Hz}, \mathrm{q}, \mathrm{C}-3\right), 59.71(\mathrm{~s}, \mathrm{C}-6), 90.83\left(\mathrm{~d},{ }^{1} J_{\mathrm{F}}=193.9\right.$ Hz, q, C-5) IR $\left(\mathrm{CCl}_{4}\right):$ 543, 923, 1094, 1136, 1290, 1322, 1355, 1454, 2868, $2948 \mathrm{~cm}^{-1}$; MS

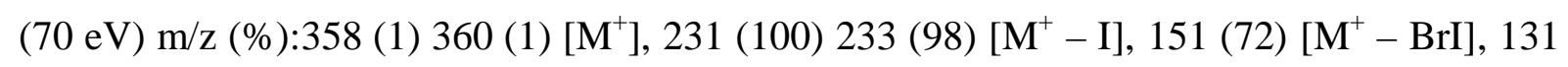
(22) $\left[\mathrm{M}^{+}\right.$- FBrI], 91 (28), 77 (14), 65 (10), 55 (8) EA: $\mathrm{C}_{10} \mathrm{H}_{13} \mathrm{FBrI}$ (359.02) Ber.: C 33.45, H 3.65 Gef.: C 33.72, H 3.45 Schmp.: $84-85^{\circ} \mathrm{C}$ 53: ${ }^{1} \mathbf{H}-\mathbf{N M R}\left(250 \mathrm{MHz}, \mathrm{CDCl}_{3}\right): \delta=2.38-$ 
$2.87(\mathrm{~m}, 12 \mathrm{H}) ;{ }^{13} \mathbf{C}-\mathbf{N M R}\left(62.9 \mathrm{MHz}, \mathrm{CDCl}_{3}\right): \delta=30.31\left(\mathrm{~d},{ }^{4} J_{\mathrm{F}}=11.1 \mathrm{~Hz}, \mathrm{q}, \mathrm{C}-7\right), 50.19$ $\left(\mathrm{d},{ }^{2} J_{\mathrm{F}}=20.3 \mathrm{~Hz}, \mathrm{~s}, \mathrm{C}-6, \mathrm{C}-10\right), 52.62\left(\mathrm{~d},{ }^{2} J_{\mathrm{F}}=20.2 \mathrm{~Hz}, \mathrm{~s}, \mathrm{C}-8\right), 53.57\left(\mathrm{~d},{ }^{3} J_{\mathrm{F}}=11.8 \mathrm{~Hz}, \mathrm{q}\right.$, C-3, C-5), 55.20 (s, C-4), 57.52 (s, C-2, C-9), 90.04 (d, $\left.{ }^{1} J_{\mathrm{F}}=198.2 \mathrm{~Hz}, \mathrm{q}, \mathrm{C}-7\right)$; IR $\left(\mathrm{CCl}_{4}\right)$ : 545, 927, 1039, 1099, 1332, 1456, 2868, $2965 \mathrm{~cm}^{-1}$; MS (70 eV); m/z (\%): 436 (1) 438 (2)

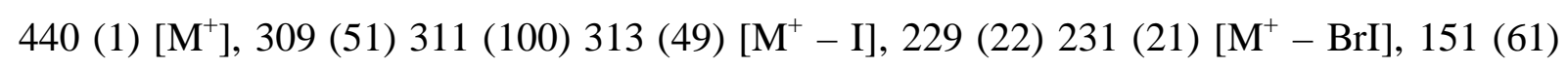
$\left[\mathrm{M}^{+}-\mathrm{Br}_{2} \mathrm{I}\right], 132$ (9) $\left[\mathrm{M}^{+}-\mathrm{FBr}_{2} \mathrm{I}\right], 109$ (48), 91 (44), 77 (10), 65 (12), 51 (11); EA: $\mathrm{C}_{10} \mathrm{H}_{12} \mathrm{FBr}_{2} \mathrm{I}$ (437.91) Ber.: C 27.43, H 2.76 Gef.: C 27.74, H 2.92; Schmp.: $114^{\circ} \mathrm{C}$

AV14m. 1-Chlor-3-phenyltricyclo[3.3.1.1 $\left.1^{3,7}\right]$ decan (57), 1,3-Dichlor-5-phenyltricyclo$\left[3.3 .1 .1^{3,7}\right]$ decan $(\mathbf{6 4})$ :

$212 \mathrm{mg}$ (1.0 mmol) 56 und $32 \mathrm{mg}(0.1 \mathrm{mmol}, 10 \mathrm{~mol} \%)$ TBABr wurden in $10 \mathrm{~mL}$ Tetrachlormethan gelöst und $3 \mathrm{~mL}$ Natriumhydroxidlösung (aq. 50\%) zugegeben. Die Mischung wurde 8 Tage bei intensivem Rühren refluxiert. Nach der Phasentrennung wurde die wäßrige Phase mit $n$-Pentan $(4 \times 30 \mathrm{~mL})$ extrahiert. Die vereinigten organischen Phasen wurden über Natriumsulfat getrocknet und am Rotationsverdampfer unter reduziertem Druck weitestgehenden vom Lösungsmittel befreit. Die erhaltenen 250 mg Rohprodukt wurden einer säulenchromatographischen Trennung unterzogen ( $n$-Pentan, 2.5 x $50 \mathrm{~cm}, \mathrm{R}_{\mathrm{f}}(\mathbf{5 7})=0.23$, $\left.\mathrm{R}_{\mathrm{f}}(\mathbf{6 4})=0.15\right)$. Es wurden $138 \mathrm{mg}(0.56 \mathrm{mmol}, 56 \%)$ von 57 und $28 \mathrm{mg}(0.1 \mathrm{mmol}, 10 \%)$ von 64 als weiße Feststoffe erhalten. $50 \mathrm{mg}$ von 56 konnten zurückgewonnen werden. 57: ${ }^{1} \mathbf{H}-\mathbf{N M R}\left(250 \mathrm{MHz}, \mathrm{CDCl}_{3}\right): \delta=1.71\left(\mathrm{~d},{ }^{3} J_{7-\mathrm{H}}=3.0 \mathrm{~Hz}, 2 \mathrm{H}, 6-\mathrm{H}\right), 1.88\left(\mathrm{~d},{ }^{3} J_{7-\mathrm{H}}=2.8 \mathrm{~Hz}\right.$, 4 H, 4-H, 10-H), 2.18 (s, 4 H, 8-H, 9-H), 2.31 (s, 2 H, 2-H), 2.29 - 2.34 (m, 2 H, 5-H, 7-H), $7.21-7.28$ (m, 1 H, 4'-H), $7.33-7.35$ (m, 4 H, 2'-H, 3'-H, 5'-H, 6'-H); ${ }^{13} \mathbf{C}-\mathbf{N M R}(62.9$ $\mathrm{MHz}, \mathrm{CDCl}_{3}$ ): $\delta=31.86$ (t, C-5, C-7), 34.73 (s, C-6), 40.54 (q, C-3), 41.34 (s, C-4, C-10), 46.86 (s, C-8, C-9), 52.72 (s, C-2), 68.84 (q, C-1), 124.73 (s, C-2', C-6'), 126.11 (s, C-4'), 128.33 (s, C-3', C-5'), 148.73 (q, C-1'); IR $\left(\mathrm{CCl}_{4}\right):$ 538, 684, 795, 863, 907, 950, 1041, 1147 , 1310, 1333, 1446, 2858, 2946, 3034, 3060, $3090 \mathrm{~cm}^{-1}$; MS (70 eV) m/z (\%): 246 (90) 248 (31) $\left[\mathrm{M}^{+}\right], 211(61)\left[\mathrm{M}^{+}-\mathrm{Cl}\right], 155$ (100) $\left[\mathrm{M}^{+}-\mathrm{ClPh}\right], 128$ (29), 115 (32), 91 (62), 77 (33), 65 (11), 51 (8); EA: $\mathrm{C}_{16} \mathrm{H}_{19} \mathrm{Cl}$ (246.77) Ber.: C 77.87, H 7.76 Gef.: C 78.05, H 7.65 Schmp.: $91^{\circ} \mathrm{C}$ 64: ${ }^{1} \mathbf{H}-\mathbf{N M R}\left(250 \mathrm{MHz}, \mathrm{CDCl}_{3}\right): \delta=1.84\left(\mathrm{~d},{ }^{3} J_{7-\mathrm{H}}=4.6 \mathrm{~Hz}, 2 \mathrm{H}, 6-\mathrm{H}\right), 2.11\left(\mathrm{~d},{ }^{3} J_{7-\mathrm{H}}\right.$ $=5.0 \mathrm{~Hz}, 4 \mathrm{H}, 8-\mathrm{H}, 9-\mathrm{H}), 2.27(\mathrm{~s}, 4 \mathrm{H}, 4-\mathrm{H}, 10-\mathrm{H}), 2.51\left(\mathrm{qq},{ }^{3} J_{6-\mathrm{H}}=4.6 \mathrm{~Hz},{ }^{3} J_{8-\mathrm{H}}, 9-\mathrm{H}=5.0\right.$ $\mathrm{Hz}, 1 \mathrm{H}, 7-\mathrm{H}), 2.53$ (s, $2 \mathrm{H}, 2-\mathrm{H}), 7.22-7.41$ (m, arom. $5 \mathrm{H}) ;{ }^{13} \mathbf{C}-\mathbf{N M R}\left(62.9 \mathrm{MHz}, \mathrm{CDCl}_{3}\right)$ : $\delta=33.10$ (t, C-7), 39.63 (s, C-6), 43.05 (q, C-5), 44.88 (s, C-8, C-9), 51.10 (s, C-4, C-10), 
55.65 (s, C-2), 66.66 (q, C-1, C-3), 124.67 (s, C-2', C-6'), 126.68 (s, C-4'), 128.57 (s, C-3', C-5'), 146.53 (q, C-1'); IR $\left(\mathrm{CCl}_{4}\right)$ : 540, 695, 872, 1023, 1042, 1137, 1323, 1447, 2864, 2946,

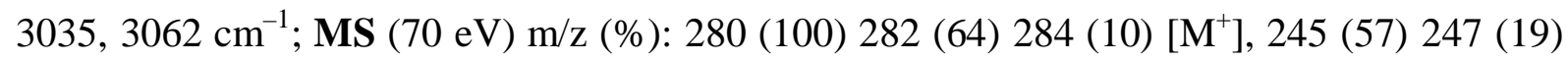
$\left[\mathrm{M}^{+}-\mathrm{Cl}\right], 209$ (19) $\left[\mathrm{M}^{+}-\mathrm{Cl}_{2}\right], 189$ (88), 91 (62) $\left[\mathrm{C}_{7} \mathrm{H}_{7}{ }^{+}\right], 77(23)\left[\mathrm{C}_{6} \mathrm{H}_{5}{ }^{+}\right] ; \mathbf{E A}: \mathrm{C}_{16} \mathrm{H}_{18} \mathrm{Cl}_{2}$ (281.22) Ber.: C 68.33, H 6.45 Gef.: C 68.05, H 6.36; Schmp.: $74^{\circ} \mathrm{C}$

AV14n. 1-Brom-3-phenyltricyclo[3.3.1.1 $\left.1^{3,7}\right]$ decan (59), 1,3-dibrom-5-phenyltricyclo[3.3.1.1 $\left.1^{3,7}\right]$ decan (65): 1,3,5-tribrom-7-phenyltricyclo[3.3.1.1 $\left.1^{3,7}\right]$ decan (66)

$212 \mathrm{mg}$ (1.0 mmol) 56, $1.33 \mathrm{~g}$ (4.0 mmol) Tetrabrommethan und $32 \mathrm{mg}(0.1 \mathrm{mmol}, 10 \mathrm{~mol} \%)$ TBABr wurden in $10 \mathrm{~mL}$ Fluorbenzol gelöst und $3 \mathrm{~mL}$ Natriumhydroxidlösung (aq. 50\%) zugesetzt. Die Mischung wurde 4 Tage bei $70^{\circ} \mathrm{C}$ intensiv gerührt. Anschließend wurden die Phasen getrennt und die wäßrige Phase mit $n$-Pentan $(4$ x $10 \mathrm{~mL})$ extrahiert. Die vereinigten organischen Phasen wurden über Natriumsulfat getrocknet und am Rotationsverdampfer bei reduziertem Druck vom Lösungsmittel befreit. Die erhaltenen $450 \mathrm{mg}$ Rohprodukt wurden einer säulenchromatographischen Trennung unterzogen $\left(n\right.$-Pentan, $2.5 \times 50 \mathrm{~cm}, \mathrm{R}_{\mathrm{f}}(\mathbf{5 9})=0.25$, $\left.\mathrm{R}_{\mathrm{f}}(\mathbf{6 5})=0.16, \mathrm{R}_{\mathrm{f}}(\mathbf{6 6})=0.31\right)$. Es wurden $180 \mathrm{mg}(0.61 \mathrm{mmol}, 61 \%)$ von 59 und $74 \mathrm{mg}(0.20$ mmol, 20\%) von $\mathbf{6 5}$ als weiße Feststoffe erhalten. 50 mg von 56 konnten zurückgewonnen werden. Bei exakt gleicher Versuchsdurchführung aber längerer Reaktionszeit (8 Tage) wurden $120 \mathrm{mg}(0.32 \mathrm{mmol}, 32 \%)$ von 65 und $112 \mathrm{mg}(0.24 \mathrm{mmol}, 24 \%)$ von 66 isoliert. In diesem Fall konnten weder Ausgangsprodukt 56 noch 59 mehr nachgewiesen werden. 59: Die NMR Spektraldaten von 59 entsprechen den Literaturdaten. ${ }^{[200]}$ 65: ${ }^{\mathbf{1}} \mathbf{H}-\mathbf{N M R}(250 \mathrm{MHz}$, $\left.\mathrm{CDCl}_{3}\right): \delta=1.94\left(\mathrm{~d},{ }^{3} J_{7-\mathrm{H}}=2.8 \mathrm{~Hz}, 2 \mathrm{H}, 6-\mathrm{H}\right), 2.34\left(\mathrm{~d},{ }^{3} J_{7-\mathrm{H}}=3.0 \mathrm{~Hz}, 4 \mathrm{H}, 8-\mathrm{H}, 9-\mathrm{H}\right), 2.42$ $\left(\mathrm{qq},{ }^{3} J_{6-\mathrm{H}}=2.8 \mathrm{~Hz},{ }^{3} J_{8-\mathrm{H}, 9-\mathrm{H}}=3.0 \mathrm{~Hz}, 1 \mathrm{H}, 7-\mathrm{H}\right), 2.50(\mathrm{~s}, 4 \mathrm{H}, 4-\mathrm{H}, 10-\mathrm{H}), 2.92$ (s, $2 \mathrm{H}, 2-$ H), $7.23-7.40$ (m, arom. 5H); ${ }^{13} \mathbf{C}-\mathbf{N M R}\left(62.9 \mathrm{MHz}, \mathrm{CDCl}_{3}\right): \delta=34.68(\mathrm{t}, \mathrm{C}-7), 39.40(\mathrm{~s}$, C-6), 44.42 (q, C-5), 46.18 (s, C-8, C-9), 52.39 (s, C-4, C-10), 58.15 (s, C-2), 61.65 (q, C1, C-3), 124.59 (s, C-2', C-6'), 126.73 (s, C-4'), 128.60 (s, C-3', C-5'), 146.42 (q, C-1'); IR $\left(\mathrm{CCl}_{4}\right):$ 540, 667, 907, 958, 1040, 1135, 1286, 1319, 1341, 1446, 2862, 2945, 3035, $3061 \mathrm{~cm}^{-}$

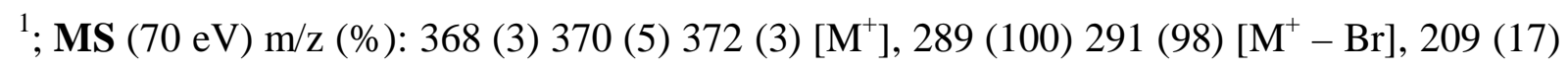
$\left[\mathrm{M}^{+}-\mathrm{Br}_{2}\right], 154$ (41), 91 (54) $\left[\mathrm{C}_{7} \mathrm{H}_{7}^{+}\right], 77$ (19) $\left[\mathrm{C}_{6} \mathrm{H}_{5}^{+}\right]$; EA: $\mathrm{C}_{16} \mathrm{H}_{18} \mathrm{Br}_{2}$ (370.12) Ber.: C 51.92, H 4.90 Gef.: C 51.71, H 4.90; Schmp.: $76-78^{\circ} \mathrm{C}$ 66: ${ }^{1} \mathbf{H}-\mathbf{N M R}\left(250 \mathrm{MHz}, \mathrm{CDCl}_{3}\right): \delta$ $=2.45(\mathrm{~s}, 6 \mathrm{H}, 6-\mathrm{H}, 8-\mathrm{H}, 9-\mathrm{H}), 2.82(\mathrm{~s}, 6 \mathrm{H}, 2-\mathrm{H}, 4-\mathrm{H}, 10-\mathrm{H}), 7.27-7.40(\mathrm{~m}$, arom. $5 \mathrm{H})$; ${ }^{13} \mathbf{C}-\mathbf{N M R}\left(62.9 \mathrm{MHz}, \mathrm{CDCl}_{3}\right.$ ): $\delta=46.32$ (q, C-7), 50.56 (s, C-6, C-8, C-9), 56.01 (s, C-2, 
C-4, C-10), 56.69 (q, C-1, C-3, C-5), 124.49 (s, C-4'), 127.22 (s, C-3', C-5'), 144.44 (q, C1'); IR $\left(\mathrm{CCl}_{4}\right)$ : 542, 696, 784, 1042, 1133, 1318, 1447, 2863, 2962, 3036, 3062, $3092 \mathrm{~cm}^{-1}$;

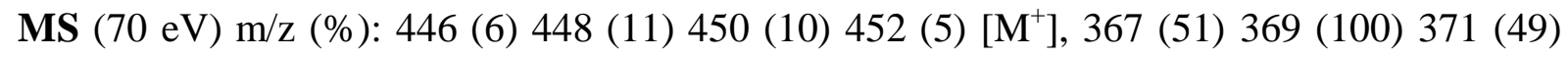
$\left[\mathrm{M}^{+}-\mathrm{Br}\right], 287$ (17) 289 (16) $\left[\mathrm{M}^{+}-\mathrm{Br}_{2}\right], 208$ (31) $\left[\mathrm{M}^{+}-\mathrm{Br}_{3}\right], 167$ (27), 115 (32), 91 (71) $\left[\mathrm{C}_{7} \mathrm{H}_{7}{ }^{+}\right], 77$ (20) $\left[\mathrm{C}_{6} \mathrm{H}_{5}^{+}\right]$; EA: $\mathrm{C}_{16} \mathrm{H}_{17} \mathrm{Br}_{3}$ (449.02) Ber.: C 42.80, H 3.82 Gef.: C 42.67, H 3.64; Schmp.: $119^{\circ} \mathrm{C}$

AV14o. 1-Iod-3-phenyltricyclo[3.3.1.1 $\left.1^{3,7}\right]$ decan $\quad(60), \quad 1,3-D i i o d-5-$ phenyltricyclo[3.3.1.1 $\left.1^{3,7}\right]$ decan $(\mathbf{6 7})$ :

$220 \mathrm{mg}$ (1.0 mmol) 56, $1.97 \mathrm{~g}$ (5.0 mmol) Iodoform und $32 \mathrm{mg}(0.1 \mathrm{mmol}, 10 \mathrm{~mol} \%) \mathrm{TBABr}$ in $10 \mathrm{~mL}$ Fluorbenzol gelöst und $2 \mathrm{~mL} \mathrm{NaOH} \mathrm{Lsg.} \mathrm{(aq.} 50 \%$ ) zugegeben. Die Mischung wurde 4 Tage bei $70^{\circ} \mathrm{C}$ intensivst gerührt. Anschließend wurden die Phasen getrennt und die wäßrige Phase mit $n$-Pentan extrahiert (4 x $10 \mathrm{~mL}$ ). Die vereinigten organischen Phasen wurden über Natriumsulfat getrocknet und am Rotationsverdampfer unter reduziertem Druck weitestgehend von Lösungsmitteln befreit. Das erhaltene Rohprodukt wurde unter den o.a. Bedingungen erneut der Reaktion unterworfen und nach vier weiteren Tagen Reaktionszeit wieder entsprechend aufgearbeitet. Das erhaltene Rohprodukt wurde einer säulenchromatographischen Trennung unterworfen ( $n$-Pentan, $2.5 \times 50 \mathrm{~cm}, \mathrm{R}_{\mathrm{f}}(\mathbf{6 0})=0.28$, $\left.\mathrm{R}_{\mathrm{f}}(\mathbf{6 7})=0.19\right)$. Es wurden $217 \mathrm{mg}(0.64 \mathrm{mmol}, 64 \%)$ von 60 und $93 \mathrm{mg}(0.20 \mathrm{mmol}, 20 \%)$ von 67 als weiße Feststoffe erhalten. $35 \mathrm{mg}$ von 56 konnten zurückgewonnen werden. 60: ${ }^{1} \mathbf{H}-\mathbf{N M R}\left(250 \mathrm{MHz}, \mathrm{CDCl}_{3}\right): \delta=1.83-1.87$ (m, $\left.2 \mathrm{H}, 6-\mathrm{H}\right), 2.03-2.04$ (m, 4 H, 8-H, 9-H), $2.15-2.18$ (m, 2 H, 5-H, 7-H), $2.62-2.74$ (m, 4 H, 4-H, 10-H), 2.81 (s, 2 H, 2-H), 7.21 7.32 (m, arom. 2H), $7.34-7.41\left(\mathrm{~m}\right.$, arom. 3H); ${ }^{13} \mathbf{C}-\mathbf{N M R}\left(62.9 \mathrm{MHz}, \mathrm{CDCl}_{3}\right): \delta=31.10(\mathrm{t}$, C-5, C-7), 34.79 (s, C-6), 41.17 (s, C-4, C-10), 41.35 (q, C-3), 49.70 (q, C-1), 51.53 (s, C-8, C-9), 57.53 (s, C-2), 124.63 (s, C-2', C-6'), 126.16 (s, C-4'), 128.36 (s, C-3', C-5'), 148.68 (q, C-1'); IR $\left(\mathrm{CCl}_{4}\right):$ 536, 669, 944, 1039, 1305, 1330, 1447, 2856, 2937, $3060 \mathrm{~cm}^{-1}$; MS (70 eV) m/z (\%): 211 (100) $\left[\mathrm{M}^{+}-\mathrm{I}\right], 169$ (7), 155 (36), 91 (20) $\left[\mathrm{C}_{7} \mathrm{H}_{7}{ }^{+}\right], 77$ (8) $\left[\mathrm{C}_{6} \mathrm{H}_{5}^{+}\right]$; EA: $\mathrm{C}_{16} \mathrm{H}_{19} \mathrm{I}$ (338.23) Ber.: C 56.82, H 5.66 Gef.: C 56.76, H 5.82; Schmp.: $94{ }^{\circ} \mathrm{C}$; 67: ${ }^{1} \mathbf{H}-\mathbf{N M R}$ $\left(250 \mathrm{MHz}, \mathrm{CDCl}_{3}\right): \delta=2.09$ (s, $\left.2 \mathrm{H}, 6-\mathrm{H}\right), 2.10-2.15$ (m, $\left.1 \mathrm{H}, 7-\mathrm{H}\right), 2.63$ (s, $4 \mathrm{H}, 8-\mathrm{H}, 9-$ H), 2.80 (s, 4 H, 4-H, 7-H), 4.59 (s, 2 H, 2-H), 7.23-7.40 (m, arom. 5 H); ${ }^{13}$ C-NMR (62.9 $\mathrm{MHz}, \mathrm{CDCl}_{3}$ ): $\delta=36.20$ (t, C-7), 39.26 (s, C-6), 43.51 (q, C-1, C-3), 45.35 (q, C-5), 49.08 (s, C-8, C-9), 55.28 (s, C-4, C-10), 63.37 (s, C-2), 124.46 (s, C-2', C-6'), 126.71 (s, C-4'), 
128.59 (s, C-3', C-5'), 146.43 (q, C-1'); IR $\left(\mathrm{CCl}_{4}\right):$ 539, 689, 952, 1039, 1283, 1316, 1340, 1447, 2858, 2938, $3061 \mathrm{~cm}^{-1}$; MS (70 eV); m/z (\%): 337 (100) $\left[\mathrm{M}^{+}-\mathrm{I}\right], 210(20)\left[\mathrm{M}^{+}-\mathrm{I}_{2}\right]$, 169 (21), 155 (46), 91 (40) $\left[\mathrm{C}_{7} \mathrm{H}_{7}^{+}\right], 77$ (13) $\left[\mathrm{C}_{6} \mathrm{H}_{5}^{+}\right]$; EA: $\mathrm{C}_{16} \mathrm{H}_{18} \mathrm{I}_{2}$ (464.12) Ber.: C 41.41, H 3.91 Gef.: C 41.20, H 3.65; Schmp.: $108^{\circ} \mathrm{C}$

AV14p. 1-Iod-3-chlor-5-phenyltricyclo[3.3.1.1 $\left.{ }^{3,7}\right]$ decan (58)

$800 \mathrm{mg}(3.5 \mathrm{mmol}) \mathbf{5 7}, 2.78 \mathrm{~g}(7.0 \mathrm{mmol})$ Iodoform und $112 \mathrm{mg}(0.35 \mathrm{mmol}, 10 \mathrm{~mol} \%)$ $\mathrm{TBABr}$ in $20 \mathrm{~mL}$ Fluorbenzol gelöst und $5 \mathrm{~mL} \mathrm{NaOH} \mathrm{Lsg.} \mathrm{(aq.} 50 \%$ ) zugegeben. Die Mischung wurde 5 Tage bei $50^{\circ} \mathrm{C}$ intensivst gerührt. Anschließend wurden die Phasen getrennt und die wäßrige Phase mit $n$-Pentan extrahiert (4 x $10 \mathrm{~mL})$. Die vereinigten organischen Phasen wurden über Natriumsulfat getrocknet und am Rotationsverdampfer unter reduziertem Druck weitestgehend von Lösungsmitteln befreit. Die erhaltenen 2 g Rohprodukt wurde einer säulenchromatographischen Trennung unterworfen ( $n$-Pentan, 4 x $60 \mathrm{~cm}, \mathrm{R}_{\mathrm{f}}(\mathbf{5 8})$ = 0.21). Es wurden $300 \mathrm{mg}(0.81 \mathrm{mmol}, 23 \%)$ von 58 als weißer Feststoff erhalten. $420 \mathrm{mg}$ von 57 konnten zurückgewonnen werden. 58: ${ }^{1} \mathbf{H}-\mathbf{N M R}\left(250 \mathrm{MHz}, \mathrm{CDCl}_{3}\right): \delta=1.95\left(\mathrm{~d},{ }^{3} J_{7-}\right.$ $\mathrm{H}=2.9 \mathrm{~Hz}, 2 \mathrm{H}, \mathrm{H}-9), 2.21(\mathrm{~s}, 2 \mathrm{H}, 6-\mathrm{H}), 2.63\left(\mathrm{qq},{ }^{3} J_{8-\mathrm{H}}=3.0 \mathrm{~Hz},{ }^{3} J_{9-\mathrm{H}}=2.9 \mathrm{~Hz}, 1 \mathrm{H}, 7-\mathrm{H}\right)$, $2.36(\mathrm{~s}, 2 \mathrm{H}, 4-\mathrm{H}), 2.51\left(\mathrm{~d},{ }^{3} J_{7-\mathrm{H}}=3.0 \mathrm{~Hz}, 2 \mathrm{H}, 8-\mathrm{H}\right), 2.67$ (s, $\left.2 \mathrm{H}, 10-\mathrm{H}\right), 2.94(\mathrm{~s}, 2 \mathrm{H}, 2-\mathrm{H})$, 7.22-7.39 (m, arom. $5 \mathrm{H}) ;{ }^{13} \mathbf{C}-\mathbf{N M R}\left(62.9 \mathrm{MHz}, \mathrm{CDCl}_{3}\right): \delta=34.49$ (t, C-7), 39.42 (s, C-6), 42.67 (q, C-1), 44.07 (q, C-5), 44.87 (s, C-8), 49.05 (s, C-9), 50.89 (s, C-4), 55.48 (s, C10), 59.68 (s, C-2), 66.24 (q, C-3), 124.54 (s, C-2', C-6'), 126.67 (s, C-4'), 128.55 (s, C-3', C-5'), 146.43 (q, C-1'); IR $\left(\mathrm{CCl}_{4}\right)$ : 541, 665, 955, 1040, 1134, 1285, 1318, 1446, 2860, 2940, $3061 \mathrm{~cm}^{-1}$; MS (70 eV); m/z (\%): 372 (1) $\left[\mathrm{M}^{+}\right], 245$ (100) $247(32)\left[\mathrm{M}^{+}-\mathrm{I}\right], 209(8)\left[\mathrm{M}^{+}-\right.$ $\mathrm{Br}, \mathrm{Cl}], 127$ (29), 91 (35) $\left[\mathrm{C}_{7} \mathrm{H}_{7}{ }^{+}\right], 77$ (14) $\left[\mathrm{C}_{6} \mathrm{H}_{5}{ }^{+}\right.$]; EA: $\mathrm{C}_{16} \mathrm{H}_{18} \mathrm{ClI}$ (371.66) Ber.: C 51.71, H 4.61 Gef.: C 51.78, H 4.77; Schmp.: $38^{\circ} \mathrm{C}$

AV14q. ( \pm )-1-Brom-3-chlor-5-phenyltricyclo[3.3.1.1 $\left.{ }^{3,7}\right]$ decan (62), 1,3-Dibrom-5-chlor7-phenyltricyclo[3.3.1.1 $\left.1^{3,7}\right]$ decan $(\mathbf{6 8})$ :

$500 \mathrm{mg}$ (1.5 mmol) 57, $1.49 \mathrm{~g}$ (4.5 mmol) Tetrabrommethan und $48 \mathrm{mg}$ (0.15 mmol, 10 mol\%) TBABr wurden in $12 \mathrm{~mL}$ Fluorbenzol gelöst und $5 \mathrm{~mL}$ Natriumhydroxidlösung (aq. $50 \%$ ) zugegeben. Die Mischung wurde 4 Tage bei $70^{\circ} \mathrm{C}$ intensiv gerührt. Anschließend 
wurden die Phasen getrennt und die wäßrige Phase mit $n$-Pentan (4 x $10 \mathrm{~mL})$ extrahiert. Die vereinigten organischen Phasen wurden über Natriumsulfat getrocknet und am Rotationsverdampfer unter reduziertem Druck weitestgehend von Lösungsmitteln befreit. Das erhaltene Rohprodukt wurde unter den o.a. Bedingungen erneut der Bromierung unterworfen, nach weiteren 4 Tagen Reaktionszeit in gleicher Weise aufgearbeitet und die $1.1 \mathrm{~g}$ an finalem Rohprodukt einer säulenchromatographischen Trennung unterzogen ( $n$-Pentan, 2 x $45 \mathrm{~cm}$, $\left.\mathrm{R}_{\mathrm{f}}(\mathbf{6 2})=0.08, \mathrm{R}_{\mathrm{f}}(\mathbf{6 8})=0.17\right)$. Es wurden $300 \mathrm{mg}(0.92 \mathrm{mmol}, 61 \%)$ von 62 und $104 \mathrm{mg}(0.26$ mmol, 17\%) von 68 als weiße Feststoffe erhalten. 100 mg von 57 konnten zurückgewonnen werden. 62: ${ }^{1} \mathbf{H}-\mathbf{N M R}\left(250 \mathrm{MHz}, \mathrm{CDCl}_{3}\right): \delta=1.88\left(\mathrm{~d},{ }^{3} J_{7-\mathrm{H}}=3.0 \mathrm{~Hz}, 2 \mathrm{H}, 9-\mathrm{H}\right), 2.14\left(\mathrm{~d},{ }^{3} J_{7-}\right.$ $\mathrm{H}=3.1 \mathrm{~Hz}, 2 \mathrm{H}, 8-\mathrm{H}), 2.30(\mathrm{~s}, 4 \mathrm{H}, 4-\mathrm{H}, 6-\mathrm{H}), 2.45(\mathrm{~s}, 2 \mathrm{H}, 10-\mathrm{H}), 2.47\left(\mathrm{qq},{ }^{3} J_{8-\mathrm{H}}=3.0 \mathrm{~Hz}\right.$, $\left.{ }^{3} J_{9-\mathrm{H}}=3.1 \mathrm{~Hz}, 1 \mathrm{H}, 7-\mathrm{H}\right), 2.72(\mathrm{~s}, 2 \mathrm{H}, 2-\mathrm{H}), 7.22-7.39(\mathrm{~m}$, arom. $5 \mathrm{H}) ;{ }^{13} \mathbf{C}-\mathbf{N M R}(62.9$ $\mathrm{MHz}, \mathrm{CDCl}_{3}$ ): $\delta=33.83$ (t, C-7), 39.50 (s, C-6), 43.69 (q, C-5), 44.82 (s, C-8), 46.21 (s, C9), 50.97 (s, C-10), 52.49 (s, C-9), 55.92 (s, C-2), 61.56 (q, C-1), 66.60 (q, C-3), 124.61 (s, C-2', C-6'), 126.68 (s, C-4'), 128.56 (s, C-3', C-5'), 146.46 (q, C-1'); IR ( $\left.\mathrm{CCl}_{4}\right)$ : 539, 696, 869, 899, 958, 1040, 1109, 1286, 1320, 1447, 1497, 2863, 2947, 3035, 3061, $3091 \mathrm{~cm}^{-1}$; MS $(70 \mathrm{eV}) ; \mathrm{m} / \mathrm{z}(\%): 324$ (4) 326 (5) 328 (2) $\left[\mathrm{M}^{+}\right], 245$ (100) 247 (32) $\left[\mathrm{M}^{+}-\mathrm{Br}\right], 209$ (14) $\left[\mathrm{M}^{+}-\right.$ BrCl], 127 (29), 91 (48) $\left[\mathrm{C}_{7} \mathrm{H}_{7}{ }^{+}\right], 77$ (18) $\left[\mathrm{C}_{6} \mathrm{H}_{5}{ }^{+}\right]$; EA: $\mathrm{C}_{16} \mathrm{H}_{18} \mathrm{BrCl}$ (324.66) Ber.: C 59.19, H 5.28 Gef.: C 59.34, H 5.63; Schmp.: $61^{\circ} \mathrm{C} \mathrm{68:}{ }^{1} \mathbf{H}-\mathbf{N M R}\left(250 \mathrm{MHz}, \mathrm{CDCl}_{3}\right): \delta=2.28(2 \mathrm{H}$, 6-H), 2.41 (s, 4 H, 8-H, 9-H), 2.65 (s, 4 H, 4-H, 10-H), 2.79 (s, 2 H, 2-H), 7.25-7.40 (m, arom. $5 \mathrm{H}$ ); ${ }^{13} \mathbf{C}-\mathbf{N M R}\left(62.9 \mathrm{MHz}, \mathrm{CDCl}_{3}\right): \delta=45.49$ (q, C-7), 49.32 (s, C-6), 50.68 (s, C-8, C-9), 54.93 (s, C-4, C-10), 56.05 (s, C-2), 57.53 (s, C-1, C-3), 64.43 (s, C-5), 124.52 (s, C2', C-6'), 127.19 (s, C-4'), 128.78 (s, C-3', C-5'), 144.46 (q, C-1'); IR $\left(\mathrm{CCl}_{4}\right)$ : 544, 632, 694, 909, 1042, 1200, 1343, 1450, 1493, 1530, 1548, 1640, 1666, 1692, 2865, 2963, $3062 \mathrm{~cm}^{-1}$; MS (70 eV); m/z (\%): 402 (6) 404 (10) 406 (5) 408 (1) [M+], 323 (77) 325 (100) 327 (25) $\left[\mathrm{M}^{+}-\mathrm{Br}\right], 243$ (24) 245 (8) [ $\left.\mathrm{M}^{+}-\mathrm{Br}, \mathrm{Cl}\right], 207$ (27) $\left[\mathrm{M}^{+}-\mathrm{Br}_{2}, \mathrm{Cl}\right], 167$ (30), 115 (33), 91 (74) $\left[\mathrm{C}_{7} \mathrm{H}_{7}^{+}\right]$, 77 (22) $\left[\mathrm{C}_{6} \mathrm{H}_{5}^{+}\right.$]; EA: $\mathrm{C}_{16} \mathrm{H}_{17} \mathrm{Br}_{2} \mathrm{Cl}$ (404.57) Ber.: 47.50, H. 4.24 Gef.: C 47.56, H 4.11; Schmp.: $118-119^{\circ} \mathrm{C}$

AV14r. ( \pm )-1-Iod-3-brom-5-phenyltricyclo[3.3.1.1 $\left.1^{3,7}\right] \operatorname{decan}(\mathbf{6 1})$

$65 \mathrm{mg}(0.2 \mathrm{mmol}) \mathbf{6 0}, 120 \mathrm{mg}(0.4 \mathrm{mmol})$ Tetrabrommethan und $10 \mathrm{mg}$ (0.03 mmol, $15 \mathrm{~mol}$ $\%$ ) TBABr wurden in $5 \mathrm{~mL}$ Fluorbenzol gelöst und $2 \mathrm{~mL}$ Natriumhydroxidlösung (aq. 50\%) zugegeben. Die Mischung wurde 3 Tage bei $70^{\circ} \mathrm{C}$ intensiv gerührt. Anschließend wurden die 
Phasen getrennt und die wäßrige Phase mit $n$-Pentan $(5 \times 4 \mathrm{~mL})$ extrahiert. Die vereinigten organischen Phasen wurden über Natriumsulfat getrocknet und am Rotationsverdampfer unter reduziertem Druck weitestgehend von Lösungsmittel befreit. Das erhaltene Rohprodukt wurde einer säulenchromatographischen Trennung unterzogen ( $n$-Pentan, 1.5 x $50 \mathrm{~cm}, \mathrm{R}_{\mathrm{f}}(\mathbf{6 1})$ $=0.18)$. Es wurden $30 \mathrm{mg}(0.07 \mathrm{mmol}, 35 \%)$ von 61 als weißer Feststoffe erhalten. $27 \mathrm{mg}$ von 60 konnten zurückgewonnen werden. 61: ${ }^{1} \mathbf{H}-\mathbf{N M R}\left(250 \mathrm{MHz}, \mathrm{CDCl}_{3}\right): \delta=2.0\left(\mathrm{~d},{ }^{3} J_{7-\mathrm{H}}=\right.$ $3.0 \mathrm{~Hz}, 2 \mathrm{H}, \mathrm{H}-9), 2.27$ (qq, $\left.{ }^{3} J_{9-\mathrm{H}}=3.0 \mathrm{~Hz},{ }^{3} J_{8-\mathrm{H}}=3.2 \mathrm{~Hz}, 1 \mathrm{H}, 7-\mathrm{H}\right), 2.39$ (bs, $\left.2 \mathrm{H}, 6-\mathrm{H}\right)$, 2.55 (s, 4 H, 4-H, 8-H), 2.71 (s, 2 H, 10-H), 3.13 (s, 2 H, 2-H), 7.21-7.39 (m, arom. 5 H); ${ }^{13}$ C-NMR (62.9 MHz, $\mathrm{CDCl}_{3}$ ): $\delta=35.38$ (t, C-7), 39.30 (s, C-6), 42.90 (q, C-1), 44.84 (q, C-5), 46.21 (s, C-9), 48.99 (s, C-8), 52.28 (s, C-4), 55.34 (s, C-10), 60.80 (s, C-2), 61.55 (q, C-3), 124.49 (s, C-2', C-6'), 126.69 (s, C-4'), 128.56 (s, C-3', C-5'), 146.39 (q, C-1'); IR $\left(\mathrm{CCl}_{4}\right):$ 540, 664, 696, 955, 1039, 1284, 1317, 1340, 1446, 1602, 1665, 1944, 2858, 2941,

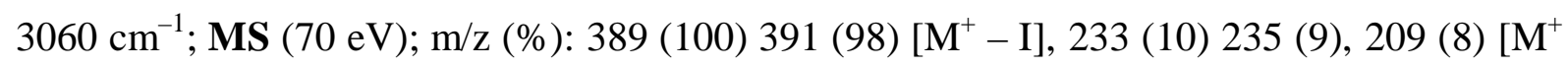
- Br,I], 169 (50), 155 (71), 128 (26), 91 (92) $\left[\mathrm{C}_{7} \mathrm{H}_{7}{ }^{+}\right], 77$ (41) $\left[\mathrm{C}_{6} \mathrm{H}_{5}{ }^{+}\right]$; EA: $\mathrm{C}_{16} \mathrm{H}_{18} \mathrm{BrI}$ (416.11) Ber.: C 46.18, H 4.12 Gef.: C 46.23, H 4.36; Schmp.: $78^{\circ} \mathrm{C}$

AV14s. ( \pm )-1-Iod-3-brom-5-phenyltricyclo[3.3.1.1 $\left.1^{3,7}\right]$ decan (61)

$950 \mathrm{mg}$ (3.3 mmol) 60, $2.78 \mathrm{~g}$ (7.0 mmol) Iodoform und $103 \mathrm{mg}(0.33 \mathrm{mmol}, 10 \mathrm{~mol} \%)$ TBABr wurden in $20 \mathrm{~mL}$ Fluorbenzol gelöst und $5 \mathrm{~mL} \mathrm{NaOH} \mathrm{Lsg.} \mathrm{(aq.} 50 \%$ ) zugegeben. Die Mischung wurde 6 Tage bei $50^{\circ} \mathrm{C}$ intensivst gerührt. Anschließend wurden die Phasen getrennt und die wäßrige Phase mit $n$-Pentan extrahiert $(4 \times 15 \mathrm{~mL})$. Die vereinigten organischen Phasen wurden über Natriumsulfat getrocknet und am Rotationsverdampfer unter reduziertem Druck von Lösungsmitteln befreit. Die erhaltenen $2.1 \mathrm{~g}$ Rohprodukt wurde einer säulenchromatographischen Trennung unterworfen ( $n$-Pentan, 3.5 x $\left.60 \mathrm{~cm}, \mathrm{R}_{\mathrm{f}}(\mathbf{6 1})=0.17\right)$. Es wurden $320 \mathrm{mg}(0.76 \mathrm{mmol}, 23 \%)$ von 61 als weißer Feststoff erhalten. $350 \mathrm{mg}$ von $\mathbf{6 0}$ konnten zurückgewonnen werden. 61: Die Analytik von 27 entspricht der unter AV14r wiedergegebenen. 
AV14t. ( \pm )-1-Iod-3-brom-5-chlor-7-phenyltricyclo[3.3.1.1 $\left.1^{3,7}\right]$ decan (63)

$100 \mathrm{mg}(0.27 \mathrm{mmol}) \mathbf{5 8}, 400 \mathrm{mg}(1.2 \mathrm{mmol})$ Tetrabrommethan und $10 \mathrm{mg}$ (0.03 mmol, 10 mol\%) $\mathrm{TBABr}$ wurden in $5 \mathrm{~mL}$ Fluorbenzol gelöst und $1 \mathrm{~mL} \mathrm{NaOH} \mathrm{Lsg.} \mathrm{(aq.} 50 \%$ ) zugegeben. Die Mischung wurde 4 Tage bei $60^{\circ} \mathrm{C}$ intensivst gerührt. Anschließend wurden die Phasen getrennt und die wäßrige Phase mit n-Pentan extrahiert (4 x $3 \mathrm{~mL})$. Die vereinigten organischen Phasen wurden über Natriumsulfat getrocknet und am Rotationsverdampfer unter reduziertem Druck von Lösungsmitteln befreit. Das erhaltene Rohprodukt wurde unter den o.a. Bedingungen erneut der Bromierung unterworfen, nach weiteren 4 Tagen Reaktionszeit in gleicher Weise aufgearbeitet und die $70 \mathrm{mg}$ an finalem Rohprodukt einer säulenchromatographischen Trennung unterzogen ( $n$-Pentan, 1 x $50 \mathrm{~cm}$, $\left.\mathrm{R}_{\mathrm{f}}(\mathbf{6 3})=0.25\right)$. Es wurden $10 \mathrm{mg}(0.02 \mathrm{mmol}, 7 \%)$ von 63 als weißer Feststoff erhalten. 35 mg von 58 konnten zurückgewonnen werden. 63: ${ }^{1} \mathbf{H}-\mathbf{N M R}\left(250 \mathrm{MHz}, \mathrm{CDCl}_{3}\right): \delta=2.33(\mathrm{~s}$, 2H, 6-H), 2.47 (s, 2H, 10-H), 2.60 (s, 2H, 8-H), $2.63-2.71$ (m, 2H, 4-H), 2.84 (s, 2H, 9-H), $2.99(\mathrm{~s}, 2 \mathrm{H}, 2-\mathrm{H}), 7.26-7.40\left(\mathrm{~m}, 5 \mathrm{H}\right.$, arom.-H); ${ }^{13} \mathbf{C}-\mathbf{N M R}\left(62.9 \mathrm{MHz}, \mathrm{CDCl}_{3}\right): \delta=29.69$ (q, C-1), 36.69 (q, C-7), 46.23 (q, C-3), 49.23 (s, C-6), 50.59 (s, C-10), 53.46 (s, C-8), 54.97 (s, C-4), 57.53 (s, C-9), 58.55 (s, C-2), 64.23 (q, C-5), 124.45 (t, C-2'), 127.17 (t, C4'), 128.76 (t, C-3'), 144.41 (C-1'); IR ( $\left.\mathrm{CCl}_{4}\right)$ : 543, 632, 695, 854, 1041, 1198, 1317, 1343, 1447, 1498, 1742, 2862, 2957, $3061 \mathrm{~cm}^{-1}$; MS (70 eV) m/z (\%): 323 (77) 327 (100) 329 (25)

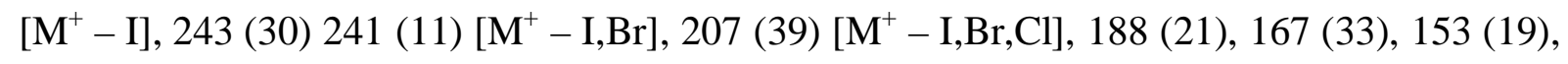
129 (19), 115 (34), 91 (86), 77 (26), 55 (13); EA: $\mathrm{C}_{16} \mathrm{H}_{17} \mathrm{ClBrI}$ (451.57) Ber.: C 42.56, H 3.79 wurde wegen zu geringer Substanzmengen nicht durchgeführt. Hochauflösende EI/MS und $\mathrm{CI} / \mathrm{MS}$ waren erfolglos, da bei der Ionisierung Iod zu leicht abgespalten wird; Schmp.: $42^{\circ} \mathrm{C}$

AV14u. ( \pm )-1-Iod-3-brom-5-chlor-7-phenyltricyclo[3.3.1.1 $\left.1^{3,7}\right]$ decan (63)

$300 \mathrm{mg}$ (0.88 mmol) 1-Brom-3-chlor-5-phenyladamantan 62, $584 \mathrm{mg}$ (1.76 mmol) Iodoform und $32 \mathrm{mg}$ (0.1 mmol, $11 \mathrm{~mol} \%)$ TBABr in $8 \mathrm{~mL}$ Fluorbenzol gelöst und $4 \mathrm{~mL} \mathrm{NaOH} \mathrm{Lsg.}$ (aq. $50 \%$ ) zugegeben. Die Mischung wurde 10 Tage bei $50^{\circ} \mathrm{C}$ intensivst gerührt. Anschließend wurden die Phasen getrennt und die wäßrige Phase mit $n$-Pentan extrahiert (4 x $10 \mathrm{~mL}$ ). Die vereinigten organischen Phasen wurden über Natriumsulfat getrocknet und am Rotationsverdampfer unter reduziertem Druck von Lösungsmitteln befreit. Das erhaltene Rohprodukt wurde unter den o.a. Bedingungen erneut der Reaktion unterworfen und diese 
Prozedur noch sechsmal wiederholt. Nach insgesamt 70 Tagen Reaktionszeit wurde das finale Rohprodukt einer säulenchromatographischen Trennung unterworfen ( $n$-Pentan, 2.5 x $50 \mathrm{~cm}$, $\left.\mathrm{R}_{\mathrm{f}}(\mathbf{6 3})=0.25\right)$. Es wurden $12 \mathrm{mg}(0.03 \mathrm{mmol}, 3 \%)$ von 63 als weißer Feststoff erhalten. 110 mg von 62 konnten zurückgewonnen werden. 63: Die Analytik von 63 entspricht der unter AV14t wiedergegebenen.

AV14v. 1-Chlor-3-phenyltricyclo[3.3.1.1 $\left.{ }^{3,7}\right]$ decan $\quad$ (57), 1,3-Chlor-5-phenyltricyclo[3.3.1.1 $1^{3,7}$ decan $(\mathbf{6 4}$, radikalisch initiiert):

$4.00 \mathrm{~g}$ (0.019 mol) 56 und $1 \mathrm{~g}$ (4.2 mmol) Dibenzoylperoxid wurden in $60 \mathrm{~mL}$ Tetrachlormethan gelöst. Die Mischung wurde $23 \mathrm{~h}$ refluxiert. Anschließend wurden am Rotationsverdampfer unter reduziertem Druck der größte Teil des Lösungsmittels entfernt. Die erhaltenen $4.6 \mathrm{~g}$ Rohprodukt wurden einer säulenchromatographischen Trennung unterzogen ( $n$-Pentan, 5 x $\left.60 \mathrm{~cm}, \mathbf{R}_{\mathrm{f}}(\mathbf{5 7})=0.23, \mathrm{R}_{\mathrm{f}}(\mathbf{6 4})=0.15\right)$. Es wurden $2.0 \mathrm{~g}(0.81 \mathrm{mmol}$, $43 \%)$ von 57 und $0.7 \mathrm{~g}(0.25 \mathrm{mmol}, 13 \%)$ von $\mathbf{6 4}$ als weiße Feststoffe erhalten. $0.7 \mathrm{mg}$ von $\mathbf{5 6}$ konnten zurückgewonnen werden. Die Charakterisierung der isolierten Verbindungen entsprechen der unter AV14m angegebenen.

AV14w. 1-Methoxytricyclo[3.3.1.1 $\left.1^{3,7}\right]$ decan (69):

4.0 g Natriumhydrid (60\% in Mineralöl, ca. 0.01 mol) wurden durch Extraktion mit $n$-Pentan (Trocken) vom Mineralöl befreit und in $100 \mathrm{~mL}$ wasserfreiem THF suspendiert. Unter Stickstoffatmosphäre und Rühren wurden portionsweise $10 \mathrm{~g}$ (0.066 mol) 1-Adamantanol (46) hinzugegeben und anschließend noch $2 \mathrm{~h}$ bei RT, dann $2 \mathrm{~h}$ unter Rückfluß weitergerührt. Nach dem Abkühlen der Reaktionsmischung wurden 14.1 g (0.01 mol) Methyliodid hinzugefügt und $30 \mathrm{~min}$ bei RT und danach $1 \mathrm{~h}$ unter Rückfluß gerührt. Nach erneutem Abkühlen der Reaktionsmischung wurde durch tropfenweise Zugabe von Wasser $(60 \mathrm{~mL})$ nicht umgesetztes $\mathrm{NaH}$ hydrolysiert, bis keine Wasserstoffentwicklung mehr stattfand. Nach der Phasentrennung wurde die wäßrige Phase mit Diethylether (3 x $50 \mathrm{~mL})$ extrahiert. Die vereinigten organischen Phasen wurden mit Salzsäure (aq. $3 \%$ ) gewaschen, über Natriumsulfat getrocknet und am Rotationsverdampfer unter reduziertem Druck von Lösungsmitteln befreit. Das Rohprodukt wurde einer Säulenfiltration unterworfen ( $n$-Pentan : 
Ether $\left.=95: 5,7 \times 15 \mathrm{~cm}, \mathrm{R}_{\mathrm{f}}(\mathbf{6 9})=0.19\right)$ und $10.41 \mathrm{~g}(0.063 \mathrm{~mol}, 96 \%)$ von $\mathbf{6 9}$ wurden als farbloses viskoses Öl erhalten. 13. Die Verbindung ist literaturbekannt.[200]

AV14x. 1-Chlor-3-methoxytricyclo[3.3.1.1, $]$ decan (70):

$4.00 \mathrm{~g}(0.019 \mathrm{~mol}) 69$ und $1.5 \mathrm{~g}(6.3 \mathrm{mmol})$ Dibenzoylperoxid wurden in $50 \mathrm{~mL}$ Tetrachlormethan gelöst. Die Mischung wurde 43 h unter Stickstoffatmosphäre refluxiert. Anschließend wurden am Rotationsverdampfer unter reduziertem Druck der größte Teil des Lösungsmittels entfernt. Die erhaltenen $4.6 \mathrm{~g}$ Rohprodukt wurden einer säulenchromatographischen Trennung unterzogen $(n$-Pentan : Ether $=95: 5,7 \times 60 \mathrm{~cm}$, $\left.\mathrm{R}_{\mathrm{f}}(\mathbf{7 0})=0.14\right)$. Es wurden $1.10 \mathrm{~g}(5.5 \mathrm{mmol}, 22 \%)$ von $\mathbf{7 0}$ als farbloses viskoses Öl erhalten. $3.7 \mathrm{~g}$ von 69 konnten zurückgewonnen werden. $\mathbf{7 0 :}{ }^{1} \mathbf{H}-\mathbf{N M R}\left(250 \mathrm{MHz}, \mathrm{CDCl}_{3}\right): \delta=1.50-$ 1.55 (m, 2H, 6-H), $1.59-1.74$ (m, 4H, 4-H, 10-H), 2.02 (bs, 4H, 8-H, 9-H), 2.10 (bs, 2H, $2-\mathrm{H}), 2.29-2.32(\mathrm{~m}, 2 \mathrm{H}, 5-\mathrm{H}, 7-\mathrm{H}), 3.22\left(\mathrm{~s}, 3 \mathrm{H},-\mathrm{CH}_{3}\right) ;{ }^{13} \mathbf{C}-\mathbf{N M R}\left(62.9 \mathrm{MHz}, \mathrm{CDCl}_{3}\right): \delta=$ 31.97 (t, C-5, C-7), 34.43 (s, C-6), 39.27 (s, C-4, C-10), 46.53 (s, C-8, C-9), 48.36 (p, $\mathrm{CH}_{3}$ ), 50.71 (s, C-2), 68.01 (q, C-1), 73.95 (q, C-3); IR $\left(\mathrm{CCl}_{4}\right): 662,787,927,1044$, 1155,1349, 1446, 1653, 1724, 1770, 1792, 2090, 2670, $2833 \mathrm{~cm}^{-1}$; MS (70 eV); m/z (\%): 200

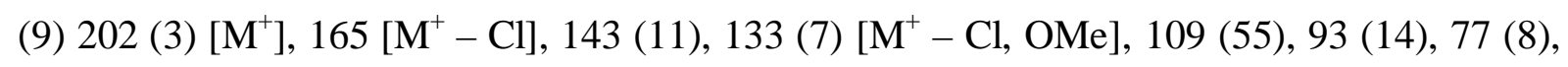
65 (4), 57 (4) EA: $\mathrm{C}_{11} \mathrm{H}_{17} \mathrm{ClO}$ (200.70) Ber.: C 65.83, H 8.54 Gef.: C 66.01, H 8.60

AV14y. 1-Iod-3-methoxytricyclo[3.3.1.1 $\left.1^{3,7}\right]$ decan (73):

$1.00 \mathrm{~g}(0.06 \mathrm{~mol})$ 69, $2.36 \mathrm{~g}$ (0.06 mol) Iodoform und $193 \mathrm{mg}$ (6.0 mmol, $10 \mathrm{~mol} \%) \mathrm{TBABr}$ wurden in $30 \mathrm{~mL}$ Fluorbenzol gelöst und $15 \mathrm{~mL} \mathrm{NaOH} \mathrm{Lsg.} \mathrm{(aq.} 50 \%$ ) zugegeben. Die Mischung wurde 3 Tage bei $50^{\circ} \mathrm{C}$ intensivst gerührt. Anschließend wurden die Phasen getrennt und die wäßrige Phase mit $n$-Pentan extrahiert $(4 \times 10 \mathrm{~mL})$. Die vereinigten organischen Phasen wurden über Natriumsulfat getrocknet und am Rotationsverdampfer unter reduziertem Druck von Lösungsmitteln befreit. Das erhaltene Rohprodukt wurde einer säulenchromatographischen Trennung unterworfen ( $n$-Pentan : Ether $=95: 5,1.5 \times 50 \mathrm{~cm}$, $\left.R_{\mathbf{f}}(\mathbf{7 3})=0.16\right)$. Neben einigen Mischfraktionen wurden $290 \mathrm{mg}(9.9 \mathrm{mmol}, 15 \%)$ von 73 als farbloses, leicht viskoses farbloses Öl erhalten. $800 \mathrm{mg}$ von 69 konnten zurückgewonnen werden. 73: ${ }^{1} \mathbf{H}-\mathbf{N M R}\left(250 \mathrm{MHz}, \mathrm{CDCl}_{3}\right): \delta=1.64-1.67(\mathrm{~m}, 2 \mathrm{H}, 6-\mathrm{H}), 1.77-1.79(\mathrm{~m}, 4 \mathrm{H}$, 
4-H, $10-\mathrm{H}), 2.05-2.16(\mathrm{~m}, 2 \mathrm{H}, 5-\mathrm{H}, 7-\mathrm{H}), 2.41-2.52$ (m, 4H, 8-H, 9-H), 2.56 (s, 2H, 2$\mathrm{H}), 3.22\left(\mathrm{~s}, 3 \mathrm{H},-\mathrm{CH}_{3}\right) ;{ }^{13} \mathrm{C}-\mathbf{N M R}\left(62.9 \mathrm{MHz}, \mathrm{CDCl}_{3}\right): \delta=33.42(\mathrm{t}, \mathrm{C}-5, \mathrm{C}-7), 34.44(\mathrm{~s}, \mathrm{C}-$ 6), 39.36 (s, C-4, C-10), 46.39 (q, C-1), 48.32 (p, $-\mathrm{CH}_{3}$ ), 50.99 (s, C-8, C-9), 55.30 (s, C2), 73.46 (q, C-3); IR $\left(\mathrm{CCl}_{4}\right)$ : 558, 666, 893, 1042, 1093, 1119, 1297, 1325, 1345, 1454, 2828, 2858, $2945 \mathrm{~cm}^{-1}$; MS (70 eV) m/z (\%): 293 (1) $\left[\mathrm{M}^{+}\right], 165\left[\mathrm{M}^{+}-\mathrm{I}\right], 143$ (11), 133 (4) [M+ - I, OMe], 109 (72), 93 (32), 77 (10), 73 (11), 65 (4), 53 (3); EA: $\mathrm{C}_{11} \mathrm{H}_{17} \mathrm{IO}$ (292.16) Ber.: C 45.22, H 5.86 Gef.: C 44.92, H 5.66

AV14z. ( \pm )-1-Brom-3-chlor-5-methoxytricyclo[3.3.1.1 $1^{3,7}$ decan (71), 1,3-Dibrom-5-chlor7-methoxytricyclo[3.3.1.1 $\left.1^{3,7}\right]$ decan (75)

$300 \mathrm{mg}$ (1.1 mmol) 70, $1.50 \mathrm{~g}$ (4.5 mmol) Tetrabrommethan und $35 \mathrm{mg}$ (0.11 mmol, 10 mol\%) TBABr wurden in $8 \mathrm{~mL}$ Fluorbenzol gelöst und $5 \mathrm{~mL} \mathrm{NaOH} \mathrm{Lsg.} \mathrm{(aq.} 50 \%$ ) zugegeben. Die Mischung wurde 4 Tage bei $75^{\circ} \mathrm{C}$ intensivst gerührt. Anschließend wurden die Phasen getrennt und die wäßrige Phase mit n-Pentan extrahiert (4 x $6 \mathrm{~mL})$. Die vereinigten organischen Phasen wurden über Natriumsulfat getrocknet und am Rotationsverdampfer unter reduziertem Druck von Lösungsmitteln befreit. Das erhaltene Rohprodukt wurde mit den o.a. Bedingungen erneut der Bromierung unterworfen, nach weiteren 4 Tagen Reaktionszeit in gleicher Weise aufgearbeitet und diese Prozedur abschließend noch einmal wiederholt. Nach insgesamt 12 Tagen Reaktionszeit wurden 290 mg an finalem Rohprodukt einer säulenchromatographischen Trennung unterzogen ( $n$-Pentan $:$ Ether $\left.=95: 5,1.5 \times 50 \mathrm{~cm}, \mathrm{R}_{\mathrm{f}}(\mathbf{7 1})=0.15, \mathrm{R}_{\mathrm{f}}(\mathbf{7 5})=0.26\right)$. Es wurden $70 \mathrm{mg}(0.25 \mathrm{mmol}, 22$ \%) von 71 als farbloses, viskoses Öl und $25 \mathrm{mg}(0.07 \mathrm{mmol}, 6 \%)$ von $\mathbf{7 5}$ als weißer Feststoff isoliert. 71: ${ }^{1} \mathbf{H}-\mathbf{N M R}\left(250 \mathrm{MHz}, \mathrm{CDCl}_{3}\right): \delta=1.69\left(\mathrm{~d},{ }^{3} J_{7-\mathrm{H}}=3.5 \mathrm{~Hz}, 2 \mathrm{H}, 6-\mathrm{H}\right), 1.99\left(\mathrm{~d},{ }^{3} J_{7-}\right.$ $\mathrm{H}=3.1 \mathrm{~Hz}, 2 \mathrm{H}, 9-\mathrm{H}), 2.09-2.16(\mathrm{~m}, 4 \mathrm{H}, 2-\mathrm{H}, 8-\mathrm{H}), 2.19-2.32(\mathrm{~m}, 2 \mathrm{H}, 10-\mathrm{H}), 2.40$ (qq, $\left.{ }^{3} J_{6-\mathrm{H}}=3.5 \mathrm{~Hz},{ }^{3} J_{9-\mathrm{H}}=3.1 \mathrm{~Hz}, 1 \mathrm{H}, 7-\mathrm{H}\right), 2.57(\mathrm{~s}, 2 \mathrm{H}, 4-\mathrm{H}), 3.25\left(\mathrm{~s}, 3 \mathrm{H},-\mathrm{CH}_{3}\right) ;{ }^{13} \mathbf{C}-\mathbf{N M R}$ $\left(62.9 \mathrm{MHz}, \mathrm{CDCl}_{3}\right): \delta=32.82(\mathrm{t}, \mathrm{C}-7), 37.75$ (s, C-6), 44.59 (s, C-10), 45.92 (s, C-8), 48.94 $\left(\mathrm{p},-\mathrm{CH}_{3}\right), 49.20$ (s, C-4), 50.57 (s, C-9), 56.65 (s, C-2), 59.26 (q, C-1), 65.34 (q, C-3), 74.98 (q, C-5); IR $\left(\mathrm{CCl}_{4}\right)$ : 566, 727, 902, 959, 1045, 1140, 1189, 1289, 1327, 1451, 1583,

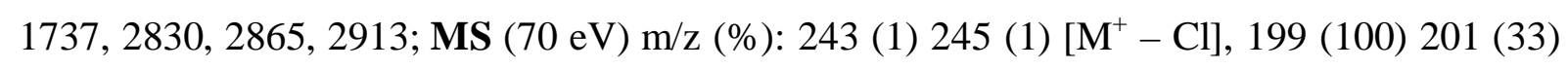
$\left[\mathrm{M}^{+}-\mathrm{Br}\right], 143$ (28) [M+ - Cl,Br], 127 (22), 123 (18), 109 (11), 91 (20), 73 (14) 65 (7), 55 (4); EA: $\mathrm{C}_{11} \mathrm{H}_{16} \mathrm{OClBr}(279.60)$ Ber.: C 47.25, H 5.77 Gef.: C 46.96, H 5.64; 75: ${ }^{1} \mathbf{H}-\mathbf{N M R}(250$ $\mathrm{MHz}_{\mathrm{CDCl}}$ ): $\delta=2.08$ (s, 2H, 6-H), 2.23 (s, 4H, 8-H, 10-H), 2.51 (s, 4H, 5-H, 9-H), 2.64 
(s, 2H, 2-H), 3.27 (s, 3H, -CH3); ${ }^{13} \mathbf{C}-\mathbf{N M R}\left(62.9 \mathrm{MHz}, \mathrm{CDCl}_{3}\right): \delta=47.78(\mathrm{~s}, \mathrm{C}-6), 49.00$ (s, $\mathrm{C}-8, \mathrm{C}-10), 49.42$ (p, $-\mathrm{CH}_{3}$ ), 54.75 (s, C-4, C-9), 54.98 (q, C-1, C-3), 55.81 (s, C-2), 62.83 (q, C-5), 75.25 (q, C-7); IR $\left(\mathrm{CCl}_{4}\right):$ 567, 859, 906, 1038, 1121, 1187, 1335, 1452, 1547, 1580, 1678, 2286, 2832, 2867, $2963 \mathrm{~cm}^{-1}$; MS (70 eV) m/z (\%): 277 (77) 279 (100) 281 (25)

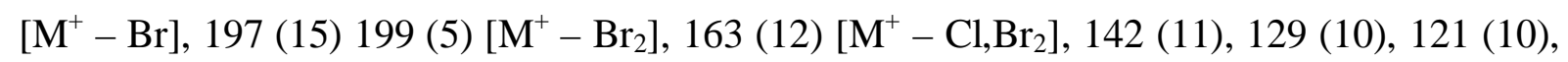
105 (8), 91 (25), 77 (17), 65 (8), 51 (6); EA: $\mathrm{C}_{11} \mathrm{H}_{15} \mathrm{OClBr}_{2}$ (358.50) Ber.: C 36.85, H 4.22 Gef.: C 37.02, H 4.19; Schmp.: $110-113^{\circ} \mathrm{C}$

AV14ä. ( \pm )-1-Iod-3-chlor-5-methoxytricyclo[3.3.1.1 $\left.1^{3,7}\right]$ decan (72), 1,3-Diiod-5-chlor-7methoxytricyclo[3.3.1.1 $\left.1^{3,7}\right]$ decan (76):

$300 \mathrm{mg}$ (1.1 mmol) 70, $1.50 \mathrm{~g}$ (3.8 mmol) Iodoform und $35 \mathrm{mg}(0.11 \mathrm{mmol}, 10 \mathrm{~mol} \%)$ TBABr wurden in $8 \mathrm{~mL}$ Fluorbenzol gelöst und $5 \mathrm{~mL} \mathrm{NaOH} \mathrm{Lsg.} \mathrm{(aq.} 50$ \%) zugegeben. Die Mischung wurde 9 Tage bei $50^{\circ} \mathrm{C}$ intensivst gerührt. Anschließend wurden die Phasen getrennt und die wäßrige Phase mit $n$-Pentan extrahiert $(4$ x $6 \mathrm{~mL})$. Die vereinigten organischen Phasen wurden über Natriumsulfat getrocknet und am Rotationsverdampfer unter reduziertem Druck von Lösungsmitteln befreit. Das erhaltene Rohprodukt wurde mit den o.a. Bedingungen erneut der Iodierung unterworfen, nach weiteren 9 Tagen Reaktionszeit in gleicher Weise aufgearbeitet und diese Prozedur abschließend noch viermal wiederholt. Nach insgesamt 54 Tagen Reaktionszeit wurden $210 \mathrm{mg}$ an finalem Rohprodukt einer säulenchromatographischen Trennung unterzogen ( $n$-Pentan : Ether $=95: 5,1.5$ x $60 \mathrm{~cm}$, $\left.\mathrm{R}_{\mathrm{f}}(\mathbf{7 2})=0.24, \mathrm{R}_{\mathrm{f}}(\mathbf{7 6})=0.35\right)$. Es wurden $75 \mathrm{mg}(0.23 \mathrm{mmol}, 21 \%)$ von 72 als farbloses, leicht viskoses Öl und $94 \mathrm{mg}(0.21 \mathrm{mmol}, 19 \%)$ von 76 als weißer Feststoff isoliert. 72: ${ }^{\mathbf{1}} \mathbf{H}-\mathbf{N M R}$ $\left(250 \mathrm{MHz}, \mathrm{CDCl}_{3}\right): \delta=1.72-1.74(\mathrm{~m}, 2 \mathrm{H}, 6-\mathrm{H}), 2.05$ (bs, $\left.2 \mathrm{H}, 9-\mathrm{H}\right), 2.13$ (s, $\left.2 \mathrm{H}, 8-\mathrm{H}\right)$, $2.21-2.25$ (m, $1 \mathrm{H}, 7-\mathrm{H}), 2.32$ (bs, 2H, 4-H), $2.40-2.48$ (m, 2 H, 10-H), 2.77 (s, 2 H, 2-H), $3.24(\mathrm{~s}, 3 \mathrm{H},-\mathrm{OMe}) ;{ }^{13} \mathrm{C}-\mathrm{NMR}\left(62.9 \mathrm{MHz}, \mathrm{CDCl}_{3}\right): \delta=33.73(\mathrm{t}, \mathrm{C}-7), 37.80(\mathrm{~s}, \mathrm{C}-6), 39.13$ (q, C-1), 44.62 (s, C-9), 48.69 (s, C-8), 48.88 (p, - $\mathrm{OCH}_{3}$ ), 49.26 (s, C-4), 53.42 (s, C-10), 59.35 (s, C-2), 65.11 (q, C-3), 74.61 (q, C-5); IR $\left(\mathrm{CCl}_{4}\right)$ : 564, 630, 660, 900, 958, 1042, 1120, 1138, 1189, 1286, 1325, 2291, 2831, 2863, $2946 \mathrm{~cm}^{-1}, \mathbf{M S}(70 \mathrm{eV}) \mathrm{m} / \mathrm{z}(\%): 199$ (100)

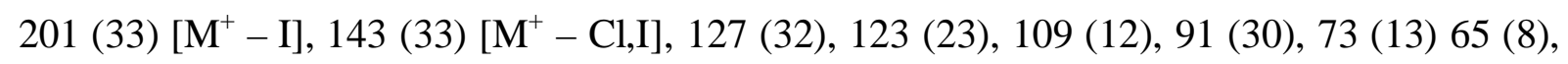
55 (3); EA: $\mathrm{C}_{11} \mathrm{H}_{15} \mathrm{OCII}$ (326.60) Ber.: C 40.45, H 4.94 Gef.: C 39.92, H 4.73; 76: ${ }^{\mathbf{1}} \mathbf{H}-\mathbf{N M R}$ $\left(250 \mathrm{MHz}, \mathrm{CDCl}_{3}\right): \delta=2.16(\mathrm{~s}, 2 \mathrm{H}, 6-\mathrm{H}), 2.39-2.51$ (m, 4 H, 4-H, 8-H, 9-H, 10-H), 2.67 -2.79 (m, 4 H, 4-H, 8-H, 9-H, 10-H), 2.98 (s, 2 H, 2-H), 3.24 (s, $\left.3 \mathrm{H},-\mathrm{OCH}_{3}\right)$; IR $\left(\mathrm{CCl}_{4}\right)$ : 
630, 795, 901, 978, 1033, 1115, 1185, 1211, 1312, 1334, 1450, 1549, 2832, 2958; MS (70 eV) m/z (\%): 325 (100) 327 (33) [M+ - I], 197 (21) 199 (7) $\left[\mathrm{M}^{+}-\mathrm{I}_{2}\right], 163$ (31) $\left[\mathrm{M}^{+}-\mathrm{ClI}_{2}\right]$, 141 (11), 131 (12), 105 (10), 91 (42), 65 (8), 51 (7); EA: $\mathrm{C}_{11} \mathrm{H}_{14} \mathrm{OCII}_{2}$ (452.50) Ber.: C 29.29, H 3.34 Gef.: C 29.17, H 3.30; Schmp.: $127^{\circ} \mathrm{C}$

AV140̈. ( \pm )-1-Iod-3-brom-5-methoxytricyclo[3.3.1.1 $1^{3,7}$ decan (74), 1-Iod-3,5-dibrom-7me-thoxytricyclo[3.3.1.1 $\left.1^{3,7}\right]$ decan (77):

$292 \mathrm{mg}$ (1.0 mmol) 1-Iod-3-methoxyadamantan 73, $1.99 \mathrm{~g} \mathrm{(6.0} \mathrm{mmol)} \mathrm{Iodoform} \mathrm{und} 32 \mathrm{mg}$ (0.10 mmol, $10 \mathrm{~mol} \%)$ TBABr wurden in $8 \mathrm{~mL}$ Fluorbenzol gelöst und $5 \mathrm{~mL} \mathrm{NaOH} \mathrm{Lsg.} \mathrm{(aq.}$ $50 \%$ ) zugegeben. Die Mischung wurde 3 Tage bei $70^{\circ} \mathrm{C}$ intensivst gerührt. Anschließend wurden die Phasen getrennt und die wäßrige Phase mit $n$-Pentan extrahiert (4 x $6 \mathrm{~mL}$ ). Die vereinigten organischen Phasen wurden über Natriumsulfat getrocknet und am Rotationsverdampfer unter reduziertem Druck von Lösungsmitteln befreit. Das erhaltene Rohprodukt wurde mit den o.a. Bedingungen erneut der Bromierung unterworfen, und nach weiteren 4 Tagen Reaktionszeit in gleicher Weise aufgearbeitet. Nach insgesamt 7 Tagen Reaktionszeit wurde das finale Rohprodukt einer säulenchromatographischen Trennung unterzogen $\left(n\right.$-Pentan : Ether $\left.=95: 5,1.5 \times 60 \mathrm{~cm}, \mathrm{R}_{\mathrm{f}}(\mathbf{7 4})=0.13, \mathrm{R}_{\mathrm{f}}(\mathbf{7 6})=0.25\right)$. Es wurden $80 \mathrm{mg}(0.21 \mathrm{mmol}, 21 \%)$ von 74 als farbloses, leicht viskoses Öl und $120 \mathrm{mg}$ (0.26 mmol, 26 \%) von 76 als weißer Feststoff isoliert. 74: ${ }^{1} \mathbf{H}-\mathbf{N M R}\left(250 \mathrm{MHz}, \mathrm{CDCl}_{3}\right): \delta=1.72-1.83(\mathrm{~m}$, 2H, 6-H), 2.22 (s, 3H, 7-H, 9-H), 2.34 (s, 2H, 8-H), 2.36 (s, 2H, 4-H), $2.44-2.55$ (m, 2H, 10-H), 2.95 (s, 2H, 2-H), $3.24\left(\mathrm{~s}, 3 \mathrm{H},-\mathrm{CH}_{3}\right) ;{ }^{13} \mathbf{C}-\mathbf{N M R}\left(62.9 \mathrm{MHz}, \mathrm{CDCl}_{3}\right): \delta=34.69(\mathrm{t}$, C-7), 37.79 (s, C-6), 39.34 (q, C-1), 45.90 (s, C-9), 48.62 (s, C-8), 48.87 (p, $-\mathrm{CH}_{3}$ ), 50.61 (s, C-4), 53.39 (s, C-10), 59.35 (q, C-3), 60.41 (s, C-2), 74.78 (q, C-5); IR $\left(\mathrm{CCl}_{4}\right)$ : 564, 694, 897, 955, 1040, 1138, 1187, 1286, 1334, 1351, 1453, 2830, 2863, $2947 \mathrm{~cm}^{-1} ; \mathbf{M S}(70 \mathrm{eV}) \mathrm{m} / \mathrm{z}$

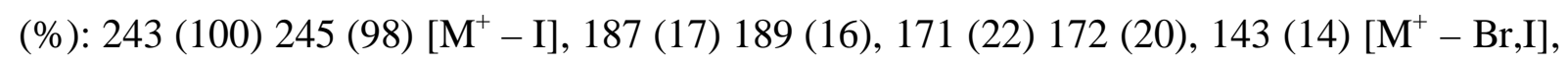
131 (12) [M+ - Br,I,OMe], 123 (29), 121 (24), 109 (38), 108 (34), 91 (40), 73 (15), 55 (11); EA: $\mathrm{C}_{11} \mathrm{H}_{16} \mathrm{OBrI}(371.05)$ Ber.: C 35.61, H 4.35 Gef.: C 35.57, H 4.10; 76: ${ }^{1} \mathbf{H}-\mathbf{N M R}(250$ $\left.\mathrm{MHz}, \mathrm{CDCl}_{3}\right): \delta=2.25-2.35(\mathrm{~m}, 4 \mathrm{H}, 6-\mathrm{H}, 9-\mathrm{H}), 2.43$ (s, 2H, 8-H), $2.64-2.77$ (m, 2H, 4H), $2.84(\mathrm{~s}, 4 \mathrm{H}, 2-\mathrm{H}, 10-\mathrm{H}), 3.25\left(\mathrm{~s}, 3 \mathrm{H},-\mathrm{OCH}_{3}\right) ;{ }^{13} \mathbf{C}-\mathbf{N M R}\left(62.9 \mathrm{MHz}, \mathrm{CDCl}_{3}\right): \delta=33.28$ (q, C-1), 49.02 (s, C-6, C-9), 49.36 (p, - $\mathrm{CH}_{3}$ ), 51.64 (s, C-8), 55.36 (q, C-3, C-5), 55.79 (s, C-4), 58.19 (s, C-2, C-10), 75.40 (q, C-7); IR $\left(\mathrm{CCl}_{4}\right): 566,649,901,1036,1123,1185$, 1334, 1451, 1653, 2833, 2864, $2950 \mathrm{~cm}^{-1}$; MS (70 eV) m/z (\%): 321 (51) 323 (100) 324 (49) 
$\left[\mathrm{M}^{+}-\mathrm{I}\right], 241(20) 243(19)\left[\mathrm{M}^{+}-\mathrm{I}, \mathrm{Br}\right], 163(25)\left[\mathrm{M}^{+}-\mathrm{I}_{,} \mathrm{Br}_{2}\right], 131\left[\mathrm{M}^{+}-\mathrm{I}_{,} \mathrm{Br}_{2}, \mathrm{OMe}\right], 105$ (14), 91 (55), 78 (15), 65 (10), 51 (8); EA: $\mathrm{C}_{11} \mathrm{H}_{15} \mathrm{OBr}_{2} \mathrm{I}$ (449.59) Ber.: C 29.36, H 3.36 Gef.: C 29.22, H 3.31; Schmp.: $140-141^{\circ} \mathrm{C}$

\section{AV15. Die Durchführung der Shift-Experimente}

Alle Shiftexperimente wurden in Chloroform als Lösungsmittel auf die folgenden Weise durchgeführt: Eine bestimmte Menge der zu untersuchenden Substanz wurde in $0.4 \mathrm{~mL}$ $\mathrm{CHCl}_{3}$ in einem NMR-Röhrchen und separat eine molar äquivalente Menge des jeweiligen LSR ebenfalls in $\mathrm{CHCl}_{3}(1 \mathrm{~mL})$ gelöst. Anschließend wurde durch schrittweise Zugabe einer definierten Menge der LSR-Lösung dessen Konzentration im NMR-Röhrchen systematisch erhöht und jeweils ein ${ }^{1} \mathrm{H}-\mathrm{NMR}$ Spektrum aufgenommen, bis keine wesentliche Erhöhung einer Verschiebung mehr zu beobachten war. In den anderen Fällen erfolgte die einmalige Zugabe von ca. $30 \mathrm{~mol} \%$ an entsprechendem Shiftreagenz.

AV15a. 1-Fluoradamantan + Dy(fod $)_{3}: 10 \mathrm{mg}+64 \mathrm{mg} \mathrm{Dy}(\mathrm{fod})_{3}=(0.06494 \mathrm{mmol})$

Tabelle 27. Shift von 2-H in 1-Fluoradamantan bei steigender Konz. von Dy(fod $)_{3}$

\begin{tabular}{cc}
$\mathrm{mol} \%$ & $\Delta \delta(-\mathrm{ppm})$ \\
\hline 0 & 0.000 \\
5 & 0.110 \\
10 & 0.250 \\
20 & 0.370 \\
25 & 0.470 \\
30 & 0.590 \\
\end{tabular}


AV15b. 1-Fluoradamantan $+\mathrm{Yb}(\text { fod })_{3:} 11.5 \mathrm{mg}+71.1 \mathrm{mg} \mathrm{Yb}(\text { fod })_{3}=(0.07464 \mathrm{mmol})$

Tabelle 28. Shift von $2-\mathrm{H}$ in 1-Fluoradamantan bei steigender Konz. von $\mathrm{Y}(\text { fod })_{3}$

\begin{tabular}{cc}
$\mathrm{mol} \%$ & $\Delta \delta(-\mathrm{ppm})$ \\
\hline 0.0 & 0.0 \\
8.2 & 0.06 \\
16.4 & 0.11 \\
24.6 & 0.15 \\
41.0 & 0.20 \\
57.4 & 0.26 \\
73.8 & 0.30 \\
90.0 & 0.31
\end{tabular}

AV15c. 1-Fluor-3-chloradamantan $+\mathrm{Yb}(\text { fod })_{3}: 9.4 \mathrm{mg}+52.9 \mathrm{mg} \mathrm{Yb}(\mathrm{fod})_{3}=(0.04982 \mathrm{mmol})$

Tabelle 29. Shift von $2-\mathrm{H}$ in 1 -Fluor-chloradamantan bei steigender Konz. von $\mathrm{Y}(\mathrm{fod})_{3}$

\begin{tabular}{cc} 
mol\% & $\Delta \delta(-\mathrm{ppm})$ \\
\hline 0 & 0.00 \\
10 & 0.04 \\
20 & 0.08 \\
30 & 0.11 \\
40 & 0.13 \\
50 & 0.14 \\
70 & 0.15
\end{tabular}

AV16. Die Synthese und Halogenierung von 2-(spiro-1',4'-dioxocyclopentyl)tricyclo[3.3.1.1 ${ }^{3,7}$ decan (79)

AV16a. 2-(spiro-1',4'-dioxocyclopentyl)tricyclo[3.3.1.1 $\left.1^{3,7}\right]$ decan (79)

$2.60 \mathrm{~g}$ an 78 (0.017 mol), $1.20 \mathrm{~g}$ Diethylenglycol (0.019) und $50 \mathrm{mg} p$-Toluolsulfonsäure wurden in $60 \mathrm{~mL}$ Toluol gelöst und am Wasserabscheider refluxiert bis kein Wasser mehr entstand (24h). Die Reaktionsmischung wurde mit $30 \mathrm{~mL}$ wäßriger $\mathrm{NaHCO}_{3}$ Lsg. (konz.) sowie $30 \mathrm{~mL}$ Wasser gewaschen und die organische Phase über $\mathrm{MgSO}_{4}$ getrocknet. Das Lösungsmittel wurde bei reduziertem Druck am Rotationsverdampfer entfernt und der Rückstand zweimal aus $n$-Hexan umkristallisiert. Es wurden $2.65 \mathrm{~g}(0.014 \mathrm{~mol}, 82 \%)$ von 79 als weiße Kristalle erhalten. 79: ${ }^{1} \mathbf{H}-\mathbf{N M R}\left(250 \mathrm{MHz}, \mathrm{CDCl}_{3}\right): \delta=1.65-1.79(\mathrm{~m}, 10 \mathrm{H}, 4-\mathrm{H}$, 
6-H, 8-H, 9-H, 10-H), 1.95 (s, 2H, 5-H, 7-H), 1.98 (s, 2H, 1-H, 3-H), 3.94 (s, 4H, 2'-H, 3'$\mathrm{H}) ;{ }^{13} \mathrm{C}-\mathbf{N M R}\left(62.9 \mathrm{MHz}, \mathrm{CDCl}_{3}\right): \delta=26.90$ (t, C-5, C-7), 34.88 (s, C-4, C-8, C-9, C-10), 36.39 (t, C-1, C-3), 37.13 (s, C-6), 64,11 (s, C-2', C-3'), 111.44 (q, C-2); IR $\left(\mathrm{CCl}_{4}\right)$ : 664, 922, 944, 1043, 1145, 1319, 1361, 1383, 1452, 1469, 1665, 1692, 1725, 2043, 2348, 2667,

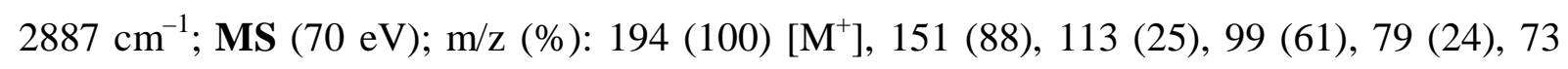
(65), 55 (23) EA: $\mathrm{C}_{12} \mathrm{H}_{18} \mathrm{O}_{2}$ (194.27) Ber.: C 74.19, H 9.34 Gef.: C 73.91, H 9.16 Schmp.: $58^{\circ} \mathrm{C}$

AV16b. 1-Brom-4-(spiro-1',4'-dioxocyclopentyl)tricyclo[3.3.1.1 $\left.1^{3,7}\right]$ decan (82), 1,3-Dibrom6-(spiro-1',4'-dioxocyclopentyl)tricyclo[3.3.1.1 $\left.1^{3,7}\right]$ decan $(\mathbf{8 3})$

$388 \mathrm{mg}$ (2.0 mmol) 79, $2.66 \mathrm{~g}$ (8.0 mmol) Tetrabrommethan und $64 \mathrm{mg}(0.2 \mathrm{mmol}, 10 \mathrm{~mol} \%)$ TBABr wurden in $15 \mathrm{~mL}$ Fluorbenzol gelöst und $6 \mathrm{~mL}$ Natriumhydroxidlösung (aq. 50\%) zugesetzt. Die Mischung wurde 7 Tage bei $60^{\circ} \mathrm{C}$ intensiv gerührt. Anschließend wurden die Phasen getrennt und die wäßrige Phase mit $n$-Pentan $(4$ x $10 \mathrm{~mL})$ extrahiert. Die vereinigten organischen Phasen wurden über Natriumsulfat getrocknet und am Rotationsverdampfer bei reduziertem Druck vom Lösungsmittel befreit. Die erhaltenen 470 mg Rohprodukt wurden einer säulenchromatographischen Trennung unterzogen ( $n$-Pentan : Ether $=95: 5,2.5 \times 55$ $\left.\mathrm{cm}, \mathbf{R}_{\mathrm{f}}(\mathbf{8 2})=0.22, \mathbf{R}_{\mathrm{f}}(\mathbf{8 3})=0.18\right)$. Es wurden $120 \mathrm{mg}(0.44 \mathrm{mmol}, 22 \%)$ von 82 als klare viskose Flüssigkeit und $70 \mathrm{mg}(0.20 \mathrm{mmol}, 10 \%)$ von 83 als weißer Feststoff erhalten. $30 \mathrm{mg}$ von 79 konnten zurückgewonnen werden. 82: Die NMR Daten sind literaturidentisch. ${ }^{[201]}$ 83: ${ }^{1} \mathbf{H}-\mathbf{N M R}\left(250 \mathrm{MHz}, \mathrm{CDCl}_{3}\right): \delta=1.92-1.98(\mathrm{~m}, 2 \mathrm{H}, 5-\mathrm{H}, 7-\mathrm{H}), 2.12-2.18$ (m, 4H, exo(4-H, 8-H, 9-H, 10-H)), $2.12-2.18$ (m, 4H, endo-(4-H, 8-H, 9-H, 10-H)), 2.86 (s, 2H, 2H), 3.94 (s, 4H , 2'-H, 3'-H); ${ }^{13} \mathbf{C}-\mathbf{N M R}\left(62.9 \mathrm{MHz}, \mathrm{CDCl}_{3}\right): \delta=42.29$ (t, C-5, C-7), 44.00 (s, C-4, C-8, C-9, C-10), 58.49 (s, C-2), 59.76 (q, C-1, C-3), 64.64 (s, C-2', C-3'), 107.27 (q, C-6); IR $\left(\mathrm{CCl}_{4}\right):$ 518, 694, 947, 1042, 1161, 1291, 1323, 1336, 1381, 1446, 1584, 2884,

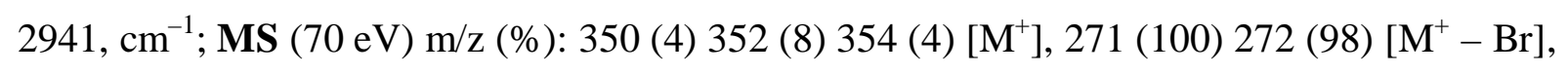
$191(10)\left[\mathrm{M}^{+}-\mathrm{Br}_{2}\right], 147$ (8), 130 (7), 119 (19), 105 (8), 99 (17), 91 (14), 77 (8), 65 (6), 55 (12); EA: $\mathrm{C}_{12} \mathrm{H}_{16} \mathrm{Br}_{2} \mathrm{O}_{2}$ (352.06) Ber.: C 40.94, H 4.58 Gef.: C 40.44, H 4.16; Schmp.: 147 $148^{\circ} \mathrm{C}$ 
AV16c. 1-Iod-4-(spiro-1',4'-dioxocyclopentyl)tricyclo[3.3.1.1 $\left.1^{3,7}\right]$ decan (84)

$0.388 \mathrm{~g}(2.0 \mathrm{mmol}) \mathbf{7 9}, 1.576 \mathrm{~g}(4.0 \mathrm{mmol})$ Iodoform und $64 \mathrm{mg}(0.2 \mathrm{mmol}, 10 \mathrm{~mol} \%)$ $\mathrm{TBABr}$ in $15 \mathrm{~mL}$ Fluorbenzol gelöst und $6 \mathrm{~mL} \mathrm{NaOH} \mathrm{Lsg.} \mathrm{(aq.} 50 \%$ ) zugegeben. Die Mischung wurde 7 Tage bei $60^{\circ} \mathrm{C}$ intensivst gerührt. Anschließend wurden die Phasen getrennt und die wäßrige Phase mit $n$-Pentan extrahiert $(4 \times 10 \mathrm{~mL})$. Die vereinigten organischen Phasen wurden über Natriumsulfat getrocknet und am Rotationsverdampfer unter reduziertem Druck von Lösungsmitteln befreit. Das erhaltene Rohprodukt wurde einer säulenchromatographischen Trennung unterworfen ( $n$-Pentan : Ether $=95: 5,1.5 \times 55 \mathrm{~cm}$, $\left.R_{f}(84)=0.19\right)$. Neben einigen Mischfraktionen wurden $185 \mathrm{mg}(0.57 \mathrm{mmol}, 29 \%)$ von 84 als farbloses, leicht viskoses farbloses Öl erhalten. $112 \mathrm{mg}$ von $\mathbf{7 9}$ konnten zurückgewonnen werden. 84: ${ }^{1} \mathbf{H}-\mathbf{N M R}\left(250 \mathrm{MHz}, \mathrm{CDCl}_{3}\right): \delta=1.68-1.76(\mathrm{~m}, 4 \mathrm{H}, 3-\mathrm{H}, 5-\mathrm{H}$, exo- $(6-\mathrm{H}, 10$ H)), 1.85 (bs, 1H, 7-H), $2.02-2.09$ (m, 2H, endo-(6-H, 10-H)), $2.43-2.48$ (m, 2H, exo-(2H, 9-H)), 2.57 (bs, 2H, 8-H), $2.84-2.89$ (m, 2H, endo-(2-H, 9-H)), 3.91 - 3.94 (m, 4H, 2'$\left.\mathrm{H}, 4^{\prime}-\mathrm{H}\right) ;{ }^{13} \mathbf{C}-\mathbf{N M R}\left(62.9 \mathrm{MHz}, \mathrm{CDCl}_{3}\right): \delta=31.43$ (t, C-7), 32.96 (s, C-6, C-9), 40.82 (t, C3, C-5), 46.78 (q, C-1), 49.25 (s, C-2, C-10), 51.77 (s, C-8), 64.32 (s, C-2'), 64.40 (s, C-3'), 109.12 (q, C-4); IR $\left(\mathrm{CCl}_{4}\right)$ : 534, 688, 920, 947, 1040, 1096, 1140, 1186, 1288, 1349, 1383, 1446, 1470, 1563, $2912 \mathrm{~cm}^{-1}$; MS (70 eV) m/z (\%): $319(0.1)\left[\mathrm{M}^{+}\right], 193(100)\left[\mathrm{M}^{+}-\mathrm{I}\right], 148$ (10), 137 (22), 131 (20), 105 (6), 99 (19), 91 (14), 79 (17), 55 (26); EA: $\mathrm{C}_{12} \mathrm{H}_{17} \mathrm{IO}_{2}$ (320.17) Ber.: C 45.02, H 5.35 Gef.: C 44.71, H 5.12

\section{AV17. Die Iodierung von 1-(1'-methyl)ethyltricyclo[3.3.1.1 $\left.{ }^{3,7}\right]$ decan (85)}

1-Iod-3-(1'-methyl)ethyltricyclo[3.3.1.1 $\left.1^{3,7}\right]$ decan

(86), 1,3-Iod-5-(1'-methyl)ethyltricyclo[3.3.1.1 $\left.1^{3,7}\right]$ decan $(\mathbf{8 8})$

$267 \mathrm{mg}(1.5 \mathrm{mmol}) \mathbf{8 5}, 1.182 \mathrm{~g}$ (3.0 mmol) Iodoform und $48 \mathrm{mg}(0.15 \mathrm{mmol}, 10 \mathrm{~mol} \%)$ $\mathrm{TBABr}$ in $10 \mathrm{~mL}$ Fluorbenzol gelöst und $10 \mathrm{~mL} \mathrm{NaOH} \mathrm{Lsg.} \mathrm{(aq.} 50 \%$ ) zugegeben. Die Mischung wurde 7 Tage bei $70^{\circ} \mathrm{C}$ intensivst gerührt. Nach $40 \mathrm{~h}$ wurden weitere $1.182 \mathrm{~g}$ Iodoform hinzugegeben und die Reaktion nach weiteren $48 \mathrm{~h}$ Reaktionszeit beendet. Anschließend wurden die Phasen getrennt und die wäßrige Phase mit $n$-Pentan extrahiert (4 x $12 \mathrm{~mL}$ ). Die vereinigten organischen Phasen wurden über Natriumsulfat getrocknet und am Rotationsverdampfer unter reduziertem Druck von Lösungsmitteln befreit. Das erhaltene 
Rohprodukt wurde einer säulenchromatographischen Trennung unterworfen ( $n$-Pentan $1.5 \mathrm{x}$ $\left.40 \mathrm{~cm}, \mathbf{R}_{\mathrm{f}}(\mathbf{8 6})=0.46, \mathrm{R}_{\mathrm{f}}(\mathbf{8 8})=0.40\right)$. Es wurden $182 \mathrm{mg}(0.60 \mathrm{mmol}, 40 \%)$ von 86 als gelblicher Feststoff und $110 \mathrm{mg} 88(0.26 \mathrm{mmol}, 15 \%)$ als farbloses, viskoses Öl isoliert werden. $12 \mathrm{mg}$ von 85 wurden zurückgewonnen. 86: ${ }^{1} \mathbf{H}-\mathbf{N M R}\left(250 \mathrm{MHz}, \mathrm{CDCl}_{3}\right): \delta=0.81$ $\left(\mathrm{d},{ }^{3} J_{1^{\prime}-\mathrm{H}}=6.9 \mathrm{~Hz}, 6 \mathrm{H}, 2^{\prime}-\mathrm{H}, 3^{\prime}-\mathrm{H}\right), 1.24\left(\mathrm{hept},{ }^{3} J_{2^{\prime}-\mathrm{H}, 3^{\prime}-\mathrm{H}}=6.9 \mathrm{~Hz}, 1 \mathrm{H}, 1^{\prime}-\mathrm{H}\right), 1.54-1.56(\mathrm{~m}$, $4 \mathrm{H}, 4-\mathrm{H}, 10-\mathrm{H}), 1.68-1.71(\mathrm{~m}, 2 \mathrm{H}, 6-\mathrm{H}), 1.93-2.02(\mathrm{~m}, 2 \mathrm{H}, 5-\mathrm{H}, 7-\mathrm{H}), 2.52-2.55$ (m, $4 \mathrm{H}, 8-\mathrm{H}, 9-\mathrm{H}), 2.60-2.64(\mathrm{~m}, 2 \mathrm{H}, 2-\mathrm{H}) ;{ }^{13} \mathbf{C}-\mathbf{N M R}\left(62.9 \mathrm{MHz}, \mathrm{CDCl}_{3}\right): \delta=16.32(\mathrm{p}, \mathrm{C}-2$ ', C-3'), 33.09 (t, C-5, C-7), 35.31 (s, C-6), 37.27 (s, C-4, C-10), 37.33 (t, C-1'), 40.65 (q, C1), 52.17 (s, C-8, C-9), 52.42 (q, C-3), 54.37 (s, C-2); IR (CCl $)$ : 669, 916, 952, 1097, 1180,

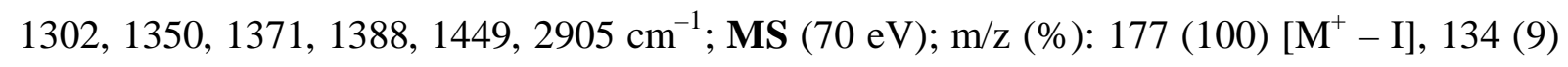
[M+ - I, i-Pr], 121 (19), 107 (11), 93 (22), 79 (18), 69 (4), 55 (3); EA: $\mathrm{C}_{13} \mathrm{H}_{21} \mathrm{I}$ (304.21) Ber.: C 51.33, H 6.96 Gef.: C 51.24, H 6.68; Schmp.: $36^{\circ} \mathrm{C}$; 88: ${ }^{\mathbf{1}} \mathbf{H}-\mathbf{N M R}\left(250 \mathrm{MHz}, \mathrm{CDCl}_{3}\right): \delta=$ $0.83\left(\mathrm{~d},{ }^{3} J_{1^{\prime}-\mathrm{H}}=6.8 \mathrm{~Hz}, 6 \mathrm{H}, 2^{\prime}-\mathrm{H}, 3^{\prime}-\mathrm{H}\right), 1.24\left(\mathrm{hept},{ }^{3} J_{2^{\prime}-\mathrm{H}, 3^{\prime}-\mathrm{H}}=6.8 \mathrm{~Hz}, 1 \mathrm{H}, 1^{\prime}-\mathrm{H}\right), 1.62-$ $1.64(\mathrm{~m}, 2 \mathrm{H}, 6-\mathrm{H}), 1.96-2.01(\mathrm{~m}, 1 \mathrm{H}, 7-\mathrm{H}), 2.36-2.38(\mathrm{~m}, 4 \mathrm{H}, 4-\mathrm{H}, 10-\mathrm{H}), 2.51-2.53$ $(\mathrm{m}, 4 \mathrm{H}, 8-\mathrm{H}, 9-\mathrm{H}), 3.25-3.28(\mathrm{~m}, 2 \mathrm{H}, 2-\mathrm{H}) ;{ }^{13} \mathbf{C}-\mathbf{N M R}\left(62.9 \mathrm{MHz}, \mathrm{CDCl}_{3}\right): \delta=16.34(\mathrm{p}$, C-2', C-3'), 35.46 (s, C-6), 36.29 (t, C-7), 37.02 (t, C-1'), 45.41 (q, C-5), 45.45 (q, C-1, C3), 49.67 (s, C-4, C-10), 52.10 (s, C-8, C-9), 64.06 (s, C-2); IR $\left(\mathrm{CCl}_{4}\right)$ : 659, 687, 918, 952, 1061, 1098, 1179, 1283, 1320, 1341, 1372, 1389, 1448, 2859, $2947 \mathrm{~cm}^{-1} ; \mathbf{M S}(70 \mathrm{eV}) ; \mathrm{m} / \mathrm{z}$

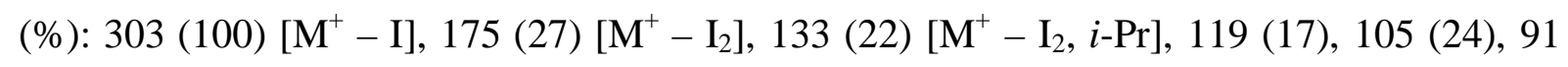
(33), 79 (10), 69 (8), 55 (6); EA: $\mathrm{C}_{13} \mathrm{H}_{21} \mathrm{I}$ (430.11) Ber.: C 36.30, H 4.69 Gef.: C 35.88, H 4.69 


\section{Anhang A. Die Enthalpien und Energien aller computeroptimierten Strukturen}

Vergleich mit experimentellen Daten zur Bestätigung der Anwendbarkeit der Computermethoden [6-311+G** (C,H,Br,Cl), 3-21G* (I)//6-31G** (C,H,Br,Cl), 3-21G* (I)]

\begin{tabular}{|c|c|c|c|c|c|c|}
\hline Spezies & Eo $[\mathrm{au}]$ & $\mathbf{S P} \mathbf{E}_{0}[\mathrm{au}]$ & $\begin{array}{c}\text { ZPVE } \\
{\left[\mathrm{kcal} \mathrm{mol}^{-1}\right]}\end{array}$ & $\begin{array}{c}\Delta \boldsymbol{H}_{\mathbf{0}} \\
{\left[\mathrm{kcal} \mathrm{mol}^{-1}\right]}\end{array}$ & $\begin{array}{c}\Delta \boldsymbol{H}_{\mathbf{2 9 8 K}} \\
{\left[\mathrm{kcal} \mathrm{mol}^{-1}\right]}\end{array}$ & 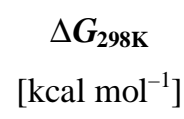 \\
\hline $\mathrm{CCl}_{3}$ & -1418.612594 & -1418.7216703 & 4.44852 & 7.25841 & 7.85141 & -14.28401 \\
\hline $\mathrm{C}_{6} \mathrm{H}_{12}$ & -235.8928684 & -235.9447166 & 107.05395 & 110.64 & 111.23 & 89.41 \\
\hline$t-\mathrm{C}_{4} \mathrm{H}_{12}$ & -158.4698053 & -158.4697501 & 82.71874 & 86.31 & 86.90 & 66.02 \\
\hline ÜZ_C ${ }_{6} \mathrm{H}_{12} \_\mathrm{CI}_{3}$ & -1654.4830797 & -1654.4829935 & 108.52994 & 115.82 & 116.42 & 82.76 \\
\hline$\ddot{\mathrm{UZ}} \_t$-Bu-H_CI${ }_{3}$ & -1577.0632765 & -1577.0632483 & 84.04850 & 91.36 & 91.96 & 59.69 \\
\hline
\end{tabular}

Berechnung des Kinetischen Isotopeneffektes [B3LYP/6-31G** (C,H,Br); 3-21G*(I)]

\begin{tabular}{|c|c|c|c|c|}
\hline Spezies & Eo $[\mathrm{au}]$ & 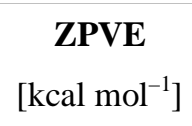 & $\begin{array}{c}\Delta \boldsymbol{H}_{\mathbf{2 9 8 K}} \\
{\left[\mathrm{kcal} \mathrm{mol}^{-1}\right]}\end{array}$ & $\begin{array}{c}\Delta \boldsymbol{G}_{\mathbf{2 9 8 K}} \\
{\left[\mathrm{kcal} \mathrm{mol}^{-1}\right]}\end{array}$ \\
\hline Adamantan & -390.7416019 & 153.58650 & 0.252526 & 0.216410 \\
\hline Adamantan_D 4 & -390.7416019 & 129.82653 & 0.216224 & 0.177728 \\
\hline $\mathrm{C}_{6} \mathrm{H}_{12}$ & -235.8928524 & 106.99845 & 0.177192 & 0.143401 \\
\hline $\mathrm{C}_{6} \mathrm{D}_{12}$ & -235.8928524 & 88.52322 & 0.149828 & 0.111801 \\
\hline $\mathrm{CBr}_{3}$ & -7724.0740459 & 3.45685 & 0.011618 & -0.026652 \\
\hline $\mathrm{CI}_{3}$ & -20708.447521 & 2.75766 & 0.011209 & -0.031396 \\
\hline $\mathrm{Br}$ & -2561.9616407 & 0 & 0.002360 & -0.016830 \\
\hline ÜZ_ $\mathrm{C}_{6} \mathrm{H}_{12} \mathrm{CBr}_{3}$ & -7959.9445764 & 107.32671 & 0.184457 & 0.126283 \\
\hline ÜZ_C $\mathrm{C}_{6} \mathrm{D}_{12} \mathrm{CBr}_{3}$ & -7959.9445764 & 83.37030 & 0.147668 & 0.086968 \\
\hline ÜZ_C $\mathrm{C}_{6} \mathrm{H}_{12} \mathrm{CI}_{3}$ & -20944.3157671 & 106.67050 & 0.183832 & 0.123682 \\
\hline ÜZ_C $\mathrm{C}_{6} \mathrm{H}_{12} \mathrm{CI}_{3}$ & -20944.3157671 & 82.72677 & 0.147074 & 0.084359 \\
\hline ÜZ_ax_C6H12_Br & -2797.8384572 & 102.55796 & 0.172524 & 0.127534 \\
\hline ÜZ_ax_C6D12_Br & -2797.8384572 & 78.73044 & 0.136029 & 0.088211 \\
\hline ÜZ_tert_Adamantan_CBr 3 & -8114.7963004 & 153.03101 & 0.257947 & 0.200929 \\
\hline ÜZ_tert_Adamantan_CBr ${ }_{3} \_\mathrm{D}_{4}$ & -8114.7963004 & 130.21930 & 0.223430 & 0.163073 \\
\hline ÜZ_sec_Adamantan_CBr 3 & -8114.7925177 & 153.43572 & 0.259222 & 0.199609 \\
\hline ÜZ_sec_Adamantan_CBr ${ }_{3} \mathrm{D}_{4}$ & -8114.7925177 & 129.66173 & 0.223206 & 0.160104 \\
\hline ÜZ_tert_Adamantan_CI $I_{3}$ & -21099.1673495 & 152.38143 & 0.257331 & 0.197487 \\
\hline ÜZ_tert_Adamantan_CI $I_{3} \mathrm{D}_{4}$ & -21099.1673495 & 129.66564 & 0.222965 & 0.159747 \\
\hline ÜZ_sec_Adamantan_CI 3 & -21099.1621873 & 152.65296 & 0.258506 & 0.196053 \\
\hline ÜZ_sec_Adamantan_CI ${ }_{3} \mathrm{D}_{4}$ & -21099.1621873 & 128.87308 & 0.222499 & 0.156478 \\
\hline
\end{tabular}


Monohalocubylradikale [6-311+G** $\left.(\mathrm{C}, \mathrm{H}, \mathrm{Br}, \mathrm{Cl}), 3-21 \mathrm{G}^{*}(\mathrm{I}) / / 6-31 \mathrm{G} * *(\mathrm{C}, \mathrm{H}, \mathrm{Br}, \mathrm{Cl}), 3-21 \mathrm{G} *(\mathrm{I})\right]$

\begin{tabular}{lcccccc}
\hline \multicolumn{1}{c}{ Spezies } & $\mathbf{E o}[\mathrm{au}]$ & $\mathbf{S P} \mathbf{E}_{\mathbf{0}}[\mathrm{au}]$ & $\begin{array}{c}\mathbf{Z P V E} \\
{\left[\mathrm{kcal} \mathrm{mol}^{-1}\right]}\end{array}$ & $\begin{array}{c}\Delta \boldsymbol{H}_{\mathbf{0}} \\
{\left[\mathrm{kcal} \mathrm{mol}^{-1}\right]}\end{array}$ & $\begin{array}{c}\Delta \boldsymbol{H}_{\mathbf{2 9 8 K}} \\
{\left[\mathrm{kcal} \mathrm{mol}^{-1}\right]}\end{array}$ & $\begin{array}{c}\Delta \boldsymbol{G}_{\mathbf{2 9 8 K}} \\
{\left[\mathrm{kcal} \mathrm{mol}^{-1}\right]}\end{array}$ \\
\hline$m$-CubBr_rad_ & -2879.6007798 & -2882.4090699 & 69.70735 & 73.49648 & 74.08885 & 49.46850 \\
$m$-CubCl_rad & -768.3895482 & -768.488106 & 70.02698 & 73.65838 & 74.25075 & 50.51518 \\
$m$-CubI_rad & -7198.2775213 & -7198.3524206 & 69.53675 & 73.40486 & 73.99723 & 48.77949 \\
$o$-CubBr_rad & -2879.5994132 & -2882.4078926 & 69.68527 & 73.47954 & 74.07191 & 49.43022 \\
$o$-CubCl_rad & -768.3882424 & -768.4868213 & 70.03162 & 73.66528 & 74.25765 & 50.50828 \\
$o$-CubI_rad & -7198.2765074 & -7198.3513389 & 69.51024 & 73.38667 & 73.97904 & 48.73054 \\
$p$-CubBr_rad & -2879.598904 & -2882.4071711 & 69.80060 & 73.57618 & 74.16854 & 50.22778 \\
$p$-CubCl_rad & -768.387677 & -768.4862283 & 70.08047 & 73.70921 & 74.30158 & 51.21988 \\
$p$-CubI_rad & -7198.2756936 & -7198.3506047 & 69.59848 & 73.46385 & 74.05685 & 49.48858
\end{tabular}

Die Chlorierung von Cuban [6-311+G** $(\mathrm{C}, \mathrm{H}, \mathrm{Br}, \mathrm{Cl}) / / 6-31 \mathrm{G} * *(\mathrm{C}, \mathrm{H}, \mathrm{Br}, \mathrm{Cl})]$

\begin{tabular}{|c|c|c|c|c|c|c|}
\hline Spezies & Eo $[\mathrm{au}]$ & $\mathbf{S P} \mathbf{E}_{0}[\mathrm{au}]$ & $\begin{array}{c}\text { ZPVE } \\
{\left[\mathrm{kcal} \mathrm{mol}^{-1}\right]}\end{array}$ & $\begin{array}{c}\Delta \boldsymbol{H}_{\mathbf{0}} \\
{\left[\mathrm{kcal} \mathrm{mol}^{-1}\right]}\end{array}$ & $\begin{array}{c}\Delta \boldsymbol{H}_{\mathbf{2 9 8 K}} \\
{\left[\mathrm{kcal} \mathrm{mol}^{-1}\right]}\end{array}$ & $\begin{array}{c}\Delta \boldsymbol{G}_{\mathbf{2 9 8 K}} \\
{\left[\mathrm{kcal} \mathrm{mol}^{-1}\right]}\end{array}$ \\
\hline Cuban & -309.468188 & -309.5327217 & 84.19385 & 87.02560 & 87.61797 & 68.38792 \\
\hline Cubylradikal & -308.79192 & -308.8574607 & 75.94511 & 78.78137 & 79.37374 & 57.87525 \\
\hline $\mathrm{CCl}_{3}$ & -1418.612594 & -1418.7216703 & 4.44852 & 7.25841 & 7.85141 & -14.28401 \\
\hline $\mathrm{CHCl}_{3}$ & -1419.2695203 & -1419.3796556 & 12.46440 & 15.26983 & 15.26983 & -5.22277 \\
\hline $\mathrm{Cl}$ & -460.132788 & -460.1668781 & 0 & 0.88855 & 0.88855 & -9.83747 \\
\hline $\mathrm{HCl}$ & -460.7974624 & -460.8338454 & 4.21998 & 5.70093 & 5.70093 & -7.00427 \\
\hline ÜZ1 CubC_CCl ${ }_{3}$ & -1728.0408654 & -1728.2159889 & 87.92560 & 94.45281 & 94.45281 & 63.71799 \\
\hline ÜZ1 Cub_C_Cl & -769.6033451 & -769.702816 & 82.92122 & 87.05509 & 87.05509 & 62.65625 \\
\hline ÜZ2 Cub_C_CCl ${ }_{3}$ & -1728.1281007 & -1728.3047634 & 88.59803 & 94.95544 & 94.95544 & 65.14495 \\
\hline ÜZ2 Cub_C_Cl & -769.6518669 & -769.7533013 & 83.11030 & 87.25276 & 87.25276 & 63.03024 \\
\hline ÜZ Cub_H_CCl 3 & -1728.0585351 & -1728.2319552 & 85.32082 & 91.46460 & 92.05760 & 61.53112 \\
\hline ÜZ Cub_H_Cl & -769.6039376 & -769.7038844 & 79.56516 & 83.58182 & 84.17419 & 59.54380 \\
\hline init $\mathrm{Cub} \_\mathrm{C} \_\mathrm{CCl}_{3}$ & -1728.0808505 & -1728.2551323 & 88.74071 & 96.01405 & 96.01405 & 58.15763 \\
\hline init Cub_C_Cl & -769.6010019 & -769.6997317 & 84.29540 & 88.72176 & 88.72176 & 62.13604 \\
\hline init Cub_H_CCl 3 & -1728.0808122 & -1728.255335 & 88.68930 & 95.41290 & 95.41290 & 59.29844 \\
\hline init Cub_H_Cl & -769.614692 & -769.7133236 & 82.09489 & 86.47339 & 86.47339 & 60.79129 \\
\hline min1Cub_C_CCl 3 & -1728.1463243 & -1728.3213396 & 89.94834 & 88.53350 & 96.31086 & 66.61583 \\
\hline min 1Cub_C_Cl & -769.6695199 & -769.769095 & 84.41121 & 96.31086 & 88.53350 & 64.44779 \\
\hline min2Cub_C_CCl ${ }_{3}$ & -1728.1545526 & -1728.3325891 & 88.78512 & 95.78375 & 95.78375 & 64.50238 \\
\hline min2Cub_C_Cl & -769.6777115 & -769.7805631 & 83.28709 & 88.05346 & 88.05346 & 62.41654 \\
\hline
\end{tabular}


Die Bromierung von Cuban [6-311+G** $(\mathrm{C}, \mathrm{H}, \mathrm{Br}, \mathrm{Br}) / / 6-31 \mathrm{G} * *(\mathrm{C}, \mathrm{H}, \mathrm{Br}, \mathrm{Br})]$

\begin{tabular}{lcccccc}
\hline \multicolumn{1}{c}{ Spezies } & $\mathbf{E o}[\mathrm{au}]$ & $\mathbf{S P} \mathbf{E}_{\mathbf{0}}[\mathrm{au}]$ & $\mathbf{Z P V E}$ & $\begin{array}{c}\Delta \boldsymbol{H}_{\mathbf{0}} \\
{\left[\mathrm{kcal} \mathrm{mol}^{-1}\right]}\end{array}$ & $\begin{array}{c}\Delta \boldsymbol{H}_{\mathbf{2 9 8 K}} \\
{\left[\mathrm{kcal} \mathrm{mol}^{-1}\right]}\end{array}$ & $\begin{array}{c}\Delta \boldsymbol{G}_{\mathbf{2 9 8 K}} \\
{\left[\mathrm{kcal} \mathrm{mol}^{-1}\right]}\end{array}$ \\
\hline Cuban & -309.468188 & -309.5327217 & 84.19385 & 87.02560 & 87.61797 & 68.38792 \\
Cubylradikal & -308.79192 & -308.8574607 & 75.94511 & 78.78137 & 79.37374 & 57.87525 \\
CBr $_{3}$ & -7752.2442258 & -7760.4848643 & 3.40342 & 6.64721 & 7.24021 & -16.78652 \\
CHBr $_{3}$ & -7752.9002096 & -7761.1409924 & 11.25049 & 14.49485 & 15.08785 & -8.54229 \\
Br & -2571.3592042 & -2574.1055419 & 0 & 0.88855 & 1.48092 & -10.56099 \\
HBr & -2572.0065178 & -2574.753076 & 3.80516 & 5.28614 & 5.87914 & -8.25803 \\
ÜZ1 CubC_CBr 3 & -8061.6732186 & -8069.9758632 & 86.68318 & 93.80521 & 94.39758 & 60.37713 \\
ÜZ1 Cub_C_Br & -2880.822556 & -2883.6328028 & 82.83232 & 87.02748 & 87.61985 & 61.92394 \\
ÜZ2 Cub_C_CBr 3 & -8061.6904978 & -8070.0628984 & 87.39760 & 94.33985 & 94.93222 & 62.05258 \\
ÜZ2 Cub_C_Br & -2880.8646989 & -2883.6761028 & 82.75046 & 87.04379 & 87.63616 & 61.93900 \\
ÜZ Cub_H_CBr 3 & -8061.6904978 & -8069.9938082 & 84.07339 & 91.33847 & 91.93084 & 56.14897 \\
ÜZ Cub_H_Br & -2880.813531 & -2883.6242592 & 78.93473 & 83.13817 & 83.73054 & 58.17206 \\
init Cub_C_CBr 3 & -8061.7127937 & -8070.0169785 & 87.68588 & 94.82680 & 95.41917 & 57.42721 \\
init Cub_C_Br & -2880.8277852 & -2883.6244699 & 83.43346 & 87.426575 & 88.018945 & 61.352907 \\
init Cub_H_Br & -2880.8361395 & -2883.6463696 & 82.59816 & 87.07141 & 87.66377 & 60.13428 \\
min1Cub_C_CBr 3 & -8061.7773929 & -8070.0788464 & 88.62820 & 95.59989 & 96.19226 & 63.35655 \\
min1Cub_C_Br & -2880.8809797 & -2883.6902743 & 84.03820 & 88.30321 & 88.89620 & 63.37035 \\
min2Cub_C_CBr 3 & -8061.7860238 & -8070.0910321 & 87.51528 & 95.10793 & 95.70030 & 61.29643 \\
min2Cub_C_Br & -2880.8910281 & -2883.7040833 & 82.96476 & 87.88654 & 88.47891 & 61.31400
\end{tabular}


Die Zweithalogenierung von Halocubanen $\left[6-311+\mathrm{G}^{* *} \quad(\mathrm{C}, \mathrm{H}, \mathrm{Br}, \mathrm{Cl}), 3-21 \mathrm{G}^{*} \quad(\mathrm{I}) / / 6-31 \mathrm{G}^{* *}\right.$ $\left.(\mathrm{C}, \mathrm{H}, \mathrm{Br}, \mathrm{Cl}), 3-21 \mathrm{G}^{*}(\mathrm{I})\right]$

\begin{tabular}{|c|c|c|c|c|c|c|}
\hline Spezies & Eo $[\mathrm{au}]$ & $\mathbf{S P} \mathbf{E}_{0}[\mathrm{au}]$ & $\begin{array}{c}\text { ZPVE } \\
{\left[\mathrm{kcal} \mathrm{mol}^{-1}\right]}\end{array}$ & $\begin{array}{c}\Delta \boldsymbol{H}_{\mathbf{0}} \\
{\left[\mathrm{kcal} \mathrm{mol}^{-1}\right]}\end{array}$ & $\begin{array}{c}\Delta \boldsymbol{H}_{\mathbf{2 9 8 K}} \\
{\left[\mathrm{kcal} \mathrm{mol}^{-1}\right]}\end{array}$ & $\begin{array}{c}\Delta \boldsymbol{G}_{\mathbf{2 9 8 K}} \\
{\left[\mathrm{kcal} \mathrm{mol}^{-1}\right]}\end{array}$ \\
\hline $\mathrm{CBr}_{3}$ & -7752.2442258 & -7760.4848643 & 3.40342 & 6.64721 & 7.24021 & -16.78652 \\
\hline $\mathrm{CCl}_{3}$ & -1418.612594 & -1418.7216703 & 4.44852 & 7.25841 & 7.85141 & -14.28401 \\
\hline $\mathrm{CI}_{3}$ & -20708.2748605 & -20708.2950264 & 2.78082 & 6.38554 & 6.97791 & -19.81677 \\
\hline Cub_Br & -2880.2777286 & -2883.0852129 & 77.92648 & 81.71247 & 82.30547 & 58.73243 \\
\hline Cub_Cl & -769.0666737 & -769.1644368 & 78.22275 & 81.85742 & 82.45042 & 59.74585 \\
\hline Cub_I & -7198.9541074 & -7199.0282637 & 77.75450 & 81.62650 & 82.21950 & 58.03024 \\
\hline Cubane & -309.468188 & -309.5327217 & 84.19385 & 87.02560 & 87.61797 & 68.38792 \\
\hline Cubane_rad & -308.79192 & -308.8574607 & 75.94511 & 78.78137 & 79.37374 & 57.87525 \\
\hline ÜZ_Cubane_CBr 3 & -8061.6904978 & -8069.9938082 & 84.07339 & 91.33847 & 91.93084 & 56.14897 \\
\hline ÜZ_Cubane_CCl 3 & -1728.0585351 & -1728.2319552 & 85.32082 & 91.46460 & 92.05760 & 61.53112 \\
\hline ÜZ_Cubane_CI ${ }_{3}$ & -21017.7197475 & -21017.81356 & 83.39346 & 90.93122 & 91.52359 & 54.36433 \\
\hline ÜZ_mCubBr_CBr 3 & -10632.497951 & -10643.5440413 & 77.81963 & 85.48380 & 86.07617 & 49.42394 \\
\hline ÜZ_mCubBr_CCl 3 & -4298.8659754 & -4301.7823725 & 79.09940 & 86.79656 & 87.38893 & 50.37713 \\
\hline ÜZ_mCubBr_CI ${ }_{3}$ & -23588.5271145 & -23591.3642338 & 77.06517 & 85.03137 & 85.62374 & 47.13667 \\
\hline ÜZ_mCubCl_CBr ${ }_{3}$ & -8521.2868194 & -8529.6233005 & 78.17311 & 86.23744 & 86.83044 & 48.04342 \\
\hline ÜZ_mCubCl_CCl${ }_{3}$ & -2187.6547948 & -2187.8615923 & 79.38221 & 86.34600 & 86.93900 & 53.67281 \\
\hline ÜZ_mCubCl_CI $I_{3}$ & -21477.3159718 & -21477.3158863 & 77.46759 & 85.81639 & 86.40875 & 46.76581 \\
\hline ÜZ_mCubI_CBr ${ }_{3}$ & -14951.1746159 & -14959.4883841 & 77.63664 & 85.38152 & 85.97389 & 48.83910 \\
\hline ÜZ_mCubI_CCl 3 & -8617.5426167 & -8617.7267074 & 78.89635 & 86.10127 & 86.69364 & 52.10529 \\
\hline ÜZ_mCubI_CI 3 & -27907.2037734 & -27907.3082389 & 76.91070 & 84.96046 & 85.55283 & 46.59199 \\
\hline ÜZ_oCubBr_CBr 3 & -10632.4964535 & -10643.5420863 & 77.93934 & 86.14959 & 86.74259 & 47.99071 \\
\hline ÜZ_oCubBr_CCl 3 & -4298.8645848 & -4301.7806813 & 79.18296 & 86.86558 & 87.45795 & 51.24937 \\
\hline ÜZ_oCubBr_CI ${ }_{3}$ & -23588.525397 & -23591.3628558 & 77.21892 & 85.73167 & 86.32404 & 45.94063 \\
\hline ÜZ_oCubCl_CBr 3 & -8521.2851282 & -8529.6214614 & 78.17557 & 86.23933 & 86.83170 & 48.92821 \\
\hline ÜZ_oCubCl_CCl 3 & -2187.6531959 & -2187.8599804 & 79.40829 & 86.35855 & 86.95092 & 53.87299 \\
\hline ÜZ_oCubCl_CI ${ }_{3}$ & -21477.314052 & -21477.4421974 & 77.45564 & 85.81136 & 86.40436 & 45.66265 \\
\hline ÜZ_oCubI_CBr 3 & -14951.173499 & -14959.4880394 & 77.79737 & 86.09312 & 86.68549 & 47.51568 \\
\hline ÜZ_oCubI_CCl ${ }_{3}$ & -8617.5415526 & -8617.7266076 & 79.03102 & 86.79656 & 87.38955 & 50.72414 \\
\hline ÜZ_oCubI_CI $I_{3}$ & -27907.2025326 & -27907.3078312 & 77.01395 & 85.04329 & 85.63566 & 47.11345 \\
\hline ÜZ_p CubBr_CBr ${ }_{3}$ & -10632.496852 & -10643.5429964 & 77.82697 & 85.51267 & 86.10504 & 49.82806 \\
\hline ÜZ_p CubBr_CCl ${ }_{3}$ & -4298.8648757 & -4301.7812566 & 79.07219 & 86.22489 & 86.81726 & 53.11998 \\
\hline ÜZ_pCubBr_CI $I_{3}$ & -23588.525998 & -23591.3631816 & 77.14239 & 85.10855 & 85.70092 & 47.73155 \\
\hline ÜZ_pCubCl_CBr 3 & -8521.2856328 & -8529.622137 & 78.15557 & 85.67080 & 86.26317 & 50.85969 \\
\hline ÜZ_pCubCl_CCl 3 & -2187.6536916 & -2187.8604692 & 79.42699 & 86.39746 & 86.98983 & 53.67532 \\
\hline ÜZ_pCubCl_CI ${ }_{3}$ & -21477.3147802 & -214774423703 & 77.47313 & 85.85090 & 86.44327 & 46.84550 \\
\hline ÜZ_pCubI_CBr ${ }_{3}$ & -14951.1737185 & 14959.4870234 & 77.63070 & 85.99648 & 86.58885 & 47.29794 \\
\hline ÜZ_pCubI_CCl ${ }_{3}$ & -8617.5416915 & -8617.725263 & 78.86806 & 86.69678 & 87.28915 & 50.72101 \\
\hline ÜZ_pCubI_CI ${ }_{3}$ & -27907.2028855 & -27907.3068695 & 76.92944 & 84.99874 & 85.59111 & 46.99799 \\
\hline
\end{tabular}




\section{Anhang B. Molekülverzeichnis}

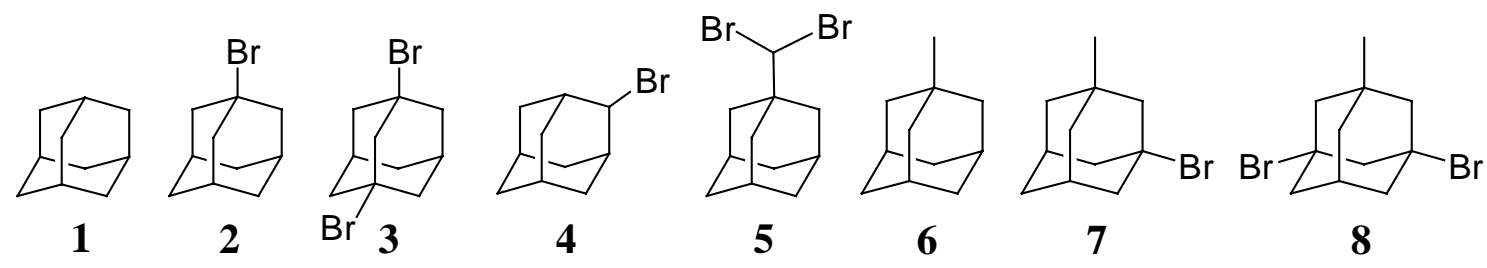

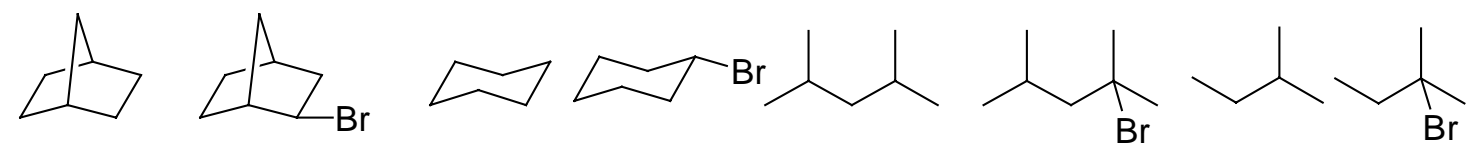

$\begin{array}{llllllll}9 & 10 & 11 & 12 & 13 & 14 & 15 & 16\end{array}$

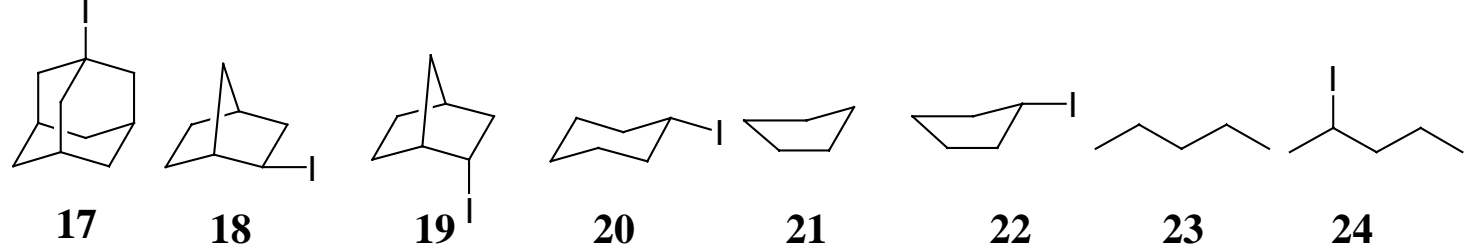<smiles>CCC(I)CC</smiles>

25

26<smiles>ClC1C2CC3CC(C2)CC1C3</smiles><smiles>ClC12CC3CC(C1)CC(Cl)(C3)C2</smiles>

28

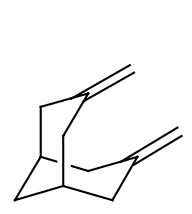

29

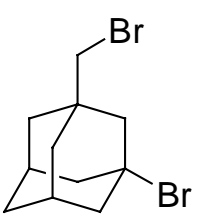

30

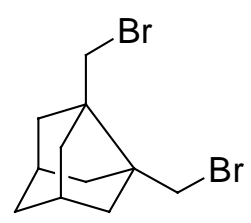

31

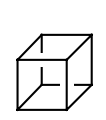

32<smiles>BrC1C(Br)C2CCCC1C2Br</smiles>

33

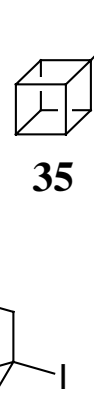

40

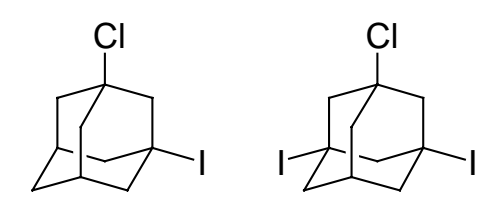

41

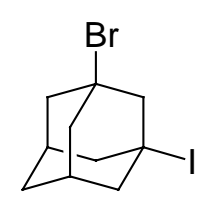

42

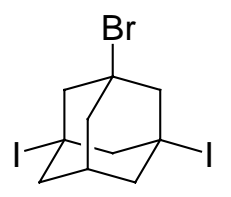

43

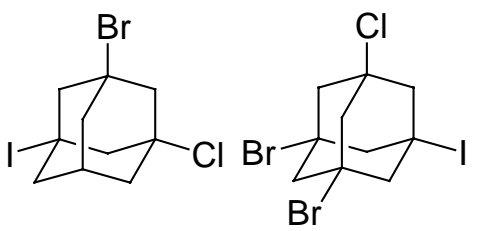

44

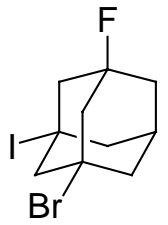

$( \pm)-51$<smiles>FC12CC3(Cl)CC(Br)(C1)CC(Br)(C2)C3</smiles>

52

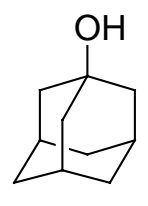

46

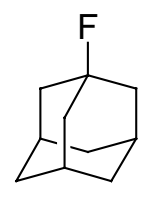

47

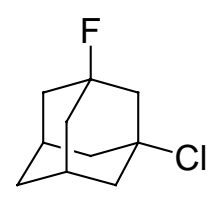

48

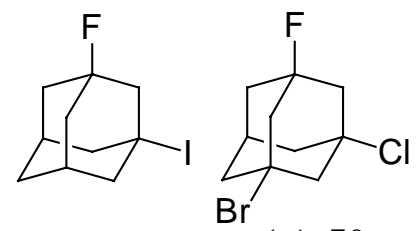

49

$( \pm)-50$<smiles>FC12CC3(F)CC(Br)(C1)CC(Cl)(CC1(Br)CC4(F)CC5(Br)CC(I)(C4)CC1(Br)C5)CC(I)(C2)C3</smiles>
53
$( \pm)-54$
$( \pm)-55$ 


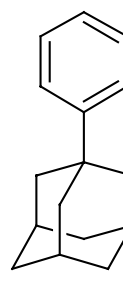
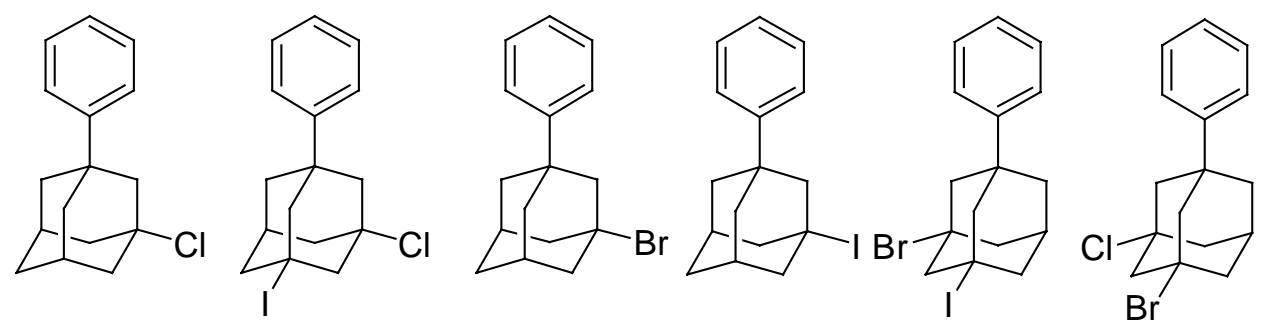

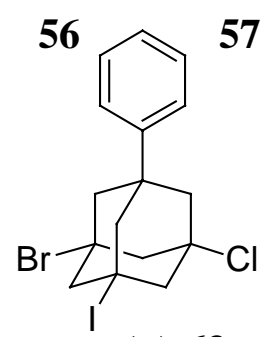

$( \pm)-63$

(土)-58

59

60

( \pm -61

$( \pm)-62$<smiles>ClC12CC3CC(Br)(C1)CC(c1ccccc1)(C3)C2(c1ccccc1)c1ccccc1</smiles>

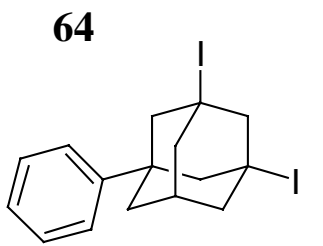

67<smiles>ClC12CC3(Br)CC(Br)(C1)CC(c1ccccc1)(C2)C3</smiles>

68<smiles>COC12CC3CC(CC(C3)C1)C2</smiles>

69<smiles>COC12CC3CC(CC(Cl)(C3)C1)C2</smiles>

70

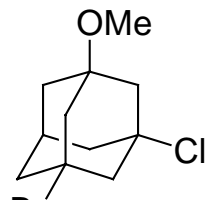

$\mathrm{Br}_{( \pm)-71}$

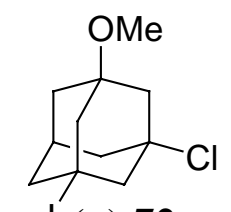

I $( \pm)-72$

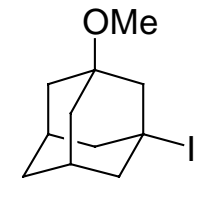

73

66

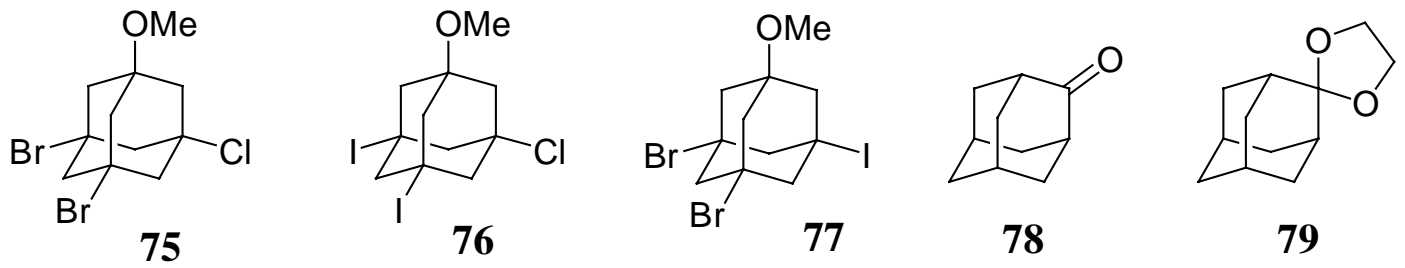<smiles>ClC12CC3CC(Cl)(CC(C1)C31OCCO1)C21OCCO1</smiles>

80

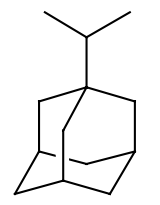

85

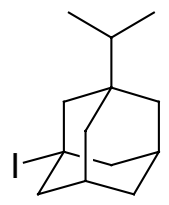

86
81

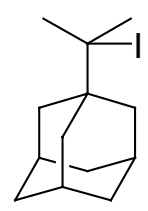

87<smiles>BrC1C2CC3CC(C2)C2(OCCO2)C1(Br)C3</smiles>

82

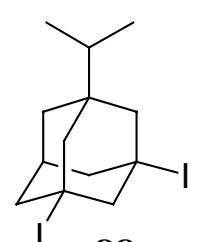

88<smiles>BrC1CC2CC3(Br)OCCOC34CC2CC14Br</smiles>

83

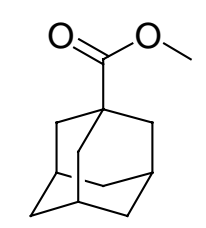

89

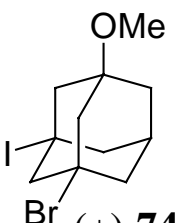

$\mathrm{Br}_{( \pm)-74}$ 


\section{Anhang C. Abbildungen der ${ }^{1}$ H-NMR Spektren}

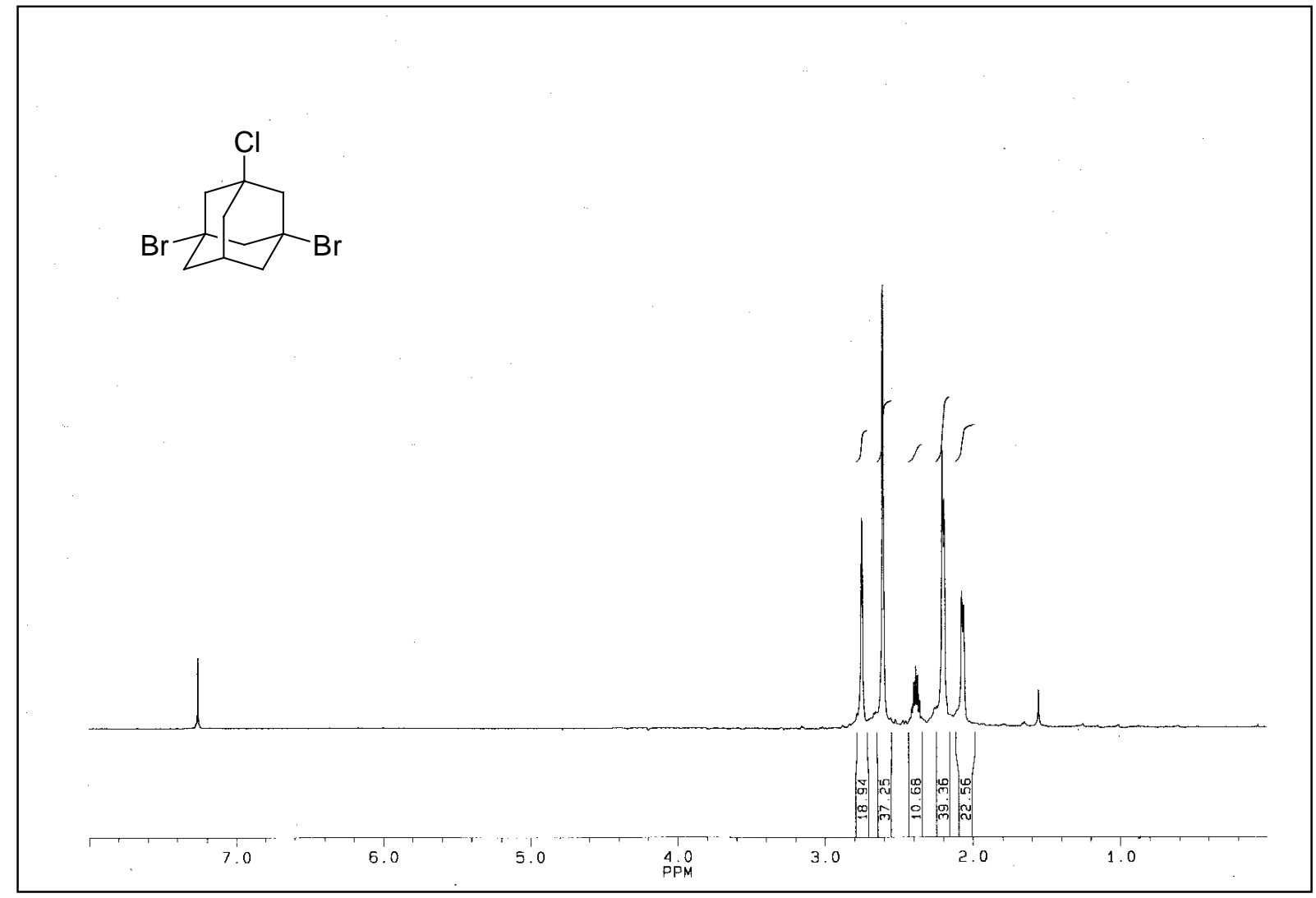

1,3-Dibrom-5-chlortricyclo[3.3.1.1 $\left.1^{3,7}\right]$ decan (39)

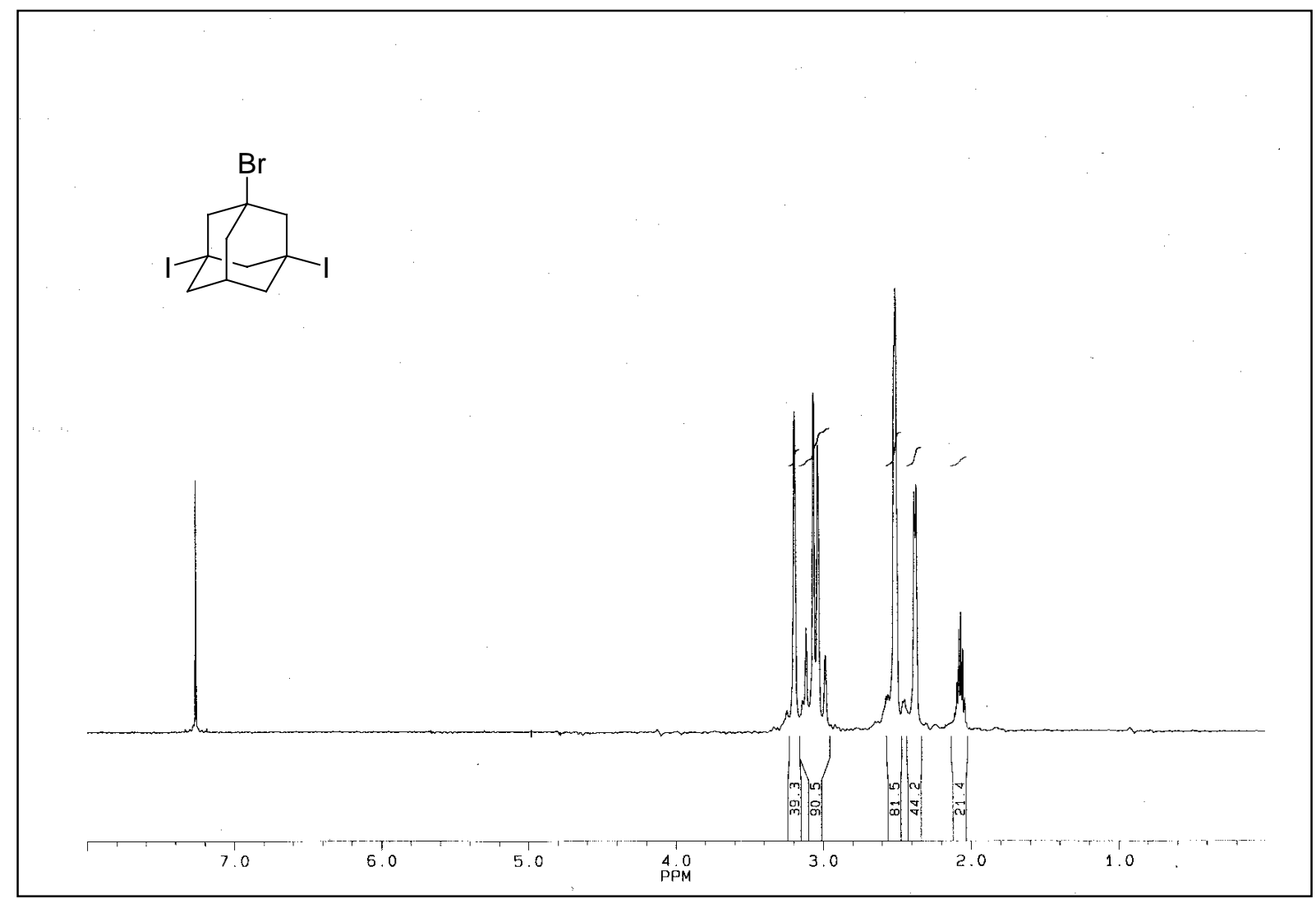

1,3-Diiod-5-bromtricyclo[3.3.1.1 $\left.1^{3,7}\right] \operatorname{decan}(\mathbf{4 3})$ 


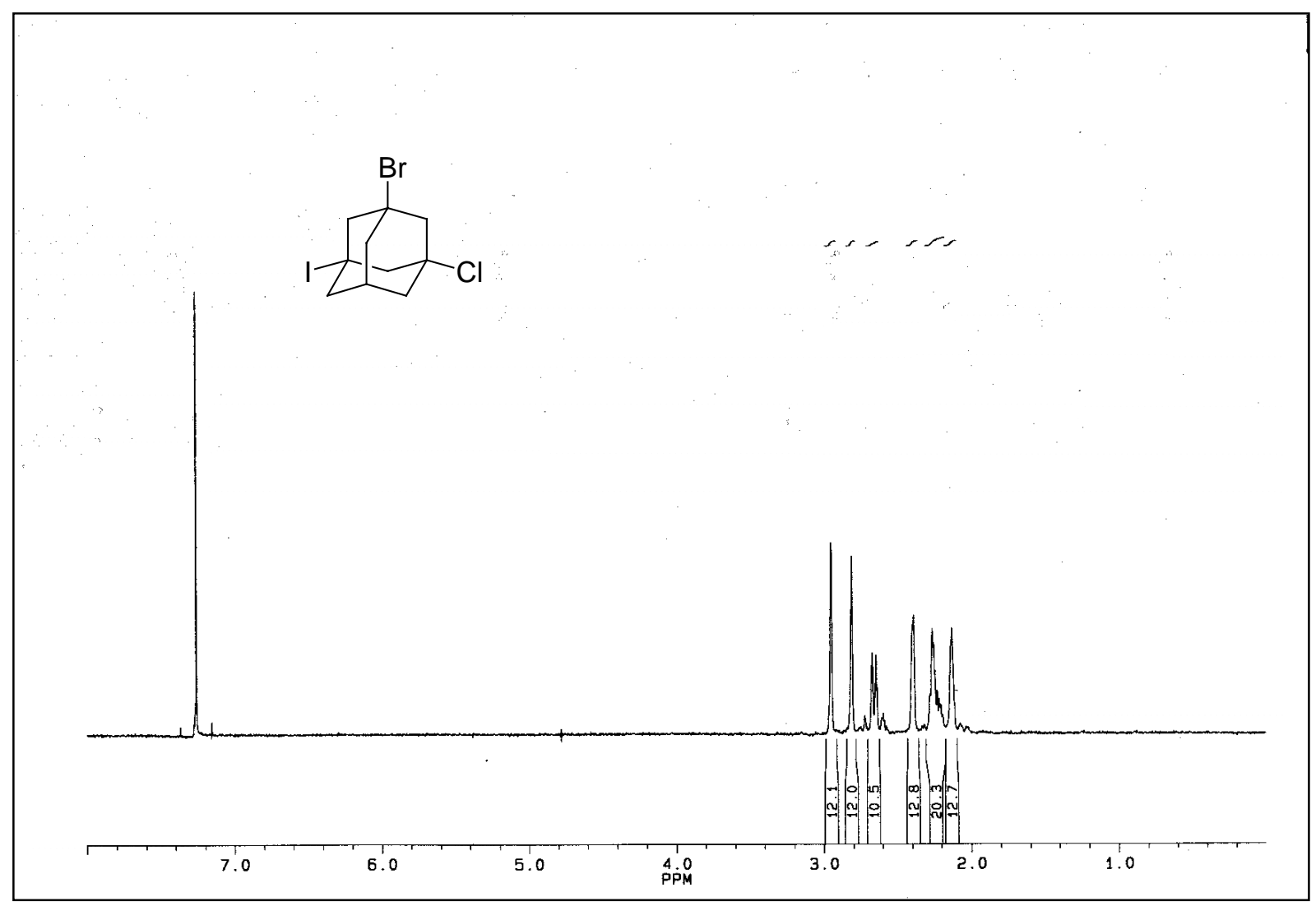

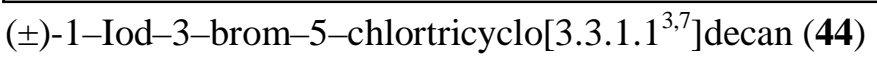

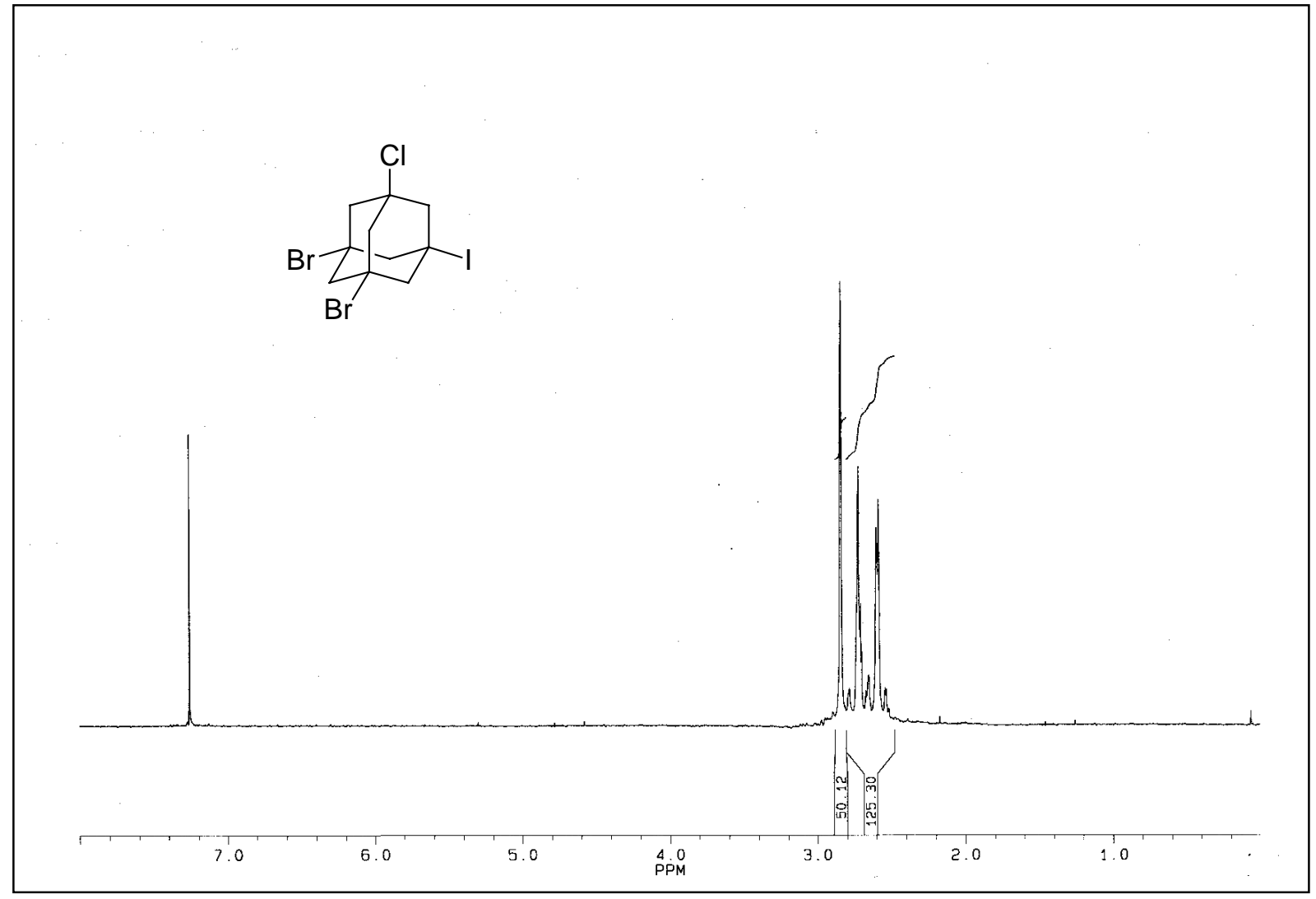

1-Iod-3,5-dibrom-7-chlortricyclo[3.3.1.1 $\left.1^{3,7}\right]$ decan (45) 


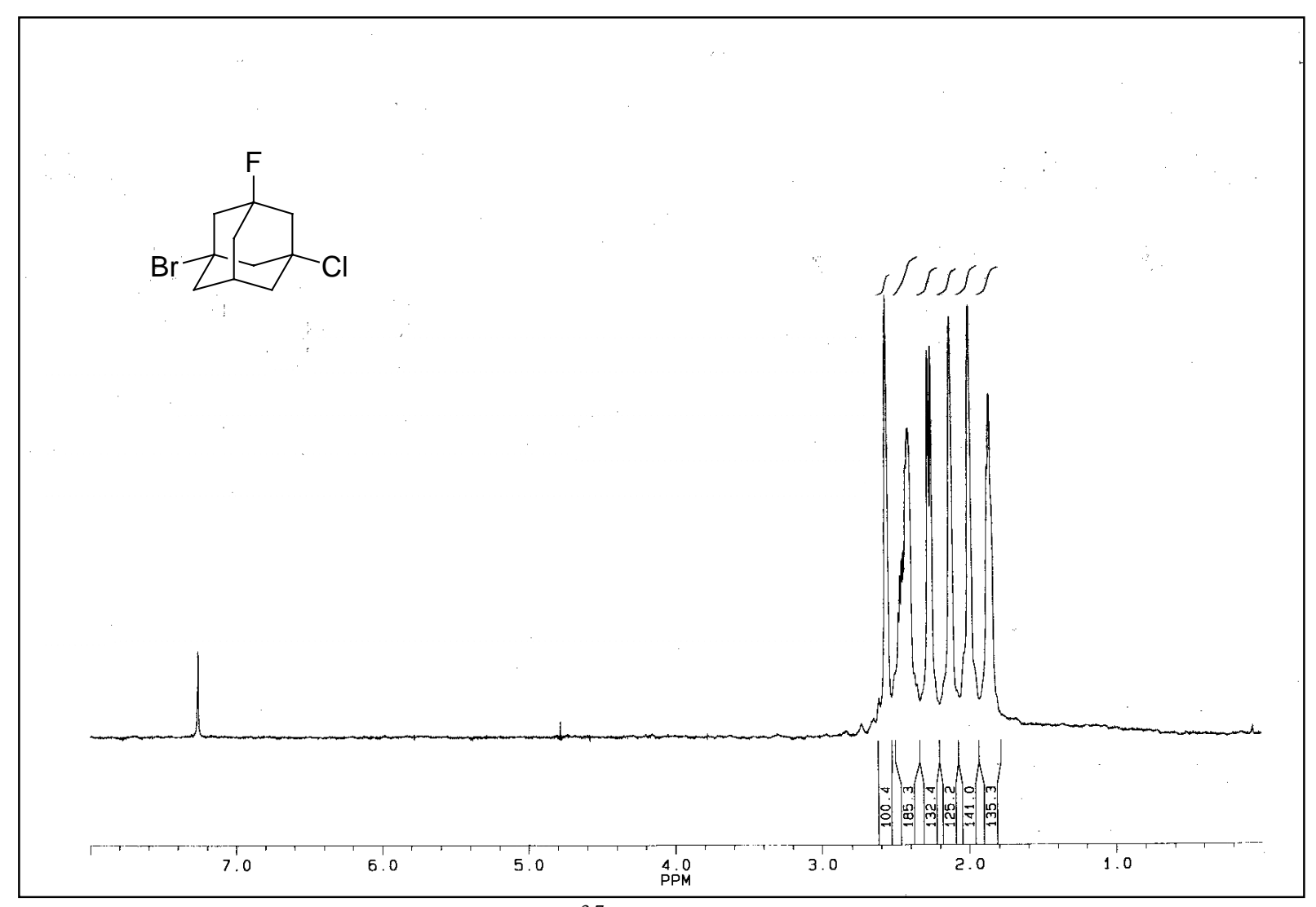

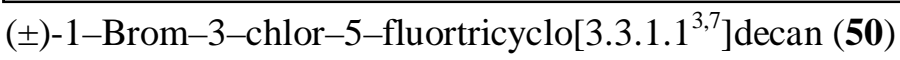

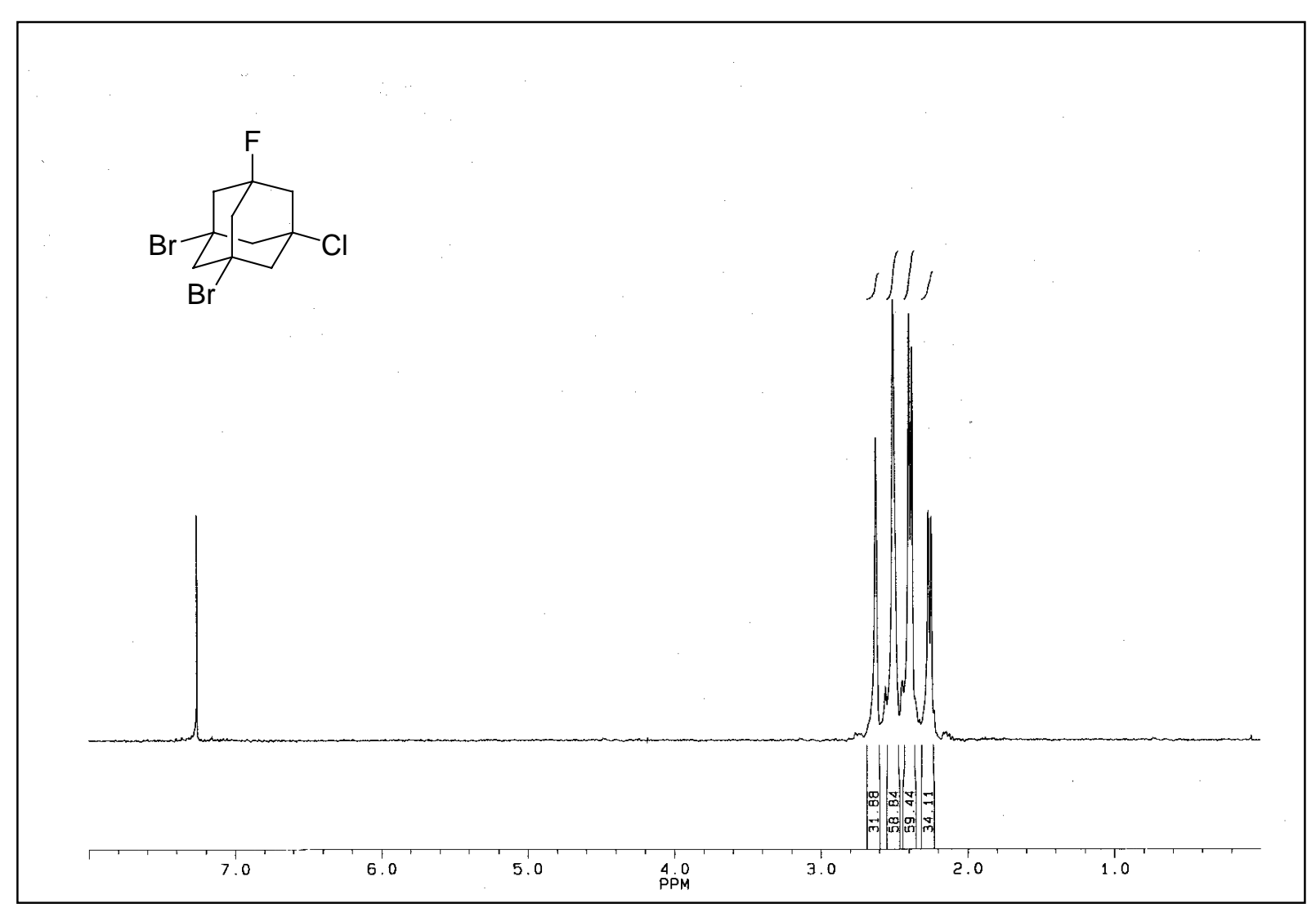

1,3-Dibrom-5-chlor-7-fluortricyclo[3.3.1.1 $\left.{ }^{3,7}\right]$ decan (52) 


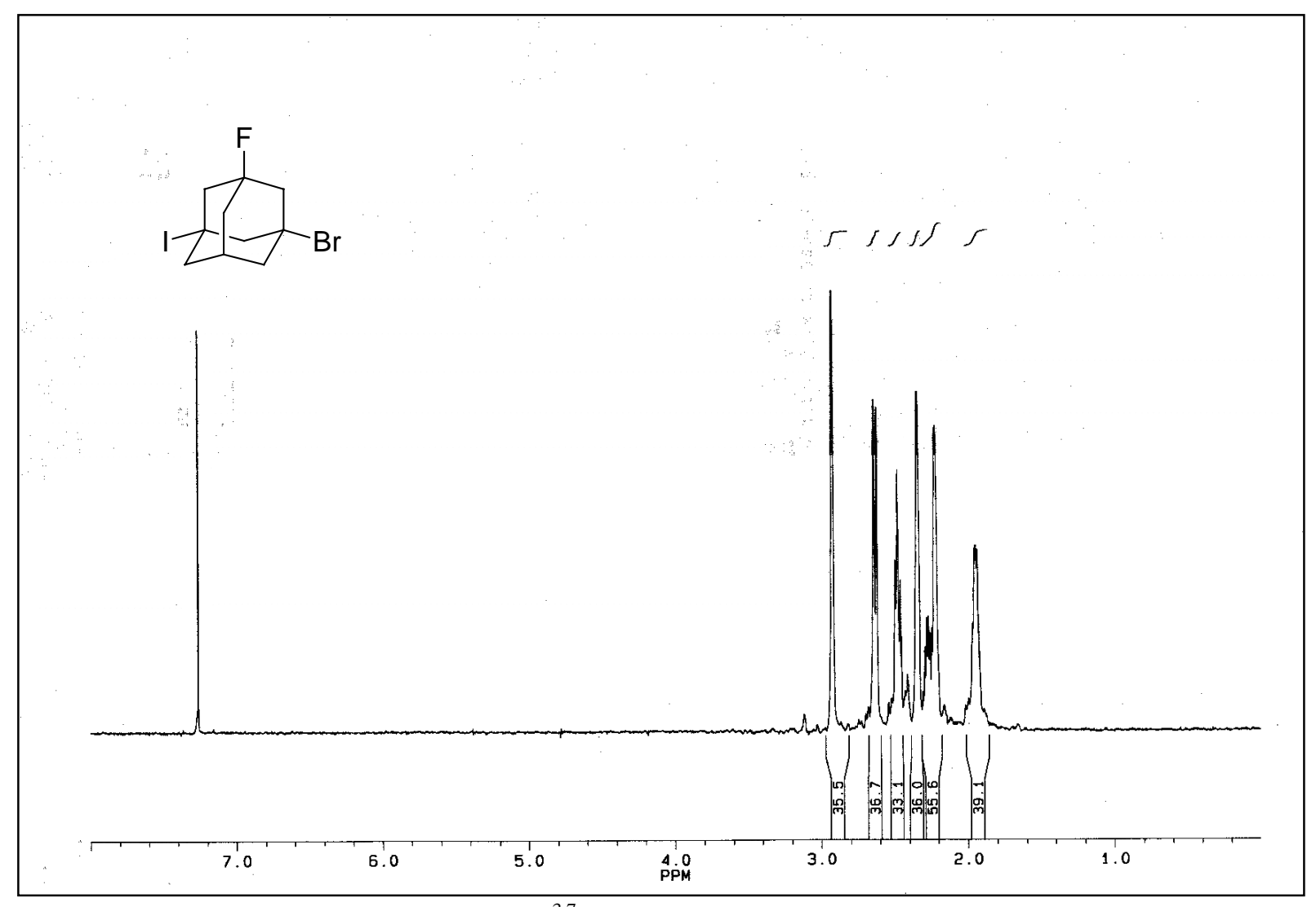

( \pm )-1-Iod-3-brom-5-fluortricyclo[3.3.1.1 $\left.{ }^{3,7}\right]$ decan (51)

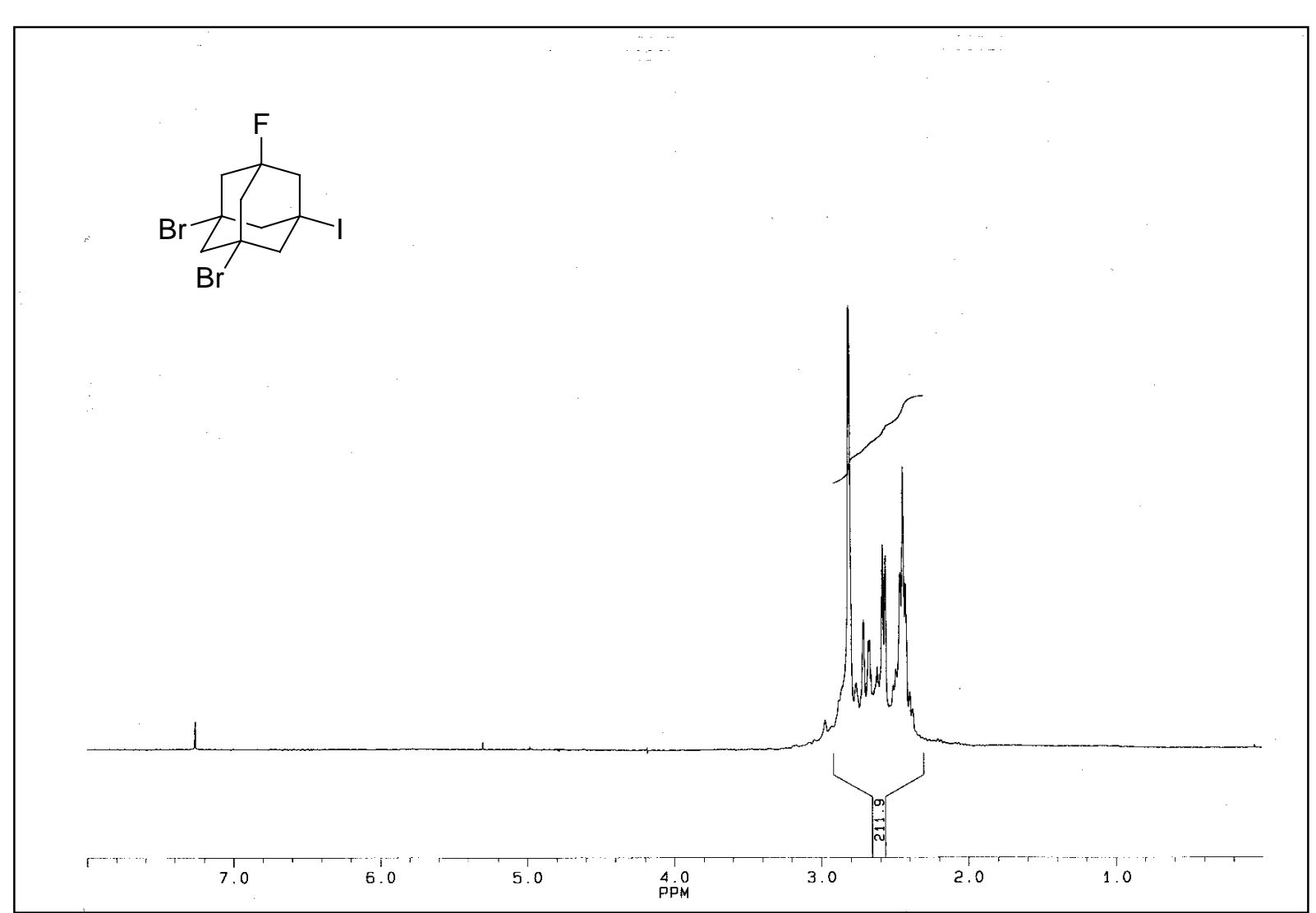

1-Iod-3,5-dibrom-7-fluortricyclo[3.3.1.1 $\left.{ }^{3,7}\right]$ decan (53) 
Anhang C. Abbildungen der ${ }^{l} H-N M R$ Spektren

155

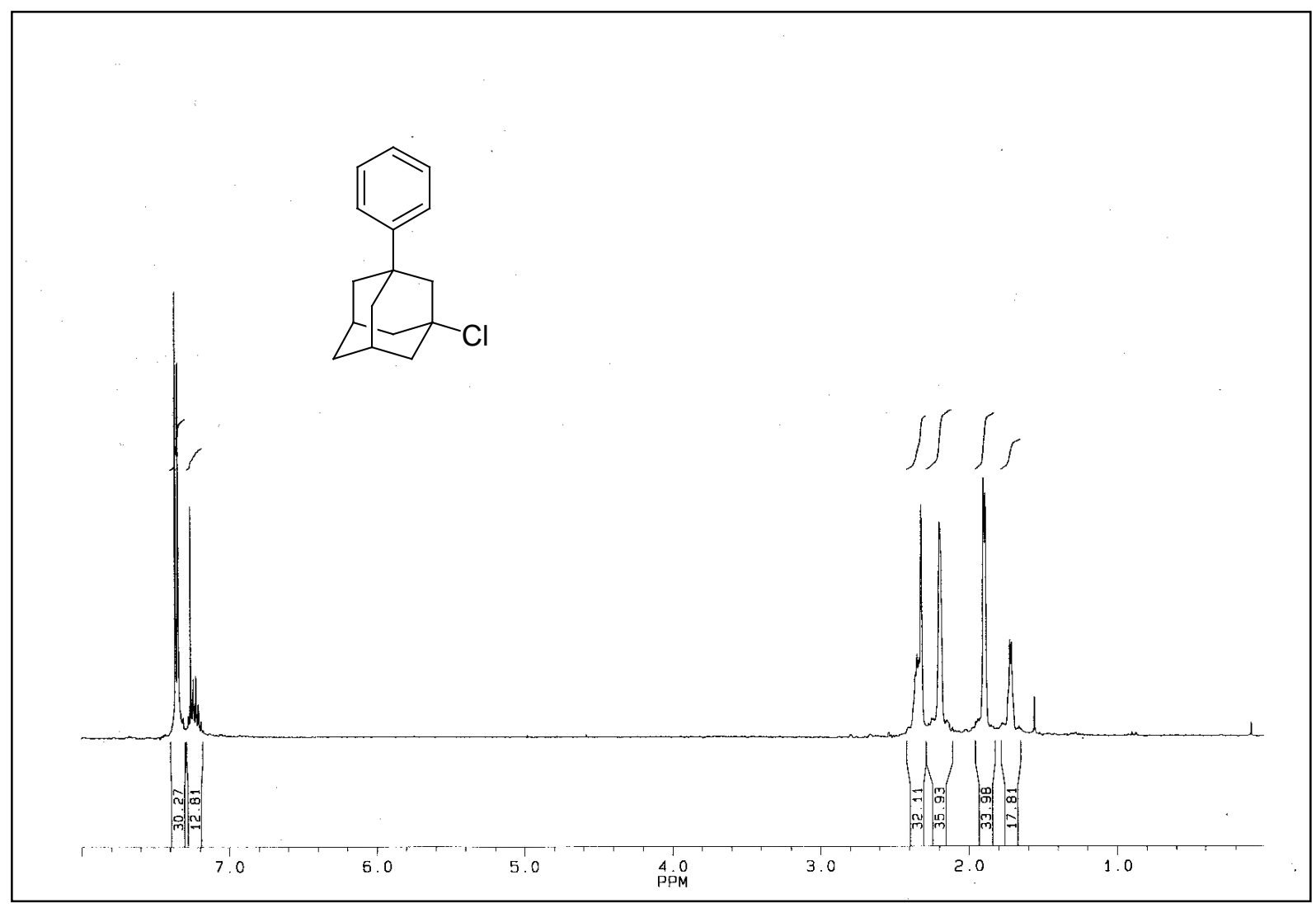

1-Chlor-3-phenyltricyclo[3.3.1.1 $\left.{ }^{3,7}\right]$ decan (53)

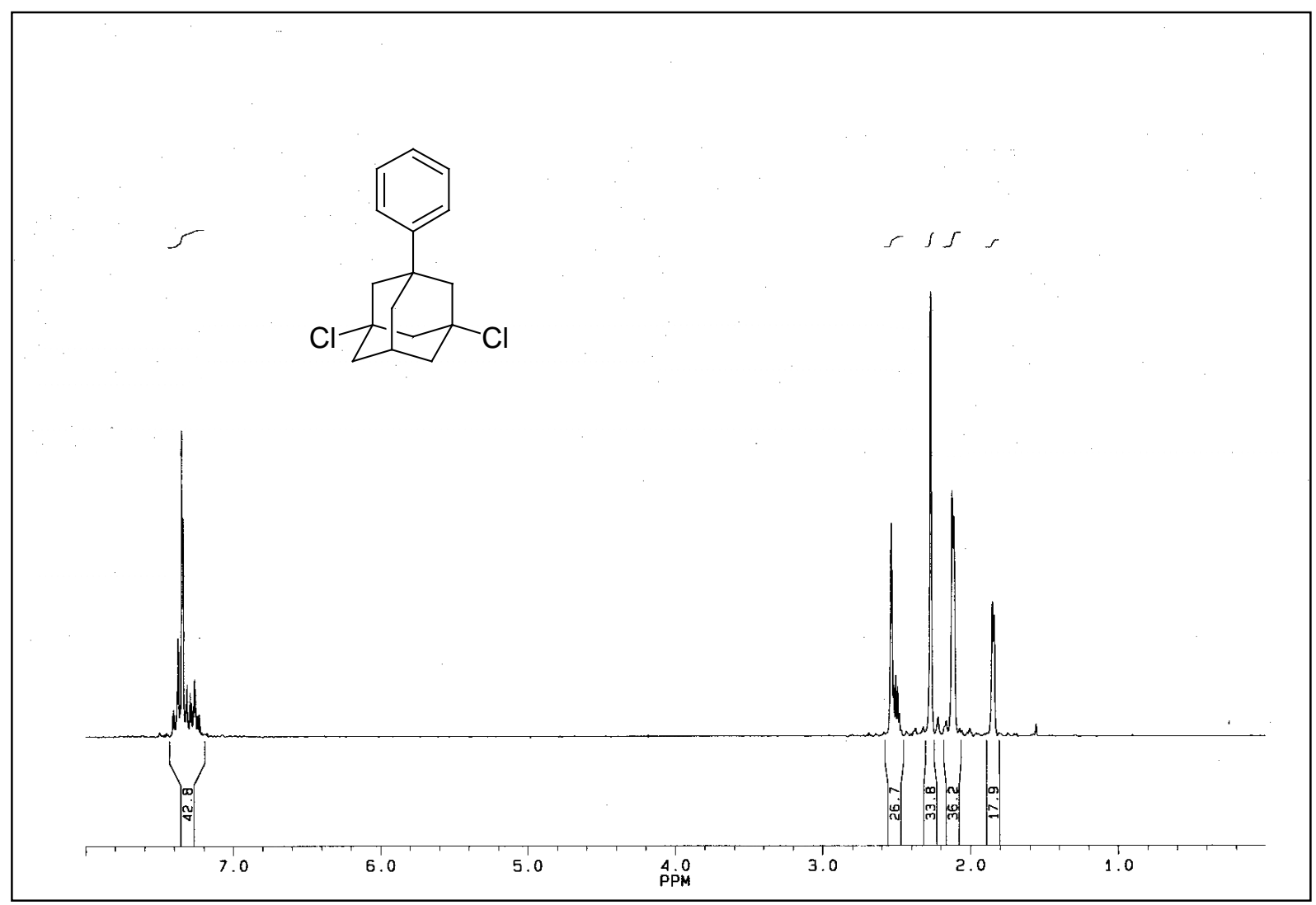

1,3-Dichlor-5-phenyltricyclo[3.3.1.1 $\left.1^{3,7}\right] \operatorname{decan}(\mathbf{6 4})$ 


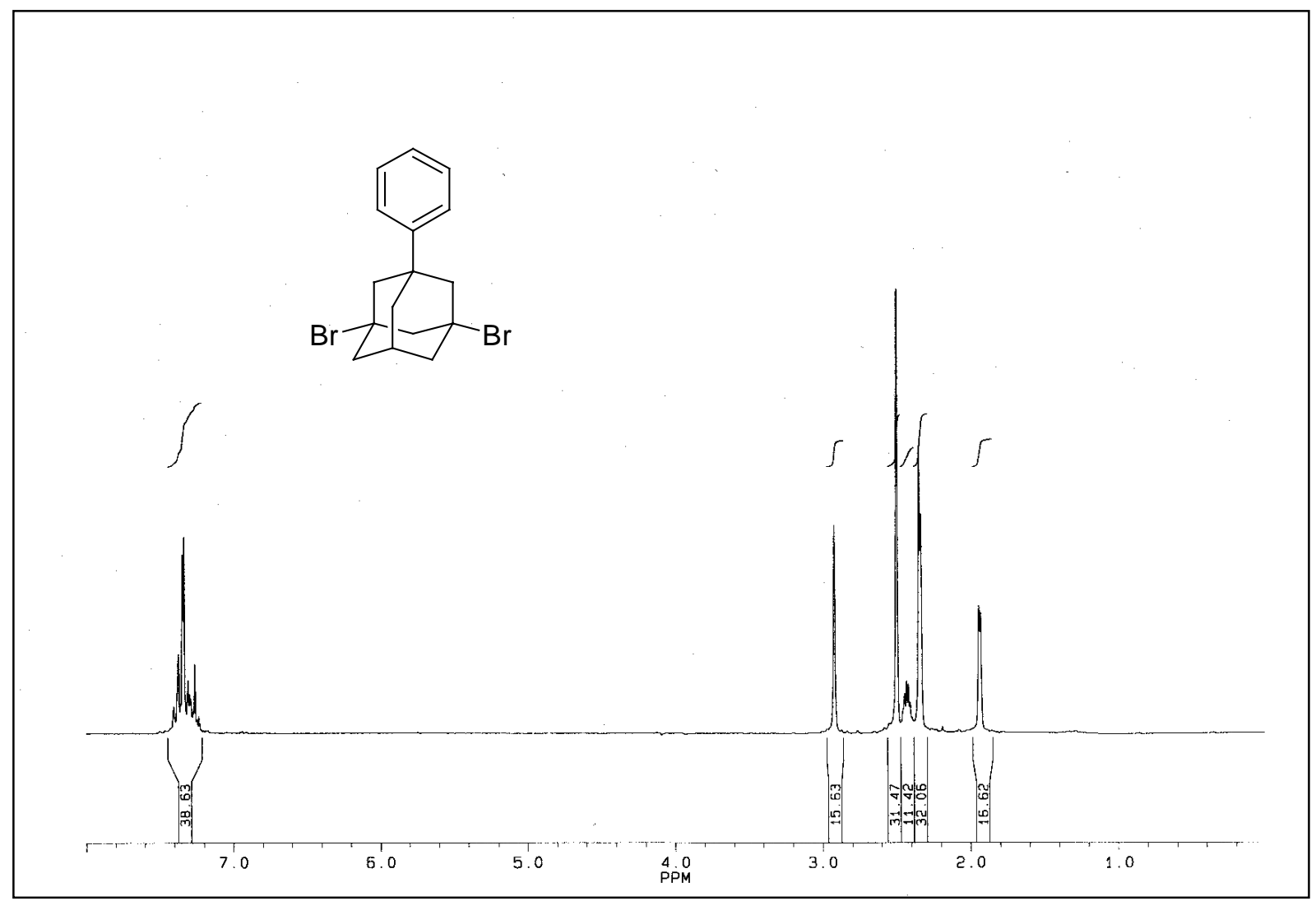

1,3-Dibrom-5-phenyltricyclo[3.3.1.1, 3 decan (65)

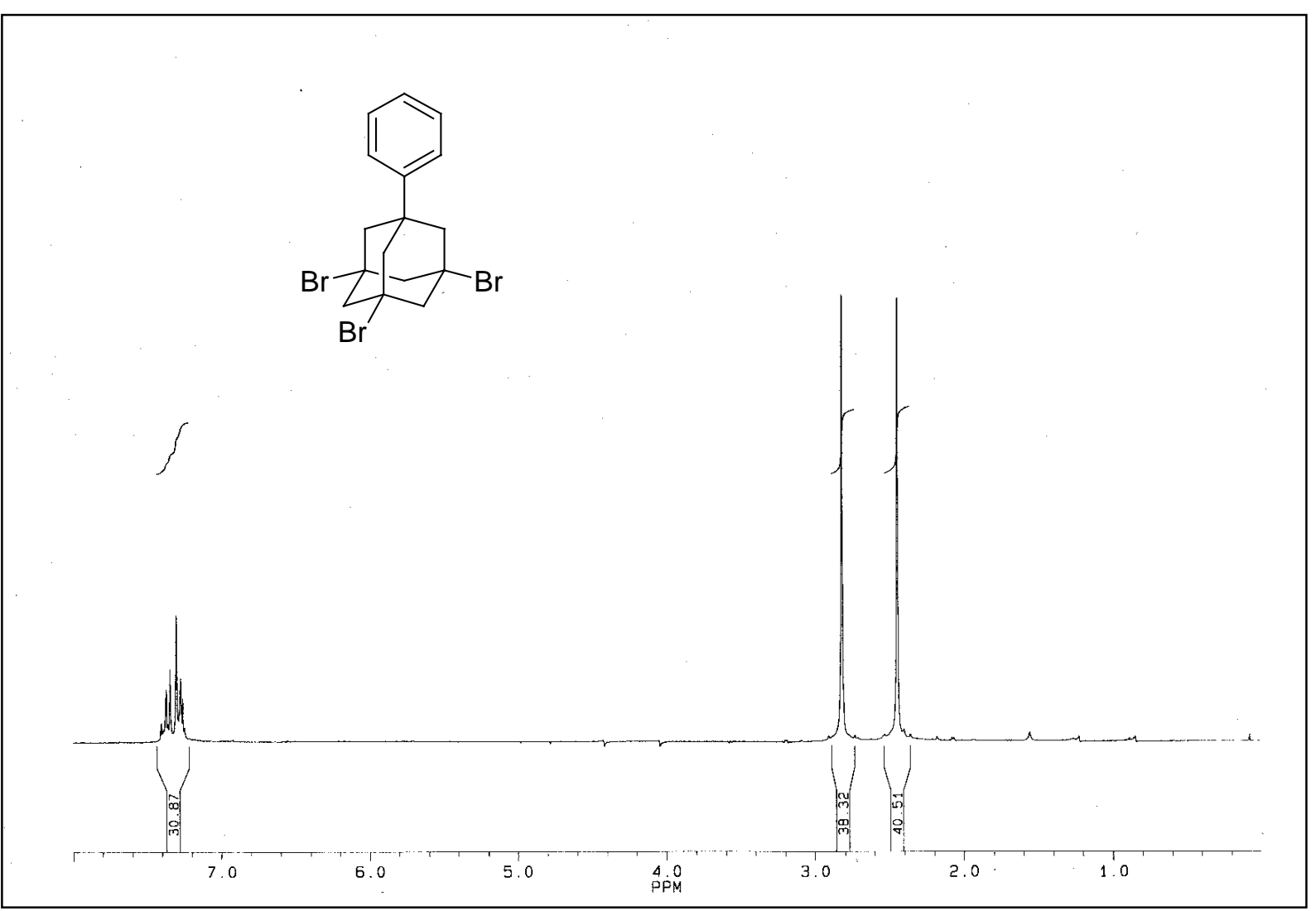

1,3,7-Tribrom-7-phenyltricyclo[3.3.1.1 $\left.1^{3,7}\right] \operatorname{decan}(\mathbf{6 6})$ 


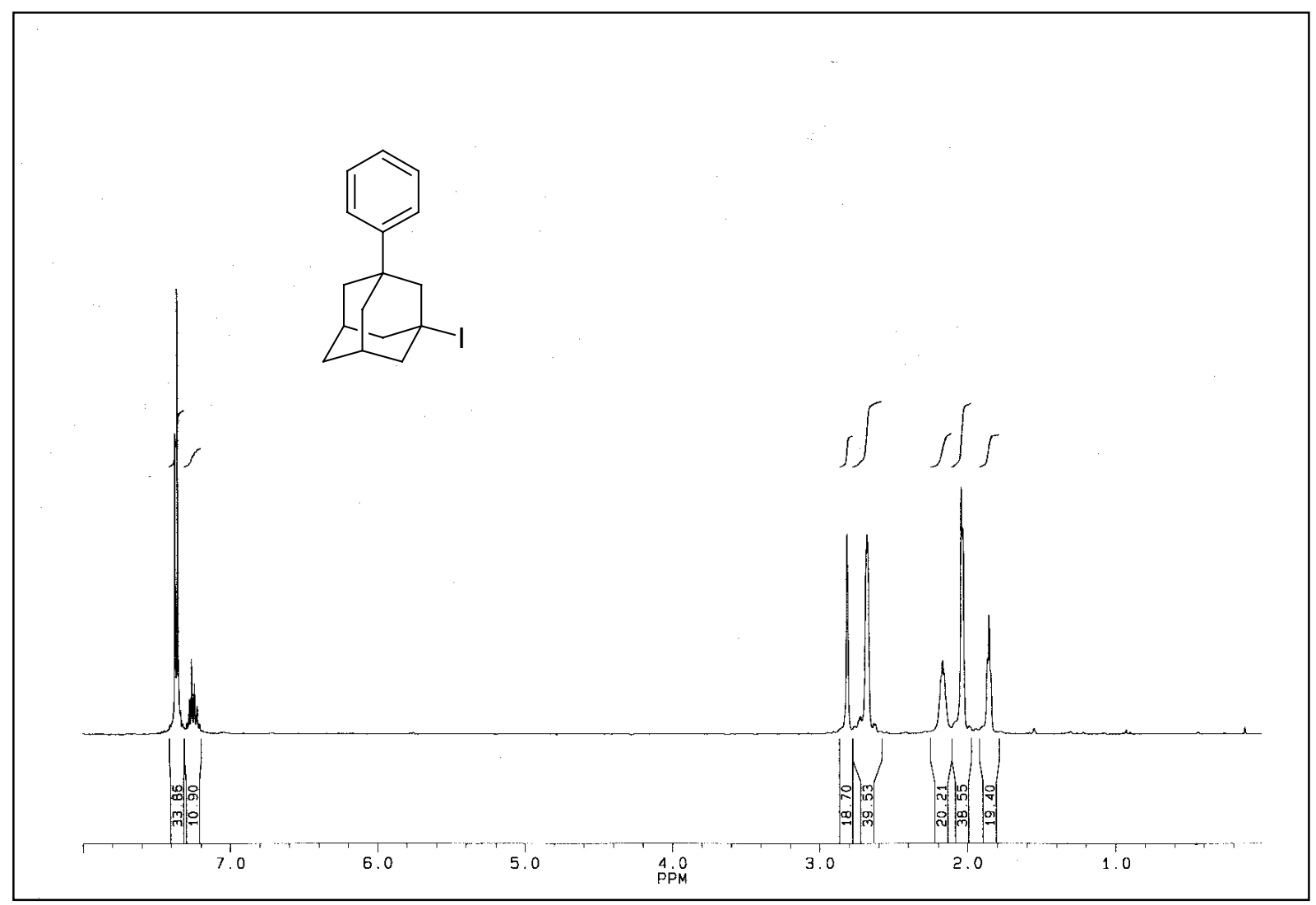

1-Iod-3-phenyltricyclo[3.3.1.1 $\left.1^{3,7}\right]$ decan (60)

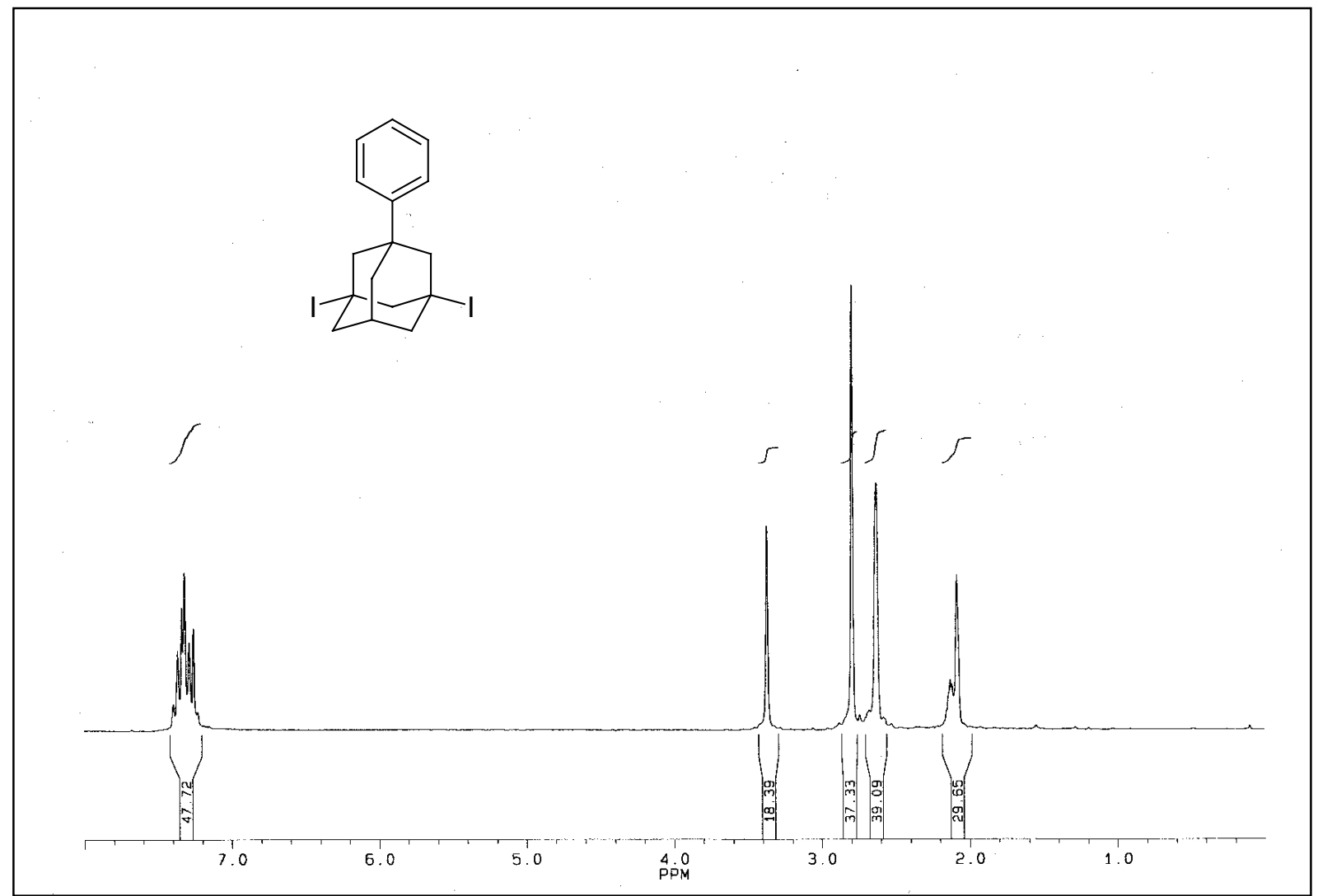

1,3-Diiod-7-phenyltricyclo[3.3.1.1 $\left.1^{3,7}\right]$ decan (67) 


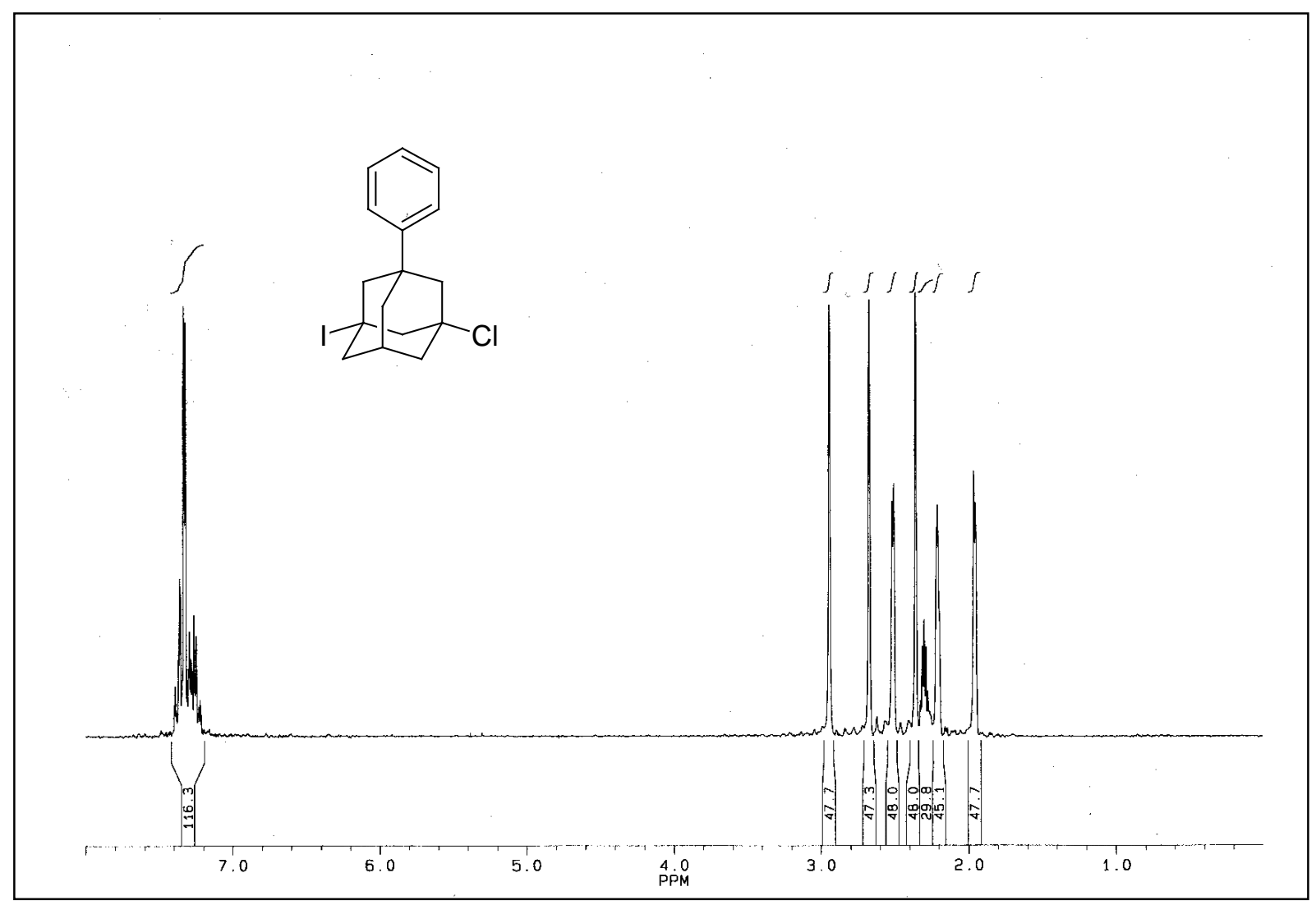

( \pm -1-Iod-3-chlor-7-phenyltricyclo[3.3.1.1 $\left.1^{3,7}\right] \operatorname{decan}(\mathbf{5 8})$

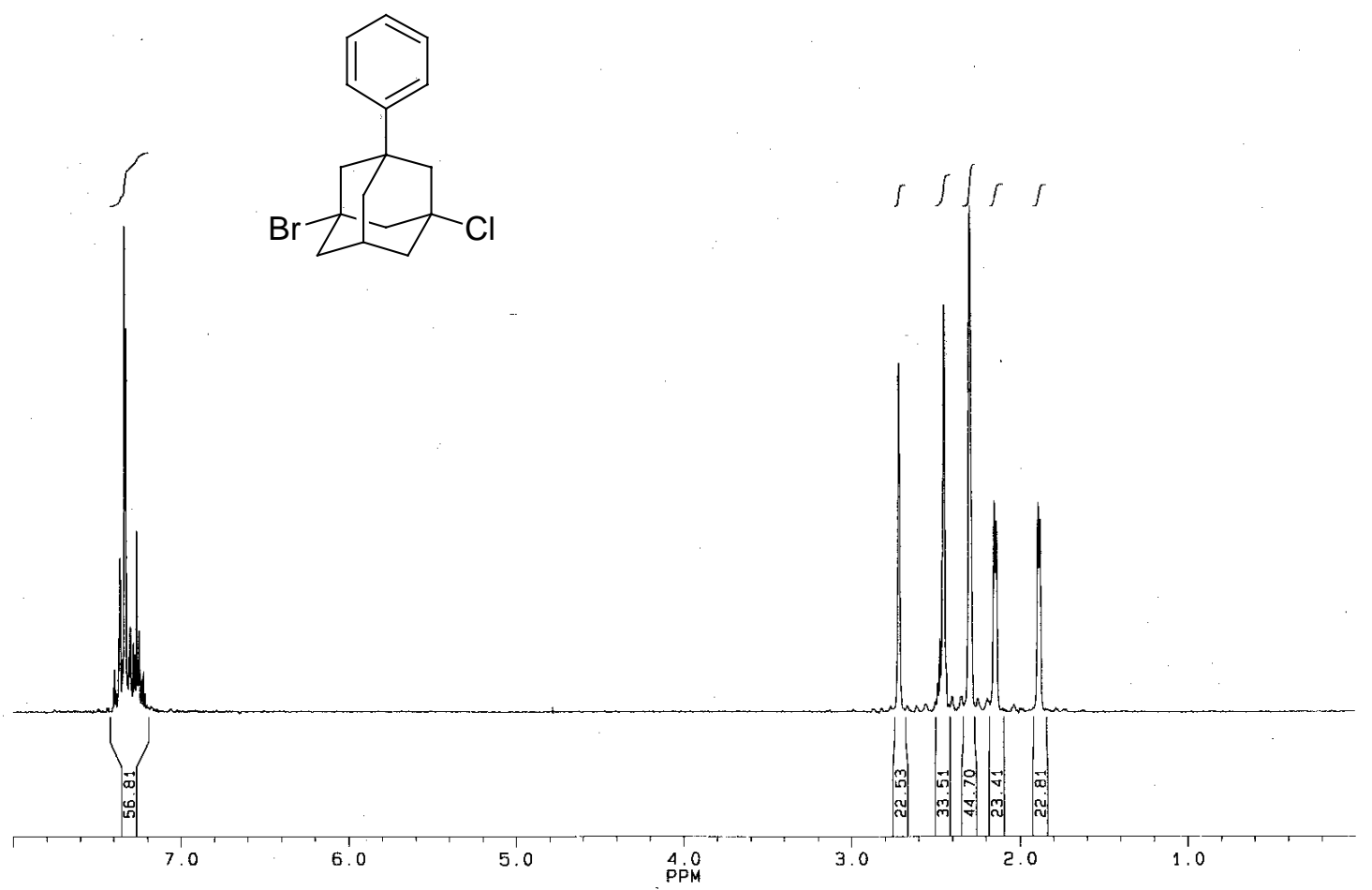

( \pm -1-Brom-3-chlor-5-phenyltricyclo[3.3.1.1 $\left.1^{3,7}\right] \operatorname{decan}(\mathbf{6 2})$ 


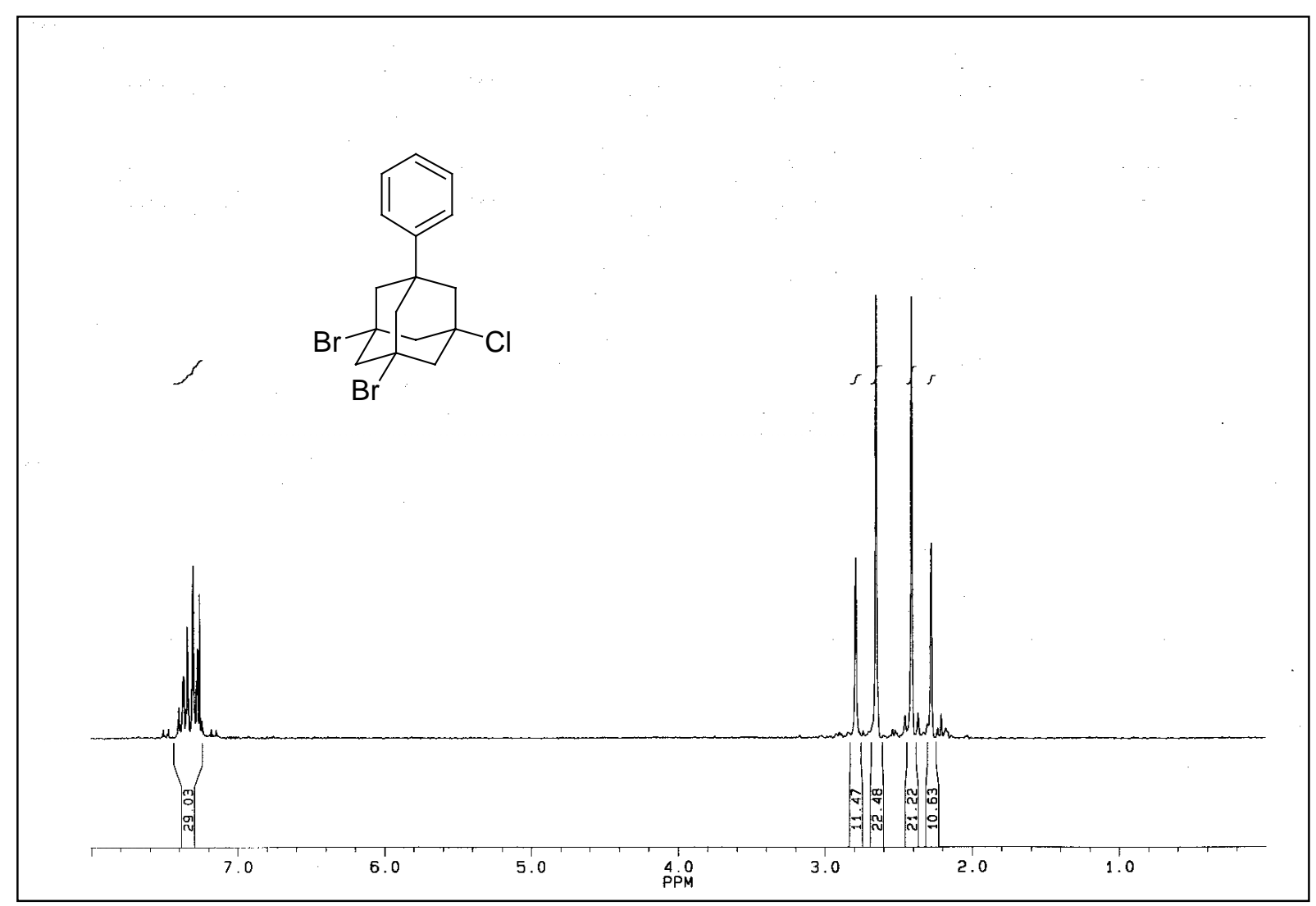

1,3-Brom-5-chlor-7-phenyltricyclo[3.3.1.1 $\left.1^{3,7}\right] \operatorname{decan}(\mathbf{6 2})$

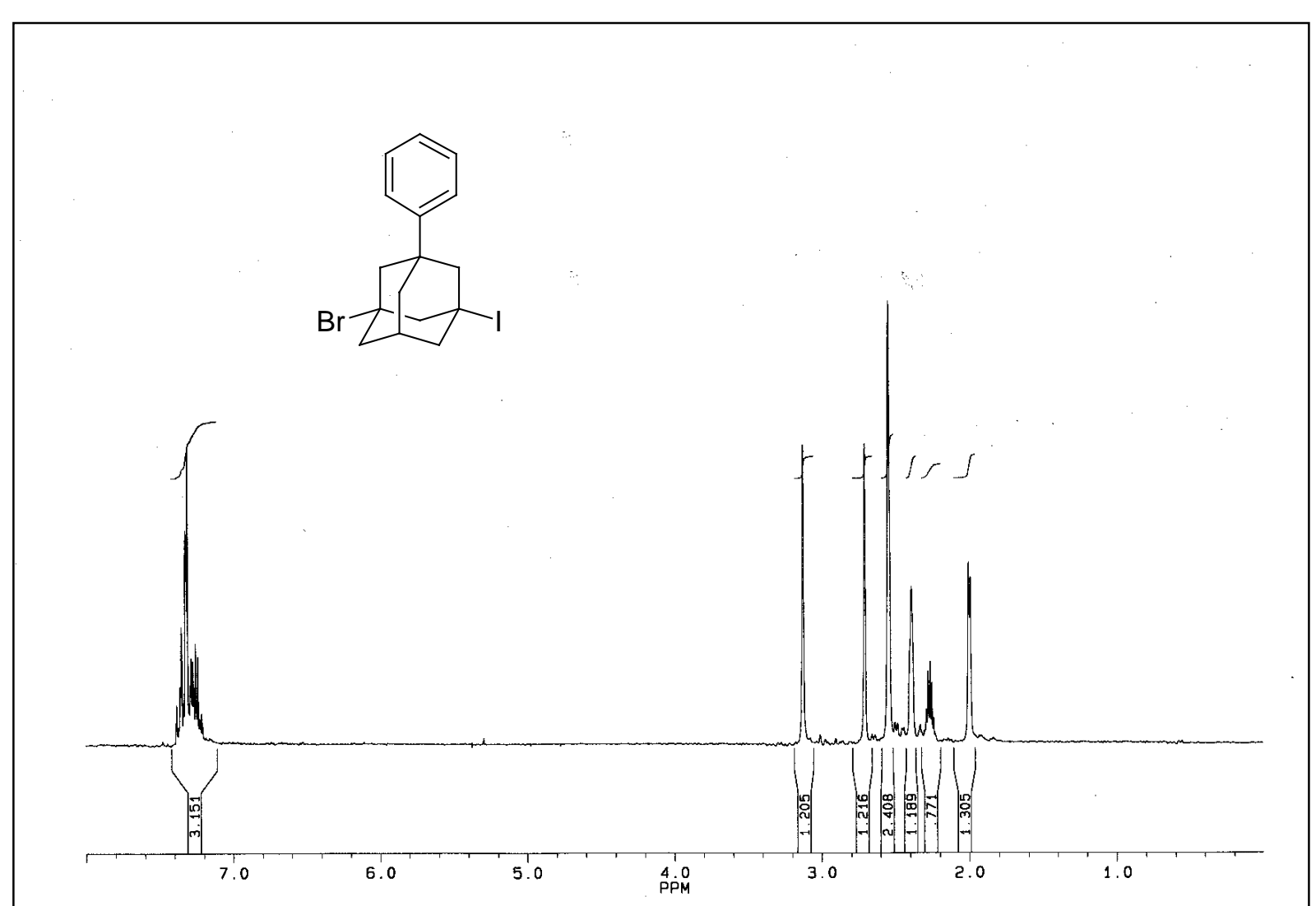

( \pm )-1-Iod-3-brom-5-phenyltricyclo[3.3.1.1 $\left.1^{3,7}\right]$ decan (61) 


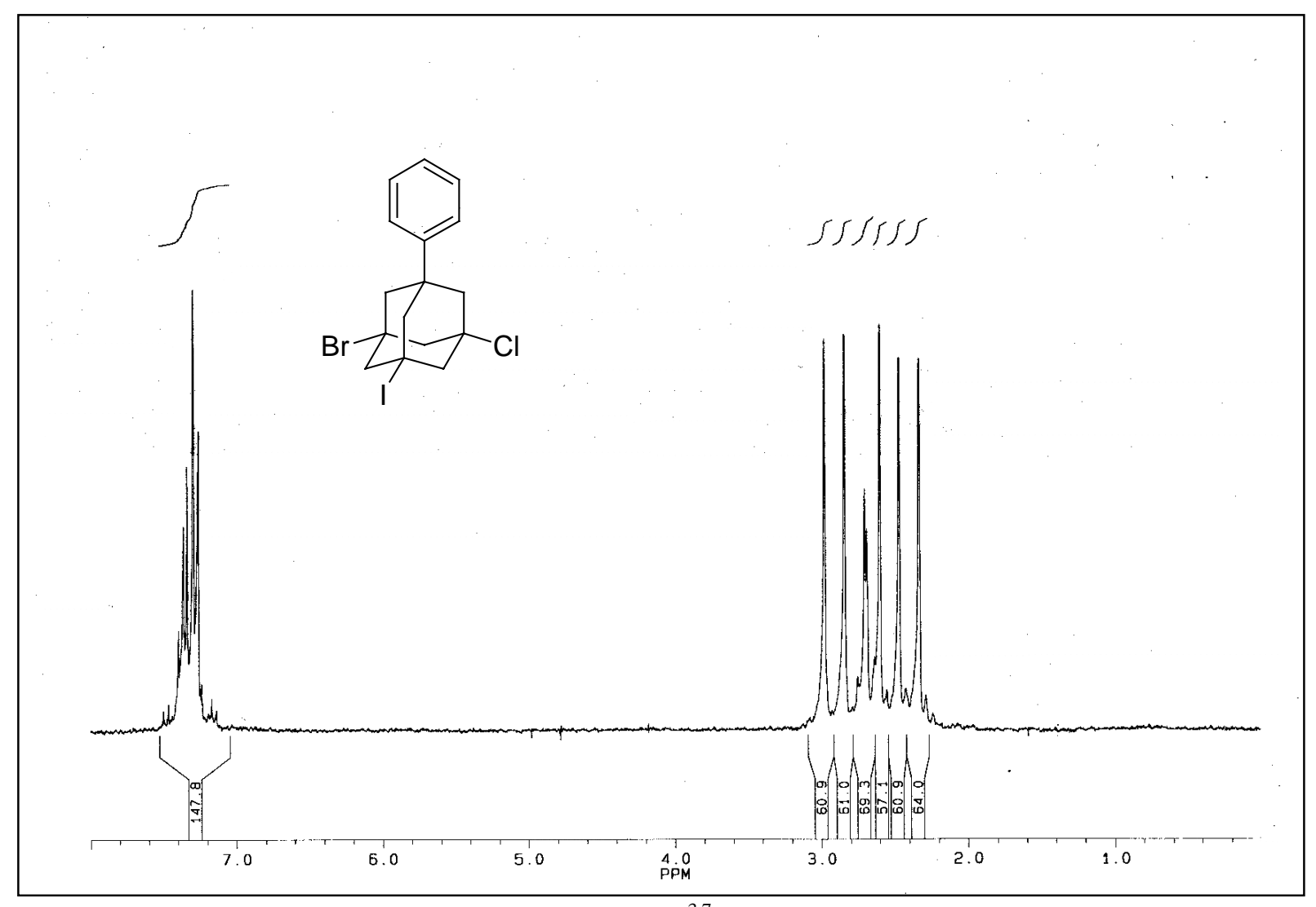

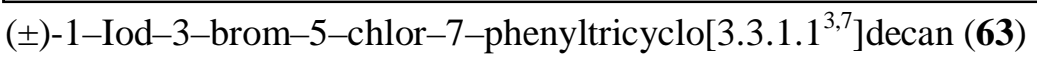

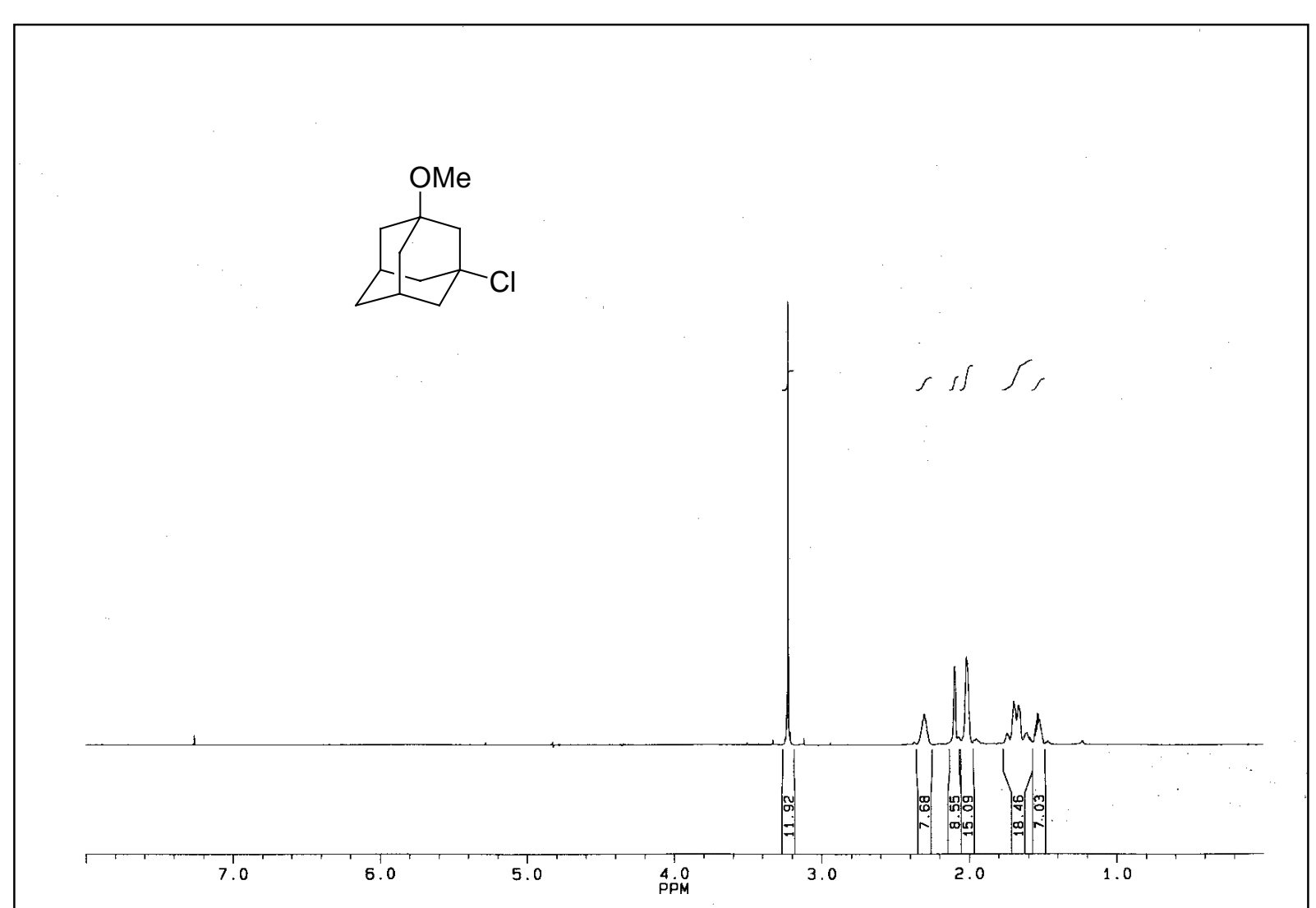

1-Chlor-3-methoxytricyclo[3.3.1.1 $\left.1^{3,7}\right]$ decan (70) 


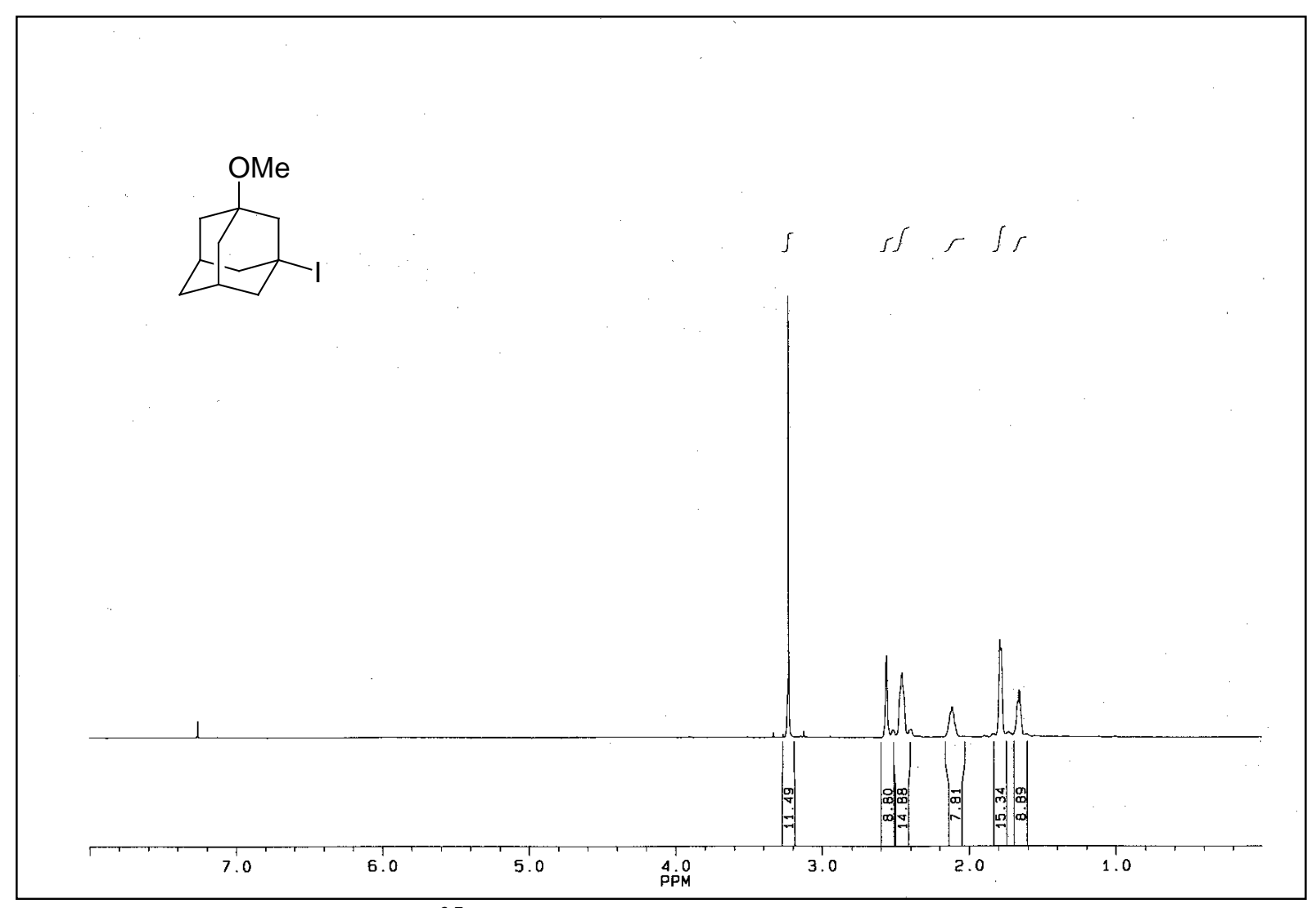

1-Iod-3-methoxytricyclo[3.3.1.1, $]$ decan (73)

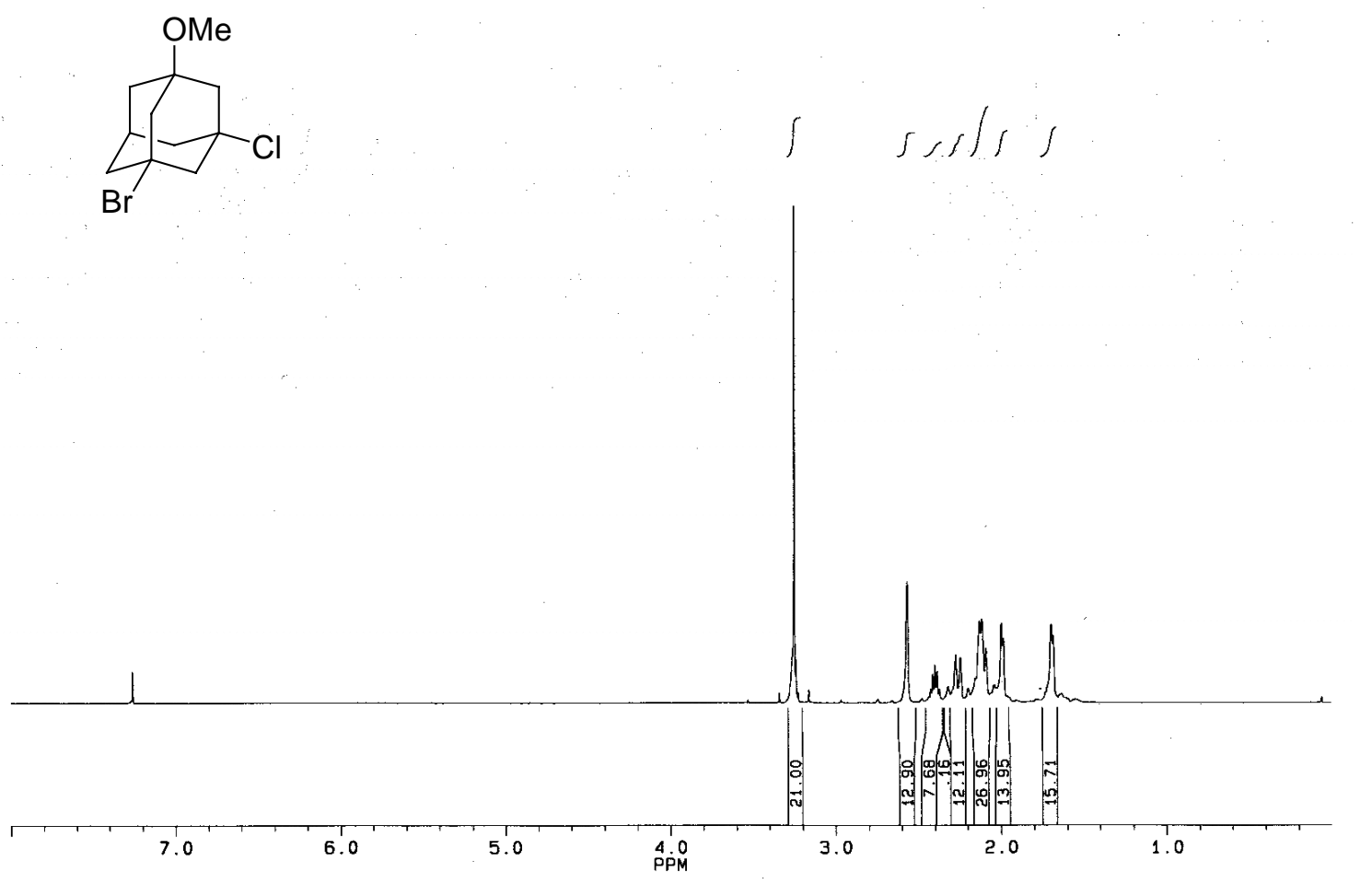

( \pm -1-Brom-3-chlor-5-methoxytricyclo[3.3.1.1 $\left.1^{3,7}\right]$ decan (71) 


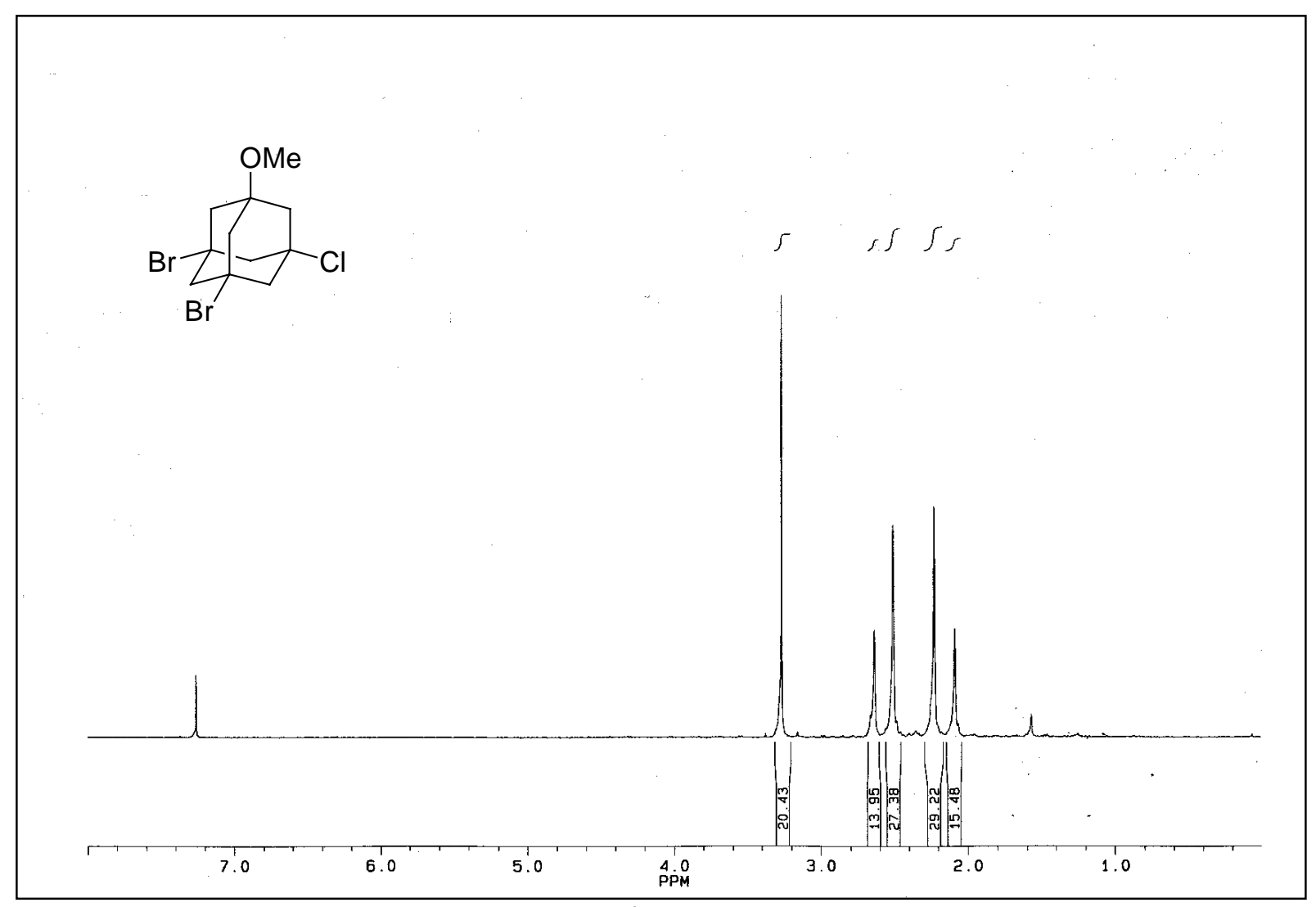

1,3-Dibrom-5-chlor-7-methoxytricyclo[3.3.1.1 $\left.1^{3,7}\right]$ decan (75)

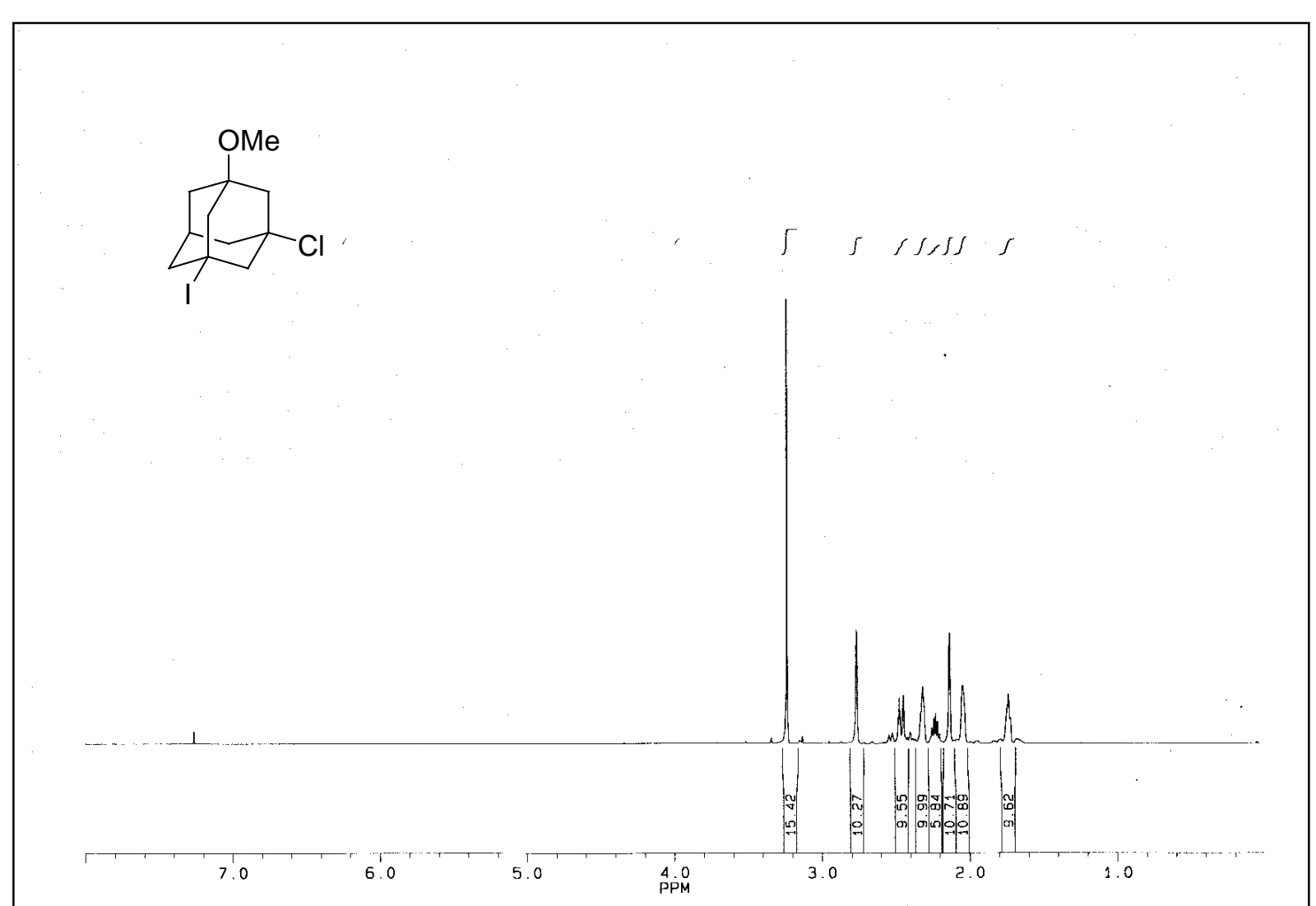

( \pm -1-Iod-3-chlor-5-methoxytricyclo[3.3.1.1 $\left.{ }^{3,7}\right]$ decan (72) 


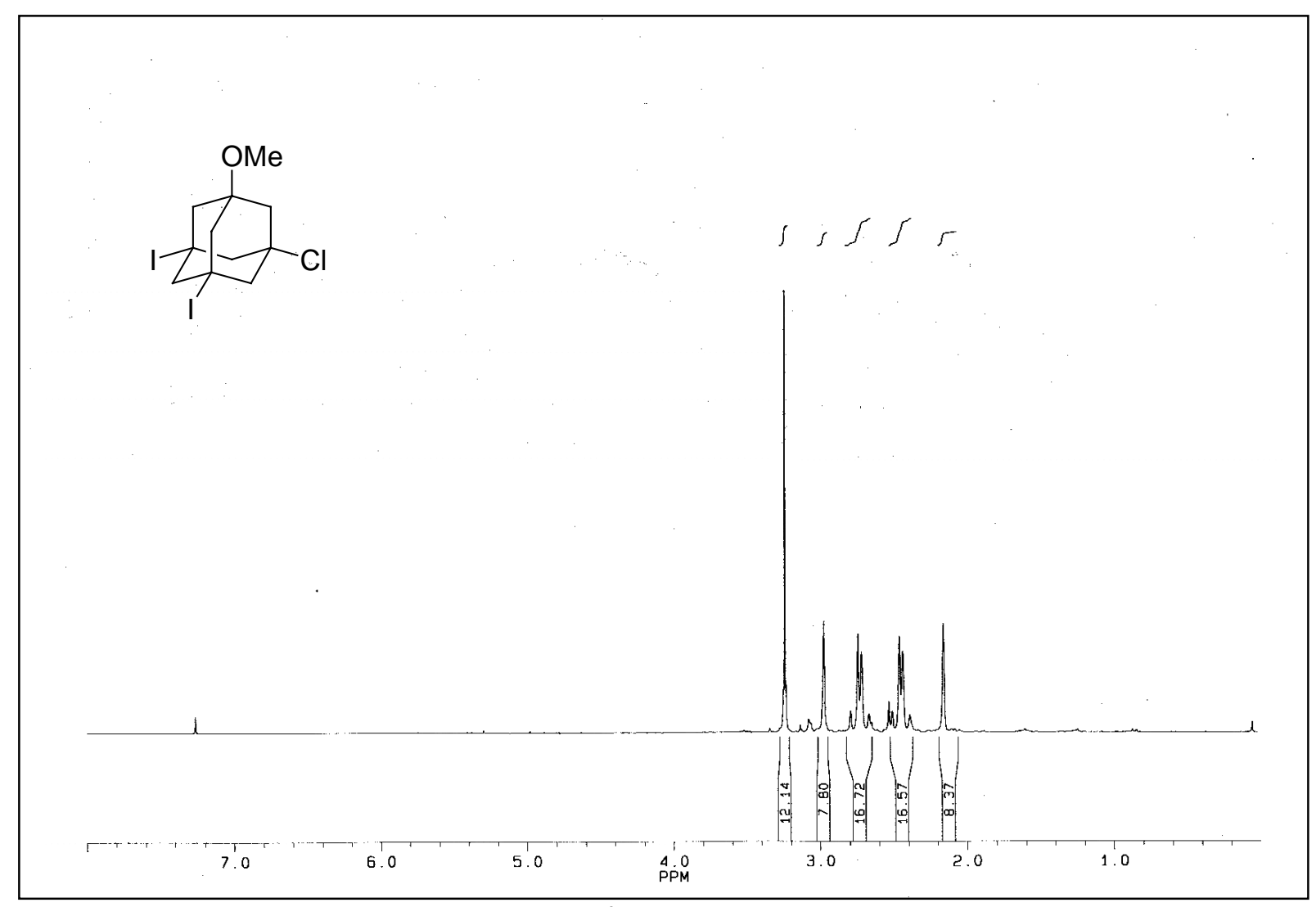

1,3-Diiod-5-chlor-7-methoxytricyclo[3.3.1.1 $\left.1^{3,7}\right]$ decan (76)

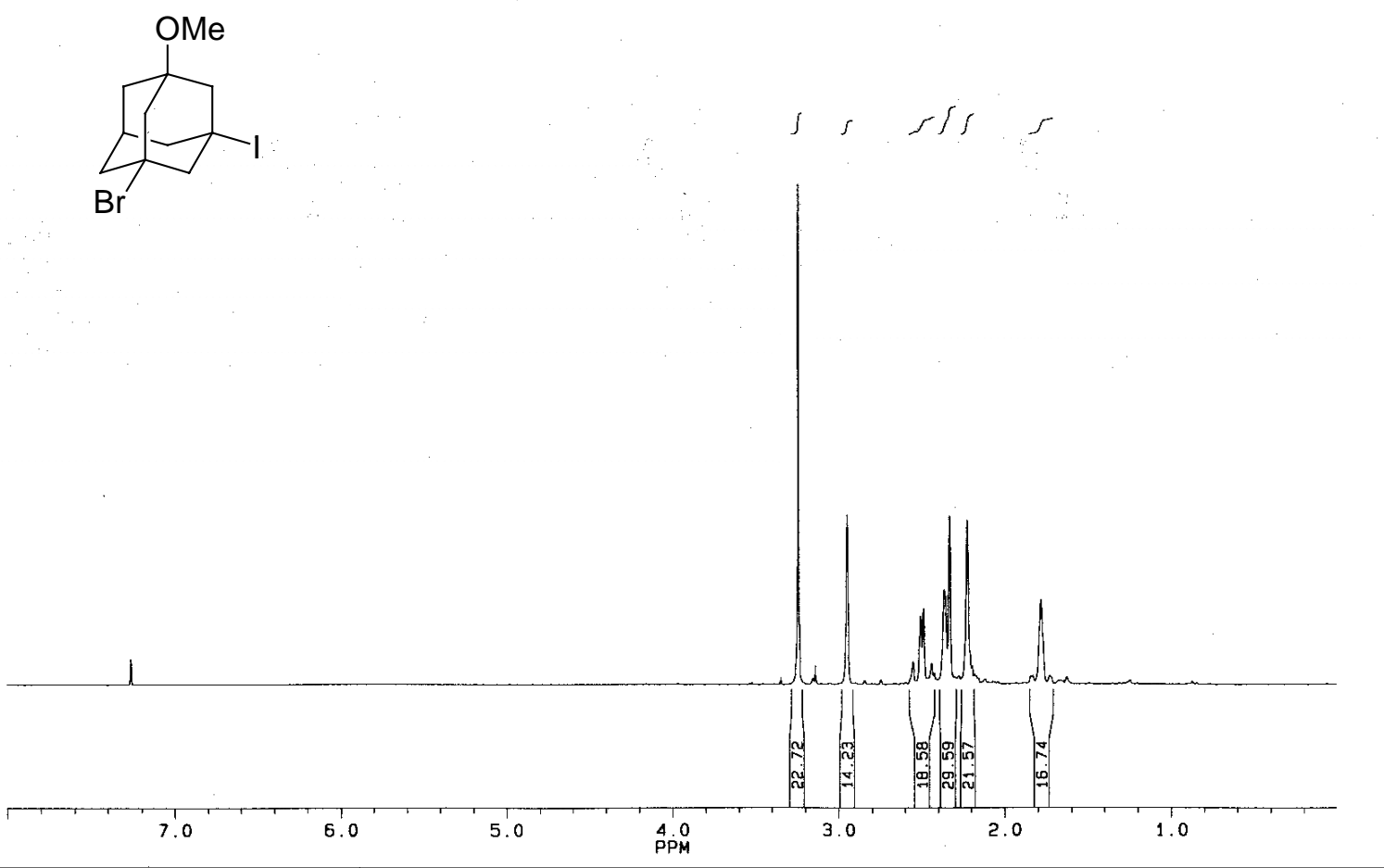

( \pm -1-Iod-3-brom-5-methoxytricyclo[3.3.1.1 $\left.1^{3,7}\right]$ decan (74) 


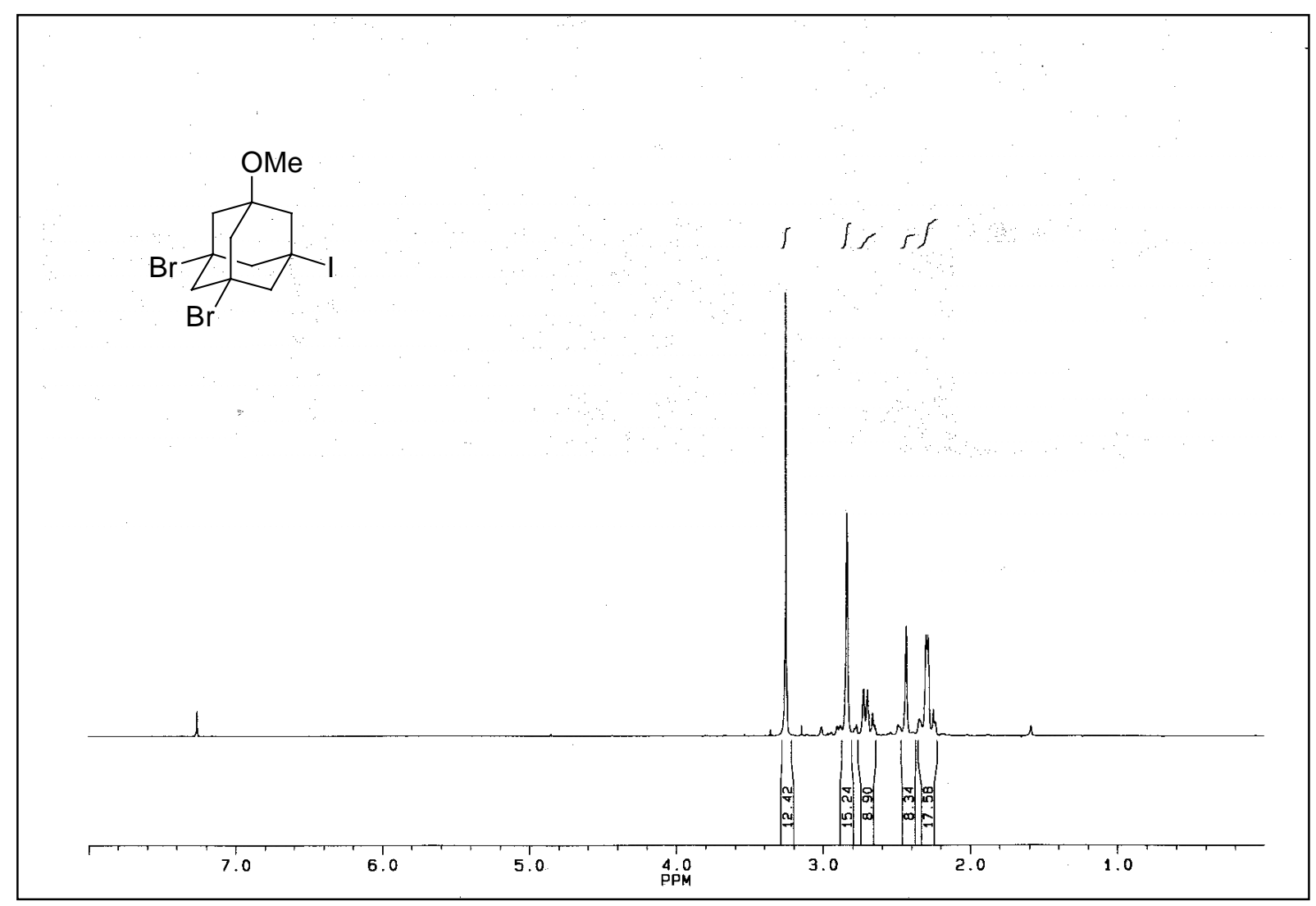

1-Iod-3,5-brom-7-methoxytricyclo[3.3.1.1 $\left.1^{3,7}\right]$ decan (77)

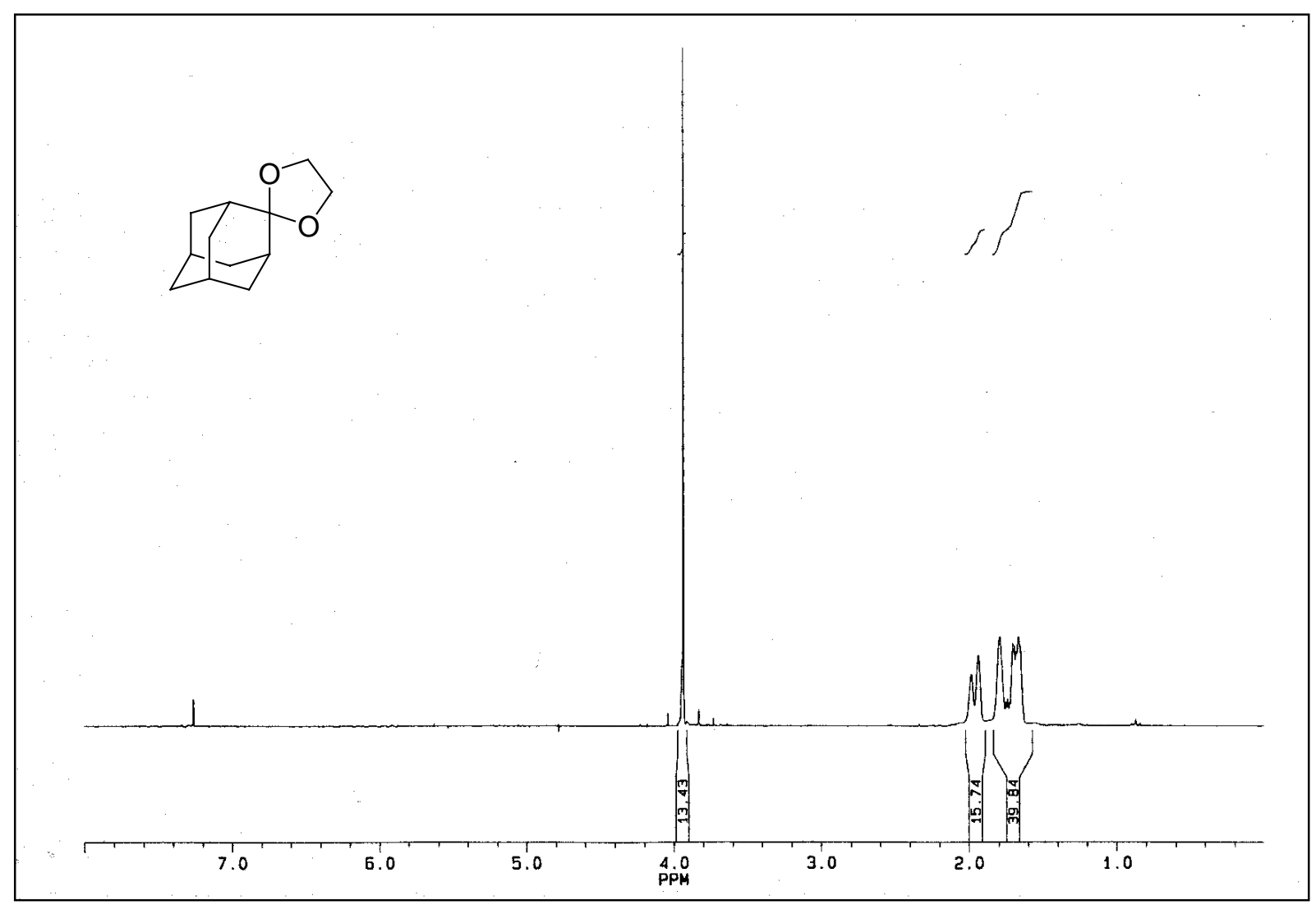

2-(spiro-1',4'-dioxocyclopentyl)tricyclo[3.3.1.1 $\left.{ }^{3,7}\right]$ decan (79) 


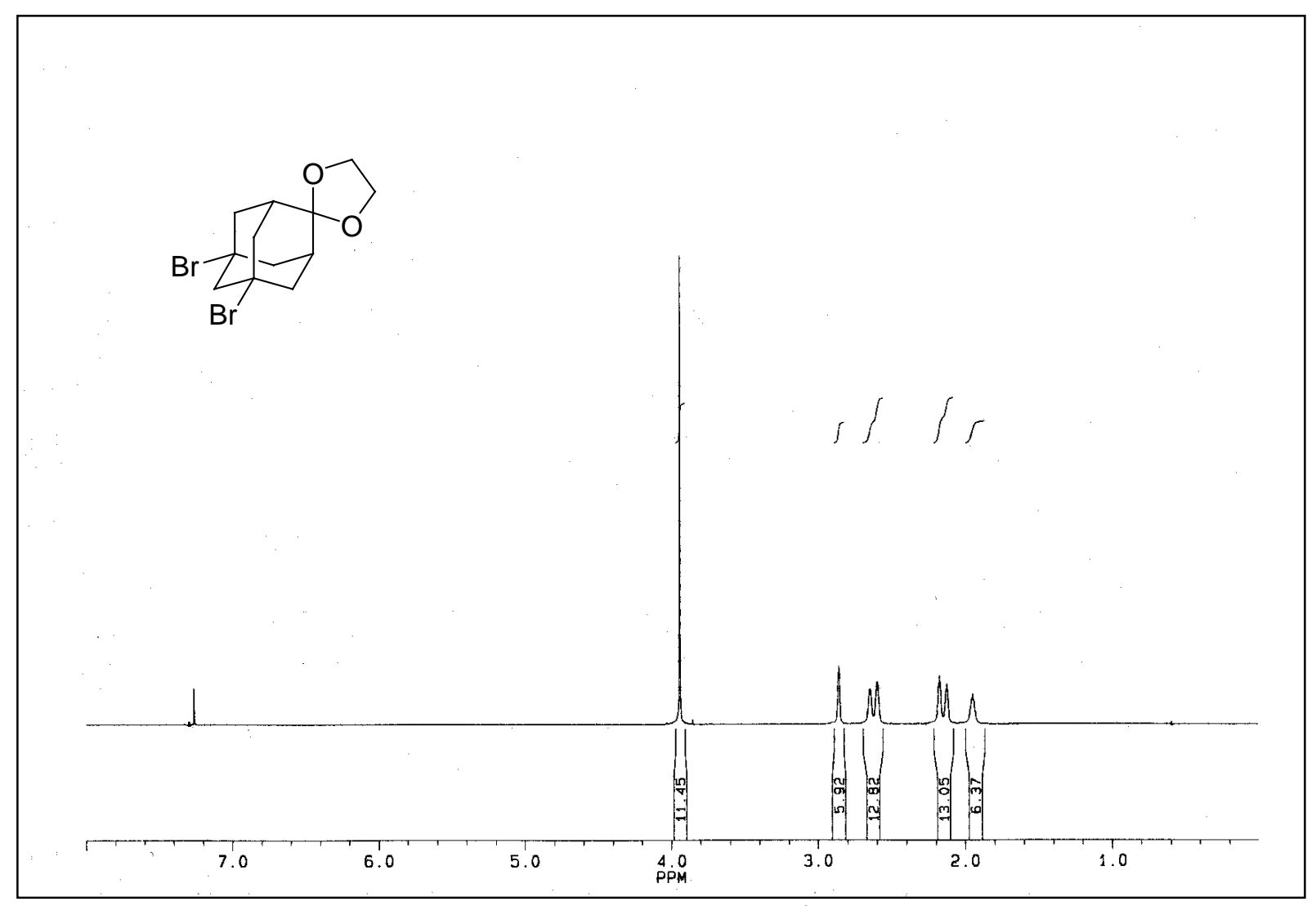

1,3-Dibrom-6-(spiro-1',4'-dioxocyclopentyl)tricyclo[3.3.1.1 $\left.{ }^{3,7}\right] \operatorname{decan}(\mathbf{8 3})$

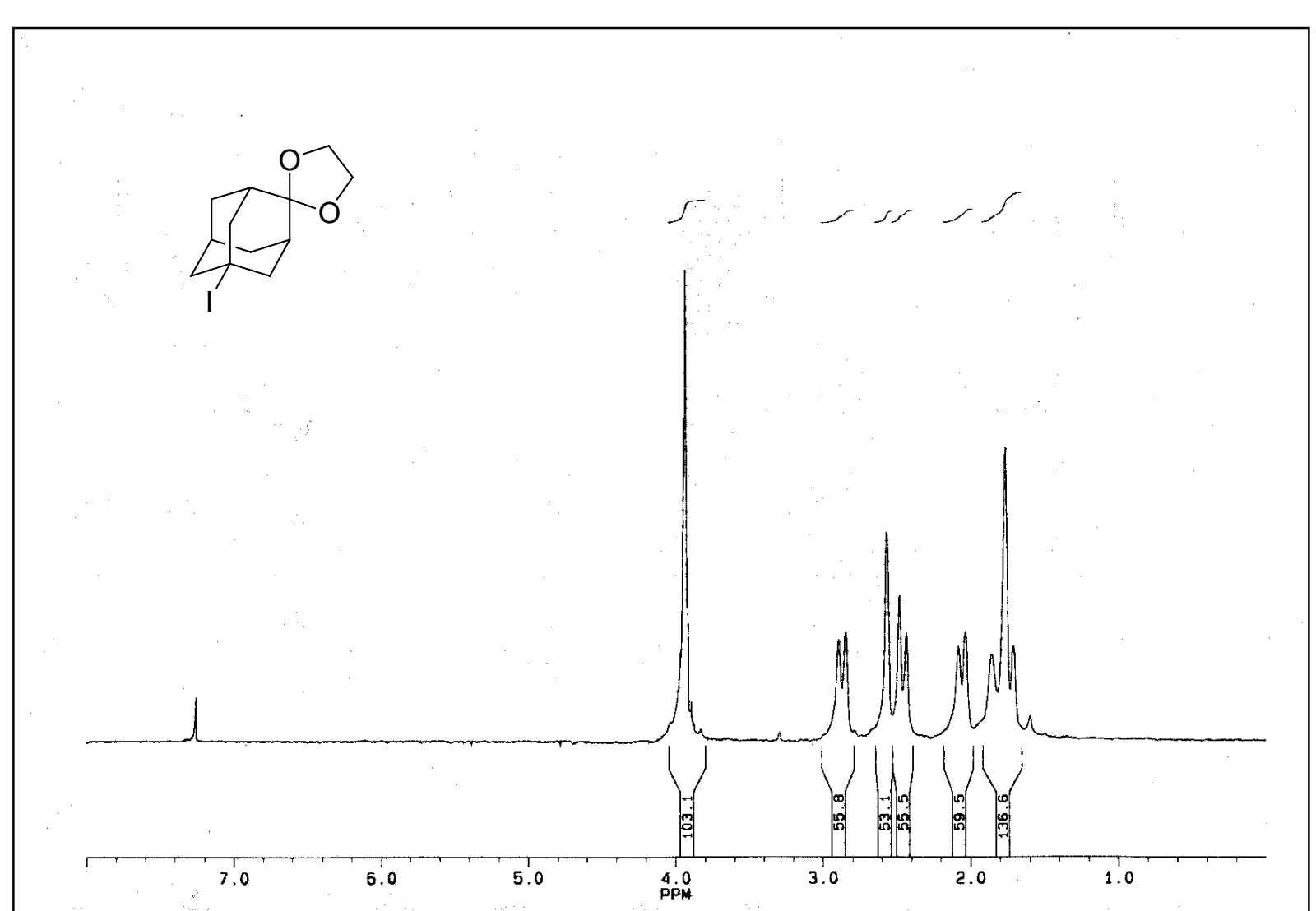

1-Iod-4-(spiro-1',4'-dioxocyclopentyl)tricyclo[3.3.1.1 $\left.{ }^{3,7}\right]$ decan (84) 


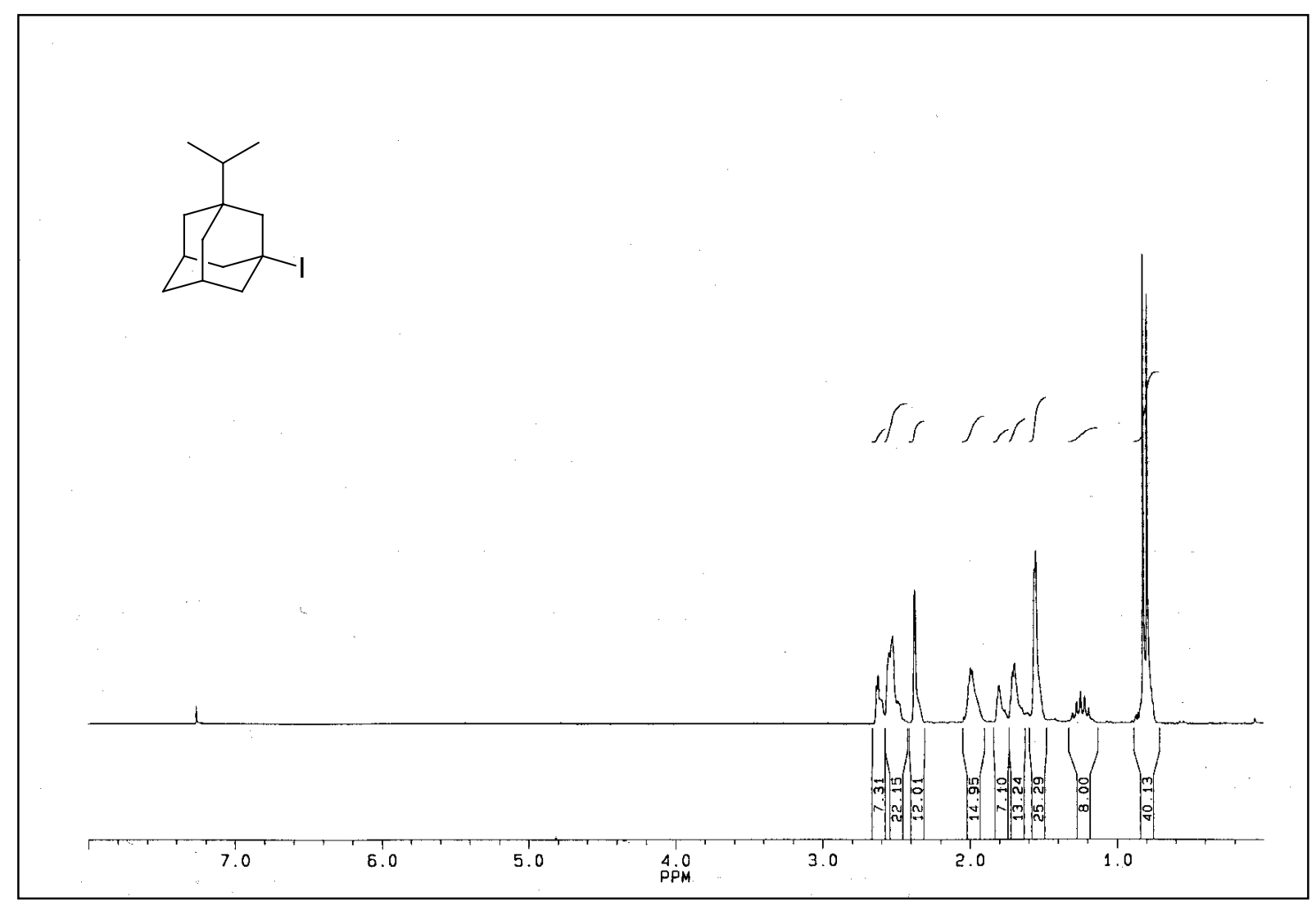

1-Iod-3-(1'-methyl)ethyltricyclo[3.3.1.1,7] decan (86)
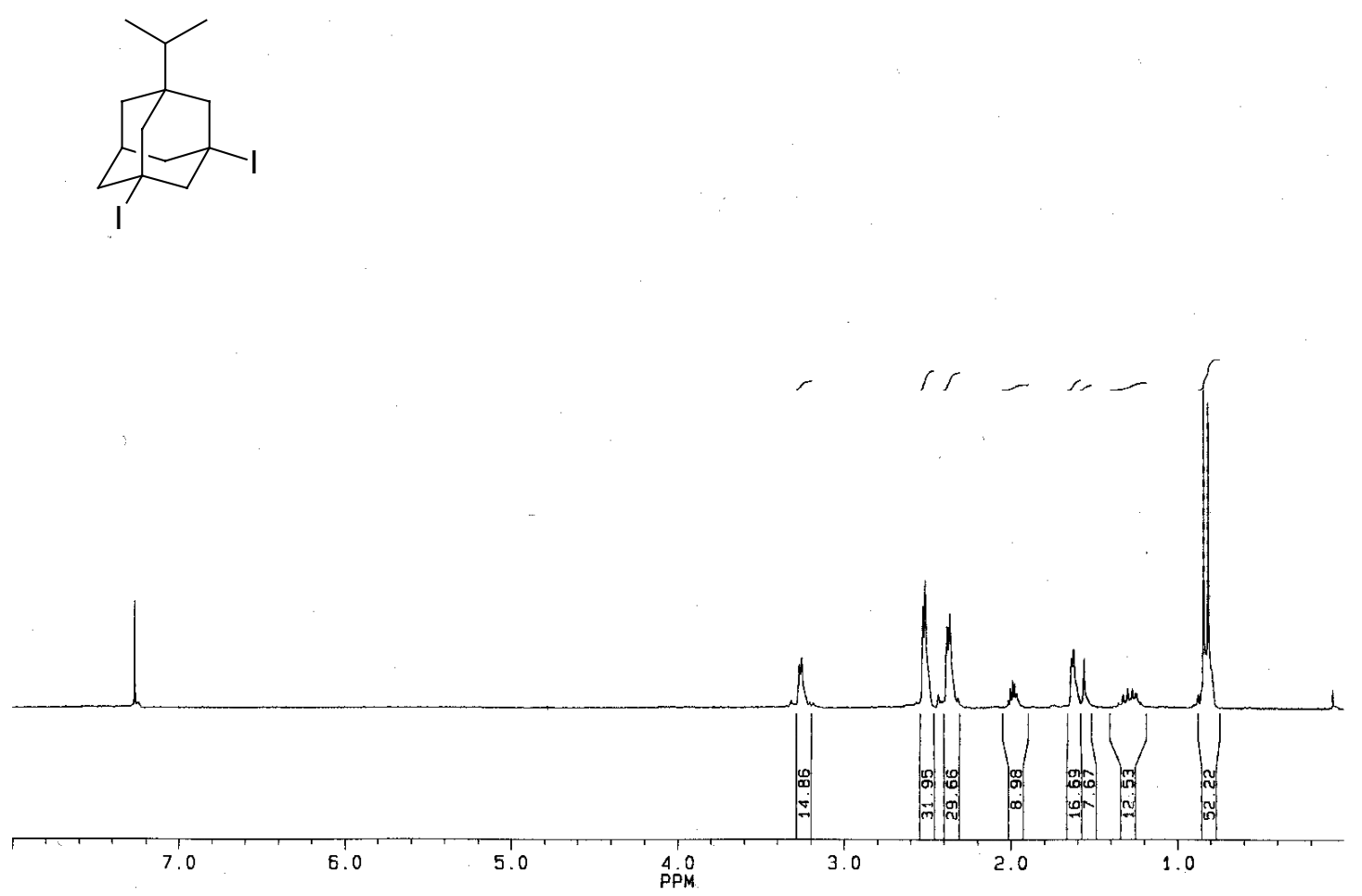

1,3-Diiod-5-(1'-methyl)ethyltricyclo[3.3.1.1 $\left.1^{3,7}\right]$ decan (88) 
Anhang D. Abbildungen der ${ }^{13}$ C-NMR Spektren

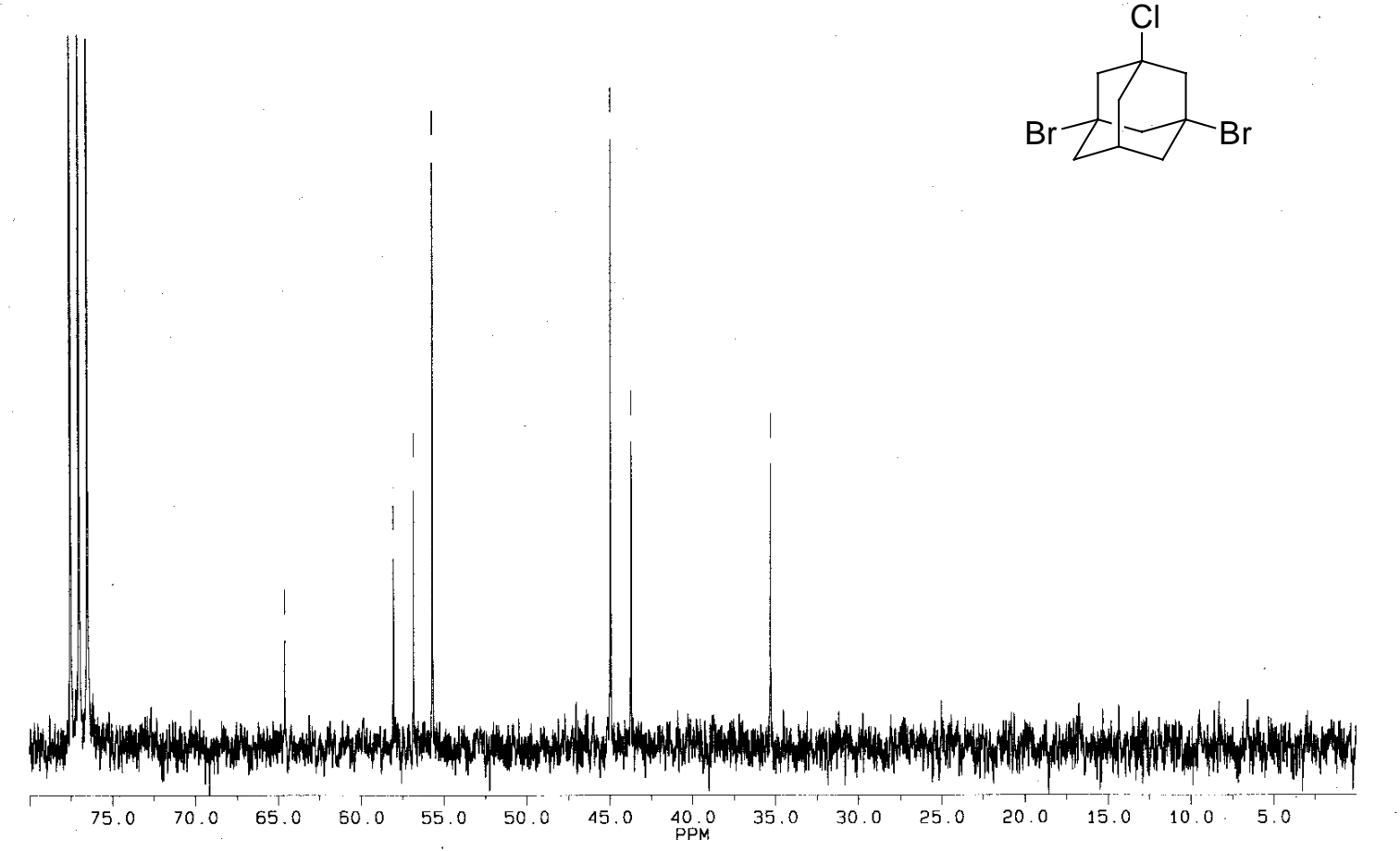

1,3-Dibrom-5-chlortricyclo[3.3.1.1 $\left.1^{3,7}\right] \operatorname{decan}(\mathbf{3 9})$
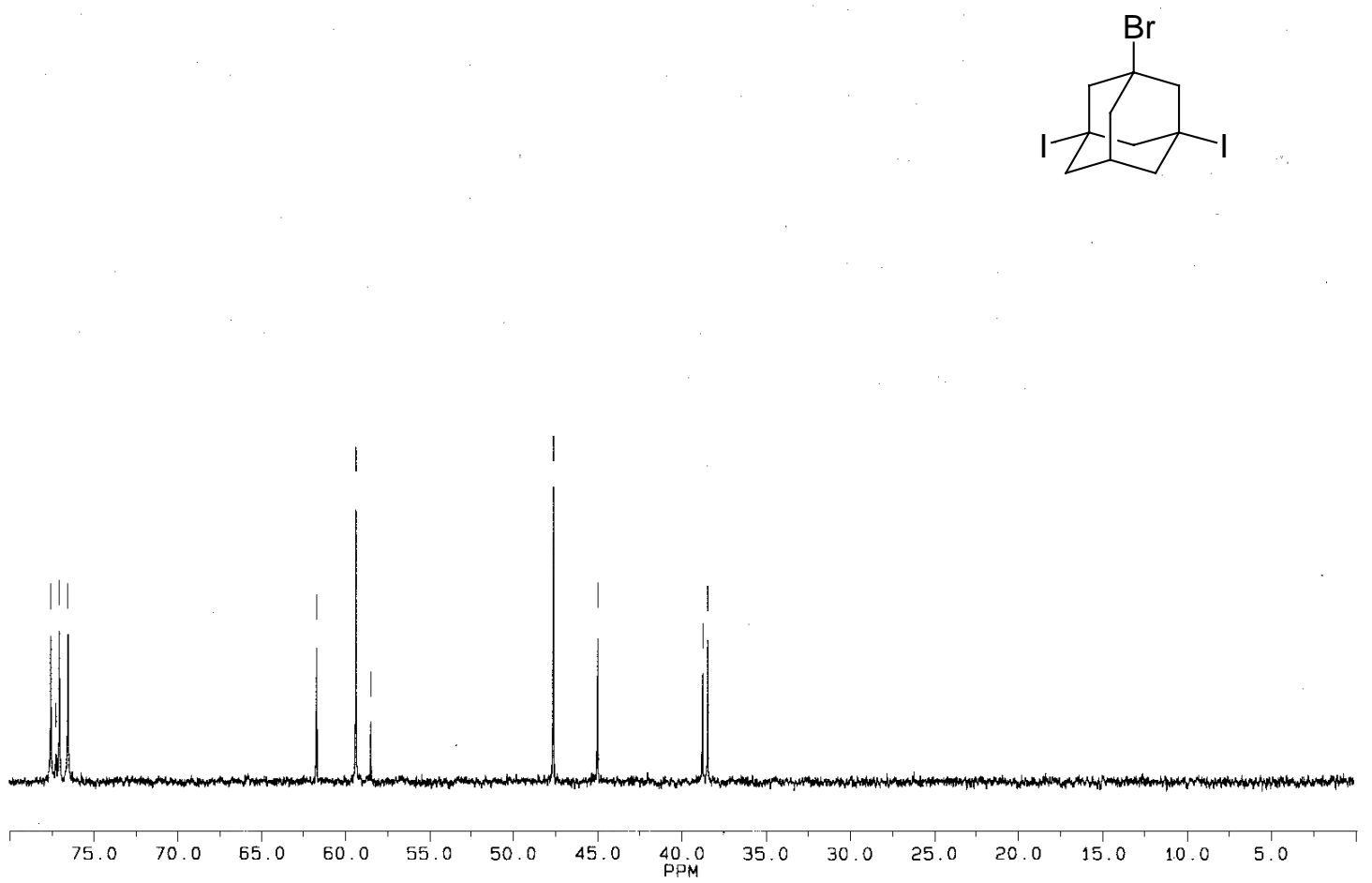

1,3-Diiod-5-bromtricyclo[3.3.1.1 $\left.1^{3,7}\right]$ decan (43) 

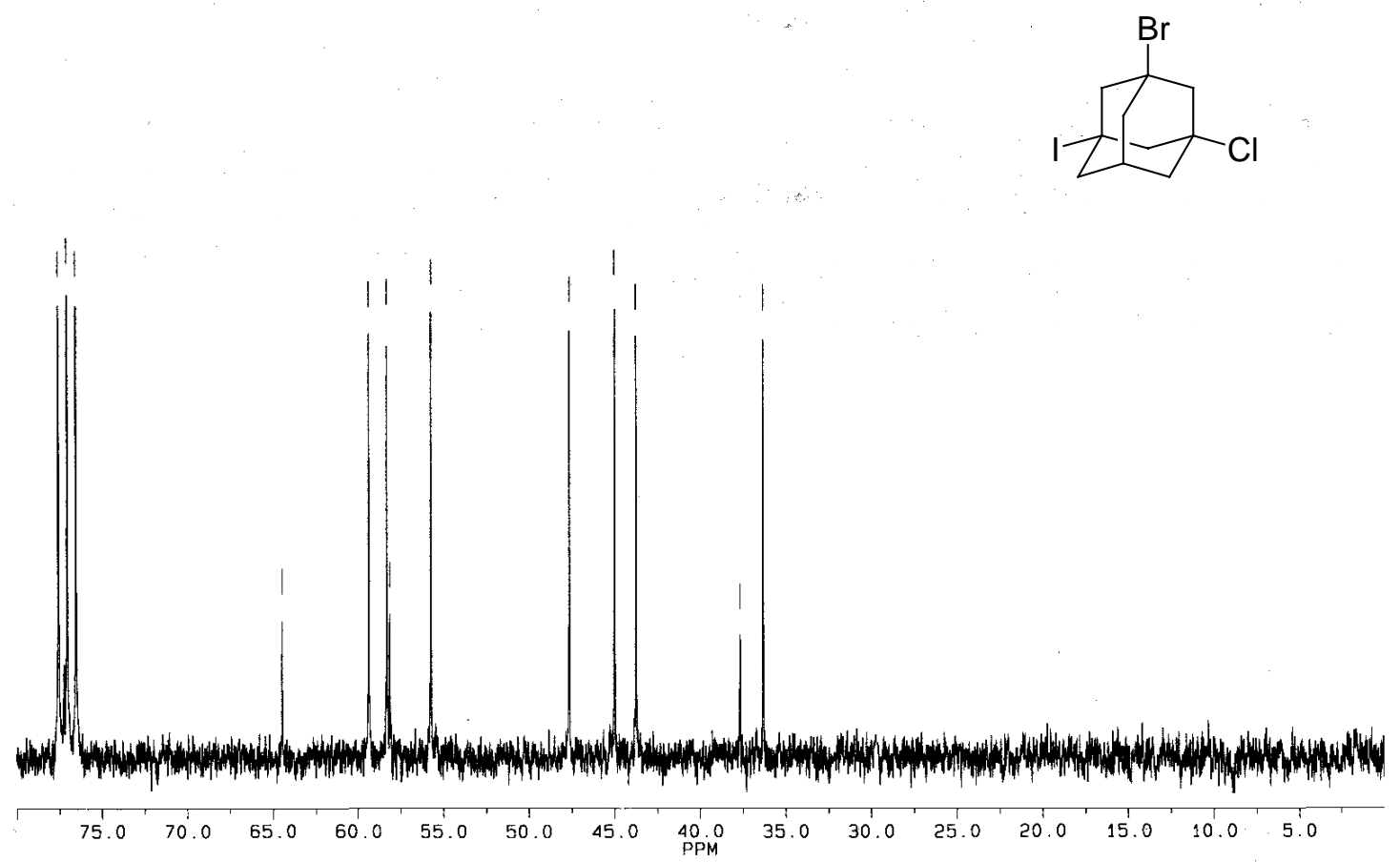

(士)-1-Iod-3-brom-5-chlortricyclo[3.3.1.1 $\left.1^{3,7}\right]$ decan (44)

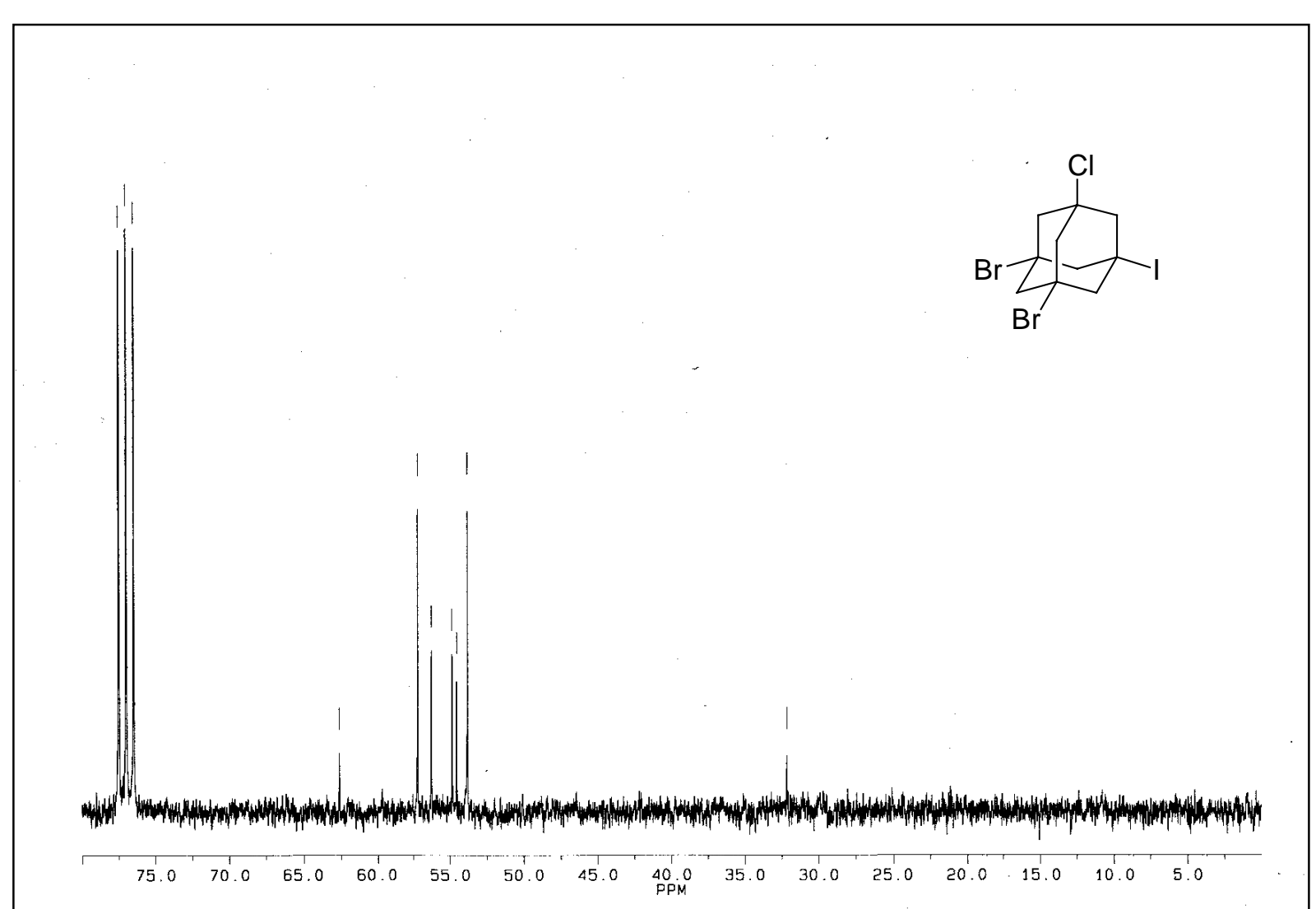

1-Iod-3,5-dibrom-7-chlortricyclo[3.3.1.1 $\left.1^{3,7}\right]$ decan (45) 


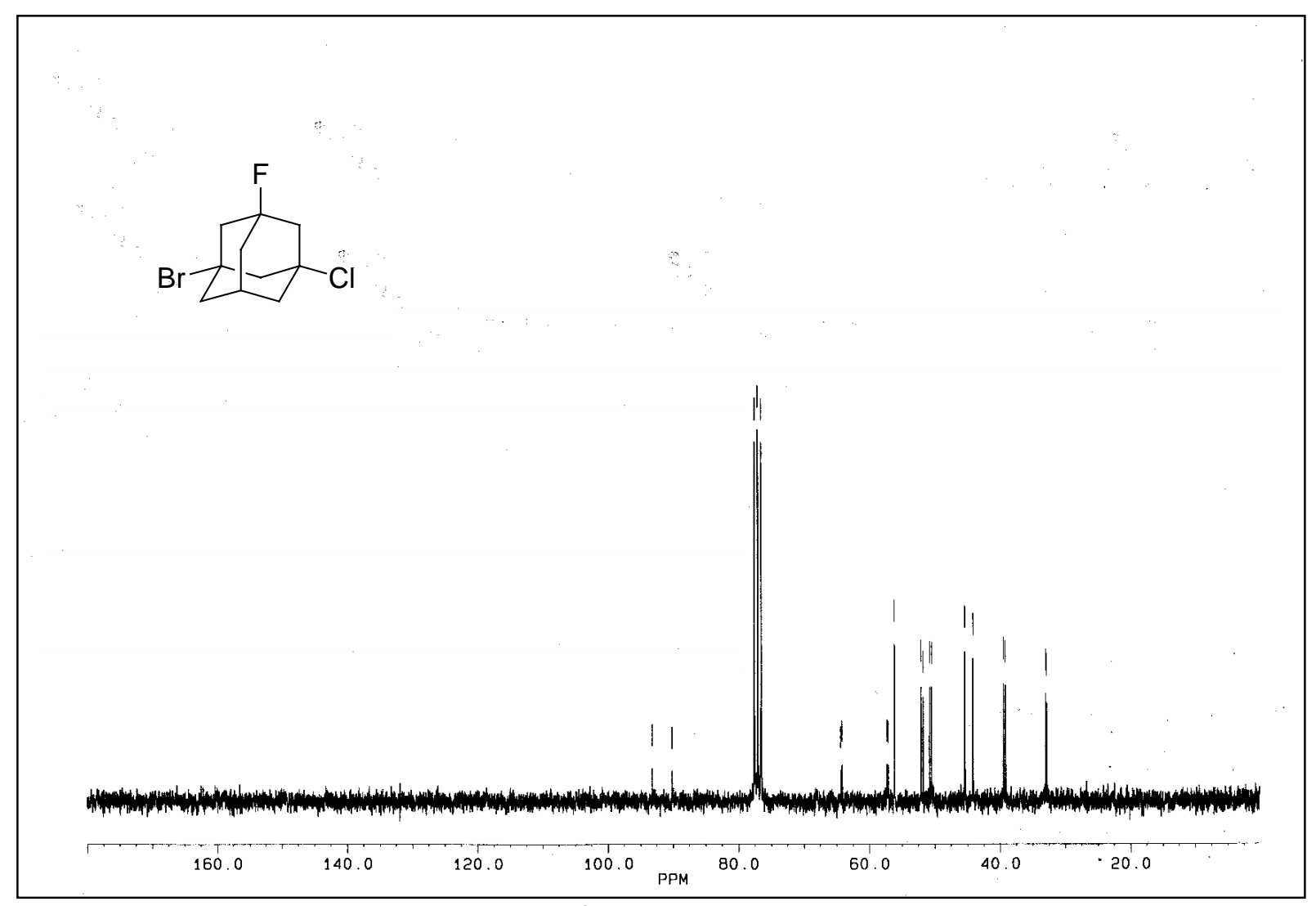

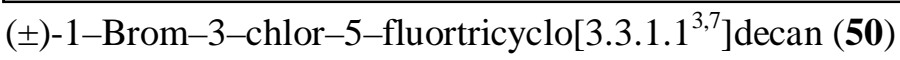

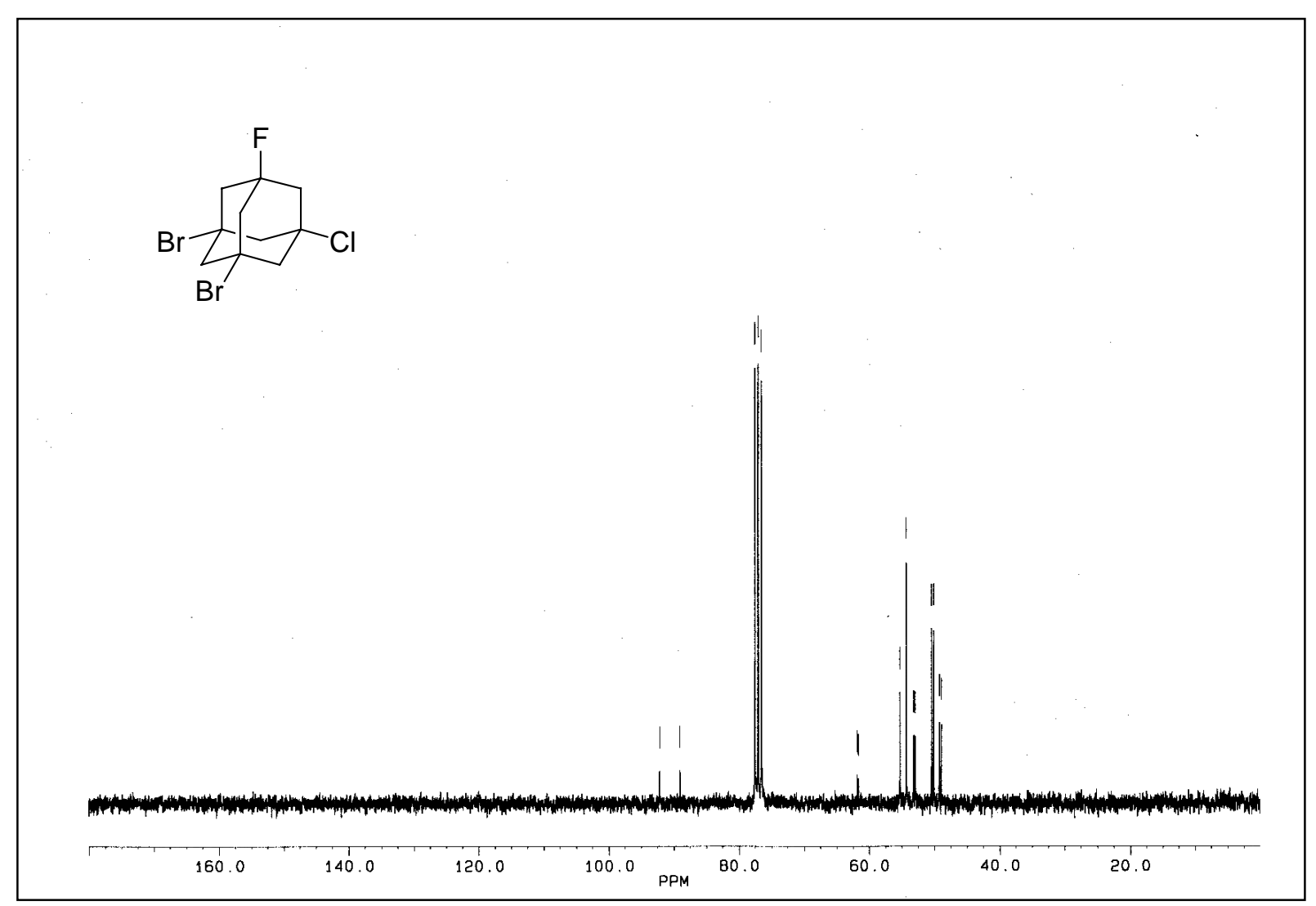

1,3-Dibrom-5-chlor-7-fluortricyclo[3.3.1.1 $\left.{ }^{3,7}\right]$ decan (52) 


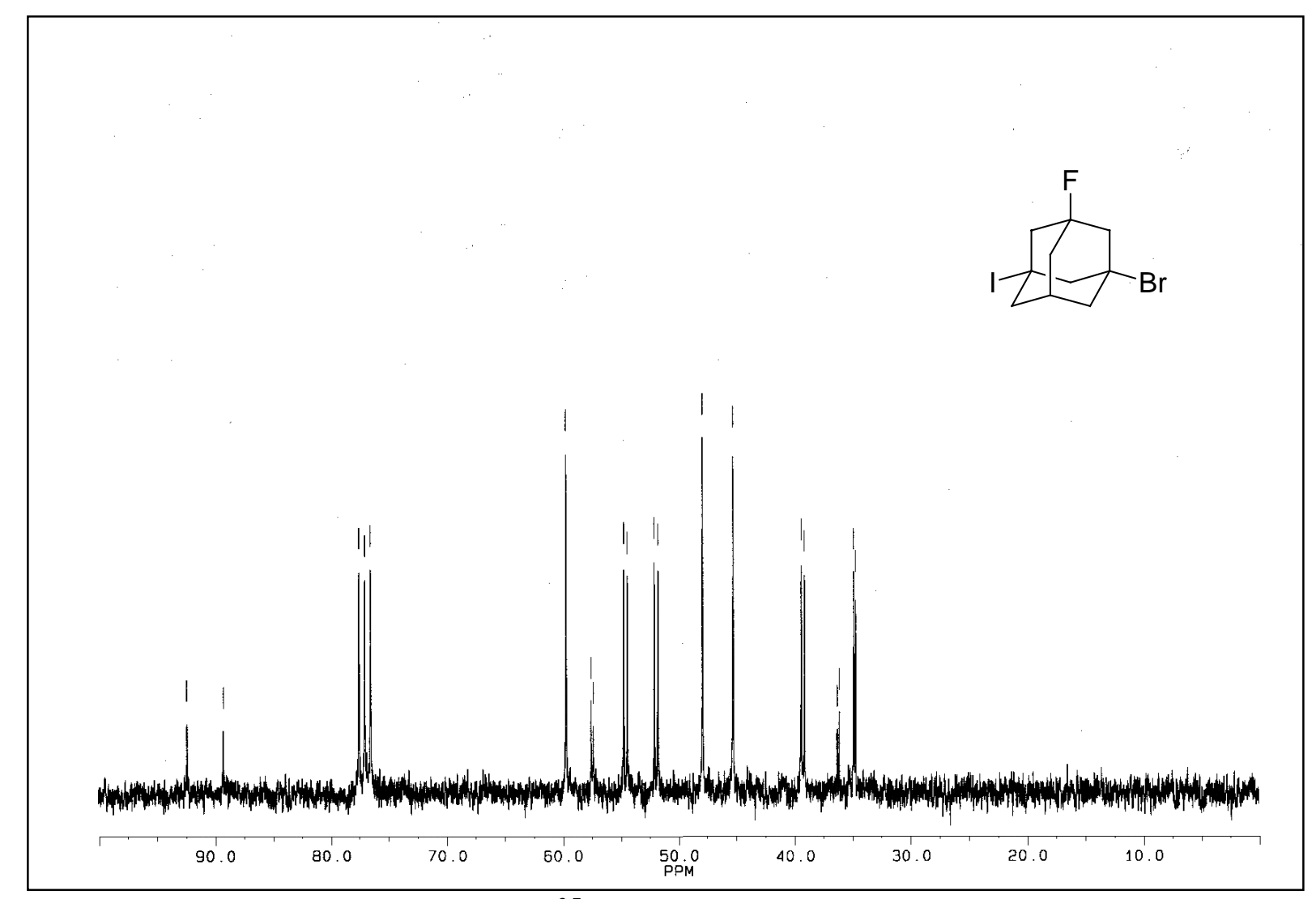

( \pm )-1-Iod-3-brom-5-fluortricyclo[3.3.1.1 $\left.{ }^{3,7}\right]$ decan (51)

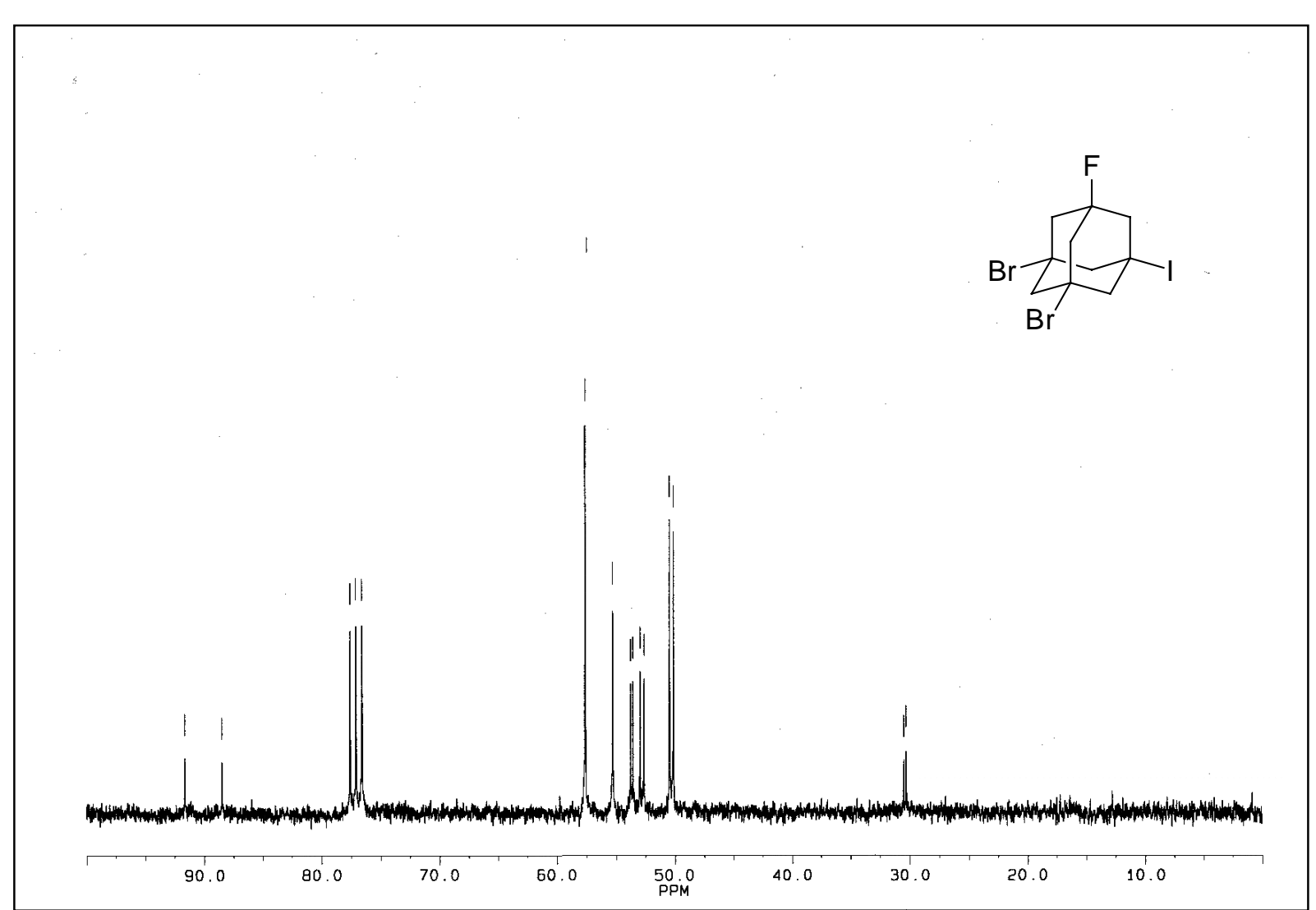

1-Iod-3,5-dibrom-7-fluortricyclo[3.3.1.1 $\left.1^{3,7}\right]$ decan (53) 
Anhang D. Abbildungen der ${ }^{13} \mathrm{C}-\mathrm{NMR}$ Spektren

171

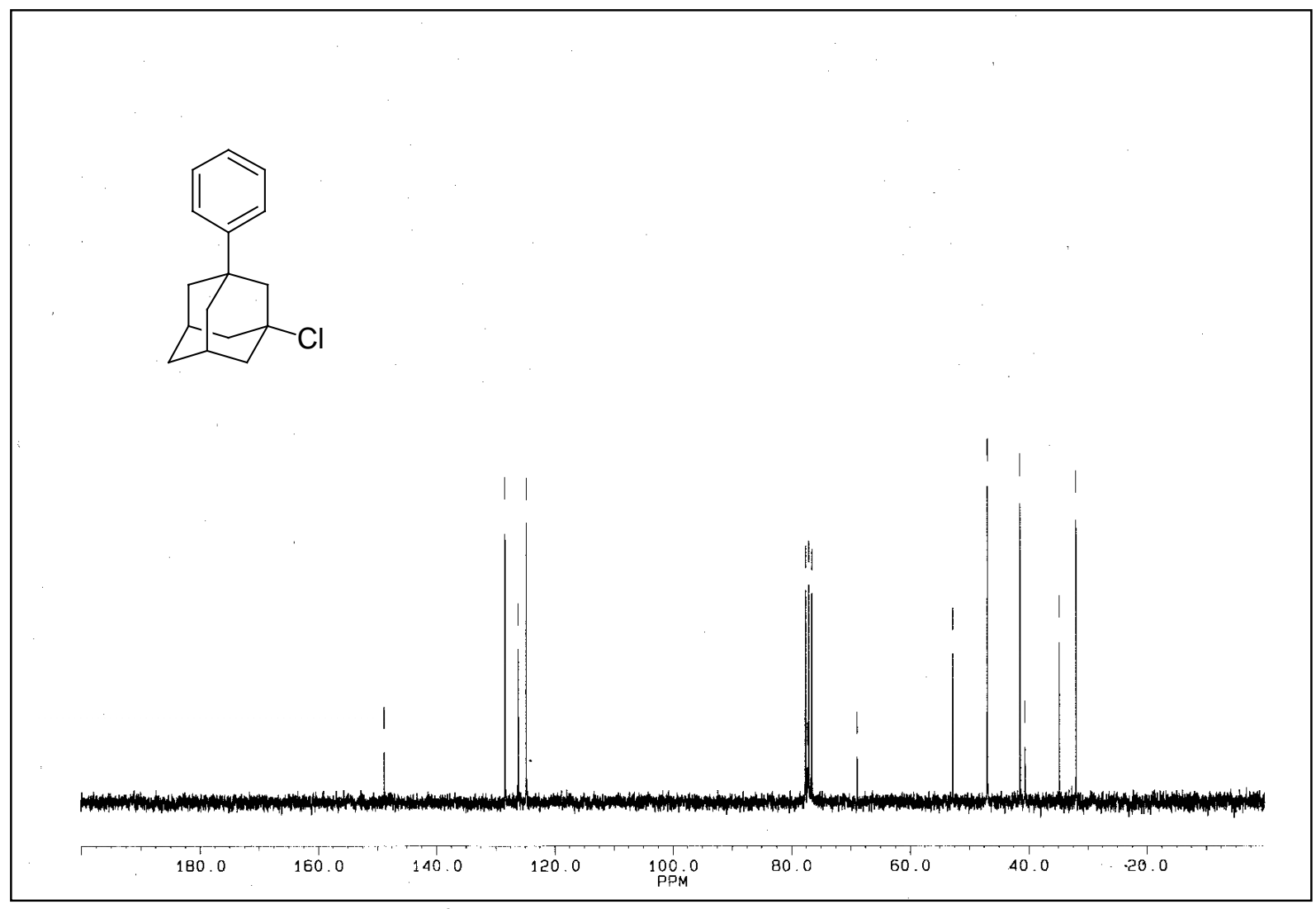

1-Chlor-3-phenyltricyclo[3.3.1.1 $\left.1^{3,7}\right]$ decan (53)
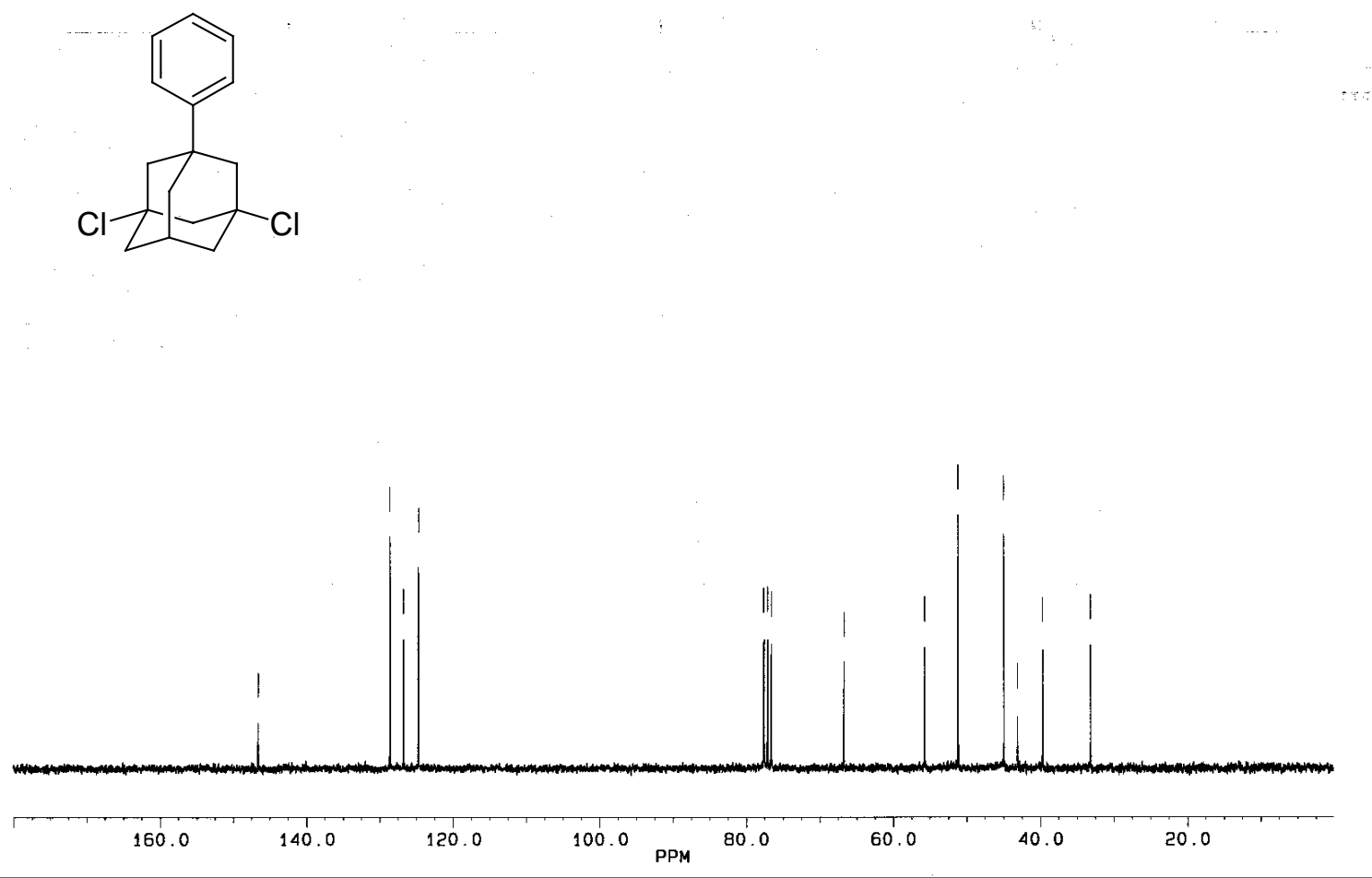

1,3-Dichlor-5-phenyltricyclo[3.3.1.1.1, $] \operatorname{decan}(\mathbf{6 4})$ 


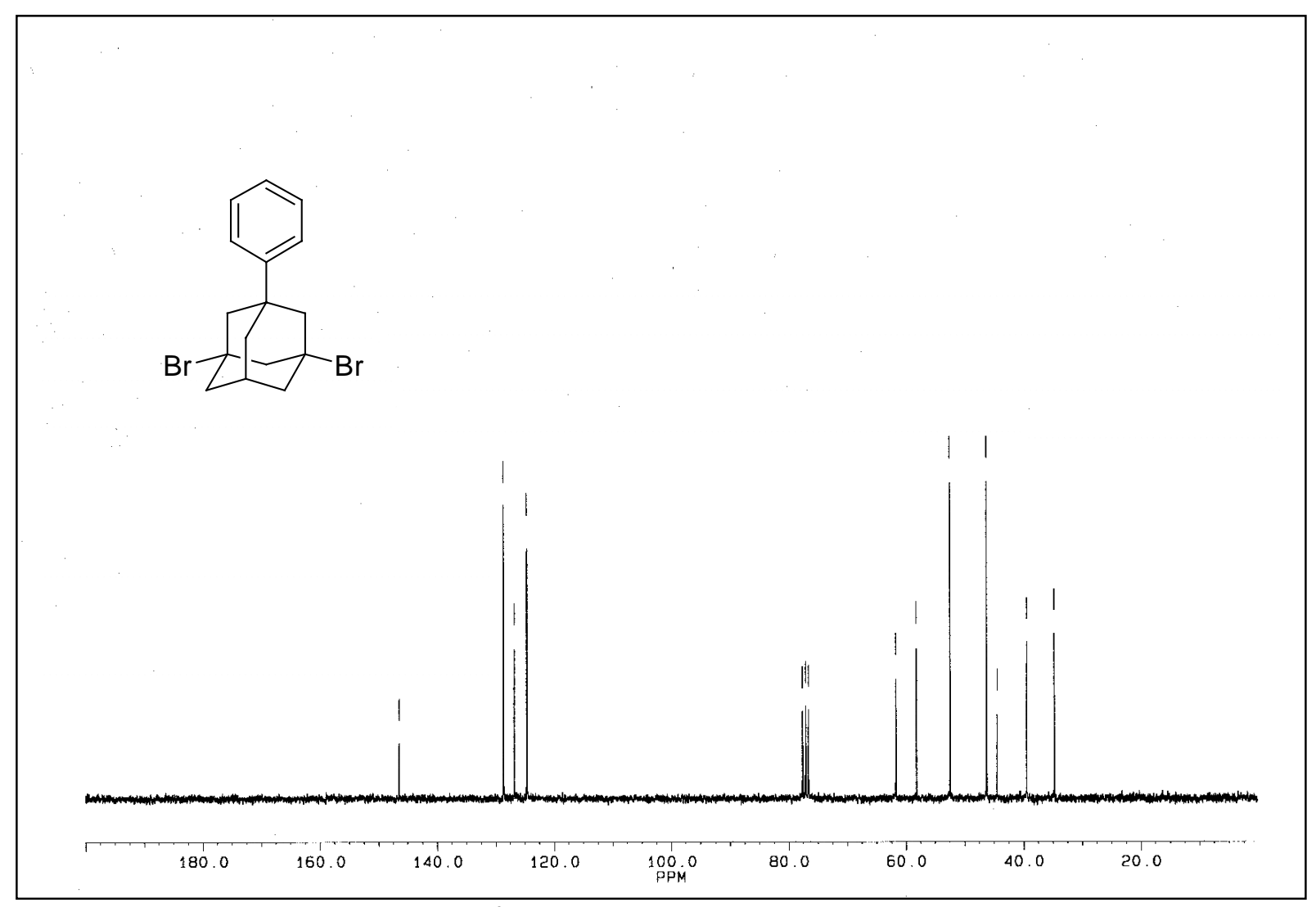

1,3-Dibrom-5-phenyltricyclo[3.3.1.1 $\left.1^{3,7}\right]$ decan (65)

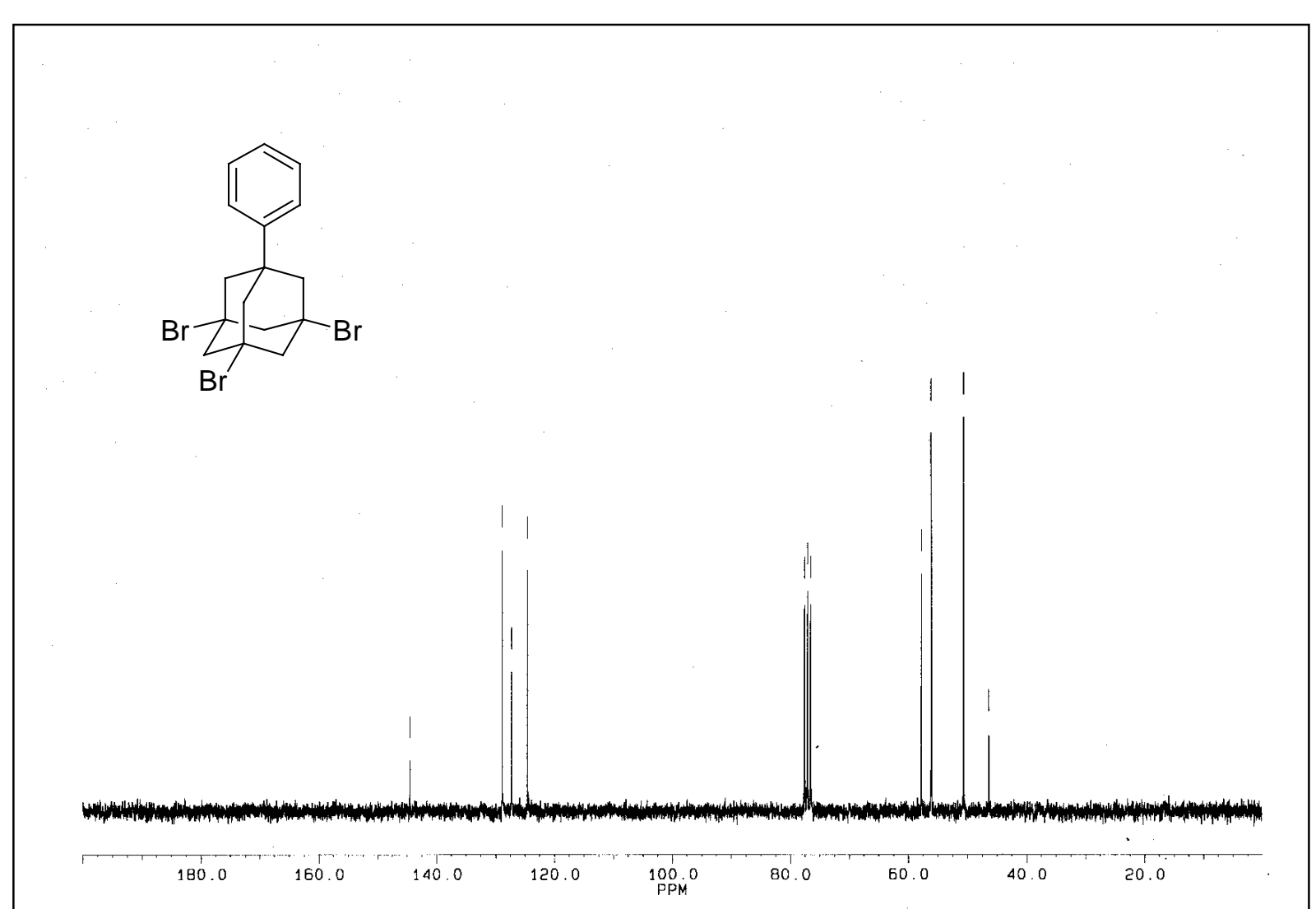

1,3,7-Tribrom-7-phenyltricyclo[3.3.1.1 $\left.1^{3,7}\right] \operatorname{decan}(\mathbf{6 6})$ 


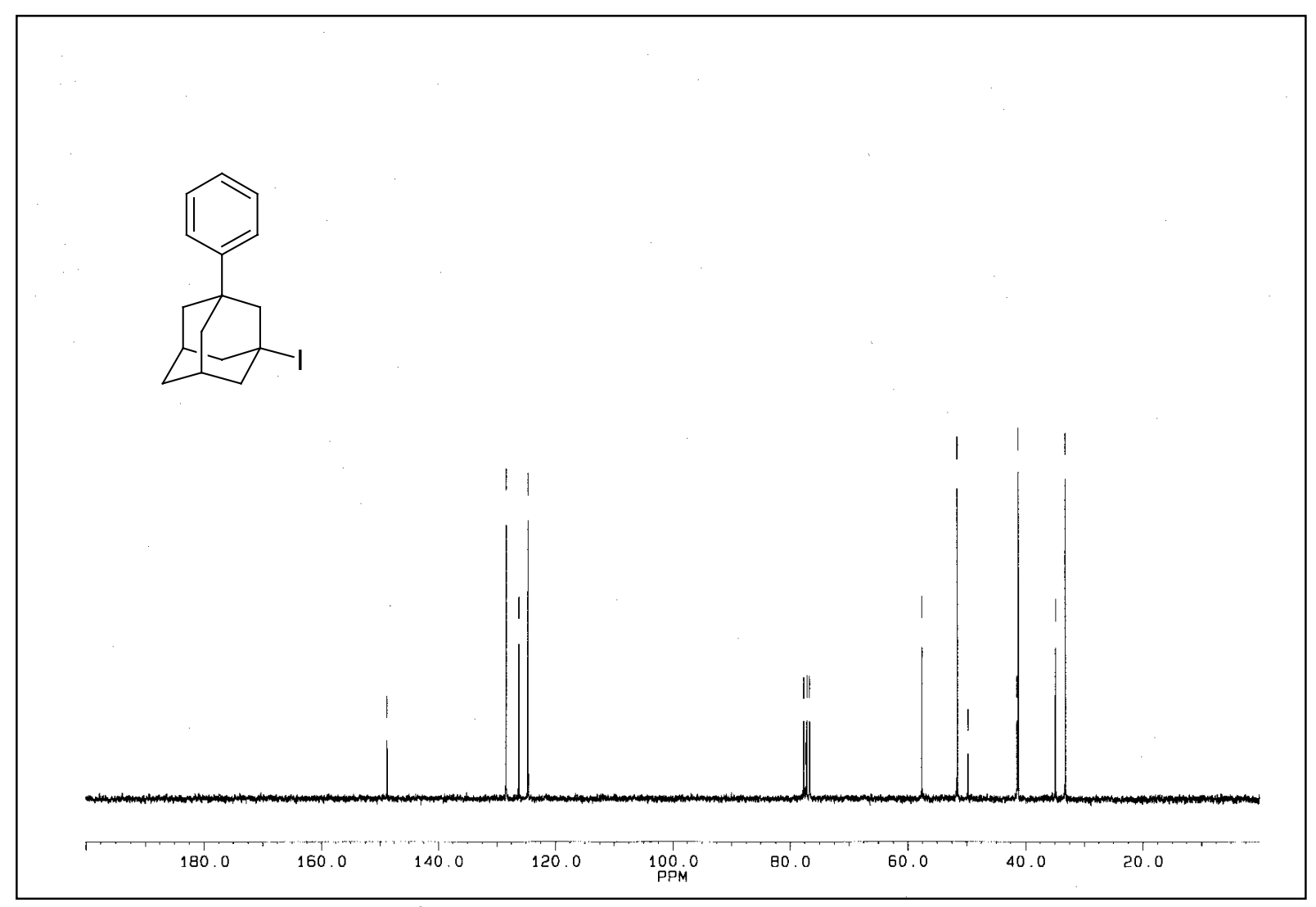

1-Iod-3-phenyltricyclo[3.3.1.1 $\left.1^{3,7}\right]$ decan (60)

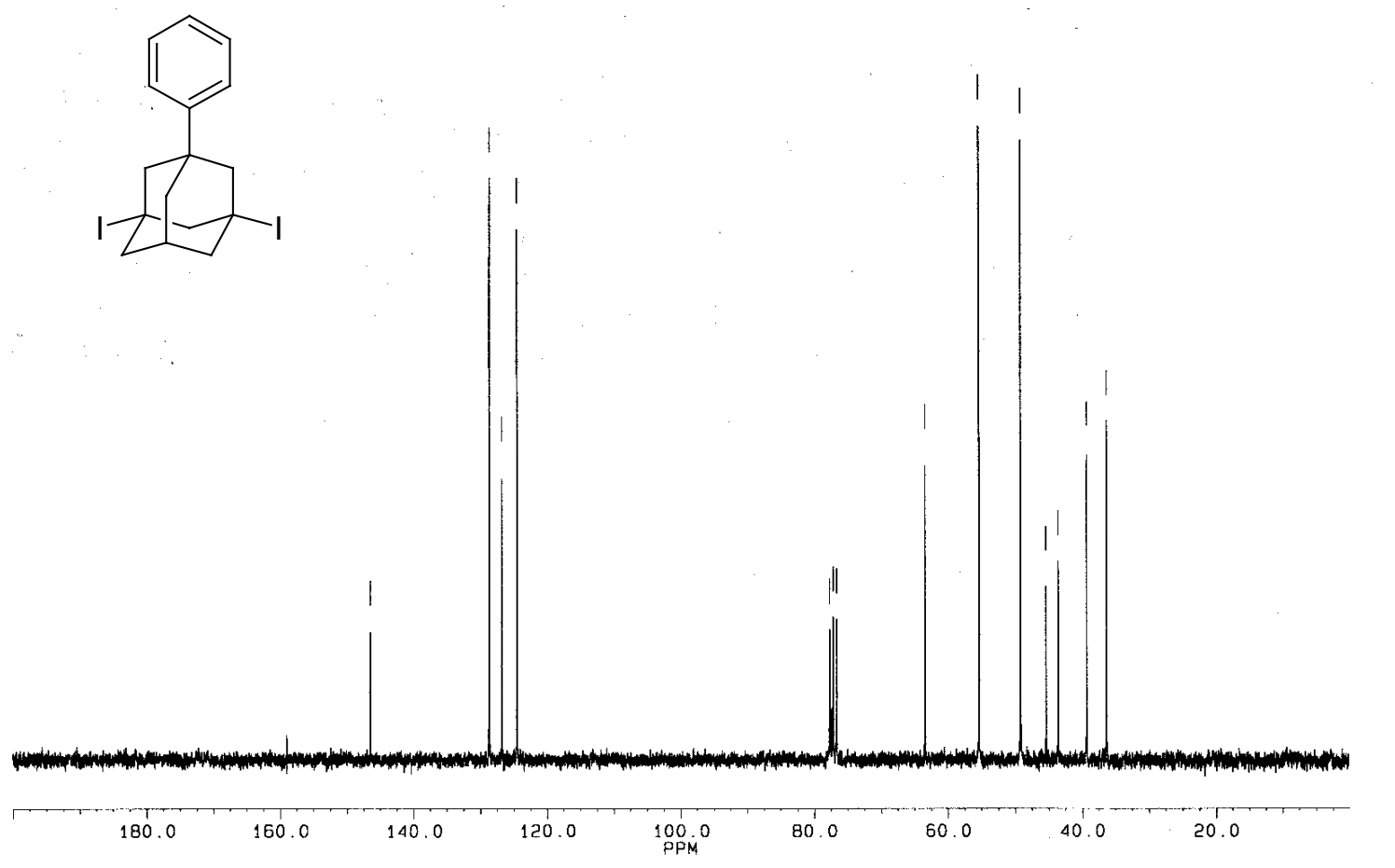

1,3-Diiod-7-phenyltricyclo[3.3.1.1 $\left.1^{3,7}\right]$ decan (67) 

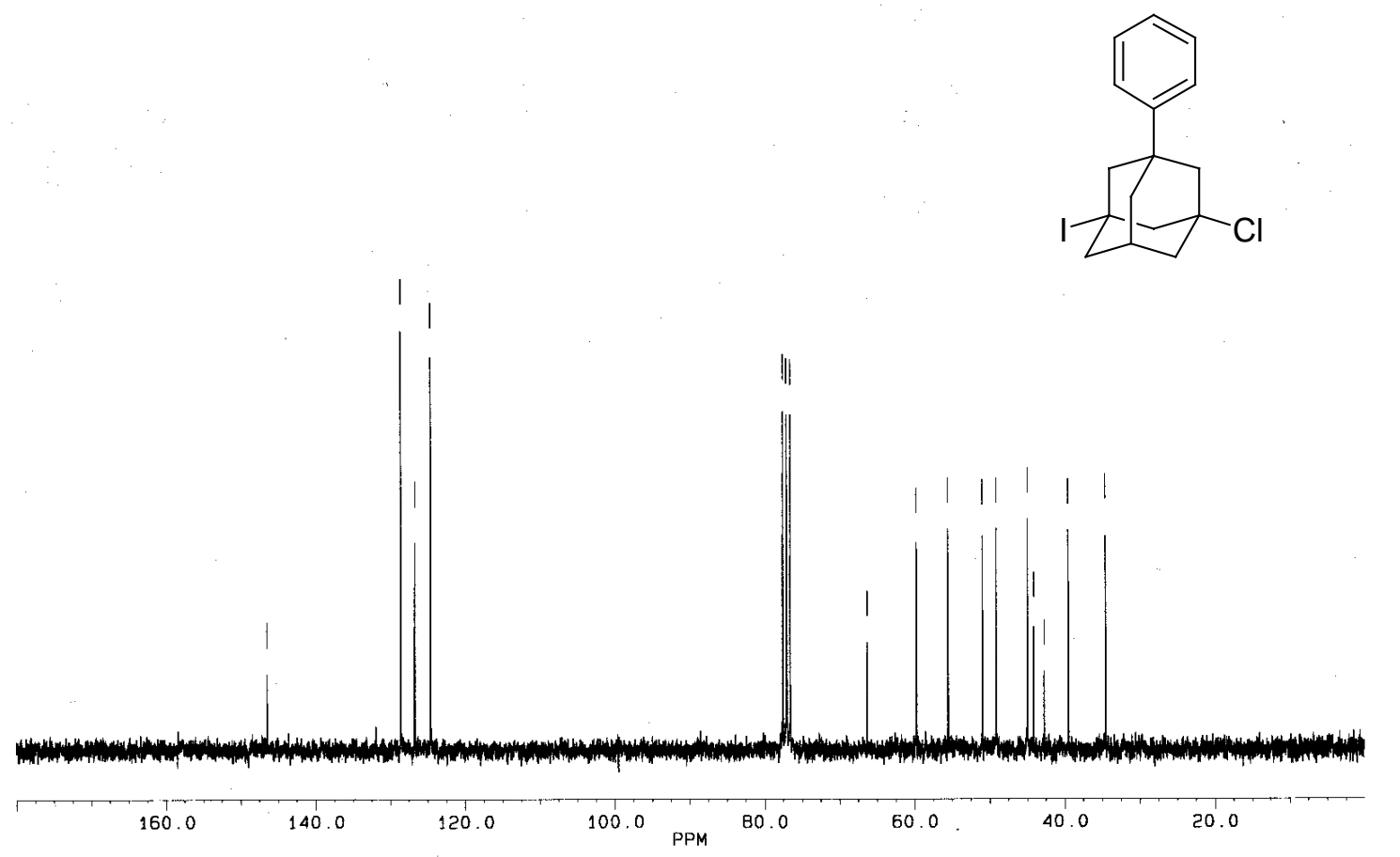

( \pm )-1-Iod-3-chlor-7-phenyltricyclo[3.3.1.1 $\left.1^{3,7}\right]$ decan (58)
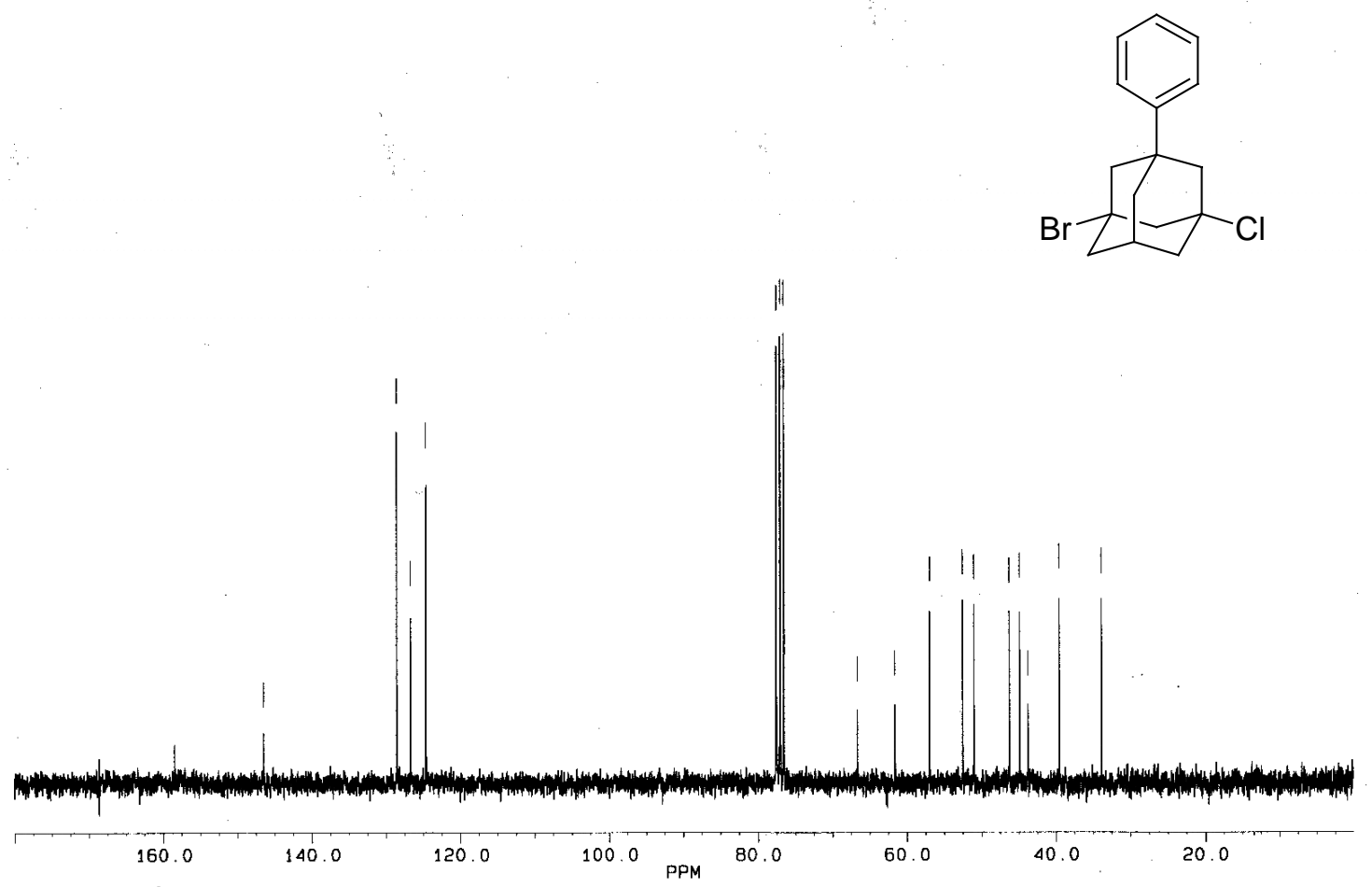

( \pm )-1-Brom-3-chlor-5-phenyltricyclo[3.3.1.1 $\left.1^{3,7}\right]$ decan (62) 


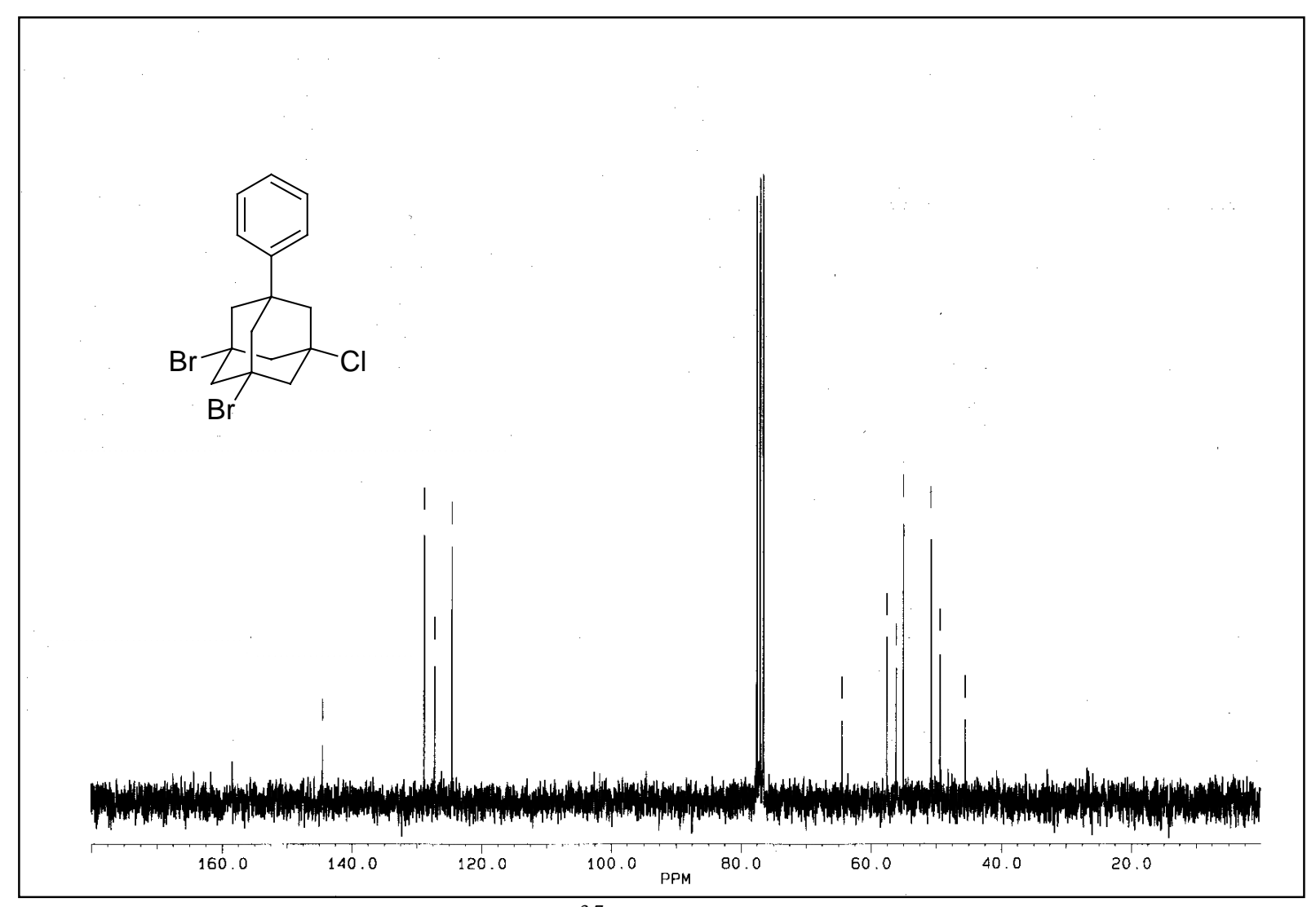

1,3-Brom-5-chlor-7-phenyltricyclo[3.3.1.1 $\left.{ }^{3,7}\right] \operatorname{decan}(\mathbf{6 2})$

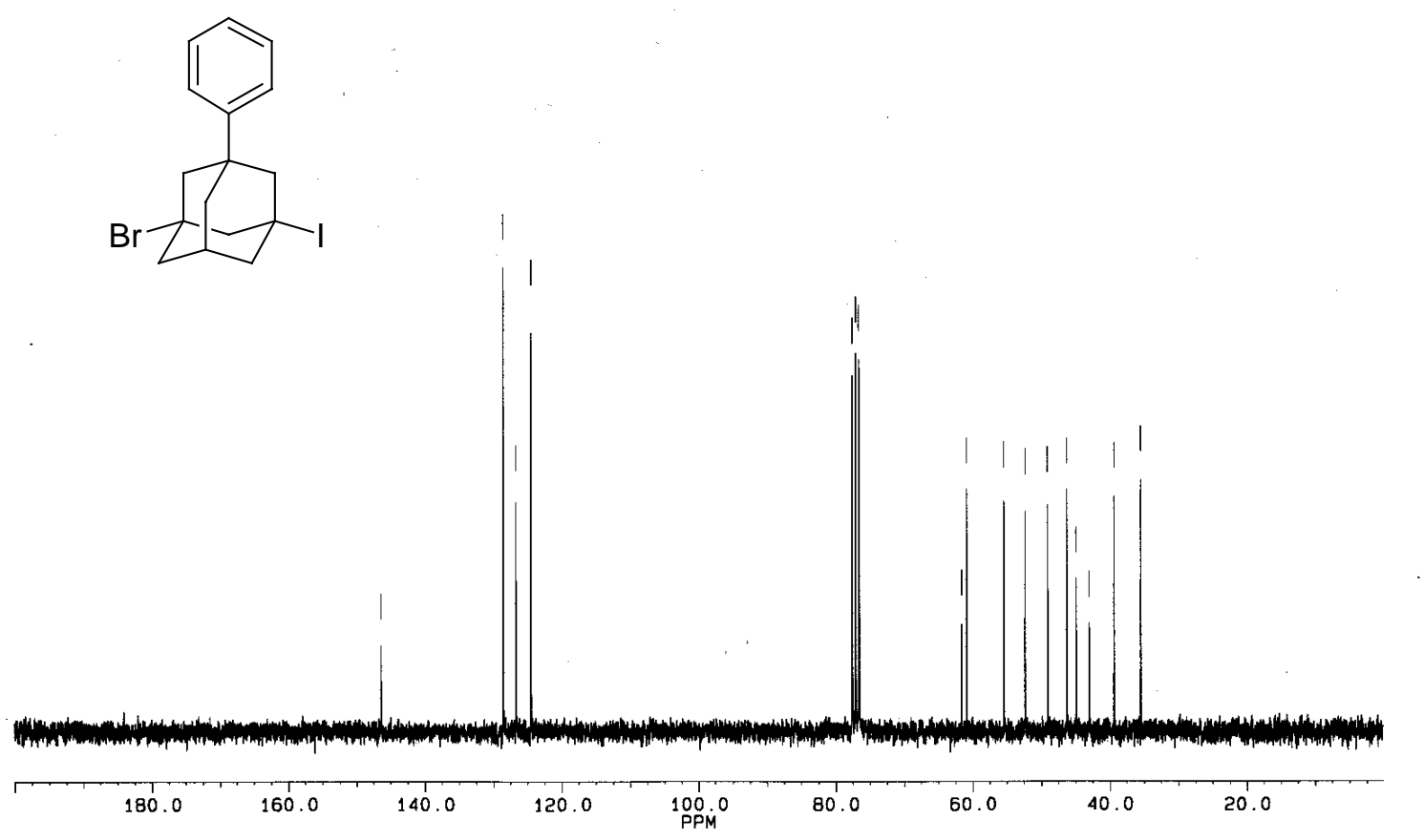

( \pm )-1-Iod-3-brom-5-phenyltricyclo[3.3.1.1 $\left.1^{3,7}\right]$ decan (61) 


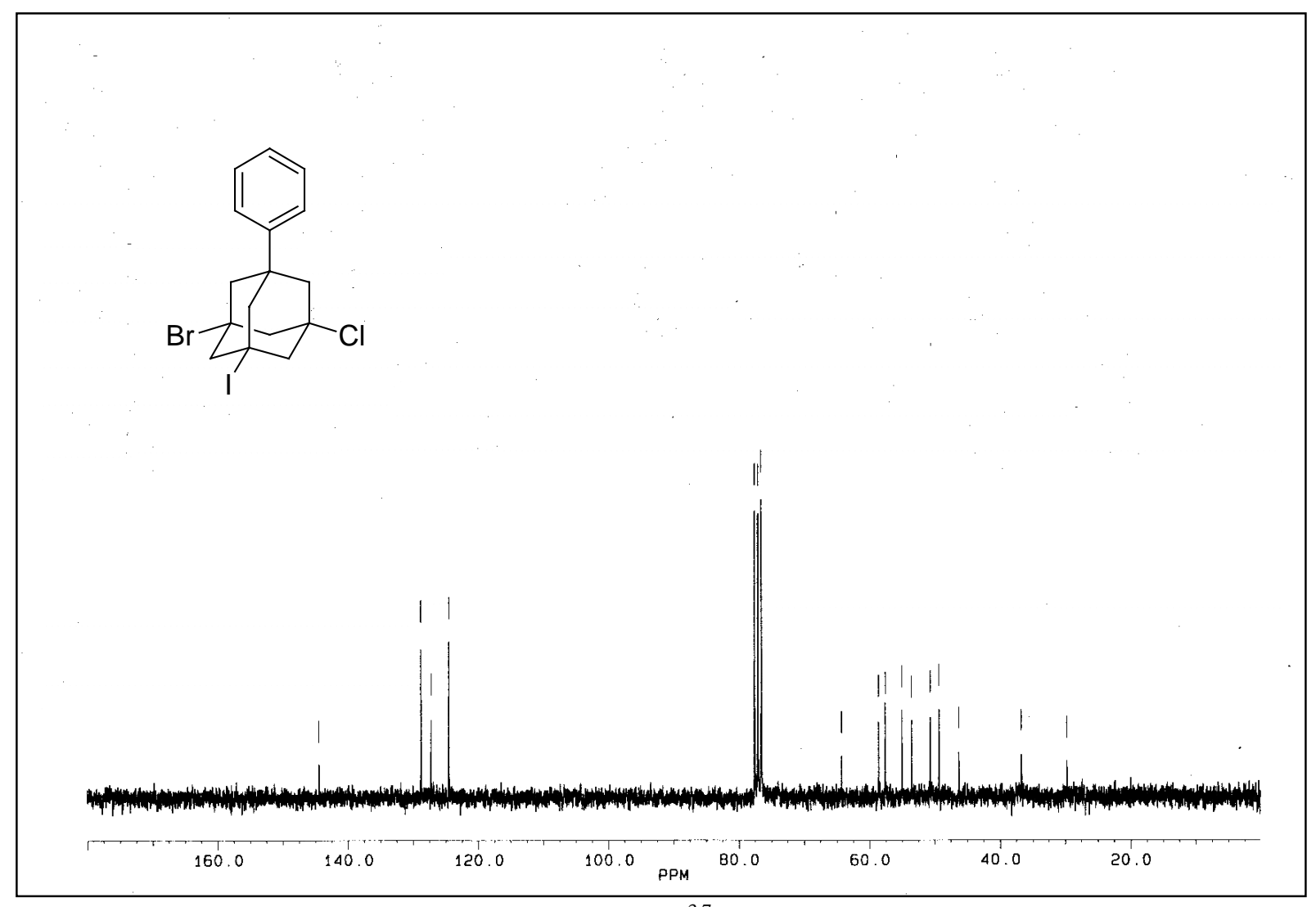

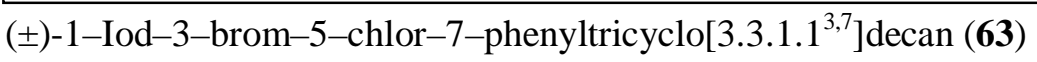

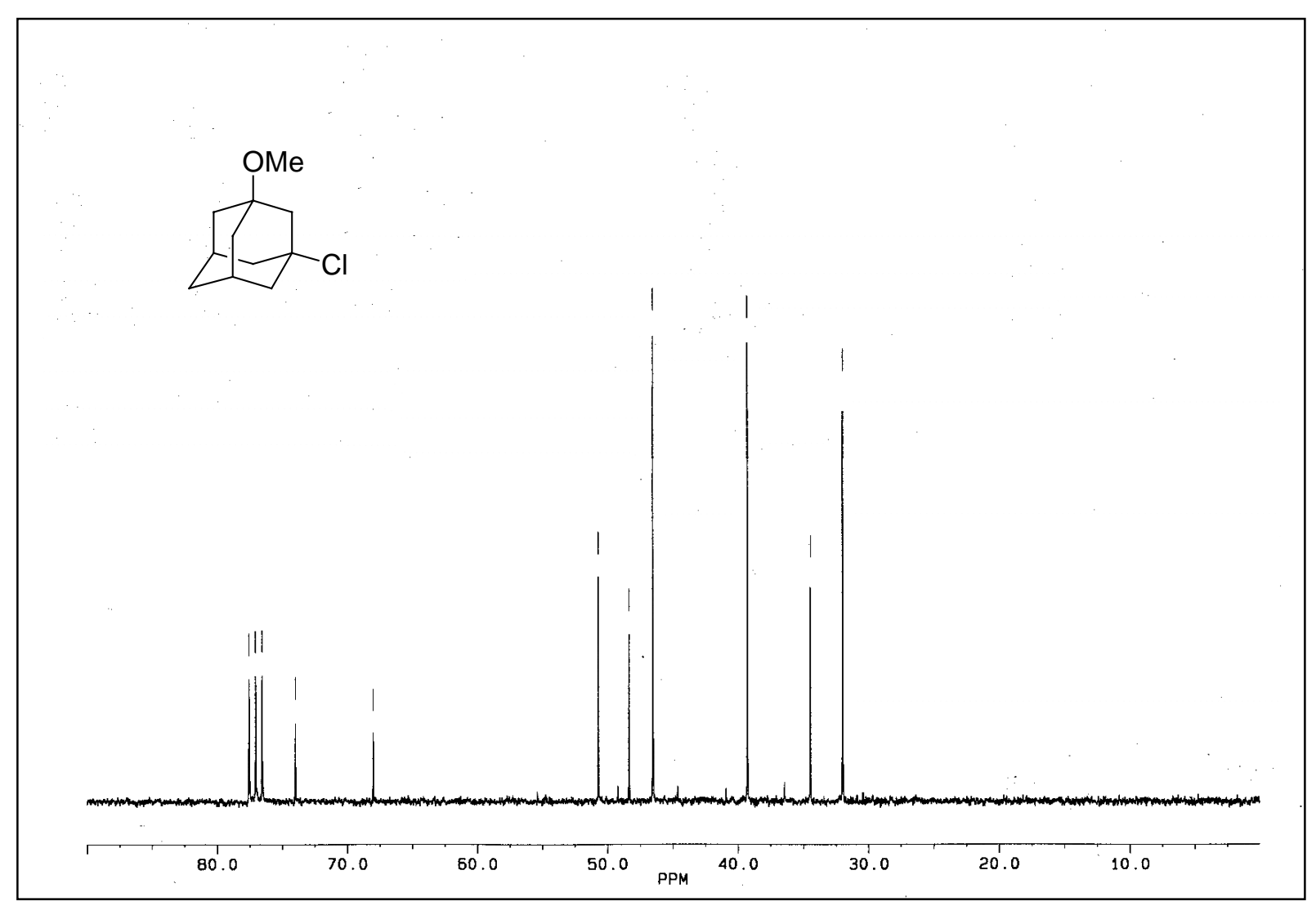

1-Chlor-3-methoxytricyclo[3.3.1.1 $\left.1^{3,7}\right]$ decan (70) 


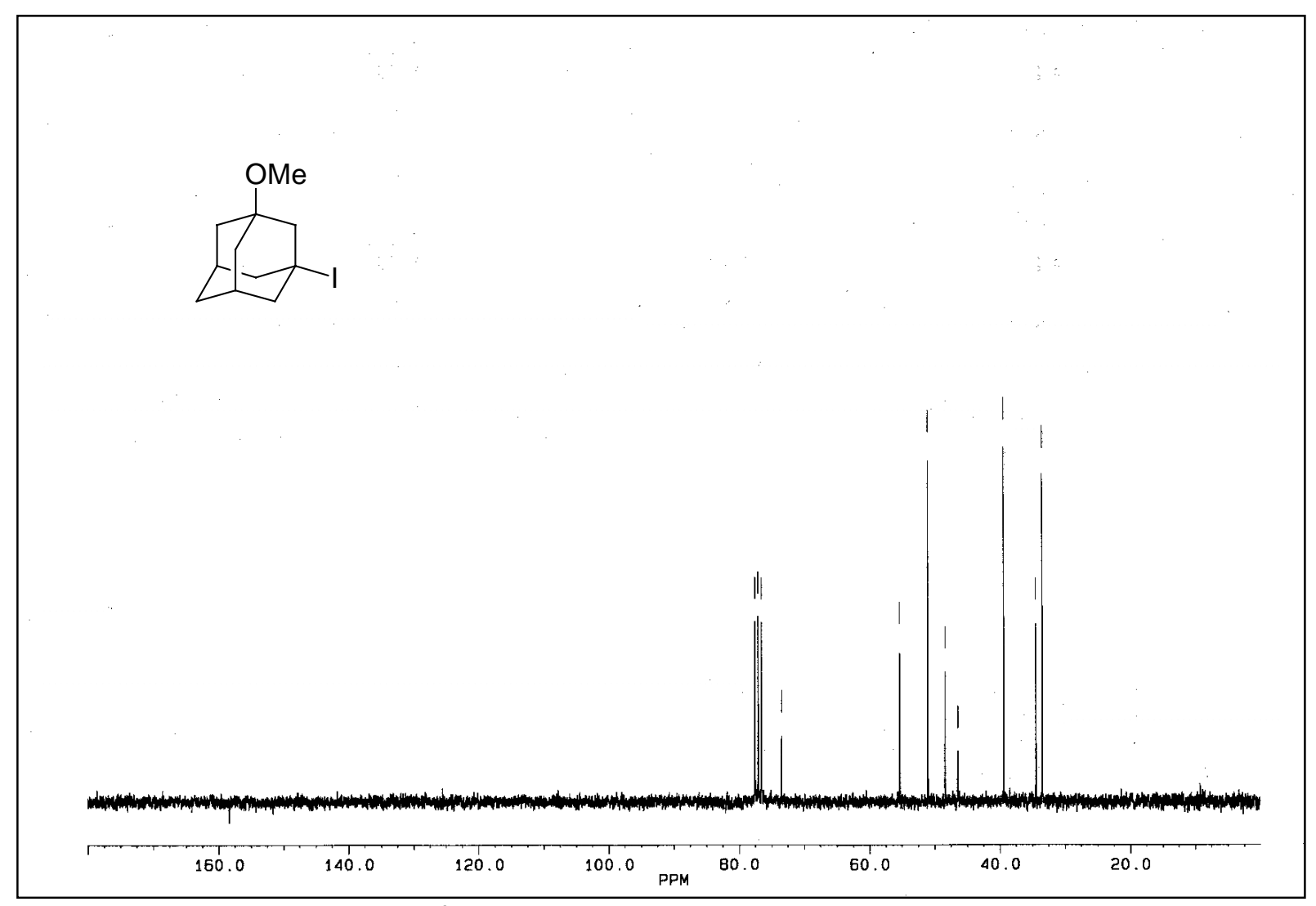

1-Iod-3-methoxytricyclo[3.3.1.1 $\left.1^{3,7}\right]$ decan (73)<smiles>COC12CC3CC(Cl)(CC(Br)(C3)C1)C2</smiles>

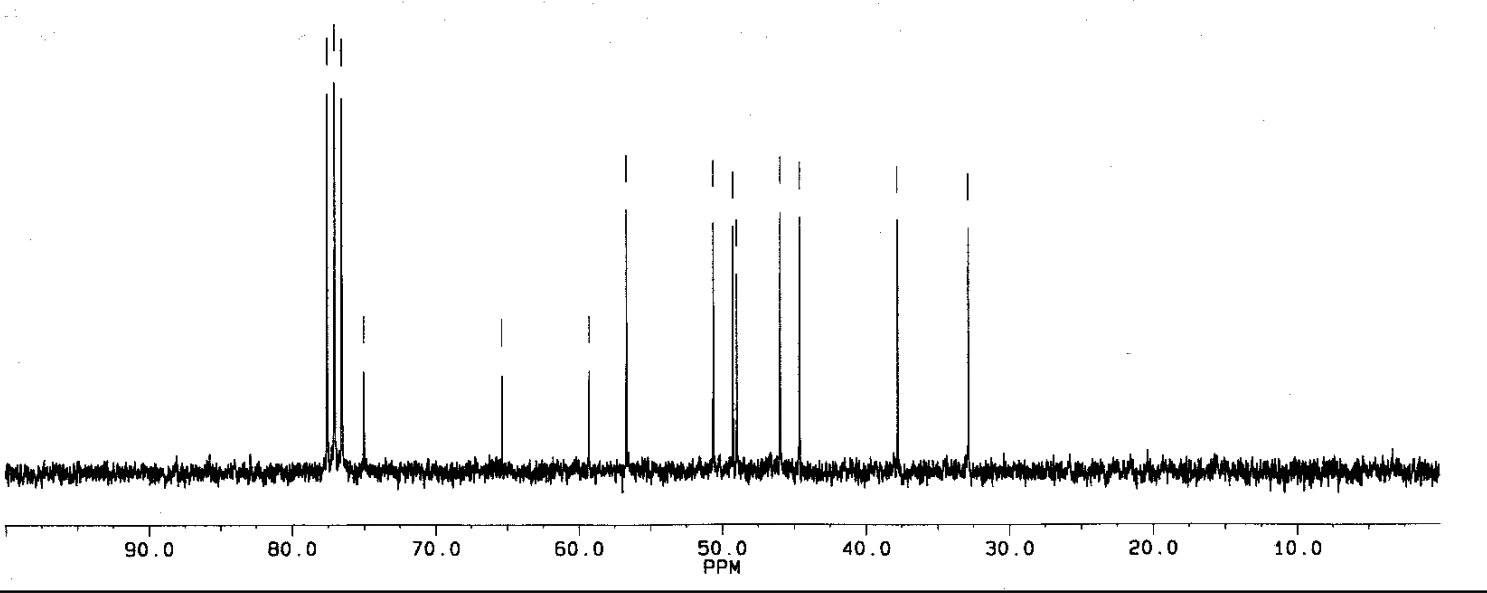

( \pm -1-Brom-3-chlor-5-methoxytricyclo[3.3.1.1 $\left.1^{3,7}\right]$ decan (71) 


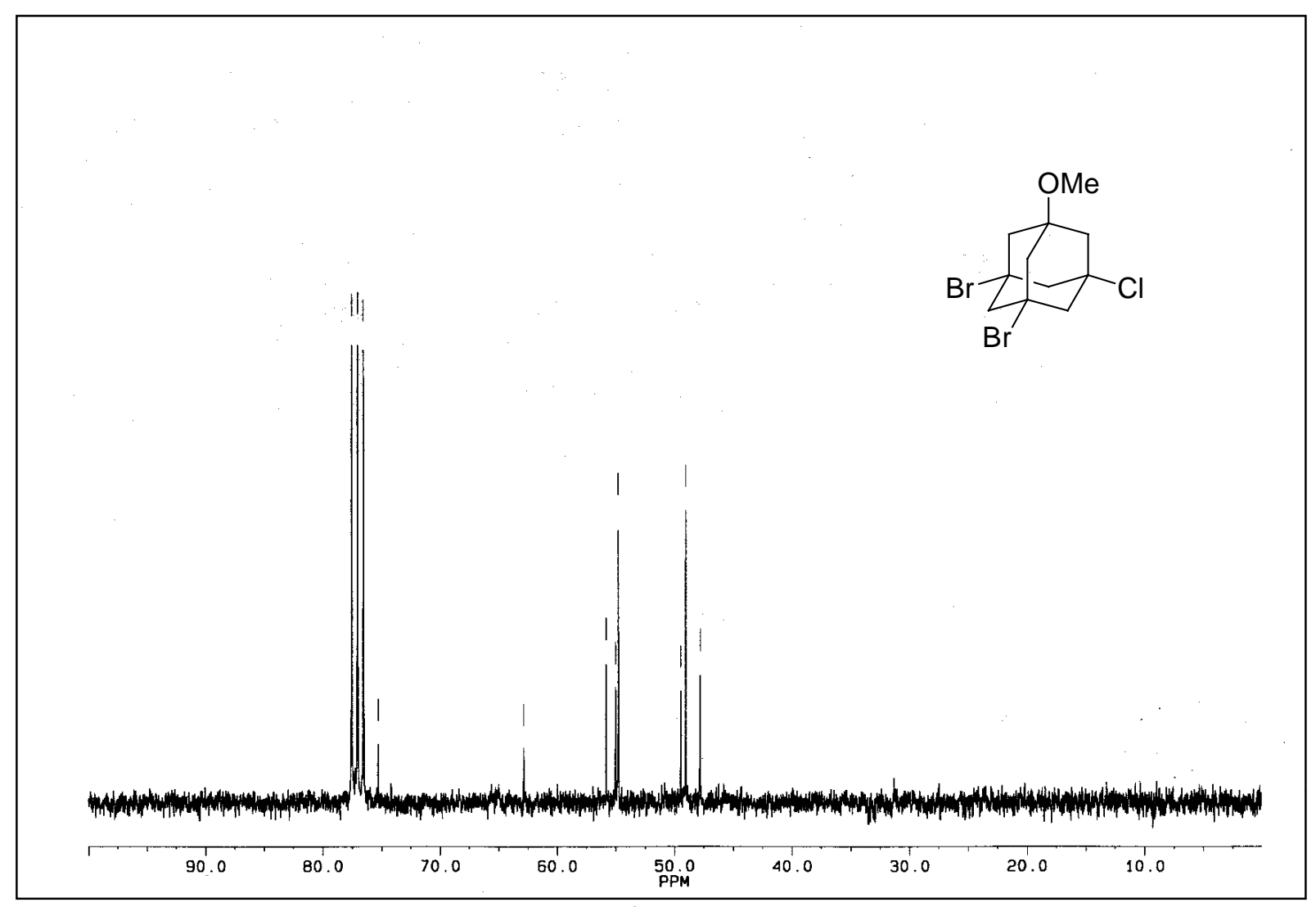

1,3-Dibrom-5-chlor-7-methoxytricyclo[3.3.1.1 $\left.1^{3,7}\right]$ decan (75)<smiles>COC12CC3CC(C)(CC(Cl)(C3)C1)C2</smiles>

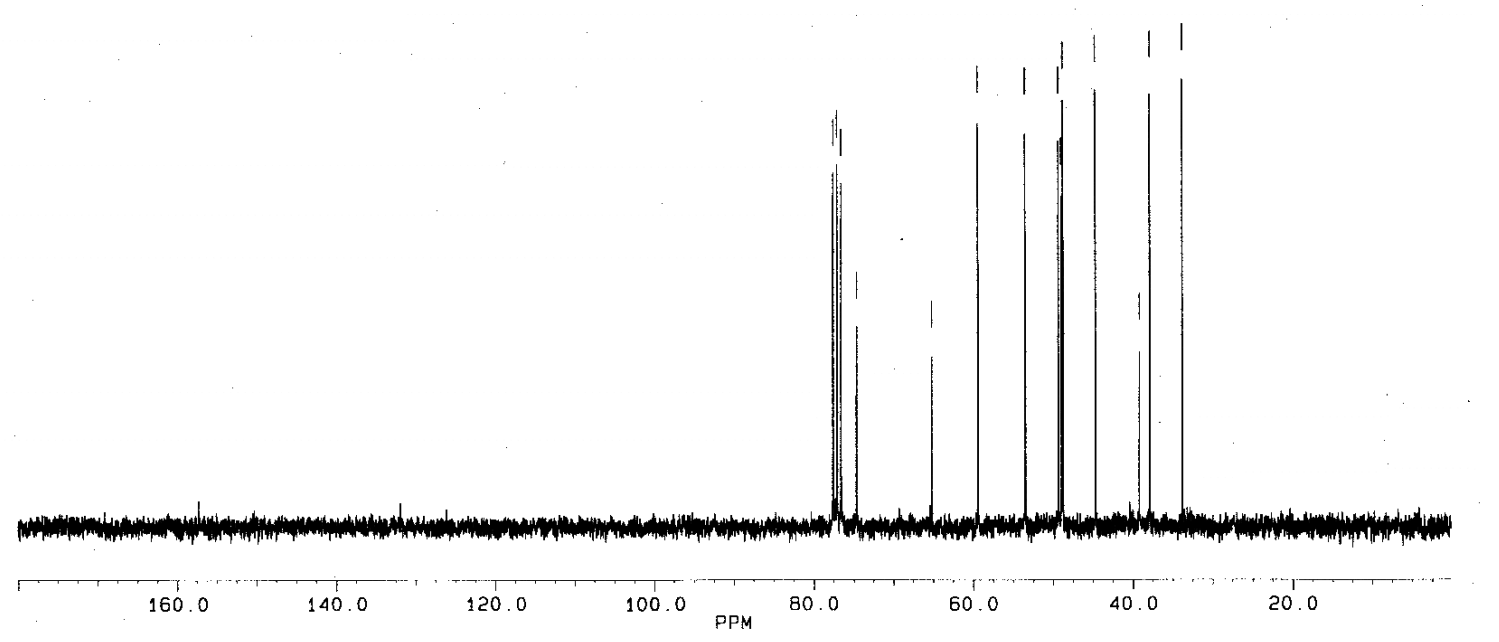

( \pm -1-Iod-3-chlor-5-methoxytricyclo[3.3.1.1 $\left.1^{3,7}\right]$ decan (72) 


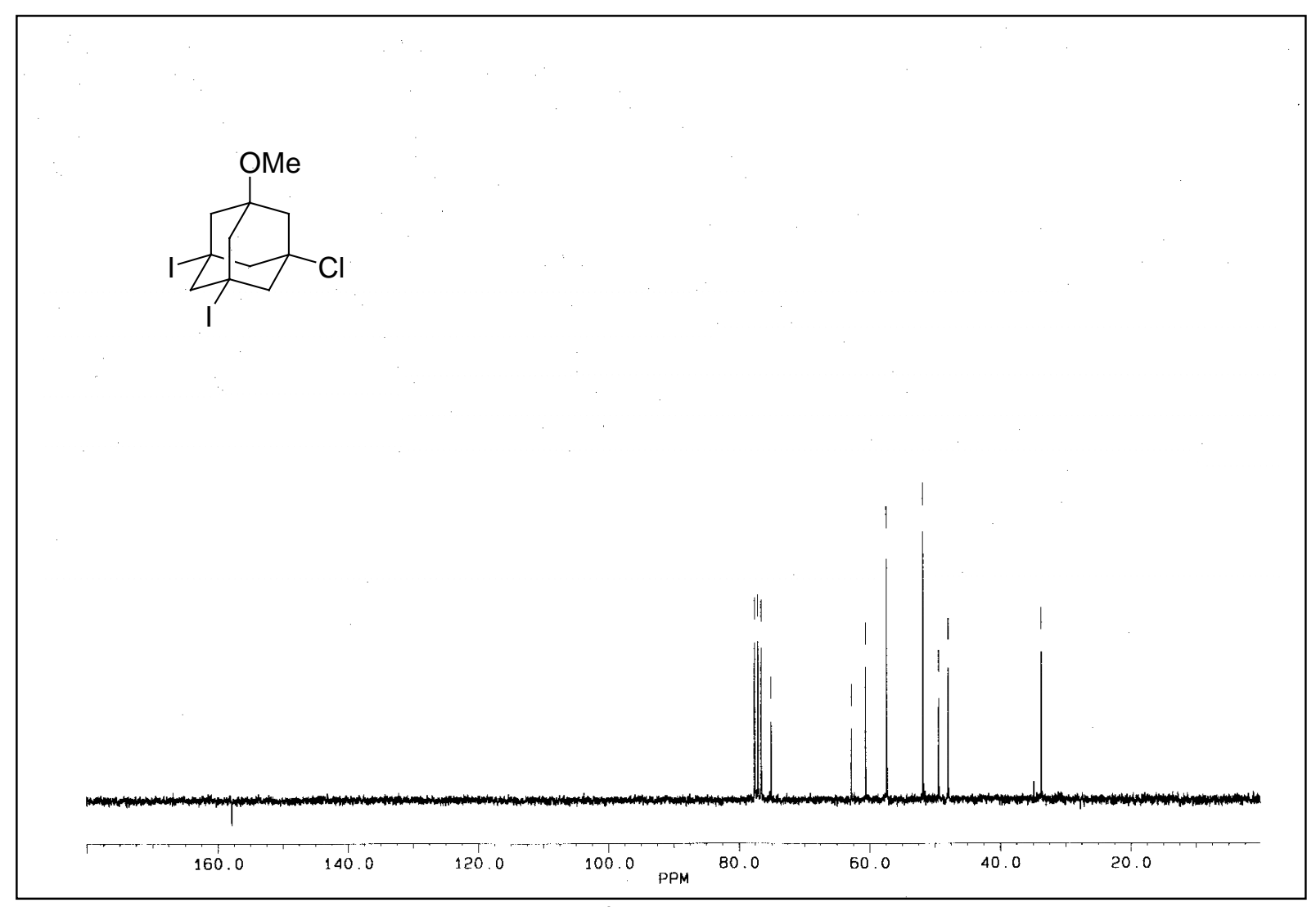

1,3-Diiod-5-chlor-7-methoxytricyclo[3.3.1.1 $\left.{ }^{3,7}\right]$ decan (76)<smiles>COC12CC3CC(Br)(CC(I)(C3)C1)C2</smiles>

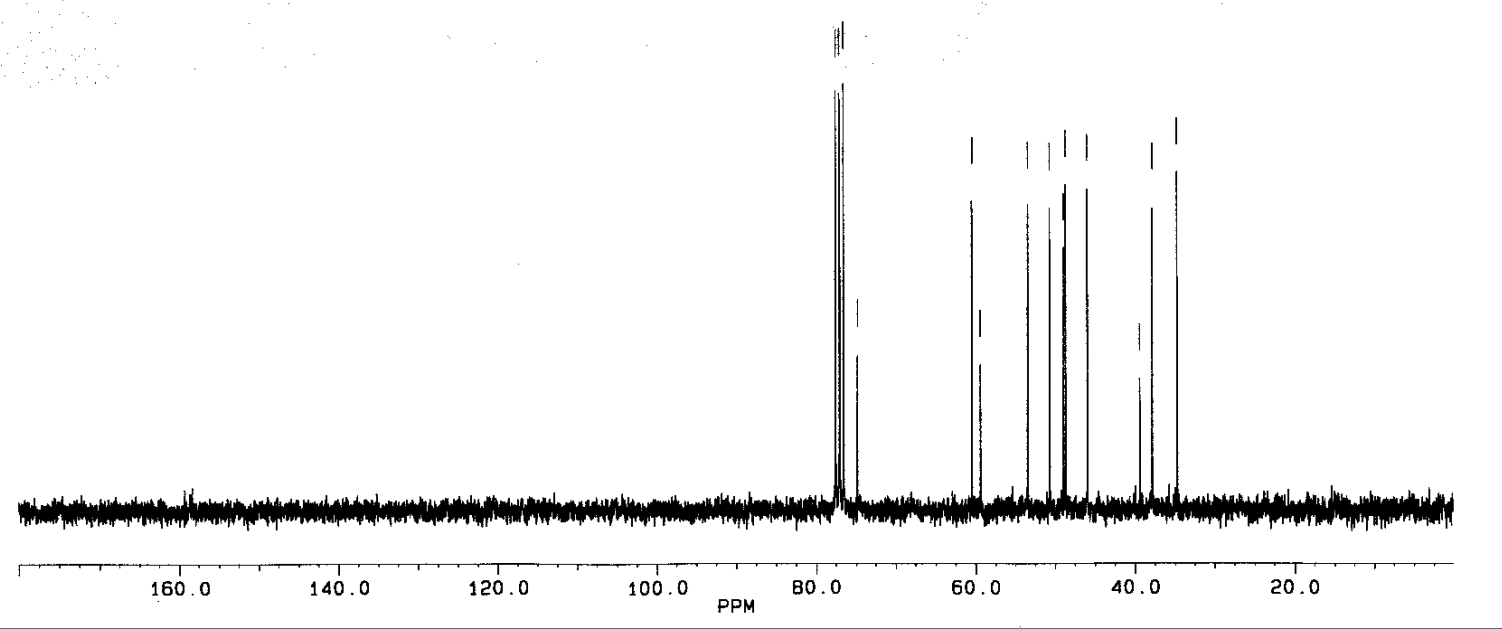

( \pm )-1-Iod-3-brom-5-methoxytricyclo[3.3.1.1 $\left.1^{3,7}\right]$ decan (74) 


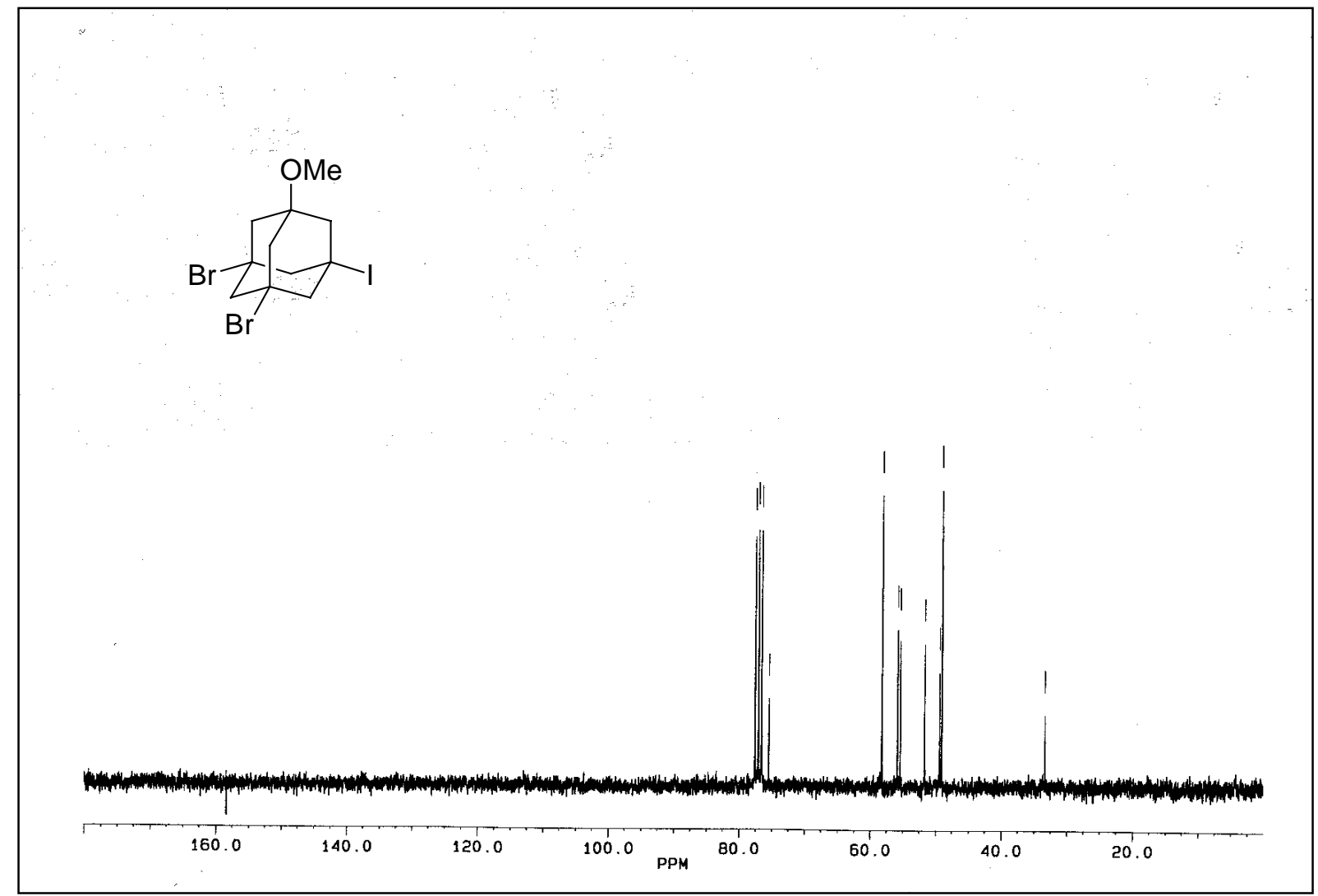

1-Iod-3,5-brom-7-methoxytricyclo[3.3.1.1 $\left.1^{3,7}\right]$ decan (77)

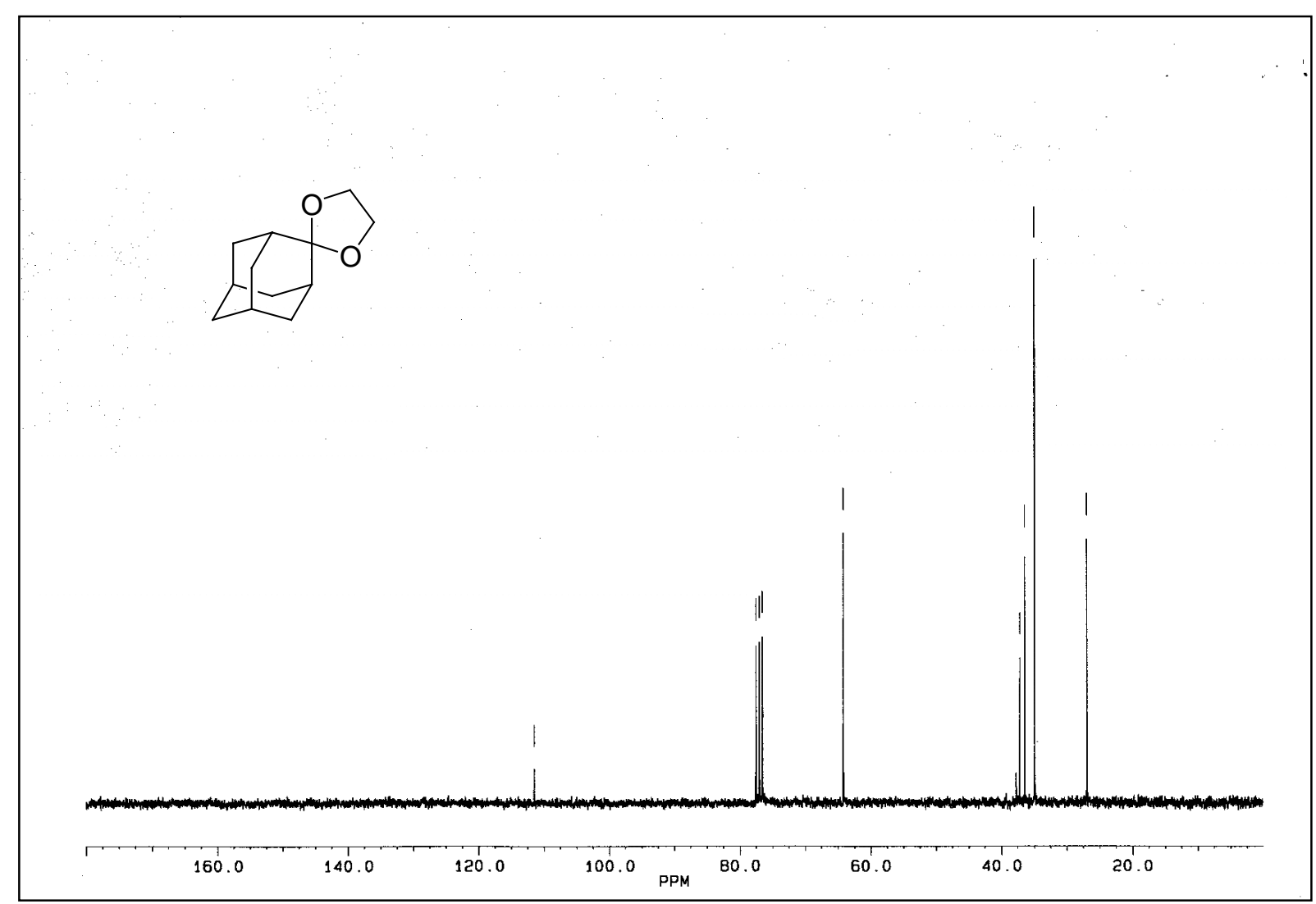

2-(spiro-1',4'-dioxocyclopentyl)tricyclo[3.3.1.1 $\left.1^{3,7}\right]$ decan (79) 


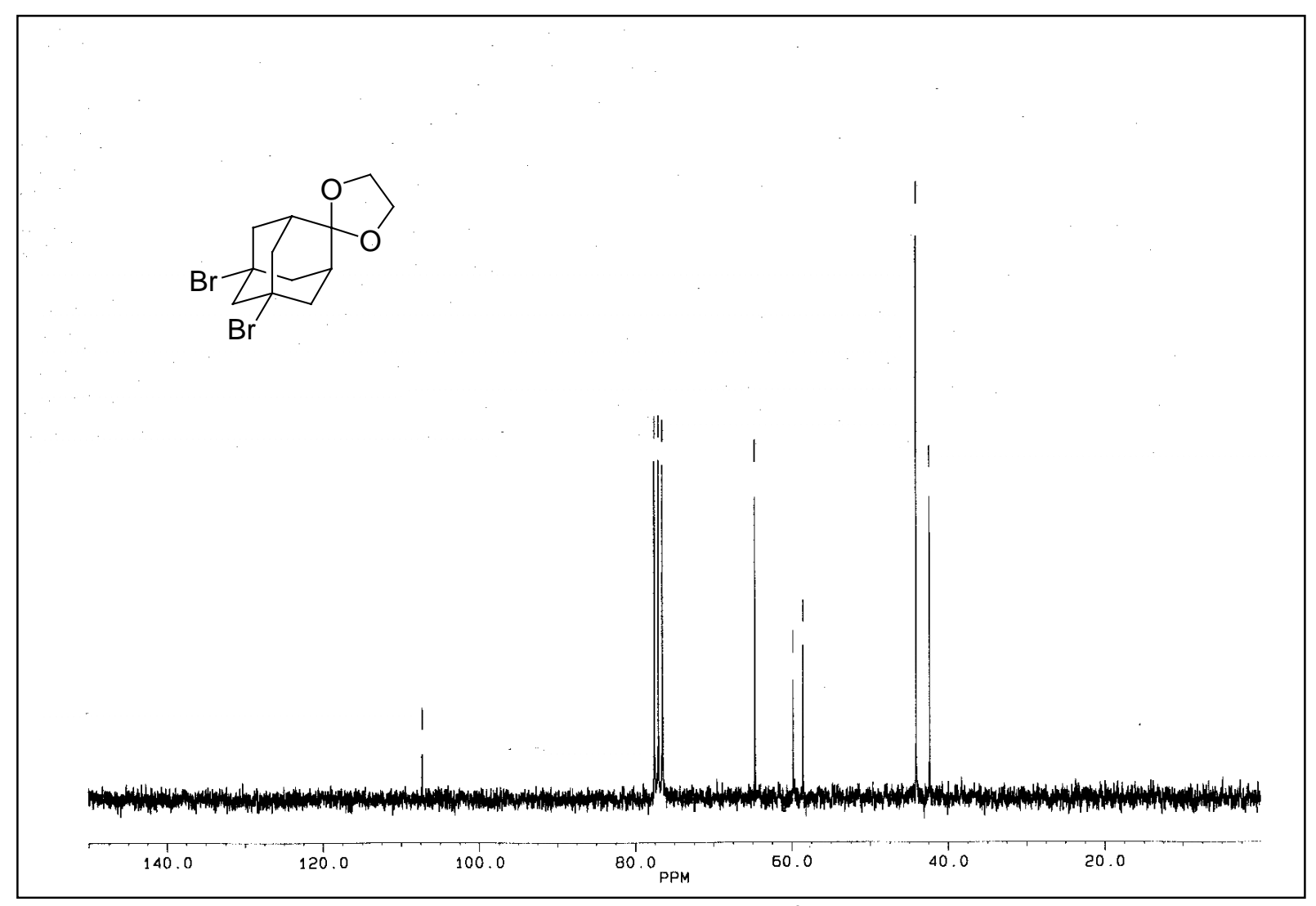

1,3-Dibrom-6-(spiro-1',4'-dioxocyclopentyl)tricyclo[3.3.1.1 $\left.1^{3,7}\right] \operatorname{decan}(\mathbf{8 3})$<smiles>IC12CC3CC(CC(C3)C13OCCO3)C2</smiles>

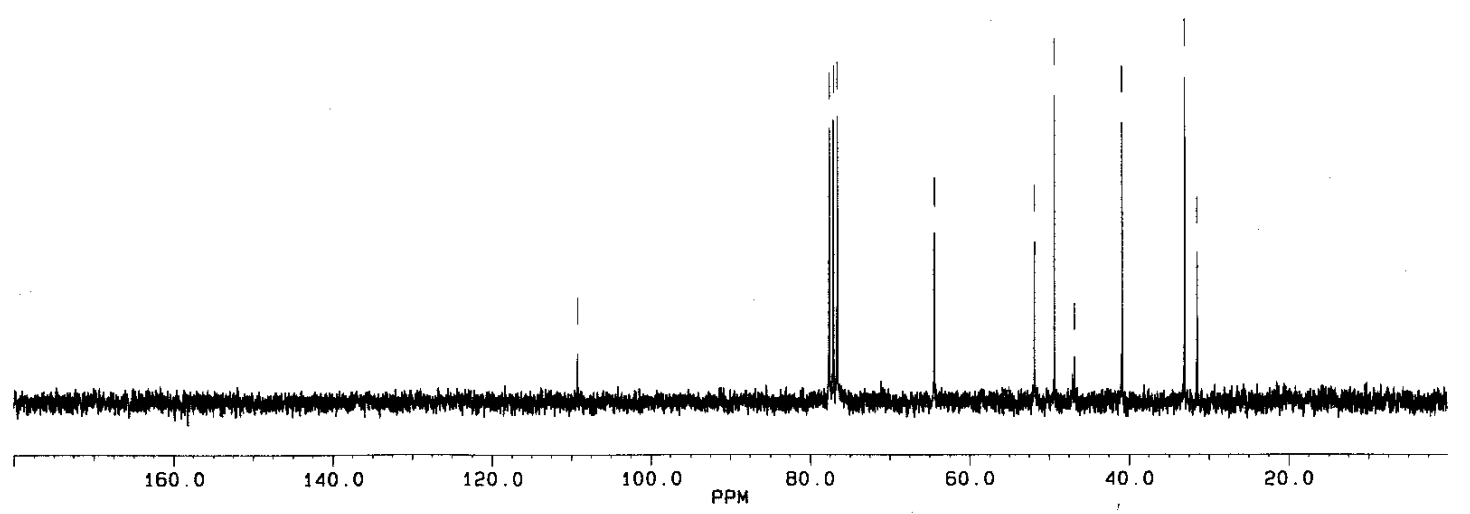

1-Iod-4-(spiro-1',4'-dioxocyclopentyl)tricyclo[3.3.1.1 $\left.1^{3,7}\right]$ decan (84) 


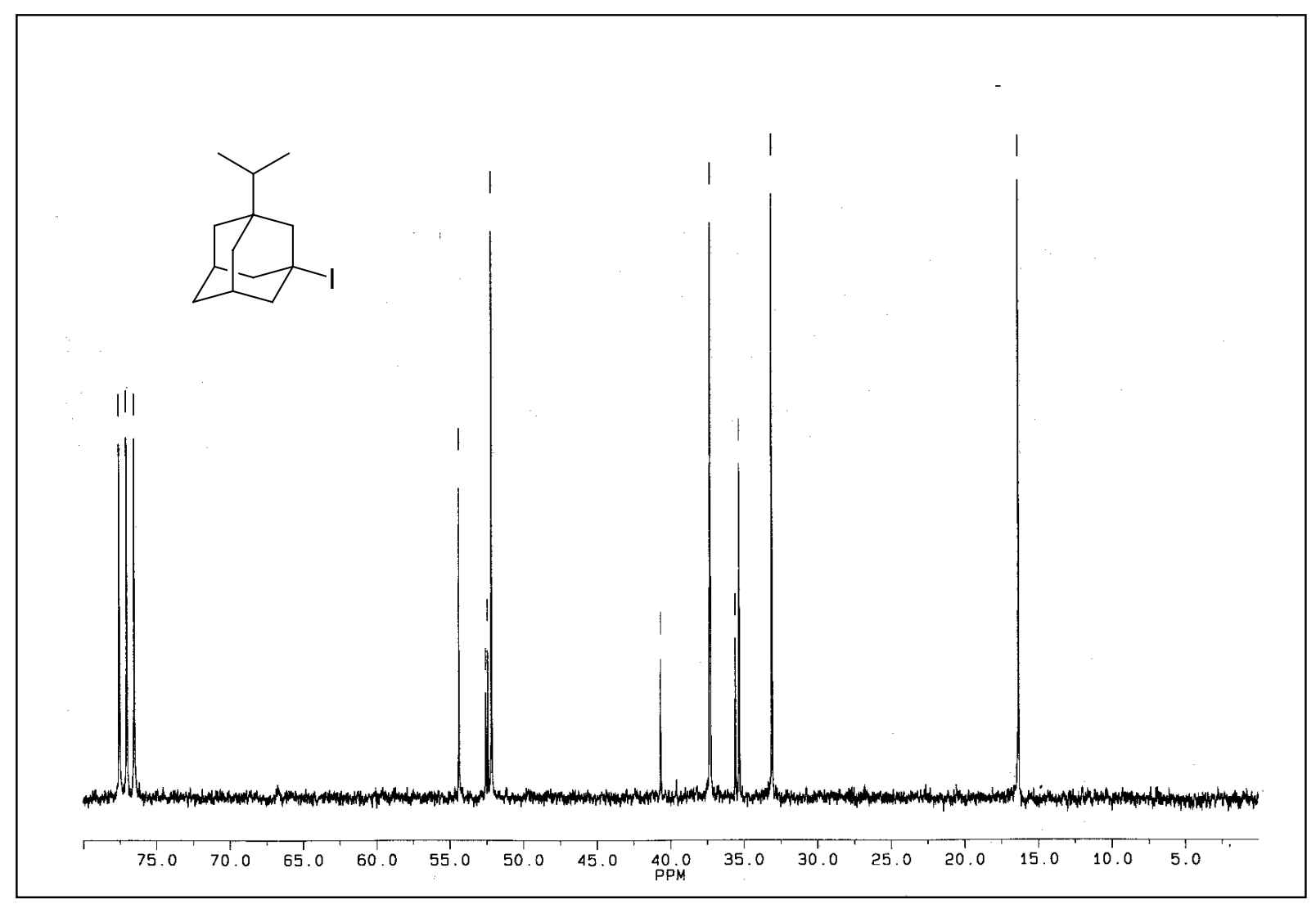

1-Iod-3-(1'-methyl)ethyltricyclo[3.3.1.1,7] decan (86)

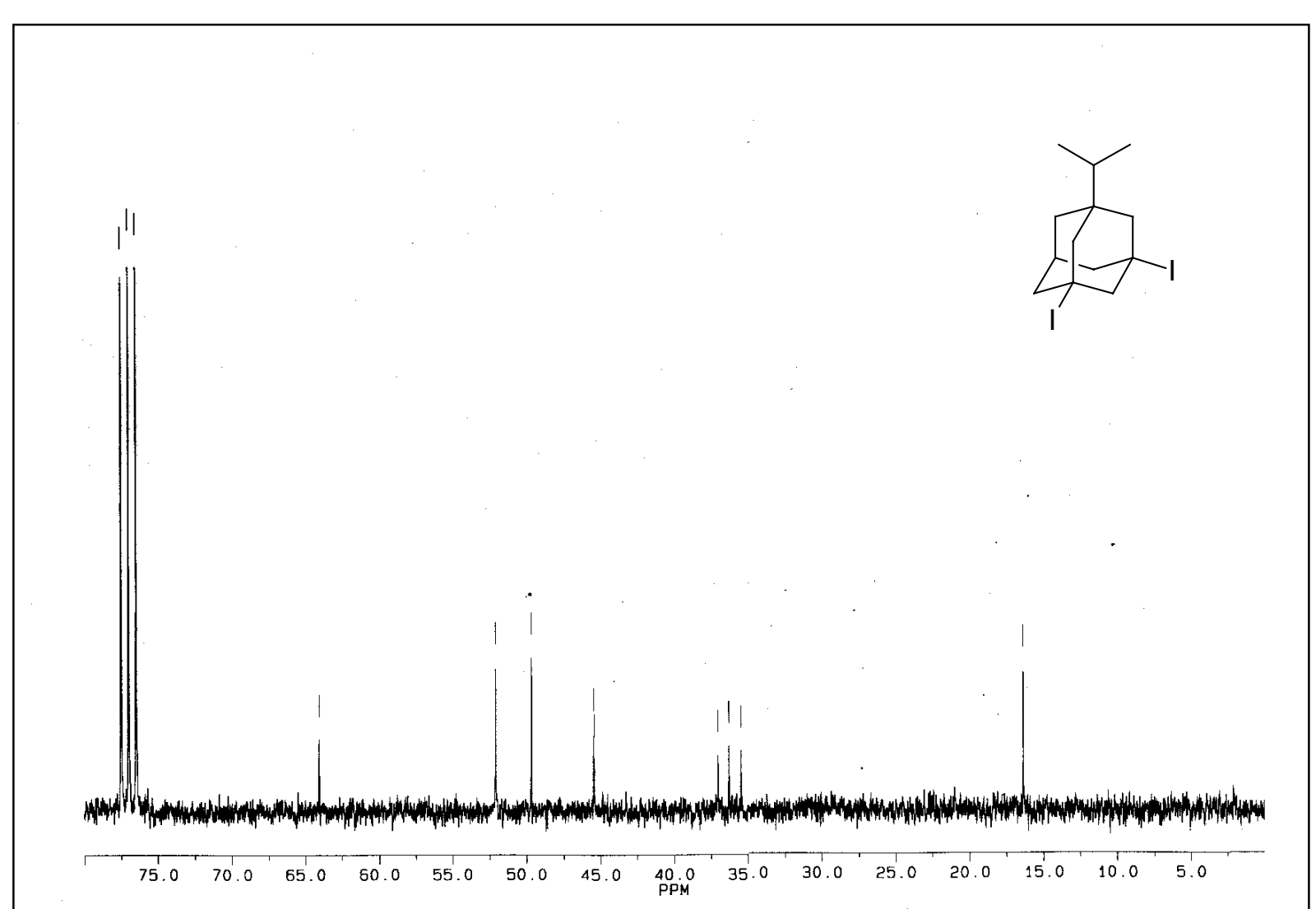

1,3-Diiod-5-(1'-methyl)ethyltricyclo[3.3.1.1 $\left.1^{3,7}\right]$ decan $(\mathbf{8 8})$ 


\section{Anhang E. Literaturverzeichnis}

[1] C. L. Hill, Activation and Functionalization of Alkanes, Wiley, New York 1989.

[2] G. A. Olah, O. Faooq, G. K. S. Prakash, Activation and Functionalization of Alkanes, Wiley, New York 1989.

[3] G. A. Olah, A. Molnár, Hydrocarbon Chemistry, Wiley, New York 1995.

[4] J. A. Davies, P. L. Watson, A. Greenburg, J. F. Liebman, Selective Hydrocarbon Activation, VCH, New York 1990.

[5] S. Murai, Topics in Organometallic Chemistry, Vol. 3, Springer, Berlin 1999.

[6] C. Rüchardt, Angew. Chem. 1970, 82, 845-858.

[7] E. S. Huyser, Free Radical Chain Reactions, Wiley-Interscience, New York 1970.

[8] M. B. Tyabin, A. E. Shilov, A. A. Shteinman, Dokl. Akad. Nauk. SSR 1971, 198, 381.

[9] R. H. Crabtree, Chem. Rev. 1995, 95, 987-1007.

[10] R. H. Crabtree, Chem. Rev. 1985, 85, 245-269.

[11] W. D. Jones, F. J. Feher, J. Am. Chem. Soc. 1984, 106, 1650-1663.

[12] H. L. Holland, Organic Synthesis with Oxidative Enzymes, VCH, Weinheim 1991.

[13] B. A. Arndtsen, R. G. Bergman, Science 1995, 270, 1970-1973.

[14] S. E. Bromberg, H. Yang, M. C. Asplund, T. Lian, B. K. McNamara, K. T. Kotz, J. S. Yeston, M. Wilkens, H. Frei, R. B. Bergman, C. B. Harris, Science 1997, 278, 260263.

[15] H. G. Davis, D. R. Kelly, R. H. Green, S. M. Roberts, Biotranformations in Preparative Organic Chemistry: The Use of isolated Enzymes and Whole-Cell Systems in Synthesis, Academic Press, Boston 1989.

[16] C. L. Hill, J. A. Smegal, T. J. Henly, J. Org. Chem. 1983, 48, 3277-3281.

[17] G. A. Olah, G. K. S. Prakash, R. E. Williams, L. D. Field, K. Wade, Hydrocarbon Chemistry, Wiley-Interscience, New York 1987.

[18] P. R. Schreiner, O. Lauenstein, P. Gunchenko, A. Wittkopp, I. V. Kolomitsyn, G. Feder, E. D. Butowa, A. A. Fokin, Chem. Eur. J. 2001, submitted for publication.

[19] J. Fossey, D. Lefort, J. Sorba, Free Radicals in Organic Chemistry, Wiley, New York 1995

[20] T. Linker, M. Schmittel, Radikale und Radikalionen in der Organischen Synthese, Wiley-VCH, Weinheim 1998.

[21] E. V. Dehmlow, S. S. Dehmlow, Phase-Transfer Catalysis, VCH, Weinheim 1993.

[22] M. E. Halpern, ACS Symposium Series 1995, 659.

[23] C. M. Starks, C. Liotta, Phase-Transfer Catalysis, Academic Press, New York 1978. 
[24] C. M. Starks, ACS Symposium Series 1985, 326.

[25] W. P. Weber, G. W. Gokel, Phase-Transfer Catalysis in Organic Synthesis, Springer, Berlin 1977.

[26] Y. Sasson, R. Neumann, Handbook of Phase-Transfer Catalysis, Blacki Academic \& Professional, London 1997.

[27] C. Starks, C. Liotta, M. Halpern, Phase-Transfer Catalysis: Fundamentals, Applications and Industrial Perspectives, Chapman \& Hall, New York 1994.

[28] D. T. Sawyer, J. L. Roberts, Acc. Chem. Res. 1988, 21, 469-476.

[29] P. R. Schreiner, O. Lauenstein, I. V. Kolomitsyn, S. Nadi, A. A. Fokin, Angew. Chem. 1998, 110, 1993-1995.

[30] P. R. Schreiner, O. Lauenstein, E. D. Butowa, A. A. Fokin, Angew. Chem. 1999, 111, 2958-2961.

[31] O. Lauenstein, Diplomarbeit, Universität Göttingen 1998.

[32] S. Patai, Z. Rappoport, The Chemistry of Alkanes and Cycloalkanes, John Wiley \& Sons, Chichester 1992.

[33] Y. M. Slobodin, L. A. Ashikanzi, G. N. Klimchuk, J. Org. Chem. (USSR) Engl. Trans. 1984, 20, 1225-1229.

[34] S.-W. Goh, K.-C. Han, T.-S. Kam, H.-L. Chong, Aust. J. Chem 1975, 28, 381-384.

[35] I. Tabushi, Z.-I. Yoshida, N. Takahashi, J. Am. Chem. Soc. 1970, 92, 6670-6672.

[36] E. V. Dehmlow, Tetrahedron 1971, 27, 4071-4075.

[37] I. Tabushi, Y. Aoyama, S. Kojo, J. Hamuro, Z.-I. Yoshida, J. Am. Chem. Soc. 1972, 94, 1177-1183

[38] F. Minisci, F. Fontana, L. Zhao, Tetrahedron Lett. 1994, 35, 8033-8036.

[39] Y. Sasson, F. Kitson, O. W. Webster, Bull. Soc. Chim. Fr. 1993, 130, 599-600.

[40] E. V. Dehmlow, M. Slopianka, Liebigs Ann. Chem. 1979, 1465-1472.

[41] E. V. Dehmlow, W. Broda, Chem. Ber. 1982, 115, 3894-3897.

[42] J. A. Orvik, J. Org. Chem. 1996, 61, 4933-4936.

[43] K. S. Root, C. L. Hill, L. M. Lawrence, G. M. Whitesides, J. Am. Chem. Soc. 1989, $111,5405-5412$

[44] C. Starks, J. Am. Chem. Soc. 1971, 93, 195-199.

[45] M. Makosza, Pure and Appl. Chem. 1975, 43, 439-462.

[46] M. Makosza, E. Bialecka, Tetrahedron Lett. 1977, 183-186.

[47] R. G. Pearson, J. Am. Chem. Soc. 1963, 85, 3533-3539.

[48] M. Rabinovitz, Y. Cohen, M. Halpern, Angew. Chem. 1986, 98, 958-968. 
[49] D. K. Bohme, G. I. Mackay, J. Am. Chem. Soc 1981, 103, 978-979.

[50] D. Landini, A. Maia, Chem. Soc., Chem. Commun. 1984, 1041-1042.

[51] J. C. Fiaud, Tetrahedron Lett. 1975, 40, 3495-3496.

[52] E. V. Dehmlow, P. S.; J. Heider, J. Chem. Research (S) 1981, 292-293.

[53] E. J. Corey, F. Xu, M. C. Noe, J. Am. Chem. Soc. 1997, 119, 12414-12415.

[54] S. Juliá, A. Ginebreda, J. Guixer, A. Tomás, Tetrahedron Lett. 1980, 21, 3709-3712.

[55] U.-H. Dolling, P. Davis, E. J. J. Grabowski, J. Am. Chem. Soc. 1984, 106, 446-447.

[56] D. L. Hughes, U.-H. Dolling, K. M. Ryan, E. F. Schoenewaldt, E. J. J. Grabowski, J. Org. Chem. 1987, 52, 4745-4752.

[57] M. J. O'Donnell, W. D. Bennett, S. Wu, J. Am. Chem. Soc. 1989, 111, 2353-2355.

[58] B. Lygo, P. G. Wainwright, Tetrahedron Lett. 1998, 39, 1599-1602.

[59] S. Arai, T. Shioiri, Tetrahedron Lett. 1998, 39, 2145-2148.

[60] E. J. Corey, M. C. Noe, F. Xu, Tetrahedron Lett. 1998, 39, 5347-5350.

[61] Y. N. Belokon, K. A. Kochetkov, T. D. Churkina, N. S. Ikonnikov, A. A. Chesnokov, O. V. Larionov, V. S. Parmár, R. Kumar, H. B. Kagan, Tetrahedron: Asymmetry 1998, 9, 851-857.

[62] J. J. Eddine, M. Cherqaoui, Tetrahedron: Asymmetry 1995, 6, 1225-1228.

[63] T. Ooi, M. Kameda, K. Maruoka, J. Am. Chem. Soc. 1999, 121, 6519-6520.

[64] M. I. Poutsma, J. A. Kochi, Free Radicals, Wiley-Interscience, New York 1973.

[65] F. Minisci, Substituent Effects in Free Radical Chemistry, Dordrecht 1986.

[66] S. Steenken, Top. Curr. Chem. 1996, 177, 126-145.

[67] N. J. Maraschin, D. B. Catsikis, L. H. Davis, G. Jarvinen, R. J. Lagow, J. Am. Chem. Soc. 1975, 97, 513-517.

[68] D. D. Tanner, H. Oumar-Mahamat, C. P. Meintzer, E. C. Tsai, T. Lu, D. Yang, J. Am. Chem. Soc. 1991, 113, 5397-5402.

[69] A. S. Dneprovskii, D. V. Kuznetsov, E. V. Eliseenkiv, B. Fletcher, J. M. Tanko, J. Org. Chem. 1998, 63, 8860-8864.

[70] B. H. Eckstein, H. A. Sheraga, E. R. van Artsdalen, J. Chem. Phys. 1954, 22, 28-35.

[71] G. C. Fettis, J. H. Knox, A. F. Trotman-Dickenson, J. Chem. Soc. 1960, 82, 41774185 .

[72] J. J. Russel, J. A. Seetula, D. J. Gutman, J. Am. Chem. Soc. 1988, 110, 3092-3099.

[73] G. S. Hammond, J. Am. Chem. Soc. 1955, 77, 334-338.

[74] T. Ito, J. H. Lunsford, Nature 1985, 314, 721-722.

[75] V. L. Lobachev, E. S. Rudakov, Kinetics and Catalysis 2000, 41, 186-195. 
[76] C. Walling, Acc. Chem. Res. 1998, 31, 135-157.

[77] S. K. Wong, J. Am. Chem. Soc. 1979, 101, 1235-1239.

[78] A. S. Dneprovskii, E. V. Eliseenkiv, D. V. Kuznetsov, Zhurn. Org. Khim. 1994, 30, 232-235.

[79] C. Walling, Pure Appl. Chem. 1967, 15, 69-80.

[80] C. Walling, V. P. Kurkov, J. Am. Chem. Soc. 1967, 89, 2455-2458.

[81] D. D. Tanner, G. C. Gidley, J. Am. Chem. Soc. 1968, 90, 808-809.

[82] J. G. Traynham, Y.-S. Lee, J. Am. Chem. Soc. 1974, 96, 3590-3594.

[83] G. Merényi, J. Lind, L. Eberson, Acta Chem. Scand. 1998, 52, 62-66.

[84] B. Fell, L.-H. Krug, Chem. Ber. 1965, 98, 2871-2886.

[85] P. R. Perkins, R. E. Pincock, Can. J. Chem. 1978, 56, 1269-1272.

[86] V. V. Smirnov, V. M. Zelikman, I. P. Beletskaya, M. M. Levitskii, M. A. Kazankovka, Mendeleev. Commun. 2000, 175-176.

[87] A. B. Shtarev, F. Tian, W. R. Dolbier Jr., B. E. Smart, J. Am. Chem. Soc. 1999, $121,7335-7341$.

[88] M. D. Bartberger, W. R. Dolbier Jr., J. Lusztyk, K. U. Ingold, Tetrahedron 1997, $53,9857-9880$.

[89] D. M. Camaioni, J. T. Bays, W. J. Shaw, J. C. Linehan, J. C. Birnbaum, J. Org. Chem. 2001, 66, 789-795.

[90] L. Liguori, H.-R. Bjørsvik, A. Bravo, R. Fontana, F. Minisci, Chem. Comm. 1997, 1501-1502.

[91] R. G. Parr, W. Yang, Density Functional Theory of Atoms and Molecules, Oxford University Press, Oxford 1989.

[92] R. G. Parr, W. Yang, Annu. Rev. Phys. Chem. 1995, 46, 701-728.

[93] P. v. R. Schleyer, N. L. Allinger, T. Clark, J. Gasteiger, P. A. Kollman, H. F. Schaefer III., P. R. Schreiner, The Encyclopedia of Computational Chemistry, John Wiley \& Sons, Chichester 1998.

[94] C. C. J. Roothan, Rev. Mod. Phys. 1951, 23, 69.

[95] W. Kohn, L. J. Sham, Phys. Rev. A 1965, 140, 864-871.

[96] A. D. Becke, Phys. Rev. A. 1988, 38, 3098.

[97] A. D. Becke, J. Chem. Phys. 1993, 98, 5648-5652.

[98] R. McWeeny, G. Dierksen, J. Chem. Phys. 1968, 49, 4852-4856.

[99] B. Miehlich, A. Savin, H. Stoll, H. Preuss, Chem. Phys. Lett. 1989, 157, 200-216.

[100] E. R. Davidson, D. Feller, Chem. Rev. 1986, 86, 681-696. 
[101] S. F. Boys, F. Bernardi, Mol. Phys. 1970, 19, 553-566.

[102] W. J. Hehre, R. F. Stewart, J. A. Pople, J. Chem. Phys. 1969, 51, 2657-2664.

[103] J. S. Binkley, J. A. Pople, W. J. Hehre, J. Am. Chem. Soc. 1980, 102, 939-947.

[104] G. A. Petersson, A. Bennett, T. G. Tensfeldt, M. A. Al-Laham, W. A. Shirley, J. Mantzaris, J. Chem. Phys. 1988, 89, 213.

[105] M. J. Frisch, J. A. Pople, J. S. Binkley, J. Chem. Phys. 1984, 80, 3265-3269.

[106] T. Clark, J. Chandrasekhar, G. W. Spitznagel, P. v. R. Schleyer, J. Comput. Chem. 1983, 4, 294-301.

[107] A. D. McLean, G. S. Chandler, J. Chem. Phys. 1980, 72, 5639-5648.

[108] M. J. Frisch, G. W. Trucks, H. B. Schlegel, G. E. Scuseria, M. A. Robb, J. R. Cheeseman, V. G. Zakrzewski, J. A. J. Montgomery, R. E. Stratmann, J. C. Burant, S. Dapprich, J. M. Millam, A. D. Daniels, K. N. Kudin, M. C. Strain, O. Farkas, J. Tomasi, V. Barone, M. Cossi, R. Cammi, B. Mennucci, C. Pomelli, C. Adamo, S. Clifford, J. Ochterski, G. A. Petersson, P. Y. Ayala, Q. Cui, K. Morokuma, D. K. Malick, A. D. Rabuck, K. Raghavachari, J. B. Foresman, J. Cioslowski, J. V. Ortiz, B. B. Stefanov, G. Liu, A. Liashenko, P. Piskorz, I. Komaromi, R. Gomperts, R. L. Martin, D. J. Fox, T. Keith, M. A. Al-Laham, C. Y. Peng, A. Nanayakkara, C. Gonzales, M. Challacombe, P. M. Gill, B. Johnson, W. Chen, M. W. Wong, J. L. Andres, C. Gonzales, M. Head-Gordon, E. S. Replogle, J. A. Pople, Gaussian98 Revision A.7, Gaussian Inc., Pittsburgh, PA 1998.

[109] I. Gracia-Cruz, M. E. Ruiz-Santoyo, J. R. Alvarez-Idaboy, A. Vivier-Bunge, J. Comp. Chem. 1999, 20, 845-856.

[110] F. Ogliaro, M. Filatov, S. Shaik, Eur. J. Inorg. Chem. 2000, 2455-2458.

[111] L. Fan, T. Ziegler, J. Am. Chem. Soc. 1992, 114, 10890-10897.

[112] I. Matheson, J. Tedder, H. Sidebottom, Int. J. Chem. Kinet. 1982, 14, 1033-1045.

[113] J. A. Hawari, S. Davis, P. S. Engel, B. C. Gilbert, D. J. Griller, J. Am. Chem. Soc. 1985, 107, 4721-4724.

[114] F. S. Fawcett, Chem. Rev. 1950, 47, 219-274.

[115] D. J. Raber, J. M. Harris, R. E. Hall, P. v. R. Schleyer, J. Am. Chem. Soc. 1971, 93, 4821-4828.

[116] D. R. Lidl, Handbook of Chemistry and Physics; 75. Ed., CRC Press, Boca Raton 1994.

[117] G. A. Olah, G. K. S. Prakash, J. Sommer, Superacids, Wiley, New York 1985.

[118] C. Aubry, J. L. Holmes, J. C. Walton, J. Phys. Chem. A 1998, 102, 1389-1393. 
[119] G. A. Kruppa, J. L. Beauchamp, J. Am. Chem. Soc. 1986, 108, 2162-2169.

[120] G. A. Olah, Cage Hydrocarbons, Wiley-Interscience, New York 1990.

[121] R. C. Fort Jr., Adamantane: The Chemistry of Diamond Molcules, Marcel Dekker Inc., New York 1976.

[122] A. G. Yurchenko, L. A. Zosim, N. L. Dovgan, N. S. Verpovsky, Tetrahedron Lett. 1976, 52, 4843-4846.

[123] O. Lauenstein, A. A. Fokin, P. R. Schreiner, Org. Lett. 2000, 2, 2201-2204.

[124] F. H. Westheimer, Chem. Rev. 1961, 61, 265-273.

[125] K. B. Wiberg, Chem. Rev. 1955, 55, 713-743.

[126] J. Bigeleisen, M. Wolfsberg, in I. Prigogine (Ed.): Advances in Chemical Physics, Interscience, New York 1958.

[127] L. Melander, W. H. Saunders Jr., Reaction Rates of Isotopic Molecules, John Wiley \& Sons, New York 1980.

[128] A. V. Willi, Isotopeneffekte bei chemischen Reaktionen, Georg Thieme Verlag, Stuttgart 1983.

[129] C. J. Collins, Phys. Org. Chem. 1964, 2, 62.

[130] C. J. Collins, H. M. Lietzke, J. Am. Chem. Soc. 1959, 81, 5379-5383.

[131] J. Bigeleisen, Pure Appl. Chem. 1964, 8, 217-232.

[132] H.-J. Bartsch, Mathematische Formeln, Buch und Zeit, Leipzig 1992.

[133] D. Landini, D. Albanese, S. Mottadelli, M. Penso, J. Chem. Soc. Perkin Trans. 1 1992, 2309-2311.

[134] M. Namavari, N. Satyamurthy, M. E. Phelps, J. R. Barrio, Tetrahedron Lett. 1990, 31, 4973-4976.

[135] T. Imamoto, T. Matsumoto, T. Kusumoto, M. Yokoyama, Synthesis 1983, 6, 460-461.

[136] H. Eyring, A. Sherman, J. Chem. Phys. 1933, 1, 435.

[137] D. H. R. Barton, D. Doller, Y. V. Geletii, Tetrahedron Lett. 1991, 32, 3811-3814.

[138] D. D. Tanner, T. Ochiai, T. Pacé, J. Am Chem. Soc. 1975, 97, 6162-6165.

[139] A. A. Fokin, O. Lauenstein, P. A. Gunchenko, P. R. Schreiner, J. Am. Chem. Soc. 2001, 123, 1842-1847.

[140] P. E. Eaton, J. Cole, T. W., J. Am. Chem. Soc. 1964, 86, 3157-3158.

[141] K. D. Kybett, S. Carroll, P. Natalis, D. W. Bonnell, J. L. Margrave, J. L. Franklin, J. Am. Chem. Soc. 1966, 88, 626.

[142] W. E. v. Doering, W. R. Roth, R. Breuckmann, L. Figge, H.-W. Lennartz, H. Prinzbach, Chem. Ber. 1988, 121, 1-9. 
[143] G. W. Griffin, A. P. Marchand, Chem. Rev. 1989, 89, 997-1010.

[144] P. E. Eaton, Angew. Chem. 1992, 104, 1447-1462.

[145] P. A. Eaton, G. Castaldi, J. Am. Chem. Soc. 1985, 107, 724-726.

[146] P. A. Eaton, H. Higuchi, R. Millikan, Tetrahedron Lett. 1987, 28, 1055-1058.

[147] P. A. Eaton, R. M. Martin, J. Org. Chem. 1988, 53, 2728-2732.

[148] A. Bashir-Hashemi, J. Am. Chem. Soc. 1988, 110, 7234-7235.

[149] T.-Y. Luh, L. M. Stock, J. Org. Chem. 1972, 37, 338-339.

[150] R. M. Moriarty, J. S. Khosrowshahi, T. M. Dalecki, Chem. Commun. 1987, 1987, 675676.

[151] P. E. Eaton, K. Pramod, T. Emrick, R. Gilardi, J. Am. Chem. Soc. 1999, 121, 41114123.

[152] J. S. Murray, T. Brinck, P. Politzer, Chem. Phys. 1996, 204, 289-299.

[153] M.-X. Zhang, P. E. Eaton, R. Gilardi, Angew. Chem. 2000, 112, 422-426.

[154] T.-Y. Luh, L. M. Stock, J. Am. Chem. Soc. 1974, 96, 3712-3713.

[155] L. Cassar, P. E. Eaton, J. Halpern, J. Am. Chem. Soc. 1970, 92, 3515-3518.

[156] G. F. Koser, Chem. Commun. 1971, 1971, 388-389.

[157] L. Cassar, P. E. Eaton, J. Halpern, J. Am. Chem. Soc. 1970, 92, 6366-6367.

[158] P. R. Schreiner, A. Wittkopp, P. A. Gunchenko, A. I. Yaroshinsky, S. A. Peleshanko, A. A. Fokin, Chem. Eur. J. 2001, accepted for publication.

[159] E. W. Della, N. J. Head, P. Mallon, J. C. Walton, J. Am. Chem. Soc. 1992, 114, 1073010738 .

[160] J. C. Walton, J. Chem. Soc. Perkin. Trans. 2 1988, 1371-1376.

[161] P. E. Eaton, G. H. Temme, J. Am. Chem. Soc. 1973, 95, 7508-7510.

[162] D. S. Reddy, M. Maggini, J. Tsanaktsidis, P. E. Eaton, Tetrahedron Lett. 1990, $31,805-806$.

[163] S. Landa, Chem. Listy 1933, 27, 415.

[164] P. v. R. Schleyer, J. Am. Chem. Soc. 1957, 79, 3292.

[165] F. D. Ayres, S. I. Khan, O. L. Chapman, Tetrahedron Lett. 1994, 35, 8561-8564.

[166] V. R. Reichert, L. J. Mathias, Macromolecules 1994, 27, 7030-7034.

[167] V. R. Reichert, L. J. Mathias, Macromolecules 1994, 27, 7024-7029.

[168] T. G. Archibald, A. A. Malik, K. Baum, M. R. Unroe, Macromolecules 1991, $24,5261-5265$.

[169] V. J. Jasys, F. Lombardo, T. A. Appleton, J. Bordner, M. Ziliox, R. A. Volkmann, J. Am. Chem Soc. 2000, 122, 466-473. 
[170] J. Kirschbaum, Analytical Profiles of Drug Substances, Vol. 12, Academic Press, New York 1983.

[171] N. Kolocouris, A. Kolocouris, G. B. Foscolos, G. Fytas, J. Neyts, E. Padalko, J. Balzarini, R. Snoeck, G. Andrei, E. De Clerq, J. Med. Chem. 1996, 39, 3307-3318.

[172] R. Raag, T. L. Poulos, Biochemistry 1991, 30, 2674-2684.

[173] J. Applequist, P. Rivers, D. E. Applequist, J. Am. Chem. Soc. 1969, 91, 5705.

[174] H. Hamill, M. A. McKervey, Chem. Commun. 1969, 864.

[175] F. N. Stepanov, V. F. Baklan, Zh. Org. Khim. Engl. Trans. 1966, 2, 1611-1612.

[176] M. Ohno, K. Shimizu, K. Ishizaki, T. Sasaki, S. Eguchi, J. Org. Chem. 1988, 53, 729733.

[177] E. Negeshi, Organometallics in Organic Synthesis, Wiley, New York 1980.

[178] K. Muraoka, M. Nojima, S. Kusabayashi, J. Chem Soc. Perkin. Trans. 2 1986, 76-79.

[179] W. Adcock, I. Clark, J. Org. Chem. 1993, 58, 7341-7349.

[180] G. A. Olah, J. T. Welch, D. V. Yashwant, M. Nojima, I. Kerekes, J. A. Olah, J. Org. Chem. 1979, 44, 3872-3882.

[181] H. Stetter, M. Schwarz, A. Hirschhorn, Chem. Ber. 1959, 92, 1629-1635.

[182] C. Allan, D. Barton, S. Sivaram, Synthesis 1979, 434-436.

[183] D. Landini, D. Albanese, S. Mottadelli, M. Penso, J. Chem. Soc. Perkin Trans. 1 1992, 2309-2311.

[184] M. Namavari, N. Satyamurthy, M. E. Phelps, J. R. Barrio, Tetrahedron Lett. 1990, 31, 4973-4976.

[185] W. N. Setzer, P. v. R. Schleyer, Adv. Organomet. Chem. 1985, 23, 353-451.

[186] G. A. Molander, C. R. Harris, Chem. Rev. 1996, 96, 307-338.

[187] M. D. Levin, S. J. Hamrock, P. Kaszynski, A. B. Shtarev, G. A. Levina, B. C. Noll, M. E. Ashley, R. Newmark, G. G. I. Moore, J. Michl, J. Am. Chem. Soc. 1997, $119,12750-12761$.

[188] T. C. Morrill, Lanthanide Shift Reagents in Stereochemical Analysis, VCH Publishers Inc., New York 1986.

[189] D. Parker, Chem. Rev. 1991, 91, 1441-1457.

[190] E. L. Eliel, N. L. Allinger, Topics in Stereochemistry 1978, 10, 288-325.

[191] J. K. Sanders, D. H. Williams, Nature 1972, 240, 385-390.

[192] T. J. Wenzel, L. J. D. R., J. Org. Chem. 1983, 48, 1951-1954.

[193] T. J. Wenzel, R. E. Sievers, J. Am. Chem. Soc. 1982, 104, 382-388.

[194] T. J. Wenzel, R. E. Sievers, Anal. Chem. 1981, 53, 393-399. 
[195] J. San Filipppo Jr., R. G. Nuzzo, L. J. Romano, J. Am. Chem. Soc. 1975, 97, 2546.

[196] W. A. König, R. Krebber, P. Mischnick, J. High Resolut. Chromatogr. 1989, 12, 732738.

[197] Y. Okamoto, E. Yashima, Angew. Chem. 1998, 110, 1072-1095.

[198] K. Lorenz, E. Yashima, Y. Okamoto, Angew. Chem. 1998, 110, 2025-2028.

[199] S. Chalais, A. Cornélis, A. Gerstmans, W. Kolodziejski, P. Laszlo, A. Mathy, P. Métra, Helv. Chim. Acta. 1985, 68, 1196-1203.

[200] W. Fischer, C. A. Grob, H. Katayama, Helv. Chim. Acta. 1976, 59, 1953-1962.

[201] M. Xie, W. J. le Noble, J. Org. Chem. 1988, 54, 3836-3839. 


\section{Abkürzungen und Akronyme}

$\AA$

AIBN

äq.

AV

ax.

B3LYP

BSSE

$\mathrm{Bz}$

DBP

DBPO

DFT

E

ESP

Et

et al.

eq.

$\mathrm{G}$

GC/MS

GZ

$\mathrm{H}$

$\mathrm{h}$

$\mathrm{HF}$

HOMO

$\mathrm{Hz}$

$i$

IR

J

K

Kat.

KIE

L

LSR

LUMO

m

$\mathrm{Me}$

MO

NBO

NMR

$o$

$p$

$\mathrm{Ph}$

PPSE

PT

PTK

RT

S

Schmp.

Sdp.

sek.
Ångstrom

Azobisisobutyronitil

äquartorial

Arbeitsvorschrift

axial

Becke-3-Lee-Young-Parr

Basissatzsuperpositionsfehler

Benzyl

Di- $t$-Butylperoxid

Dibenzoylperoxid

Dichtefunktionaltheorie

Energie

elektrostatisches Potential

Ethyl

und andere

Äquivalent

Gibbs'che Energie

Gaschromatographie/Massenspektroskopie

Grundzustand

Enthalpie

Stunde

Hartree Fock

Höchstes besetztes Molekülorbital

Hertz

iso

Infrarot

Joule

Kelvin

Katalysator

Kinetsicher Isotopeneffekt

Liter

Lanthanid-Verschiebungs Reagenz

Niedrigstes nicht besetztes Molekülorbital

meta

Methyl

Molekülorbital

Natürliche Bindungsordnung

Kernmagnetische Resonanz

ortho

para

Phenyl

Polyphophasiloxyether

Phasentransfer

Phasentransferkatalysator

Raumtemperatur

Entropie

Schmelzpunkt

Siedepunkt

sekundär 


$\begin{array}{ll}\text { SET } & \text { Single-Electron-Transfer } \\ \text { SOMO } & \text { Einfach besetztes Molekülorbital } \\ \text { SP } & \text { Single Point } \\ \text { T } & \text { Temperatur } \\ \text { TBABr } & \text { Tetra- } n \text {-butylammoniumbromid } \\ \text { TEABr } & \text { Tetraethylammoniumbromid } \\ \text { TEBABr } & \text { Triethylbenzylammoniumbromid } \\ \text { tert. } & \text { tertiär } \\ \text { TOctABr } & \text { Tetra- } n \text {-octylammoniumbromid } \\ \text { UV } & \text { Ultraviolett } \\ \ddot{U Z Z} & \text { Übergangszustand } \\ \text { vs. } & \text { gegen } \\ \text { X } & \text { Halogen } \\ \text { ZPVE } & \text { Nullpunktsenergie } \\ 1^{\circ} & \text { primär } \\ 2^{\circ} & \text { sekundär } \\ 3^{\circ} & \text { tertiär }\end{array}$




\section{Publikationsliste}

1. Halogenation of Cubane under Phase-Tranfer Conditions: Single and Double C-H-Bond Substitution with Conservation of the Cage Structure. Andrey A. Fokin, Oliver Lauenstein, Pavel Gunchenko, and Peter R. Schreiner* J. Am. Chem. Soc. 2001, 123, 1842-1847.

2. Kinetic Isotope Effects for the C-H Activation Step in the Phase-Transfer Halogenation of Alkanes. Oliver Lauenstein, Andrey A. Fokin, and Peter R. Schreiner* Org. Lett. 2000, 2, 2201-2204.

3. The First Efficient Iodination of Unactivated, Aliphatic Hydrocarbons Peter R. Schreiner,* Oliver Lauenstein, Ekatarina D. Butova, and Andrey A. Fokin* Angew. Chem. 1999, 111 2956-2958. Angew. Chem. Int. Ed. Engl. 1999, 38, 2786-2788.

Coverpicture: Angewandte Chemie, 1999, Ausgabe 18.

Chemical and Engineering News 1999 September, 20, 44.

Süddeutsche Zeitung 1999, 55, Oktober, 12, S. V2/12.

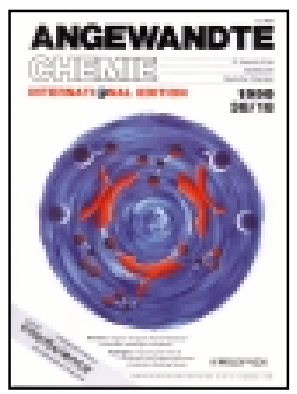

4. Deutsches Patent: P. R. Schreiner, O. Lauenstein, E. D. Butova, A. A. Fokin, 2000 Nr. 19844865.1-42

5. Selective $\mathrm{C}-\mathrm{H}$ bond Activation of Aliphatic Hydrocarbons under Phase-Transfer Conditions. Peter R. Schreiner,* Oliver Lauenstein, Igor V. Kolomitsyn, Suad Nadi, and Andrey A. Fokin Angew. Chem. 1998, 110, 1993-1995. bzw. Angew. Chem. Int. Ed. Engl. 1998, 37, 1895-1897.

Nachr. Chem. Tech. Lab. 1998, 7/8, 706.

Chemical and Engineering News 1998 July, 13, 55.

6. Concept Paper: Phase-Transfer Halogenations of Alkanes Peter R. Schreiner, ${ }^{*}$ Oliver Lauenstein, Pavel Gunchenko, Alexander Wittkopp, Gerald Feder, Ekatarina D. Butowa, Igor V. Kolomitsyn, Andrey A. Fokin, Chem. Eur. J. 2001 Zur Veröffentlichung eingereicht

7. The Direct Iodination of Unactivated $C-H$ Bonds under Phase-Transfer Conditions: Iodination of Cyclohexane and Adamantane Oliver Lauenstein, Andrey A. Fokin, Boryslav A. Tkachenko, Peter R. Schreiner* Organic Synthesis 2001 Zur Veröffentlichung eingereicht 


\section{Danksagungen}

Für den wissenschaftlichen Unterricht danke ich meinen akademischen Lehrern, den Herrn Professoren (in alphabetischer Reihenfolge) U. Beifuss, R. Brückner, M. Buback, F. Edelmann, U. Groth, K.-H. Hoyermann, G. F. Kahl, U. Klingelbiel, H. Laatsch, K. Luther, A. de Meijere, A. Meller, E. Pushmann, H. W. Roesky, P. R. Schreiner, E. Schwarzmann, P. Susse, L.-F. Tietze, J. Troe, W. G. Wagner und A. Zeeck.

Für die Aufnahme von NMR-Spektren und die Hilfestellung bei diversen diesbezüglichen Problemstellungen danke ich Dipl.-Chem. R. Machinek. Für die Aufnahme der Massenspektren danke ich Herrn Dr. G. Remberg und für die Durchführungen der Elementaranalysen Herrn. F. Hambloch sowie Fr. E. Pfeil für die Anfertigung der UVSpektren. Ferner möchte ich mich ganz besonders bei Herrn O. Senge bedanken, der mir bei diversen chromatographischen Trennproblemen immer mit Rat und Tat zur Seite stand. Großer Dank gilt den Herrn Professoren W. A. König (Universität Hamburg) und Y. Okamoto (Universität von Nagoya/ Japan) für die andauernde Kooperation bezüglich der Racemattrennungen (GC, HPLC) der verschiedenen chiralen Adamantanderivate.

Meinen Mitarbeitern danke ich für angenehme Arbeitsatmosphäre, und dabei ganz besonders Herrn M. Prall nicht nur für das Korrekturlesen der vorliegenden Arbeit.

Nicht zuletzt gilt mein Dank Herrn Prof. A. de Meijere für die fortwährende Unterstützung.

Mein größter Dank gilt Herrn Prof. Dr. A. A. Fokin (Kiev Polytechnic Institute) für die immerwährende Unterstützung und die zahlreichen, vehement geführten aber immer fruchtbaren Diskussionen, sowie die allgemeine Einführung in die ukrainischen Geheimnisse der chemischen Improvisation. 


\section{Lebenslauf}

Geboren am 31.08.1968 als Sohn von Rüdiger Lauenstein (Kaufmann) und Barbara Lauenstein (Krankenschwester) geb. Tischer in Kassel

1974

1978

1984

1987

1987-1988

1988

1989

1994

1997 bis 1998

1998

1998 bis 2001

1999

2000

2001
Besuch der Grundschule Landwehrhagen

Besuch der Mittelstufe an der Wilhelm-Leuschner-Schule (integrierte Gesamtschule) in Niestetal

Besuch der Gymnasialen Oberstufe an der Herder-Schule in Kassel Erlangung der Allgemeinen Hochschulreife

Ableistung des Grundwehrdienstes

Beginn des Studiums Informatik (Diplom) an der Universität Kaiserslautern

Beginn des Studiums Chemie (Diplom) an der Universität Göttingen

Diplomvorprüfung für Chemie mit Gut bestanden

Diplomarbeit bei Prof. PhD. Dr. Peter R. Schreiner am Institut für Organische Chemie der Universität Göttingen zum Thema: Eine neue, selektive Methode zur Aktivierung von aliphatischen Kohlenwasserstoffen: Bromierungen und Iodierungen in Mehrphasensystemen.

Diplomprüfung für Chemie mit Sehr Gut bestanden

Dissertation im AK von Prof. PhD. Dr. Peter R. Schreiner am Institut

für Organische Chemie der Universität Göttingen zum Thema: Selektive

Halogenierungen unter Phasentransferbedingungen: Mechanistische

Untersuchungen und Synthetische Anwendungen.

Teilnahme an der Winter School on Organic Reactivity (WISOR VIII)

Brixen/ Italien

Viermonatiger Forschungsaufenthalt an der University of Georgia / USA; Teilnahme am ACA Summer Course for Crystallography und 2. Platz bei der Procter \& Gamble Poster Competition 2000 Promotionsprüfung für Chemie 